\title{
ANTHROPOLOGY OF COLOR
}

EDITED BY

ROBERT E. MACLAURY

GALINA V. PARAMEI

DON DEDRICK 
Anthropology of Color 



\section{Anthropology of Color Interdisciplinary multilevel modeling}

Edited by

Robert E. MacLaury

University of Pennsylvania

\section{Galina V. Paramei}

Technische Universität Darmstadt

\section{Don Dedrick}

University of Guelph

John Benjamins Publishing Company 
The paper used in this publication meets the minimum requirements of American National Standard for Information Sciences - Permanence of Paper for Printed Library Materials, ANSI Z39.48-1984.

\section{Library of Congress Cataloging-in-Publication Data}

Anthropology of color : interdisciplinary multilevel modeling / edited by Robert E. MacLaury, Galina V. Paramei and Don Dedrick.

p. $\quad \mathrm{cm}$.

Includes bibliographical references and index.

1. Color--Terminology. 2. Colors, Words for. 3. Semiotics. I. MacLaury, Robert E., 1944- II. Paramei, Galina V. III. Dedrick, Don.

P305.19.C64A58 2007

306.44--dc22

2007026159

ISBN 9789027232434 (Hb; alk. paper)

ISBN 9789027291707 (Eb)

An electronic version of this book is freely available, thanks to the support of libraries working with Knowledge Unlatched. KU is a collaborative initiative designed to make high quality books Open Access for the public good.

The Open Access IsBN for this book is 9789027291707.

(C) 2007 - John Benjamins B.V.

This e-book is licensed under a Creative Commons CC BY-NC-ND license. To view a copy of this license, visit https://creativecommons.org/licenses/by-nc-nd/4.o/. For any use beyond this license, please contact the publisher.

John Benjamins Publishing Company · https://benjamins.com 


\section{Table of contents}

Foreword

Luisa Maffi

Color naming research in its many forms and guises

Don Dedrick and Galina V. Paramei

Full color illustrations

XVII

\section{Color perception}

Hue categorization and color naming: Cognition to language to culture

Marc H. Bornstein

Individual and population differences in focal colors

Michael A. Webster and Paul Kay

Russian color names: Mapping into a perceptual color space

Olga V. Safuanova and Nina N. Korzh

Russian 'blues': Controversies of basicness

Galina V. Paramei

Color term research of Hugo Magnus

107

Roger Schöntag and Barbara Schäfer-Prieß

\section{Color cognition}

Categories of desaturated-complex color: Sensorial, perceptual, and cognitive models

Robert E. MacLaury

Relative basicness of color terms: Modeling and measurement

Seija Kerttula

The ambiguity of brightness (with special reference to Old English)

and a new model for color description in semantics

Carole P. Biggam

Color naming in Estonian and cognate languages

Vilja Oja

Color terms in ancient Egyptian and Coptic

Wolfgang Schenkel

Basic color term evolution in light of ancient evidence from the Near East 
Basic color terms from Proto-Semitic to Old Ethiopic

Maria Bulakh

Towards a history and typology of color categorization in colloquial Arabic

Alexander Borg

Japanese color terms, from 400 CE to the present: Literature, orthography,

and language contact in light of current cognitive theory

James M. Stanlaw

Color terms in Colonia Tovar, an Alemannisch Enclave in Venezuela

Albert C. Heinrich

Mien (Yao) color terms

Theraphan L-Thongkum

\section{Color semiosis}

The semiosis of Swedish car color names: Descriptive and amplifying functions

Gunnar Bergh

Colors and emotions in English

Anders Steinvall

Linguistic construal of colors: The case of Russian

Ekaterina V. Rakhilina

Color words in painting descriptions: Some linguistic evidence

for entity-like conceptualization

Alena V. Anishchanka

Metaphors as cognitive models in Halkomelem color adjectives

Brent D. Galloway

Prototypical and stereotypical color in Slavic languages: Models based on folklore Lyudmila Popovic

Color terms in fashion

Dessislava Stoeva-Holm

To have color and to have no color: The coloring of the face in the Czech

linguistic picture of the world

Irena Vaňková

Gender, age, and descriptive color terminology in some Caucasus cultures

Liudmila V. Samarina

Towards a new topology of color

Barbara Saunders

Index 


\title{
Foreword
}

\section{Dedication to Robert E. MacLaury}

\author{
Luisa Maffi \\ Terralingua, Salt Spring Island, BC, Canada
}

I first heard about the making of Anthropology of Color when Rob MacLaury contacted me in the fall of 2003 to explore my interest in contributing to this book, which had its roots in a session by the same name held in 2002 at the 7th Biennial Conference of the European Association of Social Anthropologists in Copenhagen. Rob and I had met at the University of California, Berkeley, in 1986, where I was starting my doctoral program in anthropology and he was finishing his. We shared an interest in color categorization and naming and had the same mentors, Brent Berlin and Paul Kay. Since then, Rob and I remained in contact over time about color categorization matters, and I followed with admiration his intense dedication to the topic, as witnessed by the steady flow of his publications on the matter, and the growth of his knowledge of this topic to truly encyclopedic proportions.

Although in 2003 I was unable to accept Rob's invitation to contribute to Anthropology of Color, I once again admired Rob's unstinting pursuit of the development of a comprehensive, solidly grounded, and integrated field of color anthropology. I didn't know then that this volume would turn out to be his last effort in this pursuit. Rob died in early 2004, leaving a void that his co-editor Galina Paramei has valiantly undertaken to fill with the generous collaboration of Don Dedrick.

It is thus a strange and moving irony that Galina and Don, unaware of my earlier contact with Rob about this book, have now asked me to write the foreword for it. It is hard not to do so without turning this piece into a eulogy for Rob. Yet it must be said at the outset that Anthropology of Color is a truly collaborative work, and one of a rare kind: one that seeks to make available for the first time in the English language a body of scholarship on this topic produced in Eastern Europe and Russia that has so far remained isolated from the developments in this field in Western Europe and North America. Bridging the gap between these two traditions of work is a goal very much in the spirit of Rob MacLaury, whose enthusiasm for color categorization knew no borders, and whose encyclopedic knowledge of the literature in this field was constantly nourished by the discovery of new, untapped references. Galina Paramei, whose own professional trajectory brought her from Eastern to Western Europe, has been highly instrumental in support- 
ing this bridging effort for the purposes of this book. The chapters included herein were contributed by authors from Eastern, Central, and Northern Europe, North America, the Middle East, and Asia.

The collaborative nature of Anthropology of Color is also evident in the effort that the editors put into integrating work by authors from anthropology, linguistics, psychology, semiotics, and a variety of other fields, around the central issue of modeling. The field of color categorization has always been intrinsically multi- and inter-disciplinary, since its beginnings in the nineteenth century. The main contribution of this book, however, is to foster a new level of integration among different approaches to the anthropological study of color. The universalist evolutionary tradition of color categorization research spawned by Brent Berlin and Paul Kay's Basic Color Terms (1969) is grounded in color perception. Rob MacLaury's own research, culminating in his magnum opus Color and Cognition in Mesoamerica (1997), brought to the fore a focus on the dynamics of color cognition, on which basis he elaborated a full-fledged cognitive theory he named Vantage Theory. (See his 2002 guest-edited issue of Language Sciences, "Vantage Theory: Applications in Linguistic Cognition"; see also a website devoted to Vantage Theory that has been created by Adam Glaz 2005.) The main tenet of Vantage Theory is that categories (whether of color or other) are constructed not simply on the basis of perception, but in addition on the basis of a process of cognitive engagement with fixed and mobile coordinates that produces different vantages or points of view (analogous to the way people track their physical position in space). By its nature, Vantage Theory is also open not only to the universals of color categorization, but also to its specifics grounded in particular languages, as well as to semiotic aspects such as color connotation, metaphor, and symbolism, color in discourse and cultural practice, and so forth. The latter topics had so far been pursued separately in color ethnography and semiotics. In this edited collection MacLaury, Paramei, and Dedrick have endeavored to bring together these distinct approaches by promoting the exploration of the different but interacting and complementary ways in which these various perspectives model the domain of color experience. By so doing, they significantly promote the emergence of a coherent field of the anthropology of color.

Nobody can tell where Rob MacLaury's inquisitive mind would have led him in years to come in his passionate quest of an anthropology of color. But this collection is certainly a testament to what he had set out to accomplish. Galina Paramei and Don Dedrick are to be commended for seeing it through and making it available to an interdisciplinary and international public that will no doubt benefit from it and further advance this complex and fascinating field.

\section{References}

Berlin, Brent \& Paul Kay (1969). Basic color terms. Berkeley: University of California Press.

Glaz, Adam (2005). Vantage theory and linguistics. A website dedicated to linguistic applications of Robert E. MacLaury's Vantage Theory. http://klio.umcs.lublin.pl/ adglaz/vt.htm. 
MacLaury, Robert E. (1997). Color and cognition in Mesoamerica: Constructing categories as vantages. Austin: University of Texas Press.

MacLaury, Robert E. (Ed.) (2002). Vantage theory: Applications in linguistic cognition [Special issue]. Language Sciences, 24 (5-6). 



\title{
Color naming research in its many forms and guises
}

\author{
Don Dedrick and Galina V. Paramei
}

\section{The history of this volume}

This idea for this book originated in a conference workshop, "Anthropology of colour: Colour as a phenomenon of culture" at the 7th Biennial Conference of the European Association of Social Anthropologists in August, 2002. The workshop was initiated and organized by Liudmila Samarina and Galina Yavorska (the latter, unfortunately, was unable to participate) and carried out with the very active support of Robert MacLaury and Galina Paramei. There were seven one-hour talks and a very fruitful and interdisciplinary discussion ensued. As a result, Robert MacLaury suggested a book which would include contributions from various perspectives, levels of analysis, and disciplines (anthropology, linguistics, psychology, semantics, culture studies). This book, as MacLaury saw it, would go well beyond the modest list of original conference speakers. And this work has grown, as the reader can see from the table of contents, to a large, comprehensive presentation of contemporary color naming research from a variety of disciplines.

To the great sadness of all the contributors to this volume, Robert MacLaury died before the volume could be published. We hope his spirit lingers in this book, nonetheless, and we dedicate this book to Rob, a fine social scientist who devoted a good and great part of his life to the complex topic explored here.

\section{Introductory remarks}

The interdisciplinary or multidisciplinary nature of the investigation into color naming has always been its great attraction. Here is a field of inquiry that requires psychophysicists and physiologists, cognitive psychologists and linguists, ethnographers and ethnoscientists, computer scientists and philosophers and neuropsychologists and ophthalmologists and literary scholars to take each other seriously, on pain of mistake or irrelevance. This has been - and still is - accomplished with greater or lesser success. It is hard enough to understand what is happening in one's own discipline, without trying to figure out the nuances - the "spin," as the politicos would have it - of other epistemic practices. And yet 
some accommodations have been made. C. L. Hardin, a well known philosopher who has written extensively about color, was probably the first to bring together practitioners from a variety of disciplines, and to get them to talk to one another. Since that landmark event, at Asilomar in California in 1993 (see Hardin and Maffi 1997), a number of other excellent conferences and workshops and special journal editions and books ${ }^{1}$ have come into existence. What seems clear to us, at least, is that the investigation of color naming, in an interdisciplinary context, is a rather good model for how to do cognitive science. We - and here we include all those who take the interdisciplinary project seriously - are involved in exactly the sort of inquiry that any serious study of cognition needs to engage. What is the basic science that is involved? How do individuals and groups within and across different cultures respond to controlled stimuli? What is the relevant physiology like? Can we design cross-cultural (and cross-species) investigations that bear on what humans do, both experimentally and "on the ground?" How do human babies categorize? What is going on in the brain when humans categorize? What are the specifically cultural details we need to account for, and which details can be ignored? (an extremely difficult question!) How much of the basic (and other) science actually matters, and why? What are the culturally specific semantics of a color vocabulary like? This list could be made longer. Indeed, such a list is itself a controversial specification in an interdiscipline where there is no full agreement as to what "matters" (see Barbara Saunders' comments along this line, in the final chapter of this book, Ch. 26). That said, some things clearly do matter, and this book is about many of those things.

When Robert MacLaury and Galina Paramei conceived of the Anthropology of Color, they were interested in exposing an English speaking audience to a rich tradition in Russia and more generally Eastern Europe that is concerned with color naming. Our book serves this need admirably, as the reader can tell, just from looking at the articles and authors in all parts of this volume. But the Anthropology of Color goes beyond the simple need for a consideration of more Eastern European input to the debate. It addresses fundamental issues that are at stake in the color naming literature. Thus we find important papers by historically significant participants in the debate. Marc Bornstein's canonical work on infant color psychophysics (Bornstein, Kessen and Weiskopf 1976) has been used to provide a baseline justification for a physiological account of color naming. Bornstein starts us off, here, with a survey of the perceptualist account of color naming (though not only this, as Bornstein is sensitive to more "cultural" issues), and there is related work, updating the cross-cultural tradition, by Michael Webster and Paul Kay. Kay is the most significant single figure in the ongoing discussion about color naming, so it is good to have an upto-date account of his views on offer. It is also worth pointing out that Bornstein's paper and Kay's move in somewhat different directions. Bornstein argues for the possibility of very strong perceptual constraints on color categorization, while Kay and Webster point out that we do not have a good understanding of the relationship between individual data

1. Two recent special issues concerned with color categorization: The Journal of Cognition and Culture, 5 (3-4), 2005; Cross-Cultural Research, 39 (1-2), 2005. See also Progress in Colour Studies, Volume I, Eds. C. P. Biggam and C. J. Kay; Volume II, Eds. N. J. Pitchford and C. P. Biggam. John Benjamins, 2006. 
and data that is normalized across speakers and languages. This does not contradict the perceptualist position - as Kay argues - yet it does leave open a rather striking question: given there is significant individual difference, how is such difference ameliorated to the larger and more uniform cross-cultural scheme?

Bornstein's and Webster and Kay's papers can be found in Part I of this book, a section concerned with perceptual modeling. Other researchers represented in this section provide a variety of more or less specific work, mapping perceptual data into color naming practices. Galina Paramei extends and enriches our understanding of the "two blues" one finds in Russian - an issue of long-standing interest to color naming research (Berlin and Kay mentioned the possibility of two blues for Russian in the first edition of Basic Color Terms 1969). Safuanova and Korzh, assuming a perceptual basis for color naming, provide a perception-to-naming map for Russian, and a comparison to American and British English speakers, while Schäfer-Prieß and Schöntag help us to understand the rich history of color naming research, with special attention to the work of the German ophthalmologist Hugo Magnus.

Part II begins, fittingly, with a paper by Robert MacLaury. "Fittingly" because MacLaury has argued, for years, that one needs an account of the cognitive dimension of color naming (e.g. MacLaury 1997; Dedrick has made a similar argument, Dedrick 1998). The core idea here is that color categorizers are processing information, and that a story that explains how they map their perceptual experience into their naming practices is a desideratum for an adequate, ultimate account of color naming. Some applications of MacLaury's Vantage Theory of categorization are presented here and they are used to help us understand and explain individual differences in color categorization, a concern that came to preoccupy MacLaury. The other authors represented in Part II (Kerttula, Biggam, Oja, Schenkel, Warburton, Bulakh, Borg, Stanlaw, Heinrich, L-Thongkum) cover an amazing amount of ground, in terms of both their theoretical interests and in terms of actual geography - we find studies of languages from Japan, Venezuela, Egypt and other Middle-East countries, England, Finland, Estonia, and Thailand. These are not, one needs to emphasize, simple descriptive studies. To cite two examples here: James Stanlaw writes about his anthropological understanding of Japanese color terms in light of current "cognitive theory," and Carole Biggam is interested not just in a concordance of color names, but an integration of her linguistic evidence with the theoretical literature concerned with brightness classification.

Part III contains research in a much neglected realm: that of color semiotics or, more broadly, color term meaning. One criticism of the perceptual modeling tradition is that it often abstracts color words from their contexts (again, the reader is directed to the chapter by Saunders) a procedure which, as a psychologist might say, affects the "ecological validity" of the research. This is an issue for anthropologists as well. What would an anthropology of color be like without detailed descriptions of color vocabularies and their cultural and societal functions? In Part III we have presented a range of semiotically important research, that covers topics such as color and metaphor (Galloway), the linguistic construal of color (Rakhilina), color in artist's discourse (Anishchanka) and in fashion (Stoeva-Holm), color language and emotion (Steinvall), face coloring and cultural con- 
notations (Vaňková) as well as gender and age (Samarina), color terms for marketing cars (Bergh), and in folklore (Popovic). This list is somewhat misleading. The authors are not just writing about the cognitive-cultural function of color terms within a language, English, say. They are writing about their specific cognitive-cultural concerns in the context of a specific language. As with the preceding part of this book, Part II, a range of cultural groups and languages are discussed (German, Czech, Caucasus, Slavic, Halkomelem North West Coast (Canada) - even English!). The papers presented here are important, partly because they help to fill the aforementioned lacuna in the interdisciplinary research tradition, and partly because they address a concern that is of great interest in its own right: what are the different ways and roles in which color terms function in specific languages and cultures?

We hope that the readers of this volume will come away with two messages from the text as a whole. First, it is important not to forget that color words are culturally encoded and embodied. Parts II and III of this book should make that clear. Second, we must not forget that there is a more abstract science of color and of color naming that needs to be considered and integrated into a larger whole. Rob MacLaury, a cognitive anthropologist, conceived of this book in that light. We think it is fair to say that MacLaury - and there is no question he had the best grasp of this detailed interdisciplinary literature - never thought that abstract science could trump the cultural detail, or vice-versa. He thought, as anyone with a serious interest in color naming is bound to think, that the broad range of sciences (of the mind, of the brain, of language, culture, and cognition) are all grist for this mill. While we do not know how this interdiscipline is to be resolved, we are confident that its interdisciplinary project is worthwhile, and that this volume, Anthropology of Color, is a milestone on an uncertain, challenging road.

Luisa Maffi introduced this book with a remembrance of Robert E. MacLaury. We would like to close this introduction with a quotation from MacLaury's major work, Color and Cognition in Mesoamerica (1997):

Modeling categories as points of view incorporates a commitment to what categorization is and to what it is not. It is a process that a person undertakes, maintains, and changes in order to comprehend the world. It is a way of organizing what one senses by continuously projecting oneself. It is a method consisting of specific procedures. It wholly depends on human agency. It is not a metaphysical container, nor is it a neural reflex that deserves a name; it has no existence apart from the person who produces it on the basis of an edited selection of external reality. The selections are not taken from a boundless store of equally related possibilities; rather, they are limited by the organs of perception and motivated by social and physical environments, such as those that are easy to live in versus others that demand close attention to difficulties and unpredictable events.

(MacLaury 1997:393) 


\section{Acknowledgments}

Galina V. Paramei gratefully acknowledges the support of the Hanse-Wissenschaftskolleg, Delmenhorst, Germany, whose Fellow she was during a decisive part of the preparation of the book manuscript.

Don Dedrick would like to acknowledge the help of two research assistants, Michal Arciszewski, M. A., and, especially, Alan Belk, Ph.D. Alan did much of the manuscript formatting, prior to its submission to John Benjamins. Dedrick also acknowledges the support of the Social Sciences and Humanities Research Council, Canada, Grant \# 4102005-0148.

\section{References}

Berlin, B. \& Kay, P. (1969). Basic color terms: Their universality and evolution. Berkeley: University of California Press.

Bornstein, M. H., Kessen, W. \& Weiskopf, S. (1976). Color vision and hue categorization in young infants. Journal of Experimental Psychology: Human Perception and Performance, 1: 115-129.

Dedrick, D. (1998). Naming the rainbow: Colour language, colour science, and culture. Dordrecht: Kluwer Academic Publishers.

Hardin, C. L. \& Maffi, L. (Eds.) (1997). Color categories in thought and language. Cambridge: Cambridge University Press.

MacLaury, R. E. (1997). Color and cognition in Mesoamerica: Constructing categories as vantages. Austin: University of Texas Press. 



\section{Full color illustrations}

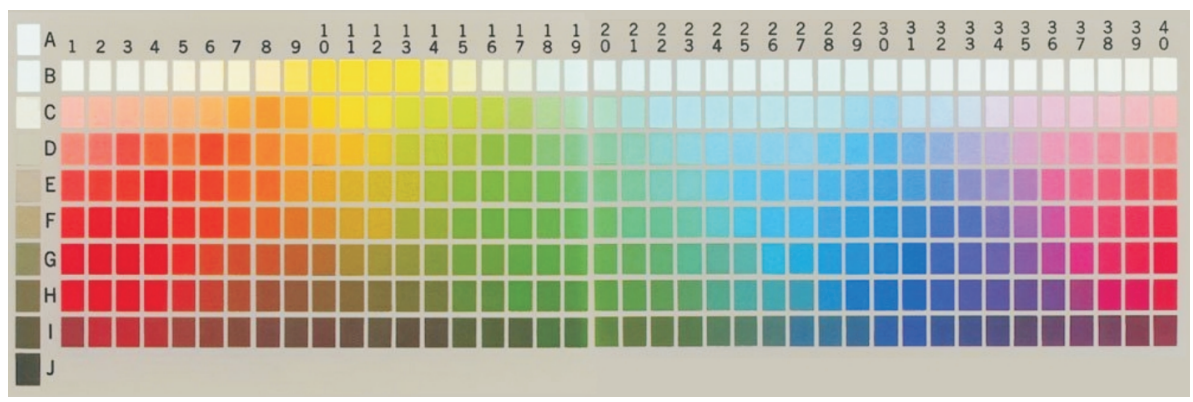

Munsell chart
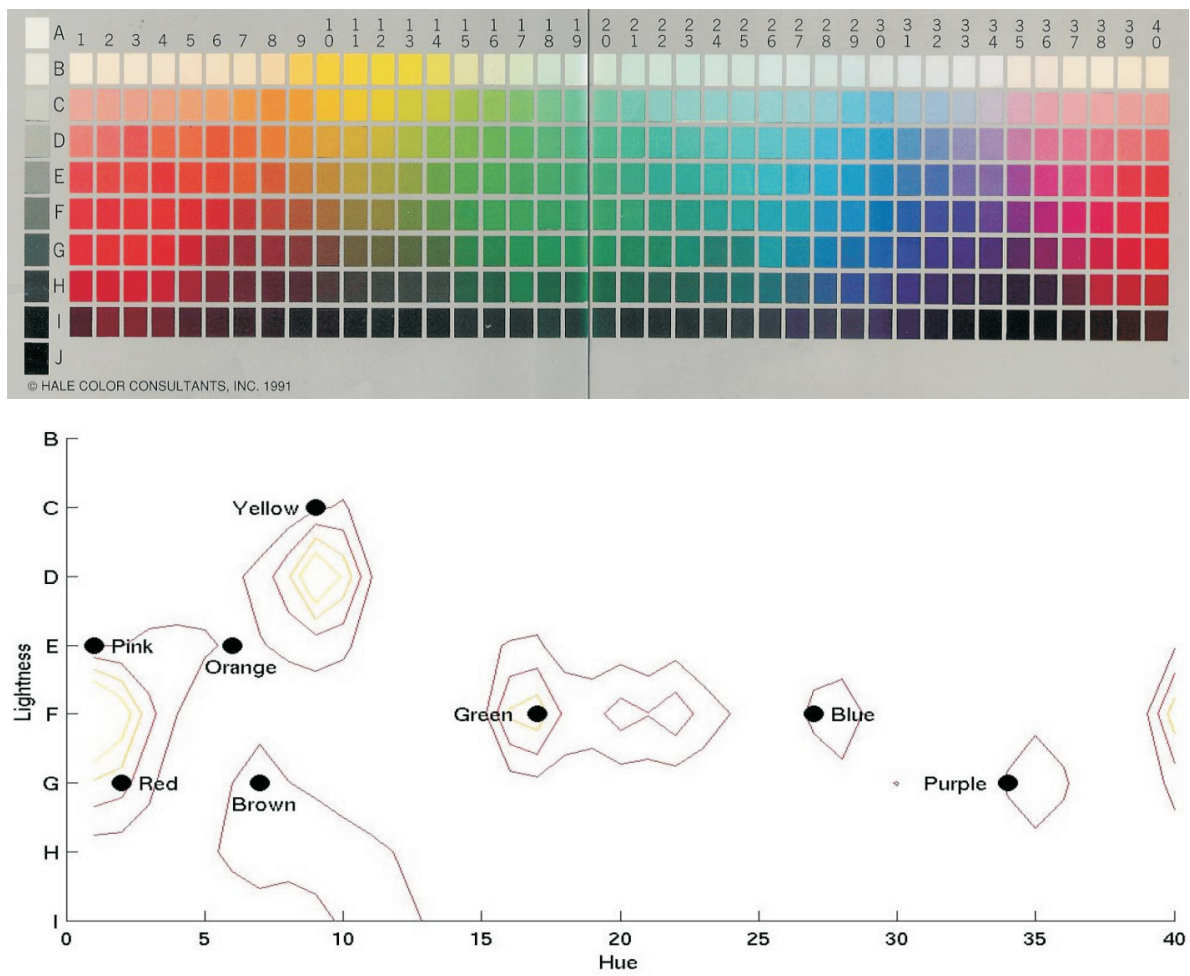

Figure 1 (Webster \& Kay p. 32). Contour plot, over the Munsell stimulus space (illustrated by the color panel), of the number of naming centroids in the WCS data set. Each contour represents centroids of 100 individual speakers. Filled circles represent average English focal choices observed by Sturges and Whitfield (1995). [Adapted from Kay and Regier, 2003, Figure 4b.] 


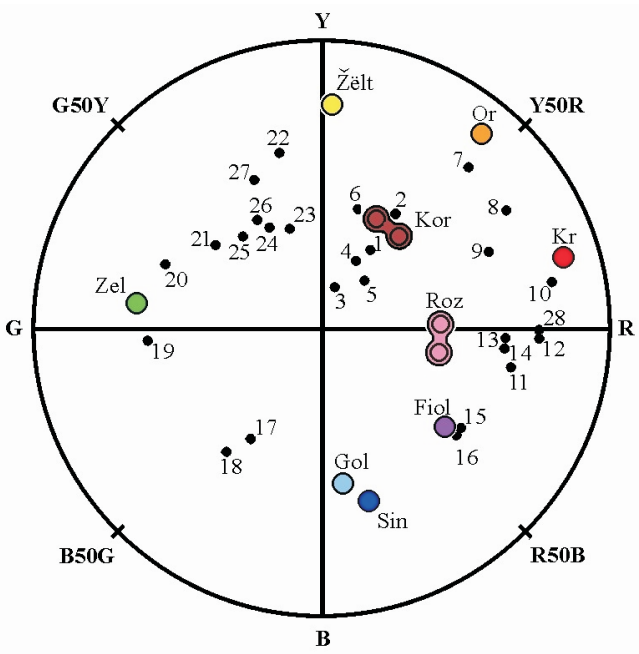

Figure 3 (Safuanova \& Korzh p. 65). Location of focal colors of the eight Russian chromatic basic terms in projection into the NCS color circle (clockwise): žëltyj 'yellow' (Žëlt), oranževyj 'orange' (Or), koričnevyj 'brown' (Kor), krasnyj 'red' (Kr), rozovyj 'pink' (Roz), fioletovyj 'purple' (Fiol), sinij 'dark blue' (Sin), goluboj 'light blue' (Gol), and zelënyj 'green' (Zel). Locations of the frequent nonbasic terms are designated by numbers (for legend see Table 2). Adapted from Korzh et al. (1991).

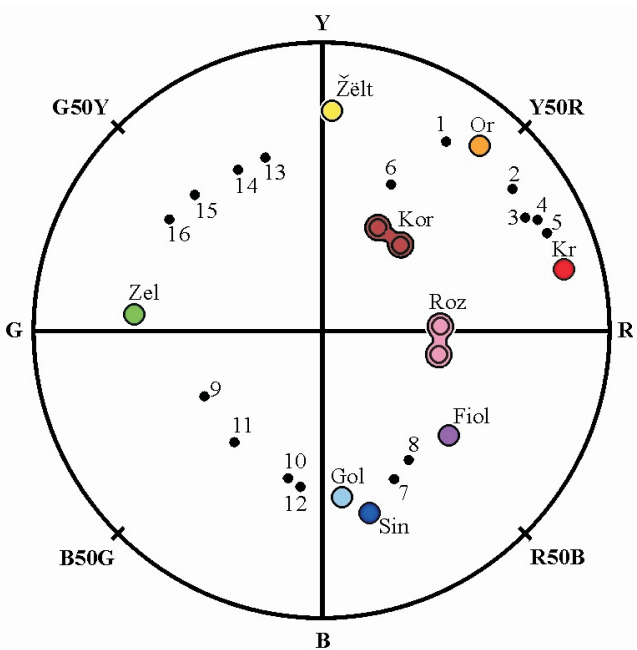

Figure 5 (Safuanova \& Korzh p. 69). Location of focal colors for the Russian chromatic basic color terms (letter designation as in Figure 2) and compound chromatic terms (indicated by numbers as in Table 3) in projection into the NCS color circle. Adapted from Korzh et al. (1991). 

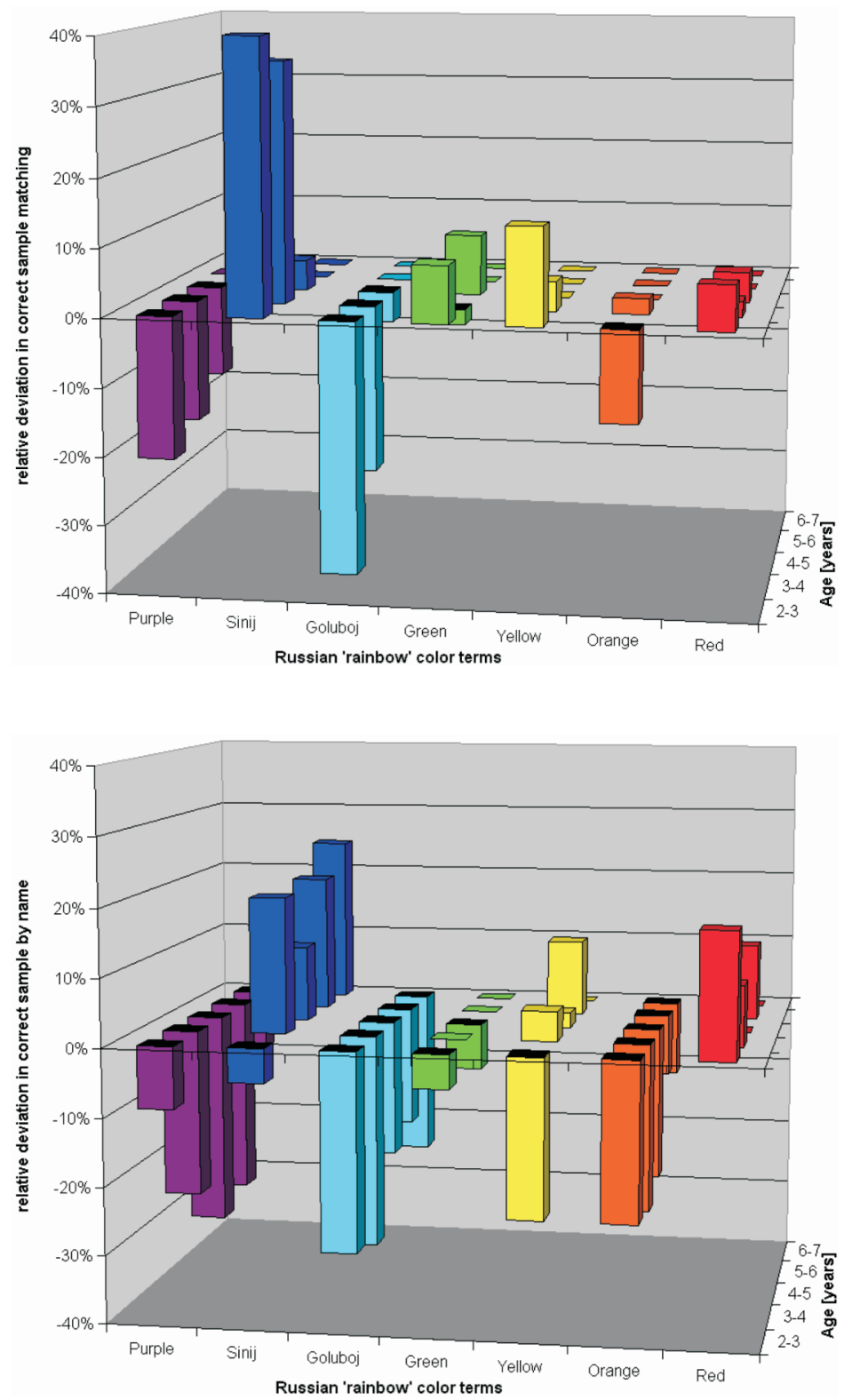

Figure 2 (Paramei p. 82). Relative deviation from correct recognition of six spectral colors and goluboj by Russian children, from early childhood to preschool age: (a) matching task, (b) comprehension task. Color terms other than the Russian 'blues' are indicated by English glosses. Based on Istomina (1960a, 1960b, 1963). 


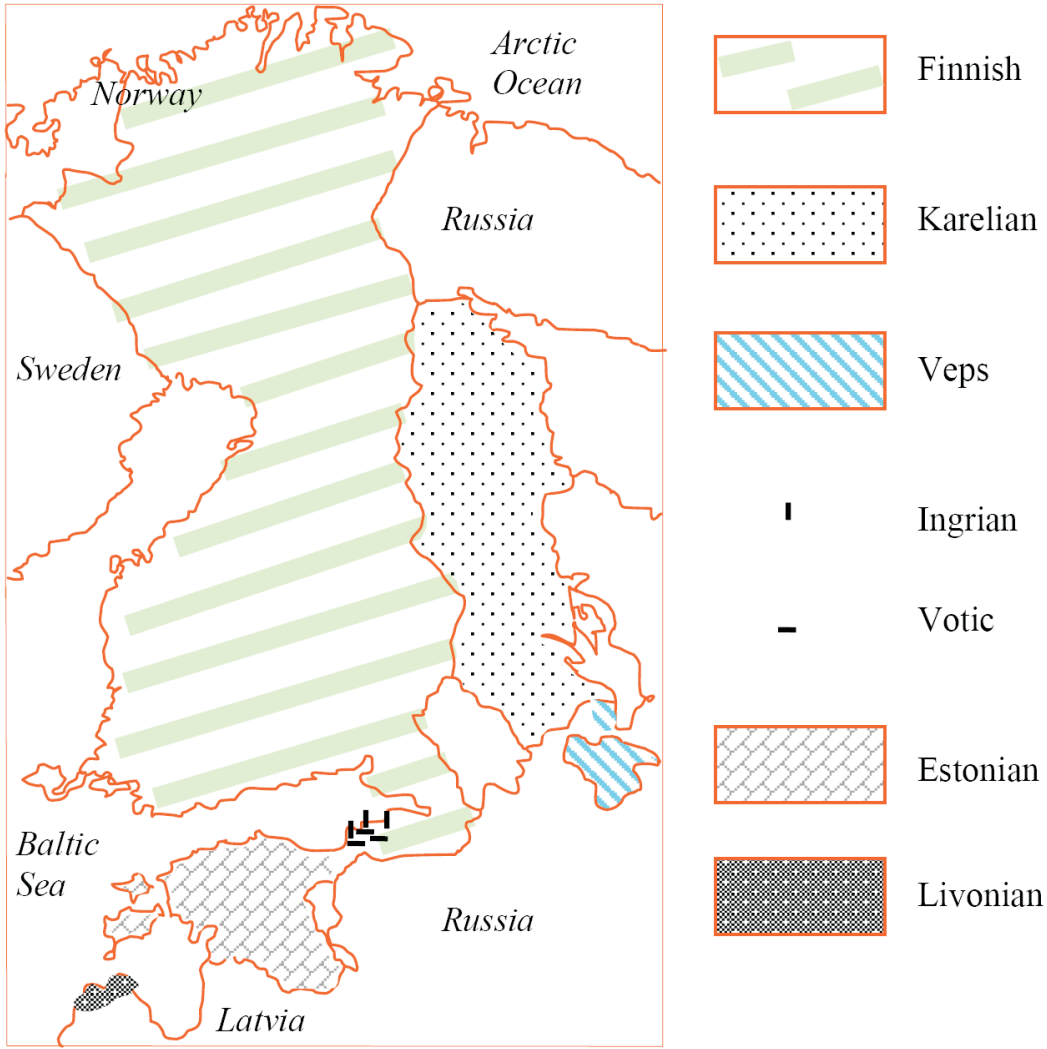

Map 1 (Oja p. 190). Finnic languages. 
PART I

\section{Color Perception}

The studies in this Part emphasize perception, although some refer as well to cognition 



\title{
Hue categorization and color naming \\ Cognition to language to culture
}

\author{
Marc H. Bornstein \\ National Institute of Child Health and Human Development, \\ National Institutes of Health, USA
}

\section{Introduction}

This survey and update about basic hue categorization and basic color naming proceeds to four principal goals. In a companion chapter (Bornstein 2006), I first reviewed practical and theoretical characteristics and functions of psychological categorization, including infants' capacities to categorize. These considerations identified, asserted, and highlighted the essentialness and adaptive significance of categorization to mental life. Second, I addressed the psychological, biological, developmental, and ethological manifestations of basic hue categorization, and its connections to color vision. In this companion chapter, I discuss cultural variation in basic color naming and its possible biological and experiential substrates. Toward the final goal of this survey and update, I assess the development of categorization to naming regularities and possible mechanisms for how we get from the start - universal biologically grounded basic categorizations of hue - to the end - cultural variation in basic color naming.

The overarching aim of these companion chapters is to bring rudimentary order to the literature on basic hue categorization and color naming. These chapters are heuristic and selective and are not intended to constitute a comprehensive review of every topic covered or associated with color phenomena. Hue categorization and color naming involve physical, sensory, perceptual, cognitive, linguistic, and cultural considerations. Moreover, I do not take as my purpose here the final adjudication of the origins or the development of hue categorization and color naming. However, the contemporary study of basic hue categorization and color naming wants clarity and demystefication. The backdrop to the present exposition is provided in Bornstein (2006). There, the significance of categorization in mental life is discussed, and four independent lines of argument are detailed that clarify, highlight, and embolden a universalist perspective on basic hue categorization that encompasses red, yellow, green, and blue. Caterogization normally refers to the treatment of a set of physically different and psychologically indiscriminable or discrim- 
inable entities as equivalent in some way (for example, by giving a group of discriminable colors the same color name). The four arguments for the basicness of red, yellow, green, and blue categories include psychophysical and perceptual studies with adults from different cultures and language communities, infancy studies, studies of diverse infrahuman species, and studies of the physiological structure and function of the visual system. First, a high degree of uniformity and regularity obtains among the four basic color names and in experimental studies that use color stimuli even when (some) different languages and cultures are sampled. Second, human infants, long before the acquisition of language or inculcation of even the rudiments of culture, partition the spectral continuum into basic categories of hue. Third, various infrahuman species that see color, but are devoid of language and culture, partition the spectrum regularly as well; primates with visual systems like human beings see similar basic hues. Last, the physiological responsiveness of neural pathways in the visual system shows patterns of wavelength discriminability that appear compatible with (and perhaps determinative of) basic hue categorization. Thus, anatomy, physiology, and psychology contribute integrally to patterning basic hue categorizations of the color world and point to the existence of a small set of natural divisions of the chromatic spectrum.

All that said, an apparent paradox in the color literature obtains: Perceptual categorization (color categorization without the involvement of language) supports a universal system, whereas color naming (color categorization in which language plays a role) points to widespread diversity and, in specific, to exceptions and variants in basic color naming. This chapter (companion to Bornstein 2006) attempts to address this paradox.

\section{Exceptions and variants to basic color naming}

Every known culture appears to have a primary color-naming system of some sort. However, cross-linguistic reports cite a wide variation in color-naming systems. Some investigators have focused on the universality of basic hue categorization. Many others, however, have been drawn to exceptions and variants. Nearly two centuries of ethnographic investigation have pointed to numerous cultures around the world that linguistically partition in the visible spectrum, but do so in diverse ways.

\section{Perspectives on variation in color naming}

What exceptions and variants to basic hue categorization exist across cultures and languages? What accounts for these exceptions and variants? I first review the second question and after return to the first. Historically, philosophers, linguists, anthropologists, and psychologists have proffered separate explanations, which can be grouped into three distinguishable stances. One perspective is that language and perception are one, and observed or reported differences in language reflect differences in perception. A second perspective shares the underlying assumption of perceptual-linguistic isomorphism, but ascribes 
language primacy over perception. A third perspective divorces language from perception and sees variations in language independent from variations in perception.

Probably the oldest view in the first school of thought is a biological evolutionary one that proposed that in the course of human history color vision itself and, attendant to it, color naming have evolved. This evolutionary view is principally associated with William Gladstone, the nineteenth-century British PM. Gladstone was a Greek scholar avocationally, and in his studies of Homer and the Homeric Age Gladstone (1858:488) recognized a paucity of color terminology in Homer and in classical Greek from which he concluded that: "the organ of color and its impressions were but partially developed among the Greeks in the heroic age." In short, Gladstone proposed that color language reflects color perception, that Greeks in the third century BC were (at least marginally) color deficient, and that between third-century BC Greece and nineteenth-century AD England human beings (must have) evolved from color deficiency to color trichromacy and, thus, from using incomplete and undifferentiated color vocabularies to complete and differentiated ones. However, it appears untenable that color vision per se evolved in human beings in this way, or could have evolved in so short a time. It is also worth noting that dimensions other than hue (e.g., saturation) may have served as a basis for naming in ancient Greek and no likely form of inherited deficiency could have clearly yielded the color partitions that classical Greek named.

The evolutionary view was supplanted early in the twentieth century by the predominance of a cultural and linguistic relativism. First associated with the German polymath Humboldt and the American anthropologist Boas, relativism is perhaps most commonly identified with the anthropologist-linguist-insurance adjuster, Benjamin Lee Whorf $(1950,1964)$. The Whorfian hypothesis holds that languages organize properties of the world pragmatically and, further, that their organization in turn influences perception (Lucy 1992). The relativist position sees human activities as driving perceptual categorization and not the other way around: That is, perception is shaped by cultural knowledge and practices (Dubois 1997), and unique local or cultural factors influence the development of lexical distinctions such as for colors (Pastoureau 2000). In relativism, language usage and cultural practice exert joint effects on the experience of color by selectively directing attention toward or away from perceived features of the chromatic ecology (Gellatly 1995).

Whorf himself commented on color vocabulary and perception specifically but only infrequently. Another anthropologist of the Whorfian school, however, Ray $(1952,1953)$, crystallized the Whorfian hypothesis on color in the following way: "The color patterning of man's world is not psychological, anatomical or physiological; there exist no natural divisions of the spectrum. Cultures divide it arbitrarily (Ray 1952:43)," and "Each culture has taken the spectral continuum and divided it upon a basis which is quite arbitrary except for pragmatic considerations (Ray 1953:102).” Later, Krauss (1968:268-269) reiterated that "our partitioning of the spectrum consists of the arbitrary imposition of a category system upon a continuous physical domain." So, for example, in a 1955 report of color naming among the Hanunóo people of the Philippines, the anthropologist Conklin (1955:341) suggested that four colors predominated in this Stone Age culture: (ma)biru 
"relative darkness (of shade of color); blackness" (black). (ma)lagti $\sqsupset$ "relative lightness (or tint of color); whiteness" (white). (ma)rara $\sqsupset$ "relative presence of red; redness" (red); and (ma)latuy "relative presence of light greenness; greenness" (green)". These four categories, Conklin further observed, spring from a taxonomy of vegetation and food stuffs that are materially important to the Hanunóo. In a nutshell, the relativist theory is that color categorization is linguistically constructed (e.g., Roberson 2005): Experience shapes the perceptual system which as we see later is probably an active force in some exceptions and variants to basic hue categorization.

Another force in relativism is diffusion. Tornay (1978), for example, suggested that nineteenth- and twentieth-century Western colonialism might account for the varying semantics of color worldwide. However, the color-naming systems of local languages cannot all have formed through the insinuation of colonial languages because there is nothing in the major languages of Western colonialism (Dutch, English, French, German, or Spanish) that could give rise to the particular exceptions and variations in hue categorization in the languages of colonized peoples (Kay \& Berlin 1997). Moreover, many native languages were reported to have idiosyncratic systems of color naming at the time of their initial contact with the West.

In the third view, color-naming systems per se are thought to evolve apart from color vision. This position was first asserted by W. H. R. Rivers, an English anthropologist who accompanied the expeditions of the Royal Anthropological Institute to Australia, Africa, and India at the turn of the nineteenth century. In studying the color vision and color vocabulary of various peoples in those parts of the world, Rivers (1901a, 1901b:47) concluded that different peoples (mostly) possess color normal vision, but from a sociotechnological perspective that "the order in which [peoples] are placed on the grounds of the development of their color languages corresponds with the order with which they would be placed on the grounds of their general intellectual and cultural development." Rivers's position strictly cleaves vision and vocabulary. A variant of this position (paradoxically) is found in the contemporary work of Berlin, Kay, and their associates on the culturally ordered encoding and acquisition of color terms. In this perspective, languages evolve from partitioning colors into only two categories, "dark" and "light," and then add new color words in a fixed order from a universal set of 11 basic color terms. Ceteris paribus, Berlin and Kay (1969) contend that, as a culture becomes technologically more complex, speakers of its language have more frequent need to distinguish objects and other entities by their colors. Many contemporary authors likewise believe that color categorization gains its primary impetus from sociocultural mechanisms (e.g., Dedrick 1998; Eco 1985; Gage 1997; Hardin 1993; Kay \& Maffi 2000; Saunders \& van Brakel 1997).

Cultural relativism dominated philosophical, linguistic, anthropological, and even psychological thought about color (as well as related cognitive issues) for the better part of the twentieth century. With respect to color, how can universalism (Bornstein 2006) and relativism be reconciled? 


\section{Experimental observation}

In the 1970s some systematicity was brought to the haphazard literature of cultural exceptions and variants in basic color naming (Bornstein 1973; Kay \& McDaniel 1978). These reviews showed that color-naming systems in the world's languages were not random, but that languages that constitute exceptions or variants with respect to basic hue categorization showed systematic and explainable kinds of exceptions and variations. Bornstein (1973) first taxonomized and then plotted a world-wide geographic distribution of the major types of color-naming systems. His survey revealed that a minority of societies (only about $25 \%$ ) semantically distinguishes among the four basic hues, whereas the majority (about 75\%) does not. Furthermore, he identified specific kinds of exceptions and variants. First, there were no cases of single names (or categories) denoting red=green or yellow=blue (that is, when a single word denotes both red and green), an observation consistent with opponent color theory (Bornstein 2006). Second, he found that languages that do not have separate basic terms for each of the four basic chromatic primaries tend to have terms that cover certain perceptually adjacent sensations (e.g., a single word that denotes red and yellow: red=yellow; or green and blue: green=blue). Third, the majority of languages that do not distinguish the four basic hues normally collapse among a very small set of the same basic categories.

On his world map, Bornstein displayed the color-naming systems of 145 societies in terms of "semantic identities" among the four basic hue categories of red, yellow, green, and blue. Three main kinds of semantic color identities appeared in this worldwide survey of basic color-naming systems. The most frequent semantic identity ( $50 \%$ of the total sample) equates green with blue (that is, the society has one word that denotes both green and blue); several societies (15\%) utilize a single term for blue and black; and nearly as many (12\%) possess only one word to encompass the experience of green, blue, and black. Later, Kay and McDaniel (1978) similarly proposed that hue categories denoted by the basic color terms of the world's languages divide into three types: (1) primary color categories are red, yellow, green, and blue; (2) derived color categories are intersections or mixtures of primaries, for example, orange (red-and-yellow) and purple (red-and-blue); and (3) composite color categories are unions of two or more primaries, for example, red=yellow, green=blue. Data from the World Color Survey confirm that all categories denoted by basic color terms fall into one of these three types (Kay, Berlin, Maffi, \& Merrifield 1997). Later still, Lindsey and Brown (2002) replicated and expanded Bornstein's (1973) observations to 203 languages.

The most frequent semantic identity or composite (by far) is a term covering the percepts of green and blue. Indeed, the term grue has been applied to languages that do not have independent terms for green and blue (Kay 1975:258-260). As early as 1928, Spier referred to the "familiar blue-green confusion." Likewise, languages that do not distinguish blue and black or green, blue, and black have been termed "dark" languages. In overview, although peoples all over the world distinguish black and white semantically, they sometimes, though not often, subsume red and yellow under the same name. The majority of societies, however, fail to distinguish semantically between green and blue, or between blue 
and black, or among green and blue and black. Apparently, many cultures around the world perceptually and/or semantically equate select basic hue categories.

\section{Universalist-relativist reconciliations}

\section{Prolegomenon}

In some languages and cultures, an isomorphism obtains between basic hue categorization and basic color naming. However, diversity in basic color naming and perhaps basic hue categorization appears to represent a dominant mode of human color sensitivity. The vision, ontogenetic, and experimental data suggest that this diversity probably develops out of a shared uniformity (Bornstein 2006). In the face of these data, a central ontogenetic/diachronic question arises: How might human beings proceed from the uniformity of hue categorization in infancy to the diversity that characterizes some systems of adult color naming? Is basic categorical perception an artifact of verbal coding, learned through a process of linguistic and cultural training (Ratner 1989; Saunders \& van Brakel 1997), or does verbal coding describe basic categorical perception that itself arises out of the neuropsychology of visual system structure and function (Bornstein 1973, 1976, 1987; Kay \& McDaniel 1978; Roberson, Davidoff, \& Braisby 1999)?

A gap has emerged between the biological cum perceptual level and the linguistic cum cultural level that needs to be bridged by an account of the rules that people (consciously or unconsciously) use to move from the one to the other. In shorter words, the cluttered path that takes us from basic hue categories to basic color naming needs to be cleared. Furthermore, the translation rules must be stated with some precision and yet be flexible enough to account for other major variations in color language that are commonly found (Dedrick 1997). Finally, how this system of mappings between color percepts and color nominals is implemented during the developmental period in which children are learning color terms needs to be clarified (Bornstein 1985; Pitchford \& Mullen 2003). Although children may "know" where the color space is cut up and how many portions there are, they must still await external tuition to acquire the lexical labels that map on to each portion. One-hundred percent of words in a language are learned, and children learn the words they hear: The evidence is clear that children's earliest vocabularies share variance with their mothers' vocabularies (Anglin 1977; Blewitt 1983). Indeed, mothers' and children's uses of specific color words are correlated (e.g., Andrick \& Tager-Flusberg 1986). The role of parental input on children's learning color words is evident even in the classical speech transcripts of Adam, Eve, and Sarah (Brown 1973). Thus, children have to learn the perceptual foci, plateaus, and boundaries of each basic color term in their language, and these boundaries might be determined by the culture in which the child is reared. Moreover, this perceptual learning is flexible, and it appears that people can "unlearn" preexisting categories while learning novel ones (Özgen \& Davies 2002). The effects of linguistic categories on discrimination and memory are not immutable, but subject to experience (Bornstein 1976; Bornstein \& Korda 1984; Boynton et al. 1989). In 
short, biological and experiential forces define and redefine the basic hue-categorization and color-naming systems.

In what possible ways could development proceed to overlay the homogeneity of hue categorization with a heterogeneity of color naming? What roles do forces of "Nature" and "Nurture" play in hue categorization and color naming development? It is possible to identify a set of logical principles by which infant perceptions and cognitions about color are transformed during ontogenesis into the small variety of adult modes of categorizing and verbalizing about color. The field of developmental science is concerned with just such ontogenetic questions (Bornstein \& Lamb 2005). Developmental change, such as in perception, can be attributable to (1) neural, anatomical, or sensory maturation (Johnson 2005); (2) improvements in attention which plays a role in perceptual learning (Ahissar \& Hochstein 1993; Shiu \& Pashler 1992), and differential attention to category-relevant information may result in the acquired equivalence or distinctiveness of different dimensions (Nosofsky 1986); (3) alterations in motivation or improvements in task performance; (4) learning and experience; or most probably (5) combinations and interactions of "all of the above." Some of the foregoing explanations, however, are only appropriate to individual-level analysis (attention, motivation), and therefore less likely to apply to populations that embody language and culture. Here I concentrate on those explanations that are appropriate to the level of the population (biology, experience, and their interaction). Despite the fact that they are sometimes conceived as either-or contributors to development, nature and nurture inevitably and invariably interact: That is, (perceptual) development fuses organismic factors with environmental experiences (Overton 1998).

\section{Models of development}

To simplify the argument as well as clarify the potential ways in which the forces of nature and nurture possibly interact to influence the trajectory of categorization-to-naming, the course of development can be conceptualized in a straightforward but comprehensive manner. Three different possible courses of development of a psychological structure or function like basic hue categorization before the onset of experience can be identified: The structure or function can be (1) undeveloped, (2) partially developed, or (3) fully developed. In addition, there are three possible ways that experience might influence eventual outcome afterward: (A) induction, (B) modification, or (C) deprivation. The three developmental courses interact with the three mechanisms of experience (Gottlieb 1981). The onset of experience, and not birth, is used intentionally as a benchmark.

First, there is the possibility that a structure or function is undeveloped at the onset of experience, but can be induced by relevant experience, the 1A combination; without relevant experience, the structure or function is presumed never to emerge (1C). Induction is the most dramatic experiential form of influence: Induction means that experience completely determines whether the structure or function emerges (Fahle \& Poggio 2002). There is reason to suppose that some perceptual categories of color can be induced. In English, blue covers a large region in color space (Uchikawa \& Boynton 1987). However, 
some languages possess more than one color term to describe the blue region. Russian has two color terms for blue: goluboj (light blue) and sinij (dark blue), but depending on context, sinij appears to have two meanings - either blue or dark blue (Berlin \& Kay 1969; Davies \& Corbett 1994; Paramei 2005). Category segmentation of blue, similar to that in Russian, is also observed in at least two East Slavonic languages - Ukrainian and Belarusian (Hippisley 2001; MacLaury 2001; Moskovič 1968). This usage is also similar to the two blues in Japanese: ao may be used as a generic blue, whereas mizu is a secondary term limited to light blue. This natural induction has been modeled in the laboratory. Özgen and Davies (2002) had one group of participants learn to categorize across a hue boundary and a second group across a lightness boundary. Lightness and hue discrimination were then measured for both groups in the training region of color space. Learning either hueor lightness-based categories induced categorical perception. Their experiments provide evidence that color discrimination is flexible and that it is possible to induce categorical perception effects on same-different judgments through a relatively fast learning regime based on either hue or lightness. Furthermore, the effects of existing color categories on same-different judgments can be reversed through training. Following this account, colorname acquisition might be viewed as a similar process of categorization induction that takes place over a much longer time.

That said, the induction of the basic hue categories of red, yellow, green, and blue is unlikely and unnecessary in human beings on extensive universalist physiological and developmental arguments (Bornstein 2006). Visual system operation yields a fluctuating $\Delta \lambda$ function that contributes to basic hue categorization, and young human infants without language, experience, or culture categorize basic hues. To be fair, it is possible, if unlikely, that infants completely learn the four basic hue categories. Even 3-month-old infants are capable of learning categories (Bornstein 2006), so that tender age alone is insufficient proof that experience plays no inductive part in the hue categories exhibited by infants. However, the basic hue categories in infants (Bornstein et al. 1976a, 1976b) are uniform, predictable on biological accounts, and comparable cross-culturally (Franklin \& Davies 2004). It could also be that infant perceptual categories and adult naming categories are wholly different phenomena. However, this is extremely unlikely as well, because there is good evidence for similarity in adult and infant perception.

Second, a structure or function such as basic hue categories may partially develop before the onset of experience (2), after which experience could operate in one of three ways. Relevant experience may modify the partially developed structure or function (2B) facilitating further development or attuning the structure or function; experience may maintain the structure or function at its partial level of development attained before the onset of experience (2); or, in the absence of relevant experience, the partially developed structure or function may be lost (2C). (Of course, experience per se may not be altogether necessary where the structure or function would continue to mature as a reflection of the genetic blueprint.)

A close analysis of the structure of hue categories shows that categorization processes do indeed change after the onset of experience in identifiable ways. Two prominent ways in which hue categories appear to alter developmentally entail perceptual "sharpening" 
and "broadening" (Raskin, Maital, \& Bornstein 1983). Sharpening manifests itself in the narrowing of boundary regions between hues, and broadening manifests itself in the complementary expansion of category plateaus. Between 3 and 4 years of age the boundary regions between children's hue categories narrow and reciprocally their plateau regions broaden; after 4 years of age, both categories and boundaries appear to remain stable through the balance of the life-span. This early change might be attributable to continuing maturation or to experience. At the very least, cultural and linguistic training can affect low-level perception. The internal color space is not fixed and static (Davidoff 1991), and some distances within linguistic categories can stretch or modify. Matsuno, Kawai, and Matsuzawa (2004) found that, despite equivalent high levels of accuracy in color matching, Pendesa, a chimpanzee with little experience in discriminative learning of colors and/or acquisition training of color names, showed low consistency and indistinctive response times for color responses in contrast to $\mathrm{Ai}$, a chimpanzee which had years of experience and showed distinctive consistent and inconsistent response times. Ai perceived colors with a clearer distinction of being inside or outside of a color group and hesitated in responses to colors located on the borders of color groups. However, these structural developmental changes in hue categories are really marginal and do not, for example, alter hue foci or the positions of wavelength cross-over points between hues, that is the general organization of hue categories along the wavelength continuum.

We know that the system of basic hue categorization is essentially fully developed even early in infancy (Bornstein 2006), and the marginal (biological or experiential) attunement (sharpening, broadening) that takes place later does not alter the number or placement of basic hue categories. Third, then, a structure or function like hue categorization may develop fully before the onset of experience (3). Afterward, the structure or function requires experience to be maintained; without relevant experience or because of biological change, the structure or function may be lost (3C). Experience can also attune a mature structure or function if it is plastic (3B)(as seems to be the case with color; see above). This third scenario appears to account best for the status of basic hue categorization in infancy and for the exceptions and variants to basic color-naming systems after infancy. Let's explore two prominent ways how.

\section{Grue and dark languages revisited}

In Western societies it appears that the four basic categories of hue that are fully developed before the onset of experience (Bornstein et al. 1976a, 1976b) are maintained during development so that they are essentially the same as those for adults (Bornstein 1973; Bornstein et al. 1976a, 1976b). However, in most other societies, where it is common for color-naming systems to differ from the basic four-fold hue category system, some processes in biology and experience modulate basic hue categorization during development. As we have seen, categories extant in infancy may be lost; existing categories may be tuned; or new categories may be induced. How these biological and experiential changes are effected requires further exacting research. The pace of neurophysiological to cultural research makes it difficult to survey accurately the state of theory (Simpson 1997) 
and shortsighted to question existing theories just because the data and phenomena are complex (Stanlaw 1997). Nonetheless, the balance of available evidence renders certain possibilities more likely than others (Van Laar 1997). Divining the linkages between hue categorization and color naming will provide a model realm for understanding the fused roles of biology and experience in development.

Most Indo-European languages distinguish blue from green and blue from black; so do languages in many other language families. However, grue languages have one basic color term to denote green and blue, and dark languages have one basic color term to denote blue and black, or, green and blue and black. In either case, infants' categorization of green and blue as separates seems inconsistent with the absence of the green-blue distinction in adult speakers (Davies et al. 1998; Roberson et al. 2000). The apparent contradiction might be resolved in a number of ways following the comprehensive logic of the developmental scheme detailed earlier. First, it could be that for infants in these cultures or linguistic communities the basic green-blue hue distinction is undeveloped in the first place, and remains one that does not develop. But, as has been shown (Bornstein 2006; Bornstein et al. 1976a, 1976b; Catherwood, Crassini, \& Freiberg 1987; Franklin \& Davies 2004), infants do categorize green from blue, and they are likely to do so universally. Second, it could be that the green-blue distinction is really only partially developed, and that it is not later attuned by language experience. Again, however, the green-blue distinction, which is both qualitative and categorical, is present and mature long before infants acquire language.

Third, then, the green-blue distinction might be present before the onset of experience, but in peoples who speak grue languages it is (mis)attuned or lost. It could be attuned or lost for experiential (e.g., utilitarian) reasons or for biological (e.g., visual) ones. For example, it could be that infants possess the categories of green and blue but the distinction is lost during development because it is not used. There is some precedent for this possibility. For example, Werker (1990) showed that infants from English-speaking families discriminate Hindi phonemic speech contrasts that are not used in English and they do so as well as do Hindi-speaking adults; however, English speakers 4 years of age and older do not. Thus, a perception present in infancy is lost. Apparently, infants stop being "universal listeners" for certain phoneme distinctions around the end of the first year of life (Werker \& Tees 1984). It also appears that experience can "maintain" those perceptual sensitivities that are already evident in the young infant, but without such exposure initial abilities are lost (Best et al. 1988). Together, these observations suggest that sometime between infancy and maturity a perceptual discrimination that is present at birth weakens on account of lack of experience, even if it is not wholly or permanently lost. Studies such as these underscore the diverse interactions that biology and experience play in perceptual development.

Several authors have argued that color categorization is principally motivated by sociocultural mechanisms (Dedrick 1998; Eco 1985; Gage 1997, Hardin 1993; Kay \& Maffi 2000; Saunders \& van Brakel 1997). Such learning views are compatible with a determinative role of experience vis-à-vis biology: induction where development had not yet occurred (1A) or attunement or loss where partial development obtained (2B, 2C) or at- 
tunement or loss after full development $(3 \mathrm{~B}, 3 \mathrm{C})$. I return to these possibilities at the end of this chapter.

\section{Biological explanations for grue}

Normally, however, before appealing to experiential (cultural and linguistic) explanations it is reasonable to explore and to rule out possible biological (visual and physiological) explanations. To be concrete, psychological aspects of differential color naming should come into consideration only when the assumption that no differences exist in actual visual processing by different peoples is eliminated. Pragmatic explanations of color naming will suffice only if no significant explanatory differences in biology that could underlie semantic identities or confusions exist among groups shown to vary in their color naming. Cultural and linguistic explanations necessarily suppose prior assumptions about equivalent biology and physiology. Perhaps some differences in color lexicons among some languages are related to physiological and, concomitantly, perceptual discriminations among the peoples who speak them.

Cross-cultural regularities in the use of grue and dark could emerge because of variation in visual sensitivity specific to the region of the visible spectrum associated with green and blue. The prominent types of semantic identities or confusions which investigations of color-naming systems have unearthed - namely, green with blue, blue with black, green and blue with black, as well as red with yellow - are in fact consistent with known deficiencies in color vision. Most people possess normal color vision: They are trichromats (having the three cone photoreceptors). Some are deficient in color vision: They are dichromats (having only two of the three). Three classical kinds of dichromats are protanopes (lacking the LWS “red" receptor), deuteranopes (lacking the MWS “green” receptor), and tritanopes (lacking the SWS "blue" receptor). Protanopia and deuteranopia are the most common and best understood color vision deficiencies in Western medicine. Tritanopia is the most rare $(0.00092 \%$ of the population; Wright 1952$)$. Tritanopes notoriously confuse greens and blues, reds and yellows.

Pickford (1951) conducted extensive studies with tritan observers and described their color-naming behavior in detail: In the blue blind, and even the blue weak, forms of the deficiency, blue and sometimes yellow are diminished in saturation compared with the normal trichromat. Dark grey invades blue and light grey or white invades yellow. The intermediate colors, violet and blue-green "join hands" across blue, and orange and yellow-green join across yellow. Thus, tritans have difficulty in accepting distinctions made by color normal persons about any of the four intermediate colors: blue-green, violet, orange, and yellow-green. The tritan person reports having disputes or differences of opinion about why a blue-green should not be called blue or green, about why red should be distinguished from orange, and so on. The tritan may confuse yellow with white, and call orange yellow and yellow white; to a tritan broadband stimuli that look pale blue, bluish, cyan, or turquoise reportedly look greenish. As Wright (1952) and Walls (1964) pointed out, in tritanopia all wavelengths short of the neutral point (around $570 \mathrm{~nm}$ ) are seen as uniform bluish-green and give rise to naming confusions or semantic identi- 
ties except at extremely short wavelengths where the visible spectrum is sharply curtailed or darkened.

It so happens that tritan and tritan-like vision is distributed geographically in a way that overlaps the geographic distribution of grue and dark languages. To explore this argument, first, I briefly discuss the distribution of sunlight on the earth; next, the transmission and filtering of sunlight in the eye and in aging, and their effects on vision; after that, the adaptive significance of intraocular pigmentation; and, last, I return to the linkages between color vision and color naming systems around the world.

The highest values of ambient light intensity (Fischer 1960) as well as largest mean annual amounts of solar radiation (Ashbel 1961; Kimball 1935; Osborn 1929) have been recorded in the tropics, with a steady decrease toward the poles. Sunlight reaching global areas of equatorial proximity (or of greater altitude) is richer in ultraviolet-B (UV-B: $280 \mathrm{~nm}<\lambda<315 \mathrm{~nm}$ ) radiation (Golding 1954; Henderson 1970; Kimball 1935; Osborn 1929), and exposure to UV-B has two notable phototoxic effects that in turn likely influence color and detail vision. Specifically, high UV-B exposure in sunlight naturalistically accelerates aging of the ocular media especially the crystalline lens (Javitt \& Taylor 1994; Werner, Peterzell, \& Scheetz 1990; Young 1991). In accord with these physical facts, the incidence of cataract increases with proximity to the equator (Young 1991); the incidence of cataract is often used to estimate the incidence of damage to the lens (Young 1994). Moreover, as the lens ages, it becomes denser and more opaque (a process known as brunescence), permitting less light to reach the retina, especially at shorter wavelengths (Weale 1988). Finally, exposure to UV-B can also cause direct selective damage to the SWS ("blue") cone photoreceptors (Harwerth \& Sperling 1971; Werner, Steele, \& Pfoff 1989). In turn, these phototoxic effects produce tritan color vision defects or circumstances that essentially mimic tritan color vision deficiency.

Before light reaches the retina, isomerizes photoreceptor pigment molecules, and thereby becomes an effective stimulus for vision, it passes through several optical structures including the cornea, the lens, the aqueous and vitreous humors, and the macula lutea. In essence, our visual world is filtered through these several ocular media. Each of these structures has some density, and so each attenuates incoming light by reflection, absorption, and scattering. Optical structures are typically pigmented yellow, and therefore they selectively absorb, and so deprive the visual system of, short-wavelength light. Furthermore, light scattering in the eye operates according to Rayleigh's law: Optical scattering is inversely proportional to the fourth power of the incident wavelength. Therefore, shorter visible wavelengths are scattered most. Longstanding studies of the attenuation characteristics of the eye converge on the fact that intraocular structures combine selectively and cumulatively to absorb and scatter incoming short-wavelength radiation (Ludvigh \& McCarthy 1938; Norren \& Vos 1974).

Changes in vision often also accompany aging, particularly changes in the transmissivity of the lens and preretinal ocular media. Yellow pigmentation normally accumulates throughout the lens as it ages, selectively absorbing short-wavelength light. During the normal life span, the transmissivity of the lens also reduces due to cumulative damage (caused in part by UV-B light; Werner 1991). This process sometimes even eventuates in 
the development of cataracts (Young 1991). High exposure to UV-B accelerates aging of the lens (Javitt \& Taylor 1994).

The reduction in transmissivity of light on account of exposure to UV-B and aging is greatest for the short wavelengths and so results in decreasing sensitivity to short wavelengths or tritan vision. By screening short-wavelength light, intraocular pigmentation thus contributes in varying degrees to mimicking the tritan color-vision complex. Physiological differences between observers in how strongly their lens filters light depend both on their environment and their particular exposure to it (Werner 2000).

However, yellow intraocular pigmentation is also biometeorologically adaptive in two ways. First, yellow filtering of ambient daylight serves the important protective function of screening potentially damaging actinic blue and ultraviolet radiation from retinal tissue (Frost 1948). Several different natural preretinal ocular filters are yellow and have (presumably) evolved to protect the retina from UV-B radiation by selectively absorbing near ultraviolet and shorter visible wavelength light before it arrives at the retina (Pokorny, Smith, Verriest, \& Pinckers 1979; Young 1994). Second, by filtering short-wavelength light before the retina, yellow pigmentation enhances pattern definition in vision and visual acuity. On account of Rayleigh's law, short-wavelengths are the most out of focus or chromatically aberrant (Millodot 1976; Wald 1967). Chromatic aberration increases linearly from 750 to $550 \mathrm{~nm}$, but then rises exponentially as wavelength shortens further. Thus, preretinal yellow filtration that cuts shortwaves down serves, under natural conditions, to sharpen retinal images (Foley-Fisher 1968; Luckiesh \& Moss 1925; Reading \& Weale 1974 ) and to increase target visibility and contrast (Luria 1972). As photographers know, yellow filters reduce the blue haze associated with distance vision. It is noteworthy that, at the fundus of the fovea, the part of the retina of highest acuity, SWS cone photoreceptors are absent - the fovea is blue blind (Wald 1967) - and color naming study shows that short-wavelength vision is suppressed foveally (Bornstein \& Monroe 1978).

Thus, yellow filtering in the eye curtails visibility in the short-wavelength end of the spectrum and adversely affects chromatic vision but, as a trade-off, generally enhances visual acuity and secures some biological protection for UV-B-sensitive retinal structures. In the context of adaptive evolutionary radiation, the vertebrate eye of diurnal animals (such as man) has pressed for better acuity (Walls 1942). The greater reduction of axial chromatic aberration through the elimination of short wavelengths must mean for many peoples better detail vision. Evolution appears to favor detail over color, quantitative over qualitative aspects of vision. The adaptive significance of this tradeoff, as Wald (1967) noted, cannot be overemphasized.

The color-naming system of the identifiably blue-weak or blue-blind encompasses the same complex of semantic color identities (grue and dark) as is commonly found to occur in the investigation of color-naming systems around the world. Are these two phenomena related? Quite possibly.

The effects of yellow ocular pigmentation in most areas of color testing and color experience are identical to the effects of tritanomaly or tritanopia (Judd, Plaza, \& Farnsworth 1950; Kalmus 1955; Pickford 1951; Verriest 1963; Wright 1952). Indeed, the main cause of the acquired tritan defect appears to be accelerated yellowing of the lens due to the 
high prevailing levels of UV-B radiation, augmented by reflected UV from the ground. Following this line of argument, Bornstein (1973) suggested that people living in high sunshine areas, such as the tropics, would be advantaged if they filter high levels of UV-B radiation that otherwise damage the retina and attenuate visual acuity. As a product of yellow intraocular filtering, damaging and out-of-focus short wavelengths reaching their retinae would be attenuated, but as a by-product visible short wavelengths (blue) would also be reduced.

A number of experimental studies conducted during the twentieth century have reported lower sensitivity to visible wavelengths shorter than $500 \mathrm{~nm}$ in individuals living or originating in geographic areas subjected to high UV-B radiation. Rivers (1901a, 1901b, 1903, 1905) found that sensitivity to blue was greatest for Englishmen, much less for Murray Islanders and Indians, and least for Central Africans. Ishak reported that chromaticity coordinates for the standard illuminants (1951), photopic luminosity curves (1952a), and spectral chromaticity coordinates (1952b) were lower for comparative groups of Egyptian than British observers. Sperling and Hsia (1957) reported that Hsia, a native of Hunan Province, China, was the considerably less sensitive of the two in the blue (430-460 nm). Dodt, Coperhaver, and Gunkel (1959) found that "darkly pigmented" eyes displayed consistently lower sensitivity at blue wavelengths below $460 \mathrm{~nm}$ than did "lightly pigmented" eyes. Fitzpatrick (1964) found a dark-pigmented group of observers significantly less sensitive than a light-pigmented group when absolute thresholds for 430-530 nm blues were tested. Eckhardt (1966) reported that thresholds for African Americans were slightly, but significantly, higher than those for European Americans at short blue wavelengths (400$440 \mathrm{~nm}$ ). Webster et al. (2002) tested for similar population differences by comparing color judgments in sets of observers in India and the United States: Compared to the U.S. observers, the Indian observers tended to choose yellows and reds that were significantly shifted toward orange, and they chose blues that were even more strongly shifted toward green (although it is possible that for some of these observers, the term interpreted as blue was really grue). Each one of the methods used by these investigators carries with it an appreciable amount of reliability and validity. First, each of the studies is internally consistent. The same experimenters used the same apparatus and applied the same procedures; only the experimental eyes (participant populations) varied. Second, each of the studies produced vision data (e.g., luminosity coefficients) for European or European American groups that compared well to those already established for Europeans. However, third, when researchers experimentally investigated directly or indirectly international or interracial differences in spectral sensitivity, their results indicated consistent and significant differences in the blue regions of the spectrum.

Although it is well established that the incidence of red-green color deficiency varies around the world, tritan conditions are generally less well known. Indeed, the likely incidence of congenital tritanopia was deemed so small as to make estimate variations difficult or not worthwhile, and most field assessments of color vision have used the Ishihara Test Plates which do not even include tests for tritan errors (Fletcher \& Voke 1985: 191; for examples see Simon 1951, and Reuning 1988). However, when sought, a higher incidence of tritan defects has consistently emerged in equatorial-dwelling populations. For example, 
peoples from southern and central Africa show a high incidence of blue errors: Pickford (1951) found that West Africans suffered significantly more frequent blue weakness than Europeans. Collier (1966) administered the Farnsworth-Munsell Dichotomous Test for Color Blindness to a handful of his Zinacantecan observers. He reported "in all cases, maximum bulging (and poorest discrimination) seems to occur in the blue-green range ... [p. 11]." Furthermore, Davies and colleagues (Davies, Corbett, Mtenje, \& Snowden 1995; Davies et al. 1992; Davies, Laws, Corbett, \& Jerrett 1998) recorded high incidences (at least $20 \%)$ of tritan vision in three independent samples from southern and Central Africa. About one-fifth of an African sample, and almost one-fifth of a Greek sample (remember Gladstone), made one or more color vision errors, and the errors they made were predominantly tritan errors. None of Davies's age-random British or Spanish samples made any such errors; and their Irish sample revealed the expected level of red-green errors. Notably, however, the pattern of errors made by the African sample resembled the pattern exhibited by an elderly British sample. Recently, in a pilot study, Wortman (2004) developed tests to assess variations in color discrimination in people living at different distances from the equator in Brazil, the longest country in the world North to South. He hypothesized that, holding language (Portuguese) constant, discrimination of short-wavelength hues would be worse for residents nearer the equator than for Brazilians farther from the equator. He conducted experiments in three coastal cities: $1^{\circ}, 8^{\circ}$, and $24^{\circ}$ south of the equator. Wortman found a statistical trend toward impoverished performance in ability to discriminate hues in the blue and green regions of the spectrum the closer to the equator his observers lived.

Intraocular yellowing reduces transmissity and decreases sensitivity to short wavelengths thereby producing predictable shifts in perceived hue: With sensitivity to blue reduced, blue-greens look greener, purples look redder, and dark blues look blacker (Fairchild 1998:5). Pickford (1951) noted that blue weakness owing purely to preretinal yellow pigmentation is associated with darkened violet. Few of these stimuli would look predominantly blue. In short, tritan defects render blues and greens less discriminable and blues blacker, and there would be less "perceptual pressure" to encode these color differences in language (Davies, Laws, Corbett, \& Jerrett 1998).

Is short-wavelength perception or discrimination restricted in speakers of grue or dark languages? The short answer may be yes. The distributions of grue and dark languages are nonrandom: Most languages of temperate-dwelling peoples distinguish the four basic categories of hue, whereas many other languages, and in specific those of more intraocularly pigmented and tropical-dwelling peoples, do not. Bornstein (1973) reported that the distributions of grue and dark languages related to a culture's residential proximity to the equator. For example, an internal analysis of African color-naming systems showed that the average deviation (north or south) from the equator of the green=blue semantic identity (having a single color term to describe greens and blues) is $16^{\circ}$, of the blue $=$ black identity is $11^{\circ}$, and of the green $=b l u e=b l a c k$ identity is $9^{\circ}$. (Lindsey \& Brown 2002, later reconfirmed this relation with a somewhat larger data set.) For example, Chichewa and Setswana are two grue languages spoken in southern Africa by people who receive 3.25 times more UV-B radiation than people who live in Greenwich, England (Davies, Corbet, 
Mtenje, \& Snowden 1995; Davies et al. 1992). If lens aging is linearly related to lifetime UV-B exposure, then a 98-year-old Englishman might have a lens as dense as the average 30-year-old South African (Lindsey \& Brown 2002). The density of yellow intraocular pigmentation and attendant tritan vision parallel the worldwide distribution of collapsed color-naming systems (Bornstein 1973; Lindsey \& Brown 2002).

Some time ago, Verriest (1963) showed that yellow filters in front of younger eyes cause a diminution of color discrimination which resembles the senile decrease and the tritan (blue-deficient) type of congenital color-vision deficiency. Further to this point, Lindsey and Brown (2002) experimentally tested these kinds of phototoxicity effects on color naming in a study that used computerized, colorimetric simulations of Munsell colors viewed through clear versus brunescent lenses. Young native American-English speakers used blue as a color name when the simulated lens through which they viewed colors was clear, but they named colors as though they were speaking grue or dark languages rather than American English when the simulated lens was dense. At the densest lens values, blue was replaced by green, analogous to grue, and to a lesser extent by gray, analogous to dark. Hardy, Frederick, Kay, and Werner (2005) later replicated Lindsey and Brown's (2002) lens-brunescence simulation (however, when the same physical stimuli were presented to younger and older observers, the two groups named the relevant stimuli in virtually the same way). Some boundaries between color-name loci, particularly between yellow and green and between green and blue, changed position along the color axis as denser lenses were simulated. These experimental within-subjects designs preclude cultural or language explanations for the result. Although they should not be accepted at face value (Regier \& Kay 2004), the qualitative similarity between color naming by speakers of grue and dark languages and color naming at the densest lens values in these experiments is arresting.

Finally, it is important to note that grue or dark could become established as predominant color terms in a language even if only a minority of a community were affected by tritan-like color anomalies because communication of color information requires color competence in speakers as well as listeners. The names for colors learned by children overlap those spoken by their mothers (Andrick \& Tager-Flusberg 1986). Lindsey and Brown (2002) argued that, if the fraction of individuals in the general population who are affected is $\alpha$, then $(1-\alpha)^{2}$ random dyads will involve a speaker and a listener who both have a color vocabulary that is not affected. For example, if $10 \%$ of the population were affected, then about $20 \%$ of all conversational partners would use grue; if $25 \%$ were affected, then about $45 \%$ would. This effect would be even more pronounced for triads or larger groups of people. In this way, grue or dark could become established and acceptable in a language even if some members of the community retained the ability to distinguish green from blue from black.

\section{Experiential explanations for grue}

Interactions among culture, language, cognition, and physiology that ultimately determine the usage of color terms are doubtlessly complex. Yellow intraocular filtration, which 
aids visual acuity and selectively attenuates short-wavelength visible radiation reaching the photoreceptors thereby decreasing the distinctive sensation of blueness, may help to explain in the paucity and polysemy of extant color terms and the promotion and distribution of grue and dark languages around the world. Of course, this biological account is only one, and it is not exclusive. As discussed, there is a variety of complementary experiential pathways to hue categorization and color naming that may be equally valid. Categorical perception of hue could be innate, as the infant research suggests, and later modified by language and experience along the lines outlined earlier. For example, Franklin, Davies, and their colleagues compared English and Himba groups on various color naming and search tasks. The Himba tribe is found in the Kaokoveld region of Northern Namibia. The Himba language, Otjihimba, has five basic color terms: otjivapa (white), otijzoozu (black), otjiserandu (red, orange, and pink), otjidumbu (yellow), and otjimburou (blue, green, and some purples). (It also has a few secondary and borrowed terms.) These authors predicted that, as there is a single Himba term for blue and green, if color naming affects color perception, search for one blue target among green distractors (and vice versa) would be faster and more accurate than search for one blue target among other blue distractors (or one green target among other green distractors) among English speakers who have distinct linguistic categories for blue and green but not among Himba speakers because search conditions would be within the one Himba green-blue category. Franklin, Clifford, Williamson, and Davies (2005) tested for categorical responding in English 2- to 4-yearolds, the age range for the onset of color term knowledge and in Himba toddlers. They found categorical perception irrespective of naming - that is, there were no cross-cultural differences in the extent of categorical perception - and categorical responding was not stronger in those children with more developed color-term knowledge. Their findings support universalist models of color categorization and suggest that color term knowledge does not modify categorical perception, at least during early childhood.

The authors also tested English and Himba adults (and they included a green-yellow set for which the Himba possessed separate terms). They found a significant category advantage (cross-category search was faster and more accurate than within-category search) in the English group, but not the Himba group. The adult language group that had a linguistic boundary for two colors performed their searches categorically; the adult group that categorized colors under a single name did not. Together, these results support robustness to the claim that the origins of hue categorization lay in perception not language.

Of course, language experience operates later in development. A complete account of hue categorization and color naming must take into account the constraints of vision system function and trichromacy, but it also needs to take into account our rich color experience as well as the pragmatics of cultural linguistic communication. Languages differ semantically, but obviously not without constraint; people cannot just call anything anything, after all. These constraints, however, are often a complex interface of both human cognitive universals and the particulars of cultures and languages (Stanlaw 1997). Basic hue categorization and color naming within and across cultures are as much influenced by constraints and commonalities imposed by visual-processing mechanisms as they are 
influenced by common culturally learned naming practices and the ways cultures come to form their naming systems.

Categorical perception in infants cannot be due solely to naming, but verbal labelling can explain some cross-cultural differences in categorical perception. Perhaps, then, there is an innate predisposition for categorization, but language or other types of learning can modify categorical perception and reorganize the representation of perceptual color space. If color perception is both innate and plastic, then these twin processes could result in compression and expansion of perceptual space for the areas of color space that are given the same term and for the areas of color space where there is a linguistic boundary. Such perceptual learning could also account for new categorical perceptions across newly learned boundaries and the attenuation of others, altering the structure of perceptual categorization. In this way, categorical perception present in infancy can be lost if a language does not reinforce the perception, and categorical perception may be induced and sharpened if a language does. Finally, categorical perception not present during infancy may later be induced if the child's ambient language distinguishes between color regions. Category training studies attest to the flexibility of categorical perception (Goldstone 1994; Goldstone, Lippa, \& Shiffrin 2001; Guenther, Husain, Cohen, \& ShinnCunningham 1999; Livingston, Andrews, \& Harnad 1998). Notably, Özgen and Davies (2002) showed that color discrimination could be improved by training and that learning could induce subdivision of a preexisting basic color category.

\section{Conclusions}

Physical attributes and properties of the world are complex and, like biological processes in the observer, constantly in flux. Through categorization, perception and cognition are organized so that psychological unity and coherence replace physical variety and biological instability. This chapter focuses on hue categorization and color naming. All physical stimuli in the color domain are not perceived as equally different from one another psychologically; rather, some are categorized together and treated as equivalent (but not necessarily as identical) based on their perceptual similarity. Whether they are adults or infants at the beginning of life, human beings perceive a set of such basic categories of hue. Psychophysical, developmental, and comparative findings further support this universalist position on basic hue categories and their biological substrates. Data on cultural diversity in hue categorization, and anthropological, linguistic, philosophical, and psychological opinion, point to systematic exceptions and variants in basic color naming, however. In this chapter, I attempted to reconcile the unity of native categorization processes with the diversity of cultural naming through a consideration of different possible developmental pathways between the two and the interplay of nature and nurture. There are nontrivial biological constraints on color categorization and color experience, and the initial phenomenology and psychophysics of color can be adequately explained by neurophysiologically identifiable mechanisms in color vision processing. The available evidence 
seems compatible with a position of universality that leads to expectations of probabilistic rather than deterministic cross-cultural correspondence.

The psychological structures that characterize color perception have been revealed in a series of psychophysical studies with adults, with infants, and with various infrahuman species, and they show that basic hue categorization possesses an identifiable physiological substrate. The data that these studies have yielded supplant an older psycholinguistic and anthropological literature that posited that language and culture alone influence or direct perceptual processes and thought even at the most basic level. Indeed, modern studies invert the traditional view and point to the influence of perceptual and cognitive forces on shaping language. Thus, modern psychophysical and perceptual data help to resolve an anthropological-linguistic question of direct and manifest importance to the study of neonate and infant cognition: Does perception influence language, or does language influence perception? Hue is a model domain for how categorical perception, linguistic relativity, and perceptual learning relate to one another. The existence in infancy of basic hue categories that appear to be firmly rooted in physiology strongly favors the primacy of perception. In color, however, relativism can overlay and modulate this universalist foundation. Languages exploit hard-wired perceptual discontinuities in forming color categories and other, presumably social, forces drive how many and (to some degree) which categories are formed. However, it is also the case that adaptive visual information processing alters perception and affects hue categorization, color naming, or both. A critical point of future research will be to further disentangle linguistic and physiological influences on conceptual hue categories.

\section{Acknowledgments}

This chapter summarizes selected aspects of my research, and portions of the text have appeared in previous scientific publications cited in the references. I thank C. Varron for assistance. Address correspondence to: Marc H. Bornstein, Child and Family Research, National Institute of Child Health and Human Development, National Institutes of Health, Suite 8030, 6705 Rockledge Drive, Bethesda MD 20892-7971, U.S.A. Email: Marc_H_Bornstein@nih.gov.

\section{References}

Ahissar, M., \& Hochstein, S. (1993). Attentional control of early perceptual learning. Proceedings of the National Academy of Sciences, USA, 90, 5718-5722.

Andrick, G. R., \& Tager-Flusberg, H. (1986). The acquisition of colour terms. Journal of Child Language, $13,119-134$.

Anglin, J. M. (1977). Word, object, and conceptual development. New York: Norton.

Ashbel, D. (1961). New world maps of global solar radiation (1957-1958). Jerusalem: Hebrew University, Department of Climatology and Meterology. 
Berlin, B., \& Kay, P. (1969). Basic color terms: Their universality and evolution. Berkeley, CA: University of California Press.

Best, C. T., McRoberts, G. W., \& Sithole, N. M. (1988). Examination of perceptual reorganization for nonnative speech contrasts: Zulu click discrimination by English-speaking adults and infants. Journal of Experimental Psychology: Human Perception \& Performance, 14, 345-360.

Blewitt, P. (1983). Dog versus collie: Vocabulary in speech to young children. Developmental Psychology, 19, 602-609.

Bornstein, M. H. (1973). Color vision and color naming: A psychophysiological hypothesis of cultural difference. Psychological Bulletin, 80, 257-285.

Bornstein, M. H. (1976). Name codes and color memory. American Journal of Psychology, 89, 269-279.

Bornstein, M. H. (1985). On the development of color naming in young children: Data and theory. Brain and Language, 26, 72-93.

Bornstein, M. H. (1987). Perceptual categories in vision and audition. In Harnad, S. (Ed.), Categorical perception: The groundwork of cognition. Cambridge, MA: Cambridge University Press.

Bornstein, M. H. (2006). Hue categorization and color naming: Physics to sensation to perception. In C. P. Biggam \& N. Pitchford (Eds.), Progress in colour studies: Psychological aspects. Amsterdam and Philadelphia: John Benjamins.

Bornstein, M. H., Kessen, W., \& Weiskopf, S. (1976a). The categories of hue in infancy. Science, 191, 201202.

Bornstein, M. H., Kessen, W., \& Weiskopf, S. (1976b). Color vision and hue categorization in young human infants. Journal of Experimental Psychology: Human Perception and Performance, 2, 115-129.

Bornstein, M. H., \& Korda, N. O. (1984). Discrimination and matching within and between hues measured by reaction times: Some implications for categorical perception and levels of information processing. Psychological Research, 46, 207-222.

Bornstein, M. H., \& Lamb, M. E. (Eds.). (2005) Developmental science: An advanced textbook (5e). Mawah, NJ: Lawrence Erlbaum Associates.

Bornstein, M. H., \& Monroe, M. D. (1978). Color-naming evidence for tritan vision in the fovea. American Journal of Optometry \& Physiological Optics, 55, 627-630.

Boynton, R. M., Fargo, L., Olson, C. X., \& Smallman, H. S. (1989). Category effects in color memory. Color Research \& Application, 14, 229-234.

Brown, R. (1973). A first language: The early stages. London: George Allen.

Catherwood, D., Crassini, B., \& Freiberg, K. (1987). The nature of infant memory for hue. British Journal of Developmental Psychology, 5, 385-394.

Collier, G. (1966). Categorías del color en Zinacantecan. In E. Z. Vogt (Ed.), Los Zinacatecos. Mexico City: Instituto Nacional Indigenista.

Conmklin, H. C. (1955). Hanunóo color categories. Southwestern Journal of Anthropology, 11, 339-344.

Davidoff, J. B. (1991). Cognition through color. Cambridge, MA: MIT Press.

Davidoff, J. B., Davies, I. R. L., \& Roberson, D. (1999). Colour categories in a stone-age tribe. Nature, 398, 203-204.

Davies, I. R. L., \& Corbett, G. (1994). The basic color terms of Russian. Linguistics, 32, 65-89.

Davies, I. R. L, Corbett, G., Mtenje, A., \& Snowden, P. (1995). The basic color terms of Chichewa. Lingua, 95, 259-278.

Davies, I. R. L., Laws, G., Corbett, G. C., \& Jerrett, D. J. (1998). Cross-cultural differences in colour vision: Acquired "colour-blindness" in Africa. Personality and Individual Differences, 25, 1153-1162.

Davies, I. R. L., MacDermid, C., Corbett, G. G., McGurk, D., Jerrett, D., Jerrett, T. \& Snowden, P. (1992). Color terms in Setswana: A linguistic and perceptual approach. Linguistics, 30, 1065-1103.

Dedrick, D. (1997). Colour categorization and the space between perception and language. Behavioral and Brain Sciences, 20, 187-188. 
Dedrick, D. (1998). Naming the rainbow. Colour language, colour science, and culture. Dordrecht: Kluwer Academic.

Dodt, E., Copenhaver, R. M., \& Gunkel, R. D. (1959). Electroretinographic measurement of spectral sensitivity in albinos, Caucasians, and Negroes. Archives of Ophthalmology, 62, 795-803.

Dubois, D. (1997). Cultural beliefs as nontrivial constraints on categorization: Evidence from colors and odors. Behavioral and Brain Sciences, 20, 188-189.

Eckhardt, R. A. (1966). Foveal luminosity functions of five Negroes in relation to macular and skin pigmentation. (Doctoral dissertation, Fordham University) Ann Arbor, MI: University Microfilms, No. 66-7091.

Eco, U. (1985). How culture conditions the colors we see. In M. Blonsky (Ed.), On signs (pp. 157-175). Oxford, UK: Blackwell.

Fahle, M., \& Poggio, T. (Eds.). (2002). Perceptual learning. Cambridge, MA: MIT Press.

Fairchild, M. D. (1998). Color appearance models. Reading, MA: Addison-Wesley.

Fischer, A. G. (1960). Latitudinal variations in organic diversity. Evolution, 14, 64-81.

Fitzpatrick, G. E. (1964). An investigation of the role of macular pigmentation in the spectral sensitivity to the short wavelengths. (Doctoral dissertation, Fordham University) Ann Arbor, MI.: University Microfilms, No. 64-13, 212.

Fletcher, R., \& Voke, J. (1985). Defective colour vision: Fundamentals diagnoses and management. Bristol: Adam Hilger Ltd.

Foley-Fisher J. A. (1968). Measurements of vernier acuity in white and coloured light. Vision Research, 8, 1055-1065.

Franklin, A., Clifford, A., Williamson, E., \& Davies, I. (2005). Color term knowledge does not affect categorical perception of color in toddlers. Journal of Experimental Child Psychology, 90, 114-141.

Franklin, A., \& Davies, I. R. L. (2004). New evidence for infant colour categories. British Journal of Developmental Psychology, 22, 349-377.

Frost, D. V. (1948). The relation of nutritional deficiencies to graying. Physiological Review, 28, 368-382.

Gage, J. (1997). Color and culture. In T. Lamb \& J. Bourriau (Eds.), Color: Art \& science (pp. 175-193). Cambridge, UK: Cambridge University Press.

Gellatly, A. (1995). Colourful Whorfian ideas: Linguistic and cultural influences on the perception and cognition of colour, and on the investigation of them. Mind \& Language, 10, 199-225.

Gladstone, W. E. (1858). Homer and Homeric age (Vol. 3). Oxford: Oxford University Press.

Golding, L. (1954). The absorption spectrum of the atmosphere. In G. P. Kuiper (Ed.), The earth as a planet. Chicago: University of Chicago Press.

Goldstone, R. L. (1994). Influences of categorisation on perceptual discrimination. Journal of Experimental Psychology: General, 123, 178-200.

Goldstone, R. L., Lippa, Y., \& Shiffrin, R. M. (2001). Altering object representations through category learning. Cognition, 78, 27-43.

Gottlieb, G. (1981). Roles of early experience in species-specific perceptual development. In R. N. Aslin, J. R. Alberts, \& M. R. Peterson (Eds.), Development of perception (Vol. 1). New York, NY: Academic Press.

Guenther, F. H., Husain, F. T., Cohen, M. A. , \& Shinn-Cunningham, B. G. (1999). Effects of categorisation and discrimination training on auditory perceptual space. Journal of the Acoustical Society of America, 106, 2900-2912.

Hardy, J. L., Frederick, C. M., Lay, P., \& Werner, J. S. (2005). Color naming, lens aging, and grue: What the optics of the aging eye can teach us about color language. Psychological Science, 16, 321-327.

Hardin, C. L. (1993). Color for philosophers: Unweaving the rainbow. Cambridge, MA: Hackett.

Harwerth, R. S., \& Sperling, H. G. (1971). Prolonged color blindness induced by intense spectral lights in rhesus monkeys. Science, 174, 520-523. 
Henderson, S. T. (1970). Daylight and its spectrum. New York: American Elsevier Co.

Hippisley, A. (2001). Basic blue in East Slavonic. Linguistics, 39, 15-179.

Ishak, I. G. H. (1951). The chromaticity co-ordinates for the standard illuminants, $S_{A}, S_{B}$, and $S_{C}$ of one British and fifteen Egyptian observers. Journal of Physiology, 115, 25-33.

Ishak, I. G. H. (1952a). The photopic luminosity curve for a group of fifteen Egyptian trichromats. Journal of the Optical Society of America, 42, 529-534.

Ishak, I. G. H. (1952b). The spectral chromaticity co-ordinates for one British and eight Egyptian trichromats. Journal of the Optical Society of America, 42, 534-539.

Javitt, J. C., \& Taylor, H. R. (1994). Cataract and latitude. Documenta Ophthalmologica, 88, 302-325.

Johnson, M. H. (2005). Developmental neuroscience. In M. H. Bornstein \& M. E. Lamb (Eds.), Developmental science: An advanced textbook (5 ed., pp. 187-222). Mahwah, NJ: Lawrence Erlbaum Associates.

Judd, D. B., Plaza, I., \& Farnsworth, D. (1950). Tritanopia with abnormally heavy ocular pigmentation. Journal of the Optical Society of America, 40, 833-841.

Kalmus, H. (1955). The familial distribution of congenital tritanopia. Annals of Human Genetics, 20, 3956.

Kay, P. (1975). Synchronic variability and diachronic change in basic color terms. Language in Society, 4, 257-270.

Kay, P., \& Berlin, B. (1997). Science $\neq$ imperialism: There are nontrivial constraints on color naming. Behavioral and Brain Science, 20, 196-201.

Kay, P., Berlin, B., Maffi, L., \& Merrifield, W. (1997). Color naming across languages. In C. L. Hardin \& L. Maffi (Eds.), Color categories in thought and language (pp. 21-56), Cambridge University Press.

Kay, P., \& Maffi, L. (2000). Color appearance and the emergence and evolution of basic color lexicons. American Anthropologist, 101, 743-760.

Kay, P., \& McDaniel, C. K. (1978). The linguistic significance of the meanings of basic color terms. Language, 54, 610-646.

Kimball, H. H. (1935). Intensity of solar radiation at the surface of the earth, and its variations with latitude, altitude, season and time of day. Monthly Weather Review, 63, 1-4.

Krauss, R. M. (1968). Language as a symbolic process. American Scientist, 56, 265-278.

Lin, H., Luo, M. R., MacDonald, L. W., \& Tarrant, A. W. S. (2001). A cross-cultural color-naming study: Part I-Using an unconstrained method. Color Research \& Application, 26, 40-60.

Lindsey, D. T., \& Brown, A. M. (2002). Color naming and the phototoxic effects of sunlight on the eye. Psychological Science, 13, 506-512.

Livingston, K. R., Andrews, J. K., \& Harnad, S. (1998). Categorical perception effects induced by category learning. Journal of Experimental Psychology: Learning, Memory, and Cognition, 24, 732-753.

Luckiesh, M., \& Moss, F. K. (1925). The effect on visual acuity of shortening the spectrum at the blue end. Journal of Optical Society of America, 10, 275-282.

Lucy, J. A. (Ed.). (1992). Language diversity and thought: A reformulation of the linguistic relativity hypothesis. Cambridge, MA: Cambridge University Press.

Ludvigh, E., \& McCarthy, E. F. (1938). Absorption of visible light by the refractive media of the human eye. Archives of Ophthalmology, 20, 37-51.

Luria, S. M. (1972). Vision with chromatic filters. American Journal of Optometry \& Archives of American Academy of Optometry, 49, 818-829.

MacLaury, R. E. (2001). Color terms. In M. Haspelmath, E. König, W. Oesterreicher, \& W. Raible (Eds.), Language typology and language universals (Vol. 2, pp. 1227-1251). Berlin: Walter de Gruyter.

Matsuno, T., Kawai, N., \& Matsuzawa, T. (2004). Color classification by chimpanzees (Pan troglodytes) in a matching-to-sample task. Behavioural Brain Research, 148, 157-165. 
Millodot, M. (1976). The influence of age on the chromatic aberration of the eye. Albrecht Von Graefes Archiv für Klinische und Experimentelle Ophthalmologie, 198, 235-243.

Moskovič, V. A. (1968). Iz polesskoj termiologii cvetooboznačenij [On Polesje lexicon of color terms]. In V. V. Martynov \& N. I. Tolstoj (Eds.), Polesje (Lingvistika. Arxeologija. Toponimika) (pp. 126-161). Moscow: Nauka.

Norren D. V., \& Vos J. J. (1974). Spectral transmission of the human ocular media. Vision Research, 14, 1237-1244.

Nosofsky, R. M. (1986). Attention, similarity, and the identification-categorization relationship. Journal Experimental Psychology: General, 115, 39-57.

Osborn, W. B. (1929). Preliminary observations to determine the ultra-violet content of South African sunlight. South African Journal of Science, 26, 527-540.

Overton, W. F. (1998). Developmental psychology: Philosophy, concepts, and methodology. In R. M. Lerner (Ed.), W. Damon (Series Ed.), Handbook of child psychology: Vol. 1. Theoretical models of human development (5e, pp. 107-188). New York: Wiley.

Özgen, E., \& Davies, I. R. L. (2002). Acquisition of categorical color perception: A perceptual learning approach to the Linguistic Relativity Hypothesis. Journal of Experimental Psychology: General, 131, 477-493.

Paramei, G. V. (2005). Singing the Russian blues: An argument for culturally basic color terms. CrossCultural Research, 39, 10-34.

Pastoureau, M. (2000). Le bleu, histoire d'une couleur. Paris: Éditions du Seuil.

Pickford, R. W. (1951). Individual differences in colour vision. London: Routledge and Kegan Paul.

Pitchford, N. J., \& Mullen, K. T. (2003). The development of conceptual colour categories in pre-school children: Influence of perceptual categorization. Visual Cognition, 10, 51-77.

Pokorny, J. Smith, V. C., Verriest, G., \& Pinckers, A. J. L. G. (1979). Congenital and acquired colour vision defects. London: Grune-Stratton.

Raskin, L., Maital, S., \& Bornstein, M. H. (1983). Perceptual categorization of color: A life-span study. Psychological Research, 45, 135-145.

Ratner, C. (1989). A sociohistorical critique of naturalistic theories of colour perception. Journal of Mind and Behavior, 10, 361-373.

Ray, V. F. (1952). Techniques and problems in the study of human color perception. Southwestern Journal of Anthropology, 8, 251-259.

Ray, V. F. (1953). Human color perception and behavioral response. Transactions of the New York Academy of Science, 16, 98-104.

Reading, V. M., \& Weale R. A. (1974). Macular pigment and chromatic aberration. Journal of the Optical Society of America, 64, 231-234.

Regier, T., \& Kay, P. (2004). Color naming and sunlight: Commentary on Lindsey and Brown (2002). Psychological Science, 15, 289-290.

Reuning, H. (1988). Testing Bushmen in the central Kalahari. In S. H. Irvine \& J. W. Berry (Eds.), Human abilities in cultural context. Cambridge: Cambridge University Press.

Rivers, W. H. R. (1901a). Introduction. In A. C. Haddon (Ed.), Reports on the Cambridge anthropological expedition to the Torres Straits. Cambridge: Cambridge University Press.

Rivers, W. H. R. (1901b). Primitive colour vision. Popular Science Monthly, 59, 44-58.

Rivers, W. H. R. (1903). Observations on the vision of the Uralis and Sholagas. Bulletin of the Madraas Government Museum, 5, 3-18.

Rivers, W. H. R. (1905). Observations on the senses of the Todas. British Journal of Psychology, 1, 321-396.

Roberson, D. (2005). Color categories are culturally diverse in cognition as well as in language. CrossCultural Research, 39, 56-71. 
Roberson, D., Davidoff, J., \& Braisby, N. (1999). Similarity and categorization: Neuropsychological evidence for a dissociation in explicit categorization tasks. Cognition, 71, 1-42.

Roberson, D., Davies, I., \& Davidoff, J. (2000). Color categories are not universal: Replications and new evidence from a stone-age culture. Journal of Experimental Psychology: General, 129, 369-398.

Sacks, O. W. (1997). The island of the colorblind and Cycad Island. New York: Knopf.

Saunders, B. A. C., \& van Brakel, J. (1997). Are there nontrivial constraints on colour categorization? Behavioral and Brain Sciences, 20, 167-179.

Shiu, L., \& Pashler, H. (1992). Improvement in line orientation discrimination is retinally local but dependent on cognitive set. Perception \& Psychophysics, 52, 582-588.

Simon, K. (1951). Colour vision of Bugandan Africans. The East African Medical Journal, 74-79.

Simpson, C. (1997). The irrelevance of the psychophysical argument. Behavioral and Brain Sciences, 20, 207.

Sperling, H. G., \& Hsia, Y. (1957). Some comparisons among spectral sensitivity data obtained in different retinal locations and with two sizes of foveal stimulus. Journal of the Optical Society of America, 47, $707-713$

Spier, L. (1928). Hauasupai ethnography. Anthropological Papers on the American Museum of Natural History, 24, 81-408.

Stanlaw, J. (1997). Two observations on culture contact and the Japanese color nomenclature system. In C. L. Hardin \& L. Maffi (Eds.), Color categories in thought and language (pp. 240-260). New York, NY: Cambridge University Press.

Tornay, S. (1978). Introduction. In S. Tornay (Ed.), Voir et nommer les couleurs. Laboratoire d'Ethnologie et de Sociologie Comparative.

Uchikawa, K., \& Boynton, R. M. (1987). Categorical color perception of Japanese observers: Comparison with that of Americans. Vision Research, 27, 1825-1833.

Van Laar, D. (1997). Ekphrasis in colour categorisation: Time for research, or time for revolution? Behavioral and Brain Sciences, 20, 210.

Verriest, G. (1963). Further studies on acquired deficiency of color discrimination. Journal of the Optical Society of America, 53, 185-195.

Wald, G. (1967). Blue-blindness in the normal fovea. Journal of the Optical Society of America, 57, 12891301.

Walls, G. L. (1942). The vertebrate eye and its adaptive radiation. Oxford, UK: Cranbrook Institute of Science.

Walls, G. L. (1964). Notes on four tritanopes. Vision Research, 4, 3-16.

Weale, R. A. (1988). Age and the transmittance of the human crystalline lens. Journal of Physiology, 395, 577-587.

Webster, M. A., Webster, S. M., Bharadwadj, S., Verma, R., Jaikumar, J., Madan, G., \& Vaithilingam, E. (2002). Variations in normal color vision III. Unique hues in Indian an United States observers. Journal of the Optical Society of America A, 19, 1951-1962.

Werker, J. F. (1990). Cross-language speech perception: Developmental change does not involve loss. In H. Nusbaum \& J. Goodman (Eds.), The transition from speech sounds to spoken words: The development of speech perception. Cambridge, MA: MIT Press.

Werker, J. F., \& Tees, R. C. (1984). Cross-language speech perception: Evidence for perceptual reorganization during the first year of life. Infant Behavior and Development, 7, 49-63.

Werner, J. S. (2000). Senescence of color vision mechanisms from the first days of life. Vision Science and its Applications. Optical Society of America Technical Digest Series, 71-74.

Werner, J. S. (1991). The damaging effects of light on the eye and implications for understanding changes in vision across the life span. In P. Bagnoli \& W. Hodos (Eds.), The changing visual system (pp. 295309). New York: Plenum Press. 
Werner, J. S., Peterzell, D. H., \& Scheetz, A. J. (1990). Light, vision, and aging. Optometry and Vision Science, 67, 214-229.

Werner J. S., Steele V. G., \& Pfoff, D. S. (1989). Loss of human photoreceptor sensitivity associated with chronic exposure to ultraviolet radiation. Ophthalmology, 96, 1552-1558.

Whorf, B. L. (1950). Four articles on metalinguistics. Washington, DC: Foreign Service Institute.

Whorf, B. L. (1964). Language, thought and reality. Cambridge MA: MIT Press.

Wortman, J. B. (2004). Effects of latitudinal position on color perception: The case of Brazil. Unpublished manuscript. University of California, San Diego.

Wright, W. D. (1952). The characteristics of tritanopia. Journal of the Optical Society of America, 42, 509521.

Young, R. W. (1991). Age-related cataract. New York: Oxford University Press.

Young, R. W. (1994). The family of sunlight-related eye diseases. Optometry and Vision Science, 71, 124144. 



\title{
Individual and population differences in focal colors
}

\author{
Michael A. Webster ${ }^{a}$ and Paul Kay ${ }^{b}$ \\ a Department of Psychology, University of Nevada, Reno, USA \\ ${ }^{b}$ International Computer Science Institute, Berkeley, CA, and University \\ of California, Berkeley, USA
}

\begin{abstract}
Using the data of the World Color Survey, variation in the selection of focal colors both within languages and across languages was studied. The variation within languages was found to be much greater than the variation across languages. For example, for color terms in different languages that roughly translate as English 'red', focus placements were found to cluster to a much greater extent across languages than within languages, although statistically significant differences in cross-language average focal choices were found. The resulting picture is one of constrained but not identical focus placements across languages and impressively greater variation in focus placements within than across languages.
\end{abstract}

\section{Introduction}

Perhaps the central problem in understanding color appearance is that the only available measures of it are subjective. Much is now known about the neural mechanisms underlying the early encoding of color information in the human visual system, yet few clear links have been identified between these mechanisms and the perceptual organization of color experience [For recent reviews see (Gegenfurtner \& Sharpe 1999; Mausfeld \& Heyer 2004)]. Without an objective test for "red", studies of color appearance have instead had to rely on characterizing how observers describe the stimulus, and this has left open the debate of whether these descriptions reflect the characteristics of perceptual or linguistic processes. Arguments in this debate have hinged largely on the question of whether different individuals label spectral stimuli in similar or different ways. Comparisons of color naming across languages point to a remarkably consistent structure of color categories (Berlin \& Kay 1969; Kay \& Regier 2003). Though exceptions and counter-arguments have been noted by a number of authors (Davidoff 2001; Davidoff et al. 1999; Gage 1993; Jameson \& D'Andrade 1997; Lucy 1997; Lyons 1995; Roberson et al. 2002; Saunders \& van Brakel 1997), the observed similarities have been taken by many as strong evidence for universal tendencies in color experience that are shaped by common perceptual processes (Boynton \& Olson 1990; Chapanis 1965; Hardin 1988; Kaiser \& Boynton 1996; Kay \& 
McDaniel 1978; Ratliff 1976; Shepard 1992). Yet within-language studies of color naming, and in particular of the loci of perceptually pure or unique hues, have also shown that these can vary widely from one individual to another within a given population (Kuehni 2004), and this suggests that something about the underlying processes is highly labile. In this chapter we examine the potential sources and nature of inter-individual and interlanguage variability and what these mean for the notion of universal color categories. While individual differences have been taken by some as evidence for linguistic relativity (Saunders \& van Brakel 1997), we argue that they instead suggest that the vagaries of particular languages may often place only a weak constraint on color categories, and that the factors that underlie within-language differences may themselves show strong universal tendencies.

\section{The World Color Survey}

Berlin and Kay (1969) advanced a hypothesis of universals in cross-language color naming in a study that compared color naming across 20 languages. Naming was assessed by asking individuals to label the colors they saw in a palette of Munsell chips that varied in hue and lightness (Munsell Value) at a high saturation (Munsell Chroma; see Figure 1A). The chips chosen as the best examples of color terms by speakers of these languages were also elicited. The responses of the participants of each language were aggregated to produce a naming pattern and a best example pattern for each language. The best example ("focal") choices clustered in small regions of the palette, leading to the proposal that color categories are similar across languages and are restricted to a set of 11 basic terms. The primary difference found among languages was in the number of basic color terms, and these varied in a consistent pattern: languages with the same number of terms tended to have terms of similar denotation. The resulting typology of basic color term systems formed an implicational hierarchy. For example, languages with only three basic color terms tended strongly to have terms focused in black, white and red. This in turn suggested that color terms are added to the lexicon in a strongly constrained order. These results were taken to imply a strongly universal pattern in color naming.

Since Berlin and Kay (1969) there has been a large number of studies arguing both for and against the notion of color universals (see, e.g., the papers in Hardin \& Maffi 1997). Criticisms of the 1969 study included the points that in most cases only one speaker was tested for each language, that these speakers also spoke English and were living in the US, and that the languages were mostly restricted to written languages of industrialized societies. Thus the similarities in color naming might have resulted from the shared history and similar experiences of the speakers and not been representative of the world's languages. The World Color Survey (WCS) was in part undertaken to address these concerns, by collecting color naming data for a much larger sample of languages and speakers (Kay et al. 1997). The data, which are now available online at http://www.icsi.berkeley.edu/wcs/data.html, include color naming and focal (best example) responses for 110 unwritten languages, with an average of 24 speakers per language. 
The respondents were the most monolingual persons obtainable in the communities studied and were tested in their own languages and in their local communities. The 110 WCS languages represent 45 different language families (see Kay \& Regier 2003:9087 for the list).

Recently, Kay and Regier (2003) analyzed the WCS data to evaluate whether the terms of different languages cluster in similar ways, that is, to perform explicit statistical tests on the evident, but primarily impressionistic, clustering observed by Berlin and Kay in a smaller sample of languages. For each speaker they calculated the centroid (i.e., the average value on each dimension) of the set of chips that were labeled with each term (after transforming the Munsell coordinates of the stimulus chips into the CIEL ${ }^{*} a^{*}{ }^{*}$ uniform color space). Figure 1 reproduces from their paper the resulting contour plot of these centroids, and also shows the location of the focal colors for English terms (Sturges \& Whitfield 1995). The English focal choices fall at or near the peaks of the naming centroid distribution for the WCS languages. Moreover, the relatively small discrepancies between the English focal choices and WCS naming centroid peaks are plausibly explained by the fact that the WCS data pictured are for naming centroids, while the English data are average focal (i.e., best example) choices. Most of the WCS languages included 'green' and 'blue' under the same name, and similarly for 'yellow' and 'orange'; one would expect a naming centroid for a 'green-or-blue' 'grue' term to fall between focal 'green' and focal 'blue' (as observed in Figure 1). For the same reason one would expect the naming centroid for a term covering 'yellow' and 'orange' to fall somewhere between the best examples of 'yellow' and 'orange' terms in languages which have them (as may also be observed in Figure 1). Kay and Regier also show statistically that significantly more of the WCS speaker centroids fell on the chips representing the English foci than on the remaining chips in the palette.

To test whether the centroids for different WCS languages tend to cluster, Kay and Regier first calculated the distance $D$ between each term centroid in the WCS and the closest term centroid in each other WCS language and summed these distances. Intuitively, the smaller the value of $D$ the greater the clustering in the WCS dataset. To perform a Monte Carlo test of the $D$ statistic, they then created hypothetical WCS datasets by taking each WCS language, as represented by its term centroids in CIEL ${ }^{\star} \mathrm{a}^{*} \mathrm{~b}^{*}$ space, and rotated the data for the language in the hue dimension by a randomly selected angle - the same angle for each term in a language and a different angle for each language. The $D$ statistic was then calculated for each of the 1,000 hypothetical WCS datasets. The value of $D$ in the actual WCS dataset was found to be lower than the lowest $D$ value of any of the 1,000 hypothetical WCS-like datasets. This Monte Carlo test established that the WCS naming centroids are significantly clustered in color space, with $p<.001$. A similar Monte Carlo analysis showed that the WCS clusters are in fact similar to the locations for the original Berlin and Kay terms $(p<.001)$, demonstrating that the clusters of favored colors in unwritten languages of low-technology societies are close to the favored colors of the written languages of high-technology societies. On the whole, these results demonstrate that different languages tend to parse color space similarly and that written languages, including English, are not exceptional in this regard. 

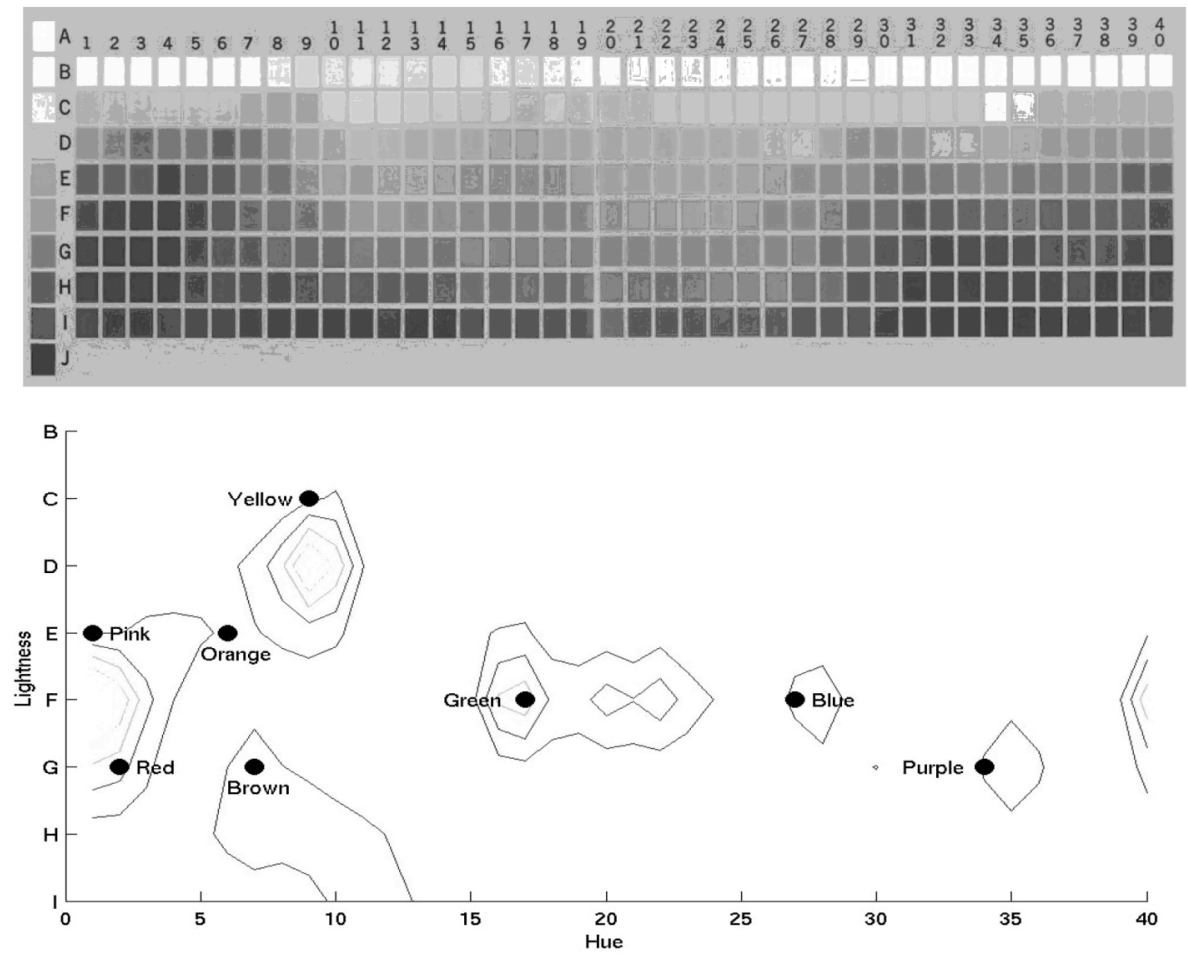

Figure 1. Contour plot, over the Munsell stimulus space (illustrated by the color panel), of the number of naming centroids in the WCS data set. Each contour represents centroids of 100 individual speakers. Filled circles represent average English focal choices observed by Sturges and Whitfield (1995). [Adapted from Kay and Regier, 2003, Figure 4b. See text for further explanation.] A version of this figure in full color can be found on p. xvii.

\section{Individual differences in color naming}

In their 1969 study Berlin and Kay also drew attention to the large individual differences within a language. In particular, they noted that the focal colors for corresponding terms from speakers of the different languages they tested were more similar than were the foci obtained in a separate study of 40 respondents from a single language (the Mayan language Tzeltal). Individual differences like these have been extensively investigated in psychophysical studies of color appearance. Most of this work has concentrated on the unique hues the stimuli that are perceived as pure red, green, blue, or yellow, and which are assumed to represent landmarks of color coding in many models of human color vision (De Valois \& De Valois 1993; Hurvich \& Jameson 1957; Kaiser \& Boynton 1996). The loci for these hues can differ dramatically from one person to the next (Jordan \& Mollon 1995; Kuehni 2004; Pridmore 1999; Schefrin \& Werner 1990; Volbrecht et al. 1997). For example, the wavelength that observers select as unique green can vary by up to $80 \mathrm{~nm}$ between sub- 


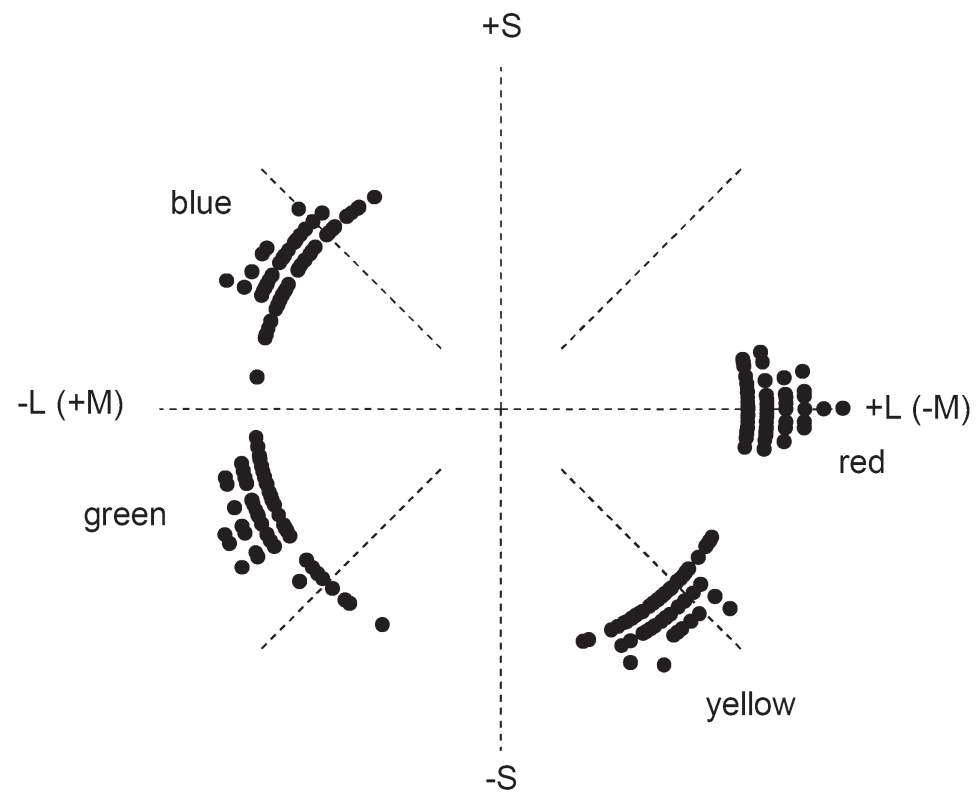

Figure 2. Individual differences in unique hues for 51 observers (Adapted from Webster et al., 2000, Figure 4). Points are plotted in terms of a color space whose two axes represent changes in the ratio of long- to middle-wavelength cone response (horizontal axis) and variation in short wavelength cone response (vertical axis), at constant luminance.

jects, that is, over a quarter of the visible spectrum. Comparable differences are also large for the broader spectra characteristic of natural color signals (Kuehni 2001; Webster et al. 2000), and thus do not simply reflect observers' inexperience with monochromatic lights. For example, Figure 2 plots the distribution in a cone-response space of hue angles chosen as unique red, green, blue or yellow for moderately saturated stimuli for 51 color-normal observers (Webster et al. 2000). The settings for unique green span a range of $60 \mathrm{degs}$, and thus include a large fraction of the color circle, and this range remains large $(\sim 45 \mathrm{deg})$ even when the settings are restricted only to the subset of subjects who set the unique hues reliably. For the remaining hues the variation is less, but still striking, in the sense that what some English speakers would call a pure yellow will be described as clearly too reddish or too greenish by others. Similar variations in perceived hue and lightness are also found for intermediate or binary hues such as orange and purple (Malkoc et al. 2002). In this chapter we consider possible sources of this variation and how it influences the focal colors measured both within and between different populations of observers.

Before exploring this question, it is worth pausing to note the location of the stimuli that observers select for unique hues. In Figure 2 these loci are plotted by their coordinates within a color plane whose two dimensions correspond to the two color-opponent axes that characterize color coding at early post-receptoral stages of the visual system (Derrington et al. 1984; MacLeod \& Boynton 1979). While reds tend to cluster around one of 
these axes, the remaining hues fall at intermediate angles in the space (Krauskopf et al. 1982). It is in fact this discrepancy - between the axes defined by the subjective unique hues versus the axes defined by psychophysical and physiological measures of chromatic sensitivity - which is at the heart of the explanatory gap between the neural and phenomenological organization of color vision, a point which has been made previously with specific regard to basic color terms (Jameson \& D'Andrade 1997). That is, the stimuli that are thought to isolate pure chromatic sensations are not the stimuli that isolate the cardinal chromatic axes of early color vision, nor do the known transformations of color signals in visual cortex suggest that the unique hues are special (Lennie et al. 1990). Yet these hue sensations seem special in the phenomenological experience of color.

\section{Sources of variation in normal color vision}

The bases for the prominent individual differences in color appearance remain unknown, and controversial. This is not surprising, because again it has not been possible to link the hue sensations to independently identifiable processes in the visual system. Speculations about the sources of normal variation include differences in the physiology, visual environment, and cultural environment of individuals. We consider each of these factors in turn, though it should be emphasized that they are frequently not separable. For example, the colors in an observer's environment are shaped by how color is used by the observer's culture, and physiological differences between observers (e.g., in how strongly their lens filters the light) depend both on their environment and their particular exposure to it (Werner 2000). It is important to note also that to the extent that any of these factors affect differences in color appearance, they are also important for understanding the similarities or universal tendencies in color naming. For example, if differences in physiology or environment are capable of causing differences in color naming, then uniformities in color naming imply uniformities in the color-relevant aspects of physiology or environment.

\section{Physiological differences}

Differences in chromatic sensitivity among individuals with normal color vision are large. Most of these differences have been characterized at the level of the retina and the optics of the ocular media (the substructures of the eye, such as the lens, which physically affect the light striking the retina), though there is no reason to expect that important differences do not occur throughout the visual pathway. The light reaching the photoreceptors is filtered by inert screening pigments in the lens and, around the fovea (where vision is best), by the macular pigment. Both these media selectively absorb at shorter wavelengths, reducing the intensity of "blue" light available at the receptors. Individual differences in lens and macular density are pronounced (Bone \& Sparrock 1971; Van Norren \& Vos 1974; Werner 1996), and are a primary source of normal variation in color matching (Webster \& MacLeod 1988). The spectral sensitivity of the photoreceptors can also vary in the wave- 
length of peak sensitivity because of polymorphisms in the genes encoding the pigments (Sharpe et al. 1999), and can vary in bandwidth because of differences in pigment density (Smith et al. 1976). Jameson et al. (2001), using a novel procedure, reported that the color experience of women who may possess more than three retinal photopigments by virtue of X-chromosome-inherited opsin gene dimorphisms may be richer than that of other humans, although other efforts to find perceptual effects of inferred human female tetrachromacy have not succeeded (e.g., Jordan \& Mollon 1993; Nagy et al. 1981). There are also striking differences in the relative numbers of the three different types of cone receptors. For example, humans have on average twice as many $\mathrm{L}$ cones as $\mathrm{M}$ cones, yet in individual subjects this ratio has been found to vary by over a 30-fold range (Carroll et al. 2002).

These physiological differences lead to obvious differences in visual sensitivity, for example in the relative sensitivity to different wavelengths. However, it is much less obvious how they contribute to color appearance. Webster et al. (2000) compared the individual differences in unique hues to the variations that would be predicted from estimates of normal variations in the lens and macular pigment and in the cone spectra. Because these factors affect a broad range of wavelengths they should influence more than one hue in similar ways in different observers, and thus they predict high correlations across observers between the settings for different unique hues. Yet the observed variations in the unique hues are remarkably independent. The effects of these sensitivity factors on color appearance have also been assessed by studying changes in color judgments with aging. The density of the lens pigment increases with age, so that the same spectrum that is achromatic to the young eye will be greatly attenuated at short wavelengths in the older eye. Yet the achromatic locus and the unique hues instead remain surprisingly stable across the lifespan (Schefrin \& Werner 1990; Werner \& Schefrin 1993). Finally, a number of studies have also tested whether the unique hues can be tied to variations in the relative numbers of different cones, and recent work has established that the differences in the L:M ratio are far too large to account for the range of individual differences in the unique hues (Brainard et al. 2000; Miyahara et al. 1998).

Despite these negative examples, there are some cases where differences in spectral sensitivity could play a role. One interesting case involves the unique hues of red-green anomalous trichromats, who have three types of cones like color normal observers but with altered photopigments so that the difference in spectral sensitivity between the $\mathrm{L}$ and $\mathrm{M}$ cones is very small. Deuteranomalous individuals set unique yellow to much longer wavelengths than normals, and this is important because it suggests there are limits to the degree to which constant color appearance can be maintained despite sensitivity differences (Neitz et al. 2002). A more controversial case is the recent proposal by Lindsey and Brown (2002) that differences in color naming may be tied to differences in lens pigmentation and retinal damage due to differences in exposure to sunlight. They observed that languages that lack a distinct 'blue' term tend to be spoken in areas of the world that have a high exposure to ultraviolet light, and suggested that the phototoxic effects of UV exposure might have reduced short-wavelength sensitivity to the point where blues effectively drop out of the spectrum and the color lexicon. This account was intended to explain dif- 
ferences between languages (those with and without a separate term for blue), and not within them, and whether it succeeds in this regard has been questioned on the basis of cross-language color naming patterns (Regier \& Kay 2004; Lazar-Meyn 2004; see also a rejoinder by Lindsey \& Brown 2004). A recent study casts further doubt on this explanation of the absence of 'blue' terms in equatorial languages and, in any case, shows it is unlikely to provide a plausible explanation for individual differences within a population. As we have noted these differences are at most weakly related to lens density. In a direct test, Hardy et al. (2004) show that older and younger observers do not differ in how blue they rate spectral stimuli, despite their large differences in lens density. Hardy et al. also show that lens density itself - which they measured directly - appears unrelated to the degree to which spectral stimuli are rated as blue.

\section{Environmental differences}

The failure to find clear correlates between color appearance and chromatic sensitivity has led a number of authors to suggest that the factors shaping color perception are in the environment rather than the observer (Shepard 1992). For example, Pokorny and Smith (1977) and Mollon (1982) argued that unique yellow - the putative null point of the red-green opponent process - may correspond to the average illuminant in the observer's environment. This could readily explain how individuals with very different cone ratios can have similar unique yellows (Brainard et al. 2000), or how a single observer can maintain stable color percepts despite aging (Werner \& Schefrin 1993) or a developing cataract (Delahunt et al. 2004). In each of these cases color perception could be calibrated according to a similar pattern of stimulation. Such proposals hinge on the idea that the visual system can adapt in order to compensate for variations in the observer, and there is abundant evidence for these adaptive adjustments, beginning as early as the receptors and extending throughout the visual system (Webster 2003).

In an elegant extension of these ideas, Yendrikhovskij (2001) used the statistical technique of cluster analysis to examine the distribution in color space of the colors that can be observed in natural images and showed that the resulting clusters - in effect, the regions of color space most often represented in natural images - fell near the foci for basic color terms. This suggests that the special status of these terms may be because they correspond to the relative frequencies of the spectral properties of objects rather than to privileged axes in the mechanisms encoding color. The analysis also provided a possible account of the relative salience of different color terms. For example, 'red' is the earliest chromatic color term to emerge in the evolutionary sequence for languages identified by Berlin and Kay (1969) and in individual development (Kay \& Maffi 1999), and this could be predicted by supposing that the distinctiveness of 'red' results because of the frequency of these stimuli and the distinctness of the cluster they form (Yendrikhovskij 2001).

If color perception reflects an adaptation to the color statistics of the environment, then variations in these statistics could underlie differences in color judgments. Natural environments do in fact vary widely in their color properties. For example, Webster and Mollon (1997) measured the color distributions for a range of outdoor scenes from dif- 
ferent environments in the US and India, and showed that these distributions varied both in the mean color and in how the set of colors were distributed around the mean. In lush environments color differences within a scene tend to lie along an axis that varies signals in the short-wave sensitive cones (the vertical axis of Figure 2), while in arid environments the distributions are instead rotated toward bluish-yellowish axes (the negative diagonal of Figure 2). Webster and Mollon also showed that measures of color appearance were strongly affected when observers were adapted to the set of colors drawn from these distributions. More recently, a similar pattern of variation was found to occur within the same environments as the seasons change (Mizokami et al. 2003). One interesting aspect of this seasonal difference is that changes in vegetation cause the average color in scenes to vary along the L vs. M axis of cone-opponent space (the horizontal axis of Figure 2). This is the chromatic dimension that distinguishes ripening fruits from foliage (Regan et al. 2001), which may have been the driving force in the evolution of trichromacy in primate color vision (Mollon 1989; Polyak 1957). (An alternative suggestion is the L vs. M axis evolved to allow primates to judge the complexion of conspecifics; Changizi et al. 2006; Mollon 1989.) As shown in Figure 2, the $+\mathrm{L}$ pole of the $\mathrm{L}$ vs. $\mathrm{M}$ axis is also the chromatic angle that unique reds tend to cluster along [though precisely how close the mean red settings are to the $+\mathrm{L}$ pole is uncertain; Webster et al. (2000, 2002); Wuerger et al. (2005)]. These observations jointly make it tempting to speculate that the special salience of red may be related to the special importance of this axis for our foraging primate ancestors. That is, red may have achieved special salience for our primate ancestors because it served the uniquely important evolutionary function of signaling ripeness (or skin tone). However, whatever value this speculation may have, it is not apparent how it might be reconciled with the large differences in hue settings of contemporary humans.

\section{Cultural differences}

The concordance of basic color terms across languages provides strong evidence for universal tendencies in color naming, but does not preclude all cultural or linguistic influence. This is an aspect of color naming that has been extensively debated elsewhere (e.g., Hardin \& Maffi 1997; Saunders \& van Brakel 1997). Most of this debate has focused on how linguistic or cultural differences might or might not contribute to differences between groups, and it is less obvious how they might give rise to variations within a population. However, color judgments are inherently subjective, and consequently may be susceptible to criterion effects that can be biased by individually experienced linguistic or cultural contexts. It is also possible that variability in focal hues arises because the latter are only weakly constrained by linguistic categories. For the physiological or environmental variables we considered above, we implicitly assumed that an individual's focal setting would be pinpointed by characteristics of the visual system or the world and that the differences between observers would thus be closely tied to these differences. However, to the extent that color categories are conceptual, they might also vary according to how much information an observer has about them, and the specific prototypes this allows them to form (Rosch 1975). 


\section{Focal color differences in Indian and United States observers}

Given the large variation in color naming among individuals and the fact that these variations could plausibly arise from many different factors, it would be remarkable if the focal colors for different populations did not show some amount of nonrandom variation. Webster et al. (2002) tested for these population differences by comparing color judgments for a set of observers in India and the United States. The US observers were college students in Reno, Nevada, tested in English. The Indian participants included college students in the city of Chennai (formerly Madras) who were fluent in and also tested in English, additional urban residents of Chennai tested in Tamil, and two groups of monolingual rural farmers tested in regions of Tamil Nadu (in Tamil) or Maharashtra (in Marathi).

In one task, observers selected the best examples of the corresponding terms for 'red', 'green', 'blue', or 'yellow' (as well as the binary hues 'orange' and 'purple') from a Munsell palette that was very similar to the palette used by Berlin and Kay (1969). (See Figure 1a). In a second task, specially printed palettes were used to display a finely graded series of hues spanning a range of reds, of greens, of blues, and of yellows, and subjects were instead asked to make a unique hue judgment for each of the four terms (e.g. by pointing to the blue that did not appear too reddish or too greenish). Figure 3 shows histograms of the focal color choices from the Munsell palette, formed by collapsing across different lightness levels to find the hue (palette column) that each observer chose for each term. The different panels are for different groups or testing conditions, with the US observers shown in the bottom two panels. There are two notable features in these results. First, the modal values of the focal hues for the different groups fall along similar columns in the palette, and in fact were identical for all groups for selections corresponding to 'red' and 'yellow'. This is consistent with a strong common basis for color naming across the different groups. The second feature is that - at a finer level - the means of the distributions are not the same. For example, compared to the US observers, the Indian observers tended to choose yellows and reds that were significantly shifted toward orange, and chose blues that were even more strongly shifted toward green (although it is possible that for some of these observers, the term interpreted as a 'blue' term was really a 'grue' term). Similar differences were also found for the unique hue judgments. These differences suggest that while basic color terms are similar across the groups, their specific foci can, and probably often do, vary in real, if modest, ways across different populations.

The Indian and US groups tested by Webster et al. (2002) differed along many of the dimensions we considered in the preceding section, in that they were drawn from different ethnicities, different languages, and different color environments. Thus, it is not clear what the basis of the focal color differences might be, and the aim was not to identify the source of any potential differences, but rather to maximize the chance of finding them. However, one potential factor that probably can be safely excluded is differences in the immediate testing environment. The college students in Chennai and in Reno were each tested under both natural outdoor lighting and incandescent lighting, illuminants with very different spectra [The top two panels of Figure 3 show the results for the Indian (ESO) subjects, tested in(doors) and out(doors), respectively, and the bottom two rows 

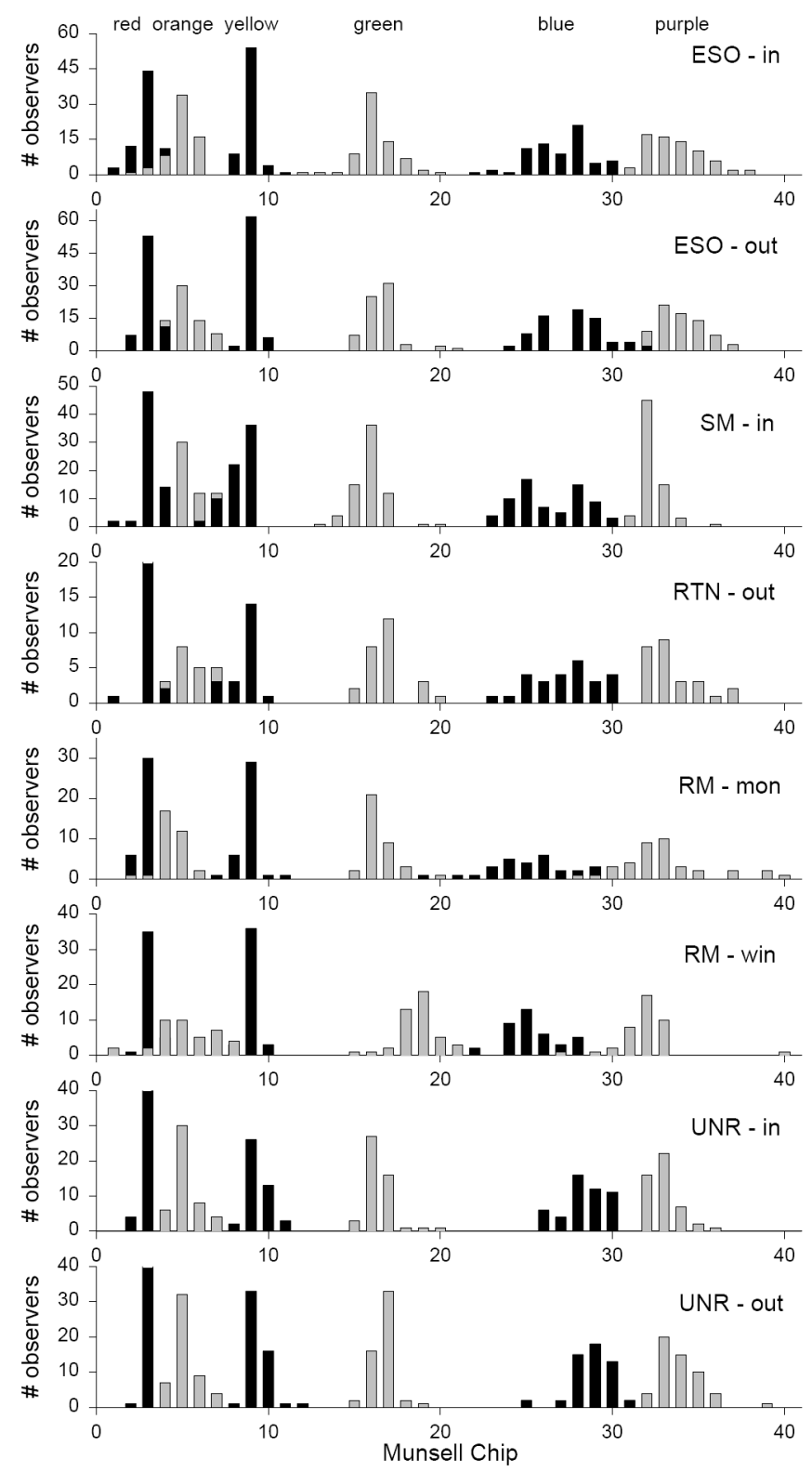

Figure 3. Color naming in Indian and US observers. Histograms show the number of observers that selected a particular Munsell hue (from the palette of 40 chips shown in Figure 1) as the best example of the color indicated. The different panels plot the choices for different groups of Indian and US observers. ESO-in and ESO-out: college students in Chennai tested in indoor or outdoor lighting; SM: sari merchants in Chennai; RTN: residents of rural Tamil Nadu; RM-mon and RMwin: residents of rural Maharashtra tested in monsoon or winter seasons; UNR-in and UNR-out: US college students tested in Reno, NV under indoor or outdoor lighting. (Adapted from Webster et al., 2002, Figure 5.) 
for the Reno (UNR) students, similarly varied by testing environment.] Within-observer group results were quite constant across changes in lighting conditions and the differences between the U.S. and India groups were thus maintained under both indoor and outdoor lighting. This suggests that whatever the bases for the differences between the two populations, they probably reflect some longer-term influences on color naming.

\section{Focal color differences in the World Color Survey}

In the preceding study, comparing focal and unique hues for Indian and US observers, the subjects were instructed to select the stimuli corresponding to what we assumed were corresponding terms across the languages. That is, they were asked to choose the best examples of 'red', 'green', 'blue' or 'yellow'. Again, the results showed that these focal choices clustered within very similar regions of color space, but also that there were significant group differences in the mean foci within these clusters (e.g., in the precise location of the mean 'red' or 'yellow' for the different groups).

These results led us to explore whether there are similar patterns of within-cluster variability across the 110 languages sampled as part of the WCS. In this case the question of equivalent color terms becomes admittedly more problematic, in part because the WCS includes languages that have different numbers of basic color terms. We consider this problem below. However, for the purpose of the present analysis, we assumed that a subset of languages in the WCS data set have terms with foci that are close to the foci for the English terms red, green, blue, and yellow. This allowed us to examine individual and group differences in the foci for these nearest-neighbor terms, in the same way that we compared differences across corresponding terms for Indian and US speakers. That is, it allowed us to ask how focal colors might vary within the similar-focus clusters, and specifically, to test whether the terms that fall within a common cluster are characterized by a common mean focal stimulus or by a range of stimuli that vary across the different populations.

The corresponding terms for each language were identified in the following steps. First, to be included in the analysis a term had to be used by more than half the speakers of the language sample. Then, for each speaker, the mean (i.e. centroid) focal point for each term was compared to the various foci for English basic color terms. The 'closest' English term was defined as the English term whose focal point was closest to the speaker's mean focal chip, as given by the Euclidean distance within the palette array. (The nominal Munsell foci for the English terms were similar to the foci shown in Figure 1.) We next tallied across the speakers within a language to find the English term that was most often closest to the focus of each term in the language. To be included in a cross-language focus cluster, a term had to be closest to the same English term for more than half of the speakers. Consensus by a simple majority may seem an overly liberal criterion for defining a term as part of a focus cluster. However, in most cases consensus was in fact much higher. Finally, once a term was assigned to a cluster, the data from all speakers of the language were 


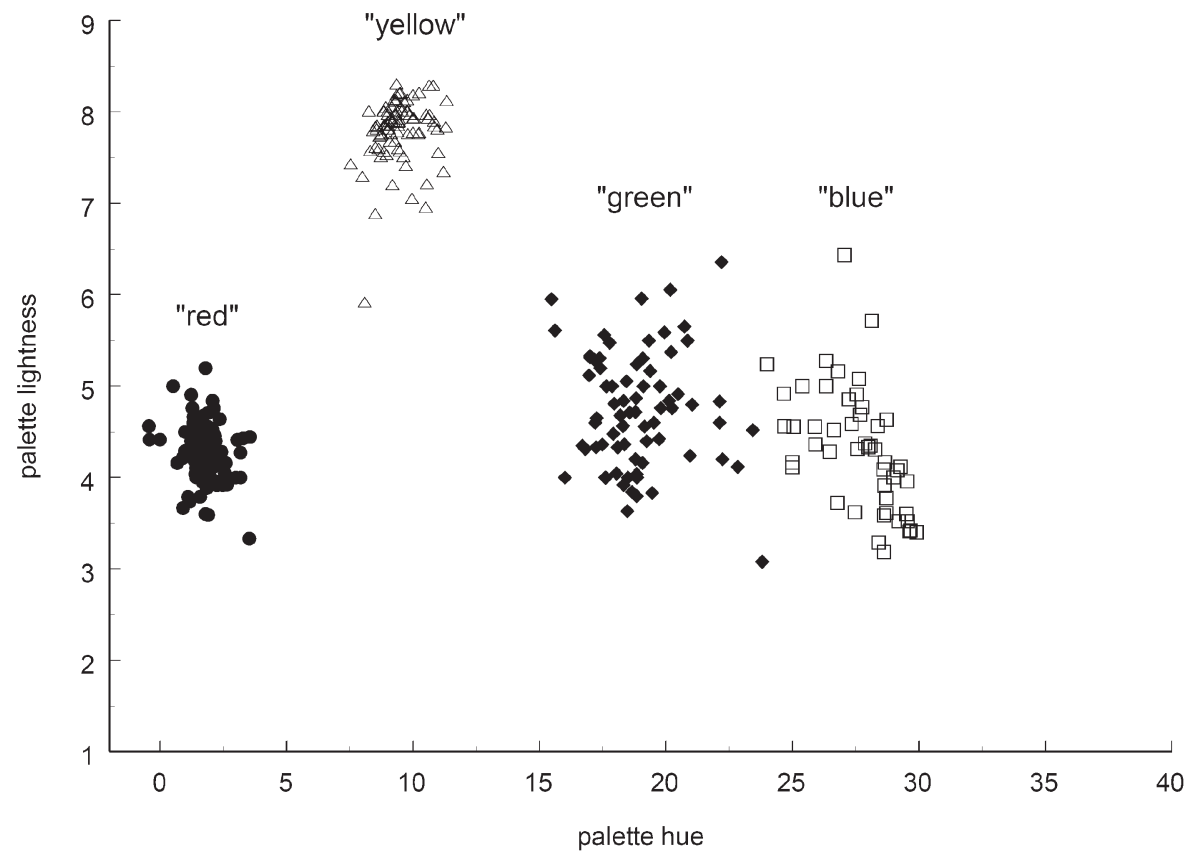

Figure 4. Location of focal colors for WCS languages that were close to the focal colors for the English terms red (filled circles), yellow (unfilled triangles), green (filled diamonds) or blue (unfilled squares). Coordinates give the location of chips in the Munsell array of Figure 1 (Kay \& Regier, 2003). [See Figure 1 for the location of English focal choices. Lightness level 9 here corresponds to lightness level B in Figure 1.]

included in locating the cluster, whether their own individual foci were consistent with this classification or not.

We analyzed only the sets of terms clustering near red, green, blue and yellow. The achromatic English terms (white, black, and gray) correspond to a small set of neutral chips, and the remaining color terms (orange, pink, brown, and purple) were the closest terms for only a small subset of the languages (Kay \& Regier 2003). Figure 4 plots the foci for the 'red', 'green', 'blue', and 'yellow' clusters, with each point showing the mean selection for speakers of a single language. This is similar to the contour plot derived for the WCS data by T. Regier (available at www.icsi.berkeley.edu/wcs/foci-20030418.html). In Figure 5, the foci have been replotted to also show the variability within each language. In this case the two panels plot the hue and lightness of the mean focal point for each language, while the error bars show the standard deviation in the foci within the language.

Note that the hue clusters in the upper panel of Figure 5 are similar to the focal color settings we discussed above for the Indian and US observers (Figure 3 ) in that the individual differences within the languages are pronounced. We again asked whether there are also significant differences between foci for different languages. To assess this, for each cluster we compared the observed variance in the foci to the variance predicted by ran- 

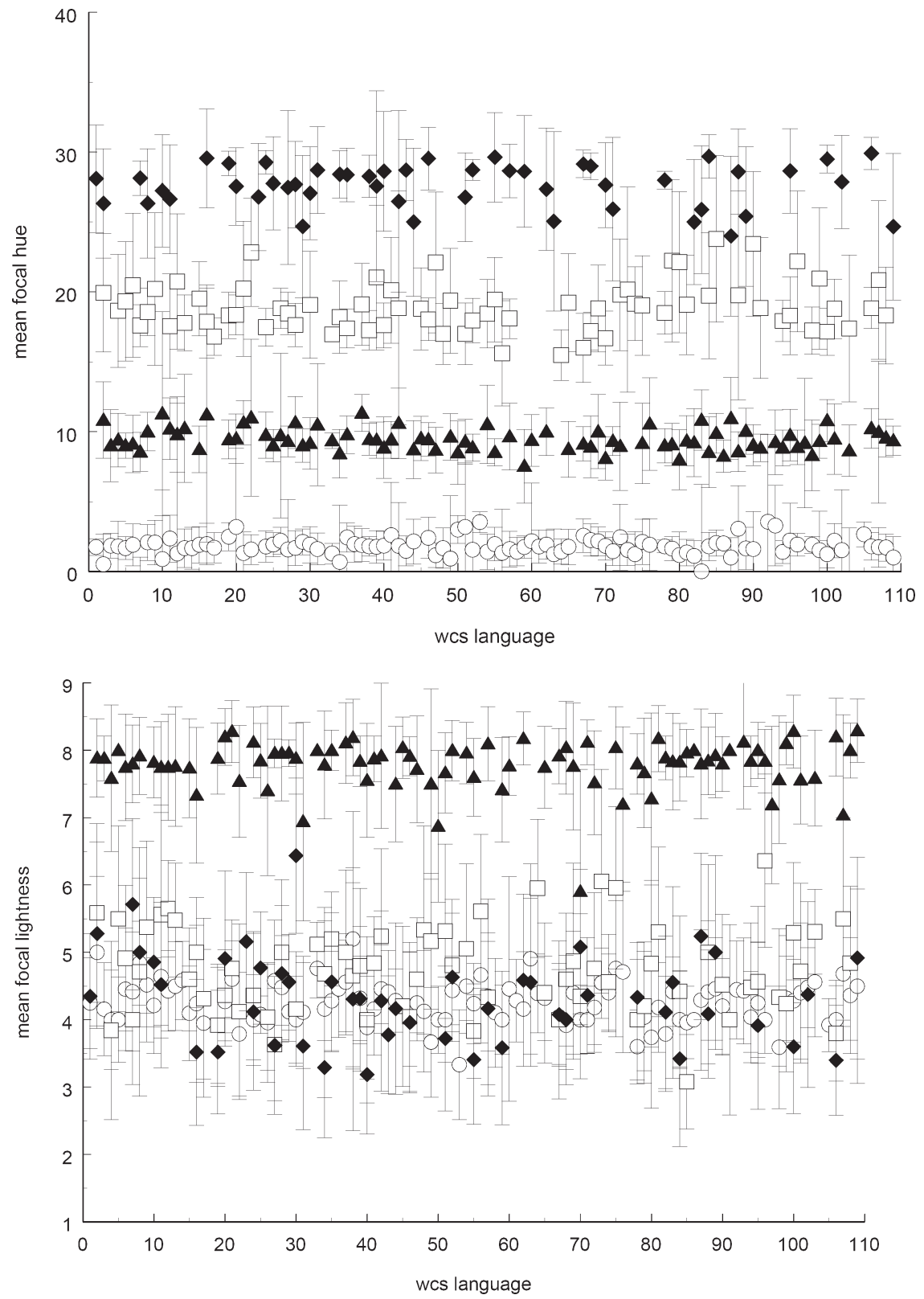

Figure 5. Color foci of Figure 4 replotted to show the separate hue and lightness levels for each language. Error bars show the range ( \pm 1 standard deviation) of foci within each language. "Red" (unfilled circles), "yellow" (filled triangles), "green” (unfilled squares), "blue” (filled diamonds). 
Table 1a. Variance in the mean foci for WCS terms closest to $r, g, b$, or $y$, compared to the variance predicted by random sampling across languages.

\begin{tabular}{|c|c|c|c|c|c|c|c|c|c|c|c|}
\hline \multirow[b]{2}{*}{ term } & \multirow[b]{2}{*}{ \# } & \multicolumn{5}{|c|}{ focal hue } & \multicolumn{5}{|c|}{ focal lightness } \\
\hline & & mean & var & pred & $F$ & $\mathrm{p}$ & mean & var & pred & $\mathrm{F}$ & $\mathrm{p}$ \\
\hline $\mathrm{r}$ & 100 & 1.78 & .45 & .25 & 1.81 & $<.002$ & 4.26 & .097 & .040 & 2.41 & $<\mathrm{e}-5$ \\
\hline $\mathrm{g}$ & 72 & 18.9 & 3.05 & .96 & 3.16 & $<\mathrm{e}-8$ & 4.74 & .41 & .099 & 4.12 & $<\mathrm{e}-10$ \\
\hline b & 49 & 27.6 & 2.38 & .93 & 2.56 & $<\mathrm{e}-5$ & 4.32 & .45 & .093 & 4.84 & $<\mathrm{e}-10$ \\
\hline y & 85 & 9.46 & .65 & .31 & 2.13 & $<.0002$ & 7.79 & .13 & .038 & 3.38 & $<\mathrm{e}-8$ \\
\hline
\end{tabular}

Table $1 \mathrm{~b}$. Variance in the mean foci for WCS terms closest to $\mathrm{g}$ and $\mathrm{b}$, for the 31 languages that included distinct $\mathrm{b}$ and $\mathrm{g}$ terms.

\begin{tabular}{|c|c|c|c|c|c|c|c|c|c|c|c|}
\hline \multirow[b]{2}{*}{ term } & \multirow[b]{2}{*}{ \# } & \multicolumn{5}{|c|}{ focal hue } & \multicolumn{5}{|c|}{ focal lightness } \\
\hline & & mean & var & pred & $\mathrm{F}$ & $\mathrm{p}$ & mean & var & pred & $\mathrm{F}$ & $\mathrm{p}$ \\
\hline g & 31 & 18.1 & 1.12 & .58 & 1.93 & $<.01$ & 4.56 & .30 & .074 & 4.06 & $<\mathrm{e}-7$ \\
\hline b & 31 & 28.3 & 1.93 & .60 & 1.93 & $<.01$ & 4.23 & .56 & .088 & 6.37 & $<\mathrm{e}-11$ \\
\hline
\end{tabular}

domly sampling across the languages. To generate these predictions, we drew repeated random samples of 20 speakers from the entire WCS sample, which is equivalent to treating all of the WCS speakers as a single population. The predicted variance in the means was estimated from 100 random samples. Figures 6-9 replot the mean hue and lightness settings for each cluster and compare these to the predictions for a single population. To help visualize the differences across the languages, the settings have been sorted in ascending order. In all cases, the spread of focal settings is larger than that predicted by a single population. This was verified by $F$-tests comparing the observed variance vs. the variance predicted by sampling across the languages. The observed variation is roughly 2 to 4 times the spread that would be expected if there were no differences between the groups, a difference that in all cases is highly significant (Table 1a). Notably, the between-group differences are consistently larger (relative to the within-group variation) for the lightness than for the hue of the focal color.

Table 2 shows the correlations between the hue and lightness settings for the different color terms. These correlations are generally weak, and are consistent with the largely independent variations in unique hues for individual observers reported by Webster et al. (2000) and Webster et al. (2002). This suggests that whatever factors give rise to the variations within the WCS clusters, they do not reflect global differences in how observers classify color (e.g., in the tendency to choose a higher or lower lightness for different colors).

One potential source of variation in the focal colors of different languages is in the number of color terms. The languages sampled in the WCS generally had far fewer basic color terms than English. For example, as noted above only a small proportion of the languages had terms with foci near 'orange' or 'pink', and thus in most cases these regions are instead subsumed within terms corresponding to 'yellow' or 'red' (Kay \& Regier 2003). It is not obvious how the best example of a color term should vary with the number or 
Table 2. Correlations between the mean hue and lightness values for WCS languages with terms near English red, green, blue and yellow.

\begin{tabular}{lrrrrrrrr}
\hline & r hue & r light & g hue & g light & b hue & b light & y hue & y light \\
\hline r hue & & & & & & & & \\
r light & -.22 & & & & & & \\
g hue & -.03 & -.12 & & & & & \\
g light & -.12 & .43 & -.09 & & & & \\
b hue & .25 & -.27 & -.14 & -.44 & & & \\
b light & -.26 & .29 & .04 & .15 & -.53 & & \\
y hue & -.13 & .36 & 0 & .22 & -.17 & .15 & \\
y light & -.03 & .23 & -.04 & .02 & -.05 & .04 & .22 \\
\hline
\end{tabular}

range of different terms, since it depends on whether these differences reflect perceptual or linguistic differences (Regier \& Kay 2004). One possibility is that the focal colors might lie near the center of the region labeled by a given term, because the region is treated as a single perceptual category. This would predict that in languages that lack an 'orange' term focal yellow might shift toward orange. A second way this shift could arise is if individual speakers chose foci near one or the other foci (e.g., near orange or yellow) or both, so that the average across speakers would again lie at an intermediate point. Previous studies have analyzed these patterns of variation specifically for the WCS languages that lack distinct 'blue' and 'green' terms, and which thus label this region of the color space with a single 'grue' term. The best examples chosen for these 'grue' terms are bimodal, with peaks near the separate English foci for green and blue (Regier \& Kay 2004). MacLaury (1997) reported a similar pattern among Mesoamerican languages. These results are consistent with the 'grue' term representing a generalization over perceptually distinct blue and green regions. On the other hand, Lindsey and Brown (2004) found that the set of grue foci in the WCS were less bimodal than the distribution given for blue and green foci. They argued that this is because a subset of speakers treats grue as if it were an undifferentiated perceptual category.

For the present analysis we did not attempt to distinguish 'grue' terms as such, and thus it is likely that some of the observed spread in the 'blue' and 'green' distributions resulted from including speakers that did not lexically distinguish blue and green. To assess this, however, we also examined the mean foci for the 31 WCS languages that were found to have separate terms near both 'blue' and 'green', according to the criteria we described above for identifying these terms. The foci for these languages are shown on the right side of Figures 7 and 8, and are a subset of the foci shown for the independently defined 'blue' or 'green' clusters plotted to the left in the figures. Restricting the terms to this subset eliminated languages near the boundary of the 'blue' and 'green' clusters. Thus the variations in the foci for 'blue' and 'green' may be partly attributable to differences in the degree to which the 'blue' and 'green' clusters are merged by the different WCS groups. (Note that because these plots show only the mean foci for each language, they do not reveal the extent to which these intermediate foci are the best examples for individual speakers.) Yet despite this factor, the cross-language variance in the 'blue' and 'green' terms remains 

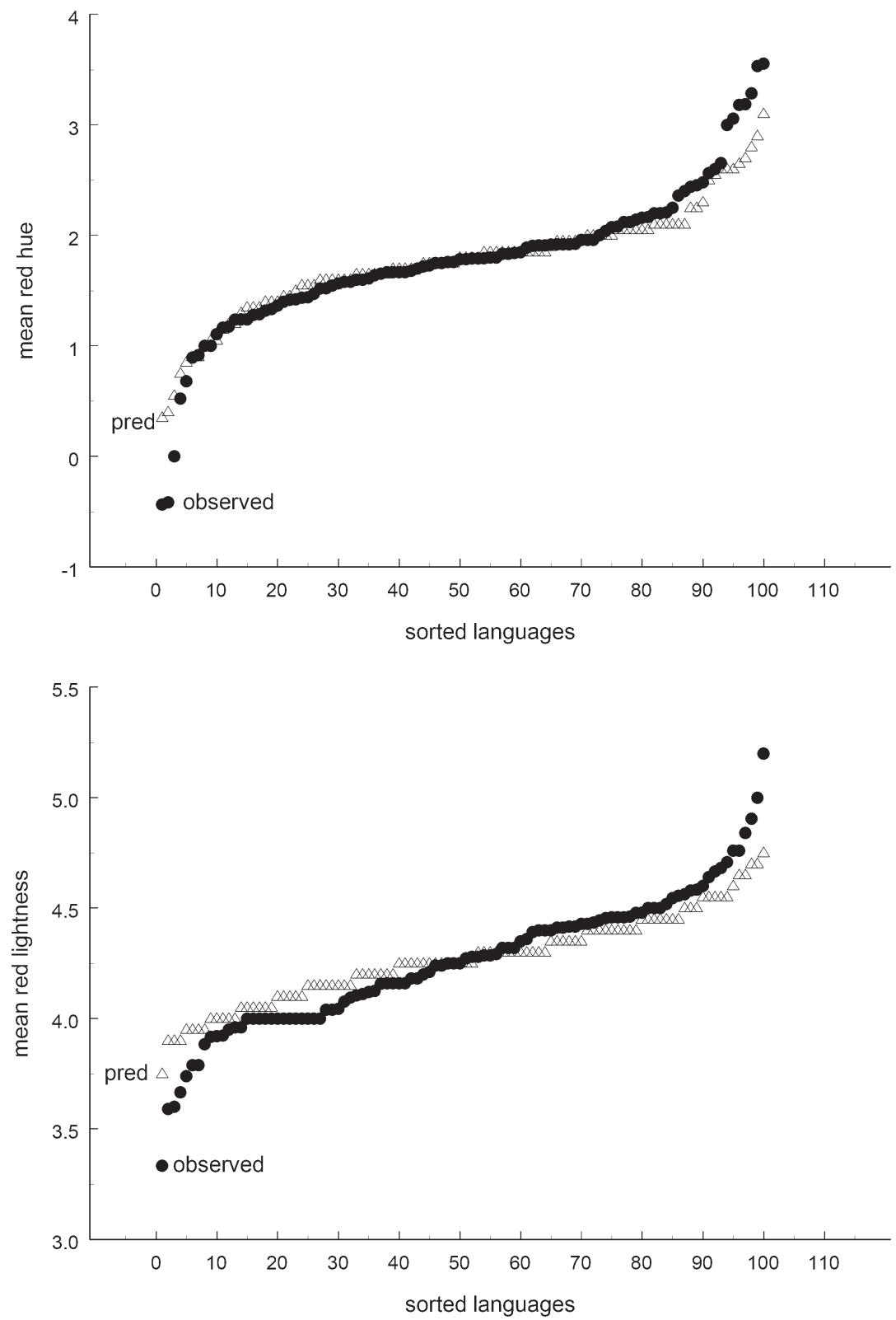

Figure 6. Average color foci for the WCS languages that had a term for stimuli near the English focal color for red, sorted in ascending order (filled circles). Predicted curves give the distribution expected if the variation in the "red" cluster is due only to the individual differences within groups (pred). The quantized steps in the predictions reflect rounding. 

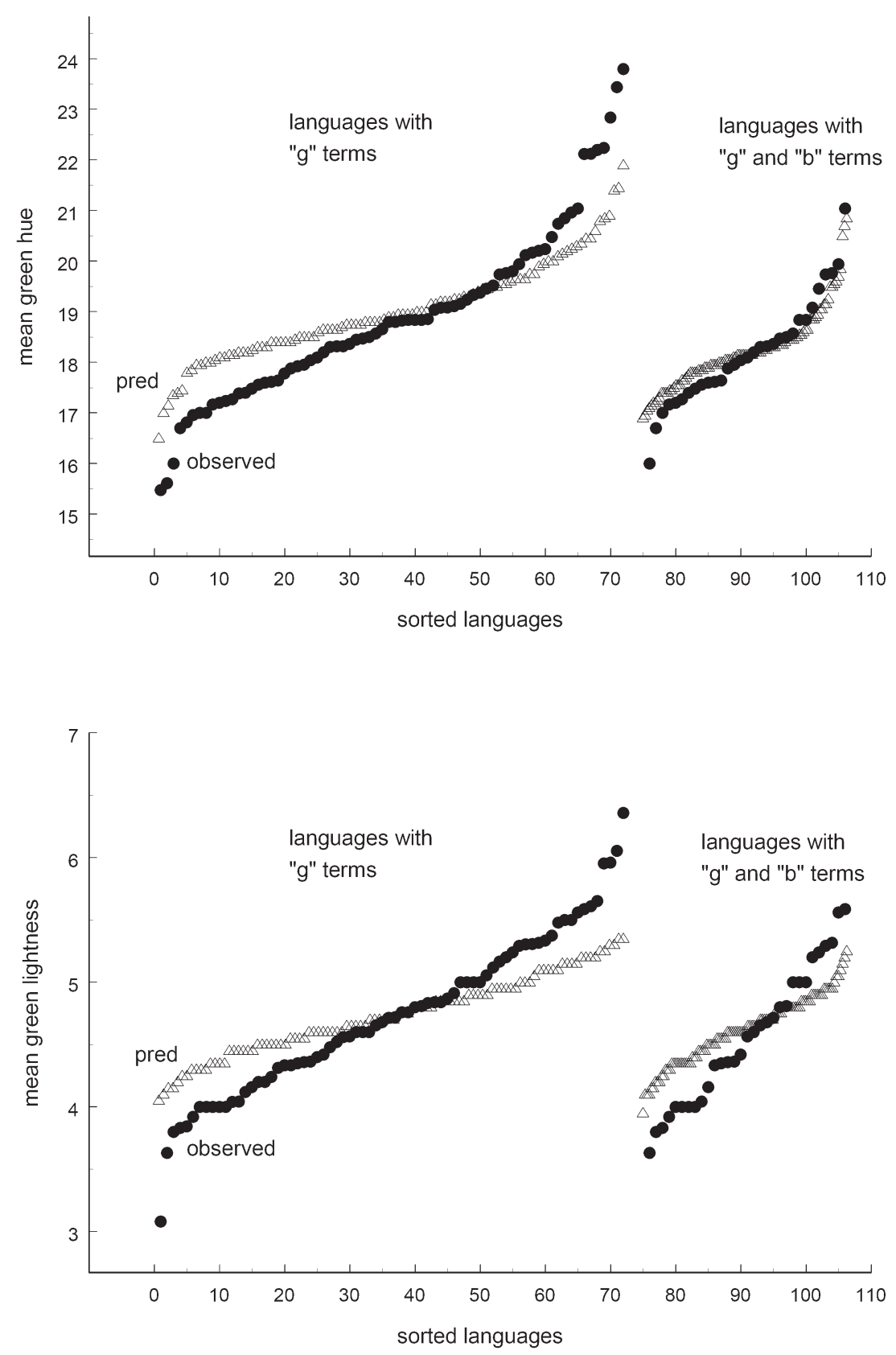

Figure 7. Sorted color foci for the WCS languages that had a term for stimuli near the English focal color for green (filled circles), compared to predictions for variations in a single population. Symbols at left plot results for all languages that included a term near "green." The right side shows the distribution for the "green" cluster for the subset of languages that also included a separate term for "blue." 

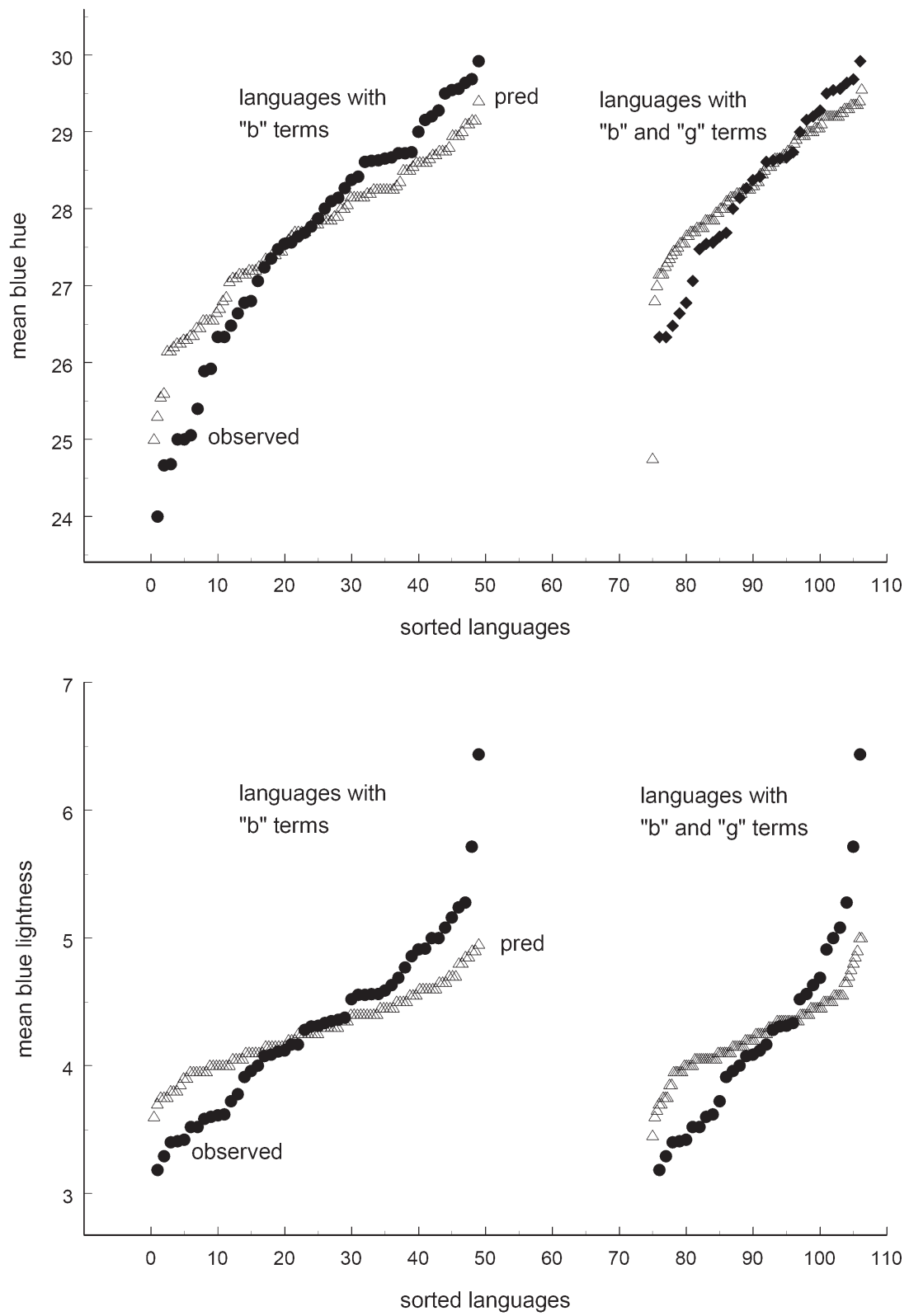

Figure 8. Sorted color foci for the WCS languages with term near English blue (filled circles), compared to predictions for variations in a single population. Left plots show all languages that included a term near "blue." Right plots are for languages that also included a term for "green." 

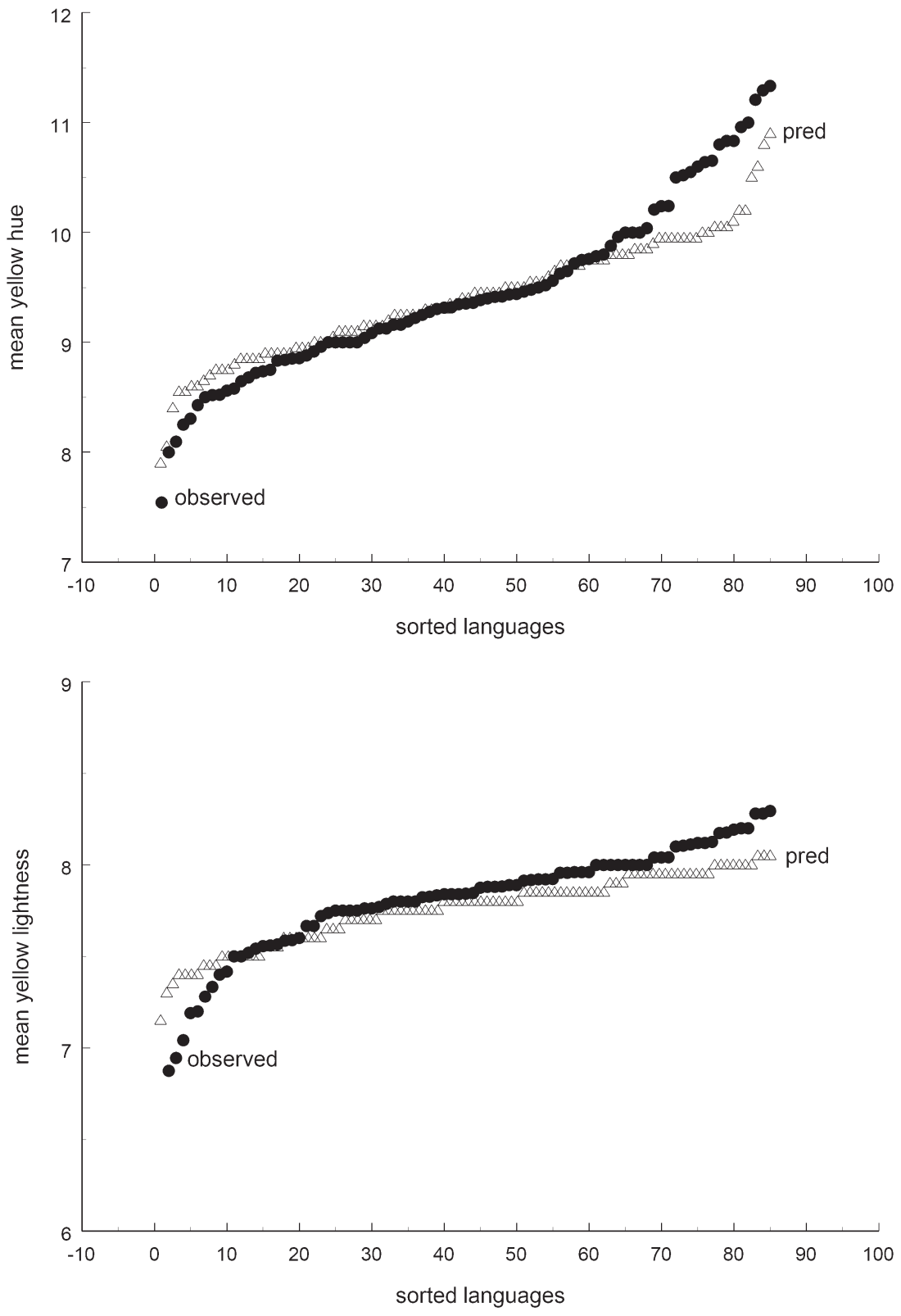

Figure 9. Sorted color foci for the WCS languages with term near English yellow (filled circles), compared to predictions for variations in a single population. 
large, and again is significantly greater than would be predicted if languages with distinct 'blue' and 'green' terms shared the focal stimuli for each term (Table 1b).

We have gone to some length to establish the fact that the foci for corresponding terms can vary across different groups. However, this finding should not overshadow the point that these differences within clusters are small compared to the differences across clusters. For example, the standard deviations of focal hues for languages within the 'red' and 'yellow' clusters were 0.67 and 0.81 , respectively, while the mean hue difference between these clusters was 7.8 steps, or roughly 10 times the within-cluster variation. This is not simply an artifact of our procedure for defining clusters by nearest-neighbor terms. For example, the color category of orange falls roughly half way between the red and yellow foci, yet only one of the 110 languages included a consensus term near orange. Similarly, despite the differences we noted for similar terms in Indian and US respondents, these differences are again small compared to the foci for different terms (Figure 3). The differences among nearest-neighbor terms are important for understanding how color naming varies across populations, but these differences reflect local dispersion within well-defined clusters and are more likely to reflect modulations of the basic color foci than categorical differences.

\section{Conclusions}

In this chapter we have approached the question of focal colors from two different traditions. Psychophysical studies of color appearance have typically started with the assumption that terms like yellow and blue correspond to special and well-defined perceptual phenomena that are shared by observers. The stimuli that elicit these states have been investigated with the aim of characterizing the visual mechanisms that might underlie these phenomena. Linguistic studies have instead focused on testing the assumption that there are corresponding color categories across languages. In the present investigation, focal colors were analyzed to determine whether they are similar enough among languages to support conclusions about universal tendencies in color naming. We have shown that at both the individual and the language level there is variation in focal colors, with markedly greater variation in the former. Speakers of a common language differ in the focal stimuli they choose for the same color term, and similarly, different languages show some, but noticeably less, variation in the average focal stimuli they choose for their nearestneighbor terms. What are the implications of these differences for the psychophysics and linguistics of color?

First, the presence of large inter-individual difference within languages does not per se bear on the issue of cross-linguistic universals in color naming. Even if the individual language samples were random samples from a single population, the Central Limit Theorem (Feller 1968:229) tells us that under reasonable assumptions the standard deviation of the distribution of any statistic calculated in each of the 110 sample languages will, on average, be a little less than one tenth the standard deviation of that statistic in the population itself - and hence less than one tenth of the value expected in any particular language sample (since the variance would be reduced by a factor equal to the number of language sam- 
ples, 110 , and the standard deviation is the square root of the variance). Of course we know from the statistics displayed in Table 1 that in fact the individual languages of the WCS are not, with respect to focal colors, drawn from a uniform population - or, more accurately, that they are highly unlikely to be so drawn. The present point is that we need Table 1 to tell us that the large variation of focus placement within individual languages is relevant to neither the issue of whether languages differ from each other in focus placement nor whether there exist universal tendencies in focus placement across languages

The present analyses of the WCS data suggest that different language groups do vary in the average focal choices for nearest-neighbor terms. These group differences place some limits on universal tendencies in color naming. In particular, they suggest that to the extent there are corresponding basic color terms, these reflect constellations of similar color categories rather than a strict equivalence, and this means that there can in fact be significant contextual influences on color naming. Our analyses have not examined the possible causes of this interlingual variation: environmental, cultural or physiological. Yet, the existence of some dispersion due to any of these factors is not surprising. For example, we have noted that there are clear differences in the physical color characteristics of different environments, and it is well established that processes of adaptation will adjust the characteristics of color perception to the observer's ambient environment. These adaptation processes alone may ensure that perceived color will differ when a single person is immersed in a lush jungle or arid desert, and thus predict that color judgments will differ on average for different inhabitants of different environments (Webster et al. 2005). What is surprising is perhaps how constrained these contextual influences are, for the foci for different populations remain strongly clustered in color space. This suggests that whatever factors give rise to the large individual differences in focal colors, they may themselves have strong universal tendencies.

\section{Acknowledgments}

Supported by EY-10834 and NSF-0130420.

\section{References}

Berlin, Brent \& Paul Kay (1969). Basic Color Terms: Their Universality and Evolution. Berkeley: University of California Press.

Bone, Richard A. \& J. M. B. Sparrock (1971). Comparison of macular pigment densities in human eyes. Vision Research, 11, 1057-1064.

Boynton, Robert M. \& Conrad X. Olson (1990). Salience of chromatic basic color terms confirmed by three measures. Vision Research, 30, 1311-1317.

Brainard, David H., Austin Roorda, Yasuki Yamauchi, Jack B. Calderone, Andrew Metha, Maureen Neitz, Jay Neitz, David R. Williams \& Gerald H. Jacobs (2000). Functional consequences of the relative numbers of L and M cones. Journal of the Optical Society of America A, 17, 607-614. 
Carroll, James, Jay Neitz \& Maureen Neitz (2002). Estimates of L:M cone ratio from ERG flicker photometry and genetics. Journal of Vision, 2, 531-542.

Changizi, Mark A., Qiong Zhang, \& Shinsuke Shimojo (2006). Bare skin, blood, and the evolution of primate color vision. Biology Letters, 2, 217-221.

Chapanis, Alphonse (1965). Color names for color space. American Scientist, 53, 327-346.

Davidoff, Jules (2001). Language and perceptual categorization. Trends in Cognitive Sciences, 5, 382-387.

Davidoff, Jules, Ian Davies \& Debi Roberson (1999). Colour categories in a stone-age tribe. Nature, 398, 203-204.

De Valois, Russell L. \& Karen K. De Valois (1993). A multi-stage color model. Vision Research, 33, 10531065.

Delahunt, Peter B., Michael A. Webster, Lei Ma \& John S. Werner (2004). Color appearance changes after cataract surgery reveal a long-term chromatic adaptation mechanism. Visual Neuroscience, 21, 301307.

Derrington, Andrew M., John Krauskopf \& Peter Lennie (1984). Chromatic mechanisms in lateral geniculate nucleus of macaque. Journal of Physiology, 357, 241-265.

Feller, William (1968). An Introduction to Probability Theory and Its Applications. Vol. 1, 3rd ed. New York: Wiley.

Gage, John (1993). Colour and Culture: Practice and Meaning from Antiquity to Abstraction. London: Thames and Hudson.

Gegenfurtner, Karl R. \& Lindsay T. Sharpe (Eds.) (1999). Color Vision: From Genes to Perception. Cambridge: Cambridge University Press.

Hardin, Clyde L. (1988). Color for Philosophers: Unweaving the Rainbow. Indianapolis: Hackett.

Hardin, Clyde L. \& Luisa Maffi (Eds.). (1997). Color Categories in Thought and Language. Cambridge: Cambridge University Press.

Hardy, Joseph L., Christina M. Frederick, Paul Kay \& John S. Werner (2005). Color naming, lens aging, and grue: What the optics of the aging eye can teach us about color language. Psychological Science, $16,321-327$.

Hurvich, Leo M. \& Dorothea Jameson (1957). An opponent-process theory of color vision. Psychological Review, 64, 384-404.

Jameson, Kimberly \& Roy G. D'Andrade (1997). It's not really red, green, blue, yellow: An inquiry into perceptual color space. In C. L. Hardin \& L. Maffi (Eds.), Color Categories in Thought and Language (295-319). Cambridge: Cambridge University Press.

Jameson, Kimberly A., Susan M. Highnote \& Linda M. Wasserman (2001). Richer color experience in observers with multiple photopigment opsin genes. Psychonomic Bulletin \& Review, 8, 244-261.

Jordan, Gabriele \& John D. Mollon (1993). A study of women heterozygous for colour deficiencies. Vision Research, 33, 1495-1508.

Jordan, Gabriele \& John D. Mollon (1995). Rayleigh matches and unique green. Vision Research, 35, 613620.

Kaiser, Peter K. \& Robert M. Boynton (1996). Human Color Vision. USA: Optical Society of America.

Kay, Paul, Brent Berlin, Luisa Maffi \& William Merrifield (1997). Color naming across languages. In C. L. Hardin \& L. Maffi (Eds.), Color Categories in Thought and Language (21-56). Cambridge: Cambridge University Press.

Kay, Paul \& Luisa Maffi (1999). Color appearance and the emergence and evolution of basic color lexicons. American Anthropologist, 101, 743-760.

Kay, Paul \& Chad K. McDaniel (1978). The linguistic significance of the meanings of basic color terms. Language, 54, 610-646.

Kay, Paul \& Terry Regier (2003). Resolving the question of color naming universals. Proceedings of the National Academy of Science, 100, 9085-9089. 
Krauskopf, John, David R. Williams \& David W. Heeley (1982). Cardinal directions of color space. Vision Research, 22, 1123-1131.

Kuehni, Rolf G. (2001). Determination of unique hues using Munsell color chips. Color Research \& Application, 26, 61-66.

Kuehni, Rolf G. (2004). Variability in unique hue selection: A surprising phenomenon. Color Research \& Application, 29, 158-162.

Lazar-Meyn, Heidi (2004). Color naming: "Grue” in the Celtic languages of the British Isles. Psychological Science, 15, 288.

Lennie, Peter, John Krauskopf \& Gary Sclar (1990). Chromatic mechanisms in striate cortex of macaque. Journal of Neuroscience, 10, 649-669.

Lindsey, Delwin T. \& Angela M. Brown (2002). Color naming and the phototoxic effects of sunlight on the eye. Psychological Science, 13, 506-512.

Lindsey, Delwin T. \& Angela M. Brown (2004). Sunlight and "blue": The prevalence of poor lexical color discrimination within the "grue" range. Psychological Science, 15, 291-294.

Lucy, John A. (1997). The linguistics of "color". In C. L. Hardin \& L. Maffi (Eds.), Color Categories in Thought and Language (320-346). Cambridge: Cambridge University Press.

Lyons, John (1995). Color in language. In T. Lamb \& J. Bourriau (Eds.), Colour: Art \& Science (194-224). Cambridge: Cambridge University Press.

MacLaury, Robert E. (1997). Color and Cognition in Mesoamerica. Austin: University of Texas Press.

MacLeod, Donald I. A. \& Robert M. Boynton (1979). Chromaticity diagram showing cone excitation by stimuli of equal luminance. Journal of the Optical Society America, 69, 1183-1186.

Malkoc, Gokhan, Paul Kay \& Michael A. Webster (2002). Individual differences in unique and binary hues. Journal of Vision, 2, 32a.

Mausfeld, Rainer \& Dieter Heyer (2004). Colour Perception: Mind and the Physical World. Oxford: Oxford University Press.

Miyahara, Eriko, Joel Pokorny, Vivianne C. Smith, Rebecca Baron \& Eleanor Baron (1998). Color vision in two observers with highly biased LWS/MWS cone ratios. Vision Research, 38, 601.

Mizokami, Yoko, Shernaaz M. Webster \& Michael A. Webster (2003). Seasonal variations in the color statistics of natural images. Journal of Vision, 3, 444a.

Mollon, John D. (1982). Color vision. Annual Review of Psychology, 33, 41-85.

Mollon, John D. (1989). “Tho' she kneel'd in that place where they grew...” Journal of Experimental Biology, $146,21-38$.

Nagy, Allen, Donald I. A. MacLeod, N. E. Heyneman \& Alvin Eisner (1981). Four cone pigments in women heterozygous for color deficiency. Journal of the Optical Society of America, 71, 719-722.

Neitz, Jay, James Carroll, Yasuki Yamauchi, Maureen Neitz \& David R. Williams (2002). Color perception is mediated by a plastic neural mechanism that is adjustable in adults. Neuron, 35, 783-792.

Pokorny, Joel \& Vivianne C. Smith (1977). Evaluation of single-pigment shift model of anomalous trichromacy. Journal of the Optical Society of America, 67, 1196-1209.

Polyak, Steven (1957). The Vertebrate Visual System. Chicago: University of Chicago Press.

Pridmore, Ralph W. (1999). Unique and binary hues as a function of luminance and illuminant color temperature, and relations with invariant hues. Vision Research, 39, 3892-3908.

Ratliff, Floyd (1976). On the psychophysiological bases of universal color terms. Proceedings of the American Philosophical Society, 120, 311-330.

Regan, Benedict C., C. Julliot, B. Simmen, Françoise Vienot, P. Charles-Dominique \& John D. Mollon (2001). Fruits, foliage and the evolution of primate colour vision. Philosophical Transactions of the Royal Society London B, 356, 229-283.

Regier, Terry \& Paul Kay (2004). Color naming and sunlight: commentary on Lindsey and Brown (2002). Psychological Science, 15, 289-290. 
Roberson, Debi, Ian Davies, \& Jules Davidoff (2002). Color categories are not universal. Journal of Experimental Psychology: General, 129, 369-398.

Rosch, Eleanor Heider (1975). Cognitive reference points. Cognitive Psychology, 4, 328-350.

Saunders, Barbara A. C. \& Jaap van Brakel. (1997). Are there nontrivial constraints on colour categorization? Behavioral and Brain Sciences, 20, 167-228.

Schefrin, Brooke E. \& John S. Werner (1990). Loci of spectral unique hues throughout the lifespan. Journal of the Optical Society of America A, 7, 305-311.

Sharpe, Lindsay T., Andrew Stockman, Herbert Jägle \& Jeremy Nathans (1999). Opsin genes, cone photopigments, color vision, and color blindness. In K. R. Gegenfurtner \& L. T. Sharpe (Eds.), Color Vision: From Genes to Perception (3-51). Cambridge: Cambridge University Press.

Shepard, Roger N. (1992). The perceptual organization of colors: an adaptation to regularities of the terrestrial world? In J. Barkow, L. Cosmides \& J. Tooby (Eds.), The Adapted Mind (495-532). Oxford: Oxford University Press.

Smith, Vivianne C., Joel Pokorny \& S. J. Starr (1976). Variability of color mixture data - I. Interobserver variability in the unit coordinates. Vision Research, 16, 1087-1094.

Sturges, Julia \& T. W. Allan Whitfield (1995). Locating basic colours in the Munsell space. Color Research \&Application, 20, 364-376.

Van Norren, Dirk \& Johannes J. Vos (1974). Spectral transmission of the human ocular media. Vision Research, 14, 1237-1244.

Volbrecht, Vicki, Janice L. Nerger \& Chrislyn E. Harlow (1997). The bimodality of unique green revisited. Vision Research, 1997, 404-416.

Webster, Michael A. (2003). Pattern selective adaptation in color and form perception. In L. M. Chalupa \& J. S. Werner (Eds.), The Visual Neurosciences. Vol. 2 (936-947). Cambridge: MIT Press.

Webster, Michael A. \& Donald I. A. MacLeod (1988). Factors underlying individual differences in the color matches of normal observers. Journal of the Optical Society of America A, 5, 1722-1735.

Webster, Michael A., Eriko Miyahara, Gokhan Malkoc \& Vincent E. Raker (2000). Variations in normal color vision. II. Unique hues. Journal of the Optical Society of America A, 17, 1545-1555.

Webster, Michael A. \& John D. Mollon (1997). Adaptation and the color statistics of natural images. Vision Research, 37, 3283-3298.

Webster, Michael A., Shernaaz M. Webster, Shrikant Bharadwadj, Richa Verma, Jaikishan Jaikumar, Gitanjali Madan \& E. Vaithilingam (2002). Variations in normal color vision III. Unique hues in Indian and United States observers. Journal of the Optical Society of America A, 19, 1951-1962.

Webster, Michael A., John S. Werner \& David J. Field (2005). Adaptation and the phenomenology of perception. In C. Clifford \& G. Rhodes (Eds.), Fitting the Mind to the World: Adaptation and Aftereffects in High Level Vision: Advances in Visual Cognition Series. Vol. 2 (241-277). Oxford: Oxford University Press.

Werner, John S. (1996). Visual problems of the retina during ageing: Compensation mechanisms and colour constancy across the life span. Progress in Retinal and Eye Research, 15, 621-645.

Werner, John S. (2000). Senescence of color vision mechanisms from the first days of life. Vision Science and its Applications. Optical Society of America Technical Digest Series, 71-74.

Werner, John S. \& Brooke E. Schefrin (1993). Loci of achromatic points throughout the life span. Journal of the Optical Society of America A, 10, 1509-1516.

Yendrikhovskij, Sergej N. (2001). Computing color categories from statistics of natural images. Journal of Imaging Science and Technology, 45, 409-417.

Wuerger, Sophie M., Philip Atkinson \& Simon Cropper (2005). The cone inputs to the unique-hue mechanisms. Vision Research, 45, 3210-3223. 



\title{
Russian color names
}

\section{Mapping into a perceptual color space}

\author{
Olga V. Safuanova and Nina N. Korzh \\ Institute of Psychology, Russian Academy of Sciences, Russia
}

\begin{abstract}
The purpose of the present study was to determine foci for the basic and frequently used non-basic, compound, and modified Russian color terms, as well as to assess their denotative precision. Such an estimate of denotative meaning indicates the area of the color space onto which the color term is mapped. It also indicates consensus, or the degree of agreement between subjects in their color naming, while enabling one to rank the colorterm conceptions in order of denotative precision. Finally, mapping these terms with a large number of color samples enabled us to ascertain nominal density of the perceptual color space, which we interpret as reflecting a distinctive elaboration of Russian speakers' conception of color.
\end{abstract}

\section{Introduction}

In the present study we are interested in mapping the denotata of frequently used Russian color terms and in the linguistic elaboration and differentiation of a perceptual color space in Russian. We approached the problem of the relationship between color perception and Russian color nomenclature, from two main theoretical perspectives.

One of these is the hypothesis of linguistic relativity articulated by Whorf (1956) and Sapir (1968). The conception is rooted in the theory of relativity originating with Wilhelm von Humboldt (1836/1971). According to his tenet, language is the subject to a permanent process of human creativity, whereby each language represents a unique worldview. The Sapir-Whorf hypothesis was elaborated within this theoretical approach; it was based on results of their ethno-linguistic studies which indicated a direct relation between language typological homogeneity and socio-cultural environment.

The idea of linguistic relativity is further developed within modern linguistics, wherein language is argued to be the determinant of modes of organizing collective and individual experience, in both cognition and communication. Categorization of color experience by means of color terms is considered a particular case of linguistic relativity. Saunders, one of its main proponents, conjectures that "... 'to see color' is not a biological 'given,' but a many-stranded socio-historical 'institution"' (Saunders 2007:474). 
Particularly relevant to our study is the proposition that to reveal a complete picture of the color reality expressed in a language, one should consider the total color-lexical content, in particular, by including a broader realm of color terms in addition to basic color terms.

An alternative framework is presented in the theory of Berlin and Kay (1969). This holds that in languages with highly elaborated color nomenclature, there exist universal 11 basic color terms (BCTs). The names of colors are considered to reflect universal characteristics of color perception and are related to opponent characteristics of the color-processing system.

Rosch (1972) underscored the insight of Berlin and Kay by demonstrating that the color continuum is subdivided into segments, or categories, perceptually as well as verbally. She tagged colors of greatest salience and verbal definitiveness within a color category focal colors; these can be defined denotatively via parameters of corresponding color samples. The focal colors have the capacity to semantically "attract" adjacent colors of the color continuum.

Since its appearance, Berlin and Kay's seminal work (1969) found support in numerous anthropological, ethno-linguistic, and psycholinguistic studies. Many of these focused on determining the basic color terms across languages according to criteria established by Berlin and Kay; on elaborating those criteria further; on investigating the structure and boundaries of color categories, and the evolution of the color-category system (e.g., Boynton \& Olson 1990; Corbett \& Davies 1997; Crawford 1982; MacLaury 2002). This rich body of research has greatly contributed to development of the theory of universal color nomenclature (Kay et al. 1991; Kay \& Maffi 2000).

Recent studies, however, reveal certain exceptions to the original assertions of Berlin and Kay's theory. In particular, there is convincing evidence that possession of linguistic categories facilitates color recognition and influences perceptual judgments, an indicator of weak linguistic relativity (e.g., Davies \& Corbett 1997a; Jameson \& Alvarado 2003; Moore et al. 2002; Roberson et al. 2000). Moreover, it was demonstrated that color-term basicness, as measured by its salience or frequency, can be graduated, thereby introducing the concept of relative basicness (Kerttula 2002). Further understanding of cognitive mechanisms of color categorization is provided by vantage theory (MacLaury 2000, 2002). Without disputing the influence of underlying physiological mechanisms of color processing, MacLaury conjectures that rather than producing "frozen" formations in individuals, color categorization is mediated by an actual cognitive viewpoint (vantage), which selectively emphasizes either perceptual similarities or differences between categorized stimuli, thus resulting in broader or narrower categories. Finally, there is accumulating evidence in favor of more than 11 basic color terms in some languages, with the additional terms being culturally specific (e.g., Hippisley 2001; Lin et al. 2001; Paramei 2005, 2007). Cultural distinctions manifest themselves, in particular, in a differentiated use of color terms for denoting variations in lightness within a single category (e.g., Uchikawa \& Boynton 1987).

Russian color nomenclature and its denotative elaboration attracted attention from many investigators during recent decades, regarding the many contentious issues named above. Russian presents a possible exception to the Berlin-Kay theory by arguably pos- 
sessing two basic terms for 'blue' - sinij 'dark blue' and goluboj 'light blue.' Though the status of goluboj as a basic term is controversial (for reviews see Paramei 2005, 2007), the predominant view among linguists and psycholinguists (Corbett \& Morgan 1988; Davies \& Corbett 1994; Frumkina 1984; Hippisley 2001), as well as the intuitive judgments of native Russian speakers justify treating it in our study as a basic color term.

In the present study, along with the basic terms, to broaden the scope we included other frequently used non-basic color terms. The rationale is the significance of non-basic terms in color naming (Frumkina 1984; Guest \& Van Laar 2000; Kerttula 2002; Korzh et al. 1991; Vasilevich 1987, 2003). Specifically for Russian, Vasilevich (1987) notes that such frequently used non-basic terms as malinovij 'raspberry' or sirenevyj 'lilac' enable native speakers to construct a differentiated color picture of their environment and cannot therefore be ignored. In addition, Russian native speakers eagerly use compound color terms, such as žëlto-zelënyj 'yellow-green' ('chartreuse') or krasnovato-koričnevyj 'reddishbrown' ('maroon'), or color terms specified by achromatic modifiers, which create color names with higher precision (cf. Alvarado \& Jameson 2002).

The purpose of the present study was to determine foci for the basic and frequently used non-basic, compound, and modified Russian color terms, as well as to assess their denotative precision. Such an estimate of denotative meaning indicates the area of the color space into which the color term is mapped. It also indicates consensus, or the degree of agreement between subjects in their color naming, while enabling one to rank the colorterm conceptions in order of denotative precision. Finally, mapping these terms with a large number of color samples enabled us to ascertain nominal density of the perceptual color space, which we interpret as reflecting a distinctive elaboration of Russian speakers' conception of color.

\section{Method}

Natural Color System (NCS): Atlas and notation system

For our psycholinguistic experiment, colors from the NCS were used. The NCS atlas contains 1526 samples in 40 hue pages (Hård \& Sivik 1981). Each page presents a single hue, corresponding to one of $2.5 \%$-steps around the color circle. On each hue page, the samples are systematically arranged with chromaticness vs. blackness presented against a white background.

The NCS perceptual space is based on Hering's opponent-color theory, according to which six colors are considered elementary and are organized in three pairs, one achromatic: white (W)-black (S), and two chromatic: red (R)-green (G) and blue (B)yellow (Y).

Within the NCS, any color is defined operationally in terms of its degree of resemblance to the six elementary attributes: whiteness $(w)$, blackness $(s)$, yellowness $(y)$, redness $(r)$, blueness $(b)$, and greenness $(g)$. The achromatic dimension blackness $(s)$ links elementary $\mathrm{W}$ and $\mathrm{B}$, implying variation of a color on a gray scale; it should not 

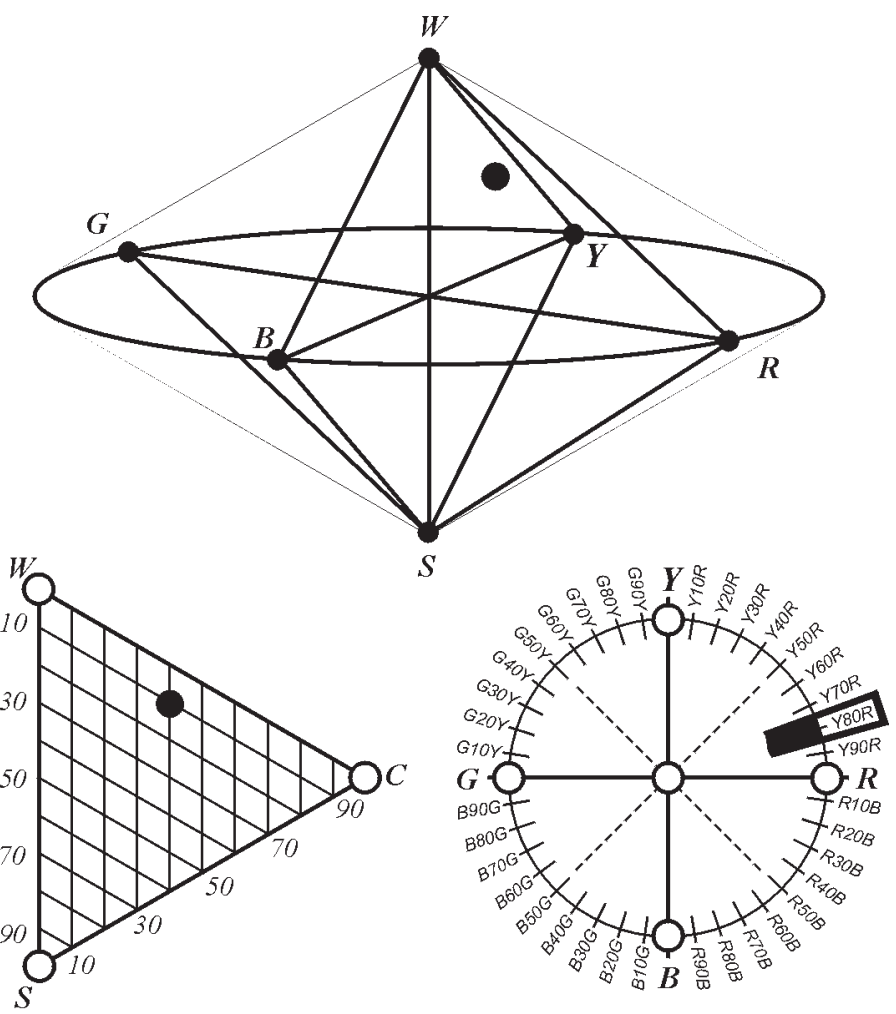

Figure 1. The NCS color space; (below) a vertical cut-through projection, blackness-chromaticness triangle (left) and the color circle (right). Adapted from Hård and Sivik (1983).

be confused with lightness dimensions in other color systems (for a discussion see Sivik 1997). The four chromatic attributes organized in two bi-polar orthogonal dimensions span the equator (Figure 1). Psychometric properties of the NCS are defined by ascribing the achromatic and each of the four chromatic attributes a metric scale with values from 0 to $100 \%$.

Since $r$ and $g$ and $b$ and $y$ are mutually exclusive, the hue notation of a given color can be compressed: for example, R60B is a purple whose visual content is $60 \%$ blueness and $40 \%$ redness. Within the NCS color circle, hue is defined, in addition, as an angular measure, $\varphi$.

Along with these dimensions, a certain color is also defined by chromaticness (c); in the NCS, this parameter reflects the resemblance (in percent) to the maximal color of the given hue, where the maximal color is a point on the NCS equatorial circumference. Note that $s+c+w=100$.

In the NCS atlas each color can thus be denoted by a digit-letter combination standing for the three parameters $s, c$, and $\varphi$. For example, 1080 Y50R indicates $10 \%$ blackness and $80 \%$ chromaticness of a color half-between elementary $\mathrm{Y}$ and $\mathrm{R}$. 
Comparing the NCS to the widely used Munsell system, differences in underlying perceptual definitions are worth noting. Munsell adopted perceptual attributes of Hue, Value (or lightness), and Chroma (saturation), where Chroma is defined as subjective difference of a certain hue from a neutral color of the same value. By comparison, in the NCS chromaticness is defined as the degree of sample's resemblance to the maximal color; it corresponds to the dimensional direction of Munsell's Chroma, but not to the scaling (Sivik 1997). In the NCS, saturation is defined as the ratio of blackness and chromaticness (Tonnquist 1986). Also unlike the NCS, Munsell-system elementary colors are not symmetrically positioned and do not belong to its equator (Valberg 2001).

\section{Color terms}

A list of Russian color names was generated in a preliminary study based on a color-term eliciting task and dictionary analysis (Penova 1991). A total of 250 color names were used in the present psycholinguistic experiment. They included Russian cognates for the eight chromatic basic color terms: krasnyj 'red,' oranževyj 'orange,' žëltyj 'yellow,' zelënyj 'green,' sinij 'dark blue,' goluboj 'light blue,' rozovyj 'pink,' fioletovyj 'purple,' and koričnevyj 'brown.'

Along with these, the following color names were included: (1) frequently used nonbasic color terms, for example, višnëvyj 'cherry' or salatovyj 'lettuce-colored'; (2) compound (double or triple) color names; (3) compound color terms with achromatic components, such as -belyj '-white,' -seryj '-gray,' -čërnyj '-black'; and (4) terms with achromatic modifiers, such as bledno- 'pale,' svetlo- 'light,' jarko- 'bright,' and tëmno- 'dark.' For English translations of the Russian color terms, we used the list suggested by Frumkina and Mikhejev (1996: 86) and Davies and Corbett (1994:73-74).

\section{Participants}

Thirty native Russian individuals (Muscovites) volunteered for the experiment. They were aged between 20 and 45 years, and had no formal training in color. All were screened for red-green abnormalities using the Rabkin Pseudoisochromatic Plates. The experiment was conducted with each participant individually.

\section{Procedure}

The color samples of the NCS atlas were presented under standard daylight illumination. For each color term, the focal color was estimated. When presented with the color term, the individual was requested to indicate in the atlas page the only sample that would best represent the term. If in the person's view, none of the samples adequately represented the color name, or the meaning of the name was unknown, no response was recorded.

The order of presentation of color names was yielded by an experimenter in the following steps. First, according to the hue of the NCS atlas page, one of the eight Russian chromatic basic color terms was named: krasnyj 'red,' oranževyj 'orange,' and so on. Second, color-term variants with the achromatic modifiers were named. Next, compound chromatic terms were presented, comprising two basic color names (or more, if 
a participant insisted), such as oranževato-krasnyj 'orangish-red' or žëlto-zelënyj 'yellowgreen.' (In Russian, the suffix -ato in an adjective indicates lower salience of the denoted quality.) Then followed the names comprising basic chromatic and achromatic components, such as rozovato-belyj 'pinkish-white', krasnovato-čërnyj 'reddish-black', rozovatoseryj 'pinkish-gray'; and finally, frequent non-basic names, such as malinovyj 'raspberry', or sirenevyj 'lilac.'

\section{Analysis}

For each color name, the following denotative indices were calculated across the individuals who participated in the study: mean value $(M)$ of each of the NCS parameters $(s, c$, $\varphi)$; standard deviation $(\sigma)$ of $M$; and, related to it, the $99 \%$ confidence interval $(C I) . M$ values parameterize the focal color of a color term; $\sigma$ indicates the color-name spread in the color space, i.e., the term's denotative precision; and CI indicates its denotative consistency. (Thus-defined denotative consistency should not be confused with consistency as within-subject replicability of a sample naming, as defined by Boynton \& Olson 1990.) In addition, for each color name, histograms representing distribution of chosen color samples were plotted for each of the three parameters $s, c, \varphi$. Finally, inter-participant consensus was calculated as percent of individuals agreeing in their color naming of the sample in question (cf. Boynton \& Olson 1990); this parameter is considered here, though, in less detail.

\section{Results and discussion}

\section{Russian basic chromatic color terms}

We found that certain basic chromatic terms map onto narrow areas in the color space, whereas other map broadly. Table 1 shows parameters for the focal colors $(M)$, their denotative consistency $(C I)$, and denotative precision $(\sigma)$. It indicates that the most precise terms are krasnyj 'red' and žëltyj 'yellow', with low variance along all three dimensions, blackness $(s)$, chromaticness $(c)$, and hue $(\varphi)$. By comparison, oranževyj 'orange' shows high denotative precision along two dimensions, $s$ and $c$. The lowest precision is revealed for zelënyj 'green,' koričnevyj 'brown,' and rozovyj 'pink.'

As Table 1 and example histograms in Figure 2 show, chromatic terms differ in precision along one or the other attribute (Korzh et al. 1991; Korzh \& Safuanova 1993). For example, for oranževyj all participants chose samples with the same blackness $\left(\sigma_{\mathrm{s}}=0\right)$, but they were not so unanimous for the hue $\left(\sigma_{\varphi}=9\right)$. Krasnyj, goluboj, and fioletovyj indicate the highest denotative consistency along hue. Rozovyj has low variance for two dimensions, while koričnevyj and zelënyj are poorly defined along all three attributes. It is noteworthy that some terms indicate split focal denotata. In Figure 2 this is illustrated by the distribution of sample choices for rozovyj, with two hue peaks: R and R20B (loosely related to 
Table 1. Russian basic chromatic terms: NCS parameters of focal colors

\begin{tabular}{|c|c|c|c|c|}
\hline $\begin{array}{l}\text { Color term/ } \\
\text { gloss }\end{array}$ & \multicolumn{3}{|c|}{$\begin{array}{c}\mathrm{M} \pm \mathrm{CI} \\
\boldsymbol{\sigma}\end{array}$} & Hue \\
\hline krasnyj & $7.8 \pm 2.5$ & $86.1 \pm 3.0$ & $84.8 \pm 3.9$ & 1090Y80R \\
\hline 'red' & 4.2 & 5.0 & 5.1 & \\
\hline oranževyj & $0.0 \pm 0.0$ & $85.8 \pm 3.3$ & $45.3 \pm 5.9$ & 0090Y40R \\
\hline 'orange' & 0.0 & 5.1 & 9.0 & \\
\hline žëltyj & $0.5 \pm 1.4$ & $77.1 \pm 4.8$ & $3.5 \pm 5.3$ & 0080Y \\
\hline 'yellow' & 2.2 & 7.8 & 8.6 & \\
\hline zelënyj & $21.1 \pm 8.1$ & $66.7 \pm 7.4$ & $7.8 \pm 7.3$ & 2070G10Y \\
\hline 'green' & 11.8 & 10.8 & 10.6 & \\
\hline sinij & $31.6 \pm 7.3$ & $63.7 \pm 4.7$ & $85.3 \pm 4.8$ & 3060R80B \\
\hline 'dark blue' & 10.7 & 6.8 & 7.0 & \\
\hline goluboj & $11.8 \pm 2.9$ & $56.2 \pm 7.5$ & $92.9 \pm 4.0$ & 1060R90B \\
\hline 'light blue' & 5.3 & 10.9 & 5.9 & \\
\hline rozovyj & $4.2 \pm 3.6$ & $41.2 \pm 4.2$ & $14.7 \pm 8.6$ & 0040R, \\
\hline 'pink' & 5.0 & 7.4 & 15.0 & 0040R20B \\
\hline fioletovyj & $29.0 \pm 5.0$ & $56.3 \pm 3.2$ & $46.0 \pm 4.3$ & 3060R50B \\
\hline 'purple' & 7.9 & 5.0 & 6.8 & \\
\hline koričnevyj & $56.7 \pm 6.6$ & $40.0 \pm 5.7$ & $43.3 \pm 6.6$ & $6030 \mathrm{Y} 50 \mathrm{R}$ \\
\hline 'brown' & 9.7 & 8.4 & 9.7 & $6030 \mathrm{Y} 50 \mathrm{R}$ \\
\hline
\end{tabular}

the English 'salmon' and 'pink'). Denotatum splitting was also found for koričnevyj (not shown here).

The data reveal high inter-subject consensus among participating individuals for certain basic chromatic names: for želtyj, the focal $0080 \mathrm{Y}$ was chosen by 13 of 30 individuals (43\%); for oranževyj, 14 of 30 (47\%) chose the focal 0090Y40R. These results are in accord with earlier findings of Frumkina (1984), who reported žëltyj, krasnyj, and oranževyj among the Russian color names with the highest consensus. It is of interest that, for some other languages studied, the terms for 'red,' 'orange,' and 'yellow' were also found to be among those with the highest consensus and/or consistency [Boynton \& Olson, 1990 (American English); Hård \& Sivik 1981 (Swedish); Lin et al. 2001 (British English and Mandarin Chinese); Sturges \& Whitfield 1997 (British English)].

The above observation prompts a deduction that the naming of the 'warm' colors in Russian follows a tendency common across languages, with their high consistency due to the primary differentiation of the 'warm' area in the color space, and hence more elaborated representation by the color nomenclature [e.g., MacLaury et al. 1998 (Hungarian); Warburton 2007 (ancient Egyptian)].

In the evolutionary vein, we assume that the differentiation of the red-yellow area can be traced to the necessity of discriminating ripeness of berries and fruits, gathered or cultivated. Further, increasing functional load in culture may have stipulated categorical 

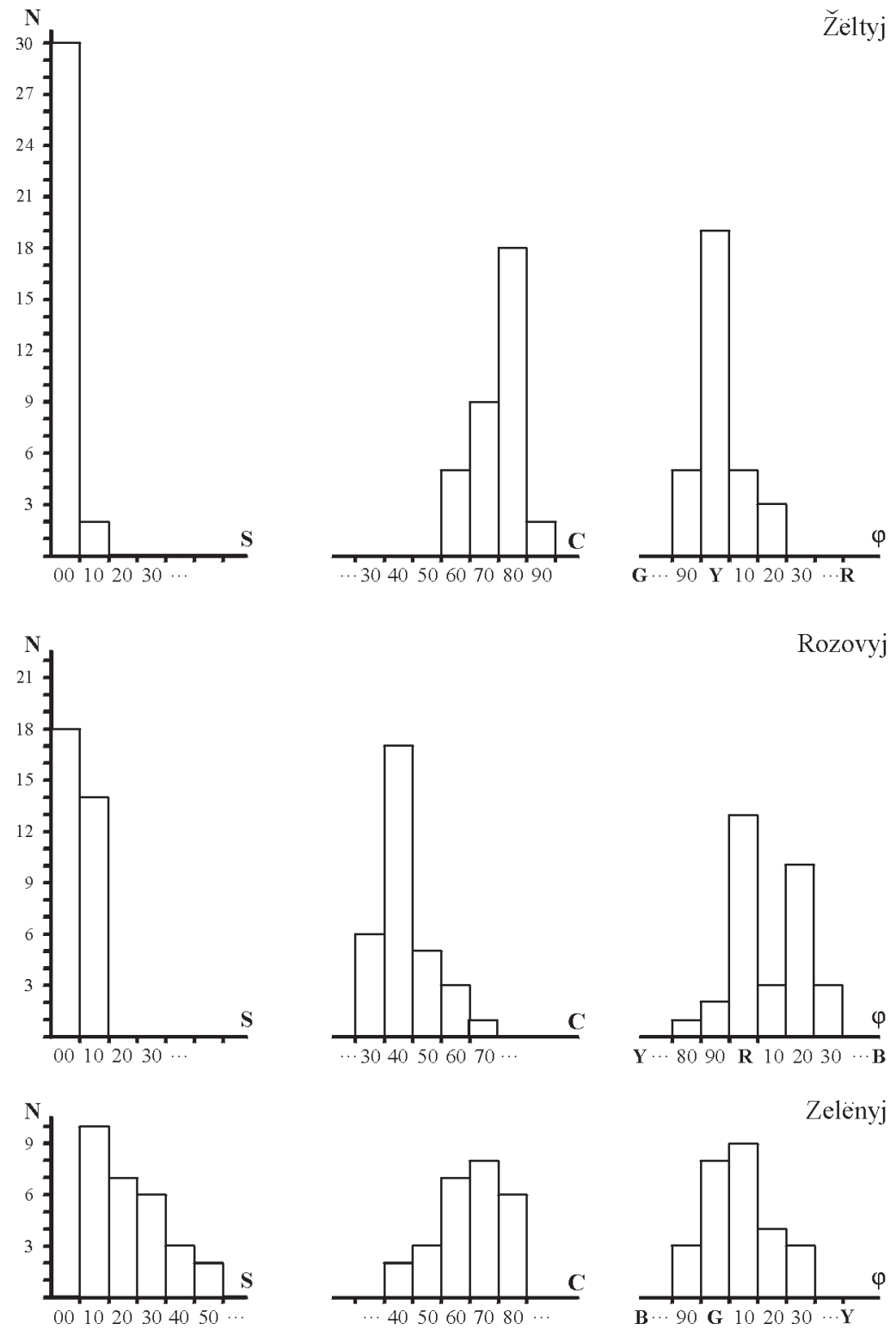

Zelënyj

Figure 2. Histograms of distributions of color-sample choices for the Russian basic color terms žëltyj 'yellow,' rozovyj 'pink,' and zelënyj 'green' along the NCS parameters blackness (s), chromaticness (c), and $h u e(\varphi)$. Ordinate indicates the number of subjects (out of 30 ) choosing the sample.

refinement of the 'warm' area and the emergence of more elaborated nomenclature, useful for making fine distinctions (see the partition principle of Kay \& Maffi 2000). 
In the 'cool' segment, the green area of the color space is represented in a less discriminative way by the basic terms for a Russian speaker, who refers to its nuances as 'light green' or 'dark green.'

By contrast, the 'blue' area is nominally well-elaborated in Russian being represented by two BCTs, sinij 'dark blue' and goluboj 'light blue' (Table 1), in accord with previous findings (Davies \& Corbett 1997; Frumkina 1984). Notably, the coordinates of the focal sinij and goluboj are separated along all three color-space attributes. The most apparent distinction, though, is along the blackness ( $s$ ) dimension: the focal sinij $(s=31.6)$ is mapped under the plane for the NCS elementary blue (the maximal color), whereas the focal goluboj $(s=11.8)$ above the plane. This distinction provides additional evidence, as noted by Paramei (2005; Figures $2 \& 5$ ), that the denotata of the Russian 'blues' differ foremost with respect to the achromatic attribute. Indeed, Lin et al. (2001:198), who also employed the NCS array, obtained $s=20.0$ for the (British) English blue.

\section{Russian non-basic chromatic terms}

The great majority of the frequently used non-basic chromatic terms presented in the experiment were familiar to the participants. However, some of these terms produced much higher denotative dispersion and were hard to relate to the color samples (see Table 2). For instance, fistaškovyj 'pistachio' and olivkovyj 'olive' reveal the highest variance, especially for hue; some individuals reported little knowledge of what pistachio and olive colors look like, others refused to indicate corresponding color samples. Notably, these two names are related to the green area of the color space.

Conversely, the best-defined non-basic chromatic terms map onto the red area, such as rubinovyj 'ruby' and kirpičnyj 'brick-red,' showing denotative consistency comparable to that for some basic chromatic terms.

Also, non-basic terms related to the purple area such as malinovyj 'raspberry', sirenevyj 'lilac', and lilovyj 'mauve' appear highly consistent; for the two former, $C I$ is smaller than for basic koričnevyj 'brown.' This might seem a surprising result, since sirenevyj was found to have widely distributed denotata (Moss et al. 1991:325) and lilovyj was reported to lack a well defined focal point, while denoting not only a variety of purple nuances (Moss 1989), but also colors as distant as green and yellow (Corbett \& Morgan 1988:60; Frumkina \& Mikhejev 1983:58).

In view of these results, Russian names for the purple area are worth closer consideration. Corbett and Morgan (1988) note that Russian possesses five terms, which map onto this area and have rather comparable salience. Among those, lilovyj and sirenevyj, rank higher in a free-list task than four basic color terms (Morgan \& Corbett 1989; Moss et al. 1991), implying their high cognitive salience. By comparison, the term used for translation of the English purple by Slobin in Berlin and Kay (1969), purpurnyj, appears a weak contender for the basic-term status: semantically it greatly overlaps with krasnyj 'red' (Allmere 1982; Vasilevich 1983) and is better translated as 'cardinal red' (Frumkina \& Mikhejev 1996: 86). Our data confirm that for Russians purpurnyj refers to a deep red color (Korzh et al. 1991; Korsch \& Safuanova 1996). Instead, fioletovyj was suggested as a semantically 
Table 2. Russian non-basic chromatic terms: NCS parameters of focal colors

\begin{tabular}{|c|c|c|c|c|c|c|}
\hline \multirow[t]{2}{*}{ No. } & \multirow[t]{2}{*}{ Color term } & \multirow[t]{2}{*}{ Gloss } & \multicolumn{3}{|c|}{$\mathrm{M} \pm \mathrm{CI}$} & \multirow[t]{2}{*}{ Hue } \\
\hline & & & $s$ & c & $\varphi$ & \\
\hline 1 & kofejnyj & coffee-colored & $47.5 \pm 11.1$ & $31.9 \pm 10.8$ & $34.4 \pm 9.1$ & $5030 Y 30 R$ \\
\hline 2 & persikovyj & peach-colored & $4.3 \pm 4.2$ & $46.4 \pm 14.4$ & $34.3 \pm 9.3$ & 0050Y30R \\
\hline 3 & slonovaja kost' & ivory-colored & $1.2 \pm 1.7$ & $12.9 \pm 6.6$ & $14.1 \pm 8.2$ & 0010Y10R \\
\hline 4 & beževyj & beige & $16.9 \pm 6.2$ & $25.3 \pm 7.6$ & $30.7 \pm 6.8$ & 020Y30R \\
\hline 5 & kremovyj (mean) & creamy & $54.8 \pm 6.4$ & $19.3 \pm 8.1$ & $46.7 \pm 7.7$ & 0020Y50R \\
\hline $5 \mathrm{a}$ & zeltovato-kremovyj & yellowish-creamy & $4.7 \pm 6.7$ & $20.3 \pm 5.6$ & $25.8 \pm 5.9$ & 0020Y30R \\
\hline $5 b$ & oranževo-kremovyj & orange-creamy, salmon & $5.0 \pm 5.3$ & $22.8 \pm 11.7$ & $43.6 \pm 8.8$ & 0020Y50R \\
\hline $5 c$ & rozovato-kremovyj & pinkish-creamy & $6.1 \pm 7.2$ & $15.3 \pm 6.9$ & $71.1 \pm 8.1$ & 0020Y70R \\
\hline 6 & pesočnyj & sandy & $8.2 \pm 4.1$ & $42.4 \pm 12.6$ & $18.8 \pm 8.9$ & 1030Y20R \\
\hline 7 & ryžij & brownish red, ginger & $8.5 \pm 4.5$ & $76.9 \pm 9.3$ & $48.2 \pm 10.5$ & 1080Y50R \\
\hline 8 & kirpičnyj & brick-colored & $13.0 \pm 6.3$ & $75.4 \pm 5.8$ & $65.9 \pm 4.5$ & 1080Y70R \\
\hline 9 & korallovyj (mean) & coral & $9.1 \pm 5.9$ & $62.5 \pm 9.6$ & $74.6 \pm 6.1$ & 1070Y70R \\
\hline $9 \mathrm{a}$ & oranževo-korallovyj & orange coral & $10.7 \pm 6.8$ & $60.7 \pm 10.2$ & $69.3 \pm 7.3$ & 1060Y70R \\
\hline $9 b$ & krasno-korallovyj & red coral & $8.0 \pm 4.9$ & $64.0 \pm 8.9$ & $83.5 \pm 7.5$ & $1070 \mathrm{Y} 80 \mathrm{R}$ \\
\hline 10 & alyj & scarlet & $8.8 \pm 3.5$ & $82.1 \pm 11.6$ & $90.0 \pm 5.6$ & 1090Y90R \\
\hline 11 & malinovyj & raspberry & $15.8 \pm 5.1$ & $69.0 \pm 4.9$ & $14.2 \pm 6.0$ & 1070R10B \\
\hline 12 & rubinovyj & ruby & $17.0 \pm 5.4$ & $76.5 \pm 6.2$ & $3.2 \pm 5.8$ & $2080 \mathrm{R}$ \\
\hline 13 & bordovyj & red whine, claret & $30.8 \pm 9.1$ & $65.3 \pm 8.2$ & $6.1 \pm 5.5$ & $4060 \mathrm{R}$ \\
\hline 14 & višnevyj & cherry & $29.7 \pm 7.7$ & $65.6 \pm 7.2$ & $7.8 \pm 5.3$ & 3070R10B \\
\hline 15 & sirenevyj & lilac & $23.2 \pm 4.4$ & $50.5 \pm 3.4$ & $46.3 \pm 3.9$ & 2050R50B \\
\hline 16 & lilovyj & mauve & $28.9 \pm 7.2$ & $50.6 \pm 6.9$ & $47.8 \pm 5.9$ & 3050R50B \\
\hline 17 & biruzovyj (mean) & turquoise & $17.5 \pm 5.1$ & $52.5 \pm 5.2$ & $33.6 \pm 10.6$ & 2050B30G \\
\hline $17 \mathrm{a}$ & sinij-birusovyj & dark-blue turquoise & $15.3 \pm 4.0$ & $52.7 \pm 6.1$ & $23.3 \pm 12.3$ & 2050B20G \\
\hline $17 b$ & zelënyj-biruzovyj & green turquoise & $20 \pm 6.0$ & $52.3 \pm 6.2$ & $45.3 \pm 13.2$ & 2050B50G \\
\hline 18 & morskaja volna (mean) & sea wave & $29.7 \pm 7$ & $54.0 \pm 5.7$ & $42.7 \pm 9.4$ & 3050B40G \\
\hline $18 \mathrm{a}$ & sinyaja morskaja volna & dark-blue sea wave & $30.6 \pm 6.2$ & $53.8 \pm 4.7$ & $31.3 \pm 7.0$ & 3050B30G \\
\hline $18 \mathrm{~b}$ & zelënaja morskaja volna & green sea wave & $22.8 \pm 9.7$ & $54.1 \pm 5.3$ & $54.2 \pm 11.1$ & 2060B50G \\
\hline 19 & izumrudnyj & emerald-green & $23.5 \pm 6.1$ & $61.8 \pm 7.2$ & $95.3 \pm 7.6$ & $2060 \mathrm{G}$ \\
\hline 20 & salatovyj & light green, lettuce & $3.1 \pm 3.5$ & $62.5 \pm 8.2$ & $28.8 \pm 7.5$ & 0060G30Y \\
\hline 21 & fistaškovyj & pistachio-green & $12.1 \pm 9.5$ & $50.0 \pm 10.4$ & $44.3 \pm 19.8$ & - \\
\hline 22 & limonnyj & lemon-colored & $0.0 \pm 0.0$ & $65.6 \pm 10.8$ & $88.1 \pm 8.6$ & 0070G90Y \\
\hline 23 & tabačnyj & tobacco & $40.0 \pm 14.4$ & $38.6 \pm 6.7$ & $81.5 \pm 6.7$ & 4040G80Y \\
\hline 24 & xaki & khaki & $42.3 \pm 7.0$ & $41.5 \pm 8.3$ & $70.8 \pm 11.7$ & 4040G70Y \\
\hline 25 & olivkovyj & olive & $36.6 \pm 12.1$ & $43.8 \pm 13.2$ & $56.3 \pm 15.7$ & - \\
\hline 26 & bolotnyj & marsh & $48.6 \pm 3.0$ & $42.1 \pm 9.1$ & $67.1 \pm 7.4$ & $5050 \mathrm{G} 70 \mathrm{Y}$ \\
\hline 27 & zaščitnyj & brown-green, khaki & $34.4+16.9$ & $47.8 \pm 7.4$ & $76.7 \pm 13.7$ & 3050G80Y \\
\hline 28 & purpurnyj & cardinal red & $16.5 \pm 5.1$ & $75.5 \pm 8.4$ & $1.0 \pm 5.4$ & $2080 \mathrm{R}$ \\
\hline
\end{tabular}




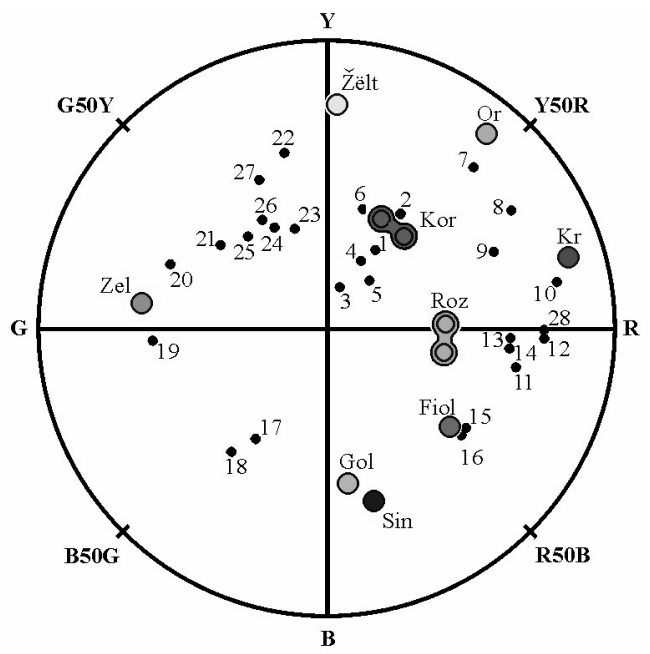

Figure 3. Location of focal colors of the eight Russian chromatic basic terms in projection into the NCS color circle (clockwise): žëltyj 'yellow' (Žëlt), oranževyj 'orange’ (Or), koričnevyj 'brown' (Kor), krasnyj 'red' (Kr), rozovyj 'pink' (Roz), fioletovyj 'purple' (Fiol), sinij 'dark blue' (Sin), goluboj 'light blue' ( $\mathrm{Gol}$ ), and zelënyj 'green' $(\mathrm{Zel})$. Locations of the frequent non-basic terms are designated by numbers (for legend see Table 2). Adapted from Korzh et al. (1991).

A full color version of this figure can be found on p. xviii

more adequate Russian counterpart for the basic purple (Corbett \& Morgan 1988; Frumkina \& Mikhejev 1996; Vasilevich 1987, 1988). This assertion is confirmed by our data: the NCS parameters for focal fioletovyj are very similar to those for purple found by Lin et al. (2001).

Interestingly, the refinement of the 'red' and 'purple' areas in the Russian color nomenclature is echoed, in respect to English, by Kerttula; she notes that "Color term categories that might divide in future are red-related and purple-related terms, which already have hyponyms and are used to define other color terms in dictionaries. Violet, which is hyponym of purple, will probably form a group of its own" (Kerttula 2002:335).

In accord with the note of Rosch (1972) that focal colors have the capacity to semantically attract adjacent colors, we, too, observe that focal colors denoted by non-basic color names tend to group around corresponding foci of the basic color terms. For instance, the foci of alyj 'scarlet' and rubinovyj 'ruby' are located near that of krasnyj 'red,' while focal sirenevyj 'lilac' and lilovyj 'mauve' abut to fioletovyj 'purple' (see Tables 1 and 2).

Figure 3 shows locations of focal colors for the chromatic basic and frequent non-basic color terms projected into the NCS color circle. The diagram illustrates a non-uniform lexical representation of the color space: as was noted above, nominative density is highest in the red-yellow area, whereas the least number of color names is associated with the blue-green area. 


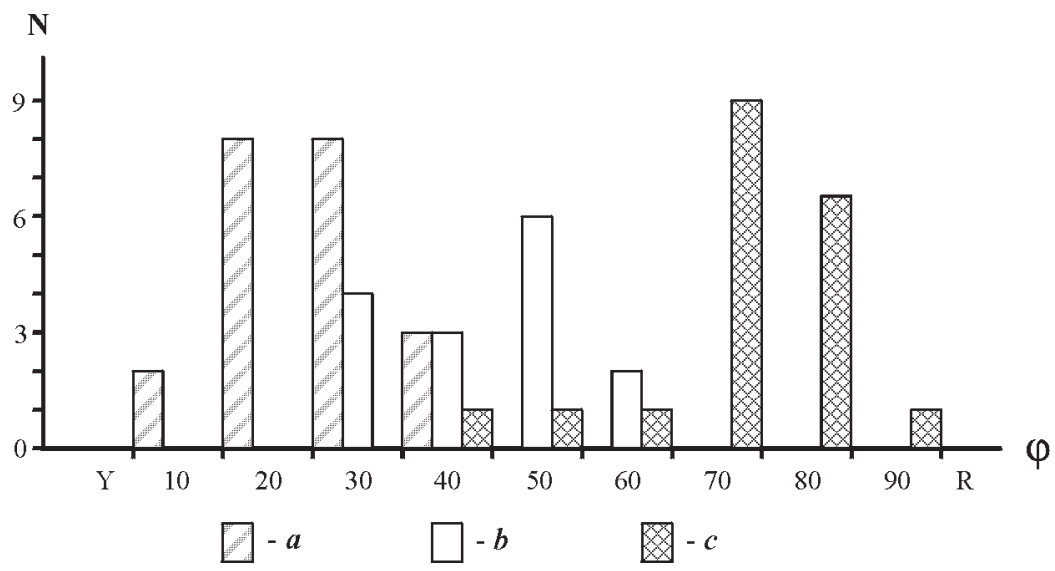

Figure 4. Histograms of three-peak distribution of color-sample choices for the non-basic name kremovyj 'creamy': a-želtovato-kremovyj 'yellowish-creamy' b-oranževo-kremovyj 'orange-creamy', and $c$-rozovato-kremovyj 'pinkish-creamy.' Abscissa indicates the NCS attribute of hue $(\varphi)$, ordinate the number of subjects (out of 30) choosing the sample.

Here we consider, in addition, those non-basic chromatic names that appeared especially resistant to referral to a single color sample. When confronted with them, Russian individuals commonly tended to introduce their own specifications, by qualifying the main term with a number of various chromatic modifiers. This frequently resulted in multiple focality of the main term. For example, when presented with kremovyj 'creamy', participants preferred to find denotata tinged with various hue nuances rather than that for pure 'creamy.' As a consequence (see Figure 4), three sub-focals were obtained that vary in their hue: želtovato-kremovyj 'yellowish-creamy' (0020Y30R), oranževo-kremovyj 'orange-creamy' (0020Y50R), and rozovato-kremovyj 'pinkish-creamy' (0020Y70R). The three best examples of kremovyj reveal, though, the same values for $s$ and $c$; they are light and low saturated.

The tendency of multiple focality was similarly observed for other non-basic terms (whose histograms are not presented here): for korallovyj 'coral' some subjects indicated color samples for red coral, whereas the other for orange; for morskaja volna 'sea wave' dark-blue and green focals were obtained; and for biruzovyj 'turquoise' dark-blue and green focals.

\section{Russian compound chromatic names}

Along with the basic and frequent non-basic terms, we estimated the precision of frequent Russian compound names, combinations of two basic color terms that denote colors neighboring in the color circle. Here only a part of those terms and their best examples are listed illustrating main tendencies (see Table 3). As expected, inter-subject consistency 
Table 3. Russian compound chromatic terms: NCS parameters of focal colors

\begin{tabular}{|c|c|c|c|c|c|c|}
\hline \multirow[t]{2}{*}{ No. } & \multirow[t]{2}{*}{ Color term } & \multirow[t]{2}{*}{ Gloss } & \multicolumn{3}{|c|}{$\mathrm{M} \pm \mathrm{CI}$} & \multirow[t]{2}{*}{ Hue } \\
\hline & & & s & c & $\varphi$ & \\
\hline 1. & želtovato-oranževyj & yellowish-orange & $0.6 \pm 1.8$ & $81.2 \pm 6.5$ & $34.4 \pm 5.4$ & 0080Y30R \\
\hline 2. & krasnovato-oranževyj & reddish-orange & $3.1 \pm 4.4$ & $84.4 \pm 4.6$ & $58.1 \pm 5.5$ & 0080Y60R \\
\hline 3. & krasno-oranževyj & red-orange & $0.9 \pm 2.9$ & $80.9 \pm 6.7$ & $65.4 \pm 5.0$ & 0080Y70R \\
\hline 4. & oranževato-krasnyj & orangish-red & $6.5 \pm 4.7$ & $84.0 \pm 4.3$ & $73.0 \pm 3.0$ & 1080Y70R \\
\hline 5. & oranževo-krasnyj & orange-red & $2.0 \pm 4.3$ & $83.0 \pm 6.9$ & $68.0 \pm 6.5$ & 0080Y70R \\
\hline 6. & želtovato-koričnevyj & yellowish-brown, $\tan$ & $35.0 \pm 8.0$ & $58.8 \pm 7.1$ & $24.4 \pm 6.5$ & $4060 Y 20 R$ \\
\hline 7. & fioletovo-sinij & purple-dark blue & $29.0 \pm 11.3$ & $55.6 \pm 8.0$ & $71.0 \pm 11.3$ & 3050R70B \\
\hline 8. & sine-fioletovyj & dark blue-purple & $31.0 \pm 3.3$ & $51.0 \pm 5.9$ & $61.0 \pm 5.8$ & 3050R60B \\
\hline 9. & golubovato-zelënyj & light-bluish green & $22.9 \pm 7.0$ & $48.8 \pm 7.9$ & $70.6 \pm 9.3$ & 2050B70G \\
\hline 10. & zelenovato-goluboj & greenish light-blue & $16.9 \pm 5.9$ & $16.9 \pm 5.9$ & $15.3 \pm 8.3$ & 1050B20G \\
\hline 11. & sine-zelënyj & dark blue-green & $33.8 \pm 10.0$ & $51.2 \pm 7.7$ & $45.6 \pm 6.9$ & 3050B50G \\
\hline 12. & zelenovato-sinij & greenish-dark blue & $37.5 \pm 8.9$ & $51.2 \pm 5.9$ & $10.6 \pm 9.1$ & 4050B10G \\
\hline 13. & zelenovato-žëltyj & greenish-yellow & $1.9 \pm 2.5$ & $67.7 \pm 6.0$ & $82.6 \pm 6.5$ & 0070G80Y \\
\hline 14. & zelëno-žëltyj & green-yellow & $4.5 \pm 5.0$ & $67.3 \pm 11.4$ & $70.0 \pm 9.6$ & 0070G70Y \\
\hline 15. & žëlto-zelënyj & yellow-green & $10.0 \pm 5.3$ & $70.6 \pm 6.7$ & $55.6 \pm 11.4$ & 1070G60Y \\
\hline 16. & želtovato-zelënyj & yellowish-green & $10.6 \pm 5.4$ & $71.2 \pm 6.6$ & $43.5 \pm 13.1$ & $1070 \mathrm{G} 40 \mathrm{Y}$ \\
\hline
\end{tabular}

for non-monolexemic names was lower then that for the basic color terms (cf. Boynton \& Olson 1990).

As a rule, the compound terms are mapped systematically in the color space; that is, their foci are located between those of corresponding basic terms (see Figure 5). For example, želtovato-zelënyj 'yellowish-green' is located closer to zelënyj 'green,' whereas zelenovato-žëltyj 'greenish yellow' is nearer to žëltyj 'yellow', while žëlto-zelënyj 'yellowgreen' is mapped between these two compound terms. The same tendency is observed for compound names related to red-blue or blue-green areas of the color space. Some exceptions are observed, though, in the area of 'warm' colors: as seen in Figure 5 and Table 3, best examples of some compound terms (krasno-oranževyj 'red-orange', oranževatokrasnyj 'orangish-red,' and oranževo-krasnyj 'orange-red') actually overlap, denoting approximately the same color. The latter is believed to result from the high nominative density of the 'warm' area, where compound terms abound and their denotative meanings are very similar.

Basic color names with achromatic modifiers

Table 4 presents coordinates of best examples for the chromatic basic terms with achromatic modifiers bledno- 'pale,' svetlo- 'light,' jarko- 'bright,' and tëmno- 'dark.' Note that for certain basic terms (krasnyj 'red,' oranževyj 'orange,' koričnevyj 'brown,' and sinij 'blue'), 'pale' variants could not be defined: lexical combinations such as bledno-krasnyj 'pale red,' bledno-oranževyj 'pale orange' or bledno-koričnevyj 'pale brown,' though possible in Rus- 


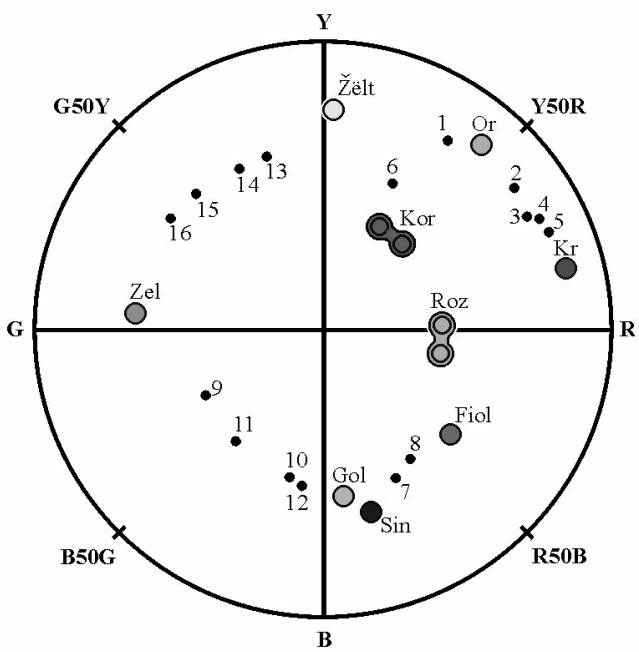

Figure 5. Location of focal colors for the Russian chromatic basic color terms (letter designation as in Figure 2) and compound chromatic terms (indicated by numbers as in Table 3) in projection into the NCS color circle. Adapted from Korzh et al. (1991).

A full color version of this figure can be found on p. xviii

Table 4. Russian compound terms consisting of basic chromatic name and an achromatic modifier: NCS hue notations of focal colors

\begin{tabular}{llcccl}
\hline & & \multicolumn{4}{c}{ Hue } \\
\cline { 3 - 6 } Color term & Gloss & svetlo- 'light' & jarko- 'bright' & tëmno- 'dark' & bledno- 'pale' \\
\hline krasnyj & red & 0080Y80R & 1090Y80R & 2080Y80R & - \\
oranževyj & orange & 0070Y40R & 0090Y50R & 1080Y50R & - \\
žëltyj & yellow & 0060Y & 0080Y & 1080Y10R & 0040Y \\
zelënyj & green & 1060G10Y & 1070G10Y & 4060G10Y & 1050G10Y \\
sinij & dark blue & 2060R90B & 2070R80B & 5060R80B & - \\
goluboj & light blue & 1040R90B & 1060R90B & 3050R90B & 1020R90B \\
rozovyj & pink & 0020R10B & 2050R10B & 2050R10B & 0020R10B \\
fioletovyj & violet & 1040R50B & 3060R50B & 4050R50B & 1040R50B \\
koričnevyj & brown & 3040Y50R & 040Y50R & 7030Y50R & - \\
\hline
\end{tabular}

sian, are used quite infrequently (Morgan \& Corbett 1989:132-134) and are subject to attributive combinability (Rakhilina 2007). We found that for non-elementary colors, their achromatically modified terms may be mapped within the modifier area of elementary colors; for example, focal goluboj 'light blue' is mapped within the area of svetlo-sinij 'light dark blue.'

Figure 6 illustrates a typical arrangement of focal colors for a basic name and its variants with achromatic modifiers, in projection into the NCS blackness-chromaticness triangle. Note that 'pale,' 'light,' and 'bright' examples of the term are systematically ar- 

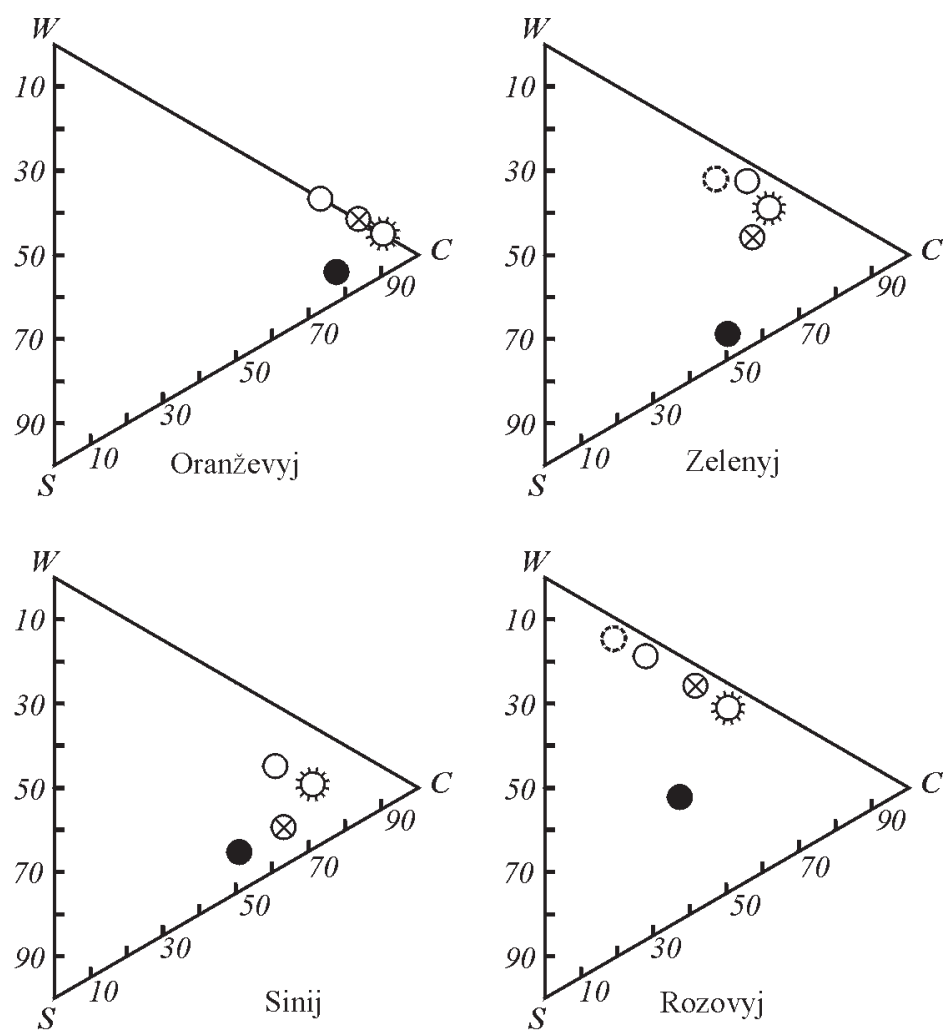

Figure 6. Location of focal colors $(\otimes)$ for the basic terms oranževyj 'orange,' zelënyj 'green,' sinij 'dark blue,' and rozovyj 'pink,' as well as for their variants with achromatic modifiers bledno- 'pale' ( ), svetlo- 'light' ( $\mathrm{O})$, jarko- 'bright' ( blackness-chromaticness triangle.

ranged along an imaginary axis, approximately parallel to either the $\mathrm{W}-\mathrm{C}$ triangle side for lighter colors - or the S-C side - for darker colors. Further, the best examples for terms with 'light' and 'dark' modifiers are located along an imaginary axis approximately parallel to the W-S side, or blackness dimension. Both indicate that, within the perceptual space, the modifier bledno- 'pale' displaces the term's focality in the direction opposite to modifier jarko- 'bright'; at the same time its denotatum is similar to that of the 'light,' though with lower chromatic component.

Achromatic basic color terms with chromatic nuances

We also estimated denotative consistency of a number of compound terms for achromatic colors with chromatic nuances (see Table 5). As denotative references for Russian basic achromatic names belyj 'white,' cërrnyj 'black,' and seryj 'gray,' the following NCS samples were used: white 0500 , black 9500 , and gray 4500 . 
Table 5. Russian compound terms consisting of achromatic names with chromatic nuances: NCS parameters of focal colors

\begin{tabular}{|c|c|c|c|c|c|}
\hline \multirow[t]{2}{*}{ Color term } & \multirow[t]{2}{*}{ Gloss } & \multicolumn{3}{|c|}{$\mathrm{M} \pm \mathrm{CI}$} & \multirow[t]{2}{*}{ Hue } \\
\hline & & $\mathbf{s}$ & c & $\varphi$ & \\
\hline rozovato-belyj & pinkish-white & $0 \pm 0$ & $9.2 \pm 1.6$ & $99.2 \pm 8.6$ & 0010R \\
\hline fioletovo-belyj & purplish-white & $0 \pm 0$ & $14.5 \pm 3.8$ & $49.0 \pm 2.8$ & 0010R50B \\
\hline zelenovato-belyj & greenish-white & $0 \pm 0$ & $9.4 \pm 4.2$ & $15.6 \pm 12.8$ & 0010G10Y \\
\hline golubovato-belyj & light-bluish-white & $0 \pm 0$ & $8.4 \pm 2.9$ & $90.0 \pm 6$ & 0010R90B \\
\hline želtovato-belyj & yellowish white & $0 \pm 0$ & $10.6 \pm 3.6$ & $98.9 \pm 8.4$ & $0010 \mathrm{Y}$ \\
\hline krasnovato-čërnyj & reddish-black & $65.0 \pm 5.7$ & $30.0 \pm 6.9$ & $92 \pm 5.7$ & 7030Y90R \\
\hline fioletovo-čërnyj & purplish-black & $69.5 \pm 5.0$ & $21.4 \pm 6.0$ & $49.5 \pm 3.1$ & 7020R50B \\
\hline koričnevato-čërnyj & brownish-black & $76.7 \pm 3.3$ & $11.9 \pm 3.1$ & $42.9 \pm 11.2$ & 8010Y50R \\
\hline zelenovato-čërnyj & greenish-black & $77.5 \pm 3.3$ & $15.0 \pm 4.7$ & $11.2 \pm 14.8$ & 8010G10Y \\
\hline sinevato-čërnyj & bluish-black & $73.8 \pm 3.7$ & $22.5 \pm 5.1$ & $83.75 \pm 7.1$ & 7020R90B \\
\hline krasnovato-seryj & reddish-gray & $31.8 \pm 8.7$ & $22.4 \pm 6.5$ & $87.7 \pm 4.9$ & $3050 \mathrm{Y} 90 \mathrm{R}$ \\
\hline fioletovo-seryj & purplish-gray & $37.8 \pm 8.9$ & $20.0 \pm 4.7$ & $45.5 \pm 5.9$ & 4020R50B \\
\hline koričnevato-seryj & brownish-gray & $48.1 \pm 6.2$ & $15.0 \pm 6.7$ & $28.7 \pm 12.7$ & $5010 \mathrm{Y} 30 \mathrm{R}$ \\
\hline želtovato-seryj & yellowish-gray & $19.5 \pm 7.0$ & $23.5 \pm 7.5$ & $97.5 \pm 8.8$ & 2020G90Y \\
\hline zelenovato-seryj & greenish-gray & $43.9 \pm 7.8$ & $18.9 \pm 4.6$ & $17.8 \pm 13.4$ & 4020G10Y \\
\hline golubovato-seryj & light-bluish-gray & $25.45 \pm 9.9$ & $16.4 \pm 4.8$ & $94.5 \pm 8.9$ & 2020R90B \\
\hline sinevato-seryj & bluish-gray & $37.0 \pm 9.2$ & $20.0 \pm 5.6$ & $86.5 \pm 4.8$ & 3020R90B \\
\hline
\end{tabular}

Among the three groups of compound terms with the achromatic components - -belyj 'white,' -čerrnyj 'black', and -seryj 'gray' - the most denotatively certain is the 'white' group. Within it, the cold golubovato-belyj 'bluish-white' and fioletovo-belyj 'purple-white' reveal the highest denotative precision, whereas the 'gray' names show the lowest (Korzh \& Safuanova 1994).

With regards to the chromatic component, compound terms with names for basic colors, krasnovato- 'reddish,' sinevato- 'dark-bluish,' and fioletovo- 'purple,' show the highest denotative precision, as opposed to that for names with zelenovato- 'greenish,' koričnevato'brownish,' and rozovato- 'pinkish.' The latter finding is of certain interest, since the order of denotative precision replicates that for the monolexemic basic chromatic terms (cf. Table 1). The finding indicates that denotative precision of compound terms with achromatic modifiers depends predominantly on the precision of their constituent chromatic component.

\section{Conclusions}

The purpose of the present study was to estimate foci of the Russian basic color terms, as well as of frequently used non-basic names and polylexemic color names with chromatic and achromatic compounds; and to ascertain each color-term's denotative precision and its 
denotative consistency. As denotata, samples of the Natural Color System were used. The psycholinguistic experiments provided the following findings.

1. The perceptual color space is represented non-uniformly by Russian color nomenclature, with color names distributed with different densities across this perceptual gamut. The red-yellow 'warm' area is represented nominally most densely. In the 'cool' segment, in Russian, unlike many other languages, the blue area is nominally well-elaborated too; it possesses two terms for blue, sinij 'dark blue' and goluboj 'light blue', distinctive in their foci and comparable in denotative consistency. By contrast, the green area reveals the fewest associated color names. These findings indicate that color nomination evolves differently across the perceptual gamut, with its 'warm' part being generally in advance. Nominative density is believed to be one of the factors that influence the denotative precision of color names.

2. Russian basic color terms differ with regards to their denotative precision in the perceptual color space, and denotative consistency. Among the nominally best defined are terms for the 'warm' area.

3. Russian color terms differ in their denotative consistency with regards to the three NCS perceptual attributes: blackness $(s)$, chromaticness $(c)$, and hue $(\varphi)$. For example, krasnyj 'red' shows high denotative consistency along all three attributes; by comparison, rozovyj 'pink' is poorly defined for hue, whereas koričnevyj 'brown' is least consistent along all three attributes.

4. The best-elaborated Russian non-basic color terms are no less consistent than some of the basic terms. (These are predominantly related to the red and purple area; for example, rubinovyj 'ruby,' kirpičnyj 'brick-colored,' sirenevyj 'lilac', malinovyj 'raspberry,' and lilovyj 'mauve.')

5. Denotative precision of compound color terms with achromatic modifies depends predominantly on the precision of the color-name constituent that designates the chromatic nuance.

\section{Acknowledgments}

We are deeply grateful to Galina Paramei, Douglas Bowden, Anna Leonova, and David Bimler for helpful and valuable comments and suggestions.

\section{References}

Allmere, R. A. (1982). K probleme perevodimosti: psixolingvističeskij podhod k anglo-russkomu perevodu cvetonaimenovanij [On the problem of translatability: A psycholinguistic approach to English-Russian translation of color terms]. In Ju. A. Sorokin (Ed.), Tezisy VII Vsesojuznogo simpoziuma po psixolingvistike $i$ teorii kommunikacii [Abstracts of the 7th All-Soviet symposium on psycholinguistics and communication theory] (119-121), Moscow: Institut Jazykoznanija AN SSSR. 
Alvarado, Nancy \& Kimberly A. Jameson (2002). The use of modifying terms in the naming and categorization of color appearances in Vietnamese and English. Journal of Cognition and Culture, 2, 5380.

Berlin, Brent \& Paul Kay (1969). Basic color terms. Their universality and evolution. Berkeley: University of California Press.

Boynton, Robert M. \& Conrad X. Olson (1990). Salience of chromatic basic color terms confirmed by three measures. Vision Research, 30, 1311-1317.

Corbett, Greville G. \& Ian R. L. Davies (1997). Establishing basic color terms: measures and techniques. In C. L. Hardin \& L. Maffi (Eds.), Color categories in thought and language (197-223). Cambridge: Cambridge University Press.

Corbett, Greville G. \& Gerry Morgan (1988). Colour terms in Russian: Reflections of typological constraints in a single language. Journal of Linguistics, 24, 31-64.

Crawford, T. D. (1982). Defining "basic color term". Anthropological Linguistics, 24, 338-343.

Davies, Ian R. L. \& Greville G. Corbett (1994). The basic color terms of Russian. Linguistics, 32, 65-89.

Davies, Ian R. L. \& Greville G. Corbett (1997). A cross-cultural study of colour grouping: Evidence for weak linguistic relativity. British Journal of Psychology, 88, 493-517.

Frumkina, Revekka M. (1984). Cvet, smysl, sxodstvo. Aspekty psixolingvističeskogo analiza [Color, meaning, and similarity: Aspects of a psycholinguistic analysis.]. Moscow: Nauka.

Frumkina, Revekka M. \& Alexei V. Mikhejev (1983). Vozmožnosti sopostavitel'nogo izučenija leksiki $\mathrm{v}$ èksperimente (na materiale russkix prilagatel'nyx-cvetoobosnačenij) [The possibilities of a comparative study of vocabulary in an experiment (on material of Russian adjectives-color terms)]. Sapostavitelno Jezykosnanie (Sofia), 8, 51-63.

Frumkina, Revekka M. \& Alexei V. Mikhejev (1996). Meaning and categorization. New York: Nova Science. Guest, Steve \& Darren Van Laar (2000). The structure of colour naming space. Vision Research, 40, 723734.

Jameson, Kimberly A. \& Nancy Alvarado (2003). The relational correspondence between category exemplars and names. Philosophical Psychology, 16, 25-37.

Hård, Anders \& Lars Sivik (1981). NCS - Natural Color System: A Swedish standard for color notation. Color Research \& Application, 4, 129-138.

Hård, Anders \& Lars Sivik (1983). Basic concept of the NCS and its use for studies of color rendering. Poster paper at the Forsius Symposium on Color Order Systems. Kungälv, Sweden.

Hippisley, Andrew (2001). Basic BLUE in East Slavonic. Linguistics, 39, 151-179.

Humboldt, Wilhelm von (1836/1971). Linguistic variability and intellectual development (G. Buck \& F. A. Raven, Trans.). Coral Gables, FL: University of Miami Press.

Kay, Paul, Brent Berlin, \& William R. Merrifield (1991). Biocultural implications of systems of color naming. Journal of Linguistic Anthropology, 1, 12-25.

Kay, Paul \& Luisa Maffi (1999). Color appearance and the emergence and evolution of basic color lexicons. American Anthropologist, 101, 743-760.

Kerttula, Seija (2002). English colour terms: Etymology, chronology, and relative basicness. [Mémoires de la Société Néophilologique de Helsinki, 60]. Helsinki: Société Néophilologique.

Korzh, Nina N., Irina V. Penova, \& Olga V. Safuanova (1991). Denotativnye značenija cvetonaimenovanij [Denotative meanings of color terms]. Psikhologicheskij Zhurnal, 12, 69-79.

Korzh, Nina N. \& Olga V. Safuanova (1993). The dynamics of perceptual image and individual-personal characteristics of the reflection of a color environment. Journal of Russian and East European Psychology, 31, 22-37.

Korzh, Nina N. \& Olga V. Safuanova (1994). Denotativnye značenija cvetonaimenovanij [Denotative meanings of color terms]. Psikhologicheskij Zhurnal, 15, 109-115. 
Korsch, Nina N. \& Olga V. Safuanova (1996). Denotative meaning of the colour name. In L. Sivik (Ed.), Colour report F50. Colour and psychology. AIC interim meeting 96 in Gothenburg (14-15). Stockholm: Scandinavian Colour Institute.

Lin, Helen, M. Ronnier Luo, Lindsay W. MacDonald, \& Arthur W. S. Tarrant (2001). A cross-cultural colour-naming study: Part 1 - Using an unconstrained method. Color Research \& Application, 26, 40-60.

MacLaury, Robert E. (2000). Linguistic relativity and the plasticity of categorization. Universalism in a new key. In M. Pütz \& M. H. Verspoor, (Eds.), Explorations in linguistic relativity (pp. 249-294). Amsterdam \& Philadelphia: John Benjamins.

MacLaury, Robert E. (2002). Introducing vantage theory. Language Sciences, 24, 493-536.

MacLaury, Robert E., Judit Almási, \& Zoltán Kövecses (1998). Hungarian Piros and Vörös: Color from points of view. Semiotica, 114, 67-81.

Moore, Carmella C., A. Kimball Romney, \& Ti-lien Hsia (2002). Cultural, gender, and individual differences in perceptual and semantic structures of basic colors in Chinese and English. Journal of Cognition and Culture, 2, 1-28.

Morgan, Gerry \& Greville G. Corbett (1989). Russian colour term salience. Russian Linguistics, 13, 125141.

Moss, Anthony E. (1989). Basic colour terms: Problems and hypotheses. Lingua, 78, 313-320.

Moss, Anthony, Ian Davies, Greville G. Corbett, \& Glynis Laws (1990). Mapping Russian basic colour terms using behavioural measures. Lingua, 82, 313-332.

Paramei, Galina V. (2005). Singing the Russian blues: An argument for culturally basic color terms. CrossCultural Research, 39, 10-38.

Paramei, Galina V. (2007). Russian 'blues': Controversies of basicness. In R. E. MacLaury, G. V. Paramei, \& D. Dedrick (Eds.), Anthropology of color: Interdisciplinary multilevel modeling (75-106). Amsterdam: John Benjamins.

Penova, Irina V. (1991). Sistema naimenovanij $i$ kodov cvetov [A system for naming and coding colors]. Moscow: VNIITI.

Rakhilina, Ekaterina V. (2007). Linguistic construal of colors: The case of Russian. In R. E. MacLaury, G. V. Paramei, \& D. Dedrick (Eds.), Anthropology of color: Interdisciplinary multilevel modeling (363-378). Amsterdam: John Benjamins.

Roberson, Debi, Ian Davies, \& Jules Davidoff (2000). Color categories are not universal: Replication and new evidence from a stone age culture. Journal of Experimental Psychology: General, 129, 369-398.

Rosch, Eleanor H. (1972). Universals in color naming and memory. Journal of Experimental Psychology, 93, 10-20.

Sapir, Edward (1968). Language and environment. In D. Mandelbaum (Ed.), Selected writings of Edward Sapir in language, culture and personality. Berkeley: University of California Press.

Saunders, Barbara A. C. (2007). Towards a new topology of colour. In R. E. MacLaury, G. V. Paramei, \& D. Dedrick (Eds.), Anthropology of color: Interdisciplinary multilevel modeling (467-479). Amsterdam: John Benjamins.

Sivik, Lars (1997). Color systems for cognitive research. In C. L. Hardin \& L. Maffi (Eds.), Color categories in thought and language (163-193). Cambridge: Cambridge University Press.

Sturges, Julia \& T. W. Allan Whitfield (1997). Salient features of Munsell colour space as a function of monolexemic naming and response latencies. Vision Research, 37, 307-313.

Sutrop, Urmas (2001). List task and a cognitive salience index. Field Methods, 13, 263-276.

Tonnquist, Gunnar (1986). Philosophy of perceptive color order systems. Color Research \& Application, $11,51-55$.

Uchikawa, Keiji \& Robert M. Boynton (1987). Categorical color perception of Japanese observers: Comparison with that of Americans. Vision Research, 27, 1825-1833. 
Valberg, Arne (2001). Unique hues: An old problem for a new generation. Vision Research, 41, 1645-1657. Vasilevich, Alexander P. (1983). Psixolingvističeskij podxod k ustanovleniju leksičeskix sootvetstvij (na materiale bolgarskix, russkix i anglijskix cvetonaimenovanij) [A psycholinguistic approach to estimation of lexical equivalents (on material of Bulgarian, Russian, and English color terms)]. Sapostavitelno Jezykosnanie (Sofia), 8, 5-17.

Vasilevich, Alexander P. (1987). Issledovanie leksiki v psixolingvističeskom èxperimente: Na materiale cvetooboznačenij v jasykax raznyx system [An investigation of nomenclature lexicon in a psycholinguistic experiment: On material of color terms in languages of different systems]. Moscow: Nauka.

Vasilevich, Alexander P. (1988). Cvetonaimenovanija i problemy perevoda teksta [Color terms and problems of text translation]. In V. N. Komissarov, L. A. Cherniakhovskaya, \& L. K. Latyshev (Eds.), Tekst i perevod [Text and Translation] (84-96). Moscow: Nauka.

Vasilevich, Alexander P. (2003). Jazykovaja kartina mira cveta. Metody issledovanija i prikladnye aspekty [A language view of the color world. Methods of investigation and applied aspects]. Post-doctoral dissertation, Moscow Institut jazykoznanija [Russian Academy of Sciences].

Warburton, David A. (2007). Basic color term evolution in light of ancient evidence from the Near East. In R. E. MacLaury, G. V. Paramei, \& D. Dedrick (Eds.), Anthropology of color: Interdisciplinary multilevel modeling (229-246). Amsterdam: John Benjamins.

Whorf, Benjamin L. (1956). Languages and logic. In J. B. Carroll (Ed.), Language, thought and reality. The collected papers of Benjamin Lee Whorf. Cambridge, MA: MIT Press. 


\title{
Russian 'blues'
}

\section{Controversies of basicness}

\author{
Galina V. Paramei \\ Technische Universität Darmstadt, Germany
}

Sinij and goluboj, the two Russian confounders for 'blue', are considered. The question addressed is whether solely sinij or both have status of basic color terms. Results along various lines of experimental research are analyzed. Developmental studies in preschoolers show that with regards to sinij, acquisition of goluboj is overdue, but comparable to that of 'orange' and 'purple'. Psycholinguistic studies indicate that both Russian 'blues' are named with high consensus and have circumscribed, though adjoining areas when mapped into a color space. Psychometric studies relate data of Russian and English speakers on color categorization and discrimination within the blue region. Results are indecisive on whether Russian speakers possess a sinij-goluboj category boundary. However, choices of stimuli and/or experimental design were suboptimal. Further controlled psychometric investigation is required. Nevertheless, linguistic (developmental, metonymic, metaphoric) and psycholinguistic analyses provide converging evidence that both Russian 'blues' have status of the basic color terms.

\section{Introduction}

The accepted cross-cultural universal inventory of basic color terms (BCTs) is comprised of 11 terms (Berlin \& Kay 1969). In particular, the inventory includes a single basic term for 'blue'; its listed Russian equivalent is sinij. For native Russians, however, it is self-evident that two terms for 'blue' are indispensable - sinij and goluboj-commonly translated as 'dark blue' and 'light blue', respectively.

This exceptional feature has generated a considerable body of research. In the linguistic domain, both terms are held to be basic; however, the basic status of goluboj is disputed on ethnographic grounds. To address the controversy about the basicness of the two Russian 'blues,' arguments are drawn from diachronic and lexical-semantic analyses, as well as from empirical linguistic and psycholinguistic studies. These are surveyed at length elsewhere (Paramei 2005); here I briefly summarize findings along those lines of research. 


\section{Diachronic perspective on the Russian 'blues'}

Diachronic analysis shows that sinij had emerged in Old Russian as early as the 11th century (Baxilina 1975:35). Derived from the same root as sijat' 'to shine,' it is of Proto-Slavic origin (Vasmer 1971:Vol. 3, 624, 629) and was used to designate color of bodies of water (sea, lakes, or rivers), skin, or eyes. However, in Old Russian the chromatic meaning of sinij had not crystallized from two other inherent meanings, (1) 'radiant,' 'sparkling,' 'glittering, for example in collocations with 'stars,' 'lightning,' or 'eyes', and (2) 'dark,' 'gloomy,' 'swarthy', 'deep shade of color' (Baxilina 1975:36, 176-179; Ivić 2000:144); the latter is traced to the name then for devil, sinec.

In comparison, the term goluboj is first registered in the early 13th century, being an East Slavic innovation; it was used as a cognate for gray horse coat (Baxilina 1975:36). The adjective descends from the Russian noun golub' 'dove.' By the 16th-17th centuries it had dissociated from its etymon altogether and attached itself exclusively to a different 'blue' modal, also attested in its current meaning, 'light blue' (Baxilina 1975: 193-195).

\section{Psycholinguistic analysis}

Psycholinguistic studies of modern Russian confirm the implied opposition of the two 'blues' along the dark-light semantic dimension. They provide converging evidence that focal goluboj and sinij are mapped as non-overlapping entities, differentiated along the lightness dimension (for details see Paramei 2005). When mapped onto the Munsell color array, the goluboj-focus corresponds to lighter blue samples, whereas the sinij-focus maps onto relatively darker samples (Figure 1). By comparison, the blue foci found in various studies partly overlap with those for sinij. Note, however, that elementary blue, i.e. the blue sample that across cultures is most often named with a 'blue' gloss (MacLaury 1997b), is located at intermediate lightness. The difference between the foci of the two Russian blue terms spans one or two steps in hue and at least two steps in lightness, as Figure 1 illustrates (see also Jameson 2005: Figure 11).

However, when along with the foci, ranges of the two 'blues' were estimated, these were found to overlap across medium lightness (Taylor et al. 1997). On these grounds the authors contend that goluboj has a recessive status in relation to dominant sinij - rather than being a complement (MacLaury 1997a).

Notably, further psycholinguistic studies reveal that the two Russian 'blues' are distinct along the saturation dimension, provided color samples varying in saturation are used. In particular, in a study by Korzh and associates, who employed the Swedish Natural Color System, the Moscow authors found that goluboj has lower chromaticness (is less saturated) than sinij (Korzh et al. 1991: Figure 4; Safuanova \& Korzh 2007, Table 2). A study by the Surrey group, who employed Color-Aid Corporation samples, revealed a pronounced separation between sinij- and goluboj-regions along a saturation dimension (Davies \& Corbett 1997:506).

This finding that the two 'blue' categories are differentiated along both achromatic dimensions - brightness (lightness) and saturation - is hardly surprising, since these 


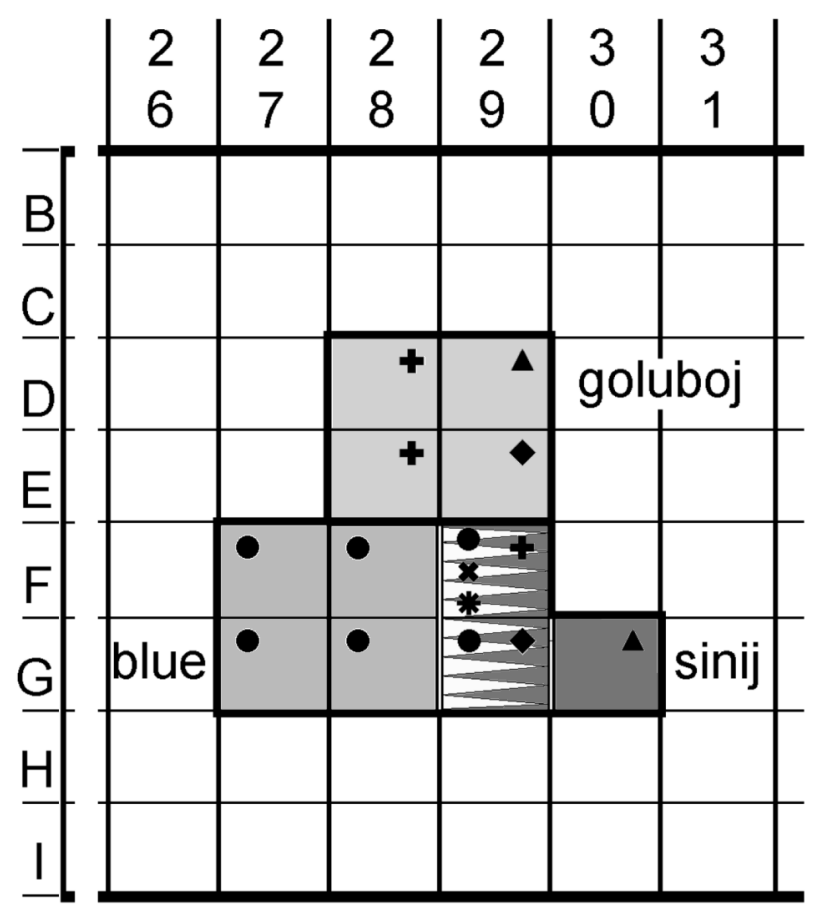

Figure 1. Munsell color array (a detail). Foci for the Russian 'blue' terms goluboj 'light blue' [light gray shaded] and sinij 'dark blue' [dark gray shaded] estimated by $\boldsymbol{\Delta}$ Frumkina (1984), $\bullet$ Morgan and Moss (1988/1989), and + Taylor et al. (1997). For comparison, foci are shown [medium gray shaded] for 'blue' by Berlin and Kay (1969) and 'Elementary blue' by $\boldsymbol{X}$ MacLaury (1997b), obtained across languages, and for English blue estimated by * Sturges and Whitfield (1995).

are integral dimensions (Burns \& Shepp 1988). They are argued to be psychologically fused, whereby selective sensitization to brightness at category boundaries spills over to saturation (Goldstone 1994).

Together, these points mean that psychometrically the category boundary between sinij and goluboj would be more accurately demarcated in a color space of higher dimensionality, and imply a need to assess lightness and its interaction with saturation.

\section{Lexical-semantic analysis}

Lexical-semantic analysis concurs that sinij and goluboj are used with near synonymy for naming an abstract color of medium-lightness blue (Alimpieva 1982:50-51). Beyond this range, darker and saturated blue is termed as sinij, as opposed to lighter and desaturated blue, named by goluboj.

More importantly, though, the two terms are not equivalent when denoting certain objects and properties, especially the coloration of natural environments (e.g., sea, sky), 
animals, and plants (Vendina 1998:281) or artifacts (Rakhilina 2000:172; 2007) in relation to their utility.

Further, the two Russian 'blues' function metonymically, implying extensions that go beyond the lightness and saturation dimensions, and invoking quite distinct sentiments and axiological connotations. In particular, while sinij connotes semantic intensity, strength, deepness of a blue color, goluboj refers to a diluted, dim, tarnished, or airy blue (Alimpieva 1987:93-94).

Sinij calls for positive associations in collocations with words like Rus' 'Russia,' nebo 'sky,' or sneg 'snow, but also for negative emotional associations of potential danger or unpleasantness, when one refers to (for example) heavy clouds, haze, or harsh, shrill light. Interestingly, the two emotionally opposite connotations are related to the two archaic meanings delineated above and inherent in the term, that is, (1) 'glitter' versus (2) 'gloom.' In contrast, goluboj conveys positive emotional expression of 'tender,' 'affectionate,' 'soft,' or 'tranquil' (Alimpieva 1987:94, 98) and is associated with 'serene' and with ethically lofty features (Gadányi 2000:67).

Both 'blue' terms in Russian are richly symbolically charged; they have specific socioethnic and socio-political loadings, providing them with distinct functions (Kul'pina 2001; Vendina 1998). Lastly, apart from the function of directly designating color experience, the two blue terms as parts of metaphors are in no way interchangeable for native speakers. This can be exemplified by the expressions sinij chulok 'bluestocking' (equivalent to and perhaps borrowed from the English expression) as opposed to golubaja krov' 'blue blood.'

This brief account of lexical-semantic analysis lends further support to the contention that sinij 'dark blue' and goluboj 'light blue' denote distinct colors in Russian, not just different shades of the same color, as an English translation might suggest (Lyons 1997:200). Along with undoubtedly basic sinij, the Russian equivalent of the universal term for 'blue' (Berlin \& Kay 1969), goluboj should also be bestowed a basic status.

\section{Linguistic indices}

The basic status of goluboj is also buttressed by linguistic studies from the Surrey group (for a review see Corbett \& Davies 1995). In elicited-list experiments, the researchers explored the psychological salience of the Russian 'blues' by assessing each term's frequency and order of occurrence: indices implied by Berlin and Kay (1969:6) in their fourth criterion of color term basicness. After a five-minute elicitation, sinij ranked 7 (Morgan 1993:11) or 1 (Davies \& Corbett 1994:73); the contentious goluboj ranked 4.5 in both studies.

In the Surrey linguistic tests, the frequency of the two Russian 'blues' in texts, and their derivational elaboration were assessed (one of Berlin and Kay's subsidiary criteria). These two indices respectively ranked sinij as 5 and 2, whereas goluboj ranked 6 (Corbett \& Morgan 1988:57). 
These briefly-surveyed lines of research show that both terms rank ahead of many other BCTs, and provide strong and converging evidence that psychological salience of goluboj is comparable to that of sinij.

The exceptional nature of goluboj, and its proposed status as a 12th basic color term, imply a more general question: whether the number of basic color categories is not restricted to eleven, and might further evolve, thereby refining a grid of categories that conceptually filter color sensations. This possibility is admitted in later modifications of the BCT theory by Kay and McDaniel (1978:640-641).

In the following, psychological studies on the Russian 'blues' are surveyed, with the intention of assembling further arguments on the terms' status. In particular, findings along the following lines of research are considered:

1. Developmental studies;

2. Acquisition of Russian color terms as reflected in semantic color space;

3. Exploration of any Whorfian effect of the Russian 'blues' upon perceptual tasks.

Perceptual-cognitive universals of color sensation serve as prerequisites for partitioning the Blue region; these are briefly addressed in conclusion - along with socio-cultural factors that foster the refined categorization of blue in Russian. The present survey also pursues a subsidiary goal of presenting, at greater length, Russian studies that are not always available in English translation.

\section{Developmental studies}

Developmental studies are complementary, and arguments from them are of high potential for elucidating the status of sinij and goluboj in Russian color nomenclature. The rationale underlying a developmental paradigm was suggested by Berlin and Kay (1969): it is essentially that children should acquire the basic color terms in hierarchical order, because of the fundamental nature of perception of the BCTs' referents, and because of greater exposure to the more frequent color terms in language. In developmental studies, acquisition of color terms is commonly assessed using a combination of color elicitation ("Which color names do you know?"), production (“What do you call this?"), comprehension ("Show me a ... ?"), color matching, grouping, and mapping tasks.

With respect to the two Russian 'blues,' Berlin and Kay (1969:35-36) questioned the basic status of goluboj, while arguing that goluboj is "less salient and less well understood by Russian children" than the other terms, citing Istomina's experiments (1960b/1963) on color perception and color naming in two- and three-year-olds.

One counter-argument to Berlin and Kay's conjecture is provided by Wierzbicka (1990:121), who notes that among Spanish children, the word for 'blue' is less salient than that for 'yellow', which in turn is less salient than the word for 'green'; and yet all these words are regarded as BCTs.

In this perspective and in the present context, Berlin and Kay's rationale could be relaxed as follows: if goluboj and sinij are both basic, then children should acquire and 
master both before acquiring any non-basic terms. Keeping this in mind, let us look closely at the results reported in the original Russian developmental studies.

In an early study, Shif (1940) assessed matching, naming, and comprehension of color by Russian children aged from two to 14 years. Her main finding is that the primary basic colors and BCTs were better recognized than derived basic colors and BCTs. In particular, she reports that in the color-naming task, goluboj-response was more prevalent than rozovyj 'pink, oranževyj 'orange,' or fioletovyj 'purple,' though all were mastered less completely than the primary BCTs. ${ }^{1}$ (For a summary see Davies et al. 1998: Appendix.)

\section{Istomina's studies}

Here I look more closely at Istomina's (1963) study cited by Berlin and Kay, as well as at her companion study published in Russian (Istomina 1960a) - with a similar design, but with three- to seven-year olds. In both studies, the researcher tested seven colors - those considered spectral (rainbow) hues in the Russian tradition: krasnyj 'red,' oranževyj 'orange,' žëltyj 'yellow, zelënyj 'green,' goluboj 'light blue,' sinij 'dark blue,' and fioletovyj 'purple.' Note that the researcher herself implicitly regarded sinij and goluboj as basic. Outcomes are considered from two of Istomina's experiments that were intended to highlight the development of perceptual versus verbal color categorization.

In the perceptual experiment, seven cards with the rainbow hues were presented, one at a time. To each card, three circles of one hue were pasted that varied in lightness (light, medium, and dark). ${ }^{2}$ When presented with a card, the child's task was to select from a set of samples $(7 \times 3=21)$ those that matched the target, without verbal designation (this does not exclude, though, their sub-vocalization). Absolute numbers of selected samples aggregated for each of the five age groups are presented in Istomina (1960a: Table 3) and Istomina (1963: Table 1).

Figure $2 \mathrm{a}$ shows in a graphic form the outcome compiled from the two studies. Data were converted into relative values [(\% actually selected $)-100 \%$ ]. These convey the degree of deviation from correct matching: positive numbers indicate an over-extension (i.e. that the target color was matched with samples from other categories) whereas negative numbers point out to under-matching to the target. The graph underscores Istomina's observation that in early childhood color perception is dominated by the primary basic color categories (BCCs) - 'red,' 'yellow, ' green,' and sinij; by contrast, goluboj and the derived BCCs 'orange' and 'purple' are under-extended. It is noteworthy that in children under 4 years old, the sinij category is markedly extended - apparently, at the expense of goluboj and fioletovyj 'purple.' Figure $2 \mathrm{a}$ also shows that in preschoolers the goluboj category undergoes continued expansion and that its dynamics is comparable to that of the

1. For English glosses, here and henceforth I use the list suggested by Frumkina and Mikhejev (1996:86) and Davies and Corbett (1994:73). Note that the Russian basic term for 'purple,' fioletovyj, includes the violet and bluish purple range (Vasilevich 1988).

2. All colors were defined in hue, saturation, and lightness in terms of Rabkin's Atlas. Unfortunately their colorimetric indices are not provided by the author. 
two derived BCCs. Notably, by the age of 5 all seven categories are perceptually recognized correctly.

Another experiment, comprehension, used the same set of 21 color samples. They were placed in front of the child, who was asked to choose all examples of the color that was named by the experimenter ("Give me the green"). Outcomes for the five age subgroups absolute number of chosen samples aggregated for each group - are presented in Istomina (1960a: Table 11) and Istomina (1963: Table 5). The data are presented here graphically as the degree of deviation from correct choice (Figure 2b). Positive and negative values, respectively, indicate over-extension and under-extension of the chosen referents of the target color name. As in the perceptual task, in the comprehension task the primary BCCs are forerunners in their elaboration; however, even in seven-year-olds only three of these - 'red,' 'yellow', and 'green' - are recognized without error. Expansion of the nonprimary color categories is markedly slower; note that the error percentage for goluboj is comparable to that for the derived BCCs.

Comparison of the results from the two tasks confirm Istomina's conclusion that preschoolers' perceptual categorization precedes their verbal-based categorization; the development of color-term comprehension leaves much room for improvement even after the age of 7.

To summarize with respect to the present issue, Istomina found that for the youngest age performance on goluboj was the poorest of the seven rainbow colors, whereby performance on sinij was of the same order as the other primary colors. However, for preschoolers performance on goluboj was absolutely correct in the matching task (from 5 years old) and in the grouping task (from 6 years old; Istomina 1960a:91). In the comprehension task, performance on goluboj was as good as on oranževyj and fioletovyj; when assessed by color-term production, it was better than fioletovyj, though less good than oranževyj (Istomina 1960a: Table 8). This more thorough examination of Istomina's results indicates that it is scant basis for disqualifying goluboj as a basic term.

In a more recent study (Davies et al. 1998), three- to six-year-old Russian children performed tasks of color-term elicitation, production, and comprehension. To a reasonable approximation, the order of color term acquisition - as a measure of basicness - was in accord with Berlin and Kay's theory. Davies et al.'s data are also consistent with the weaker claim that primary terms tend to be learned before derived BCTs.

For all age groups, goluboj was the most frequently elicited term, after the conventional primary terms vying for first place. In production and comprehension, the rank of goluboj, at worst, scored higher than fioletovyj and higher than almost all derived terms. In the production task, however - and even more in the comprehension task - the two 'blue' terms were confused more often than other neighboring pairs of terms up to age 6, with the asymmetry in errors favoring sinij. The degree of confusion declined with age, but nevertheless persisted for the oldest age group indicating that both 'blue' terms are not completely mastered in the five- to six-years-olds. Although goluboj scored poorly on the comprehension task, its performance was generally better than at least one other accepted BCT, usually fioletovyj 'purple' or seryj 'gray.' 
a

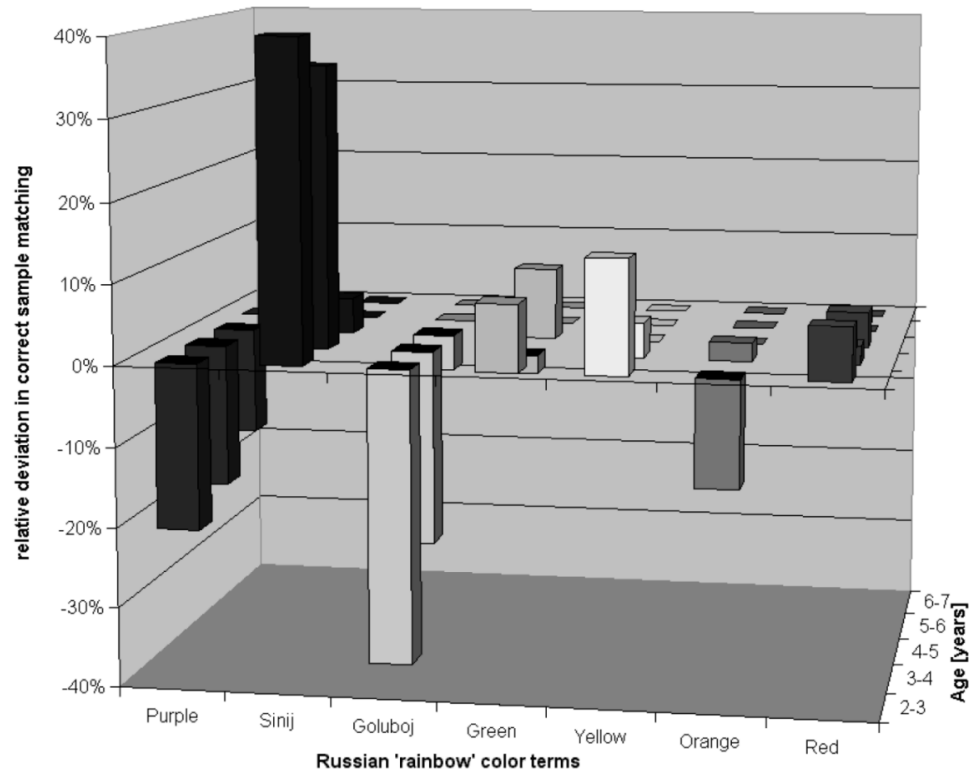

a

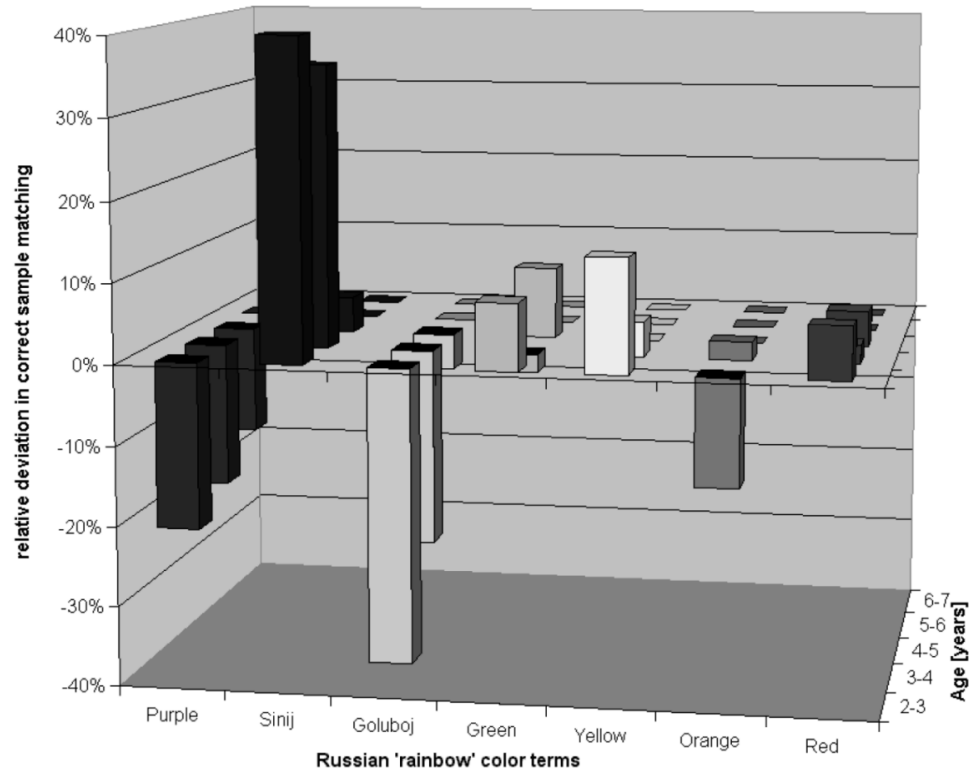

Figure 2. Relative deviation from correct recognition of six spectral colors and goluboj by Russian children, from early childhood to preschool age: (a) matching task, (b) comprehension task. Color terms other than the Russian 'blues' are indicated by English glosses. Based on Istomina (1960a, 1960b, 1963).

A full color version of this figure can be found on p. xix 
On balance, the pattern found by Davies et al., as well as in the studies of Shif and Istomina, support the contention that both 'blue' terms are genuinely basic in Russian, but this support is weaker than that from the adult data reviewed above. The data from developmental studies suggest that in terms of Kay and McDaniel's (1978) framework, goluboj belongs to the derived rather than to the primary basic color terms.

\section{Semantic color space: An indication of the lexical partition in 'blue' region}

The problem of separation of the sinij and goluboj categories can be approached psychometrically by exploring their projections within a semantic color space. Below I consider recent studies by Vartanov and colleagues aimed at reconstruction such spaces; the studies pursued different purposes but were carried out with the same experimental paradigm.

\section{Color-term acquisition by adult non-Russian speakers}

Vartanov and Nguen (1995) were interested in possible changes in the structure of the semantic color space, expected to reflect the progressive acquisition of Russian color nomenclature by non-native speakers. Five Vietnamese subjects with varying command of Russian and no special education on color nomenclature were confronted with 25 Russian color terms. The color-term set comprised 11 BCTs plus goluboj 'light blue', some BCTs with qualifiers, (e.g. bledno-rozovyj 'pale pink'), some compounds (e.g. žëlto-zelënyj 'yellow-green'), and some frequently used non-basic terms, like sirenevyj 'lilac' or cveta morskoj volny 'sea blue' (Vartanov \& Nguen 1995:31-32).

Color terms were presented in pairs. The subject's task was to estimate the subjective similarity within each pair on a 0-9 integer scale, with 0 indicating subjectively identical terms and 9 most dissimilar. Matrices of similarity were processed using non-metric multidimensional scaling (MDS). As a result, for each subject color terms were represented as points in a four-dimensional (4D) Euclidean space (based on the values of characteristic roots indicating the impact of dimensions). Here the relationship between the color terms is reflected by the configuration of points; the larger the distance between a certain pair of points the greater the dissimilarity between corresponding color terms. The dimensions stand for the perceptual (phenomenal) channels: the first two represent the opponent subsystems, red-green and blue-yellow, which contribute to the chromatic content implied by the term; two others represent the term's achromatic content, with the third dimension labeled by the authors as reflecting the "degree of grayness" and the fourth "white-black" (the two latter may be treated as saturation and brightness/lightness, respectively).

The main finding in the study is that the structure of the semantic color space becomes markedly elaborated and refined with progress in Russian language proficiency. In particular, the authors contrast configurations for two subjects - with the best and the poorest command of Russian (Vartanov \& Nguen 1995: Figure 2). These demonstrate that initial clustering around the six primary BCTs gives way to a more diversified configuration with 
larger distances between the term-points, whereby the configuration of the Vietnamese proficient subject approaches the one obtained for native speakers.

With regards to the present issue, the Russian 'blues,' Figure 3 (my adaptation of Vartanov \& Nguen's Figure 2) illustrates the relationship between the two terms. For the subject poor in Russian, it indicates apparent confusion of the meaning of goluboj: in the chromatic plane, goluboj abuts to 'green' and 'yellow-green'; and its shade, 'pale'-goluboj is mis-located between 'red' and 'orange' (Figure 3a); in the achromatic plane, points for both goluboj and sinij fall onto the same locus (Figure 3b).

By contrast, the configuration for the subject proficient in Russian clearly separates points for the two 'blue' terms along all four dimensions in the expected way. Specifically, in the chromatic plane goluboj is located midway between sinij and zelënyj 'green' (Figure $3 \mathrm{a}$ ); and in the achromatic plane it is appraised as lighter than sinij (Figure 3b). It is worth noting, though, that goluboj's location for this observer still slightly deviates from that obtained for native speakers in that it is not differentiated from non-basic 'sea blue,' and is not readily mastered when accompanied by 'pale-'modifier (for the semantic color space for Russian speakers see Vartanov \& Sokolov 1995:348, Figure 2).

Semantic disentangling of the two Russian 'blues' by this subject is especially remarkable when one takes into consideration that Vietnamese is one of the 'grue' languages, designating 'blue' and 'green' by a single category name, xahn; though it contains fixed expressions such as 'grue like the sky' and 'grue like the leaves' (Jameson \& Alvarado 2003).

\section{Representation of colors in the Russian blind}

Further arguments on the demarcation of sinij and goluboj in cognitive color representation are drawn here from Vartanov's (1997) study of a congenitally blind native Russian. The researcher followed an approach from the frequently cited study by Shepard and Cooper (1992) for US-English speaking subjects.

For the blind subject, the semantic color space preserves only a deformed vestige of the color circle, as Figure 4 shows (adapted from Figures A and B in Vartanov 1997:7879). The chromatic plane reveals, in particular, polarization of warm and cool color terms; within the latter, goluboj is clustered with sinij and 'dark'-sinij (Figure 4a). However, in the achromatic plane goluboj is clearly detached from sinij along the white-black dimension, while gravitating to terms for 'white,' 'gray,' and 'beige' (Figure $4 \mathrm{~b}$ ).

Together both studies, though indecisive about the status of the two Russian 'blue' terms, give some indication that in Russian semantic color space the 'blue' region partitions into sinij and goluboj. The partition seems to result from differential deployment of attention to the lightness and saturation dimensions, which conceivably is a function of both availability of the two color terms, and demands of communication accuracy. 

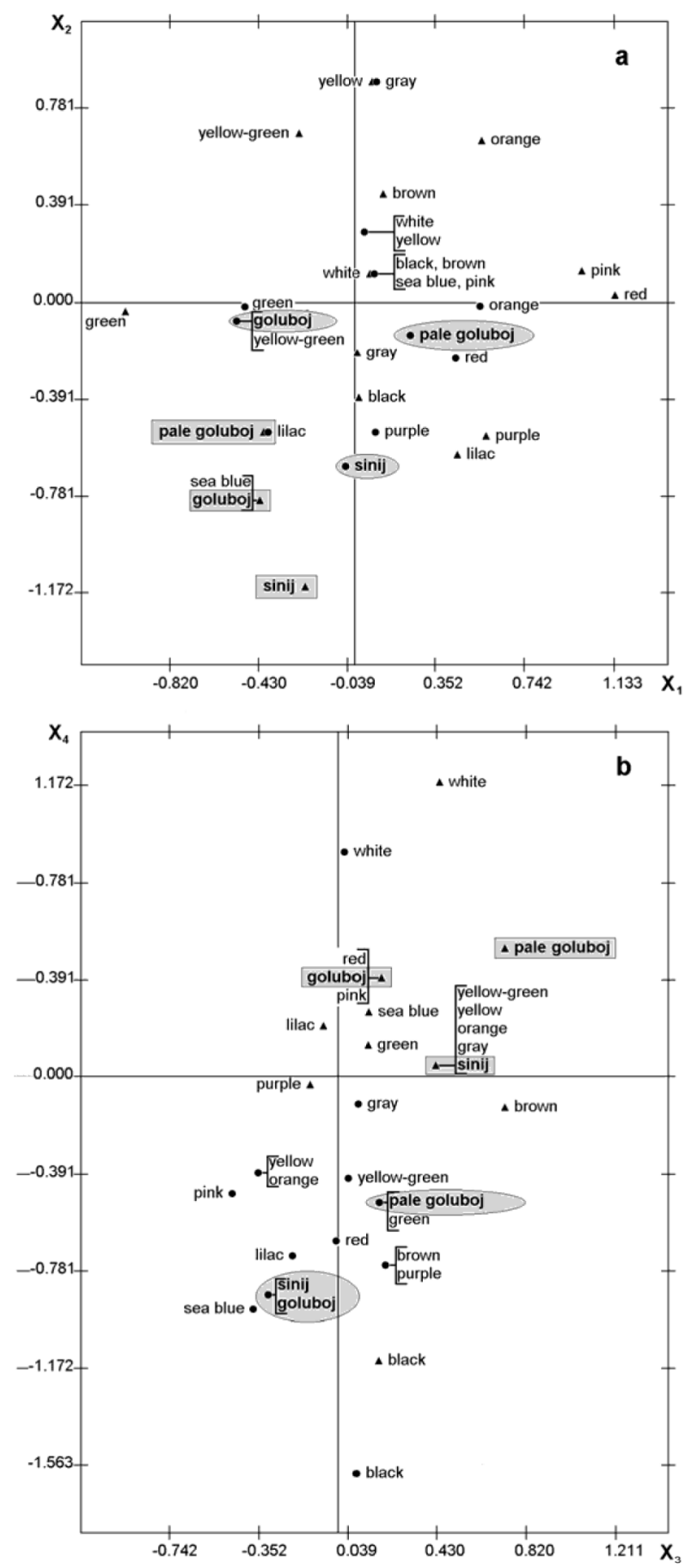

Figure 3. Semantic space of Russian color terms for two Vietnamese subjects with poor $(\bullet)$ and proficient $(\boldsymbol{\Lambda})$ command of Russian: projection on (a) chromatic plane and (b) achromatic plane. English glosses for color terms are given, except for sinij and goluboj. Dimensions represent phenomenal channels: $X_{1}$ - red-green, $X_{2}$ - blue-yellow, $X_{3}$ - 'degree of grayness,' and $X_{4}-$ 'white-black.' (Adapted from Vartanov \& Nguen 1995:37-38, Fig. 2a, b). 

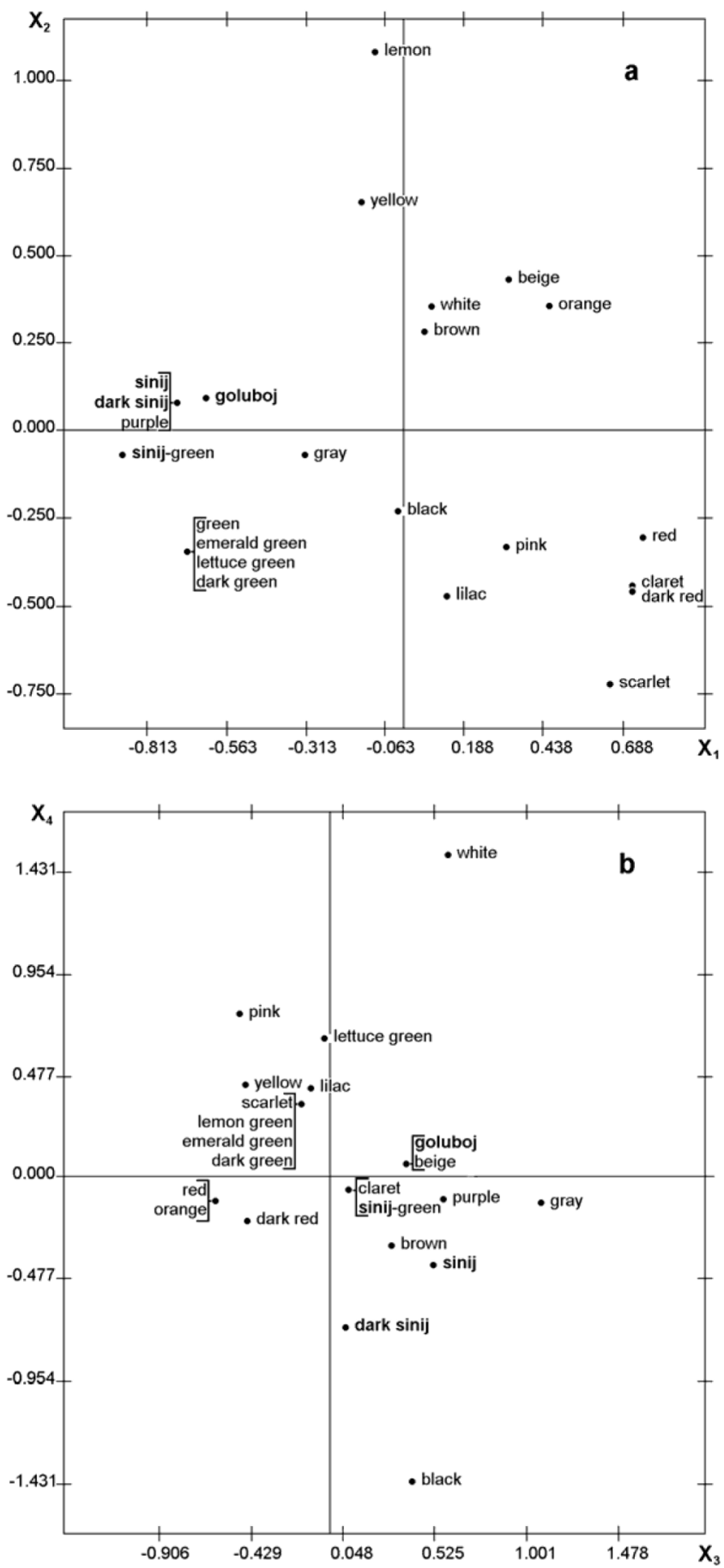

Figure 4. Semantic space of Russian color terms for a blind subject: projection on (a) chromatic plane and (b) achromatic plane. Terms and dimensions labeled as in Figure 3. (Adapted from Vartanov 1997: 78-79, Figs. A, B). 


\section{Blue region: Exploring the Whorfian effect}

Here findings from a multifaceted series of studies conducted at Surrey University are surveyed. They are considered at some length, being the only studies to bear directly on the issue; for the same reason, the findings are subjected to critical scrutiny.

The Surrey researchers compared the perceptual performance of Russian and English speakers in the Blue region of the color space. The studies were intended to investigate whether lexical categories available in Russian affect non-linguistic perception in its speakers, that is the lexical category boundary (Whorfian) effect, as formulated by Kay and Kempton (1984). Below results from the naming, triads, and free-sorting tasks are considered in more detail. Inferences from other tasks (Stroop; rating color similarities) are mentioned only briefly.

\section{Mapping the 'blue' terms}

In a psycholinguistic study (Davies \& Corbett 1997), English and Russian subjects named Color-Aid Corporation tiles that evenly sampled the color space. The focus here is performance over a sub-sample of 10 tiles, all named blue by half or more of the English subjects. In comparison, the Russian subjects used sinij for five of the tiles (including one with a 'gray-'modifier), morskoj volny 'sea blue' for a tile abutting to the green-termed region, and goluboj for the other four (Davies \& Corbett 1997: Appendix 2).

Figures 5 and 6 (adapted and extended from Davies \& Corbett 1997:500, Figures $1 \mathrm{~b} \& 1 \mathrm{c}$,) illustrate the distribution of term use in the CIE-1976 chromaticity diagram $\left(L^{\star} u^{\prime} v^{\prime}\right)$. It shows that for the Russians the Blue region is split into two termed with dominant sinij and goluboj; as expected the splitting is especially distinct along the luminance dimension, $L^{*}$.

The numbers accompanying each point indicate the naming consensus within the respondent sample, that is, the percentage of times the modal term was used (Davies \& Corbett 1997:Appendix 2). English participants were quite unanimous in tile naming (Figure 5). By comparison, for Russian participants the consensus is markedly weaker (Figure 6). A possible explanation may lie in the instruction to subjects to "name as many of the tiles as they could" (Davies \& Corbett 1997:501), without necessarily using monolexemic names. The low percentages thus imply that Russian respondents frequently used the 'blue' terms either with modifiers, or as part of compound names (like 'gray'-sinij indicated in Figure 6).

This is indeed confirmed by findings from another naming study (Laws et al. 1995). Here Russian subjects were instructed to use single words, while the Blue region was sampled a total of 35 tiles (rather than 10), and the procedure was repeated three times, increasing data stability.

Figure 7 (based on Laws et al. 1995: Tables 1 \& A1) shows the Russian 'blue' terms mapped onto the CIE-1976 chromaticity diagram. For each of the blue tiles employed, the most frequent term is indicated, along with the percentage of its use. Of the 35 blue tiles, eight were always given the same name, indicating consistency within subjects and 

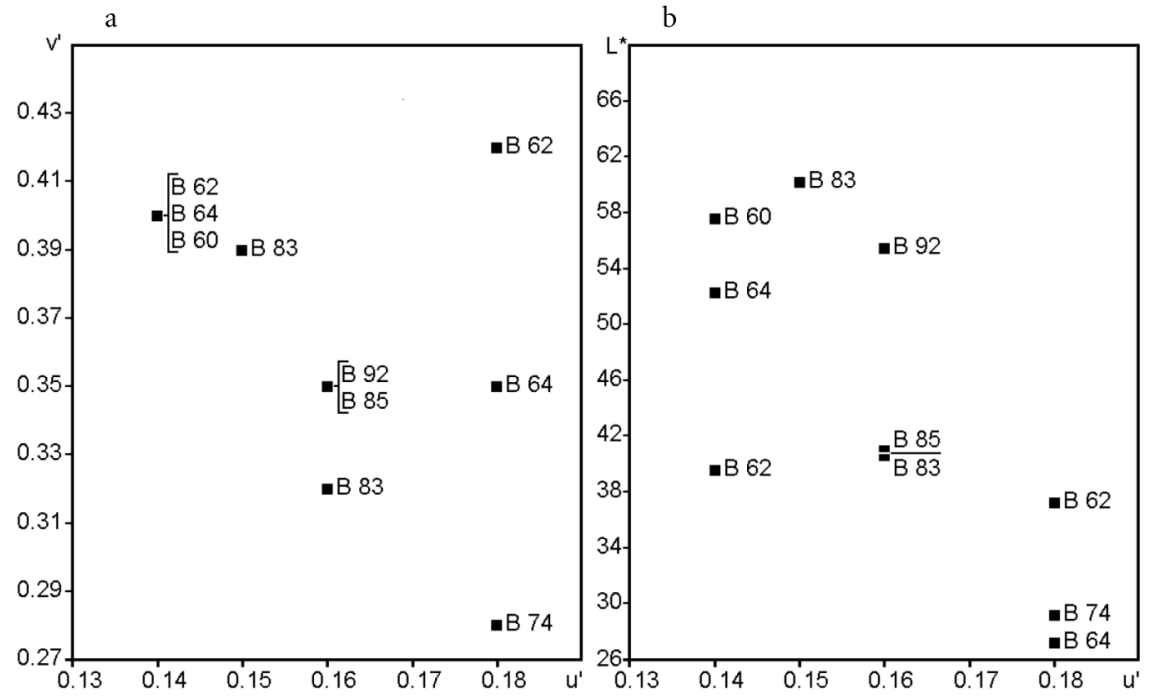

Figure 5. Tiles named as blue $(\mathrm{B}, \boldsymbol{\square})$ in English, mapped onto the $C I E L^{*} u^{\prime} v^{\prime}$ chromaticity diagram: projection onto the plane (a) $u^{\prime} v^{\prime}$ and (b) $L^{*} u^{\prime}$. Numbers indicate the naming consensus (\%) among the subjects. (Based on Davies \& Corbett 1997:516-517, Apps 1 \& 2.)

a

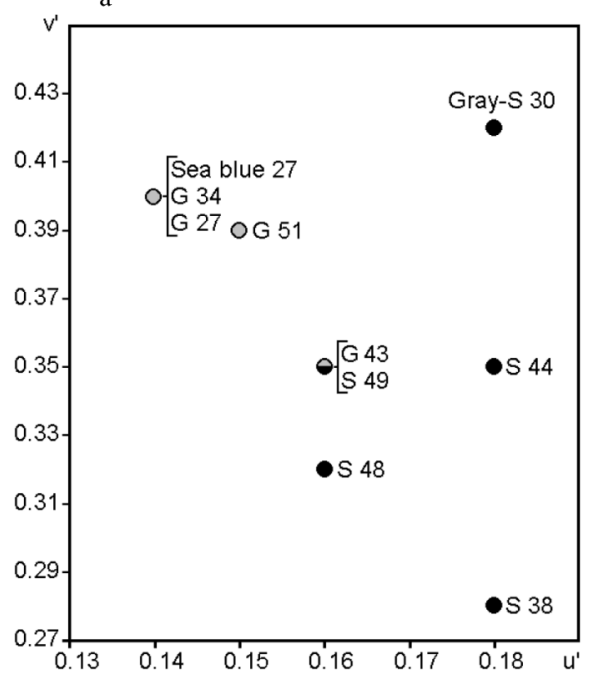

b

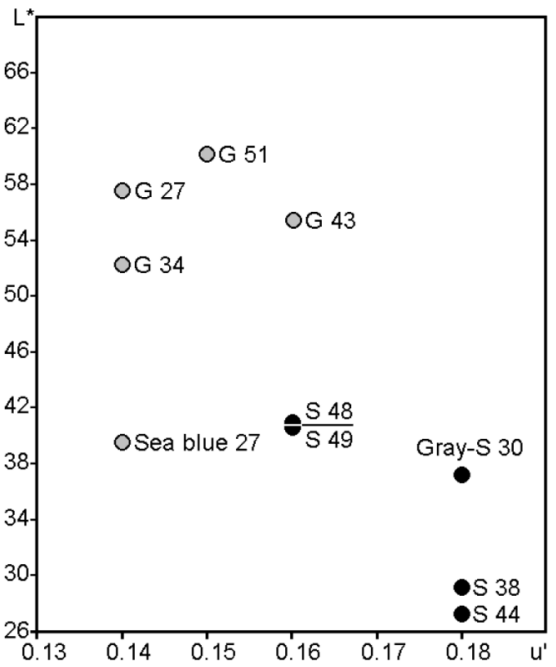

Figure 6. Tiles named as $\operatorname{sinij}(\mathrm{S}, \bullet)$ and $\operatorname{goluboj}(\mathrm{G}, 0)$ in Russian, mapped onto the $C I E L^{*} u^{\prime} v^{\prime}$ chromaticity diagram: projection onto the plane (a) $u^{\prime} v^{\prime}$ and (b) $L^{*} u^{\prime}$. Numbers indicate the naming consensus (\%). (Based on Davies \& Corbett 1997:516-517, Apps 1 \& 2.) 

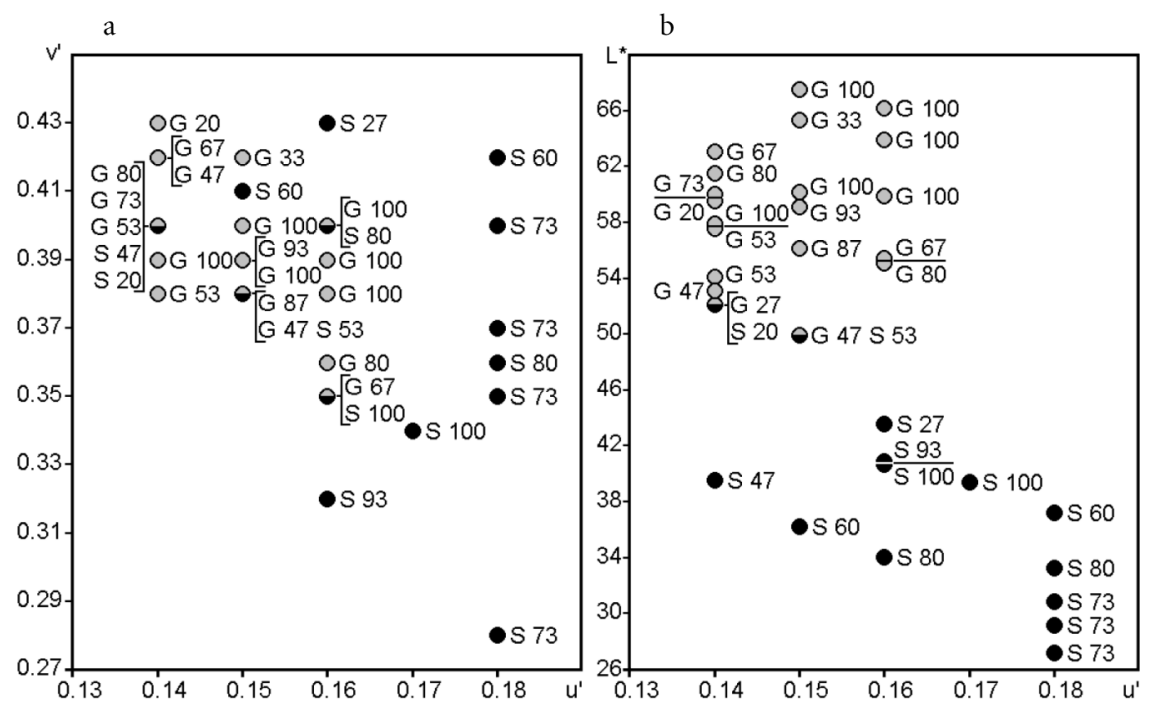

Figure 7. Tiles named as $\operatorname{sinij}(\mathrm{S}, \bullet)$ and $\operatorname{goluboj}(\mathrm{G}, \mathrm{o})$ in Russian, mapped onto the $C I E L^{*} u^{\prime} v^{\prime}$ chromaticity diagram: projection onto the plane (a) $u^{\prime} v^{\prime}$ and (b) $L^{*} u^{\prime}$. Numbers indicate the naming consensus (\%). (Based on Laws et al. 1995:66, 92-93, Tables 1 \& A1.)

consensus between subjects - sinij for two tiles and goluboj for six. Two more tiles, one sinij and one goluboj, achieved nearly this level of unanimity (93\%). Naming decisions were split between sinij and goluboj for other 12 tiles.

The plot in Figure 7 illustrates that the boundary between the two 'blue' terms becomes apparent when represented in 3D: there is a clear-cut watershed in the $L^{*} u^{\prime}$-plane. All tiles with $L^{*} \leq 50$ are named sinij and $L^{*} \geq 52$ named goluboj. In contrast, in the 2D-plane $\left(u^{\prime} v^{\prime}\right)$, within a region $u^{\prime}=0.14-0.16, v^{\prime}=0.35-0.41$, both sinij and goluboj are assigned.

The pattern of distribution of the two Russian 'blue' terms confirms that generally sinij predominates at low lightness levels and goluboj is used for lighter and greener samples. The conclusion is that the Russian 'blue' categories are structured as two small focal regions, with an intermediate region between them (Laws et al. 1995: 87), and occupy nonoverlapping regions only in three-dimensional color space - whereas the regions of other BCTs do not overlap when projected onto the color plane (Moss et al. 1990:324).

Categorical perception of blue: Behavioral measures

A number of behavioral studies compared the perceptual performance of English and Russian speakers in order to examine the possible effects of the goluboj category in the Russians. The following presents a short survey of results obtained in various perceptual tasks. 


\section{Naming times for the 'blue' terms}

Moss et al. (1990) repeated, with Russian participants and Color-Aid Corporation colors, a study that Boynton and Olson $(1987,1990)$ carried out with US-English speaking participants and OSA colors. In the Moss et al.'s study, the tiles were exposed for two seconds, while subjects were instructed to name the color with a monolexemic term as quickly as possible. Performance indices that characterize color terms were obtained: mean naming time (RT), frequency of term occurrence, intra-subject consistency, and inter-subject consensus. Here results for the 'blue' terms, the point at issue, are compared.

For American observers (Boynton \& Olson 1987: Figure 1), mean RT for blue was $1420 \mathrm{~ms}$ (across all respondents and term occurrences), second fastest; the minimal RT (reached at focal blue) was $1230 \mathrm{~ms}$. For another American sample (Boynton \& Olson 1990: Figure 5), mean RT for blue was $1940 \mathrm{~ms}$ (rank 3), with $1530 \mathrm{~ms}$ for the focal.

For the Russian sample, goluboj appeared to be second fastest, with mean RT of 1509 ms, while sinij ranked third, with RT of $1531 \mathrm{~ms}$ (Moss et al. 1990: Table 2). The authors do not provide RTs for individual tiles; therefore it is not possible to determine focal colors for the Russian terms for 'blue.' It is noteworthy, though, that of 11 tiles that gained complete consensus (out of 219), six tiles were named sinij and one tile goluboj (Moss et al. 1990:318). In sum, short RTs and high naming consistency provide converging evidence that goluboj has semantic accessibility comparable to that of sinij.

\section{Triads task: Probing the within-Blue boundary}

The triads task (Laws et al. 1995) was designed to compare the performance of English and Russian speakers in estimating perceived differences within the Blue region. Its rationale was that Russians were expected to use the name strategy (Kay \& Kempton 1984); consequently, if sinij and goluboj are both genuinely basic, subjective distances at the two terms' boundary would be larger for them compared to those for English respondents.

Blue tiles from the Color-Aid Corporation were used (ranges B, BG or BGB). Seven triads were constructed so that in each triad, two color tiles tended to share one of the Russian 'blue' terms, with the third tile attracting the other term. Subjects were presented with each triad and asked which tile was most different from the other two.

Two indices characterize the members of the triads. Perceptual Distance Index (PI), ranging from 0.5 to 1.0 , measures each tile's perceptual distance relative to the other two members. Nominal Overlap Index (NI), ranging from 0 to 1.0, reflects the degree of sinijgoluboj confusion (Laws et al. 1995:69). The tile expected to be odd-one-out is the one with highest PI and lowest NI.

For each triad, the number of times a given tile was selected as most different was calculated across nine subjects in each language group. The authors found that for four triads, in line with the prediction, the most-different tile maintained the highest PI/lowest NI relation in choices of nearly all subjects.

Three other triads were crucial, since different choices were predicted for English and Russian respondents depending on whether perceptual or name strategy would motivate 
the selection. All three triads produced split decisions from the Russian group, but (contrary to expectations) also from the English group, with the split distributed similarly. The authors interpret the result as lack of evidence for any reliable boundary effect from the Russian linguistic categories.

Since this inference is counterintuitive, the critical triads warranted closer attention. Table 1 presents indices for the three triads; specifically, for each tile it shows PI, NI, and respondents' choices (Laws et al. 1995: Table 2). Inspection of the PI reveals that the triads do not meet the primary criterion of perceptual balance crucial for this kind of test: within each triad, PIs are not equal for pairs of items, strongly indicating an odd-one-out decision on perceptual grounds.

A secondary criterion is that the two languages being compared should provide different naming-strategy contributions to subjective similarity. In the authors' terms: in one of the languages, nominal index NI should be small for one of the PI-balanced stimuli but not the other, whereas in the other language, NI should be the same. However, the sole principle of triad construction was that NI was low (in Russian) for one of the stimuli (Laws et al. 1995:68). We do not know what NI would have been in English (presumably balanced, since all stimuli were putatively from the Blue region). But because PI was ignored in the choice of the triads, they cannot be optimal.

To elucidate perceptual characteristics of the triads, the items are plotted in the $L^{*} a^{*} b^{*}$ chromaticity diagram (Figure 8). ${ }^{3}$ Projection onto the $L^{*} a^{*}$-plane (Figure $8 \mathrm{~b}$ ) illustrates that the blue tiles varied in lightness - the dimension inherent in the differentiation of sinij and goluboj.

Further, perceptual distances between each pair of items within the triads were calculated (in $L^{*} a^{*} b^{*}$-space). Prompted by the visual impression from Figure 8, in addition, hue distances (in the chromatic plane $a^{*} b^{*}$ ) and distances along the lightness dimension $L^{*}$ were separately estimated. These are shown in Table 1 , in absolute units and normalized on the smallest distance. Table 1 also presents the frequency of sinij- and goluboj-naming, ${ }^{4}$ and the location of the lexical boundary between the two 'blue' categories (depicted by a vertical double line).$^{5}$

3. Yxy coordinates of the blue tiles employed by Laws et al. (1995: Table A1) were converted into $L^{*} a^{*} b^{*}$ coordinates for the Illuminant C, 2-deg standard observer (for conversion see http://colorpro.com/info/tools/ convert.htm).

The CIE $L^{*} a^{*} b^{*}$ measures were chosen to enable a direct comparison with discrimination distances in the study of Kay and Kempton (1984). In it, Munsell color chips were employed; perceptual distances among these are expressed as $L^{\star} a^{*} b^{\star}$ measures usually (in particular, within the World Color Survey). For conversion of Munsell notation to the $L^{\star} a^{*} b^{*}$ coordinates see: http://www.gretagmacbeth.com/index/products/products_colorstandards/products_books-for-designers/products_munsell-conversion/products_munsell-conversion_sw.htm

4. Distributions of the two 'blue' terms are taken from absolute values obtained in the naming task of the same study (Laws et al. 1995: Table 1). The percent values reflect sinij- or goluboj-naming as a proportion of $\mathrm{N}=15$.

5. The lexical boundary is defined as the point where sinij- and goluboj-naming are equally frequent (cf. Kay \& Kempton 1984:68). The boundary location is inferred from Figure 7. 
Table 1. Indices for color tiles in the 'split-voice' triads A, B, D in Laws et al. (1995).

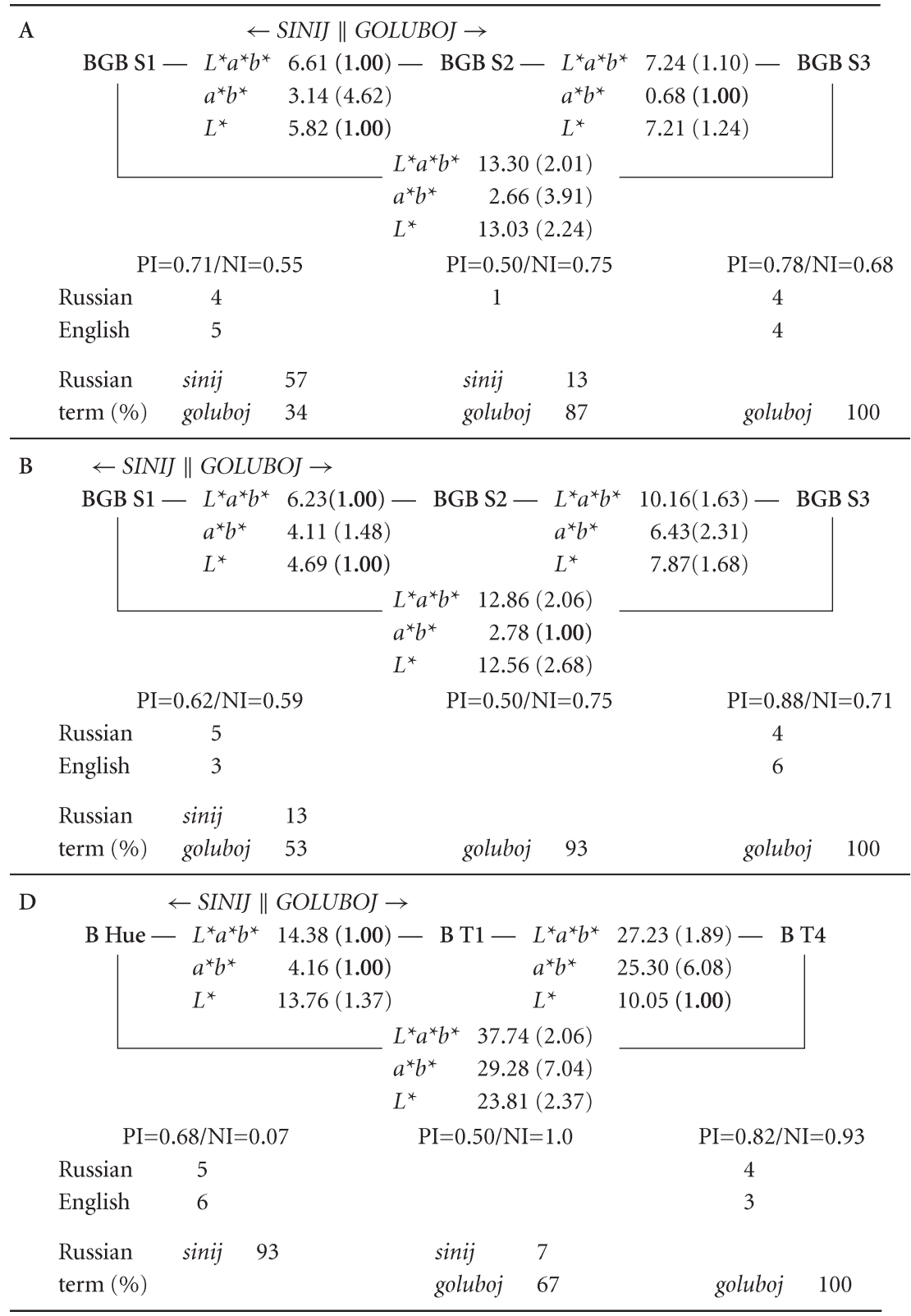

A closer inspection of the distance sub-measures $\left(a^{*} b^{*} ; L^{*}\right)$ in Table 1 (see also Figure 8) reveals a common particularity of all split-voice triads - one concealed by the overall $L^{*} a^{*} b^{\star}$-measure, as well as by the PI: among the three items, one pair is more similar in hue $\left(a^{\star} b^{\star}\right)$, whereas the other pair in lightness $\left(L^{\star}\right)$. 

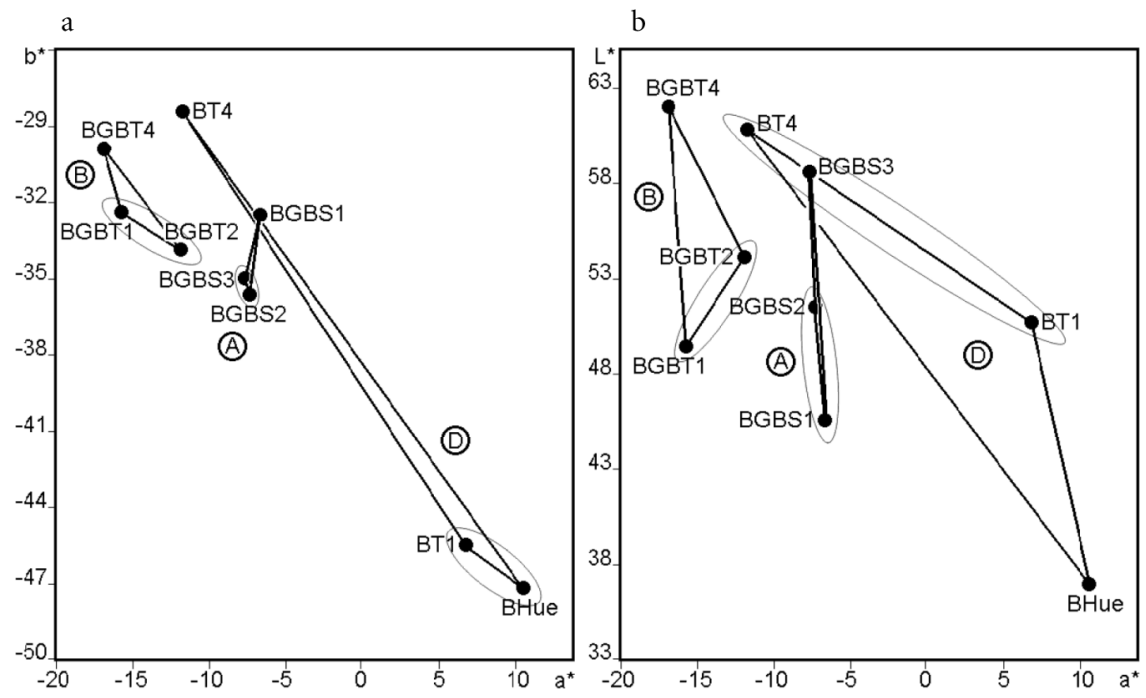

Figure 8. Crucial (and 'split-voice') triads A, B and D, mapped onto the CIE $L^{*} a^{*} b^{*}$ chromaticity diagram: projection onto the plane (a) $a^{*} b^{*}$ and (b) $L^{*} a^{*}$. Letters are tile designations; ellipses indicate within-triad pairs of items that are most similar in either (a) or (b). (Based on Laws et al. 1995:69, 92-93, Tables 2 \& A1.)

We do not know whether, in their choices of the most different tile, respondents were aware of the two color dimensions along which the tiles varied. It is conceivable that, faced with the difficult judgment, they resolved the hue versus lightness conflict by deciding on the dimension that subjectively was more salient. Paraphrasing Kay and Kempton (1984:72), the subjects might reason as follows: "It's hard to decide here which one looks the most different; are there any other kinds of clues I can use? Aha! $X$ and $Y$ are both $G O L$ UBOJ [Russian]/LIGHT BLUE [English]" Or, in an alternative, lightness based variant: "Aha! $X$ and $Y$ are both DARKER".

The two alternative outcomes - expressed as split voices - suggest that subjects may be sensitized to the two dimensions (hue and lightness), by a marked and conflicting variation of compared stimuli along them. Further, the split-voice distribution implies that a perceptual dimension strategy is pursued, independently of the language spoken. This implicit labeling of color dimensions would qualify as a naming strategy or lexical classification, but it is distinct from - and capable of overriding - the strategy of naming color categories.

From the above, two general conclusions may be drawn. First, the results of this triads experiment cannot be considered decisive for the question of whether there is a boundary effect from the two Russian 'blue' linguistic categories. Secondly, to ensure a reliable inference with respect to the Whorfian effect, each Blue triad should be perceptually balanced, with respect to the CIE metrics, along both hue and lightness - to preclude the pop-out of the color dimensions and to stop them over-ruling the color-category labeling. 


\section{Geometric representation of similarities in the Blue region}

In a further study, Davies and Corbett (1997) posed the question of how similar the colorgrouping behavior would be in English and Russian. In particular, they looked for any evidence that Russian speakers are more likely than English to form groups distinguishing dark blues (sinij) from light blues (goluboj).

A free-sorting task was used, with 65 tiles from the Color-Aid Corporation that evenly sampled the color space. Subjects were asked to sort randomly placed tiles into groups, so that members of a group looked similar to each other (Davies \& Corbett 1997:499, 501).

On average, Russian speakers produced 12.9 groups $( \pm 7.3)$, not significantly different from the $12.4( \pm 6.9)$ produced by English speakers - these are similar to the number of BCTs in both languages.

As a next step, similarity matrices were calculated for both language samples (for details see Davies \& Corbett 1997:503). The correlation between the matrices for Russian and English participants was high $(r=0.95)$. The two groups differed on an index of consensus: $S D_{m}=10.9$ for English, 14.2 (higher) for Russians; it is not clear, however, whether the difference is significant.

The inter-blue similarity between dark-blue and light-blue tiles was seen as greater by English than by Russians (Davies \& Corbett 1997:Table 2b). To assess whether the two language groups differed significantly, the authors performed a two-way ANOVA on color region ('intra-dark blue,' 'intra-light blue') by language (English, Russian). The main effect of language was highly significant. The interaction between the two factors showed the predicted pattern, but only approached significance $(p=0.07)$.

This result does not confirm the prediction that Russians would be less likely to group dark blue tiles with light blue tiles than English. Consider, however, that a form of statistical analysis was used that sets a high threshold of significance. Each class of tile pairs inter-blue, intra-sinij, and intra-goluboj - contains few cases (only six intra-goluboj pairs), and is treated by ANOVA as a distribution sampled randomly by each language group. A more powerful test (e.g. MANOVA) would use the fact that this is a repeated measures situation, where the same tile pairs are being measured in two conditions, so that the similarity for a given pair according to the Russian data can be matched with its similarity according to English. Plausibly the inter-blue category boundary for Russian respondents would then be significant.

Finally, the similarity matrices were processed using non-metric MDS to reconstruct the grouping of the tiles as geometric spaces. A 3D solution was retained for both language samples. Both solutions are similar in their basic structure, with the spatial distribution of colors resembling the color circle - reds opposing greens and blues opposing yellows.

Figure 9 (adapted from Davies \& Corbett 1997: Figures 2 \& 3) details the Blue region from each solution (plotting the first two dimensions). Inspection reveals that English have a major cluster with blue as the modal term (Figure 9a). By comparison, the Russian blue cluster splits into two abutting sub-clusters with the dominant terms of goluboj 'light blue' or sinij 'dark blue' (Figure 9b). 
a

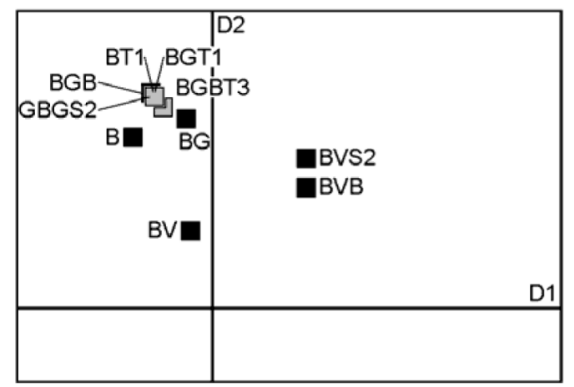

$\mathrm{b}$

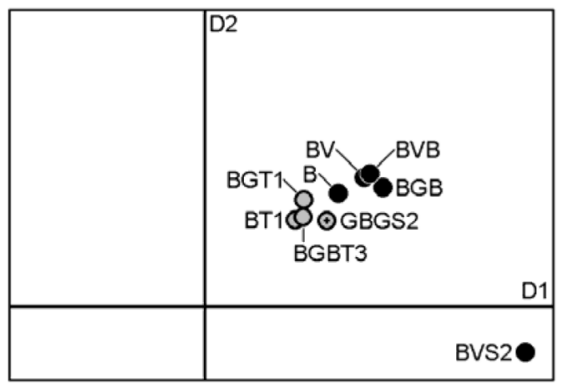

Figure 9. The blue region of the MDS solution: projection onto the first two dimensions, D1D2, for (a) English and (b) Russian. Letters are tile designations. For between-language comparison, the tiles named by Russians as sinij 'dark blue' are indicated by filled symbols and those as goluboj 'light blue’ by gray symbols. (Adapted from Davies \& Corbett 1997:505-504, Figs. 2, 3.)

The authors note that the separation of the two sub-clusters is greater when represented in the 3D solution, as the sinij-tiles have lower scores on the 3rd dimension (labeled as roughly saturation) than the goluboj-tiles. ${ }^{6}$ Even so, they report stretching of distances between the sinij and goluboj regions in the MDS solution for English speakers, the opposite of the prediction from the Sapir-Whorf hypothesis. It is not clear whether this stretching is in absolute terms, or relative to distances within the sinij and goluboj regions.

Davies and Corbett add, though, that since there are reliable differences between the two language samples in the grouping behavior (indicated above), the effect of colorcategory availability must be small relative to the inter-sample overall similarity. Davies (1998) reaches the same conclusion, from the results of a forced-sorting task.

The language effect on perceptual color structure must indeed be elusive, as was shown by Moore et al. (2002) for US-English and Chinese speakers: in a common solution, the effect size of language constituted only $1.5 \%$, whereas the effect size of the universally shared knowledge of color similarities amounted to $60 \%$ and of individual differences to $14 \%$.

Laws et al. (1995:Experiment 3) also collected sorting data, but with only nine English- and nine Russian-speaking informants, the estimated similarities are too noisy to discern any pattern in the results.

Finally, Laws et al. (1995, Experiment 2) collected direct ratings of inter-tile similarity from six English and eight Russian speakers. Here there was the complicating possibility that informants varied in response style, exacerbated by the fact that each informant was presented with a different pair of tiles as a reference or baseline for judging subsequent pairs. Even so, the English group judged inter-blue pairs to be on average more similar

6. It may be that $4 \mathrm{D}$ solutions, allowing separate lightness and saturation dimensions, would accentuate any 'stretching' between the two 'Russian blue' sub-clusters. Stress values fell in the range $12 \%$ to $17 \%$. According to common guidelines (Kruskal 1964:3), these values are 'poor,' suggesting that more than three dimensions are appropriate. 
(relative to intra-sinij and intra-goluboj pairs) than did the Russian group (Laws et al. 1995: Table 4). Again, an ANOVA analysis failed to find this difference to be significant; but a more powerful test, taking into account the repeated-measures nature of the situation, might have done so. It would be interesting to see MDS solutions for these latter data. Ideally, any MDS analysis would use the repeated measures option, keeping individuals' data matrices separate and thereby correcting for response style.

Is there a category effect from the Russian 'blues' in perceptual tasks? Pros and Contras

The Surrey group's perceptual results are briefly summarized below, in conjunction with a recent study on discriminability (Winawer et al. 2003). All these studies compared behavioral measures for English and Russian speakers, while exploring the putative category effect of the two Russian lexical labels for 'blue.'

Pros: For Russian speakers, the partitioning of the Blue region into sinij and goluboj categories reveals itself in the following.

1. The naming task: two small unique regions are separated and circumscribed in a three-dimensional color space (Davies \& Corbett 1997; Laws et al. 1995).

2. The naming times: RTs for both goluboj and sinij are comparable and faster than for almost all other BCTs (Moss et al. 1990).

3. The free-sorting task: the blue tiles are customarily separated into two groups named sinij and goluboj (Laws et al. 1995).

4. The similarity color space (derived from the free-sorting task): the Blue cluster is split into two abutting sub-clusters - comprising light blue and dark blue tiles, with goluboj and sinij, respectively, as modal terms (Davies \& Corbett 1997).

5. The two-alternative forced-choice color discrimination task: Russians have an advantage in making near-threshold discriminations among blue stimuli crossing the golubojsinij boundary. The advantage, which is reduced by verbal interference, is revealed by shorter RTs for cross-category compared to within-goluboj or within-sinij discriminations. By contrast, the English (for whom all discriminations are within-blue) do not show this pattern (Winawer et al. 2003).

Contras: No reliable evidence for the effect of the two Russian 'blues' in the Blue region is shown in the following.

1. The triads task: No difference between the Russian and English 'odd-one-out' choices (Laws et al. 1995). However, the critical triads were not perceptually balanced and contained items with conflicting hue and lightness differences; therefore they were not optimal and not necessarily capable of testing the hypothesis.

2. The similarity color space (derived from the free-sorting task): No support for 'stretching' between the sub-clusters of light blues and dark blues (Davies \& Corbett 1997). However, the MDS procedure and the ANOVA test applied cast doubt on the statistical outcome. 
3. Rating similarity between pairs of blue stimuli: No significant effect of language on mean similarity ratings for (a) intra-sinij, (b) intra-goluboj, and (c) inter-blue (Laws et al. 1995). However, the variability of mean ratings within each category was quite large, 1.71 \pm 0.81 for intra-sinij' pairs for Russian speakers (Laws et al. 1995: Table 3). As mentioned above, noise in the data might have arisen from different subject response styles. This variability, and especially the small number of pairs compared by ANOVA, might obscure any differences between the rating distributions.

4. Free-sorting and labeled-sorting tasks: No significance between English and Russian in the mean number of categories produced (Laws et al. 1995). However, there is a difference under the free-sorting instruction: Russian produced $8.8 \pm 4.0$, English $7.3 \pm 3.8$ categories (Laws et al. 1995: Table 5). Besides, in the labeled sort all Russian subjects included categories for sinij and goluboj. Last but not least, the obtained data is apparently quite noisy: the presence of noise is indicated by low correlation coefficients between sorting estimates (Laws et al. 1995: Table 9). It is highly probable that with only nine subjects in each language group, the ANOVA could not detect any differences between pairs of broad distributions.

5. Stroop task: No larger effect for the sinij/goluboj combinations with Russian subjects than for the navy/sky combinations with the English (Davies et al. 1991). For both language groups, the light blue interfered less than other colors or names (red, yellow, and dark blue). However, for the Russians, light blue had the fastest RT and dark blue the next fastest; by comparison, for the English light blue has the slowest RT (Davies et al. 1991:317). As the authors mention, it remains possible that the effect of the Russian 'blues' is obscured by the inherent variability of the RT task. ${ }^{7}$ Also, the insignificant effect for the light blue and dark blue might be due to an overall marked difference in RTs between the two languages, with the Russian group being much slower. ${ }^{8}$

To sum up the results from the surveyed perceptual tasks: sinij and goluboj are comparable with regards to naming times. Discriminative RTs indicate a category boundary separating the two 'blue' categories, which form distinct but abutting clusters in perceptual space. The categorical distinction between sinij and goluboj can be revealed reliably, provided (1) representation within a 3D space, including the lightness dimension, and (2) a dense sampling of the Blue area, that is, the differences between stimuli are near-threshold.

Since the separation of the two Russian 'blue' categories is small, any boundary effect in perceptual tasks is obscured when the differences are large, i.e. when the judgment no longer is "hard to make" (cf. Kay \& Kempton 1984). Due to the subtlety of the category effect, statistical tests appear insensitive to it when data are noisy, the number of compared stimuli is small, and/or subjects are too few.

7. Since RT data are noisy (Paramei \& Cavonius 1999:1666), obtaining a reliable RT difference requires many more trials than the number $(N=64)$ in the considered study.

8. Significantly shorter RTs of English participants are quite explicable keeping in mind that almost all were secretaries professionally used to a computer keyboard, whereas the Russian subjects from the Soviet Union, visiting teachers at the University of Surrey in the late-1980s, could hardly have had many possibilities for computer experience. 


\section{Driving forces for partitioning of the Blue region}

Driving forces for the categorical refinement of the color space may be sought within different explanatory schemes. Here I briefly review two explanatory hypotheses that shed light on the emergence of the additional 'blue' category in Russian. Together they indicate that the potential for 'blue' term development arises from the behavioral importance of differentiation that allows improvement of category codability and communication accuracy.

\section{Environmental optimality}

The environmental optimality scheme invokes the necessity of communication about perceptual regularities of terrestrial color experience, and can be traced to a concept put forth by Shepard (1992). In operational terms, this hypothesis was elaborated by Yendrikhovskij (2001), who postulated that the structure of color categories originates from the statistical distribution of colors encountered in the visual environment. Vector quantization of a large database of color scenes, while introducing a minimum distance criterion, enabled the author to compress the environment color statistics into a restricted number of clusters (= color categories). In the present context, the relevant finding is that the number of the BCCs delimited in the color space varies depending on a compromise between the distortion costs (communication accuracy, codability) and complexity costs (differentiation) of the category system. In particular, when distortion costs prevail, the result is progressive partitioning of BCCs and hence an advanced BCT nomenclature.

With respect to the Blue region, of interest is the model's prediction that beyond the 11 BCCs, there is a strong potential for further segmentation of the color space - into two or three categories between the foci for blue, white, and gray (Yendrikhovskij 2001: Figure $4 \mathrm{~d})$. This tendency might, indeed, well fall on fertile ground in a language (Russian) developed for communication in a natural environment where various shades of blue are main colors of the ambient landscape of large water areas and snow.

Implications from topological properties of the color space

Further arguments of progressive categorical segmenting of the color space are grounded in its topological properties - as suggested by the Interpoint-Distance Model (IDM; Jameson 2005; Jameson \& D'Andrade 1997). According to the model, asymmetries in the cognitive color space imply that certain existing BCCs are separated by wide perceptual gaps, a feature that fosters emergence of color categories within the gap.

Some psychophysical studies support this view (using the Munsell color solid). In particular, Indow (1988) provided evidence that the purple category bisects a great distance between red and blue. Also, Sun (1983) estimated psychological distances between the foci of the $11 \mathrm{BCTs}$ and demonstrated that the largest distance between blue and green yields 
a potential zone of difficulty for color naming, thereby promoting an additional term to fill the gap.

There is evidence that perceptual space sets the stage for a light-blue term. The World Color Survey found a fair number of 'black-or-blue' terms, but not 'black-or-green,' 'black-or-red,' or 'black-or-yellow' terms (Lindsey \& Brown 2002; Regier \& Kay 2004). The existence of 'black-or-blue' terms in several languages does suggest that blue is subjectively a darker sensation than the other named (understandable in view of the fact that the most saturated blue samples of the Munsell array barely stimulate middle- or longwavelength cones, which signal lightness). As a consequence, blue is sensed as closer to black - which makes the subjective distance between blue and white larger than it might otherwise be.

This appraisal of blue category in terms of lightness-darkness prompts a suggestion that salience of lightness, as well as perceptual separation, preconditions categorical partitioning of the blue area. This inference is in accord with another significant assertion of the IDM model - that cognitive salience of a certain perceptual dimension predetermines the location of an emerging BCC in the color space.

In this vein, the goluboj 'light blue' category emerges as a mid-point along the longest color-space stretch between basic blue and white. The partitioning of the Blue region in Russian, as Jameson (2005: Figure 11) forcefully points out, is facilitated by the fact that sinij 'dark blue' is denotatively offset from 'elementary blue.' Driven by the needs of pragmatic communication, goluboj therefore emerges as a complementary category by designating light and unsaturated blue and effectively balancing sinij's features of greater darkness and saturation.

\section{Cultural specificity: A Russian rhapsody in sinij and goluboj}

From the above it follows that goluboj basicness is quite feasible on psychometric grounds alone. By all accounts, these fulfill the necessary requirement for the Blue region to be partitioned. Though the "bottom-up" factors function as cognitive constraints, they cannot serve as exhaustive explanatory principles for conceptual refinement of the blue category and naming. This is because color terms are part of socially anchored linguistic systems (Gellatly 1995; Lucy 1997; Rakhilina 2007; Wierzbicka 1990), whereby the process of color categorization gains its primary impetus from cultural mechanisms (Dedrick 1998; Gage 1997; Hardin 1988; Kay \& Maffi 1999; Saunders \& van Brakel 1997). Here I argue that such mechanisms fulfil the sufficient requirement for fine-tuning the 'blue' category. Below some evidence is considered that the differentiation between the two Russian 'blues' was induced by semiotics originating in socio-history.

The question arises - apart from estimating basicness of the two Russian terms for 'blue' - what exceptional cultural conditions might stimulate the emergence of two such terms? Elsewhere (Paramei 2005) I surmise that this distinction is rooted in color semiotics, specifically, in the deep and sustained influence of the Russian Orthodox Church and its predecessor in the Christianization of Russia, the Byzantine Church. My view con- 
verges with a hypothesis of Moss (1988) that early Russian iconography played a crucial role in elaborating the differentiated spiritual symbolism of the two shades of blue. ${ }^{9}$ Moss conjectures, in particular, that Suzdalian iconographic art - with its predilection for light bluish and silvery range - became culturally powerful after the capital of Russia and the Patriarch's seat were transferred first from Kiev to Vladimir-Suzdal' and later to Moscow (Moss 1988: 166-167).

Blues of various shades (produced from rare lapis lazuli) acquired an exceptional salience in icons and frescoes by the two most influential iconographers of early Moscow, Theophanes the Greek and his pupil Andrei Rublev. The different blues appear to be theologically loaded, in particular, in depicting Christ's mandorla: the emanating Divine Light becomes light-bluish and silver as it recedes from its source, but is dark blue when it takes on Christ's flesh (Gage 1993:55, 59).

Consider also that in Orthodox art the Holy Spirit is symbolized by a dove. And as a derivation from golub' 'dove,' goluboj 'light blue', is symbolic of the spiritual and heavenly. Note too that the halos for symbolic figures (i.e. spiritual beings) are light blue (Kandinsky 1911/1977:38).

A rich icon palette of light and dark blues was echoed by color terms in Russian iconographic manuals - these contained an obligatory and detailed description of colors and frequently, metonymic or symbolic meanings of color terms (Lindgren 1997). In the manuals, as many as five pigment terms indicate blue shades - two of goluboj (golubec and lazor') and three of sinij (lavra, sin', and krutik). Notably, modern books on icon painting strictly follow the regimentations of the medieval Russian Orthodox Church and still use some of these old names for different shades of 'blue' (Schneider \& Fedorov 1978:55).

If one considers the expansion of the state-dominated Russian church in medieval Russia and the importance of iconography in the extremely religious population, along with the fact that icons and mural paintings in churches "probably constituted the Russians' main experience of bright colours, then it is reasonable to assume that the Russian language would quickly have acquired a basic term denoting light blue" (Moss 1988: 167).

A need to distinguish the blue shades - negligible under neutral conditions elsewhere - was encouraged by the culture, in which the percepts of dark blue and light blue appeared to function as cultural signifiers (cf. Sahlins 1976). Within this explanatory scheme, for Russian speakers goluboj emerged as a semiotic code, a historic conception, and should be considered culturally basic (Frumkina 1999:952).

Speakers of modern Russian share a tacit knowledge about the meaning of goluboj: beyond the color itself, the percept also implies the luminous dimensions of luster, glitter, and iridescence. But more important are conceptual extensions inherent in its meaning, for goluboj is associated with sentiments of tenderness and serenity. For religious Russians, it is, in addition, a symbol of spirit and grace, of transcendental tranquility and ultimate feeling of rest - to quote Kandinsky, “the typical heavenly colour” (1911/1977:38).

9. I only discovered Moss's (1988) study of the cultural history of the Russian 'blues' after completing the previous article. 


\section{Conclusions}

The present study surveyed results from various behavioral tasks to evaluate the basicness of the two Russian terms for 'blue, sinij and goluboj. All the behavioral measures are predicated on Berlin and Kay's (1969:6) fourth criterion for a color term's basicness - the notion of psychological salience. As conceptualized in the literature, these behavioral measures operationalize the color-salience construct in empirically different ways. However, the rationale for the comparability of various empirical measures as indices of salience has not been addressed explicitly.

In this context, the survey of results on the two Russian 'blue' terms sheds additional light on (1) what 'facets' of psychological salience certain behavioral measures capture, (2) which tasks optimally measure salience, and (3) how this operationalization is related to the definition of basicness. The following specific observations help clarify the use of the basic construct in analyses of the two Russian 'blue' categories:

1. Basicness of both sinij and goluboj is reliably found in naming tasks. It is manifested by short naming times and high frequency of occurrence. These behavioral measures, together with high rank in elicited lists, capture the high degree of lexical accessibility of the two Russian 'blues' and indicate that both terms are at a hyperonym level in the color nomenclature.

2. Mapping tasks provide evidence for perceptual distinction between the Russian 'blue' categories, in the form of distinct focals, and domains that are circumscribed though abutting - provided the two categories are mapped onto a 3D space and the color space is sampled densely.

3. Naming and mapping tasks show high codability of the two Russian 'blue' terms by measures of high within-subject consistency of use and high consensus among respondents.

4. Discrimination $2 A F C$ tasks reveal an $R T$ boundary advantage - a lexical category boundary between sinij and goluboj, indicating that they are proper categories. This category boundary effect disappears under verbal interference, indicating that it is specific for Russian verbal coding.

5. Both sinij and goluboj are conceptually distinct and salient. In tasks of free sorting of color samples, this is shown by the emergence of sinij and goluboj clusters. In the task of rating color term similarity, conceptual distinction of the two 'blues' is indicated by good separation in the semantic color space. Also from the developmental perspective, conceptual distinctiveness of the two 'blues' shows up as high rates of performance in naming and matching behaviors of preschool children.

The above named behavioral measures capture the terms' denotative meanings. However, to adequately appraise the conceptual distinction of the two Russian 'blues' behavioral measures should be complemented by linguistic measures - such as the terms' metonymic and metaphoric usage and combinability - to capture their attributive meanings.

As indicated above, in determining the lexical category boundary (Whorfian) effect of the two Russian 'blues' it is crucial to insure that stimuli used vary within near-threshold 
range of perceptual differences. In the absence of a fine gradient of stimuli, visual coding is likely to be promoted as a subject's choice strategy, thereby obscuring any potential lexical boundary effect. For future studies this reinforces the need for psychometric tasks, which would minimize judgments based on purely color discrimination criteria, and invoke the verbal coding strategy. The choice of tasks, stimuli, and behavioral measures is essential for understanding and reliably measuring any perceptual groupings attributable to the intrusion of lexical categorization.

What seems clear from the present survey is that the Russian 'blues' - sinij and goluboj - satisfy the abovementioned basicness criteria on the basis of both perceptual and lexical grounds.

\section{Acknowledgments}

This paper was presented at the workshop "Anthropology of colour: Colour as a phenomenon of culture" at the 7th Biennial Conference of the European Association of Social Anthropologists, Copenhagen, August 15, 2002. Preparation of the manuscript was supported by a fellowship from Hanse-Wissenschaftskolleg, Delmenhorst, Germany. I am deeply grateful to the late R. E. MacLaury for suggestions on an earlier version of the paper and to D. L. Bimler for valuable comments. The constructive advice of K. A. Jameson and P. Kay is highly appreciated. The generous help of A. Anishchanka and L. V. Samarina in supplying me with Russian sources is gratefully acknowledged. My husband, Martin, devotedly implemented the figures.

\section{References}

Alimpieva, R. V. (1982). Stanovlenie leksiko-semantičeskix grupp cvetovyx prilagatel'nyx v russkom jazyke pervoj poloviny XIX v. [Establishing of lexical-semantic groups of color adjectives in Russian of the first half of the 19th century]. In Voprosy semantiki: issledovanija po istoricheskoj semantike [Problems of semantics: Investigations in historical semantics] (49-60). Kaliningrad: Kaliningrad State University. Alimpieva, R. V. (1987). Sinonimičeskij mikrorjad "sinij - goluboj" v sopostavlenii s pol'skim błękitny - niebeski (k probleme semantičeskoj èvolucii leksičeskix èkvivalentov rodstvennyx jazykov) [Synonymic microrange sinij-goluboj in comparison with Polish błękitny - niebeski (on the problem of semantic evolution of lexical equivalents of cognate languages)]. In Semantika slova $v$ diaxronii [Semantics of the Word in Diachrony] (92-99). Kaliningrad: Kaliningrad State University.

Baxilina, Natalja B. (1975). Istorija cvetooboznačenij v russkom jazyke [The history of color terms in Russian]. Moscow: Nauka.

Berlin, Brent \& Paul Kay (1969). Basic color terms: Their universality and evolution. Berkeley: University of California Press.

Boynton, Robert M. \& Conrad X. Olson (1987). Locating basic colors in the OSA space. Color Research \& Application, 12, 94-105.

Boynton, Robert M. \& Conrad X. Olson (1990). Salience of chromatic basic color terms confirmed by three measures. Vision Research, 30, 1311-1317. 
Burns, Barbara M. \& Bryan E. Shepp (1988). Dimensional interactions and the structure of psychological space: The representation of hue, saturation, and brightness. Perception \& Psychophysics, 43, 494-507.

Corbett, Greville G. \& Gerry Morgan (1988). Colour terms in Russian: Reflections of typological constraints in a single language. Journal of Linguistics, 24, 31-64.

Davies, Ian R. L. (1998). A study of colour grouping in three languages: A test of the linguistic relativity hypothesis. British Journal of Psychology, 89, 433-452.

Davies, Ian R. L. \& Greville Corbett (1994). The basic color terms of Russian. Linguistics, 32, 65-89.

Davies, Ian R. L. \& Greville G. Corbett (1997). A cross-cultural study of colour grouping: Evidence for weak linguistic relativity. British Journal of Psychology, 88, 493-517.

Davies, Ian R. L., Greville G. Corbett, Glynis Laws, Harry McGurk, Anthony E. St. G. Moss, \& M. W. Smith (1991). Linguistic basicness and colour information processing. International Journal of Psychology, 26, 311-327.

Davies, Ian R. L., Greville G. Corbett, Harry McGurk, \& Catriona MacDermid (1998). A developmental study of the acquisition of Russian colour terms. Journal of Child Language, 25, 395-417.

Dedrick, Don (1998). Naming the rainbow. Colour language, colour science, and culture. Dordrecht, Boston, \& London: Kluwer Academic Publishers.

Frumkina, Revekka M. (1984). Cvet, smysl, sxodstvo. Aspekty psixolingvističeskogo analiza [Color, meaning, and similarity: Aspects of a psycholinguistic analysis]. Moscow: Nauka.

Frumkina, Revekka M. (1999). What does my eye tell your mind? Behavioral and Brain Sciences, 22, 951952.

Frumkina, Revekka M. \& Alexei V. Mikhejev (1996). Meaning and categorization. New York: Nova Science.

Gadányi, Károly (2000). Sopostavitel'nye xarakteristiki cveta v nekotoryx slavjanskix jazykax [Comparative characteristics of color in some Slavic languages]. In K. Gadányi, L. Mojszejenko, \& V. Mojszejenko, Slovo i cvet v slavjanskix jazykax [Word and color in Slavic languages] (59-68). Melbourne: Academia Press.

Gage, John (1993). Colour and culture. Practice and meaning from antiquity to abstraction. Singapore: Thames and Hudson.

Gage, John (1997). Colour and culture. In T. Lamb \& J. Bourriau (Eds.), Colour: Art \& science (175-193). Cambridge: Cambridge University Press.

Gellatly, Angus (1995). Colourful Whorfian ideas: Linguistic and cultural influences on the perception and cognition of colour, and on the investigation of them. Mind \& Language, 10 199-225.

Goldstone, Robert (1994). Influences of categorization on perceptual discrimination. Journal of Experimental Psychology: General, 123, 178-200.

Hardin, Clyde L. (1988). Color for philosophers: Unweaving the rainbow. Indianapolis, IN: Hackett.

Indow, Tarow (1988). Multidimensional studies of Munsell color solid. Psychological Review, 95, 456-470.

Istomina, Z. M. (1960a). O vzaimootnošenii vosprijatija i nazyvanija cveta u detej doškol'nogo vozrasta (èksperimental'noe issledovanie) [On the relationship between perception and naming of color in preschool children (an experimental study)]. Izvestija Akademii Pedagogicheskix Nauk RSFSR, Iss. 113, 76-102.

Istomina, Z. M. (1960b). Vosprijatie i nazyvanie cveta v rannem vozraste. Izvestija Akademii Pedagogicheskix Nauk RSFSR, 113, 76-102 [Translated as Istomina (1963). Page references are to the 1963 translation].

Istomina, Z. M. (1963). Perception and naming of color in early childhood. Soviet Psychology and Psychiatry, 1, 37-45.

Ivić, Milka (2000). The markedness principle and the Slavic color vocabulary. In History and perspectives of language study. Papers in honor of Ranko Bugarsk (141-145). Amsterdam: John Benjamins.

Jameson, Kimberly A. (2005). Why GRUE? An Interpoint-Distance Model analysis of composite color categories. Cross-Cultural Research: The Journal of Comparative Social Studies, 39, 159-204. 
Jameson, Kimberly A. \& Nancy Alvarado (2003). Differences in color naming and color salience in Vietnamese and English. Color Research \& Application, 28, 113-138.

Jameson, Kimberly \& Roy G. D’Andrade (1997). It's not really red, green, yellow, blue: An inquiry into cognitive color space. In C. L. Hardin \& L. Maffi (Eds.), Color categories in thought and language (295-319). Cambridge: Cambridge University Press.

Kandinsky, Wassily (1977). Concerning the spiritual in art (M. T. H. Sadler, Trans.). New York: Dover. (Original work published 1911).

Kay, Paul \& Willett Kempton (1984). What is the Sapir-Whorf hypothesis? American Anthropologist, 86, $65-79$.

Kay, Paul \& Luisa Maffi (1999). Color appearance and the emergence and evolution of basic color lexicons. American Anthropologist, 101, 743-760.

Kay, Paul \& Chad K. McDaniel (1978). The linguistic significance of the meanings of basic color terms. Language, 54, 610-646.

Korzh, Nina N., Irina V. Penova, \& Olga V. Safuanova (1991). Denotativnye značenija cvetonaimenovanij [Denotative meanings of color terms]. Psikhologicheskij Zhurnal, 12, 69-79.

Kruskal, Joseph B. (1964). Multidimensional scaling by optimizing goodness of fit to a nonmetric hypothesis. Psychometrika, 29, 1-27.

Kul'pina, Valentina G. (2001). Lingvistika cveta. Terminy cveta v pol'skom i russkom jazyke [Linguistics of color: Color terms in Polish and Russian]. Moscow: Moskovskij Licej.

Laws, Glynis, Ian R. L. Davies, \& Catherine Andrews (1995). Linguistic structure and non-linguistic cognition: English and Russian blues compared. Language and Cognitive Processes, 10, 59-94.

Lindgren, Nelli (1997). [Color terms in Russian icons (a historical-semantic aspect)]. In B. Nilsson \& E. Teodorowicz-Hellman (Eds.), Nazwy barw $i$ wymiarów [Colour and measure terms] (9-23). Stockholm: University of Stockholm.

Lindsey, Delwin T. \& Angela M. Brown (2002). Color naming and the phototoxic effects of sunlight on the eye. Psychological Science, 13, 506-512.

Lucy, John A. (1997). The linguistics of “color." In C. L. Hardin \& L. Maffi (Eds.), Color categories in thought and language (320-346). Cambridge: Cambridge University Press.

Lyons, John (1997). Colour in language. In T. Lamb \& J. Bourriau (Eds.), Colour: Art \& science (194-224). Cambridge: Cambridge University Press.

MacLaury, Robert E. (1997a) Color and cognition in Mesoamerica: Constructing categories as vantages. Austin: University of Texas Press.

MacLaury, Robert E. (1997b). Ethnographic evidence of unique hues and elemental colors. Behavioral and Brain Sciences, 20, 202-203.

Moore, Carmella C., A. Kimball Romney, \& Ti-lien Hsia (2002). Cultural, gender, and individual differences in perceptual and semantic structures of basic colors in Chinese and English. Journal of Cognition and Culture, 2, 1-18.

Morgan, Gerry (1993). Basic colour terms: Comparative results for French and Russian. Journal of French Language Studies, 3, 117.

Morgan, Gerry \& Anthony E. St. G. Moss (1988/1989). The two blues of Russian: The referents of sinij and goluboj. Die Farbe, 35/36, 353-357.

Moss, Anthony A. E. St. G. (1988). Russian blues and purples. Quinquereme, 11, 164-177.

Moss, Anthony E. St. G., Ian R. L. Davies, Greville G. Corbett, \& Glynis Laws (1990). Mapping Russian basic colour terms using behavioural measures. Lingua, 82, 313-332.

Paramei, Galina V. (2005). Singing the Russian blues: An argument for culturally basic color terms. CrossCultural Research: The Journal of Comparative Social Studies, 39, 10-38. 
Paramei, Galina V. \& C. Richard Cavonius (1999). Color spaces of color-normal and abnormal observers reconstructed from response times and dissimilarity ratings. Perception \& Psychophysics, 61, 16621674.

Rakhilina, Ekaterina V. (2000). Kognitivnyj analiz predmetnyx imen: Semantika i sočetaemost' [Cognitive analysis of object names: Semantics and combinability]. Moscow: Russkie Slovari.

Rakhilina, Ekaterina V. (2007). Linguistic construal of colors: The case of Russian. In R. E. MacLaury, G. V. Paramei, \& D. Dedrick (Eds.), Anthropology of color: Interdisciplinary multilevel modeling (363-378). Amsterdam: John Benjamins.

Regier, Terry \& Paul Kay (2004). Color naming and sunlight: Commentary on Lindsey and Brown (2002). Psychological Science, 15, 289-290.

Safuanova, Olga V. \& Nina N. Korzh (2007). Russian color names: Mapping into a perceptual color space. In R. E. MacLaury, G. V. Paramei, \& D. Dedrick (Eds.), Anthropology of color: Interdisciplinary multilevel modeling (55-74). Amsterdam: John Benjamins.

Saunders, Barbara A. C. \& Jan van Brakel (1997). Are there nontrivial constraints on colour categorization? Behavioral and Brain Sciences, 20, 167-228.

Schneider, Ivan \& Peter Fedorov (1978). Die Ikonenmalerei. Technik und Vorzeichnungen (H. Skrobucha, Trans.). Recklinghausen: Verlag Aurel Bongers.

Shepard, Roger N. (1992). The perceptual organization of colors: An adaptation to regularities of the terrestrial world? In J. Barkow, L. Cosmides, \& J. Tooby (Eds.), The adapted mind: Evolutionary psychology and the generation of culture (495-532). Oxford: Oxford University Press.

Shepard, Roger N. \& Lynn A. Cooper (1992). Representation of colors in the blind, color-blind, and normally-sighted. Psychological Science, 3, 97-104.

Shif, Zhozefina I. (1940). Podbor sxodnyx cvetovyx ottenkov i nazyvanie cvetov [Matching of similar color shades and naming of colors]. In Voprosy psixologii gluxonemyx $i$ umstvenno otstalyx detej [Psychological problems of deaf-and-dumb and learning-disabled children]. Moscow: Uchpedgiz.

Sturges, Julia \& T. W. Allan Whitfield (1995). Locating basic colours in the Munsell space. Color Research \& Application, 20, 364-376.

Sun, Richard K. (1983). Perceptual distances and the basic color term encoding sequence. American Anthropologist, 85, 387-391.

Taylor, John R., Henrietta Mondry, \& Robert E. Maclaury (1997). A cognitive ceiling of eleven basic color terms. In R. E. MacLaury, Color and cognition in mesoamerica: Constructing categories as vantages (419-429). Austin: University of Texas Press.

Vartanov, Alexander V. (1997). Cvetovoe semantičeskoe prostranstvo, sformirovavšeesja v uslovijax otsutstvija zritel'nogo opyta [Color semantic space formed in absence of visual experience]. Vestnik Moskovskogo Universiteta. Ser. 14. Psikhologija, No. 3, 76-81.

Vartanov, Alexander V. \& B. T. Nguen (1995). Formirovanie semantičeskogo cvetovogo prostranstva pri usvoenii inostrannyx jazykov [Formation of a semantic color space while learning foreign languages]. Vestnik Moskovskogo universiteta. Ser. 14. Psikhologija, No. 2, $29-46$.

Vartanov, Alexander V. \& Evgenij N. Sokolov (1995). Rol' pervoj i vtoroj signal'nyx system v sootnošenii semantičeskogo i perceptivnogo cvetovyx prostranstv [The role of the first and second signal systems in the correlation of semantic and perceptual color spaces]. Zhurnal Vysshej Nervnoj Deiatelnosti, 45, 343-356.

Vasilevich, Alexander P. (1988). Cvetonaimenovanija i problemy perevoda teksta [Color terms and problems of text translation]. In V. N. Komissarov, L. A. Cherniakhovskaya, \& L. K. Latyshev (Eds.), Tekst i perevod [Text and translation] (84-96). Moscow: Nauka.

Vasmer, Max (1964-1973). Ėtimologičeskij slovar' russkogo jazyka [Etymologic dictionary of Russian] (Vols. 1-3). Moscow: Progress. 
Vendina, Tatjana I. (1999). Cvet v ètnokul'turnoj sisteme russkogo, staroslavjanskogo i drevnerusskogo jazykov [Color in the ethno-cultural system of Russian, Proto-Slavonic, and Old Russian]. In Slavjanskij Al'manax 1998 [Slavic almanac 1998] (277-304). Moscow: Indrik.

Wierzbicka, Anna (1990). The meaning of color terms: semantics, culture, and cognition. Cognitive Linguistics, 1, 99-150.

Winawer, Jonathan A., Nathan Witthoft, Lisa Wu, \& Lera Boroditsky (2003). Effects of language on color discriminability [Abstract]. Journal of Vision, 3, 711a. http://journalofvision.org/3/9/711//, doi:10.1167/3.9.711

Yendrikhovskij, Sergej N. (2001). Computing color categories from statistics of natural images. Journal of Imaging Science and Technology, 45, 409-417. 


\title{
Color term research of Hugo Magnus
}

\author{
Roger Schöntag ${ }^{\mathrm{a}}$ and Barbara Schäfer-Prieß ${ }^{\mathrm{b}}$ \\ ${ }^{a}$ University of Munich, Germany \\ ${ }^{\mathrm{b}}$ University of Heidelberg, Germany
}

In the second part of the 19th century, there was an international scientific controversy on the development, recognition and denomination of color terms, in which participated famous scholars like William E. Gladstone, Lazarus Geiger, Grant Allen, Ernst Krause or Charles Darwin. Among the proponents of the theory that human color perception had developed gradually during the evolution of the human species was the German ophthalmologist Hugo Magnus, who formulated crucial suggestions concerning the relationship between the human capacity of perceiving different colors and the existing color terms in the languages of the world.

Revising his original point of view in further publications in consequence of the results of his ethnological inquiry, Magnus brought to light much information still relevant for current debates. Comparing some of his results like e.g. the evolutionary color term sequence to a modern scientific concept such as Berlin and Kay's, there appear to be astonishing similarities, suggesting that the contributions of Hugo Magnus to the color-term discussion have unjustly fallen into oblivion.

\section{Introduction}

In the late 18th century arose a cross-scientific controversy, which reached its peak around 1880 and generated results that can still be seen to be valid today. An interest in human perception of color and its possible reflection in different color terminologies began with philological analyses by William Ewart Gladstone (1858), when he noticed the lack of certain major color terms in ancient Greek literature, the sort later to be called basic. Starting from a detailed report on Homeric epics, ${ }^{1}$ the controversy grew with appearance of works by Lazarus Geiger (1871) and Hugo Magnus (1877a, 1877b), especially their observations

1. In Section IV (Homer's Perception and Use of Colour) of Gladstone's third volume, he lists "the signs of the immaturity" he found in the Homeric poems: "I. The paucity of colours. II. The use of the same word to denote not only different hues or tints of the same colour, but colours which according to us, are essentially different. III. The description of the same object under epithets of colour fundamentally disagreeing one from the other. IV. The vast predominance of the most crude and elemental forms of colour, black and white, over every other, and the decided tendency to treat other colours as simply intermediate modes between these extremes. V. The slight use of 
on the designations of colors in the Rigveda, the Bible, and Greek and Latin authors, such as Aristotle, Juvenal, Statius and Valerius Flacchus.

\section{Philological Research}

Gladstone (1877:366) postulated that in the time of Homer the human eye was incapable of discerning and distinguishing all colors. He based his hypothesis on the evolutionary perspectives in the tradition of Charles Darwin and Jean Baptiste Pierre Antoine Lamarck. ${ }^{2}$ Consequently, Gladstone (1877:367) imagined an "education" over time to higher color sensibility for humanity as a whole. ${ }^{3}$ He concludes his argument with the uninformed observation that the primitive human being was only equipped with the faculty to distinguish between bright and dark hues, and that the development of the visual organ was accompanied by the recognition of more colors; for example, Homer already named the colors red and orange [erythros, xanthos] (see translation in Gladstone 1878:388).

Lazarus Geiger (1871:57) was also convinced that color perception was an acquired faculty, which could be retraced through the study of ancient literature. He proposed a 'black-red-gold' age followed by a 'white-yellow-red-black' age. The more primitive stage would correspond to the color terms in the Rigveda and the latter to the stage of color perception in the time of Ionian natural philosophy. Neither Gladstone nor Geiger distinguished between the color terms they found in the ancient texts and the capacity to perceive colors.

By 1877, Hugo Friedrich Magnus, professor of ophthalmology at the University of Breslau, became aware of Geiger's work. The historical evolution of the function and capacity of different human senses had rarely been treated in medical literature, ${ }^{4}$ and Geiger's work suggested to him a way to link philological analysis and evolutionary theory with medical and physiological research. Magnus also recognized a significant and problematic contradiction in comparing the sensory faculties of 'uncivilized peoples' (Wilde) of his time with the ancients. At first thought one is inclined to believe in the famous

colour in Homer, as compared with other elements of beauty, for the purpose of poetic effect, and its absence in certain cases where we might confidently expect to find it" (Gladstone 1858:458).

2. Jean Baptiste Pierre Antoine De Monet, Chevalier de Lamarck.

3. "Painters know that there is an education of the eye for colour in the individual. The proposition, which I desire to suggest, is that this education subsists also for the race" (Gladstone 1877:367). Magnus followed him in this position (cf. 1878b, f).

4. Magnus had also shown his interest and passion for the history of ophthalmology and connected subjects in many other publications such as Geschichte des Grauen Stares [History of cataract], 1876a; Das Auge in seinen ästhetischen und cultur-geschichtlichen Beziehungen [The eye in its aesthetic and historic-cultural relationship], 1876b; Die Anatomie des Auges bei den Griechen und Römern [The anatomy of the eye at the time of the Greek and the Romans], 1878a; Culturgeschichtliche Bilder aus der Entwickelung des ärztlichen Standes [Historic-cultural images of the development of the medical profession], 1890; Die antiken Büsten des Homer: eine augenärztlicheästhetische Studie [The Ancient busts of Homer: An ophthalmologic-aesthetic study], 1896; Die Augenheilkunde der Alten [The Ophthalmology of the Ancients], 1901. 
acuteness of hearing, the sense of smell and eyesight of the hunting and gathering people who live in accord with nature (Naturleben). He asked "Why should they not be able to distinguish colors?"

Magnus tried to solve this contradiction, as he saw it, by postulating a difference between the elementary capacity of a sense organ, for example, the perception of light (eye) or sound waves (ear), and other possible functions (Functionsäusserungen), such as being able to discern different colors or melodies. The latter faculties are for Magnus higher functions, which were formed in the course of evolution and, therefore, should be seen as modern acquisitions of mankind (1877a: 1-6).

For an investigation of color terms Magnus had to rely, as did his predecessors, on philological analyses, even though he was a doctor of medicine and a scientist particularly interested in the anthropological and physiological aspects of the subject. But in accordance with his theory of the evolutionary acquisition of higher functions of the sense organs, he rejected the method of analysis practiced up to then, which had used as a point of reference the color scale of the spectrum established by Isaac Newton. Magnus instead chose a subdivision, which, in his view, was closer to the ancient capacity of color perception, because the Newton scale reflected the modern faculty to perceive and discern the color spectrum; hence he drew up light 'richness' (Lichtreichthum) as a criterion for his analyses of the ancient texts. ${ }^{5}$ Thus, unlike his scientific predecessors, he avoided a direct identification of the color terms found in ancient literature with the color nomenclature of modern European languages (Magnus 1877a:6-9). According to this concept, he structured his first inquiry: "Colors of luminous intensity" (lichtstarke Farben) like red, orange, yellow; "colors of medium luminous intensity" (Farben mittlerer Lichtstärke) like green; and "colors of little luminous intensity" (Farben geringer Lichstärke) like blue and violet (Magnus 1877a:9-41).

Finally Magnus (1877a:41-42) postulated four stages of evolution concerning the perception of color in the history of mankind. At a first primitive stage people had only a sensibility for red, which coincided with the sensibility for bright and luminous colors. For that Magnus found it more useful to speak of the capacity to discern quantities of light and not color hues in a stricter sense. ${ }^{6}$ A second stage was reached when the human retina was sufficiently developed to distinguish red from yellow, as these colors were no longer merged in perception of simple brightness. By the third stage mankind had developed the sensibility for green hues, colors of a medium luminous intensity, which were detached from, on one hand, perception of darkness (dark green) and, on the other, perception of

5. Magnus (1877:45-46) followed Aristotle and Helmholtz, who both try to explain color sensibility by focusing on color lightness. Magnus, who was fully aware of physical and physiological theories of his time - see Magnus 1881:27-50, where he discusses the theories of Young, Helmholtz, Hering and others - explicitly quotes Helmholtz (1867:267).

6. Magnus (1877b:19) deduced this first stage from the diminishing faculty of the eye to discern colors when the light is insufficient, referring to observations of Chodin (1877). 
darker yellow (light green). ${ }^{7}$ The last stage was reached with the faculty to perceive colors of weak luminous intensity like blue and violet, which were until then perceptually merged in an idea of general darkness. In sum, Magnus inferred an evolution, which followed the spectrum of color, starting at the luminous, intense colors and finding its end in the dark part of the spectrum.

For Magnus, as an ophthalmologist, the most intriguing part of the investigation might have been the possibility of a physiological explanation, which could illuminate this postulated evolution. To substantiate his assertion that at the earliest stage people could only perceive the quantity of light hitting the retina and not its quality, ${ }^{8}$ he quotes Greek testimonies like Empedocles, Aristotle and Plato, who emphasized the basic opposition of dark versus light whence all other colors emanate.

Magnus believed that over the centuries of human evolution the retina had been more and more exposed to light and the continuous stimulation by light rays had caused a gradual change in perception of lightness with respect to darkness. This path of evolution seemed to him quite logical, because his queries about the color perception of children suggested certain parallels, such as the preference for red stimuli in the young children (Magnus 1877a:43-56).

In this earliest study by Hugo Magnus, the striking element is the importance he attributes to brightness in color perception. This reminds us very much of the findings a century later of Kay and McDaniel (1978:616-617) and Kay (1975:258-260). At Stage I of the Berlin and Kay (1969) theory of color-term evolution, there is only a distinction between black and white. But with Rosch's (1972) Dugum Dani studies, we received a different picture of this first stage. People of this ethnic group seem to distinguish between light-warm colors (mola: white, red, orange, yellow, pink, red-purple) and dark-cool colors (mili: black, blue, green). Foci of mola and mili were found to be situated, respectively, in light and warm versus dark and cool hues. Especially remarkable was the focus of mola, which in $67 \%$ cases was identified with English focal 'red.' ${ }^{9}$ Magnus had not, though, introduced the opposition of light-warm hues versus dark-cool hues. His decisive criterion is the degree of brightness related to the light richness, strength (Lichtreichthum, Lichtstärke).

When Magnus brought up the argument of color brightness in order to support the evolutionary theory of Geiger, it might be considered as the right step with the wrong objective. For the remotest ages Magnus (1877a:10-11) favored red and not white as the focus of bright hues, because of the luminous intensity of the former. Thus he postulated that those people could not really perceive red hues, but just the intensity, which caused a particular sensibility to these rays of the spectrum. For him, it was the most reasonable

7. This development also implied a clearer distinction between the luminous, intense colors red and yellow. In this respect, dark green is the dark part of the luminous, intense color spectrum.

8. "This even lower stage ... in the development of the color sense could fairly be characterized as follows: at that time the human retina, when struck and excited by light of any composition, sensed only its quantity and not its quality, i.e. color" (Magnus 1877a:44, our translation).

9. Hardin (1998:211-216) offers an interpretation of these findings integrated in the Berlin \& Kay scheme and his critical observations. 
means to explain why the Rigveda makes no clear distinction between white and red. In Magnus's second publication on color terms (1877b) he mainly presents more concisely the theory of his first publication (1877a). ${ }^{10}$ As before, Magnus (1877b: 19) situates the first stage in the age of the Rigveda, characterized by a bright (red/white)-dark opposition, and the second stage, with emerging sensitivity to yellow, in the age of Homer. He adds considerations about the distinction between green and blue. At this third stage of evolution, the sensitivity is so far developed that man could finally perceive and distinguish the color green, but not yet blue. This could explain the frequent confusion of blue and green in ancient Greek texts. The following stage is characterized by the sensitivity to blue and violet. Bright blue is resolved from the formerly grey part of spectrum, dark blue from the dark/black spectrum (1877b:10-12).

As an interesting supplement, Magnus (1877b:12) reports on Bastian's (1869:89) investigation, which described the confusion of blue and green in Burma at that time as being totally normal. ${ }^{11}$ Magnus drew what was for him the logical conclusion that these people were still on a lower level of evolution and had not reached the sensibility for blue hues.

At the end of this publication, Magnus's considerations about the consequences of his thesis on color evolution lead him to speculate that the development of our physiological faculties has not come to an end, but is still in alteration. In other words, future humans will be able to perceive more in the color spectrum than current humans do, being able to see beyond the present limit at wavelengths shorter than ultraviolet.

\section{Polemics on the Gladstone-Geiger Hypothesis}

Before publication of Magnus's first study (1877a) on the development of color sensation, its scholarly discussion was broadly limited to classical philological research. He and others enriched the debate, however, by introducing physiological, ethnological, and evolutionary facets.

The theory of evolutionary change is in first place influenced by Lamarck, who denied invariability of species and attributed changes to the pressure for an animal to adapt itself in an optimal way to its habitat such that corresponding change in an organ's structure, which would be inherited, would depend on frequency of the organ's use. ${ }^{12}$

10. "The living strength of different colors should be regarded as the guiding principle of the evolution of color sense" (Magnus 1877b: 19, our translation). Hirschberg (1877:111) offers a short summary. This first publication by Magnus on color sense instigated such reverberations in the scientific world that merited its translation to French (Magnus 1878g).

11. The coincidence with the modified Berlin \& Kay scheme (Kay 1975:260) that introduces the category grue (cool), unifying the colors green and blue, cannot be ignored. The grue category appears first at Stage IIIa of their scheme.

12. "In the evolution of animals, the progression in the composition of the organization sometimes undergoes anomalies due to the influence of circumstances of habitation and adopted habits" (Lamarck 1809: 134-135, our translation). "The frequent use of an organ, becoming regular because of habits, increases the organ's faculty; it 
Darwin also supported the evolution of species but explained it with natural selection:

Nothing at first can appear more difficult to believe than the more complex organs and instincts should have been perfected, not by means superior to, though analogous with, human reason, but by the accumulation of innumerable slight variations, each good for the individual possessor. Nevertheless, this difficulty, though appearing to our imagination insuperably great, cannot be considered real if we admit the following propositions, namely, - that gradations in the perfection of any organ or instinct which we may consider, either do now exist or could have existed, each good of its kind, - that all organs and instincts are, in ever so slight a degree, variable, - and, lastly, that there is a struggle for existence leading to the preservation of each profitable derivation of structure or instinct. The truth of these propositions cannot, I think, be disputed.

(Darwin 1859/1996:371)

Magnus (1877a:44) saw the basic principle for his theory in the generally accepted fact that all organic forms tend to develop from a relatively low initial level, to perfection. ${ }^{13}$ Even though calling himself an adherent of Darwin, ${ }^{14}$ Magnus found himself in conflict with other adherents of Darwin's theory. ${ }^{15}$ According to Magnus and others, color perception as an evolutionary product developed over the last few thousand years. This notion is, however, no longer discussed in modern color science. Due to interventions of scholars like Ernst Krause (1877a, 1877b, 1878), Charles Grant Blairfindie Allen (1879), ${ }^{16}$ Anton Marty (1879), and Rudolf Hochegger (1884), who exposed gaps in this version of the theory, it was finally abandoned. ${ }^{17}$

The first objection came from Krause, who did not believe that the sense of color is such a recent acquisition among mankind: "The color-sense manifestly appears already in insects and many of the lowest vertebrates; its complete absence could therefore hardly be supposed in the very lowest race of men (1878:120).” In his preface to the German translation of Allen's The Colour-Sense, Krause made controversial comments reproaching Allen, Magnus, and Stein for not quoting his objections adequately. He also accused Magnus of having misinterpreted Darwin, as a differentiated color sense was already known to

develops by its use and acquires a size and a strength of action, which is lacking in animals, that less exercise it" (Lamarck 1809:248, our translation).

13. "The probability of this assumption ... of insensitivity to color as an original starting point of color sense can ultimately be justified by the general fact, that all organic creations, by means of a gradual evolution, raise themselves from a correspondingly low initial stage by and by to ever-greater perfection" (Magnus 1877a:44, our translation).

14. "As an advocate of the Darwinist perspective..." (Magnus 1881:171, our translation).

15. For modern comments on the founding fathers of evolutionary theory, see, for example, Bayertz et al. (1982); Heinrich (1982); Kohn (1985); Lefèvre (1997); Messerly (1996); and Riedl (2003).

16. For the announcement of his forthcoming study, see Allen (1878); for the German translation of Allen (1879), see Allen (1880).

17. The list could have been extended: Cohn (1878a), Schroeder (1879), Zehender (1878), and Dor (1878a, 1878b, 1878c) doubted the short period of evolution, because people like the Ancient Egyptians already knew how to paint in many different colors. 
be developed in animals. He even communicated his thoughts about this discussion to Charles Darwin, who was keenly interested in these observations, as is documented by his correspondence:

\section{To Ernst Krause}

Down, June 30th, 1877.

I have been very much interested by your able argument. [Editors' footnote: The interest felt by Mr. Darwin is recorded by the numerous pencil-marks on the margin of his copy against the belief that the sense of colour has been recently acquired by man.] The following observation bears on this subject. I attended carefully to the mental development of many young children, and with two, or as I believe three of them, soon after they had come to the age when they knew the names of all common objects, I was startled by observing that they seemed quite incapable of affixing the right names to the colours in coloured engravings, although I tried repeatedly to teach them. I distinctly remember declaring that they were colour-blind. But this afterwards proved a groundless fear. ${ }^{18}$

On communicating this fact to another person he told me that he had observed a nearly similar case. Therefore the difficulty, which young children experience either in distinguishing, or more probably in naming colours, seems to deserve further investigation. I will add that it formerly appeared to me that the gustatory sense, at least in the case of my own infants, and very young children, differed from that of grown-up persons. This was shown by their not disliking rhubarb mixed with a little sugar and milk, which is to us abominably nauseous; and in their strong taste for the sourest and most austere fruits, such as unripe gooseberries and crab apples.

(Darwin \& Seward 1903:47-48)

Magnus (1877c) responded to the Krause's criticisms directly in the later study Farben und Schöpfung, wherein he rejects the argument of the equivalence of animal and human color sense, while emphasizing the agreement of his color theory with Darwinist claims (Magnus 1881:138-140). ${ }^{19}$ Krause (1877a:272), however, had the same opinion as Mag-

18. Also Magnus (1881:176-177; 1883:30) mentioned this case without quoting a source. As Krause printed this (1877) letter in Kosmos, Magnus surely referred to Darwin (1877) in Kosmos.

19. The main criticism of Krause was that color sense should have been an original faculty of human beings: "These principal laws [of natural selection], manifesting as various secondary laws (mimicry etc.), are essentially based on the requirement that color sensitivity is a general and original, in other words a very early developed faculty of the sense organ" (Krause 1877a:270, our translation). Magnus (1877c:423-424) rejected this by postulating an evolution of color sense in animals. But he also denied the equivalence of the quality of animal and human color sense: "Nevertheless individual especially enthusiastic Darwinists, for example Mr. Dr. Ernst Krause, made the assertion that the theory of gradual evolution of color sense is in irreconcilable contradiction to Darwin's theory and must therefore be rejected unconditionally.... If these scientists want to derive a principal contradiction between Darwin's theory and the hypothesis of the evolution of color sense, from their purported but as-yet-unproven identity of animal and human color sense, then we can justifiably show this premise to be incorrect, out of hand" (Magnus 1881:138-139, our translation). Krause (1877b) insisted on his point of view. 
nus with regard to the stages by which color terms emerge, which reflect the order of the color spectrum. ${ }^{20}$

Allen (1878) showed two complementary ways to argue against any hypothetical development of color perception. The description of works of art and other archaeological material of pre-historic man and the ancient world proves undoubtedly (according to Allen) "that the color-sense was fully developed long before the epoch of the Iliad or the Book of Genesis" (Allen 1879:204). An inquiry sent to officials among different tribes in Europe, Asia, Africa, America, and the Pacific Islands should back up "the supposition that the colour sense is, as a whole, absolutely identical throughout all branches of the human race" (Allen 1879:205). The result of his analysis did indeed confirm his supposition: "Man is the descendant of an arboreal quadrumanous animal, of frugivorous habits, who shared the common vertebrate faculty of colour perception, and the common frugivorous taste for bright hues" (Allen 1879:21).

Criticizing Geiger, Gladstone, and Magnus because of their one-sided philological method, he addressed particularly to Magnus the reproach of arguing "a priori as to what the sensations of the savages must be like" (Allen 1879:220), without further observations or inquiry, whereas "a little more care and a little more extended search would le[a]d him [Magnus] to abandon his theory, based as it is upon the shifting sands of half-forgotten languages" (Allen 1879:220).

Marty (1879:63), ${ }^{21}$ who opposed the development theory, emphasized the need to distinguish designation and perception, as the human faculty to perceive different colors does not imply a broad classification in color terms. ${ }^{22}$ Hochegger (1884:48) also supported this necessary distinction inter alia with regard to the results brought up by examination of 'uncivilized peoples' (Naturvölker). ${ }^{23}$

20. "With regard to the sequence. . . in which color names came into use, I share absolutely the view of Dr. Magnus that the introduction of new terms should have occurred in the spectral order of colors" (Krause 1877a:272, our translation).

21. For a recent comment on Marty's point of view in the discussion on color perception, see Spinicci (2000).

22. Magnus (1881:167) rejected, however, Marty's assertion that 'uncivilized peoples' (Naturvölker) have a better developed color-sense.

23. "The adherents of the theory of color sense evolution proceeded on the basis of comparative language analysis from the following principle: color sensitivity and color terminology run side-by-side in parallel, the chronological constitution of color nomenclature implying a direct and reliable conclusion about the corresponding state of color sensitivity. This key principle and corner-stone of Geiger's theory was wrong. In particular studies of color sense of primitive peoples have shown clearly that discriminative sensitivity to all colors can exist, although a linguistic language term is missing" (Hochegger 1884:48, our translation). 


\section{Ethnological research}

Hugo Magnus (1880), ignoring (or not quoting) the investigations of Allen (1879), ${ }^{24}$ sent out more than 60 questionnaires ${ }^{25}$ to all five continents. ${ }^{26}$ He planned to compare color sense of ethnic groups with little or no contact with modern society, whence he hoped to discover relations between color perception and its dependence on culture. At this point he still favored Geiger's thesis of color evolution. ${ }^{27}$ In the semester of 1877-1878, ethnologist Eduard Pechuël-Loesche ${ }^{28}$ (Leipzig) prompted Magnus to start the project with support from the ethnological museum (Museum für Völkerkunde zu Leipzig). Magnus referred to Alarik Frithiof Holmgren $(1878 \mathrm{a}, 1878 \mathrm{~b}),{ }^{29}$ who was first ${ }^{30}$ to carry out an investigation with 'uncivilized people' (auf niederer Stufen der Civilisation stehender Völkerschaften) to gain insight into the relation between color sense and culture (Magnus 1880:1-4). For the questionnaire, Magnus chose the following colors, whose names correspond to nine of Berlin and Kay's 11 basic color terms: white, black, red, green, yellow, brown, purple,

24. Quoting Allen (1879), Magnus (1880:48-49) refuted Allen's speculations about the evolution of the animal color sense, but did not comment on his study. Only later did Magnus (1881:161-162) criticize Allen's questionnaire considering it in need of systematic chromatic description.

25. See Appendix.

26. Before he started his overseas study, first described in Magnus and Peschuël-Loesche (1878), Magnus (1878b:288-289) carried out color tests with pupils from Breslau to gain experience in the questionnaire's use (Magnus \& Cohn 1878; Magnus 1878c). Here we also see the relation between the color-sense problem and the discussion on color-blindness with respect to revealing color-sense defects (Cohn 1878b; Magnus 1878d, 1878e, 1879).

27. The description of Magnus's intentions at that time as given by Berlin \& Kay (1991:139) might be seen as ambiguous. Magnus definitely did not start the study to disprove assertions of Gladstone and Geiger with regards to the physiological evolution.

28. He was already experienced in ethnographic studies, whose results were partly published later; e.g. The Loango expedition (Güssfeldt et al. 1879/1907).

29. Holmgren, who was especially interested in color blindness, in $1877 / 1878$ carried out a study with the Lapps in the north of Sweden whose results he communicated to Magnus (see Magnus 1880:1, who quotes Holmgren, 1878a, with a comment that his new test is especially apt for uncivilized people). Holmgren developed a wellknown test for diagnosing color-blindness: "According to our method, the examiner selects from the collection of Berlin wools in a pile on a convenient table, and lays aside a skein of the especial color desired for this examination; then he requires the one examined to select to other skeins most closely resembling the color of the sample, and to place them by its side. The chromatic sense of the individual is decided by the manner in which he performs his task" (Holmgren 1878b: 182).

30. Seemingly Magnus ignores the already cited study of Grant (1879), although he was aware of the respective publication, which he discusses only in Magnus (1880:161-162). 
orange, and gray, while omitting blue and pink. Along with his own investigation, he relied on other studies to complete and control results. ${ }^{31}$

The questionnaire was distributed to and filled in with the help of missionaries, doctors, and overseas officers who had access to tribes, such as Challam, Sioux, Snake, Makah (North America); Tambukki, Pondo, Fingu, Gaika, Damara, Fulah, Madingo, Krobo (Africa); Toda, Koda, Badaga, Telugu, Batta, Olon (Asia); and tribes of territories in Australia and Europe (Magnus 1880:4-6). Having analyzed the results, he frankly admits the failure of his first assessment of color-sense evolution: lack of a color term is not directly related to perception of the corresponding color (Magnus 1880:22, 44). ${ }^{32}$

Despite the failure of his 1877 assessment of the evolution of color sense, Magnus had therein made a number of significant observations, one of which is that many ethnic groups do not provide an abstract designation of color. ${ }^{33}$ Color is often identified with form or surface properties or at least with pattern, as with arrangement and size of spots or of other markings. For example, the South African Ovaherero finely differentiate cattle by names that designate color and form in many combinations (Magnus 1880:17-21). Magnus offered his thoughts about the relation between color naming and the living conditions or environment. In this respect, he did not want to abandon completely his ideas of the physiological evolution of color sense. He postulated that under the pressure of certain external conditions, separate parts of the color sense undergo an education to higher perfection (höhere Erziehung). As a striking example for this theory (Magnus 1880: 1920), he extends his example from the Ovaherero by quoting the nomenclature among the Kaffir of the same region, who have 31 names for colors, most of which relate to their cattle, but only one expression for green and blue combined (luhlāza). Merging the other quoted empirical researches with his own inquiry, Magnus (1880:34) concludes with ten facts deduced from his worldwide overview:

1. 'Primitive peoples' have the same color sense as the civilized nations;

2. Color perception and color terms need not correspond;

31. Magnus (1880:3-4) quotes inter alia, Andree (1878), Cohn (1879), Gatschet (1879a, 1879b), Kotelmann (1879), Stein (1879), Virchow $(1878,1879)$. Also, Andree (1879) reviews Gatschet (1879a), but this review is not mentioned by Magnus. These studies further attest to liveliness of the color topic then.

32. "Therefore I have no hesitation at all about admitting without further ado my mistake concerning this question; and to accept that I was wrong about the scope of the results obtained from comparative-language studies and that the conclusions I drew from these do not correspond to actual situation. Above all, this retraction refers to the attempt to assign single stages of color-sense evolution to definite chronological boundaries, boundaries whose narrowness or width was solely defined by those eras, in which incomplete color nomenclature was deduced from contemporaneous literature" (Magnus 1880:44, our translation).

33. "The understanding of color as an abstract conception ... as we find it in the civilized nations, is probably missing in the majority of tribes that we investigated. It seems that the philosophical singling-out, the separation of the abstract feature (the color) from the concrete form (the colored object) is too difficult an intellectual operation for the majority of the primitive peoples; and therefore they dispense with developing the idea of independent color in concept and language, preferring to but merge the notion 'color' with other concepts which are more adequate and more comfortable for their intellectual sphere" (Magnus 1880:15, our translation). 
3. Color perception and color terms are often found in enigmatic discrepancy (e.g., few color terms versus well-developed sensation);

4. Cases of 'incomplete' color terminology mostly follow a set pattern;

5. Terms for long-wavelength colors are always more precise than terms for shortwavelength colors;

6. The term for 'red' is always the best developed, followed by that for 'yellow, 'green,' and finally 'blue';

7. 'Confusion' of color terms mostly involves neighboring colors of the spectrum (e.g., red and orange, yellow and green, but never colors like blue and red);

8. The most frequent confusion is between blue and green;

9. The minimal color terminology consists of 'red' for long-wavelength colors and 'dark' for short-wavelength colors;

10. Even in highly elaborated color terminology confusion of short-wavelength colors is possible; these are then subsumed under terms for 'dark' (e.g., blue and violet are denoted as 'black' or 'gray'). ${ }^{34}$

The regularities in color terminology identified by Magnus ${ }^{35}$ remind us of the Basic Color Terms theory of Brent Berlin and Paul Kay (1969:2-4) who nearly a hundred years later determined similar patterns, relying - as did Magnus - on the analysis of many language communities around the world. Table I compares the two schemes. ${ }^{36}$

Table 1. Evolutionary color-term sequences devised by Magnus in comparison to that of Berlin and Kay.

\begin{tabular}{lll}
\hline & Magnus (1877a:41-42) & Berlin \& Kay (1969:4) \\
\hline Stage I & bright (red)/dark & black/white \\
Stage II & red/yellow & red \\
Stage IIIa & green & green \\
Stage IIIb & & yellow \\
Stage IV & blue/purple & yellow and green \\
Stage V & & blue \\
Stage VI & & brown \\
Stage VII & (others) & purple/pink/orange/gray \\
\hline
\end{tabular}

34. A summary of Magnus's results in their relation to actual knowledge can be found in Berlin \& Kay (1991: 139146), earlier synopses in Parsons (1924:158-159) and Rivers (1901a, 1901b). For a short history of color ethnography see MacLaury (2001:1227-1231).

35. Although Magnus admitted his fault in assuming a development of color sense, he maintained an uneasiness about the likelihood of a relation between its regularities, culture, and culturally based education (Magnus 1880:20-21).

36. For recent developments in research on color terms see Backhaus et al. (1998) and Zollinger (1999), both including summaries inter alia of Berlin \& Kay (1969), Kay \& McDaniel (1978), and Kay (1975). 
Berlin and Kay assess the research of Magnus in reference to their data:

Magnus's work is the most comprehensive and conclusive of his time. His only major finding that requires revision now is his conclusion that yellow always appears before green. This is not justified by his own data. One is tempted to speculate that Magnus, as Geiger before him, was able to overlook this fact because of the attractiveness of maintaining a simple theory which strictly ties "primacy" of a color category to magnitude of dominant wavelength. It was not until Rivers's work, some twenty years later, that comparable research was carried out.

(1991: 145-146)

Magnus $(1881,1883)$ tried to defend this position, arguing against his critics. He quoted further studies on color ${ }^{37}$ including works of the prominent evolutionists Ernst Haeckel ${ }^{38}$ and Alfred Russel Wallace - in an attempt to show that the Darwinist evolutionary theory was not in contradiction with his own theory of color sense and that he was right in his postulated gradation of color-term acquisition.

\section{Summary}

The great achievement of Hugo Magnus is surely seen in his detailed description of his worldwide survey results that brought to light much new information, and in the postulation of a universal sequence of the emergence of color terms, which in its essence is still valid today. What is also remarkable is that his empirical research led him to give up albeit somehow reluctantly - the then-dominant Gladstone-Geiger Hypothesis, which had been his starting point.

\section{References}

Andree, Richard (1878). Ueber den Farbensinn der Naturvölker. Zeitschrift für Ethnologie, 10, 323-334.

Andree, Richard (1879). Adjectives of color in Indian languages. By Albert S. Gatschet. The American Naturalist vol. XIII, August 1879, p. 475-485. Archiv für Anthropologie, 12, 263-264.

Allen, Grant (1878). The colour-sense. Nature, 19 (November 14), 32.

Allen, Grant (1879). The colour-sense: Its origin and development: An essay in comparative psychology. London: Trübner \& Co.

Allen, Grant (1880). Der Farbensinn. Sein Ursprung und seine Entwicklung: Ein Beitrag zur vergleichenden Psychologie. Leipzig: Ernst Günther's Verlag.

Backhaus, Werner G. K., Reinhold Kliegl, \& John S. Werner (Eds.) (1998). Color vision: Perspectives from different disciplines. Berlin \& New York: Walter de Gruyter.

Bastian, Adolf (1869). Miscelle. Zeitschrift für Ethnologie, 1, 89-90.

37. E.g., Almquist (Chukchi), Birgham (Kanakas), and Pontoppidan (Sandwich Isles) carried out color testing of the indigenes, using Holmgren's wools (Magnus 1883:4). For further reports on color sensitivity in various ethnicities see also Nachtigal (1874), Virchow (1879), and Kirchhoff (1879).

38. "For example, Häckl says with regards to the gradual evolution of color sense: 'Thus, modern evolution theory, in its application to the historical evolution of sense organs, offers us a delightful vista into the whole future.' Does this sound, I would ask, like a contradiction between Darwinism and the evolution theory of color sense?" (Magnus 1881: 139-140, our translation). 
Bayertz, Kurt, Bernhard Heidtmann, \& Hans-Jörg Rheinberger (Eds.) (1982). Darwin und die Evolutionstheorie. Köln: Pahl-Rugenstein-Verlag.

Berlin, Brent \& Paul Kay (1969). Basic color terms: Their universality and evolution. Berkeley: University of California Press.

Berlin, Brent \& Paul Kay (1991). Basic color terms: Their universality and evolution (2nd ed.). Berkeley: University of California Press.

Chodin, A. (1877). Über die Abhängigkeit der Farbempfindungen von der Lichtstärke. Jena: Verlag von Hermann Dufft.

Cohn, Hermann (1878a). Bemerkung zu der Berichtigung des Herrn Dr. Magnus. Centralblatt für praktische Augenheilkunde, 2, 288.

Cohn, Hermann (1878b). Beobachtungen an 100 Farbenblinden. Beilage zum Augusthefte des 'Centralblattes für praktische Augenheilkunde,' 2 (December), XXIX-XXXVII.

Cohn, Hermann (1879). Sehschärfe und Farbensinn der Nubier. Schlesische Zeitung, 331.

Darwin, Charles (1877). Biographische Skizze eines kleinen Kindes. Kosmos, 1 (August), 367-376.

Darwin, Charles (1996). On the origin of species by means of natural selection, or the preservation of favoured races in this struggle for life (Gillian Beer, Ed.). Oxford: Oxford University Press. (Original work published 1859)

Darwin, Francis \& Albert Charles Seward (1903). More letters of Charles Darwin: A record of his work in a series of hitherto unpublished letters, Vol. 2. London: John Murray.

Dor, Heinrich (1878a). Zur geschichtlichen Entwickelung des Farbensinnes. Beihefte zu den Klinischen Monatsblättern für Augenheilkunde (Bericht über die Elfte Versammlung der ophthalmologischen Gesellschaft), 16, 120-129.

Dor, Heinrich (1878b). Gegen Magnus: Zur Entwickelung des Farbensinnes. Klinische Monatsblätter für Augenheilkunde (Offene Correspondenz), 14, 532-533.

Dor, Heinrich (1878c). Zur geschichtlichen Entwickelung des Farbensinnes. Beilage zum Augusthefte des 'Centralblattes für praktische Augenheilkunde', 2, XXXVII-XXXVIII.

Gatschet, Albert Samuel (1879a). Adjectives of color in Indian languages. American Naturalist, 13 (August), 475-485.

Gatschet, Albert Samuel (1879b). Farbenbenennungen in nordamerikanischen Sprachen. Zeitschrift für Ethnologie, 11, 293-302.

Geiger, Lazarus (1871). Ueber den Farbensinn der Urzeit, und seine Entwicklung (Gesprochen auf der Versammlung deutscher Naturforscher in Frankfurt a. M. den 24. September 1867). In L. Geiger (Ed.), Zur Entwickelungsgeschichte der Menschheit, Vorträge (45-60). Stuttgart: Verlag der J. G. Cotta'schen Buchhandlung.

Gladstone, William Ewart (1858). Studies on Homer and the Homeric age, Vol. 3. London: Oxford University Press.

Gladstone, William Ewart (1877). The colour sense. Nineteenth Century, 2, 366-388.

Gladstone, William Ewart (1878). Der Farbensinn: Mit besonderer Berücksichtigung der Farbenkenntnis des Homer. Breslau: J. U. Kern's Verlag (Max Müller).

Güssfeldt, Paul, Julius Falkenstein, \& Eduard Pechuël-Lösche (1879/1907). Die Loango-Expedition: ausgesandt von der Deutschen Gesellschaft zur Erforschung Äquatorial-Africas, Ein Reisewerk in drei Abteilungen. Leipzig: Frohberg.

Hardin, Clyde L. (1998). Basic color terms and basic color categories. In W. G. K. Backhaus, R. Kliegl, \& J. S. Werner (Eds.), Color vision: Perspectives from different disciplines (209-217). New York: Walter de Gruyter.

Heinrich, Dieter (Ed.) (1982). Evolutionstheorie und ihre Evolution: Vortragsreihe der Universität Regensburg zum 100. Todestag von Charles Darwin. Regensburg: Mittelbayerische Druckerei- und Verlagsgesellschaft mbH. 
Helmholtz, Hermann von (1869). Handbuch der physiologischen Optik. Leipzig: Voss.

Hirschberg, Julius (1877). 'Die geschichtliche Entwickelung des Farbensinns' von Dr. H. Magnus in Breslau. Leipzig, Veit \& Comp. 1877, 56 Seiten. Centralblatt für praktische Augenheilkunde, 1 (Juni), 111.

Hochegger, Rudolf (1884). Die geschichtliche Entwickelung des Farbensinnes: Eine psychologische Studie zur Entwickelungsgeschichte des Menschen. Innsbruck: Verlag der Wagner'schen UniversitätsBuchhandlung.

Holmgren, Frithiof Alarik (1878a). Zur Entdeckung der Farbenblindheit bei Massenuntersuchungen. Centralblatt für praktische Augenheilkunde, 2 (August), 177-182.

Holmgren, Frithiof Alarik (1878b). Color-blindness in its relation to accidents by rail and sea. In Annual report of the board of regents, Smithsonian Institution: Showing the operations, expenditures, and condition of the Institution for the year 1877 (131-195). Washington, DC: Smithsonian Institute.

Kay, Paul (1975). Synchronic variability and diachronic change in basic color terms. Language in Society, 4, 257-270.

Kay, Paul \& Chad K. McDaniel (1978). The linguistic significance of the meanings of basic color terms. Language, 54, 610-646.

Kirchhoff, Alfred (1879). Über Farbensinn und Farbenbezeichnung der Nubier. Zeitschrift für Ethnologie, $11,323-334$.

Kohn, David (Ed.) (1985). The Darwinian heritage. Princeton: Princeton University Press.

Kotelmann, Ludwig Wilhelm Johannes (1879). Die Augen von 9 Lappländern, 8 Patagoniern, 18 Nubiern und 1 Neger vom weissen Nil. Berliner Klinische Wochenschrift, 16 (November), 701-703.

Krause, Ernst (1877a). Die geschichtliche Entwickelung des Farbensinnes. Kosmos, 1 (Juni), 264-275.

Krause, Ernst (1877b). Vertheidigung des ablehnenden Standpunktes. Kosmos, 1 (August), 428-433.

Krause, Ernst (1878). The colour sense. Nature (December), 19, 120.

Lamarck, Jean Pierre Baptiste Antoine (1809). Philosophie zoologique. Paris: Dentu.

Lefèvre, Wolfgang (1997). Jean Baptiste Lamarck (1744-1829). Berlin: Max-Planck-Institut für Wissenschaftsgeschichte.

Magnus, Hugo (1876a). Geschichte des grauen Stares. Leipzig: Verlag von Veit \& Comp.

Magnus, Hugo (1876b). Das Auge in seinen ästhetischen und culturgeschichtlichen Beziehungen: Fünf Vorlesungen. Breslau: J. U. Kern's Verlag (Max Müller).

Magnus, Hugo (1877a). Die geschichtliche Entwickelung des Farbensinnes. Leipzig: Verlag von Veit \& Comp.

Magnus, Hugo (1877b). Die Entwickelung des Farbensinnes. Jena: Verlag von Hermann Dufft.

Magnus, Hugo (1877c). Zur Entwickelung des Farbensinnes. Kosmos, 1 (August), 423-427.

Magnus, Hugo (1878a). Die Anatomie des Auges bei den Griechen und Römern. Leipzig: Verlag von Veit \& Comp.

Magnus, Hugo (1878b). Die Erziehung des Farbensinns. Zeitschrift für Ethnologie, 10, 288-289.

Magnus, Hugo (1878c). Zur Entwickelung des Farbensinnes. Klinische Monatsblätter für Augenheilkunde, 14, 465-478.

Magnus, Hugo (1878d). Berichtigung des Cohn'schen Aufsatzes: 'Ueber die spectroscopische Untersuchung Farbenblinder' im Novemberheft dieses Centralblattes. Centralblatt für praktische Augenheilkunde, 2 (December), 287.

Magnus, Hugo (1878e). Über Farbenblindheit. Beilage zum Augusthefte des 'Centralblattes für praktische Augenheilkunde', 2, XXXVIII.

Magnus, Hugo (1878f). Ueber systematische Erziehung des Farbensinnes in den Schulen (Vorgelesen durch Hess). Beilagenheft zu den Klinischen Monatsblättern für Augenheilkunde, 16, 132-137.

Magnus, Hugo (1878g). Histoire de l'évolution du sens des couleurs (Avec une introduction par Jules Soury). Paris: C. Reinwald.

Magnus, Hugo \& Hermann Cohn (1878). Breslau. Untersuchung von 5000 Schulkindern in Bezug auf Farbenblindheit. Klinische Monatsblätter für Augenheilkunde, 16, 245-256. 
Magnus, Hugo \& Eduard Peschuël-Loesche (1878). Breslau. Museum für Völkerkunde zu Leipzig. Klinische Monatsblätter für Augenheilkunde, 16, 375-377.

Magnus, Hugo (1879). Stilling, 'Ueber Farbensinn und Farbenblindheit, Rede gehalten auf der 51. Versammlung deutscher Naturforscher' und Aerzte, Cassel 1878. Stilling, 'Die Prüfung des Farbensinnes beim Eisenbahn und Marinepersonal.' Neue Folge, Erste Lieferung. Tafeln zur Bestimmung der Roth-Grünblindheit. Cassel 1878. Magnus, 'Beiträge zur Kentniss der physiologischen Farbenblindheit.' v. Gräfe's Archiv. Bd. XXIV. H. 4. Berliner Klinische Wochenschrift (Kritiken und Referate), 16 (März), 142.

Magnus, Hugo (1880). Untersuchungen über den Farbensinn der Naturvölker. Jena: Verlag von Gustav Fischer.

Magnus, Hugo (1881). Farben und Schöpfung: Acht Vorlesungen über die Beziehungen der Farben zum Menschen und zur Natur. Breslau: J. U. Kern's Verlag (Max Müller).

Magnus, Hugo (1883). Über ethnologische Untersuchungen des Farbensinnes. Berlin: Verlag von Carl Habel. Magnus, Hugo (1890). Culturgeschichtliche Bilder aus der Entwickelung des ärztlichen Standes. Breslau: J. U. Kern's Verlag (Max Müller).

Magnus, Hugo (1896). Die antiken Büsten des Homer: eine augenärztlich-ästhetische Studie. Breslau: J. U. Kern's Verlag (Max Müller).

Magnus, Hugo (1901). Die Augenheilkunde der Alten. Breslau: J. U. Kern’s Verlag (Max Müller) [Engl. transl.: Hugo Magnus (1998/1999). Ophthalmology of the Ancients (in two parts). Oostende: Waryenborgh].

Marty, Anton (1879). Die Frage nach der geschichtlichen Entwickelung des Farbensinnes. Wien: Druck und Verlag von Carl Gerold's Sohn.

MacLaury, Robert E. (2001). Color terms. In M. Haspelmath, E. König, W. Oesterreicher, \& W. Raible (Eds.), Language typology and language universals: An international handbook, Vol. 2 (1227-1251). New York: Walter de Gruyter.

Messerly, John G. (1996). Piaget's conception of evolution: Beyond Darwin and Lamarck. Lanham, MD: Rowman \& Littlefield.

Nachtigal, Gustav (1879/1889). Sahara und Sudan. Berlin: Brockhaus.

Parsons, Sir John Herbert (1924). An Introduction to the study of colour vision (2nd ed.). Cambridge: Cambridge University Press.

Riedl, Rupert (2003). Riedls Kulturgeschichte der Evolutionstheorie: Die Helden, ihre Irrungen und Einsichten. Berlin: Springer-Verlag.

Rivers, William Halse Rivers (1901a). Primitive color vision. Popular Science Monthly, 59, 44-58.

Rivers, William Halse Rivers (1901b). Introduction and vision. In A. C. Haddon (Ed.), Reports on the Cambridge anthropological expedition to the Torres Straits, Vol. 2. London: Cambridge Univesity Press.

Schroeder, Carl (1879). Die Entwicklung des Farbensinnes am menschlichen Auge. Berliner Klinische Wochenschrift, 1 (September), 545-546, 561-564.

Rosch, Eleanor H. (1972). Probabilities, sampling and the ethnographic method: The case of Dani colour names. Man, 7, 448-466.

Spinicci, Paolo (2000). I nomi dei colori: Anton Marty e il dibattito sull'evoluzione del senso cromatico. Le parole della filosofia, Vol. 2 (http://www.apl.it/sf/saggi/martycol.htm).

Stein, von (1879). Einiges Interessantes von den Nubiern. Frankfurter Zeitung, 213.

Stilling, Jakob (1878). Über Farbensinn und Farbenblindheit. Cassel: T. Fischer.

Virchow, Rudolf (1878). In Berlin anwesende Nubier. Zeitschrift für Ethnologie, 10, 333-335.

Virchow, Rudolf (1879). Lappen. Zeitschrift für Ethnologie, 11, 143-148.

Zehender, Carl Wilhelm von (1878). Nachschrift des Herausgebers. Klinische Monatsblätter für Augenheilkunde, 14, 478-483.

Zollinger, Heinrich (1999). Color: A multidisciplinary approach. Zürich: Verlag Helvetica Chimica Acta. 


\section{Appendix}

\section{Museum für Völkerkunde zu Leipzig}

Sie würden der Wissenschaft einen Dienst leisten und die Unterzeichneten zu besonderem Danke verpflichten, wenn Sie durch möglichst sorgfältige Ausfüllung dieses Fragebogens zur Lösung des Problems beitragen wollten: bis zu welchem Grade die Naturvölker die Farben empfinden und durch Benennung unterscheiden wie die Culturvölker. Wir bitten Individuen des gleichen Stammes oder Volkes mit Hilfe der beigefügten Farbenscala zu prüfen, inwiefern diesselben helle, sowie dunkle Farbentöne als unter sich verschieden auffassen oder benennen; (ob sie z. B. Blau, Violett, Schwarz, Grün; oder Roth, Orange, Gelb mit dem nämlichen Werte bezeichnen oder nicht);

ob sie die verschiedenen Farbentöne der hellen sowie tot dunklen Gruppen auch wirklich als durchaus gleiche empfinden; oder ob sie dieselben wohl zu unterscheiden vermögen, obgleich ihnen die speciellen Benennungen für dieselben ja ihrer Muttersprache fehlenn.

Wichtig wäre es bei der Untersuchung, bald die ganze Farbenscala, bald nur eine helle oder dunkle Gruppe, bald nur eins einzelne Farbe dem Befragten zu zeigen, die übrigen einstweilen durch Papier etc. verdeckend. Die Prüfungen wären so lange und an so vielen Individuen vorzunehmen, bis man im Stande ist eine zuverlässige Auskunft am entsprechenden Orte niederzuschreiben.

Wir bitten das beigegebene Schema (welches doppelt gedruckt, für den Fall, dass Gelegenheit gefunden wird, mehr als einen Volksstamm zu prüfen) zu benutzen und zu notiren: in A - den einheimischen Namen des Stammes oder Volkes, den die befragten Individuen angehören ; in die darunter befindlichen Fächer, neben die betreffenden Farben, die für diese erhaltene Bezeichnung;

in B - die geographische Lage des Wohnplatzes der Befragten; in die darunter befindlichen Fächer, neben die FarbenBenennungen, die etwaige Ableitung dieser Worte, ob sie wie Roth, Gelb etc. - für sich allein bestehen, oder ob sie von Naturobjecten genommen sind, wie z. B. Orange;

in C - die allgemeine Bezeichnung für "Farbe" an sich, wenn überhaupt ein solches Wort vorhanden ist; in die Fächer darunter, ob die Benennungen der einzelnen Farben der Muttersprache des Befragten angehören, oder aus einer ändern Sprache entlehnt (und etwa verderbt) vielleicht mit einem Handelsartikel überkommen sind; oder sonstige auf die betreffenden Farben Bezug habende specielle Bemerkungen.

Auf der leeren Rückseite dieses Bogens, wo "Raum gelassen ist für alle sonstigen freundlichen Mittheilungen, bitten wir anzugeben:

1) Ausdrücke für "das Bunte" sowie für die verschiedenen Arten desselben: gestreift, getüpfelt, gefleckt, gesprenkelt, falls solche vorhanden sind; sowie Ausdrücke für: hell, dunkel, leuchtend, glänzend.

2) Anzahl und Geschlecht der Individuen, welche bei den Untersuchungen befragt wurden.

3) Inwiefern der Farbensinn der Befragten durch fremde Cultureinflüsse eine Veränderung erlitten hat, oder erlitten haben konnte.

4) Genaue Adresse dessen, dem wir die Untersuchungen verdanken.
You would render a great service to science. and very much oblige the undersigned, by answering the annexed questions as well as possible, and by kindly lending your assistance in solving the following problem:

do the uncivilized tribes perceive colours and distinguish them by names like civilized nations?

We beg to examine persons of the same tribe or nation by means of the annexed scale of colours, and to ascertain, whether they are able to discern hues belonging either to groups of bright, or to groups of dull colours as being different among themselves, and to give them separate names (whether they apply for example to blue, violet, black, green, or to red, orange, yellow, the same word or not);

whether they perceive the different hues either of the bright or of the dull groups of colours in reality as identi$\mathrm{cal}$ or whether they distinguish them perfectly, although they have no special names for them in their native tongue.

In order to obtain unimpeachable results it would be advisable to show them alternately the whole scale of colours, then only a bright or dull group of them, and again but one single colour in question, the others being covered with a piece of paper etc.

These examinations should be repeated as often, and in regard to as many individuals, as would make it possible finally to write down a reliable answer in the corresponding square,

We beg to make use of the annexed diagram (which is arranged doubly in case more than one tribe) should be examined, and to write

in A- the native name of the tribe or nation, to which the persons asked belong; in the squares below, corresponding to the colours, the names received for them;

in $\mathrm{B}$ - the geographical situation of the home of those asked; in the squares beneath, alongside the names of the colours, the derivations of these words, whether they are special names - as red, yellow etc. - or whether they have been taken from objects of nature, as for instance orange;

in $\mathrm{C}$ - the general name for "colour" itself, if any such word exist; in the squares beneath, please to state, whether the names of the colours belong to the native tongue of those asked, or have been derived from any other language (and perhaps corrupted) perchance with some article of trade; - or any other special notes in regard to the respective co-lours.

On the fourth page of this sheet, where room has been left for any other communications you may have the kindness to impart, we beg to add:

1) the native expressions for "coloured" as well as for different kind of patterns: striped, dotted, spotted, speckled, in case any such exist, and also any words signifying: light, dark, bright, shining;

2) the number and sex of the persons who where examined;

3) whether the ability in distinguishing colours, has been or could have been improved by foreign influences;

4) the full address of the writer, to whom we are indebted for the answers to our inquiries. 
PART II

\section{Color Cognition}

The studies in this part emphasize cognition, although some include other levels of modeling, such as the sensorial 



\title{
Categories of desaturated-complex color Sensorial, perceptual, and cognitive models
}

\author{
Robert E. MacLaury $\dagger$ \\ University of Pennsylvania, USA
}

Over sixty percent of investigated languages name a color category by which speakers combine desaturation and complexity. The category engulfs the grey core of the Munsell color solid to emerge on the least saturated but blended areas of the solid's surface, commonly brown, lavender, beige, and pale. But people vary in their construction of the category, emphasizing either its desaturation or its complexity. Many reinforce its composition with recognition of dark, light, or blue. A few who favor complexity include very complex colors that are also highly saturated, such as purple, while they still recognize desaturation and include grey as well. The category shows distinct patterns between languages, whence it undergoes various trajectories of development. Many such categories are of basic status, in spite of their variability. Yet individuals elect not to use them more often than they disregard their basic categories of hue or brightness; apprehending color in terms of its desaturation and complexity is an option that crosscuts the alternatives while not precluding them. Statistical differences in the frequency and constitution of the category appear between New World and Old World languages.

Interlocking models of sensation, perception, and cognition allow clearer understanding of this widely recurrent behavior. Their application here provides opportunity to assess the merits of each. Issues regarding the perceptual model underscore one case in which vision researchers and color ethnographers might pool resources to improve knowledge.

\section{Prior research}

Categories of desaturated-complex color have a fifty-year history in academic research. As will be developed, philologists recognized them as problematic and advanced ingenious accounts of their semiotics and etymologies (e.g., Malkiel 1953). However, it was only after Berlin and Kay (1969) published their universalist observations of basic color terms that under their instigation Janet Dougherty and I independently and simultaneously chanced on such categories by ethnographic elicitation with color chips, she in the Polynesian outlier West Futunese of the New Hebrides (1975) and I in Ayoquesco Zapotec of Oaxaca, Mexico (1975). Dougherty used genuine Munsell chips in accord with Berlin and Kay's 
acetate mapping method (cf. Lenneberg \& Roberts 1956). She found that Futunese khósi denoted the "appearance and associated feeling of skin after swimming in salt water or being smeared with ashes" but also designated "desaturation, greys, browns, pastels" (p. 97, Table 3.1) and "hues of medium saturation in the blues and purples" (118). Following Chad McDaniel's insight (personal communication, Anthropology Seminar, UC-Berkeley, Fall 1974), she adds "Consideration of the actual color solid, however, suggests that khósi denotes the internally contiguous core ... The surface sections of such a desaturated core appear at diverse locations on the Berlin and Kay array [of 320 surface chips] which correspond to the discontinuous regions in which khósi is mapped" (118-121, Figs. 3.7-9; bracketed words added). Her figures show foci throughout grey and all those surface regions, especially those below / 6 Munsell chroma, with greatest concentration at Munsell $\mathrm{F} \varnothing$ at middle lightness grey, 13 in light blue and light purple, 6 in darker blue and purple. She further notes that khósi fulfills the criteria of a basic color term (122), speakers vary in its composition (123); not all informants who know of khósi use it to name chips (124); children apply it as do adults without evidence of change across generations (123); and khósi coexists with frequent use of práon 'brown' and occasional use of kréi 'grey' (173174). Dougherty queries whether khósi primarily names skin condition or color, and notes its absence in Bill Gunn's Futunese dictionary (ca. 1915, no source).

I reported Ayoquesco Zapotec yà ač as 'dark grey' (opposed to tê 'light grey, pale') and as color de coyuche (Sp., color of [light brown] organic cotton) in my Master's thesis (1970: 17, 177). But in 1972, after I investigated with various adaptations of Nick Hale's paper copy of Munsell chips from Berlin and Kay (1969), I learned that yà?ač names grey, brown, lavender, beige, pale, and sometimes purple or chartreuse (MacLaury 1973). Its variation is identical to that of khósi, even its strong association with natural events. Those include a tom turkey puffing its feathers, referred to by the reflexive verb alboratorse in Spanish: Ayoquescans translated yà?ač in the participle as color alborotado. Like other Ayoquesco Zapotec color terms that occur in doublets (yá?as/nagas 'black', nya?aty/nàgăty 'white'), yà?ač is accompanied by nagàc 'color caluda'. Caluda is corn dough mixed with mineral lime (Sp. cal) for making tortillas, which is chartreuse in color. The concept of desaturated and complex color, then, is associated with mixtures, for example, exploded like turkey feathers, or compacted as dough with lime. The derivative verb root -yà? ač 'scatter' refers prototypically to scattering detached feathers, as would a feline that catches a bird ( $y \grave{a}{ }^{2} a c ̌$ is cognate with terms for 'white, yellow, and/or red' in other Zapotec languages and related linguistic families, and, thus, likely originates as a color adjective [MacLaury 1986a:app. 2, p. 9; 1997a:216]). I was also told that yà?ač means 'turkey-egg color', which I found on very close examination to consist of myriad flyspecks of black, white, red, and grey, giving a brownish grey impression at arm's length. And like khósi, yà?ač is known by all but used by fewer than all to name color chips. It, too, is accompanied by loanwords for brown and grey, kafé and plomado, mainly kafé (Having been unable to obtain Berlin and Kay's book, then sold out, I neglected to elicit foci. Three of Hale's prints were supplied to me by Berlin).

Malkiel's phylological encounter with prieto, preto in Iberian languages bears parallels to our experiences with khósi and yà?ač: 
Para Covarrubias, apretar 'restringir' y prieto en el sentido de 'apretado' se remontan indudablemente a premō, -ere. En cuanto a prieto 'oscuro, negro', cuyo uso y difusión define con especial acierto, confiesa con honradez: "Es vocablo de los antiguos castellanos, y derechamente yo no le hallo etimología que me quadre". Su coetáneo F. Rosal comparte la primera opinión; en cuanto a la génesis de prieto como nombre de color, ofrece al lector un alternativa: por una parte reconoce el origen idéntico de los dos empleos de prieto, porque ciertos objetos, como el polvo o la niebla, cuanto más densos (es decir, apretados), más negros parecen; por la otra, ofrece la derivación prieto 'negro' ... De estas dos suposiciones, sea dicho en defensa del benemérito humanista, juzgo más verosímil la primera (1953:6-7).

In sum, the dark color term (prieto) is associated with squeezing together (apretar), as with dense dust or haze: the more compacted, the darker; the verb might have chronologically preceded the adjective in word history, although the etymology is difficult. As we shall see in the ethnographic examples, certain desaturated, complex categories are also dark. Futunese, Zapotec, and various Iberian languages link the concept with mixture, Zapotec and the latter with compacting. Both desaturation and complexity emerge in the folk definitions.

In my diachronic study of basic color-term lexicons (MacLaury 1975:2-6, Tables 1-2), I include a section on our then-recent recognition of desaturated terms. Besides introducing their universal characteristics with a description like those of khósi and yà?ač, I cite probable reports of desaturation terms, chart the coexistence of some with terms naming brown and grey, and project that publications may fail to fully describe desaturation terms, erroneously passing them off as terms for grey or brown in absence of a pointed identification of them in the literature. The reports include Tovarese brün 'brown, light blue, grey' (Heinrich 1974:4, on a dialect of German in Venezuela), Castilian pardo 'grey, brown, dusky, cloudy' (Peers et al. 1960:604), and Ayutla Mixtec kúší 'a mixed colour, something like the colour of ashes' (Merrifield 1971:266). Later we found Australian Walbiri yuljuduyuljudu 'grey, light blue, light purple' [cf. yuljudu 'smoke'; Hale 1975:298] (MacLaury \& Greenfeld 1984:33). Tovarese brün and Ayutla Mixtec kúší coexist with brown and grey terms, respectively: braun and gri:s, yá? $\bar{a}$ and šà $2 \bar{a}$. The relations suggest that desaturation-complexity categories differ from those of brown or grey.

Probably the desaturation-complexity category has been inaccurately described in many a field report. In a section on Brown and Grey (1975:1-2), I show that 20 Numic languages of California in the C. Wright Merriam Files (data compiled by Berlin 1975) are reported to name grey, brown, or grey=brown at stages prior to Stage V of Berlin and Kay's implicational evolutionary order, and 6 name grey at Stage V - all early or 'premature' by their prediction - which suggests an alternative more consistent with that hypothesis: some or all of these reportedly grey or brown terms might have actually named a desaturation category when Merriam collected them. (Nichols 1974:269 lists such data as cognates for reconstruction to Proto-Numic. " $\mathrm{PN}{ }^{\star} k u$ and ${ }^{\star} a i$ occur throughout Numic, often on the same roots, with the resultant form containing either a color implication or a color specification, e.g. “'blue', 'grey', 'dull', 'light-colored', etc.”; he distills these data [1980:161], “ $i d w i$ 'd is from PN ${ }^{\star} a i-k$ 'wi- which gives several words for GRAY and 'smoky' in Central 
Numic, but is BROWN in some Northern Paiute areas and PURPLE in others" [related languages].) It is of descriptive and theoretical value that desaturated-complex categories be understood, including their development within color-category systems, and that the findings be commonly known.

Two examples of basic color terms from Berlin and Kay (1969:74, 89) emphasize that point, one in Apache and the other in Masai. Keith Basso (in a letter) reported Western Apache libaha (his transcription) with the gloss of 'brown', which prompted Berlin and Kay to comment "Apache ... constitutes an exception to the partial ordering ... in that libaha 'brown' appears before dukliž 'green' has segmented into green and blue." Specimens collected with Munsell chips from that dialect by Philip Greenfeld in 1979 show libaah (different transcription) to be a classic term of desaturation and complexity (Greenfeld 1986, Fig. 5; MacLaury 1997a:56, Fig. 2.18; cf. Perry 1972:25, li-baah 'dull color, grey, brown'), which would have enabled Berlin and Kay to regard the issue differently. They take their Masai basic color terms from (Hinde 1901), observing "The term for 'blue' in Masai is complex and might therefore be analyzable. If so, Masai should be treated as Stage IV rather than Stage V.' Hinde's entry for 'grey' is mbusth (p. 57) and for 'blue' mbusth ollonyori (49), grey + modifier (ol-o-nyori \{masculine singular article-relative pronounbe green, the-which-(is)green, 'the green one', said of beads, a snake-type, and the full moon] [Mol 1995:26-27, 95-98, 1996:286, 311]). A second head lexeme, gwarigoi, shares the 'grey' gloss but not the blue gloss with mbusth. It looks like gwarigoi names only 'grey' while mbusth names a desaturated-complex category that includes blue, where mbusth is modified. (Hinde might have distinctly sought a 'blue' term and featured the modifier to mark it. But Mol [1995:58-59] under "proper colours" glosses \{mbusth $>$ \} pus 'lightgrey, bluish,' \{gwarigoi > barrikoi and wuarikoi 'brown.' And he glosses ten other words as shades of grey or brown, some, if not all, of which apply to cattle. Masai may harbor more than one desaturated-complexity term.). In any case, what we now know of the T-C category ( $\mathrm{T}$ - desaturation, $\mathrm{C}$ - complexity) resolves Apache and Masai color terms as Stage $\mathrm{IV}+\mathrm{T}$, explains why Masai mbusth (pus) names grey and blue, and clarifies why Hinde glosses two terms as 'grey' with only one of them limited to that - although ninety-plus years later Mol reports apparent doublet forms of the term to mean 'brown.'

In 1982, Richard Sun (p.c.) cautioned that the T-C category might not encompass all of the color-solid core, especially in areas where certain warm colors become greyer. We were unaware that Landar, Ervin, and Horowitz (1960) had determined precisely Sun's insight with measurements of Navaho basic color terms. Navaho and Apache are closely related, and Navaho lebá is cognate with Apache libaah. Landar, Ervin, and Horowitz (1960:371-376) find that lebá occurs in 20\% of 144 responses to color chips from four informants, which suggests that lebá is basic. They gloss these responses as 'gray' (consistent with other less-than-complete recordings, e.g., The Franciscan Fathers [1910:71] gloss Navaho labá" as 'gray, roan' and the same root in dinlbá" as "stone gray, white with a tinge of black"). But Landar et al. also graph responses over a gamut of 29 Farnsworth-Munsell chips (1960:380, Fig. 1), showing highest use of lebá in bluish purple: "lebá, though commonly translated as 'it is grey', sports strong connotations of lavender and might be used as a translation equivalent to purple” (382). Thus, lebá names a desaturated-complex cat- 
egory of at least some extent. The Farnsworth-Munsell hues are uniformly of Munsell brightness value $5 /$ and chroma /2, middle lightness and low saturation that show subdued color (Farnsworth 1943:568, Fig. 1). Uses of lebá (1) pertain across all colors of this gamut, except red, and (2) crosscut the uses of all other terms. No other Navaho term does (1) or (2). In the same figure, Landar et al. (1960) diagram American English (AE) speakers' responses to the same stimuli, which show that all color terms pertain to short segments of the gamut, none nearly so widely as lebá. AE does not have such a category (Fig. 3 herein; MacLaury 1997a: Fig. 1.3). Collier (1966:422-424, Tables 1-3) diagrams Mexican Spanish and Mayan Tzotzil color-term responses to the same Farnsworth-Munsell stimuli, languages which, like AE, do not name a desaturated-complex category (cf. MacLaury 1986: App. 1, p. 2, Fig. 1, Mex. Sp.; 1991a:51, Figs. 9-11, Tzotzil). The Mexican Spanish and Tzotzil ranges, too, are confined to local segments, even though each language segments the stimuli differently. Navaho lebá is the only term for a desaturated-complex category so measured, and its unique qualities are blatant by contrast. Its crosscutting range plus its breadth of application suggest that it names a unique kind of color category which differs in behavior from those of hue or of a local blend.

MacLaury and Greenfeld (1984) analyze desaturation terminology in 50 languages from the Mesoamerican Color Survey (MCS), featuring Apache from which Greenfeld collected 31 specimens with Munsell chips. We project that the desaturation category is motivated by the difficulty of naming a residual sense of vague color caused by a perceived combination of desaturation and complexity. Yet by compiling Greenfeld's mappings, we show that Apache positively structures the desaturated-complex category in graded membership of grey $>$ brown $>$ lavender $>$ beige $>$ pale $>$ purple, which favors desaturation (e.g., grey) more than complexity (e.g., brown). Further, we claim that most of the other 49 languages also structure the desaturated-complex category in this order, and we present quantitative evidence from (a) 177 lexical idiolects and (b) naming and focus preferences across the 50 languages. We find that lexical idiolects constitute four types of combinations (of T desaturation term, Bn brown term, and Gy grey term): Type I ( $\mathrm{T}$ alone), Type II ( $\mathrm{T}$ $+\mathrm{Bn})$, Type III $(\mathrm{T}+\mathrm{Bn}+\mathrm{Gy})$, Type IV $(\mathrm{T}+\mathrm{Gy})$. Finally, we attempt to develop a perceptual model of proposed category structure. Because we deemed the model problematic, most of our conclusions have remained unpublished - save those based only on Apache data (Greenfeld 1986). But our compilation of lexical idiolects is impeccable. Segments from our Table I follow in the present Table 1. Column headings are: Language (plus location), Type (I, II, III, IV), Id (Idiolects a., b., c. etc.), Desaturation (terms that name the desaturated-complex category), Brown (terms that name only brown), Grey (terms that name only grey), Ref (reference number of each idiolect), In (number individuals manifesting each idiolect), It (number of individuals interviewed in the language).

In Guarajío, 25 individuals were interviewed, 10 of whom named the desaturatedcomplex category with one of three terms (wetapaeme, sawčame, or morisawame). Type I comprises idiolects a. and b., which each are used by 3 individuals and identified by a different term (wetapaeme or sawčame). Type II comprises idiolects c., d. and e. which each is used by one individual and differs by its combination of terms (wetapaeme / čokora vs moriswame / sawčame vs morisawame / čokora). Type III comprises idiolect f., used 
Table 1. Types of idiolects that name desaturation-complexity

\begin{tabular}{|c|c|c|c|c|c|c|c|c|}
\hline Language & Type & Id & Desaturation & Brown & Grey & Ref & In & It \\
\hline \multirow{6}{*}{$\begin{array}{l}\text { 10. Guarajío, } \\
\text { Los } \\
\text { Alamos, } \\
\text { Sonora, } \\
\text { Mexico }\end{array}$} & I & a. & wetapaeme & $\varnothing$ & $\varnothing$ & 25 & 3 & \multirow[t]{6}{*}{25} \\
\hline & “ & b. & sawčame & $\varnothing$ & $\varnothing$ & 26 & 3 & \\
\hline & II & c. & wetapaeme & čokora & $\varnothing$ & 27 & 1 & \\
\hline & “ & d. & moriswame & sawčame & $\varnothing$ & 28 & 1 & \\
\hline & “ & e. & morisawame & čokora & $\varnothing$ & 29 & 1 & \\
\hline & III & f. & wetapaeme & sawčame & wakosa & 30 & 1 & \\
\hline \multirow{10}{*}{$\begin{array}{l}\text { 22. Mixtec, } \\
\text { Diuxí, } \\
\text { Oaxaca, } \\
\text { Mexico }\end{array}$} & I & a. & nšàrá & $\varnothing$ & $\varnothing$ & 64 & 1 & \multirow[t]{10}{*}{16} \\
\hline & “ & b. & čàá & $\varnothing$ & $\varnothing$ & 65 & 1 & \\
\hline & & & nšàrá & $\varnothing$ & $\varnothing$ & & & \\
\hline & “ & c. & nšàrá & & & 66 & 1 & \\
\hline & & & nvázù & & & & & \\
\hline & & & kaféê & $\varnothing$ & $\varnothing$ & & & \\
\hline & II & d. & nšàrá & kafeêê & $\varnothing$ & 67 & 1 & \\
\hline & “ & e. & čàá & kaféê & $\varnothing$ & 68 & 1 & \\
\hline & III & f. & nšàrá & kaféê & čàá & 69 & 2 & \\
\hline & VI & g. & kaféê & $\varnothing$ & gris & 70 & 1 & \\
\hline
\end{tabular}

by one individual who names desaturated-complex, brown, and grey categories with one term each (wetapaeme / sawčame / wakosa). Idiolect b. names the desaturated-complex category sawčame, while idiolects $\mathrm{d}$. and $\mathrm{f}$. name brown sawčame. The Mixtec data show how the intricacies can proliferate. Here 6 idiolects are named by 7 individuals. Idiolect b. names the desaturated-complex terms with two terms (čàa and nšà áa), idiolect c. with three (nšà 'á, nvázù, and kaféê). All four types are used with the same terms naming different kinds of categories among the four. In some idiolects of other languages each of the three categories are named with two or more terms. The original Table 1 of all data from 50 languages is an intricate compilation indeed.

From that, we extracted these statistics on the idiolect types:

Table 2. Statistics relating to lexical idiolect types

\begin{tabular}{llllll}
\hline Types & I & II & III & IV & Totals \\
\hline Number of Idiolects & 70 & 76 & 21 & 7 & 174 \\
Number of Individuals & 144 & 100 & 23 & 8 & 275 \\
Ratio Individuals:Idiolects & 2.058 & 1.358 & 1.095 & 1.143 & \\
\hline
\end{tabular}

Individuals of the sample use the types of idiolects with decreasing frequency in the direction of Type I > Type II > Type III > Type IV. Ratios of idiolects to individuals reduce in the same direction. The rarer the type, the greater its idiolectical variability.

We offer that Type I is most common because it is the simplest. Type II is more common than Types III and IV because most desaturation-complexity categories are organized as we find in Apache, with grey of higher membership than brown, that is, with desaturation emphasized more than complexity. We assume the common maximal membership in 
grey will encourage alternative naming of brown before grey - complexity before desaturation - which foreordains higher correspondence of the desaturated-complex term with terms for only brown than with terms for only grey. Type IV may occur exclusively among desaturated-complex categories whose maximal membership is brown. (The exclusive naming of lesser membership is substantiated in other kinds of color categories, such as the "cool" category of green-with-blue wherein the prevalent focus in green throughout Mesoamerica fosters secondary naming with head lexemes only of blue [Burgess, Kempton, \& MacLaury 1983; MacLaury 1997a:Ch. 8]). Greater variability among rarer types perhaps is encouraged by their reduced opportunity to influence each other.

We compared naming preferences between major and minor desaturated-complex terms, and we also so compared focus preferences. The major term is that which an individual uses most and a minor term is another term used less by the same individual to name the category, for example, as among the options seen in idiolects 65 and 66 of Table 1 (Mol's Masai glosses suggest a major and two minor terms, pus versus barrikoi and wuarikoi). We prepared the comparison by plotting on the Munsell array the outlines of what we saw as grey, brown, lavender, beige, and pale, which, respectively, include 8, 37, 28, 23 , and 51 chips, which we called Divisors. Across our sample of 275 desaturated-complex categories, we counted the number of times major terms were applied to the chips of each color, and we divided the sum by the divisor for that color, which produced its Naming Score. We did the same for minor terms. These Naming Scores appear in Table 3. For major terms, they are highest in grey, next highest in brown, and lower in the other colors. They generally parallel the descending numbers of major-term foci, which are also included in the table. The data in Table 3 roughly match the descending Numbers of Individuals who named Types I-IV as shown in Table 2. The descending orders match our proposed gradation of membership values for the desaturated-complex category. Naming scores and numbers of foci for minor terms show complementary orders that are commensurable with numbers of alternative terms among the types, which are highest in brown, next highest in grey, and lower in other colors. We surmise that the minor terms name an opposite slant on the category, from which it is organized to favor brown (In hue categories, complementation of coextensive terms has been analyzed in accord with vantage theory, wherein it is called "the dominant-recessive pattern of coextension" [MacLaury 1997a]). As will be developed, the major and minor terms do not objectively name the category but depict different emphases within it.

Table 3. Statistics from naming

\begin{tabular}{llllll}
\hline Colors & Gray & Brown & Lavender & Beige & Pale \\
\hline Divisors & 8 & 37 & 28 & 23 & 51 \\
Major Terms & & & & & \\
Naming Scores & 103 & 62 & 31 & 22 & 22 \\
Foci & 111 & 74 & 17 & 11 & 22 \\
Minor Terms & & 32 & 8 & 9 & 3 \\
Naming Scores & 15 & 52 & 5 & 11 & 1 \\
Foci & 21 & & & & \\
\hline
\end{tabular}


Although the statistics from naming ranges (Table 3) closely correspond to the statistics relating to lexical idiolect types (Table 2), we have not rushed our findings into print. Although we were confident of our assignment of chips to grey, brown, lavender, beige, and pale - that is, the adequacy of our divisors - we lacked an objective standard. We thought anyone could disbelieve our judgment, even though it would require blatant distortion to produce such robust results invalidly.

Yet we faced the issues of modeling the desaturation-complexity category. Why is grey usually favored by major terms? Why are there exceptions, however scarce? Why do the exceptions as well as the secondary countertrends - those among minor terms and terms for only brown or grey - favor brown? Why do the countertrends on the whole not totally reverse the priorities to favor one of the other colors: pale, beige, or lavender?

While retaining our plots of the five colors over the Munsell chips, we devised a system of dual scoring that consisted of desaturation scores or "T-scores" and complexity scores or "C-scores" to constitute the model shown in Table 4.

Table 4. Membership values of the desaturated-complex category.

\begin{tabular}{llllll}
\hline Colors & T-Scores & C-Scores & Sums & Membership Values \\
\hline Grey & 7 & 1 & 8 & 8 \\
Pale & 3 & 1 & 4 & 4 \\
Lavender & 3 & 3 & 6 & 6 \\
Beige & 2 & 3 & 5 & 5 \\
Brown & 1 & 6 & 7 & & 7 \\
Summary & Grey $8>$ & Brown 7 $>$ & Lavender 6 $>$ & Beige 5 $>$ & Pale 4 \\
\hline
\end{tabular}

T-scores are inverses of Munsell chromas (saturations) 0-6 that compose most of the desaturated-complex category: Munsell $/ 0=$ T-score $7, / 1-2=6-5, / 3-4=4-3, / 5-6=2$ 1. Thus, grey of $/ 0$ receives $\mathrm{T}$-score $7 ; / 6$ at center of brown (/2-12) warrants $\mathrm{T}$-score 1 ; /4 at center of lavender begets T-score 3;/5 at center of beige (/2-6) begets T-score 2; /4 at center of pale (/2-6) begets T-score 3. C-scores sum mergers of pure sensations that compose a color: grey 1 (white+black, 1 relation), brown 6 (yellow+black+red+green, 6 relations), lavender 3 (white+blue + red, 3 relations), beige 3 (white+yellow + red), pale 1 (white+any hue with nonprevalent overlaps of 2 hues, mostly 1 relation). To enhance the differences between the summed values of grey $8>$ brown $7>$ lavender $6>$ beige $5>$ pale 4 , we invoked the notion of nonlineal progression in which an arithmetic difference between two higher gradations has exponentially greater effect than the equivalent difference between lower gradations, for example, as with degrees on the seismologic Richter scale or with subjective valuation of increasing loudness between decibels (Eugene Hammel, p.c). Thus, the difference between grey 8 and brown 7 would be subjectively much greater than the difference between lavender 6 and beige 5; 8 grey would be far and away the best example of the desaturated-complex category while 7 brown would be the next best example by a sizable increment over the other colors, which, in turn, would differ in value of membership negligibly among themselves. Nonlinear progression seemed to 
explain the distinctively stronger correspondences at the top of our scale, as shown in Tables 2 and 3. It also might explain why major and minor terms favor, respectively, grey and brown, rather than full reverses of all category members, and it might likewise explain the favoritism of brown over grey when single colors are separately named within the range of major desaturated-complex categories. Further, the notion is consistent with the indistinctive, mixed statistics among lavender, beige, and pale.

We saw problems with our model. First, T-scores and C-scores are derived from our personal plots of the five colors. Second, nothing explains why a T-unit and a C-unit should be of equivalent subjective strength. Third, no psychophysical literature pointedly measured perceptual complexities of grey, brown, purple, or other blends (Karen De Valois, p.c. 1984); rather, we inferred them from naming patterns in then-unpublished ethnographic data (later publication cited below). Fourth, our predictions and our data are not independent. Fifth, the encoding of different emphases on one category by major and minor terms requires a cognitive model, whatever the merits of our speculation about perception; the prevalence of Type II over Type IV calls for the same. So we shelved our draft for a day when we might know more.

The literature on perceived chromatic complexity deserves a sketch. At a point, the scientific world recognized the elemental, simplex compositions of white, black, red, yellow, green, and blue (as summarized by Boring 1942). Sternheim and Boynton (1966) showed that while red and yellow are irreducible, subjects compose orange as their combination the first quantitative demonstration of nonelemental complexity. Bartleson (1976:188189) asserts that brown is a three-dimensional color and distinct from a one-dimensional hue perception, as "orange might be adequately described by hue alone." In addition to hue [optimally 5.0 YR], brown consists of low lightness [optimally 1-2/] and low chroma [optimally /6]. Fuld, Werner, and Wooten (1983) initially propose that brown is elemental after finding that, unlike with orange, subjects fail to recognize its pure constituents, neither black nor yellow. But later they express misgivings in a conference paper, cited in Quinn, Wooten, and Ludman (1985: 198): “This last finding has, however, been called into question by a recent report indicating that brown is not an elemental color, although it may differ, perhaps in terms of its susceptibility to linguistic response biases, from other nonelemental colors (Rosano, Wooten, and Quinn 1984)," published as Quinn, Rosano, and Wooten (1988). Improving their methods, they find that brown is an intersection of yellow and black sensations. Indeed, English speakers confine their categories and semantics of yellow and black to exceptionally small ranges in relation to those named by languages explored by our ethnographic color surveys (e.g., Fig. 3 herein, brown = brown in English; MacLaury 1997a: 12, Fig. 1.3). Fuld, Wooten, and Whalen (1981) find purple to be nonelemental, composed of red and blue on a middle level of lightness. Quinn, Wooten, and Ludman (1985) find that grey is nonelemental, being composed of perceived black and white. Overall, these researchers distinguish elemental colors from perceptual blends. They do not exhaustively explore perceptual complexities of the blends, even though some of their results impinge on that issue. For example, Quinn, Rosano and Wooten (1988) find lesser perception of red in brown, and they cite others who regard brown to be par- 
tially composed of red (e.g., Webster's Third New International Dictionary of the English Language; Hering 1964). Neither they nor others consider the contribution of green to brown. Yet their conclusions, as far as they pertain to perceptual complexity, are consistent with the linguistic facts upon which Greenfeld and I based our projections of complexity. We specified the chromatic input of brown as yellow+black+red+green [6 relations] because speakers of different languages name brown with either a word focused in yellow or a word focused in black or, less commonly, with a word focused in red or a word focused in green (e.g., MacLaury 1997a: 100-101, Figs. 4.5 and 4.6, brown $=$ black or yellow in Tzeltal; 224, Fig. 8.1, brown $=$ red in Nahuatl; 175, Fig. 6.35, brown $=$ green in Mixtec). Some idiolects name the red and green halves of brown separately while focusing the names together in middle brown (e.g., Fig. 3b herein). Quinn, Wooten and Ludman's discussion of grey squares with our C-score of 1 for grey, while their composition of purple is simpler than our C-score of 3 for lavender, wherein we include the contribution of white. In ethnographic data, purple is named with words focused in either blue or red or white or black (respectively: p. 355, Fig. 11.14d; 78 and 267, Figs. 3.1 and 9.1; 261, Fig. 8.37; 60, Fig. 2.23), among various other means of naming. Even in grey, we and others find occasional applications of green-focused terms (e.g., pp. 100-103, Figs. 4.5-9; p. 493). We sidelined our perceptual model in 1984, but we might wait until the end of our lives for vision researchers to thoroughly quantify complexity. They and color ethnographers control different fragments of an unassembled picture and, thus, must talk to each other more than they have.

Greenfeld (1986) published the compilation of his mappings and foci of Apache libaah, comparing them to Dougherty's (1975) compilations from Futunese khósi, and at last making easily available the experimentally rendered descriptions of this kind of color category. He proves that Apaches think of it as structured rather than as an amorphous residue. I diagram four of his individual Apache results (MacLaury 1997a:56, Fig. 2.18).

MacLaury (1985) refined the remains of our project (an intended dissertation chapter that MacLaury [1986a] excludes). I came to grips with the depth and breadth of this multifarious genre of cognition, especially the ways that speakers of different languages vary it. The data to be displayed and discussed herein extend observations first outlined in that unpublished chapter. Table 5 lists them and indexes them to figures.

\section{Issues Revisited}

MacLaury and Greenfeld (1984) and MacLaury (1985) set forth ten facets and implications of the desaturated-complex category, which are up-dated here.

\section{Qualities versus Hues}

Categorization of color qualities, such as desaturation and complexity, differs from categorizing pure colors and their local intersections. The separate objectives constitute different types of color category. As a type, the desaturated-complex category is akin to categories of saturated-complex color, which are ethnographically attested to include such combi- 
Table 5. Qualities of the desaturated-complex category indexed to examples

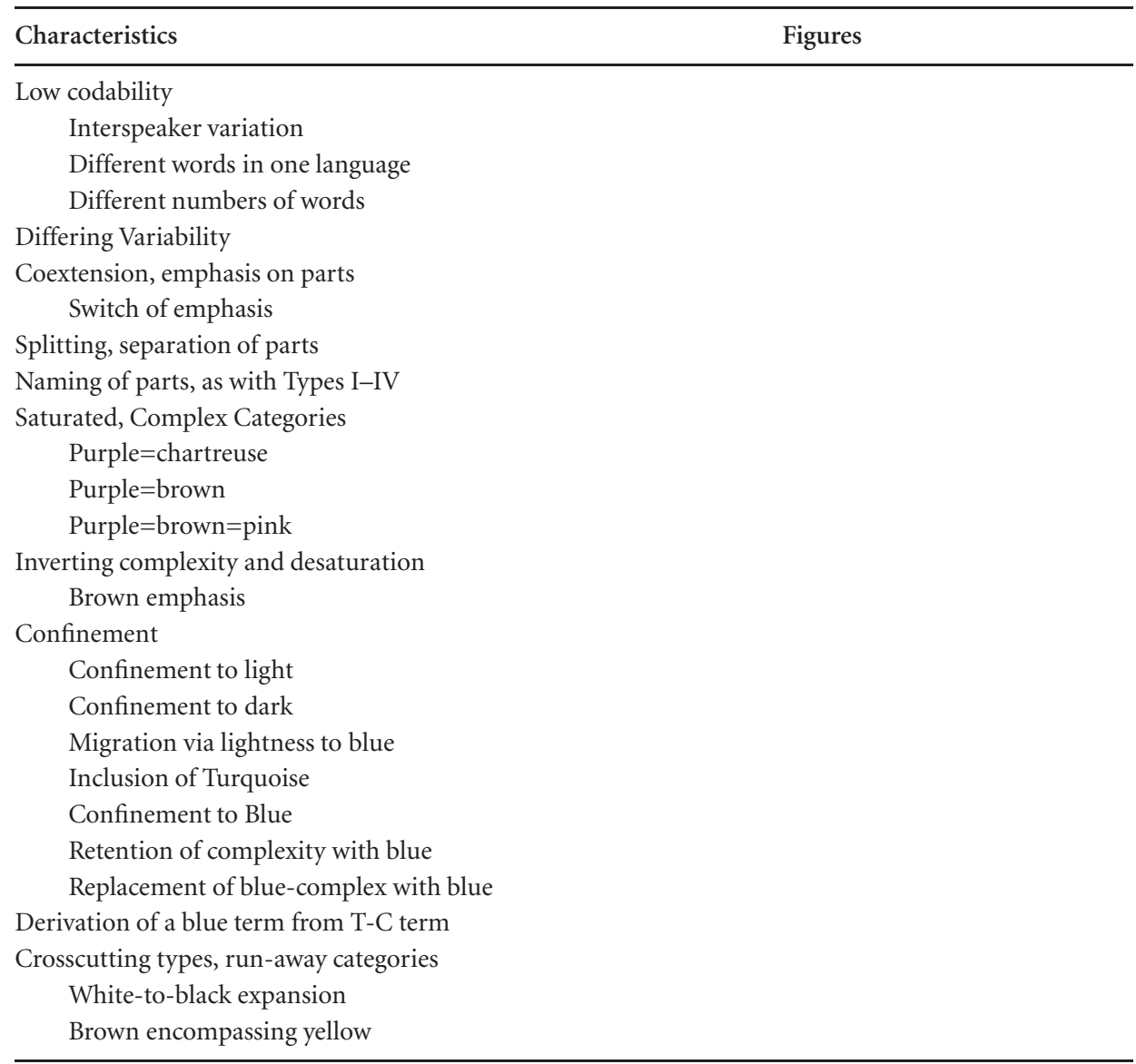

nations as brown-purple-pink or purple-chartreuse. MacLaury (1975) confounded the latter sort with desaturation-complex categories, listing among them Chayuco Mixtec ndyāá 'brown-purple' (Merrifield 1971:266-267), and Tikopia fero 'brown, brown-yellow, pink, purple' (Monberg 1970:352). We may add, "up to the end of the 18th century in the German-speaking world, braun signified both 'brown' and 'violet'... Braun then covered a wider section of the color field" (Ohmar 1953:133). The category of brown-purple is of special interest, as it appears intermediate to categories of desaturation-complexity and saturation-complexity, brown at /6 sitting on the fence. Among the data discussed herein, saturation-complexity categories are introduced. In Tlapanec in particular, they accompany the desaturated-complex category in dividing the domain.

\section{Types of Perspective Taking}

Color categories afford two kinds of perspective, first when a major term and one or more minor terms each emphasize a different part of one category without entirely excluding its other parts and, second, when separate categories are based on different perceptions that 
inhere in the same colors and while both categories pertain to the colors. Both a hue category and a desaturated-complex category can be named by a major and minor term, and thus they equally exemplify the first kind of perspective taking. The perspectives on one category are related because they apply different priorities to the same perceptions. The second kind, multi-categorical perspectives, is evident in the different bases upon which hue categories and desaturated-complexity categories are constructed - vivid points and their local intersections versus widely intermittent qualities. These kinds may simultaneously pertain to any color among grey, brown, lavender, beige, pale, sometimes purple or blue, or any hue less saturated than /6, except red. The coexisting perspectives are unrelated because their perceptual bases are distinct. The first kind of perspective was ascertained among hue categories (MacLaury 1997a), then merely shown to pertain in the same way to T-C categories. But ascertainment of the second kind has required principled differentiation between categories on separate bases.

\section{A Singular Organizing Principle}

Although color categories are based on distinct recognitions - local hue versus qualities that pervade the domain - the different types of category are organized by a singular cognitive principle. The basis of a category does not affect its organizing principle. Vantage theory models that principle, as will be specified.

\section{Basic Color Category}

Berlin and Kay (1969) defined a basic color term on criteria partial to those that name categories of hue, whether composite, primary, or derived (Kay \& McDaniel 1978). But terms that name qualities of color apart from hue will behave differently. Their status as basic or nonbasic must be assessed on a standard consistent with their distinctive properties. The crosscutting semantics of the desaturated-complex term is critical to its identity as a basic color term. Because it crosscuts, individual speakers may opt to seldom use it or not to use it, as Dougherty (1975) and I (MacLaury 1975) found with khósi and yà?ač: more speakers neglected these terms than other major color terms. However, the option provided by its special nature will be predictably exercised. Unlike other types of color terms, a desaturated-complex term cannot be ruled nonbasic or "parabasic" only because fewer speakers volunteer it while naming chips.

\section{Codability}

Codability is the assumed capacity of a color term to be used repeatedly with the same meaning (Brown \& Lenneberg 1954). The capacity is assumed on the basis of observed frequency and consistency of color-chip naming between subjects and between trials of the same subject. Brown and Lenneberg conceived the diagnostic with only hue-based terms in mind. But a basic desaturation-complexity category will encourage frequency and consistency of naming below that exhibited by hue-based terms, even though its inherent capacity is equal. This could be revealed by selecting subjects from a language that employs such a category and by restricting them to a choice between either its name or a null response during chip naming experiments. Its crosscutting character would other- 
wise create the appearance of low codabilitiy, as on criterion of consistency. Such would certainly occur on a criterion of frequency in response to color chips of predominantly high saturation, as with the 330 ethnographic Munsell chips.

\section{Brightness}

So called brightness categories encompass all the spectral hues at high saturation (MacLaury 1992, 1997a, 2002, 2005), similar to the way that the desaturation-complex category crosscuts hue categories at low saturation. But "brightness" is only a convenient rubric, for this kind of color category has never been analyzed psychophysically (MacLaury 2005). It remains to be determined what relation(s) putative brightness categories bear to categories based on hue and categories based on other color qualities: desaturation-complexity and saturation-complexity.

\section{Perceptual Determination}

As we claim of the desaturated-complex category, grey is usually a better member than brown - desaturation over complexity. However, some languages favor brown over grey, even among their major terms for the category, as is suggested by data from Mazatec, Tlapanec, Seri, Huastec, and Guajibo. Perception in itself does not always determine categorical values of membership, even though it is influential

\section{Simplification Hypothesis}

Addition to the desaturated-complex category of minor terms with contrasting emphases, alternative terms meaning only 'brown' or 'grey', curtailing emphasis on dark, light, or blue, retraction of the range from some of its parts, renaming of those parts, or splitting of the range, might constitute ways of facilitating cognitive management of the extensive desaturated-complex experience. Simplification of cognition in local regions might be the propelling motive of all these trajectories of development, even though their totality complicates color naming when beheld in overview. For example, it might be easier to comprehend desaturation-complexity if regarded mainly in terms of its lightness, even though the favoring of lightness requires a further take on the category. Simplification appears to motivate change in exceptionally expansive categories of experiences unrelated to color. For example, Zapotec languages prehistorically acknowledged a vast category of entities that are related to each other by characteristic movement, diminution, and importance to people, as is suggested by the fossilized prefix bi- on words whose meanings share these qualities (Cruz 1935:112-117). But, before the category became extinct, Ayoquesco Zapotec replaced the prefix with ni- on words of this complex that name referents that are distant from people, such as stellar constellations, or that intrude on them, as do crop pests (MacLaury 1986b, 1991b). Simplification as a motive for category change has not been examined across domains.

\section{Origins and Destination}

We lack a definitive view of how the desaturated-complex category emerges and how it might persist in highly differentiated systems of color categories, say, those of Berlin and 
Kay's Stage VII with elaboration of secondary, tertiary, and technical color terms, as with major world languages. My statement that the category reduces to the brown-grey range of English dun or Spanish pardo might be premature (MacLaury 2001: \$3.2; cf. Murray 1933:715, re. dun; MacLaury 1986a:App. 1, p. 11, Fig. 8a, re. pardo). It occurs among early-stage brightness terminology (MacLaury 1997a:69, Fig. 2.29, Cayapa ya- and ñin-).

\section{Combining Blue with Grey}

Willmer (1961) and Trezona (1970) discuss the evidence that rod signals travel in pathways carrying signals from short-wave cones, those that contribute most to blue sensation, wherein signals of blue and achromatic low luminance are summed under photopic conditions beneath those at which suppression by cones is total - as well as under scotopic and mesopic conditions. "The sensations of blue, violet and purple are often associated with twilight conditions, when rods are beginning to dominate vision" (Willmer 1961:152); "rods and 'blue' cones exist separately but share a neural pathway" (Trezona 1970:332); there is a great deal to be gained by sharing pathways... because of the limitation on the number of channels set by the small size of the optic nerve, necessary for mobility of the eyeball" (326). Reitner, Sharpe, and Zrenner (1991) show in a blue-cone monochromat that some rod and cone signals travel by separate pathways, which is why the two types of signal can be compared and discriminated. But data supporting the shared pathway might explain why speakers of some languages include blue in a grey-focused desaturated-complex category, while they so include none of the other pure hues.

\section{Levels of Modeling}

To model the desaturated-complex category, I relegate the notion of residuality to primal sensorial motivation, instate the MacLaury-Greenfeld perceptual model as a provisional placeholder, and add vantage theory as a cognitive account of the categorization process. These models integrate as three levels, which are arranged from simplest and lowest to most complex and highest (1-3) in Table 6.

These levels represent a change over our earlier modeling, which treated levels 1 and 2 on a par and paid lip service to prototype theory (à la Rosch 1973) in lieu of vantage theory - which had not been invented then.

Table 6. Integrated models of the desaturated-complex category

\begin{tabular}{ll}
\hline Experience & Models \\
\hline 3. Cognition & Vantage Theory \\
2. Perception & T-Scores, C-Scores, Nonlinear Progression \\
1. Sensation & Residuality \\
\hline
\end{tabular}




\section{Sensorial Residuality}

The range of the desaturated-complex category shows an imperfect correspondence with null responses compiled from our color surveys: Null responses prevail in grey, brown, lavender, beige, pale, and blue, although they occur elsewhere, likely for many reasons. They suggest that desaturated and complex colors are harder to name than are the pure colors. They further suggest, then, that desaturated and complex color presents the viewer with a vaguer, less identifiable experience than does distinctive color. Some people may simplify their naming task by categorically referring to all vague color as "blah color" or, collectively as speakers of a particular language, name such blah with its own color term. However, as Greenfeld (1986) shows, such term does not name a hodgepodge of leftover percepts. Although residuality might evoke a sensorial motive, higher powers of integration soon, if not instantaneously, take over. Apparently, people prefer to impose order on chaos.

Folk glosses of khósi and yà?ač refer to minor chaotic events: goose bumps and salt on the skin, puffing and scattering feathers. Navaho lebá symbolizes chaos in other ways: "(tbai, tbahigi is almost universally the color of evil, equivalent to our use of the word 'dirty' in its moralizing sense, 'despicable.' The monsters are referred to generally as gray, but after the main ones had been destroyed, 'gray gods' were said to exist, some of which were destroyed wholesale by a hail and wind storm” (Reichard 1974:202).

\section{Perception of Desaturated-Complex Color}

Elsewhere, I have equated sensation and perception, even though others equate perception and cognition (MacLaury 1997a:87-93, cf. 471). Here I separate the three. Perception is the addition of discernment to sensation, in this case breaking down the vague colors into the constituents of their vagueness: desaturation, complexity, and their combination; perception is of this low order of cognition, unlike the integrative order addressed by vantage theory. But before a sensory experience can be organized as categorical vantages, people must analyze it sufficiently such that its various gists will be extractable. As shown in our perceptual model in Table 4, desaturation and complexity are rated separately by degree. This requires some assertion on the part of the perceiver, more than a reaction. I plug in the MacLaury-Greenfeld perceptual model, with its faults.

Potential for improving this model may reside in the apparently exceptional perceptual distances among red/green/yellow/black and among blue/red/white/black. These intersections constitute the longest stretches in hue perception. MacLaury and Stewart (1984) found that brown and purple are named by MCS languages "prematurely" at Berlin and Kay's Stages IIIa-b and IV more often than are pink, orange, or grey. But measurement of large perceptual distances between Munsell chips is problematic (MacLaury 1997a: 88). We need help from vision researchers (cf. Kay 1999:957-958).

The MacLaury-Greenfeld model of desaturation-complexity perception complements Berlin and Kay's model of hue perception. The latter, which is consistent with the Hering Opponent-Process Model (Hurvich \& Jameson 1957), poses six simplex elemental colors; they are ascribed to points on the Munsell array from focus aggregates of 20 languages (Berlin \& Kay 1969: Fig. 5) and 107 languages (MacLaury 1997b: Fig. 1). 
Each model provides the perceptual foundation for an application of vantage theory: the first application to account for cognition of hue categories (MacLaury 1997a, 2002a), the second now to address cognition of desaturated-complex categories.

\section{Vantage Theory}

Vantage theory is a model of categorization. It includes the categorizer as a "viewer" who actively creates, maintains, changes, divides, and dissolves a category, and it details how the viewer accomplishes these processes. In short, it specifies the method by which any aspect of categorization is achieved. Whereas vantage theory originally accounted for hue categorization (MacLaury 1986a, 1987, 1992, 1995, 1997a, 2000, 2002a), researchers in distinct fields have applied it to problems apart from color (M. MacLaury 1989; Aoyagi 1995; Hill \& MacLaury 1995; MacLaury, ed. 2002b; MacLaury 2003; Taylor 2003). The unproven premise of vantage theory is that the categorization method is based on a deep-seated, neurally expedited analogy with the method by which people process their positions in a terrain of landmarks and motion. The analogy is so engrained and so automatic that it is instantly and unreflectively invoked for each category that a person calls to mind, verbally or silently. The analogy is drawn on the level of coordinates, which in space-time are the discrete, fixed landmarks and degrees of slow-fast. In a category, the analogical equivalents are fixed images or certain other kinds of discrete ideas, and they are the mutable degrees of attention to similarity and difference. Like slowfast, similarity-difference are separate points on one continuum that connects end-point extremes; like assessments of slow and fast, judgments of similarity and difference are reciprocally related such that a degree of magnitude assigned to one subtracts from the magnitude attributable to the other, at least within the confines of any single perception. The analogy between space-time coordinates and categorical coordinates perhaps allowed primordial people to adapt their capacity to negotiate space-time to categorical thought, thus fashioning an ability to manipulate abstractions from their primary talent to reckon the concrete. Thereby they established the basis of what would become language and culture (cf. Nyan 2002).

The space-time equation is critical to the capability of vantage theory to explain recurrent patterns among data pertaining to categorical dynamics, such as asymmetry, alternative emphases, and skewing (MacLaury 2002a, especially \$19). For now, however, we shall apply the model to the category of desaturation-complexity.

A viewer begins to categorize a gamut of perception by extracting from it certain gists, which he uses as fixed points of reference or inherently fixed coordinates. From vague, residual color, he extracts the gists of desaturation ( $\mathrm{T}$ ) and complexity $(\mathrm{C}) . \mathrm{T}$ and $\mathrm{C}$ are inherently fixed because he thinks of them, that is, retains their images, as maximal exemplars - the limiting cases. The viewer completes the category by coordinating $\mathrm{T}$ and $\mathrm{C}$ with the inherently mobile coordinates of attention to similarity (S) and attention to difference (D). As said, S and D are inherently mobile because they are weighted in relation to each other as movable points on a continuum. Whereas $\mathrm{T}$ and $\mathrm{C}$ define the desaturated-complex category, $\mathrm{S}$ and $\mathrm{D}$ recur in all categories. 


\section{\begin{tabular}{cccl}
\multicolumn{5}{c}{ Fixed } & Mobile & \\
Levels & Coordinates & Coordinates & Entailments \\
1 & T & S & focus, range \\
2 & S & C & breadth of range \\
3 & C & D & margin
\end{tabular} \\ Formula: \\ T SS C D \\ Range: grey $>$ brown $>$ lavender, beige, pale \\ Key: $\mathrm{T}$ - desaturation, $\mathrm{C}$ - complexity, $\mathrm{S}$ - attention to similarity, D - attention to difference}

Figure 1. The desaturated-complex category modeled as the zooming hierarchy of a vantage.

In Figure 1, the vantage model organizes the desaturated-complex category as three levels, each of which comprises one ground-figure relation between a fixed coordinate and a mobile coordinate. On level 1, T is the primary fixed coordinate and the ground; $\mathrm{T}$ is coordinated with $\mathrm{S}$, an inherently mobile coordinate and the figure. Their relation entails both a category focus in desaturated color and a category range that extends beyond the focus to colors that are similar to it. On level 2, S is treated as a fixed coordinate because it is established information; its treatment as fixed occurs even though $\mathrm{S}$ is inherently mobile. $\mathrm{C}$ is coordinated with $\mathrm{S}$ and treated as mobile, even though $\mathrm{C}$ is inherently fixed. $\mathrm{C}$ is treated as mobile because it is newly introduced and consequently highlighted as a figure. This relation entails that the range broadens to include complexity. On level 3, C and D are coordinated, which, respectively assume their inherent fixed and mobile roles. Their relation entails that the category will stop its extension at a margin, that it will not expand indefinitely (Indefinite expansion occurs among certain demonstrably "run-away" categories in which S is very strong and D very weak [e.g., MacLaury 1997a:340, Fig. 11.4a]). In vantage theory, every coordinate must eventually assume its inherent role, regardless of the role via which it is introduced.

The viewer may concentrate on any one of the levels and may move his concentration between them by zooming in or zooming out. Zooming in is accomplished by adopting the mobile coordinate of a higher level as the fixed coordinate of next lowest level and by adding to that a new mobile coordinate. Zooming out is accomplished in the reverse. The levels are thereby connected within a zooming hierarchy, which composes a unified frame of reference. Therein, every part implies the presence of every other part, even those that are not in concentration. Thus, all levels within the frame influence the category to some extent at any time. Yet the current level of concentration will have the strongest influence. Because the viewer can change concentration between levels, a category may differ somewhat in organization on different days, during different phases of an experiment, or at different procedures of an interview.

The arrangement of a vantage into levels of ground-figure relations is determined by the space-time analogy. Through it, certain qualities of terrestrial experience are carried over into categorical thought. One of these is that people can directly regard only one ground-figure relation at a time, although they can hold more than one such relation in short-term memory. In a vantage, then, only one ground-figure relation can be concentrated on, while the others are retained as presuppositions. They are automatically 
presupposed because they are integral to the frame. The hierarchical order accommodates the viewer's limitations while enabling him to manage the obligatory multiplicity. Even the simplest category requires no fewer than three coordinates, in the abstract X, S, and D.

The cognition of the category is completely expressed as a formula, TSSCD, which represents all of its constituting thought. S occurs twice in the formula because it is inherently mobile; its mutability makes it quantitative, which means that its occurrence on two levels will reinforce its contribution to the category, depicted as SS. This double input is opposed to the singular contribution of inherently fixed $\mathrm{C}$, whose status as a limiting case prevents $\mathrm{C}$ from waging an influence beyond that maximal threshold. This limitation, too, would apply to T, if T were not already limited by its singular appearance. $\mathrm{D}$ is limited in precisely that latter way, even though $\mathrm{D}$, like $\mathrm{S}$, is inherently mobile and quantitative (As opposed to $\mathrm{S}$ and $\mathrm{D}, \mathrm{T}$ and $\mathrm{C}$ are qualitative). In vantage theory, nothing besides TSSCD need be stated about the cognition of the desaturated-complex category (i.e., the variant modeled in Fig. 1), because the entailments are automatic.

The entailments are not the thought but consequences of the thought; only they are manifested as observable behavior. For reasons expounded in MacLaury (2002a, \$\$9, 13, $26,36)$, the entailments include gradation of category membership, whose overt range is depicted in the figure as grey > brown > lavender, beige, and pale. In brief, values of membership can be plotted on a curve that ranges from maximal (1.0) through intermediate (0.5) to nil (0.0). T S entails the height of the curve, S C its shoulder, and C D its diminishing flange. Its exact shape is entailed by the reciprocal strengths of $S$ and $D$ and by whether the relation between $S$ on level 1 and $S$ on level 2 is additive or multiplicative.

The viewer is integral to category composition because he extracts the gists, poses them as coordinates, and further coordinates them with his attentions to S and D. He also changes the size and shape of the category range by varying his attention to $\mathrm{S}$ and $\mathrm{D}$, and by concentrating on different levels of the zooming hierarchy. He may affect long-term change by permanently shifting his strengths of attention to $S$ and $D$, which over time seem always to favor D. At least across generations, as observed empirically, categories always shrink and divide; they never expand. A viewer makes minor changes to his thought as he is impacted by the influx of novelty from his surrounds and by various other mounting requirements. Major differences in thought are found between individuals, even within a small community and especially between generations (e.g., MacLaury 1997a: Figs. 6.2 vs $6.16,6.1$ vs 6.4). The variation is easy to interpret as representing a shift in emphasis from $\mathrm{S}$ to $\mathrm{D}$, which probably has been on-going among human populations since categorical vantages became part of our adaptive complex.

The contribution of vantage theory to modeling categorization becomes starkly apparent when a category is named from two perspectives, as with the major and minor terms of certain desaturated-complex categories. In vantage theory, these are said to constitute coextensive ranges, and they are formally represented as two arrangements of the same coordinates, that is, two vantages on one category that are named separately. Thus, the viewer names his vantages rather than naming the category as disengaged from himself, and he thinks of the category in that engaged manner. The vantages of any such pair 
as well as their names are called dominant and recessive, which are the theoretical ways of referring to the heretofore major and minor. Figure 2 models these.

$\begin{array}{lcccccl} & \text { Dominant } & \text { Vantage } & & \text { Recessive } & \text { Vantage } & \\ \text { Entailments } & \text { Fixed } & \text { Mobile } & \text { Level } & \text { Fixed } & \text { Mobile } & \text { Entailments } \\ \text { focus, range } & \mathrm{T} & \mathrm{S} & 1 & \mathrm{C} & \mathrm{D} & \text { focus, margin } \\ \text { broad } & \mathrm{S} & \mathrm{C} & 2 & \mathrm{D} & \mathrm{T} & \text { narrow } \\ \text { margin } & \mathrm{C} & \mathrm{D} & 3 & \mathrm{~T} & \mathrm{~S} & \text { range }\end{array}$

Formulae: $\quad$ TSSCD

CDDTS

Ranges: grey $>$ brown $>$ lavender, beige, pale $\quad$ brown $>$ grey $>$ lavender, beige, pale

Figure 2. Dominant and recessive vantages on the desaturated-complex category.

The dominant vantage is as explicated in Figure 1. The recessive vantage inverts all coordinates such that its formula is CDDTS, with DD obtaining for the same reason that SS obtains in the dominant formula. The contrast of dominant SSD and recessive DDS produces asymmetry between the vantages. The difference will be entailed by a recessive vantage whose range is more likely to be focused on a marginal member, whose name is used less and with wider dispersion, and whose name will be invoked with greater scrutiny and critical care (which in Munsell interviews is seen in smaller mappings with smaller average size of mapping steps; e.g., MacLaury 1997a:Table 6.1). There are further entailments, such as dominant consistency and stability versus recessive inconsistency and indeterminacy, prosaic use of the dominant term versus recessive poetic use, as well as greater recessive participation in tropes, enrichment of qualifying morphology, and elaborated symbolism. The recessive entailments stem from the greater analyticity afforded by DDS, which distances the viewer from the fixed coordinates and from the purview of this vantage. Moreover, the primary recessive coordinates, C D, compel the viewer to overarch the focus and margin of the category, which enhances the distance between viewer and purview. The difference between the dominant and recessive vantage commonly translates into submerged and subjective versus reflective and objective points of view (MacLaury 1995:232-36; MacLaury 1997a:Ch. 9, 2002a:\$\$26, 35; MacLaury, Almási, \& Kövecses 1997).

The asymmetry derives directly from the limitations imposed by the space-time analogy in that dominant SSD versus recessive DDS result from the inability to concentrate on more than one ground-figure relation at a time. Thus, recessive C D on level 1 must zoom to D T on level 2, accumulating in CDDTS on level 3. The constraint inherent in the purported analogical origin of vantages explains categorical asymmetry both among coextensive vantages and among cognitive psychological findings (e.g., Rips 1975). In that sense, the proposed deep-seated equation of categorical vantages with points of view in space-time is fundamental to the explanatory potential of the theory: categories behave as they do because of the method by which we compose them and ultimately because of the means by which the method itself has been constituted.

All categories involve, at least one vantage, although that requisite only becomes evident when we encounter a category that involves two vantages. When a category involves 
only one vantage, the vantage is always dominant. When a category involves two vantages, the second vantage is always recessive. A recessive vantage cannot exist apart from its dominant counterpart.

A dominant-recessive pair of vantages constitute one categorical frame, which is the upper limit for such a frame. Therein and throughout, one balance of strength pertains between S and D, whatever their combination - SSD or DDS. However, a few idiolects name the desaturated-complex category with three terms, which suggests those speakers maintain three vantages. In Table 1, for example, Diuxí Mixtec idiolect 66 names the entire category with either of nšà'á, nvázì , or kaféê. The problem is to model the third vantage, which as such must maintain a balance of different strengths - specifically, weaker $S$ and stronger D - and it must share these strengths with a second vantage that provides its counterpart. The challenge is met in modeling hue categories and categories outside the color domain (MacLaury 1997a:167-170, 2000:279-285, 2002a: \$37; Adachi 2002: \$3.4; Pishwa 2002:\$3). The solution is to relate the three vantages like links of a chain, A, B, and $\mathrm{C}$, and to distribute them across two frames, FI and FII. A and B constitute the dominant and recessive vantages of FI, $\mathrm{B}$ and $\mathrm{C}$ the dominant and recessive vantages of FII, respectively. The balance of strength between $\mathrm{A}$ and $\mathrm{B}$ is posed as strong $\mathrm{S}$ versus weak $\mathrm{D}$, that between $\mathrm{B}$ and $\mathrm{C}$ as weak $\mathrm{S}$ versus strong D. Between FI and FII, the relative degrees of strength shift (FI > FII): strong $S>\varnothing$, weak D > weak $S, \varnothing>$ strong D. Any specific balance could pertain, as long as S is stronger in FI than in FII and D is weaker in FI than in FII (Probably, real categories composed of exactly equal strengths of emphasis on S and $\mathrm{D}$ are very rare at best, although they too would fit the interframe formula). This part of the model will not be further pursued. Four terms for a whole category have not been found in one idiolect, within or outside the color domain, although there are simple ways to model a relation among more than three vantages without adding frames (cf. Adachi 2002: $\$ 3.5)$.

Figure 2 displays a full inversion between TSSCD and CDDTS. But there are cases in which only the mobile coordinates need be inverted to model data, for example, TSSCD and TDDCS, as when two vantages share a focus despite differences between the ways their names are applied (MacLaury 2002a: $\$ 21$; Adachi 2002: $\$ 3.2$ ). Such partial inversion necessarily applies in categories formed in reference to only one inherently fixed coordinate, XSSD versus XDDS (e.g., MacLaury, Almási, \& Kövecses 1997; Stanlaw 2002).

When a viewer strenthens emphasis on $\mathrm{D}$ and thereby deemphasizes $\mathrm{S}$, the range or ranges of a category shrink(s). Within a dominant-recessive pair, SSD retards shrinkage while DDS expedites it. The result is a changing trajectory of semantic relations between vantages that extends from near synonymy to coextension to inclusion to complementation, each relation requiring progressively less emphasis on $\mathrm{S}$ and more on $\mathrm{D}$ (cf. MacLaury 1992: 141-147, 1997a: 148-152, 2000:260-277, 2002a:\$32). Two terms will assume the relation that is consistent with the balance of strength between $S$ and D that prevails in the category when the second term is innovated, and their relation will change accordingly as the balance shifts thereafter. Complementation is consistent with the complete split of a category and rearrangement of coordinates constituting each half to compose dominant vantages of separate categories. 
Although most dominant ranges of the desaturation-complexity category - at least those in the New World - favor desaturation (TSSCD), some favor complexity (CSSTD). Nothing determines that $\mathrm{T}$ must constitute the primary fixed coordinate.

Many desaturated-complex categories are curtailed in range by addition to them of emphasis on either light or dark or on blue. The accretions are modeled as curtailing coordinates, L (light), K (dark), or Bu (blue), as with TSSCLD, TSSCKD, or TSSCBuD. Moreover, such categories can develop by promoting the curtailing coordinate and by demoting or discontinuing $\mathrm{T}$ or $\mathrm{C}$, for example, CSSTBuD $>$ BuSSCD or TSSCBuD $>$ BuSSTD or like developments involving L or K. Vantages of five coordinates are rarer than those of four or three, and those of five are more variable and probably less stable. They tend to evolve into vantages constituted by fewer coordinates, or they are replaced by a simpler alternative that comes complete with a new name, often borrowed from a language that uses that term to name the simpler construct. The prime example is the replacement of Old English haewen 'mold; grey, blue' in Middle English with French bleu 'blue' (documented by Biggam 1997, who interprets her data independently).

\section{Worldwide Overview}

As in Table 7, the World Color Survey (WCS) reveals differences between the Old World and New World in the frequency at which languages and individuals name the desaturated-complex category, and in the emphasis they most often place upon it.

Explanatory hypotheses of the differences might emerge if a comparison of Old World and New World color naming revealed other differences that imply a cause common to all differences.

Table 7. Desaturated-complex categories in the World color survey

\begin{tabular}{llll}
\hline World Color Survey & Old World & \multicolumn{1}{l}{ New World } \\
\hline Number of Languages & 55 & 110 & 55 \\
$\begin{array}{l}\text { Languages } \\
\text { with the T-C category }\end{array}$ & 26 & 68 & 42 \\
$\begin{array}{l}\text { Percentage of languages } \\
\text { with the T-C category }\end{array}$ & $47.27 \%$ & $61.81 \%$ & $76.36 \%$ \\
$\begin{array}{l}\text { Valid interviews } \\
\begin{array}{l}\text { Interviews yielding } \\
\text { a T-C category }\end{array}\end{array}$ & 1301 & 1279 \\
$\begin{array}{l}\text { Percentage of interviews } \\
\text { yielding a T-C category }\end{array}$ & 147 & 391 \\
$\begin{array}{l}\text { Emphasis within } \\
\text { the T-C category }\end{array}$ & $11.30 \%$ & $\begin{array}{l}30.57 \% \\
\text { Most often }\end{array}$ & $\begin{array}{l}\text { Most often } \\
\text { desaturation }\end{array}$ \\
\hline
\end{tabular}




\section{Munsell specifications}

Data were elicited with 330 separate and numbered Munsell chips and with arrays of the chips in their spectral order. The WCS used one green-centered array, while the MCS used this plus a red-centered array, both of the same chips. Each informant was interviewed in daylight shade without interference from onlookers. He or she named the loose chips one-by-one in random order, the responses recorded by number. And he indicated on an array his focus of each head lexeme that he had volunteered in chip naming. In the MCS, some informants mapped on an array the range of each head lexeme, a procedure to be described below. Data were analyzed after they were derandomized and combined on a graph that depicts the green-centered Munsell array, as shown in Figure 3. ${ }^{1}$ Its technical specifications are found in MacLaury (1997a: Fig. 1.3, 1997b: Fig. 1). The graph represents levels of lightness across the rows, A-J at left and B-I elsewhere (lightest at top, darkest at bottom), maximally saturated hues across columns $1-40$, and minimally saturated white, grey, and black in the detached left column (column $\varnothing$ in text). Each row-column intersection represents a separate chip. Maximal saturation refers to that attainable by 1976 pigment technology (when these chips were produced), which are moderate levels and not the higher levels obtained by monochromatic light or observed in nature, as with vivid flowers. The maximal saturations are uneven. Otherwise, the psychological distances between Munsell chips are equal within rows and regular between rows (Newhall, Nickerson, \& Judd 1943), distance decreasing in proximity to white or black. Because B-I 1-40 constitute the outer skin of the spheroidal color solid, their flat projection in Figure 3 exaggerates psychological distances between chips across lighter and darker rows similar to the way Mercator's projection stretches the top and bottom of the global map. The left column is innermost within the solid. Chips between that and the surface are excluded. MacLaury (1997a:415-16) chronicles decisions leading to this composition of the 330 ethnographic Munsell chips.

Figure 3 displays derandomized applications of color-term head lexemes to the chips by individual speakers of four national languages, which might provide readers with a guide to interpret the black-and-white diagrams of data from unfamiliar languages. Application of each color term is distinctively hatched. A focus is represented as a cross in an oval when it matches the application of its name to a chip, or as an oval with hatching when it does not. Because naming and focusing result from independent elicitations, such mismatches are common - especially where ranges overlap.

\section{Summary}

The sensorial model concerns the motivations for forming the category of desaturatedcomplex color and might address such motivations as fully as would be possible. However,

1. Figure 3 was absent in the manuscript of the late R. E. MacLaury: Editor. 
the MacLaury-Greenfeld perceptual model is provisional. Its unresolved issues underscore the benefits that color anthropologists and vision scientists might accrue by working in collaboration on this problem and on many others. Vantage theory models cognition of the desaturated-complex category in all its facets. This system of explanation applies here as it has to other kinds of color categories and to categories unrelated to color, which suggests the possibility that specific principles of categorization pertain to categories of many sorts regardless of what they categorize. Semiotic nuance and symbolism might accommodate to further levels of modeling, thus uniting diverse approaches to color.

\section{Acknowledgments}

When I undertook graduate study at the Language Behavior Research Laboratory in 1974, the "desaturation" category became my designated specialization. I thank those at the Lab for their insights and encouragement, including Brent Berlin, Paul Kay, Eugene Hammel, Janet W. D. Dougherty (J. W. D. Keller), Chad McDaniel, Craig Molgaard, and Richard Sun. And I am grateful to Brent Berlin, Paul Kay, and William R. Merrifield for conducting the World Color Survey and providing its data for this study. I also thank the many who participated in my Mesoamerican Color Survey, too numerous to list here but whose names appear in MacLaury (1997a:Fig. 397-407, Table I.1). Special to the present study is Philip J. Greenfeld for his collaboration and donation of his Apache data. My thanks to Bill Wooten for reprints, to Karen De Valois for her informed overview of vision research conducted and not conducted, to Galina Paramei for advise and sources on the visual relation of grey and blue, and to Liudmila Samarina and Galina Yavorska for organizing the symposium Anthropology of Colour. Responsibility rests with me.

\section{References}

Adachi, Nobuko (2002). Negotiation of speech style in Japanese women's language: Vantage theory as cognitive socioliguistics. In MacLaury, R. E., ed., 575-590.

Bartleson, C. James (1976). Brown. Color Research \& Application 1, 181-192.

Berlin, Brent (1975). Initial classification of color lexicons found in the C. Hart Merriam vocabularies. MS in possession of author.

Berlin, Brent \& Paul Kay (1969). Basic Color Terms: Their Universality and Evolution. Berkeley University of California Press.

Biggam, Carole P. (1997). Blue in Old English. Amsterdam / Atlanta: Rodopi.

Boring, E. G. (1942). Sensation and Perception in the History of Experimental Psychology. New York: Appleton-Century-Crofts.

Brown, Roger, \& Eric H. Lenneberg (1954). A study in language and cognition. Journal of Abnormal and Social Psychology 49: 454-462.

Burgess, Donald, Willett Kempton, \& Robert E. MacLaury (1983). Tarahumara color modifiers: Category structure presaging evolutionary change. American Ethnologist 10: 133-149. 
Collier, George A. (1966). Categorias del color in Zinacantan. In Evon X. Vogt, Los Zinacantecos: Un pueblo Tzotzil de los altos de Chiapas (pp. 414-432). Mexico, D.F.: Instituto Nacional Indigenista.

Cruz, Wilfredo C. (1935). El Tonalamatl Zapoteco: Ensayo Sobre su Interpretación Lingüística. Oaxaca de Juárez: Imprenta del Gobierno del Estado.

Dal, Ingrid (1938). German brun als Eptheton von Waffen. Norsk Tidskrift for Spragvidenskap 9: 219-230. Dougherty, Janet W. D. (1975). A Universalist Analysis of Variation and Change in Color Semantics. Dissertation in Anthropology. University of California, Berkeley.

Edgeworth, Robert J. (1978). What color is 'ferrugineus'? Glotta 56: 297-305.

Edgeworth, Robert J. (1979). Does purpureus mean “bright”? Glotta 57: 281-291.

Farnsworth, Dean (1943). The Farnsworth-Munsell 100-hue and dichotomous tests for color vision. Journal of the Optical Society of America 33: 568-578.

Feo, Michele (1975). Pallida no, ma puì che neve bianca. Giornale stroico della letteratura italiana 152: 321-361.

Franciscan Fathers, The (1910). An Ethnologic Dictionary of the Navaho Language. Saint Michaels, Arizona.

Fuld, Kenneth, John S. Werner, \& Bill R. Wooten (1983). The possible elemental nature of brown. Vision Research 23: 631-637.

Fuld, Kenneth, Bill R. Wooten, \& James J. Whalen (1981). The elemental hues of short-wave and extraspectral lights. Perception \& Psychophysics 29: 317-322.

Gaultier de Claubry, Isabelle (1886). Note sur le vocabulaire des couleurs chez les Arabes d'Algérie. Bulletin de la Société d'Anthropologie de Paris (Series 3) 9: 698-710.

Greenfeld, Philip J. (1986). What is grey, brown, pink, and sometimes purple: the range of "wild-card" color terms. American Anthropologist 88: 908-916.

Hale, Kenneth L. (1975). Gaps in grammar and culture. In M. D. Kinkade, K. L. Hale, and O. Werner, eds., Linguistics and Anthropology in Honor of C. F. Voegelin (pp. 296-298). Werner Lisse: Peter de Ridder.

Heinrich, Albert C. (1974). Colour in Colonia Tovar: a preliminary report on the colour terminology of an Alemannisch speaking enclave in the mountains of northern Venezuela. Paper presented at the 73rd Annual Meeting of the American Anthropological Association, Mexico, D.F.

Hering, Ewald (1964 [1920]). Outlines of a Theory of the Light Sense. Trans. L. M. Hurvich and D. Jameson. Cambridge, MA: Harvard University Press.

Hinde, H. (1901). The Masai Language. London: Cambridge University Press.

Hurvich, Leo M., \& Dorothea Jameson (1957). An opponent-process theory of color vision. Psychological Review 64: 384-404.

Kay, Paul (1999). Asymmetries in the distribution of composite and derived basic color categories. Behavioral and Brain Sciences 22: 957-958.

Kay, Paul, \& Chad K. McDaniel (1978). The linguistic significance of basic color terms. Language 54: 610-646.

Landar, Herbert J., Susan M. Ervin, \& Arnold E. Horowitz (1960). Navaho color categories. Language 36: 368-382.

Lenneberg, Eric H., \& John M. Roberts (1956). The language of experience: A study in methodology. Memoir 13, supplement to International Journal of American Linguistics 22(2).

MacLaury, Maria I. (1989). La Placita: Vantages of Urban Change in Historic Tucson. Master of Architecture Thesis. University of Arizona, Tucson. Ann Arbor: University Microfilms International (No. 1339280).

MacLaury, Robert E. (1970). Ayoquesco Zapotec: Ethnography, Phonology, and Lexicon. Master of Arts Thesis. University of the Americas, Mexico, D.F. (Cholula, Puebla).

MacLaury, Robert E. (1973). Letter to Brent Berlin and Paul Kay on Ayoquesco Zapotec yà?ač 'grey, brown, lavender, beige.' 
MacLaury, Robert E. (1975). Reconstructions of the Evolution of some (50) Basic Color Term Lexicons: A Step Toward Developing Diachronic Methods for Testing the Berlin-Kay Hypothesis." MS, UCBerkeley WCS / The German Research Society.

MacLaury, Robert E. (1985). Residual Color Categories, Chapter XI of “Color in Mesoamerica," an MS of which the first half became MacLaury (1986a, 1997a) and the second, including this chapter, remains in MS, "Color in Mesoamerica, Vol. II: Primary, Secondary, and Desaturated Categories."

MacLaury, Robert E. (1986a). Color in Mesoamerica, Vol. I., A Theory of Composite Categorization. Dissertation in Anthropology. University of California, Berkeley. Ann Arbor: University Microfilms International (No. 8718073).

MacLaury, Robert E. (1986b). Sounds of the sacred: Zapotec symbolic syllables. Paper presented at the Sound Symbolism Conference. University of California, Berkeley.

MacLaury, Robert E. (1991a). Social and cognitive motivations of change: measuring variablity in color semantics. Language 67: 34-62.

MacLaury, Robert E. (1991b). Zapotec diminution. Paper presented at the 30th Conference on American Indian Languages: Mesoamerican Languages; 90th Annual Meeting, American Anthropological Association, Chicago.

MacLaury, Robert E. (1992). From brightness to hue: An explanatory model of color-category evolution. Current Anthropology 33:137-186.

MacLaury, Robert E. (1995). Vantage theory. In J. R. Taylor and R. E. MacLaury, eds., Language and the Cognitive Construal of the World (pp. 231-276). Berlin / New York: Mouton de Gruyter.

MacLaury, Robert E. (1997a). Color and Cognition in Mesoamerica: Constructing Categories as Vantages. Austin: University of Texas Press.

MacLaury, Robert E. (1997b). Ethnographic evidence of unique hues and elemental colors. Behavioral and Brain Sciences 20:202-203.

MacLaury, Robert E. (2000). Linguistic relativity and the plasticity of categorization: Universalism in a new key. In M. Pütz and M. Verspoor, eds., Explorations in Linguistic Relativity (pp. 249-294). Amsterdam/Philadelphia: John Benjamins.

MacLaury, Robert E. (2001). Color terms. In M. Haspelmath, E. Konig, W. Oesterreicher, and W. Raible, eds., Language Typology and Language Universals: An International Handbook (pp. 1227-1251). New York / Berlin: Walter de Gruyter.

MacLaury, Robert E. (2002a). Introducing vantage theory. In MacLaury, R. E., ed., 493-536.

MacLaury, Robert E., guest editor (2002b). Vantage Theory: Applications in Linguistic Cognition. Language Sciences 24 (5-6), special issue.

MacLaury, Robert E. (2003). Vantages on the category of vertical extent: John R. Taylors' "high" and "tall". Language Sciences 25:285-288.

MacLaury, Robert E. (2005). So-called brightness in color ethnography: Potentials for LCD technology in fieldwork and categorization research. Cross-Cultural Research 39:205-227.

MacLaury, Robert E., Judit Almási, \& Zoltán Kövecses (1997). Hungarian piros and vörös: color from points of view. Semiotica 114: 67-81.

MacLaury, Robert E. \& Philip J. Greenfeld (1984). How to Say "Blah" in Fifty Languages: A Study of Residual Color Categorization. MS, UC-Berkeley WCS.

MacLaury, Robert E., \& Stephen O. Stewart (1984). Simultaneous sequences of basic color-category evolution. Paper presented at the 83rd Annual Meeting of the American Anthropological Association, Denver.

Malkiel, Yakov (1953). "Apretar", "Pr(i)eto", "Perto": Historia de un Cruce Hispanolatino. Thesavrvs: Boletín del Instituto Caro y Cuervo 9: 1-135.

Meier, Harri (1975). Brau- und Grautöne. Biblos 51: 547-564.

Merrifield, William R. (1971). Review of Berlin and Kay 1969. Journal of Linguistics 7: 259-268. 
Mol, Frans (1995). Lessons in Maa: A Grammar of the Masai Language. Lemek, Kenya: Maasai Centre Lemek; Limuru: Kolbe Press.

Mol, Frans (1996). Maasai Language \& Culture Dictionary. Lemek: Maasai Centre Lemek; Limuru: Kolbe. Monberg, Torben (1971). Tikopia color classification. Ethnology 10: 349-358.

Murray, James A. H., ed. (1933). The Oxford English Dictionary, Vol. III, D-E. Oxford: Clarendon Press.

Newhall, Sidney M., Dorothy Nickerson, \& Deane B. Judd (1943). Final report of the O.S.A. Subcommittee on the spacing of the Munsell colors. Journal of the Optical Society of America 33: 385-418.

Nichols, Michael Porter (1974). Northern Paiute Historical Grammar. Dissertation in Linguistics, University of California, Berkeley.

Nicholas, Michael Porter (1980). Renewal in Numic color systems. In K. Klar, M. Landon, and S. Silver, eds., American Indian and Indo-European Studies: Papers in Honor of Madison S. Beeler (pp. 159-167)

(Trends in Linguistics: Studies and Monographs 16, W. Winter, ed.). The Hague: Mouton.

Nyan, Thanh (2002). Vantage theory and innateness. In MacLaury, R. E., ed., 705-712.

Ohman, Susanne (1953). Theories of the linguistic field. Word 9: 123-134.

Peers, Edgar A. et al. (1960). Cassell's Spanish Dictionary. New York: Funk \& Wagnalls.

Perry, Edgar (1972). Western Apache Dictionary. Fort Apache: White Mountain Apache Tribe.

Pishwa, Hanna (2002). Language learning and vantage theory. In MacLaury, R. E., ed., 591-624.

Quinn, Paul C., J. L. Rosano, \& Bill R. Wooten (1988). Evidence that brown is not an elemental color. Perception \& Psychophysics 43: 156-164.

Quinn, Paul C., Bill R. Wooten, \& Evette J. Ludman (1985). Achromatic color categories. Perception \& Psychophysics 37: 198-204.

Reichard, Gladys A. (1974). Navaho Religion: A Study of Symbolism. Princeton: Princeton University Press.

Reitner, Andreas, Lindsay T. Sharpe, \& Eberhart Zrenner (1991). Is colour vision possible with only rods and blue-sensitive cones. Nature 352: 798-800.

Rips, Lance J. (1975). Inductive judgements about natural categories. Verbal Behavior and Verbal Learning 14: 665-681.

Rosano, J. L., Bill R. Wooten, \& Paul C. Quinn (1984). Evidence that brown in not an elemental color. Paper presented at the OSA meeting, San Diego.

Rosch, Eleanor Heider (1973). On the internal structure of perceptual and semantic categories. In T. E. Moore, ed., Cognitive Development and the Acquisition of Language (pp. 111-144). New York: Academic Press.

Stanlaw, James M. (2002). "Hire" or "Fire"? Taking AD-vantage of innovations in the Japanese syllabary. In MacLaury, R.E., ed., 537-574.

Sternheim, Charles E., \& Robert M. Boynton (1966). Uniqueness of perceived hues investigated with a continuous judgement technique. Journal of Experimental Psychology 72: 770-776.

Taylor, John R. (2003). Near synonyms as co-extensive categories: "tall" and "high" revisited. Language Sciences 25: 263-284.

Trezona, P. W. (1970). Rod participation in the 'blue' mechanism and its effect on colour matching. Vision Research 10: 317-332.

Willmer, E. N. (1961). Human colour vision and the perception of blue. Journal of Theoretical Biology 2: $141-179$. 


\title{
Relative basicness of color terms
}

\author{
Modeling and measurement
}

\author{
Seija Kerttula \\ Department of English, University of Helsinki, Finland
}

Color terms have been of great interest for nearly 150 years. After more than a century of cross-linguistic studies with relativistic emphasis, Berlin and Kay's theory switched the focus to basic color terms and a supposed universal ground. Yet, towards the end of the 20th century, research based strictly on universal basicness seemed to have come to a standstill. Relativist voices were raised, broader approaches were developed, and multidisciplinary efforts began to shed light on the nature of color naming. There was a new need to combine universal and relativist approaches. Research described here shows that a concept I call "relative basicness" is worthwhile. This concept makes it possible to trace historical layers and universal trends. If proven applicable to terms in domains apart from color, the model could be used to clarify general semantic structures and relations.

\section{Introduction}

Color terms have been of great interest for nearly 150 years. After more than a century of cross-linguistic studies and relativist emphasis, Berlin and Kay's theory (1969) turned the focus to basic color terms and their universal regulation. Towards the end of the 20th century research based on mere basicness seemed to have come to a standstill. Relativist voices were raised (e.g., Saunders 1992), broader approaches were searched (MacLaury 1992), and multidisciplinary efforts shed more light on the universalism in color naming (Hardin \& Maffi 1997). There was a new need to combine universal and relativist approaches.

When I began my diachronic research on English color terms in the late 1980s, I was unaware that the methods I had chosen reflected the new demand. First, and in a traditional sense, I sought to clarify how English color terminology had developed historically. But in addition, I looked for an alternative approach to basicness. Rather than viewing it as an all-or-none system, I wanted to find the degrees to which color terms are established in relation to each other. I named this relative basicness. To study it, I developed a model that allowed evaluating variables of basicness and combining their results. Thus, I hoped, the model would prove reliable and adjustable across languages and might even apply to terminologies apart from color. It was to be of special interest whether terms with highest 
values of relative basicness were the oldest. If so, the application of the model might extend to relative dating.

Moreover, I was interested in other universal trends that might be shown by the values of relative basicness in combination with historical data. Berlin and Kay's theory is invaluable for generally defining the role of basic color terms and universals. However, universal influence is not reflected by basic color terms only, but by all expression of color. To discover further trends, we must identify the underlying forces that direct them, and yet we must do so while they are seemingly regulated by culture specifics. This approach requires us to recognize variation, which is essential to every evolutionary process (cf. Kay 1975).

\section{The model of relative basicness}

In my dissertation (Kerttula 2002), I researched the development of English color terms throughout the past and measured the relative basicness of 100 contemporary English color terms, thereby introducing the model. For English color terms, I used a cumulative value of relative basicness that was based on the following four parameters.

1. Primacy expresses how primary is the color sense of the term compared with its other senses. This variable includes Berlin and Kay's (1969:6) criterion 2 of hyperonomy or nonhyponomy: "Its meaning is not included in that of any other color term." The value is based on definitions in advanced monolingual dictionaries. Such seem to reflect the general sense of color; for example, some English nonbasic color terms that define other color terms (such as crimson) also have high values of relative basicness. There are four levels of primacy: (1) If color is the primary sense of the term and if it is a hyperonym, it receives the highest value (e.g., red). This covers the cases in which the term is not defined by another basic term, as with orange by yellow (e.g., "orange is a reddish yellow"). However, descriptions that merely fail to specify a hyponymic relationship, too, score highest, as with "gray is between black and white." (2) If the color sense is primary and the term is a hyponym (e.g., violet defined as blue), it receives the second highest value. (3) If color is the secondary sense of the term, it gets the third highest (e.g., rust: ferric oxide, reddish brown). (4) If color is the tertiary (or later) sense of the term, it receives the lowest value (e.g., ginger: a certain plant, its rootstock, light brown).

Although primacy may be defined using dictionary definitions, comparing the proportions of different uses within a corpus might prove a more reliable method for the purposes of this study, provided that the corpus is versatile. Dictionary definitions may reflect fossilized sense hierarchies, which the corpus may reveal to be updated.

2. Frequency specifies number of occurrences in a text or discourse. The count must only include uses referring to a color or light-related sense and not proper names, idioms, animal and plant names, or metaphors (e.g., black market) and metonyms (e.g., black music). However, additional color terms used to qualify these (i.e., not included in terms themselves) are acceptable.

3. Application defines the number of referents and usually correlates with frequency. This variable is given as Berlin and Kay's (1969:6) criterion 3 of decontextualized, context- 
general, or noncontext-specific: "Its use is not restricted to certain objects." Although I used partial grouping to analyze application of English color terms, a simpler method could be adopted by which the referent numbers are directly transformed into values. Proper names and names of animals and plants as well as metaphoric and metonymic uses are precluded (yet those attributing these are accepted as in frequency). Also exact synonyms (e.g., Finnish lippis and lippalakki 'peaked cap') are excluded and proper names are included in the general object name (e.g., Rolls-Royce and Chevrolet are counted as auto 'car').

4. Derivational productivity is the number of derivative types and may include certain compounds. While doing a diachronic survey of the development of English color terms, using the Colour section of the Historical Thesaurus of English (University of Glasgow 2005), I noted that the oldest color terms have the most derivatives. This, too, constitutes evidence of basicness, and I added this criterion to the parameters. The connection between basicness and derivation is implicitly supported by Berlin and Kay's (1969:6) supplemental criterion 5: "The doubtful form should have the same distributional potential as the previously established basic terms. For example, in English, allowing the suffix -ish, e.g., reddish." Corbett and Davies (1997: 197-215) use derivation to establish basic color terms (they assess results against how these match Berlin and Kay's order).

While the first parameters (1), (2), and (3) build on historical development, the long-term rooting of a term is most reflected by its (4) derivational productivity. This renders (4) a valuable barometer for historical study of color terms, and it might suggest relative age of a term when philological means are absent. While defining derivational productivity, I counted the derivatives that carry a general color sense. For English color terms, I included certain compounds. However, derivation can be measured without using compounds.

Although parameters are four for English and Finnish, their number is open to adjustment to other languages or other types of terms for which certain parameters may be inapplicable. Relative basicness might also be measured by using fewer parameters. Primacy alone, with its few levels, is insufficient for showing difference between individual terms, although it reveals clearly basic terms. Frequency and application are interrelated and, thus, either by itself can show difference between terms. Derivational productivity alone seems adequate for confirming basic color terms, but it must be supplemented by either frequency or application to determine relative basicness. In addition to these four parameters, others might be considered, as will be discussed in the analysis of Finnish color terms.

The results produced by parameters are transformed into values, which when added form a value of relative basicness. Although this cumulative method worked well in the analyses of both English and Finnish color terms, I also introduce an alternative method in the section for Finnish color terms. This method is based on mere rank order values indicating the relative position of results within each parameter. The final rank order value is achieved by ordering the terms by the totals of rank order values for each parameter. Rank order values may bring up some differences, which are not shown by the cumulative method, and this method may also be used independently. However, the cumulative 
method, although it cannot be regarded as statistically valid in the strict sense, presents differences within a language more accurately. In general, there is a need for developing further the way of presenting total basicness to show differences in the basicness of terms in a more satisfactory way.

Evidence from existing studies shows that measuring relative basicness is worthwhile (Kerttula 2002). Apart from revealing degrees of relative basicness, the values make it possible to trace historical layers and universal trends. If proven applicable to terms in domains apart from color, the model could be used to clarify semantic structures and relations. Philologically, the results of applying the model to English and Finnish color terms conform to those obtained from chronological dating based on the etymologies and so support its use for dating. These prospects indicate that the model may be used as a tool to confirm, challenge, and check results that traditional methods have produced in both diachronic and synchronic studies of languages.

\section{Measuring relative basicness of English color terms}

By way of terminology, brightness here and in Kerttula (2002) covers lightness and darkness and sometimes shining, glittering, and luminance. Its use is rightly criticized by Biggam (2007), because it commonly refers also to saturation. But English pale expresses both saturation and lightness; in Finnish, saturation may be qualified by kirkas 'bright' and loistava 'shining.' We need a cover term for shininess, brightness, and achromatic color, as well as a cover term for both brightness and saturation. Biggam introduces a model that provides color terminologists and lexicographers with tools for consistent color term description.

The writing of English color terms spans more than 1,300 years. The quantity of Old English (OE) terms that designate shininess, gloss, and other luminous effects indicates that Anglo-Saxons emphasized this aspect of color. This preference, confirmed statistically, lacks in-depth semantic research (cf. Biggam 2007). The proportions of various Old English color terms (including derivations and compounds) - in common use as defined by the Thesaurus of Old English (Roberts et al. 1995: 140-147) - illustrate the emphasis, as in Table 1.

The table excludes terms for light by itself. It shows that OE terms designating brightness, light, and glittering are plentiful $(135=36 \%)$ and commonly used $(76=39 \%)$, as are the terms for darkness $(79=21 \% ; 34=18 \%)$; together they are more plentiful and common than terms for hue and achromatic senses $(163=43 \% ; 82=43 \%)$. Yet development of OE hue-based terms was well underway. Biggam (1998:325) suggests that the categories for white, black, red, yellow, green, and gray were basic in OE. However, certain English basic color terms (e.g., white, black, brown, yellow) originate in terms that named shininess, glowing, and other luminance, and some retain such meaning in Old English. All in all, the table gives an impression that the most prodigious, and thus the oldest, elements are those of brightness, darkness, and redness. 
Table 1. Proportions of common Old English color terms, Thesaurus of Old English

\begin{tabular}{lcc}
\hline Color term type & No. of terms & Commonly used \\
\hline Brightness, light, glittering, effulgence & 135 & 76 \\
Darkness, dimness & 79 & 34 \\
Red & 40 & 24 \\
Black & 33 & 20 \\
White & 29 & 16 \\
Paleness & 16 & 10 \\
Yellow & 15 & 6 \\
Green & 8 & 3 \\
Grey & 18 & 2 \\
Brown & 4 & 1 \\
Total & 377 & 192 \\
\hline
\end{tabular}

After the Norman conquest in 1066, English was exposed to strong French influence, by which English enriched its color terminology (Kerttula 2002:244-250, 262-265). Many color terms were borrowed, and new models for creating color terms were introduced by which native and French resources were exploited. The foment seems to have fostered the transfer of emphasis to hue. With this trend and starting from the fourteenth century, new types of color terms were created (Kerttula 2002:306-314). These were brightness and saturation qualifiers such as dark, light, deep, and pale that were newly restricted from nominal use to modifying hue terms. Color terms were also borrowed from Latin, but there were practically no loans from Germanic languages - even Scandinavian languages or from Celtic or Greek. My etymological survey of 100 English color terms shows that 32 are borrowed French color terms, while 43 represent assignment by English of color meaning to a nonchromatic French term.

In sum, the development of English color terms represents a case in which an allegedly universal process received heavy cultural intervention. It is of interest to see whether traces of the incursion emerge in results of applying relative-basicness model to 100 English color terms. They were selected from those with 10 or more occurrences in the British National Corpus (BNC), a multigenre corpus of 100 million words of present-day British English. Due to this size, the transformation of results into values (Kerttula 2002:81-92) was more complicated than in the analysis of Finnish color terms, and it is not described in full. Yet the simpler methods of the Finnish analysis could be used in any application of the model. Results for each parameter follow.

\section{Primacy in English}

To clarify the primacy of a color sense, I used the definitions of advanced dictionaries: Cambridge International Dictionary of English (Procter et al. 1995), which is built around the Cambridge Language Survey Corpus (100 million words); Longman Dictionary of Contemporary English (Summers et al. 1995), which is based on the BNC, the Longman 
Table 2. Major English color terms and their scores

\begin{tabular}{lc}
\hline Color terms & Frequency \\
\hline Black & 10 \\
White, red, dark & 9 \\
Green, blue, grey/gray & 8 \\
Pale, yellow, golden, blonde, brown, pink, purple & 7 \\
Silver, cream, orange & 6 \\
\hline
\end{tabular}

Lancaster Corpus (30 million words), comprehensive corpora of British and American speech, and the Longman Learner's Corpus (5 million words); Collins COBUILD English Dictionary (Sinclair et al. 1995), which is based on the Bank of English corpus (200 million words). Highest values (4) were given to black, white, red, yellow, green, brown, gray, orange, and blue. However, purple and pink, which are generally thought to be basic color terms, are associated with red and so got value 3 . Most other color terms received a value 3 or 2 .

\section{Frequency in English}

For counts, I used the BNC. It was impossible to count the thousands of occurrences in the BNC because this requires a manual review of data. I therefore checked all occurrences that numbered less than 1,000 , subsets of 1,000 for those of numbering more.

Black occurs most frequently. White and red are equaled by a nonbasic color term: dark. Such is the like of pale, golden, blond, silver, and cream. Most of these are used both to name color by themselves and to qualify other color terms. At the outset, I was unsure what to do with these words but included them because of their nominal, singular use. The results suggest that qualifiers, such as dark and pale, are integral to color-term evolution, although they somehow inhere differently than nonqualifiers, such as black, white, and red.

\section{Application in English}

The applied range of referents was defined by using the BNC data. As with frequency, it was impossible to inspect all the referents manually. I therefore relied on categories such that an occurrence of a certain referent or occurrences of a term in a referent group scored a credit. The English results showed that almost half the terms examined scored highest credits, including the traditional basic color term.

\section{Derivational productivity in English}

While analyzing derivations, I collected those with a general color sense as well as certain compounds. Highest derivational values are: white 20 , red 17 , yellow 13 , pink 13 , brown 
13 , green 12 , blue 12 , purple 12 , black 10 , silver 10 , golden 10 , rose 10 , dark 8 , crimson 7 , pale 6 , ruby 6 .

\section{Computing a relative basicness score for English color terms}

Adding the credits, highest values of relative basicness are: 38 white; 35 red; 29 black, yellow, green, blue, brown; 28 purple, pink; 27 grey/gray; 25 dark; 24 golden; 23 pale, silver; 20 orange, crimson; 19 rose; 17 ochre, tawny, violet; 16 cream, drab, ruddy; 15 scarlet, ruby, blond(e), azure, dun, russet, fawn, tan, lilac; 14 maroon, dusky, emerald, turquoise, beige, bronze, mauve, peach; 13 milky, ivory, light, buff, ginger, verdant, indigo, navy, amber, smoky, livid; 12 vermilion, magenta, inky, saffron, sapphire, umber, lavender, ashen; 11 carmine, burgundy, coral, ebony, aquamarine, olive, khaki, copper, chestnut, pearl, slate, grizzled.

Unexpected is the value of black. Its relatively low 29 may be explained in that Middle English black replaced Old English basic sweart (Kerttula 2002:321), which is, for example, reflected by black having fewer derivatives than white or red. Even if color naming be universally regulated, its ordering and development might be altered by intervening events.

\section{Remarks on English color terms}

The relative basicness and etymologies of English color terms produce the following results in support of universal development:

1. The basic color terms suggested by Berlin and Kay receive highest values of relative basicness.

2. There are other color terms that receive high values of relative basicness and that might develop into basic color terms. These are, for example, crimson and violet, which are used to define other color terms in dictionaries.

3. Warm colors are named earlier and more readily than cool colors. This is shown both by the values of relative basicness as well as diachronic analysis.

4. There are indications that over the centuries, when analyzing the color space to name its segments, the emphasis was first on brightness, later on hue. The transfer of emphasis to hue was followed by creation of abundant brightness and saturation qualifiers. This might constitute a third universal phase in the development of color naming.

Results (5) and (6) indicate that universal development may be altered by cultural intervention.

5. There were overall changes in color naming after the Norman Conquest of 1066: the emphasis was transferred to hue, while new models of naming colors were adopted.

6. The OE basic term for black, sweart, was supplanted by black. 
In sum, the result indicates that the universal development of color terms may be channeled in a particular direction or, at least, accelerated by culture.

\section{Testing the model: Relative basicness of Finnish color terms}

The model must be tested against another language, preferably one from another linguistic family. Finnish offers the contrast. It is of the Finno-Ugric group, part of the Uralic family, and neither French nor Latin, the two main donors to English, has directly affected its color naming. English has two cases; whereas Finnish with its fifteen cases is a synthetic inflectional language in which suffixes are used both to show grammatical relations and to derive words. However, as in English, the nominal and adjectival forms of Finnish color terms are identical, which in Finnish otherwise only occurs in the names of nationalities and tribes.

Finnish and other Finnic (Livonian, Estonian, Votian, Ingrian, Karelian, Olonetsian, Ludian, and Vepsian) color terms are extensively discussed by Koski (1983). Cognates across Finnic languages show that Late Proto-Finnic named three basic color categories, white, black, and blue (Koski 1983:246). Koski assumes that Pre-Finnic only named two basic color categories. He poses early emergence of blue, which contradicts Berlin \& Kay's diachronic sequence. But he explains that blue overlaps with black and green such that the term may have denoted only dark when it arose. In addition to Koski, Finnish color terms have also been discussed by Sutrop (2002) and Oja $(2001,2007)$ in connection with their analyses of Estonian color terms.

Apart from the oldest terms, the etymologies of the Finnish color terms (from Kulonen et al. 1990, 1995, 2000, unless otherwise indicated) show varying loans.

Musta 'black' has cognates in Finnic languages and in the Sami language (Northern Finland, the closest of the remote cognates of Finnish), with meanings such as black, dark, dirty. The origin of this term is unclear.

There are two forms of 'white', valkoinen and valkea, of which the latter also means 'light,' 'bright,' and the nominal form valkea denotes 'fire.' A derivation, valjeta, means 'to dawn.' Valkoinen and valkea are of Finno-Ugric origin; they have cognates in the daughter languages with meanings such as 'a white domestic animal,' 'light,' 'lightning,' 'bright,' 'shine,' and 'clear.'

Punainen 'red' is also Finno-Ugric. Puna originally meant 'hair,' from which it developed the meaning 'color' and later 'red.'

Keltainen 'yellow' was borrowed by Finnic languages from Baltic languages (cf. Lithuanian geltà 'yellow, 'jaundice' and geltas, geltónas 'yellow', and Latvian dzeltans, 'yellow, of the same Proto-Indo-European root as English yellow and golden).

Sininen 'blue' is the same as Erzya seń, sän and Mordvin seń, señam (Volgan languages remotely related to Finnish); sininen has cognates in many Finnic languages. The word

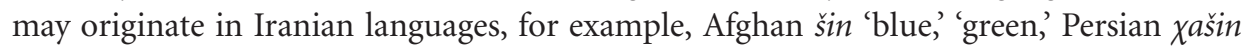
'bluish,' and Ossetian ox $x \bar{s} n$ 'dark gray.' In some Finnic languages its cognates also mean 'green,' as when they refer to verdure, and some Finnish uses indicate that it may have 
referred to blackness and darkness (Koski 1983:149-150, 246-249). Interestingly, similar older usage is mentioned of Russian sinij (Paramei 2005).

To trace the earlier meaning of sininen, I turned to the newly digitized Finnish folk poetry database (SKVR, in the Language Bank of Finland provided by the Finnish Information Technology Center for Science). The database, with oral tradition mainly collected in the nineteenth century, mirrors centuries-old Finnish usage, and parts of it were used to compile the Finnish national epic Kalevala. The data yield hundreds of occurrences of sininen, but listing its referents is as confusing as encountering Old English color use has traditionally been for modern minds. Sininen refers to earth, meadows, woods, people, eye-lashes, thighs, animals (ewe, elk, crane, duck, whitefish), dresses, silk, arrows, rocks, sea, or the sky, giving an impression that it could be associated with various properties such as darkness, brightness, greenness, and blueness. This shows another approach to color, resembling that of Old English.

Vihreä 'green' is still strongly associated with growing, whence it originates. It is related to Finnic cognates meaning 'green,' 'growth' and Volgan Mari əžar, užar 'green,' 'young' and possibly Hungarian virit, virul 'blossom,' 'grow green.'

Harmaa 'gray' is a Baltic loan.

Ruskea 'brown' is of uncertain origin, possibly Germanic or Baltic. It earlier meant 'red,' and its cognates or assimilated counterparts in various Finnic languages are basic color terms for 'red' (Koski 1983:82).

Oranssi 'orange,' turkoosi 'turquoise,' violetti 'purple' (which replaced earlier sinipunainen 'blue-red'), and lila 'lilac' are loans from Swedish, which borrowed them from French. Beige may be similar. Pinkki 'pink' is a loan from English.

Analyzed material is from the 1999 corpus of Turun Sanomat (TuSa), part of the Language Bank of Finland corpora. Turun Sanomat is a daily newspaper published in Turku; its article corpus from 1999 consists of 11 million words (vis-à-vis the 100-million word BNC corpus). The corpora differ because the BNC contains literary and spoken material absent in the TuSa. I do not see this as an obstacle for contrasting the results, since basicness should realize in a similar way across text types when large corpora are used. However, most of the corpora consist of similar types of texts. References to foreign news agencies indicate that 15 percent of the TuSa articles derive from non-Finnish dispatches.

The data show that Finnish color terms are fewer than those of English, comprising 14 established chromonyms: musta 'black,' valkoinen or valkea 'white,' punainen 'red,' keltainen 'yellow,' vihreä 'green,' harmaa 'gray,' ruskea 'brown,' sininen 'blue,' oranssi 'orange,' violetti 'purple,' turkoosi 'turquoise,' lila 'lilac,' pinkki 'pink,' and beige 'beige.' The suffix -nen marks an adjective; some newer loans ending in - $i$ may have added -nen to form an alternative: oranssi, oranssinen. When incorporated into the stem of a color term, -nen is often omitted in compounds, for example, keltainen 'yellow' > keltasirkku 'yellow hammer.'

The terms of strongest basicness are easy to discern: musta, valkoinen, punainen, keltainen, vihreä, harmaa, ruskea, and sininen. These are followed by newer terms with weaker basicness: oranssi, violetti, turkoosi, and beige. According to Koski (1983:238-9), turkoosi is a hyponym of neither 'blue' nor 'green.' The primary term for 'purple' is vio- 
letti. There also exists a rather literary and Biblical sounding term, purppuranpunainen, literally 'purple red.' Koski (1983:231) asked 45 Finns the meaning of this term, but it was most commonly defined as 'bright red,' other definitions being 'red,' 'dark red,' and 'dark red close to violet.' Pinkki 'pink' is a late loan. It is replacing an older established term, vaaleanpunainen, which literally means 'pale red.' Also lila 'lilac,' a hyponym of violetti, is a relative newcomer.

Considering the number of Finnish color terms discussed, interest in cooler colors is converse to the red-biased development of English. The preference is shown by violetti, turkoosi (older than pinkki) and lila. This may reflect environmental chromaticness due to the climate in Finland, in which warmer green periods may only last five months of the year. Winter days are short and dark but sometimes enlivened by the appearance of the sun, which makes the snow glittering white and the sky dazzling blue. Blue and white are also the colors of the Finnish flag, symbolizing the sky, lakes, and snow, which are regarded as the most beautiful natural elements of the nation. There are indications of a similar preference for coolness in Russian, which has two basic terms for 'blue' (Paramei 2005, 2007).

Finnish brightness and saturation qualifiers are limited and seldom used: vaalean 'light, pale,' tumman 'dark, kirkkaan 'bright,' and syvän 'deep.' These are normally written together with the color term, as with vaaleanpunainen, and often refer to both brightness and saturation at the same time (Koski 1983:314-9).

Vaalean 'light, pale' in TuSa distributes unevenly across basic color categories: red 55, blue 13 , brown 7 , green 6 , yellow 3 , grey 3 , turquoise 1 . The exceptionally large number of occurrences of vaaleanpunainen 'light, pale red' indicates that there is a mental readiness for a pink basic term and that the recent loan pinkki has a good chance of becoming such. Vaaleansininen 'light blue' is also rather established, which additionally supports the idea of Finnish preference for cool colors. Vaalean expresses both brightness (lightness) and saturation.

Tumman 'dark' occurs as follows: blue 20, brown 20 , red 13 , grey 6 , green 5 , bluered 2, orange 1. Although tumman occurs with certain color terms more than others, the larger numbers show that nonbasic terms covering dark brown, dark blue, and dark red might be expected to develop.

Kirkkaan 'bright' exhibits only these collocations: yellow 12, red 10, green 7, blue 4 . Kirkkaan is used with the names of purest hues, as is English bright, and it refers to both brightness and saturation.

Syvän 'deep' only expresses saturation. It shows fewest occurrences: red 6, blue 2, green 2, black 2, orange 2. According to Koski (1983:316), syvän in this sense may be a late translation loan from either Swedish or German.

There are some additional less common qualifiers, but their frequency is low. In general, it seems Finnish brightness and saturation qualifiers appeared in Finnish at a similar phase as they did in English. There is no evidence of this kind of qualifier use in the SKVR corpus of old folk poetry, which only contains few other occurrences of tumma and vaalea. Based on my diachronic analysis of English color terms, I have suggested (Kerttula 
2002:315) that brightness and saturation qualifiers are part of a universal sequence, in which segmenting and naming of color evolves in three phases:

Phase 1: Emphasis on brightness

Terms for 'brightness' and 'darkness'; first hue categories, starting with red.

Phase 2: Emphasis transferred to hue

Additional hue categories, reorganization of some existing terms for new uses.

Phase 3: Creation of brightness and saturation qualifiers

A new method for defining non-hue elements of color.

This process may also include aspects of two- and three-dimensionality. Emphasis on brightness could be related to three-dimensional or spatial color, which may be visualized by thinking of dark woods, dazzling bright sunshine over water, or flames in a fire. Spatial view of color must have been more central in the minds of early people who lived without constant light sources or easily reproducible surface colors, and so in a world where light made a difference. Thus, the transfer of emphasis to hues, which are more clearly associated with two-dimensionality, may trigger a need for describing luminance and saturation in a new way.

\section{The model of relative basicness applied to Finnish color terms}

In the following the model of relative basicness is applied to Finnish color terms. For all criteria, both cumulative and rank order values are shown.

Primacy in Finnish

To determine primacy, I used two Finnish-Finnish dictionaries, Suomen kielen perussanakirja (Itkonen \& Kulonen 1992-2000) and Uusi suomen kielen sanakirja (Nurmi 1998). Primacy is defined by scoring 4 for color terms that are not hyponyms of other color terms, 3 for hyponyms with primary color sense, 2 for hyponyms with secondary color sense, and 1 for hyponyms with less than secondary color sense.

Eight color terms (valkoinen, punainen, musta, vihreä, sininen, keltainen, harmaa, and ruskea) score 4, that is, they are not defined using any other color term. Lila and pinkki are still associated with red, violetti with blue and red. Oranssi and beige are defined in terms of yellow. These all score 3. Apart from violetti, for which one of the dictionaries gives the sense 'violet dye' as primary (which, since it contradicts practice, is ignored), turkoosi 'turquoise' is the only term with its literal sense in active use, scoring 2. 


\section{Frequency in Finnish}

The following table sums the frequencies of the Finnish color terms. Cumulative values are determined by scoring 1 point for each complete set of 50 occurrences and 1 for the partially filled remainder.

The frequencies of valkoinen/valkea 'white' and punainen 'red' are notably higher than those of other established color terms. With smaller frequencies of similar quantity follow musta 'black,' vihreä 'green,' sininen 'blue,' and keltainen 'yellow.' Of the lower frequencies,

Table 3. Primacy of Finnish color terms

\begin{tabular}{lcc}
\hline Color Term & Cumulative Value & Rank \\
\hline Valkoinen/valkea 'white' & 4 & 1 \\
Punainen 'red' & 4 & 1 \\
Musta 'black' & 4 & 1 \\
Vihreä 'green' & 4 & 1 \\
Sininen 'blue' & 4 & 1 \\
Keltainen 'yellow' & 4 & 1 \\
Harmaa 'gray' & 4 & 1 \\
Ruskea 'brown' & 4 & 1 \\
Oranssi 'orange' & 3 & 2 \\
Violetti 'purple' & 3 & 2 \\
Pinkki 'pink' & 3 & 2 \\
Lila 'lilac' & 3 & 2 \\
Beige 'beige' & 3 & 3 \\
Turkoosi 'turquoise' & 2 & 2 \\
\hline
\end{tabular}

Table 4. Frequencies of color terms in TuSa

\begin{tabular}{lrcc}
\hline Color Term & Total & Cumulative Value & Rank \\
\hline Valkoinen/valkea 'white' & $625 / 65$ & & \\
& 690 & 14 & 2 \\
Punainen 'red' & 655 & 14 & 3 \\
Musta 'black' & 554 & 10 & 4 \\
Vihreä 'green' & 393 & 8 & 5 \\
Sininen 'blue' & 386 & 8 & 6 \\
Keltainen 'yellow' & 363 & 8 & 7 \\
Harmaa 'gray' & 233 & 5 & 8 \\
Ruskea 'brown' & 149 & 3 & 9 \\
Oranssi 'orange' & 59 & 2 & 10 \\
Violetti 'purple' & 32 & 1 & 11 \\
Pinkki 'pink' & 15 & 1 & 12 \\
Lila 'lilac' & 13 & 1 & 13 \\
Turkoosi 'turquoise' & 12 & 1 & 14 \\
Beige 'beige' & 8 & & \\
\hline
\end{tabular}


Table 5. Referents of color terms in TuSa

\begin{tabular}{lccc} 
Color Term & No. of Referents & Cumulative Value & Rank \\
\hline Valkoinen/valkea 'white' & 359 & 15 & 1 \\
& 300 & 12 & 2 \\
Punainen 'red' & 269 & 11 & 3 \\
Musta 'black' & 189 & 8 & 4 \\
Sininen 'blue' & 168 & 7 & 5 \\
Vihreä 'green' & 163 & 7 & 6 \\
Keltainen 'yellow' & 121 & 5 & 7 \\
Harmaa 'gray' & 91 & 4 & 8 \\
Ruskea 'brown' & 35 & 2 & 9 \\
Oranssi 'orange' & 15 & 1 & 11 \\
Violetti 'purple' & 10 & 1 & 12 \\
Pinkki 'pink' & 5 & 1 & 13 \\
Lila 'lilac' & 3 & 1 & 14 \\
Turkoosi 'turquoise' & 2 & & \\
Beige 'beige' & & & \\
\hline
\end{tabular}

ruskea 'brown' and harmaa 'gray' represent an older layer. These are followed by later loans headed by oranssi 'orange.'

\section{Application in Finnish}

The cumulative values for application are determined by scoring 1 for each complete set of 25 referents and 1 for the remainder.

Valkoinen 'white' has notably more referents than other color terms. It is followed by punainen 'red,' musta 'black,' sininen 'blue,' vihreä 'green,' and keltainen 'yellow.' A full comparison cannot be made with the English result, which only measures certain levels of application due to a method based on counting referents grouped. However, the corresponding English terms also show the highest values.

The numbers of referents show that there are two clear layers of color terms: the oldest basic terms with numerous referents and later borrowed terms with a limited application. Of the latter, oranssi is closest to the basic color terms in score.

\section{Derivational productivity in Finnish}

The derivational data was taken from Suomen kielen perussanakirja (Haarala et al. 19901994) and updated by Internet searches (December 2003), which reflect the most recent derivational development. Derivational productivity was calculated by scoring one credit for the base form and each of the following categories if the term had one or more of these derivatives: 
adjective, 'somewhat $x$,' e.g., mustahko 'blackish';

adjective, present participle of transitive verb, e.g., mustaava 'making black';

adjective, past participle of transitive verb, e.g., punattu 'made red';

adjective, past participle of the transitive verb 'make $x$ ' negated, e.g., punaamaton, 'not made red';

adjective, present participle of intransitive verb, e.g., sinistyvä 'becoming blue';

adjective, past participle of intransitive verb, e.g., sinistynyt 'become blue';

adjective, past participle of intransitive verb 'become $x$ ' negated, e.g., kellastumaton, 'not yellowed';

noun, zero-derivation, e.g., keltainen 'yellow';

noun, formed with a suffix, e.g., keltaisuus 'yellowness';

noun formed from transitive verb, e.g., keltaaminen 'making yellow';

noun formed from intransitive verb, e.g., mustuminen 'becoming black';

transitive verb, e.g., punata 'make red';

intransitive verb, e.g., mustua 'become black.'

The basic terms have the highest derivational values as shown in Table 6 .

Derivational productivity may not be an ideal parameter in Finnish, because this agglutinative language allows freer creation of new derivatives than, say, English. However, the derivations distinguish the most established color terms from the nonbasic. The notably higher and similar numbers of the basic color terms indicate that they could be identified by derivation alone. The low value of oranssi suggests that it has not yet become basic.

Table 6. The derivatives of color terms in TuSa

\begin{tabular}{lcc}
\hline Color Term & No. of Derivatives (Cum. Value) & Rank \\
\hline Valkoinen/valkea 'white' & 14 & 1 \\
Musta 'black' & 14 & 1 \\
Punainen 'red' & 14 & 1 \\
Harmaa 'gray' & 13 & 2 \\
Keltainen 'yellow' & 13 & 2 \\
Vihreä 'green' & 13 & 2 \\
Sininen 'blue' & 12 & 3 \\
Ruskea 'brown' & 12 & 3 \\
Oranssi 'orange' & 4 & 4 \\
Violetti 'purple' & 3 & 5 \\
Lila 'lilac' & 3 & 5 \\
Pinkki 'pink' & 2 & 6 \\
Beige 'beige' & 2 & 6 \\
Turkoosi 'turquoise' & 2 & 6 \\
\hline
\end{tabular}




\section{Additional parameters considered}

Other color term uses, too, seem to differ between basic and nonbasic, providing further indication.

\section{The proportions of nouns and adjectives}

Steinvall (2002:73-78) notes that proportions of nominal and adjectival uses of English color terms differ and that this correlates in reverse order with the frequencies of terms. Table 7 shows the proportions of nouns and adjectives of Finnish color terms in TuSa. The percentages indicate that older basic color terms are predominantly adjectives rather than nouns, while the percentages change toward favoring nouns among the newest basic color terms.

Rough correlation with basicness emerges in adjective/noun percentages. However, comparison with values of the four main parameters shows that this might not mirror relative basicness in this kind of corpus research. For example, pinkki has a more advanced position than harmaa, which is a notably older and more established term. (The high noun frequency of harmaa may be explained by its frequent use in TuSa fashion texts in 1999). In general, terms for warm colors seem more advanced here than by the criteria used in the analysis. The position of pinkki may suggest that it is rapidly replacing vaaleanpunainen 'pale red.' In this respect, the proportions of adjectives to nouns might be used for predicting whether a color term is gaining or losing a footing. The four main parameters show that turkoosi, a relatively old loan, is losing its position and its noun percentage sustains this.

Table 7. The noun/adjective proportions of Finnish color terms

\begin{tabular}{lcccc}
\hline Color Term & Nouns & Adjectives & Total & Noun \% \\
\hline Musta 'black' & 56 & 498 & 554 & 10.1 \\
Valkoinen/valkea 'white' & $70 / 3$ & $555 / 62$ & 690 & 10.6 \\
Punainen 'red' & 74 & 581 & 655 & 11.3 \\
Keltainen 'yellow' & 41 & 322 & 363 & 11.3 \\
Sininen 'blue' & 63 & 323 & 386 & 16.3 \\
Vihreä 'green' & 78 & 315 & 393 & 19.9 \\
Ruskea 'brown' & 30 & 119 & 149 & 20.1 \\
Pinkki 'pink' & 4 & 11 & 15 & 26.7 \\
Oranssi 'orange' & 18 & 41 & 59 & 30.5 \\
Harmaa 'gray' & 75 & 21 & 233 & 32.2 \\
Violetti 'purple' & 11 & 7 & 32 & 34.4 \\
Lila 'lilac' & 6 & 3 & 13 & 46.3 \\
Beige 'beige' & 5 & 4 & 12 & 62.5 \\
Turkoosi 'turquoise' & 8 & & & 66.7 \\
\hline
\end{tabular}


Despite these discrepancies, this criterion might be a strong candidate for an additional parameter, provided that versatile corpus data are used, that is, one which includes spoken language.

\section{Metaphor}

Study of the metaphoric use among Finnish color names shows that only the basic color terms are employed to create metaphors. But they may not be fully reliable in defining the relative basicness of color terms for two reasons. Firstly, a metaphor does not represent the color sense of the term, but has moved elsewhere. Secondly, a metaphoric sense often generates expressions within its new sense. Thus, the term for 'black,' musta, has notably more metaphors (39) than others, as with 'dark, evil', which many tropes elaborate without apparent thought of color. Next comes vihreä 'green' with many metaphors based on ecological thinking that even outnumber occurrences of actual color sense in those contexts. Such results do not conform to those from the four main parameters. Metaphoric use may only confirm the basicness of a color term if not otherwise certain.

\section{Names of animals and plants or other nominal compounds}

TuSa and listings provided by Suomen kielen perussanakirja show that only basic Finnish color terms are used to create animal and plant names. The reason is clear: these groups are no longer very productive. For example, the relatively established term oranssi 'orange' does not occur in animal and plant names. Although these two groups reflect basicness to certain extent, they are not usable as an individual parameter. Separately they may reveal something of historical color semantics. Using both the names of animals and plants - as well as other nominal compounds - as one parameter might be useful in languages that have multiple color terms and extensive corpora.

Names of illnesses and terms for food are formed with color terms, but in TuSa they are too few for measuring relative basicness.

\section{Proper names}

Only basic color terms are used in proper names in TuSa. However, there are plenty of translations (e.g., Valkoinen talo 'White house,' Punainen mylly 'Moulin Rouge') among them, and this parameter is hardly usable with similar corpus data. It might be useful when examining anthropological and geographical corpora.

Additional more general parameters might also be considered such as measuring salience by occurrences across different source or text types. As Biggam (1995:51-65) has shown, this kind of sociolinguistic analysis is especially needed, when sources are scarce, as with ancient languages. 


\section{Scoring relative basicness of Finnish color terms}

In Table 8, the four parameters produce total values, which are given with corresponding values for English color terms. These values are only to show differences in terms within a language and not to be considered as statistically valid in the strict sense. However, the Finnish rank order values are directly based on given statistical results, not on cumulative values deducted from them.

Cumulative values show that valkoinen and punainen score notably highest on relative basicness, which corresponds to the result from English. The score of valkoinen may relate to its common Finno-Ugric association with the senses 'light' and 'shining.' That the term for 'white' in unrelated languages begets the highest value of relative basicness implies potential wide recurrence, a fact to watch in pursuing hypotheses of universality. Valkoinen is closely followed by punainen, which further corresponds to the results from English. The high value of punainen may be explained by its old meaning of 'color.' On the whole, the orders are similar in Finnish and English. Musta has a relatively higher value than English black, which may imply that it has a comparatively longer history as the basic term for black.

The cumulative values of relative basicness show that eight Finnish color terms are basic: valkoinen, punainen, musta, sininen, keltainen, vihreä, harmaa, ruskea. Of these, ruskea is the newest. However, oranssi has an outlying lowest value, which suggests that it is nonbasic, although it is probably developing into a basic color term.

Compared with English, Finnish color terms are few. Violetti, pinkki, lila, and beige are newer nonbasic color terms. The values indicate that oranssi will reach basicness prior to others, while in English purple and pink reached basicness before orange. Beige

Table 8. The cumulative and rank order values of relative basicness of Finnish color terms

\begin{tabular}{|c|c|c|c|c|c|c|c|}
\hline \multirow[b]{2}{*}{ Color Term } & \multicolumn{4}{|c|}{ Cumulative rank } & \multicolumn{2}{|c|}{ Tot. Cum. Values } & \multirow{2}{*}{$\frac{\text { Total }}{\text { (Rank)1 }}$} \\
\hline & Prim. & Freq. & Appl & Deriv. & Finnish & English & \\
\hline $\begin{array}{l}\text { Valkoinen/ } \\
\text { valkea 'white' }\end{array}$ & $4(1)$ & $14(1)$ & $15(1)$ & $14(1)$ & 47 & 38 & $4(1)$ \\
\hline Punainen 'red' & $4(1)$ & $14(2)$ & $12(2)$ & $14(1)$ & 44 & 35 & $6(2)$ \\
\hline Musta 'black' & $4(1)$ & $10(3)$ & $11(3)$ & $14(1)$ & 39 & 29 & $8(3)$ \\
\hline Vihreä 'green' & $4(1)$ & $8(4)$ & $7(5)$ & $13(2)$ & 32 & 29 & $12(4)$ \\
\hline Sininen 'blue' & $4(1)$ & $8(5)$ & $8(4)$ & $12(3)$ & 32 & 29 & $13(5)$ \\
\hline Keltainen 'yellow' & $4(1)$ & $8(6)$ & $7(6)$ & $13(2)$ & 32 & 29 & $15(6)$ \\
\hline Harmaa 'gray' & $4(1)$ & $5(7)$ & $5(7)$ & $13(2)$ & 27 & 27 & $17(7)$ \\
\hline Ruskea 'brown' & $4(2)$ & $3(8)$ & $4(8)$ & $12(3)$ & 23 & 29 & $21(8)$ \\
\hline Oranssi 'orange' & $3(2)$ & $2(9)$ & $2(9)$ & $4(4)$ & 11 & 20 & $24(9)$ \\
\hline Violetti 'purple' & $3(2)$ & $1(10)$ & $1(10)$ & $3(5)$ & 8 & 28 & $27(10)$ \\
\hline Lila 'lilac' & $3(2)$ & $1(12)$ & $1(12)$ & $3(5)$ & 8 & 15 & $31(12)$ \\
\hline Pinkki 'pink' & $3(2)$ & $1(11)$ & $1(11)$ & $2(6)$ & 7 & 28 & $30(11)$ \\
\hline Turkoosi 'turquoise' & $2(3)$ & $1(13)$ & $1(13)$ & $2(6)$ & 6 & 14 & $35(13)$ \\
\hline Beige 'beige' & $3(2)$ & $1(14)$ & $1(14)$ & $2(6)$ & 6 & 14 & $36(14)$ \\
\hline
\end{tabular}


shows no signs of deeper rooting, probably because its un-Finnish orthography hinders spontaneous derivation. Turkoosi is shedding its former popularity.

The final rank order values are fairly in line with the cumulative values, showing that this method could be used as a simple alternative to the Berlin and Kay criteria for defining relative basicness. When used together, the two methods seem to complement each other.

\section{Conclusion}

Readapted basic color term criteria substitute for those of Berlin and Kay in analyses by diachronic data and text data. Their reliability is sustained in application to English by producing a plausible ranking that, in addition, matches the order in which English basic color terms are independently known to have developed historically. As further support of their validity, they apply with equal facility and plausibility in Finnish, whose result largely matches that from English. The correspondence suggests a potential universal whereby basic color-term status accumulates over time such that its strength may be ranked within a language and rankings expected to coincide between languages. The coincidence would derive from the tendency of basic color terms to evolve everywhere in a constrained order.

\section{References}

Berlin, Brent \& Paul Kay (1969). Basic Color terms: Their Universality and Evolution. Berkeley, CA: University of California Press.

Biggam, C. P. (1995). Sociolinguistic aspects of Old English colour lexemes. Anglo-Saxon England, 24, $51-65$.

Biggam, C. P. (1998). Grey in Old English. London: Runetree Press.

Biggam, Carole P. (2007). The ambiguity of brightness (with special reference to Old English) and a new model for color description in semantics. In R. E. MacLaury, G. V. Paramei \& D. Dedrick (Eds.), Anthropology of Color: Interdisciplinary Multilevel Modeling (171-188). Amsterdam: John Benjamins.

British National Corpus. Retrieved from http://www.natcorp.ox.ac.uk.

Corbett, Greville G. \& Ian R. L. Davies (1997). Establishing basic color terms: Measures and techniques. In C. L. Hardin \& L. Maffi (Eds.), Color Categories in Thought and Language (197-223). Cambridge: Cambridge University Press.

Haarala, R. et al. (Eds.) (1990-1994). Suomen kielen perussanakirja [The Basic Dictionary of the Finnish Language]. Helsinki: Valtion painatuskeskus.

Hardin, Clyde L. \& Luisa Maffi (Eds.) (1997). Color Categories in Thought and Language. Cambridge: Cambridge University Press.

Itkonen, Erkki \& Ulla Maija Kulonen (Eds.) (1992-2000). Suomen sanojen alkuperä. Etymologinen sanakirja [The Origin of Finnish Words. Etymological Dictionary]. (Suomalaisen Kirjallisuuden Seuran toimituksia, 556; Kotimaisten kielten tutkimuskeskuksen julkaisuja, 62.) Helsinki: Suomalaisen Kirjallisuuden Seura, Kotimaisten kielten tutkimuskeskus.

Kalevala. Retrieved from http://www.finlit.fi/kalevala/indexeng.html English translation retrieved from http://www.sacred-texts.com/neu/kveng/index.htm. 
Kay, Paul (1975). Synchronic variability and diachronic change in basic color terms. Language in Society, 4, 257-270.

Kerttula, Seija (2002). English Colour terms: Etymology, Chronology, and Relative Basicness. [Mémoires de la Société Néophilologique de Helsinki, 60]. Helsinki: Société Néophilologique.

Koski, Mauno (1983). Värien nimitykset suomessa ja lähisukukielissä [Color Names in Finnish and CloselyRelated Languages]. Helsinki: Suomalaisen kirjallisuuden seura.

MacLaury, Robert E. (1992). From brightness to hue: An explanatory model of color-category evolution. Current Anthropology, 33, 137-186.

Nurmi, T. (1998). Uusi suomen kielen sanakirja [New Dictionary of the Finnish Language]. Jyväskylä: Gummerus.

Oja, Vilja (2001). Linguistic studies of Estonian colour terminology. (Dissertationes Philologiae Estonicae Universitatis Tartuensis 9). Tartu: Tartu University Press.

Oja, Vilja (2007). Color naming in Estonian and cognate languages. In R. E. MacLaury, G. V. Paramei \& D. Dedrick (Eds.), Anthropology of Color: Interdisciplinary Multilevel Modeling (189-210). Amsterdam: John Benjamins.

Paramei, Galina V. (2005). Singing the Russian 'blues': An argument for culturally basic color terms. CrossCultural Research: The Journal of Comparative Social Studies, 39, 10-38.

Paramei, Galina V. (2007). Russian 'blues’: Controversies of basicness. In R. E. MacLaury, G. V. Paramei \& D. Dedrick (Eds.), Anthropology of Color: Interdisciplinary Multilevel Modeling (75-106). Amsterdam: John Benjamins.

Procter, P. et al. (Eds.). (1995). Cambridge International Dictionary of English. Bath: Bath Press.

Roberts, J., Kay, C. \& Grundy, L. (Eds.) (1995). Thesaurus of Old English. London: King's College London. Saunders, Barbara A. C. (1992). The invention of Basic Colour Terms. Utrecht: ISOR.

Sinclair, John et al. (Eds.) (1995) Collins COBUILD English Dictionary. London: HarperCollins.

SKVR 2003. Suomen Kansan Vanhat Runot [Finnish Folk Poetry] in digital format (20,003 texts, November 2003). Compiled by the Finnish Literature Society and CSC (Finnish IT Center for Science). Retrieved from http://www.csc.fi/kielipankki

Steinvall, Anders (2002). English Color Terms in Context. Ph.D. diss. Umeå University: Skrifter från moderna språk 3.

Summers, D. et al. (Eds.). (1995). Longman Dictionary of Contemporary English. Third edition. Bungay, Suffolk: Clays Ltd.

Sutrop, Urmas (2002). The Vocabulary of Sense Perception in Estonian. Frankfurt am Main: Peter Lang.

Turun Sanomat (1999). A collection of newspaper articles (11,821,904 words). Compiled by the Department of General Linguistics, University of Helsinki, and CSC (Finnish IT Center for Science). Retrieved from http://www.csc.fi/kielipankki

University of Glasgow. (2005). Historical thesaurus of English. Retrieved from http://www2.arts.gla.ac.uk/ SESLL/EngLang/thesaur/homepage.htm 



\title{
The ambiguity of brightness (with special reference to Old English) and a new model for color description in semantics
}

\author{
Carole P. Biggam \\ Department of English Language, University of Glasgow, United Kingdom
}

The paper addresses problems of ambiguity in the use of the English words bright and brightness in certain publications on color semantics. The Old English language (records date from the late fifth century to c.1150 A.D.) is taken as a case study. After establishing which visual sensations could be described as 'bright', the usages of various authors writing on Old English color are investigated, and found to differ considerably. The evidence for the frequently encountered statement that Old English was concerned almost entirely with 'brightness', and not hue, is then investigated, and (hopefully) a more balanced conclusion is reached. Finally, a proposed metalanguage for color statements concerning historical languages (where native speakers cannot be consulted) is presented in an effort to avoid future ambiguity.

The trouble with the word "brightness" is that it has been used in a great many different senses ... Clarification of terminology is certainly in order.

(Beck 1972:14)

\section{Introduction}

The words bright and brightness have often been used ambiguously in color semantics, as others have also noticed (see MacLaury 2005). The nature of the problem will be illustrated here by using Old English (Anglo-Saxon) as a case study, and it will also be argued that the Old English (OE) color system has usually been assessed on only partial evidence. In addition, an attempt will be made to provide a standardized model and terminology for research in color semantics, with the aim of avoiding ambiguity in future work.

It is well known that any research which aims, as far as possible, at objectivity, must employ a technical vocabulary with agreed and fixed meanings (unlike popular vocabulary) to facilitate unambiguous discussion. Controlled language of this type need not consist of dedicated terms which occur in no other context, but, where vocabulary is 
pressed into technical service from everyday language, it must have a carefully delimited definition.

\section{The meaning of bright}

As a first step, the everyday usage of bright will be considered. The Oxford English Dictionary $(O E D)$ records nine major senses in which bright is, or has been used in Modern English (ModE). (I exclude sense 10, which concerns compound words with bright). Two of the nine senses are relevant to color studies, and their definitions, including subdivisions, are as follows:

1. Shining; emitting, reflecting, or pervaded by much light.

a. said of luminaries.

b. of polished metals, precious stones, and other objects whose surfaces naturally reflect light.

c. of illuminated surfaces, of the day in sunshine, etc.

d. of transparent substances: Clear, translucent.

[I exclude sense 1e, which is figurative, meaning, for example, 'lit up with happiness'].

4. a. Of vivid or brilliant color: used also with names of color, as bright red.

[Omitted is sense $4 \mathrm{~b}$, a specialized term for a color of tobacco].

When using bright in color semantics, all writers on Old English appear to be agreed that sense $4 \mathrm{a}$ above is excluded, although the majority of them do not state this exclusion. The lack of any clear statement can occasionally lead to doubt, as in the following: "... a moment's thought will show that the sensation of pale lemon yellow is very different from that of bright lemon yellow" (Lerner 1951:246). The reader is left wondering whether Lerner's "bright lemon yellow" could actually be vivid yellow, even though he appears not to use this sense of bright elsewhere in the paper. Usually, of course, the vividness sense which occurs in popular usage is considered a feature of saturation, rather than brightness.

Having removed vividness, the remaining senses of the OED's definition can be listed to show the semantic elements of ModE bright in popular usage. The elements are: lightemitting, light-reflecting (of surfaces, polished and similar), pervaded by much light (of surfaces, highly illuminated), pervaded by much light (of spaces, for example, daylight), transparent, and translucent.

The above list does not, however, include paleness, which has also been described as 'bright' in semantics. Pale colors and achromatics are perceived as reflecting more light than their dark equivalents, and, moreover, certain hues, especially yellow, are considered to be always pale. Another phenomenon of light, usually included by semanticists under the heading 'brightness', is the constancy, or lack of it, of the light source. Intermittent light can be referred to in Modern English by words such as glinting, twinkling, and flashing, and constant light by words such as gleaming and glowing. 
The list of elements which have been denoted by bright in color semantics can now be presented as follows:

1. Light-emitting: a. intermittent; b. constant.

2. Light-reflecting: a. intermittent; b. constant.

3. Pale.

4. Pervaded by light (surfaces).

5. Pervaded by light (spaces).

6. Transparent.

7. Translucent.

The list is intended to show the semantic elements which have been involved in the various published uses of the word bright, but it should be noted that the use of the noun brightness in color semantics usually implies a range extending from 'positive' brightness to 'negative' brightness (such as darkness).

\section{Mead's "brightness"}

It is now time to justify my statement that ambiguity has arisen in the past in the consideration of brightness, and that this can be demonstrated by the case of Old English. (Where brightness appears in double quotation-marks, it indicates the individual usage of the author under discussion).

The first major article on color in Old English was published by William E. Mead in 1899. Mead contrasts "words expressing light or brightness" with "words denoting or implying darkness" (1899:175). He lists all these words, in order to exclude them from the main body of his paper on "color". He interprets color as referring to hues and achromatics only, and describes the lightness, "brightness" and darkness words as "in the strictest sense colorless" (Mead 1899: 176). Although he classifies lightness with "brightness", he always uses the two terms, rather than subsuming one under the heading of the other. He lists the lightness and "brightness" words together in alphabetical order, without explaining the semantic difference between them (Mead 1899:174). Lightness is also ambiguous, meaning either 'pale' (surfaces) or 'well-lit' (spaces), but Mead's inclusion of whiteness terms with the 'color' words, shows that his 'lightness' refers to the lighting of spaces. His "brightness"/lightness list includes words which clearly refer to the pervasion of space by light (such as afenleoht 'evening light'), light-emission (sunne 'sun'), and light-reflection (goldbeorht, literally 'gold-bright').

It should be noted that "having ... cleared the ground" by excluding "brightness", lightness and darkness words, Mead proceeds to describe what he calls "color-words", and this section includes terms such as hwit 'white, radiant' and blac 'shining, pale', listed under the heading 'WHITE'. However, he writes, "Nearly all the passages where these words are used imply something bright or shining" (Mead 1899:176), but he does not include these words in his lightness/"brightness" category if he detects even the slightest hint of whiteness or paleness. 
It is now clear that, when Mead refers to "brightness", he includes the elements of light-emission, light-reflection, pervasion of spaces by light, and transparency. Inclusion of the last element is suggested by Mead's mention of gloeshluttor 'clear, transparent' when giving examples of words that "suggest brightness", but which he had left out of his already long list. Finally, it is significant, in view of later publications on Old English color, that Mead (1899: 193-4) mentions "brightness" in connection with only one potential hue term, and that is brun.

\section{Lerner's "brightness"}

The next paper on Old English color which is frequently cited was published by L. D. Lerner in 1951. Lerner lists four "sensations" which make up a color impression: hue, admixture with white, admixture with black, and "brightness". He defines "brightness" as being "determined by the amount of light falling on or emanating from it [a color]", and he gives an example of the significance of "brightness": "... we tend not to notice that different hues of a similar brightness may give very similar sensations. A piece of green corduroy, well rubbed and caught by the sun at the right angle, will be almost indistinguishable from a similar piece of yellow, orange or even red material" (Lerner 1951:246).

Lerner's "brightness" includes light-emission. His definition of "brightness" as the amount of light emanating from a color, could be taken to exclude light-sources, if he did not use a passage in Beowulf which includes "gleaming lights", as an example of "brightness". He also includes both light-reflection, since he cites referents of brun which "flash in the sunlight" as proof of this word's brightness element, and the pervasion of surfaces by light, since his corduroy samples are all "bright" because of a similar level of surface illumination. The pervasion of spaces by light is not mentioned, but would appear to be included, since he discusses the negative brightness of a dark night (1951:247). Lerner clearly excludes paleness from "brightness", since his 'admixture with white' sensation is separate from it. There is no discussion of transparency or translucency, but they would appear to be excluded by his general definition of "brightness" which involves light falling on a color, not passing through an object.

The main point which Lerner wished to convey was that, while Modern English emphasizes hue in its color vocabulary, this was not necessarily true of Old English. He makes a statement which has been extremely influential in Old English color studies, and beyond: "A study of Anglo-Saxon color words in their contexts will show, I think, that the authors were much more interested in brightness than we are" (1951:247). He then discusses OE brun, fealu and wann, suggesting that the presence of strong "brightness" elements is the reason why they had caused problems for translators. (Rough traditional definitions are: brun 'brown, dark, shining'; fealu 'yellow, tawny, grey'; wann 'dark').

Lerner's suggestion that "brightness" was more important to the Anglo-Saxons was, by the 1990s, interpreted as meaning that hue was barely recognized by them. Lerner is always cited in support of this view but it has been forgotten that, at the end of his article, he writes, "A more thorough investigation might, in fact, show that a transitional stage 
had already been reached in the Old English period, and that many of the color words which seem to have something like their modern senses did indeed have that" (1951:249). It is interesting to note that Lerner sees the need for "a more thorough investigation". (His paper was just over three pages long, and appeared as a 'Miscellaneous Note').

\section{Barley's "brightness"}

A third influential article on color in Old English was published by Nigel F. Barley in 1974. Barley lists the three variables of perceived color as hue, saturation, and "brightness". He defines brightness as "the amount of light transmitted", and writes that "The primacy of hues [in Modern English] is shown ... by the fact that we reserve the noun category for their designation and use 'pale' and 'dark' as qualifiers to express the brightness dimension". To make his meaning absolutely clear, Barley includes a diagram of the color solid, involving hues, saturation, and a "brightness" range from 'light' to dark, and he also describes the depiction of "brightness" on the Munsell color chart (Barley 1974:1516). This "brightness" is quite different from that of Lerner who describes the increasingly paler hues ending in white, and the increasingly darker hues ending in black on the color pyramid, but adds "Brightness is not represented on the pyramid ...", thus differentiating paleness/darkness from "brightness" (Lerner 1951:246). By contrast, Barley regards them as identical. He is aware that his "brightness" is not the same as Lerner's, and refers to the latter's use of the term as "somewhat vague" (Barley 1974:20).

Barley's statements and diagrams suggest that his "brightness" excludes light-emission and light-reflection (shininess), since they cannot be shown on the color solid or the Munsell chart, and his statement quoted above, that pale and dark are used as brightness qualifiers in the modern language, implies an exclusive equation of "brightness" with paleness/darkness. His Figure 4, however, appears to contradict this conclusion, as it lists the "white/light" Old English vocabulary as blac, hwit, beorht, leoht and scir (1974:18), and any Old English dictionary would include senses like 'shining' in the definitions of all of these. The reader's comprehension suffers another blow when Barley mentions Lerner's suggested "brightness" element in brun, fealu and wann, which clearly involves light-reflection (its presence or absence). Criticizing Lerner's use of brightness, however, Barley writes, “... he already has a light-dark axis in his model” (Barley 1974:20), thereby apparently reaffirming his own equation of brightness with paleness/darkness only.

It is also unclear whether the pervasion of space by light is included in Barley's "brightness", but his plotting of a "brightness" range on the color solid, makes it unlikely. However, when he writes "Even one whose interests are principally anthropological cannot but be struck by the fact that Beowulf is a poem of bright day and darkest night, light ale-hall and gloomy wasteland" (1974:17), he implies he is including the lighting of spaces, although this must remain uncertain. Whether transparency and translucency are also included is unknown. The only bright element which can be clearly demonstrated from Barley's article is paleness, and, while this may well be a misunderstanding, the very confusion of the reader supports the contention that the use of brightness is problematic. 


\section{Mead, Lerner and Barley compared}

The "brightnesses" of Mead, Lerner and Barley can now be compared, in terms of the brightness elements listed earlier in this paper. The results are:

1. Mead: light-emission, light-reflection, pervasion of spaces by light, transparency.

2. Lerner: light-emission, light-reflection, pervasion of surfaces by light, pervasion of spaces by light.

3. Barley: paleness, ?pervasion of spaces by light.

It can be seen from these results that the three most frequently cited writers on Old English color could hardly differ more in their usage of the term brightness.

The significance of Barley's article for later scholars is that he presents the suggestions put forward by Mead and Lerner as facts. He writes, "The main stress of the Old English system falls firstly, not upon hue, but upon brightness" (Barley 1974:17), and, elsewhere, "The fact that the Anglo-Saxon color system was not hue-stressing ..." (1974: 18; my italics). These confident and unambiguous statements do not reflect the tenor of Mead's paper ("I realize clearly the tentative character of the paper" (1899:205-6)), nor of Lerner's recommendation for "a more thorough investigation" (1951:249). Barley was unaware of König's more thorough collection of data (see below) until after his article had been completed (Barley 1974: 19).

\section{König's "brightness"}

Mead, Lerner and Barley are the major sources of later writers' information on Old English color vocabulary and its meaning, but some authors also refer to König and to Bragg. Günter König presented his dissertation on Old English color in 1957, and it was the first major attempt to study poetry and prose texts. The dissertation consists of a detailed and thorough listing of the Old English color vocabulary, with brief contexts, and a classification into three categories: Farben; Glanz und Schein and Hell und Dunkel. His first category consists of words denoting hues and achromatics, the second is for light-emission and light-reflection, and the third for pervasion of spaces by light, transparency, and darkness. This means that König essentially takes Mead's “"brightness"/lightness' heading and divides it into two. Like Mead, he classifies paleness with hues and achromatics, and it is not clear whether he includes translucency.

\section{Bragg's "brightness"}

By the time Lois Bragg published her paper on color words in Beowulf' in 1985, there was already a great deal of confusion concerning color in Old English. Not everyone realized that the major authors on the subject had used brightness in different ways, but it was generally accepted that the Old English color vocabulary was more about 'bright- 
ness' than hue. The fact that the surviving Old English poems chose to create atmosphere by means other than hue was often remarked upon; for example, Mills writes " ... the ancient English poets chose to film their war movies in black and white" (1976:169).

"Brightness" was now spreading everywhere. Although Mead (1899: 193-194) had suggested that brun could "possibly" mean "brightness" in certain contexts, Lerner concluded that the only element occurring in all the referents of brun was light-reflection ("they flash in the sunlight"), that the "essential quality" of fealu was "brightness", and that of wann was lack of "brightness" (1951:247-248). Barley took this tendency further, suggesting that brun, fealu, wann and groeg "are terms generally applicable to glossy things" (1974:24).

Bragg, however, was more cautious. After her review of nine words in Beowulf, she concludes "... the colors ... are used with considerable specificity as to hue" (1985:48). In particular, her verdict on brun, which Lerner and Barley had considered to be a "brightness" word with hardly any hue meaning, is even more 'hue-friendly' than Mead's, as she interprets it as exclusively 'dark brown' in some contexts, and 'dark brown plus glossy' in others.

\section{Casson's “brightness”}

In spite of Bragg's implied warning that the rejection of hue in Old English color had gone too far, the tendency continued still further. It should be noted that only König had, thus far, produced a quantity of basic data from both poetry and prose which could be used to assess the Old English color system, although even he had omitted certain sources, such as charter bounds and place-names, and he had considered contexts in a minimal fashion. Unfortunately, his work was frequently overlooked in the English-speaking world.

MacLaury's publication of 'From brightness to hue: An explanatory model of colorcategory evolution' (1992) is also of significance for the present discussion. He suggests that, in the development of most languages' color systems, the importance of "brightness" gradually decreases and that of hue increases. Accordingly, he postulates four types of color category: brightness (Type D), brightness with hue (Type C), hue with brightness (Type B), and hue (Type A). It should be noted, however, that MacLaury's "brightness", like Barley's, is tied to the Munsell color chart, a color denotation system for surface colors, on which, by definition, certain semantic features of brightness, as listed at the beginning of this article, cannot be plotted.

Also in 1992, Ronald W. Casson published a paper which supported MacLaury's views, although he prefers the terms 'pure-brightness', 'brightness-dominated', 'huedominated', and 'pure-hue' for MacLaury's four types of color category.

Casson writes "Anglo-Saxon or Old English color vocabulary focused primarily on brightness and only secondarily on hue". So what exactly is Casson's "brightness"? He describes it as follows: “. . ranging from light to dark and including quantity of luminescence (from light sources) and degree of reflectivity (from reflecting surfaces)" (1992:395). This is probably the fullest explanation that any of the writers so far mentioned have given of 
their own use of brightness. It clearly includes light-emission, although his use of the word luminescence 'the emission of light by a substance that has not been heated' (COED), if taken literally, would exclude sources like the sun. Casson's "brightness" also includes light-reflection, and the pervasion of spaces by light, the latter because his reference to the "light to dark" range must refer to the lighting of spaces rather than paleness, since he includes "admixture [of a hue] with white or black" in his saturation range (1992:395).

Casson (1992:395-6) proceeds to classify the Old English color categories according to MacLaury's four-part scheme. He recognizes a pure-brightness category, represented by words such as beorht 'bright' and deorc 'dark', and then classifies the Old English color categories of names for BLACK, WHITE, YeLLOW and BLUE as "brightness"-dominated, RED as hue-dominated, and GREEN as pure-hue. Casson's pure-brightness category is, however, hard to reconcile with MacLaury's Type D ("brightness") category. Specifically, MacLaury's "brightness" category includes the achromatics, and, as exist in some languages, color categories consisting entirely of pastel colors (1992:150), while Casson's 'pure-brightness' category consists of light-emission, light-reflection, and the pervasion of spaces by light.

Casson published an elaboration of his earlier ideas in 1997, and this paper marks the apex of the long trail from Mead's tentative suggestion that brun may have a "brightness" element in some contexts, to almost every Old English color word being, supposedly, dominated by brightness. Even the one word which Casson (1992:396) had once described as "pure-hue", namely, grene 'green', partially surrenders to "brightness" in his later paper: "Old English grene was basically a hue term, but it also had significant brightness senses" (1997:229). Casson's position is that "Hue was only minimally conceptualized in Old English ..." (1997:224).

\section{The quality of the evidence for Old English brightness}

At this stage, I am not dismissing anyone's view of brightness in Old English color, but merely pointing out that we are often talking about different things. It is now time to address the quality of the evidence which has backed the arguments. The hunt for brightness in Old English has been conducted on the basis of incomplete evidence, and an inadequate research methodology. As an illustration of this, I propose to consider OE graeg, since earlier opinions can be compared with the more recent detailed examination of this word (Biggam, 1998:31-99).

Mead: grag = 'grey' (1899:189-90).

Evidence given (including some quotations).

1. Referents: sword, mail-coat, wolf, seagull, flood of the sea, ash-spear with the grey bark still left on the shaft, curling smoke, hoar-frost.

Lerner: græeg not discussed.

König: groeg = grau 'grey' (1957: 149-50, 232-3).

Evidence given (including some brief quotations). 
1. Referents:

a. Proper application (eigentliche Verwendung): goose, seagull, swan, wolf, horse, wheat, earth, mass of water.

b. Abstract application (abstrakte Verwendung): stone, sword, mail- coat, flowers.

c. Symbolic application (symbolische Verwendung): horse (in a dream).

2. Translations: Lat. cinereus.

Barley: groeg = 'grey, glossy' $(1974: 18,24,27)$.

Evidence given (no quotations).

1. Old English referents: horses, other animals, metal, human hair, water.

2. Early Germanic (including Old English) referents: human hair, animal fur (chiefly horses and wolves), iron

Bragg: groeg = 'grey' (1985:50).

Evidence given (including brief quotations from Beowulf, the subject of the paper).

1. Referents:

a. (from Beowulf) spear, mail-coat, sword.

b. (from other sources) animals, birds, metal.

2. Translations: Lat. fuscus.

Casson (1992): grae = 'glossy, grey' (1992:396).

Evidence given (no quotations).

1. Referents:

a. (from Barley) horses, other animals (especially dogs and wolves), metals (primarily iron), possibly human hair, water.

b. (from another source, probably Mead) ash bark.

Casson (1997): grag = 'glossy, grey’ (1997:228).

Evidence given (no quotations).

1. Referents:

a. Hue sense: grey animals, animal fur, human hair, ash bark, wheat, stone and rock, hill, valley.

b. "Reflective and luminous senses": water, wave, iron, sword, spearhead, mail-coat, smoke, hoar-frost.

From the above, it can be seen that, of the major writers on Old English color, only Barley and Casson see a brightness element in grog, and Casson's (1992) evidence is taken from Barley. A review of Casson's later evidence (1997:228) for the "reflective and luminous senses" of groeg should, therefore, indicate what persuaded him to regard this word as predominantly "bright". Since none of the referents he lists is light-emitting, his "luminous" sense can be rejected, and it must be assumed that he sees light-reflection in the other referents. The only way such a belief can be tested is to consider the contexts in which these referents are found, and this has now been done (Biggam 1998). My research methodology, referred to as 'interdisciplinary semantics', aims to wring every last drop of information from the extant evidence, and is described in Biggam (1997:79-90). 
Biggam: groeg = 'grey (greyish hue)' (1998:31-99). (Secondary sense in brackets). Evidence given (including quotations from all known references).

1. Referents (including interdisciplinary studies of contexts):

a. Old English referents: spearheads, wheat (Spelt ear or glume grain), goose, seagull, wolf, sword, stone, boundary stone, pigs, horse, mail-coats, sea, mark on a sword-blade, human hair, iron, ash (from a fire), rock, wave in a storm, mass of water, water, squirrel fur, warm fur garment, colt's foot (a plant).

b. Latin referents: color, yellow/orange dye, human hair, gall dye, horse.

2. Translations: glaucus, ferrugineus, elbus, ferrugo, cycneus, cinereus, croceus, fuscus. Also considered:

3. Contrasts and comparisons with other color words.

4. Alliterative contexts.

5. Early Germanic cognates.

6. Related citations (citations with the same textual origin, which can reduce the emphasis on certain information).

It has been shown above that Casson (1997) confirmed his belief in a light-reflecting semantic element of groeg, and quoted as evidence, the following referents: water, wave, iron, sword, spearhead, mail-coat, smoke, hoar-frost. First of all, smoke and hoar-frost can be rejected, as they do not occur as referents of groeg. This was an error made by Mead, probably resulting from confusion with the smoke referent of hasu, and the hoar-frost referent of har (two other terms with grayness elements). Secondly, the referent 'wave' can also be rejected since the context is a storm, and the water is unlikely to be reflecting sunlight. This leaves the other references to water and those to metal.

The three remaining water references involve the sea, water running down from a hill, and a mass of water. The contexts do not exclude light-reflection, but they could all be understood as involving grayness or a grayish hue.

The five references to iron are all glossary entries in which groeg translates Latin ferrugo or ferrugineus. These words, in Classical Latin, indicated a color ranging from reddish-purple to near-black (OLD 1982:691), and it can be shown that this dark element continued into the early medieval period, in at least one source which was known in Anglo-Saxon England, namely, Isidore of Seville. He states "Ferrugo color est purpurae subnigrae ..."; 'Ferrugo is a blackish purple dye ...' (1911:19.28.6). Isidore's words on ferrugo are quoted in an Anglo-Saxon glossary, now called the Harley Glossary. Other Anglo-Saxons, however, appear to have understood that ferrugo is cognate with Latin ferrum 'iron', and so they translated ferrugineus into Old English as isengroeg 'iron-grey'. This translation appears alongside the 'blackish purple' glossing tradition in the Harley Glossary (Biggam 1998:84-5, 69-70; Oliphant 1966:179). With such associations, it is highly likely that 'iron-grey' was understood to indicate dark grey, and this makes sense to anyone who remembers the dark kitchen-knives which were the norm before the advent of stainless steel. The darkness which is conveyed in these uses of groeg makes it unlikely that a light-reflecting quality was involved, which is what Casson claims, as the metal appears to be unburnished. 
It can now be seen that, although Casson presents eight referents which he claims indicate light-reflecting qualities, two of them are spurious, one of them occurs in an inappropriate context, and one of the remaining five, namely iron, occurs consistently in a translation context involving non-shiny darkness. In addition, metaphorical considerations cast doubt on the "brightness" of the sword and mail-coats. The sword refers to Abraham's weapon when he set out to sacrifice Isaac. The close identification of a man with his sword led to the suggestion that the appearance and condition of Beowulf's sword reflected his own advanced age and scarred body in that particular passage (Biggam 1998: 66). If a similar identification is made of Abraham with his sword, it should be noted that the patriarch was over one hundred years old when he girded on his grog sword. It has also been pointed out that warriors are often compared with wolves in Old English poetry, in phrases such as 'sword-wolves', 'slaughter-wolves' and 'war-wolves', and the Anglo-Saxons referred to the wolf as 'the grey animal'. This may indicate that warriors in their mail-coats were seen as being clad in grey, like wolves (Biggam 1998:174-5). In other words, these metaphorical uses, if acceptable, suggest that grayness, rather than reflectivity, was, at least, the primary element evoked in these contexts, and may have been the only element.

The danger of Lerner's, Barley's and Casson's research methodology, namely, the consideration of a list of referents without their contexts, is that it ignores valuable evidence. Using a similar approach with Modern English, I can 'show' that the word grey has a brightness component. As an experiment, a random sample of fifty quotations has been taken from the British National Corpus. The grey referents are: clothes, fat (in food), street, human face, owl, chair, castle, hills (in mist), human eyes, wire, smoke, whale, bedcover, parrot, import, metal, human hair, stone barn, wind, satin, slate, wardrobe, sunlight, church tower, river, cap (tweed), horses and ponies, distant shore, car. Using the methodology which has been employed for Old English color studies, a brightness component can be claimed for ModE grey on the basis of the italicized referents in this list. Having found a substantial brightness element, I could now argue that the chair and wardrobe must be painted in gloss paint, and that, because birds' feathers have a sheen, the owl and parrot can also be added to the "brightness" list. This produces a total of twelve referents with a "brightness" element, 'proving' that ModE grey can mean 'bright'. I think not.

The purpose of this exercise is to show that listing referents without their contexts is likely to be misleading, but it is important to understand in what way it is misleading. The argument is not that OE grog was never used of, for example, a light-reflecting object, just as this is not the argument in connection with ModE grey, but this usage does not necessarily mean that light-reflection is a semantic element of either word. It is, therefore, necessary to distinguish between a word's semantic elements and the other undeniable but non-denoted aspects of the referent. An accepted semantic element, such as GREY, may be either always present, as in the case of a word meaning 'grey and shiny', or it may be sometimes present, as in a word meaning 'grey, shiny'. Both these cases must be distinguished from contexts in which a word means 'grey' but just happens to be used now and then of shiny objects (or rough objects, or soft objects, and so on), without denoting these quali- 
ties. With historical languages, such a distinction, critical for evaluating a word's meaning, can only be established by a detailed consideration of the contexts.

I have argued above that the methodology previously used to establish whether grag, and other words, have a brightness element, is inadequate to the task. My own findings suggest that the probable early Old English meaning of groeg (indicated by the secondary sense, 'grayish hue') was concerned with saturation, not brightness. Where does this leave the Old English grey color category? When Casson assessed the position on the brightnessto-hue scale of the Old English color categories, he did not mention grey, although he did refer to grey terms as unlikely to be "pure-hue or hue-dominated" (1992:396). This means he must classify the grey category as pure-"brightness" or "brightness"-dominated. This does not tally with my findings. I found only one grayness word which has a high likelihood of containing a light-reflecting element, and that is glosen. The remaining eight greyness words (including compounds) do not (Biggam 1998:312). If my evidence is accepted, this moves an entire Old English color category from a "brightness"-dominated classification to, at least, a 'hue'-dominated classification. It is probably better classified as pure-'hue', although the word hue is technically inappropriate for an achromatic. The reason for this suggested classification is that the Modern English grey category, which I presume Casson would certainly classify as pure-'hue', nevertheless includes some words with a brightness element, like silvery and pearly (Kirkpatrick 1987: Section 429).

\section{Towards a standardized terminology}

In this section, a proposal will be put forward which is intended to help with the discussion of the color meanings conveyed by individual lexemes and languages. It is intended to provide a semantic metalanguage for a less ambiguous description of natural-language color words than has previously been employed. These metalinguistic terms for use in semantic studies are not intended to conform to the principles of color-vision science or the psychology of color perception. This is because natural language does not label everything that a human perceives, still less every scientific phenomenon, and, furthermore, it is common for native speakers of a particular language to disagree on details of the meaning of individual color words. It will still be necessary to study particular color terms in depth, but it is hoped that the scheme below will facilitate general discussion and, in particular, the description of dead languages, for which our comprehension will always be imprecise.

The major constituents of the color impression are usually listed as hue, saturation and brightness, but it is now clear that brightness has been used to denote different aspects of appearance, and this has caused confusion in the discussion of at least one language's color vocabulary. For the purposes of the study and recording of natural language, we need to separate the several qualities described by hue, saturation and brightness. It cannot be stated too strongly that I am not suggesting here that a speaker of any language consciously analyzes the color elements in each word when s/he uses them, but, just as a lexicographer must identify the senses which any word can indicate, so a color semanticist needs to know the range of a particular color word, both in general terms, and in particular contexts. For 
those semanticists who write in the English language, it must be recognized that certain of the descriptive terms they use are ambiguous, and they must either explain, in each publication, precisely how they are using such terms, or refer to a standardized system. The schema below, therefore, is an attempt to provide unique identifiers for the features and ranges of phenomena which may be involved in the linguistic expression of a color.

1. HUE: red, yellow, green, blue, brown, purple, orange, pink, etc.

Other hue-terms can be used, e.g. turquoise, provided the coverage is first specified, e.g. greenish blue+bluish green.

Mixtures of hues can be indicated by, e.g. yellow-green (an apparently equal mix of the two hues), or yellowish-green (in which green is dominant).

Categories can be combined, e.g. green+blue (grue).

See below for further discussion on hue.

2. SATURATION: vivid - mid - dull

Saturation is interpreted as a fully-saturated hue (vivid), with the rest of the range including increasing amounts of grey.

3. TONE:

3.1 Achromatic tone: white - pale grey - mid grey - dark grey - black

3.2 Chromatic tone: pale or dark

Chromatic tone is interpreted as a hue with graduated admixtures of white or black.

4. BRIGHTNESS:

4.1 Light-emission: dazzling - shining-glowing

Light-emission can be further described by its constancy, i.e. intermittent or constant.

4.2 Reflectivity: shiny - lustrous - matt

Shininess and lustre can be further described by their constancy, i.e. whether reflecting light intermittently or constantly.

4.3 Surface illumination: well-lit or poorly lit

Medium illumination is taken as the norm, and need not be recorded.

4.4 Space illumination: brilliant - dim - unlit

Medium illumination is taken as the norm, and need not be recorded.

\section{TRANSPARENCY: transparent - translucent}

The use of the hue-terms requires further explanation. Red, for example, without further qualification, indicates a single-hue coverage extending to all the variations of red which are denoted by the other standard terms, namely, vivid red, mid red, dull red, pale red, dark red, shiny red, lustrous red, matt red, well-lit red, poorly lit red, transparent red and translucent red. When describing the coverage of a 'red' lexeme in a particular language, the coverage may be relatively small, e.g. pale red+shiny red, or red (minus orangey-red), or the coverage may be relatively large, e.g. red+pink+orange. The coverage of such a lexeme may be quite complex to describe, e.g. vivid (red+pink+orange)+pale (red+pink+orange) and so on, but, once described, the writer can revert to the actual lex- 
eme. It may also be necessary, of course, to describe the different coverages of the word in different contexts. The qualifier very can be used, where appropriate, with any descriptor in the schema.

Two examples of how the above terms may be used to describe isolated color words, and color words in literary or spoken contexts, are given below, here using definitions from the COED:

Chestnut, dictionary definition: 'deep reddish-brown'.

Standardized description: dark vivid reddish-brown. (Deep is taken to indicate a fully-saturated hue to which black has been added).

Chestnut in the context of a newly-groomed racehorse seen exercising on a sunny day.

Standardized description: well-lit shiny dark vivid reddish-brown.

Cream, dictionary definition: 'very pale yellow or off-white'.

Standardized description: very pale yellow or yellowish white.

Cream in the context of a jumper worn by someone on an evening walk.

Standardized description: poorly lit matt yellowish white.

The above standardized descriptions are simply examples to illustrate the purpose of the metalanguage which is to unambiguously communicate broadly-defined color senses in semantic discussion carried on in the medium of English.

My own concern is with text-restricted (dead language) studies, so what follows is based on my experience of such work. A review of a color word by means of interdisciplinary semantics (Biggam 1997:26-39) produces information on the color impression it conveys, as retrieved from each individual context. There has to be a process of weeding out insignificant information, by which I mean those aspects of the referent's appearance to which the word is not alluding. It must be borne in mind that some languages do not recognize color as an abstract concept, and the researcher may decide that such 'color' vocabulary actually belongs to a different semantic field (consider Conklin 1955, for example). With living languages, native speakers can be questioned about such things, but this is not possible with text-restricted studies. If some of the referents of a color word are shiny, for example, under what circumstances should SHINY be considered a semantic element of that word? I recommend that only two conditions be acceptable as indicating that the phenomenon is significant:

1. Where SHINY, for example, occurs in the majority of the occurrences providing the basic data, and where MATT does not occur more than once (to allow a margin for error).

2. Where SHINY, for example, occurs in a reference as the only possible meaning. If no other semantic element, including a hue, is appropriate to the context, then the word must contain a SHINY element.

On the basis of the data retrieved and the application of the above two conditions, a revised definition can be drawn up for the color word. The definition should include, of course, any contextual restrictions which have emerged from the study, and these can be expressed after the color definition, for example, 'dark blue "textiles"'. Any cultural contexts or interpretations, such as exclusive use of the word by a certain social or occupational group, or 
symbolic significance such as the modern use of blue to mean 'sad', should also be added where they can be ascertained, but this paper is chiefly concerned with the visual aspects of color words.

The word brightness has been retained in the above schema to indicate light effects, as discerned separately from features of hues, achromatics and transparency. It is to be hoped that this does not prove problematic, but the word is useful as a superordinate term. From here on in this paper, brightness, and other terms, will refer to the meaning of these words as given in the schema, except where they refer to another author's usage, in which case they will appear in quotation marks.

\section{Brightness to hue}

Where does all this leave the brightness-to-hue evolution which has been proposed? It is not the intention of this paper to disagree with the proposal that hue has become the most important aspect of color in English over the centuries. If the first color distinction was between brilliant and unlit (for day and night), then such a color system was not based on hue, but on space illumination, and it is reasonable to suggest that this early contrast was eventually extended to other aspects of brightness. It is speculative, of course, but chromatic tone forms a logical semantic 'bridge' between brightness and hue, and this would be an interesting area of investigation in future research. For example, when an informant labels pale color samples with a particular word, we need to know if s/he uses the same word for any of the brightness features (thus regarding tone and brightness as identical or closely related), or whether pale samples simply offer the nearest available color impression to the true meaning of the word, which may be, perhaps, 'dazzling'.

The historical time span between a color system based on space illumination and one based on hue is massive, and it is maintained in this paper that it is still debatable whether a brightness-dominance remained in Old English. The evidence brought forward to support such a view is fragile. Only after a thorough review of the evidence, something to which I have tried to contribute, can statements be made about the Old English color system. Although this process is at an early stage, there are already hints that Old English was more concerned with hues than we have been led to believe, just as Lerner and Bragg suggested.

The undeniable differences between the Old and Middle English color vocabularies have been explained by classifying the former as brightness-dominated and the latter as hue-dominated, but, apart from the need for further word-studies, certain factors must be taken into consideration before such a statement can be accepted:

1. The Anglo-Saxons evoked different color atmospheres in their poetry from those evoked by the Anglo-Normans, and these two styles can be roughly summarized as an atmosphere based on tone and brightness in the former, and, in the latter, one based on hue. This difference in literary style does not necessarily mean that the Anglo-Saxons had a poor hue vocabulary, nor that they avoided using it in non-poetic 
contexts. The heavy dependence on Old English poetic sources in most of the research discussed in this paper has often not been acknowledged.

2. Arguments based on the number and variety of hue words in Middle English, compared with Old English, usually avoid the undeniable fact that far fewer Old English texts survive than Middle English texts. Of especial significance is the fact that we have no Old English texts on the preparation of dyes or pigments, or the process of manuscript illustration.

3. Those who discuss the number of Old English 'pure-brightness' words which became obsolete in Middle English (Casson 1997:224), presumably implying that a hue-dominated language requires fewer such words, need to address the reason for the huge 'pure-brightness' vocabulary in Modern English, which is considered a 'purehue' language (Kirkpatrick 1987: Sections 417-420). It is noteworthy that Kerttula shows a peak in the number of achromatic/brightness terms in the 16th and 17th centuries, rather than in Old English (2002:305).

4. The argument that "The first secondary [color] terms were incorporated into the language in the late Middle English period" (Casson 1997:233) has been suggested as evidence for the perceived lack of interest in hue on the part of Old English speakers. Elsewhere in his paper, Casson limits his search for secondary color terms in English to simplex terms formed by metonymic extension, whereby the name of an entity becomes the name of a color, as in, for example, ModE orange. By restricting his analysis to simplex terms, when Old English is a language which delights in compounding, and also by restricting to metonymic extension, which is rare in Old English, Casson's argument has a built-in bias in favor of Middle and Modern English. Removing such restrictions, Old English can be shown to have plenty of secondary color terms (Roberts \& Kay 1995: Sections 3.1.14.5-3.1.14.9).

In conclusion, this paper is a general plea for less ambiguity in the specialized terminology of color semantics, and, in connection with Old English in particular, for the revision of conclusions based only on secondary evidence and misleading research methodologies. A stately progress from brightness (and, perhaps, tone) to hue is not questioned, but I maintain that this process began before Old English, perhaps long before, and that the lack of hue concepts in that language has been overstated.

\section{References}

Barley, Nigel F. (1974). Old English colour classification: Where do matters stand? Anglo-Saxon England, $3,15-28$.

Beck, Jacob (1972). Surface Color Perception. Ithaca \& London: Cornell University Press.

Biggam, C. P. (1997). Blue in Old English: An Interdisciplinary Semantic Study. Amsterdam \& Atlanta: Rodopi.

Biggam, C. P. (1998). Grey in Old English: An Interdisciplinary Semantic Study. London: Runetree Press.

Bragg, Lois (1985). Color words in Beowulf. Proceedings of the Patristic, Medieval and Renaissance Conference, 7 (1982), 47-55. 
British National Corpus (n.d.). Oxford University Computing Services, 13 Banbury Road, Oxford OX2 6NN, U.K. <www.natcorp.ox.ac.uk>

Casson, Ronald W. (1992). On brightness and color categories: Additional data. Current Anthropology, 33, 395-9.

Casson, Ronald W. (1997). Color shift: Evolution of English color terms from brightness to hue. In C. L. Hardin \& L. Maffi (Eds.), Color Categories in Thought and Language (224-239). Cambridge: Cambridge University Press.

COED see Pearsall.

Conklin, Harold C. (1955) Hanunóo color categories. Southwestern Journal of Anthropology, 2, 339-344.

Glare, P. G. W. (Ed.) (1982). Oxford Latin Dictionary. Oxford: Clarendon Press.

Isidore of Seville (1911). Etymologiarum sive originum (W. M. Lindsay, Ed.), 2 vols. Oxford: Clarendon Press.

Kerttula, Seija (2002). English Colour Terms: Etymology, Chronology and Relative Basicness. [Mémoires de la Société Néophilologique de Helsinki, 60]. Helsinki: Société Néophilologique.

Kirkpatrick, Betty (Ed.) (1987). Roget's Thesaurus of English Words and Phrases. Harlow: Longman.

König, Günter (1957). Die Bezeichnungen für Farbe, Glanz und Helligkeit im Altenglischen. Unpublished dissertation, Johannes Gutenberg University of Mainz.

Lerner, L. D. (1951). Colour words in Anglo-Saxon. Modern Language Review, 46, 246-249.

MacLaury, Robert E. (1992). From brightness to hue: An explanatory model of color-category evolution. Current Anthropology, 33, 137-186.

MacLaury, Robert E. (2005). So-called brightness in color ethnography: Potentials for LCD technology in fieldwork and categorization research. Cross-Cultural Research, 39, 205-227.

Mead, William E. (1899). Color in Old English poetry. Publications of the Modern Language Association of America, 14, 2 (new series 7, 2), 169-206.

Mills, Carl R. (1976). Stylistic applications of ethnosemantics: Basic color terms in Brunanburh and Maldon. Language and Style, 9, 164-170.

OED see Simpson \& Weiner.

OLD see Glare.

Oliphant, Robert T. (1966). The Harley Latin-Old English glossary edited from British Museum MS Harley 3376. The Hague \& Paris: Mouton.

Pearsall, Judy (Ed.) (1999). The Concise Oxford Dictionary. 10th ed. Oxford: Oxford University Press.

Roberts, Jane \& Christian Kay with Lynne Grundy (1995). A Thesaurus of Old English, 2 vols. London: King's College.

Simpson, John Andrew \& Eva S. C. Weiner (Eds.) (1989). The Oxford English Dictionary. 2nd ed., 20 vols. Oxford: Oxford University Press. 



\title{
Color naming in Estonian and cognate languages
}

\author{
Vilja Oja \\ Institute of the Estonian Language, Tallinn, Estonia
}

Modelling the Finnic color term semantics, two aspects have been considered: (1) the semantic category expressed by every color term, and (2) the background motivation of the name-giving process. We may divide the color terms of all Finnic languages into eight synonym groups, referring to the notions of 'white', 'black', 'red', 'blue', 'yellow', 'green', 'brown', and 'grey'. The hyperonyms of the groups stand for the most general color categories. Mixed tones are often designated by several hyperonyms. Most of the Finnic color names have a descriptive background: the adjectives are semantically motivated by colored objects. Many names of specific shades originate in nouns referring to a dye or its origin. Secondary qualities of hues are referred to by the adjectival part of a compound term. Color terms used by Finnic peoples belong to an open system providing for an unlimited number of acceptable terms.

\section{Introduction}

Present analysis focuses on semantic aspects of Estonian (Est.) color names and their relationships with those in other Finnic languages: Finnish (Fin.), Karelian (Kar.), Vepsian (Veps.), Ingrian (Ingr.), Votic (Vot.) and Livonian (Liv.) - all making up the Finnic branch of the Finno-Ugric language family. To facilitate the reader's insight into the color term distribution, Map 1 shows the geographical location of the seven Finnic languages in the 20th century.

The study draws on extensive authentic dialect material collected from Finnic languages. The Estonian vocabulary comes from the Database of Estonian color terms (see Oja 1998a, 1998b: 8-17). The database includes 1500 entries based on the material of more than 20,000 usage records. A considerable share of the material has been picked from the files of the Estonian Dialect Archives and the card index of standard Estonian usage, being thus representative of the usage of the 1920s-1940s. The material on the other Finnic languages has been collected by leading experts of each language and is provided in the Finnic linguistic atlas Atlas Linguarum Fennicarum (see Oja 2007). Additional material has been obtained from dictionaries and other printed sources as well as from the Finnish Dialect Archives (Helsinki, Research Institute for the Languages of Finland) and the card index of the Votic Dictionary (Tallinn, Institute of the Estonian Language - IEL). 


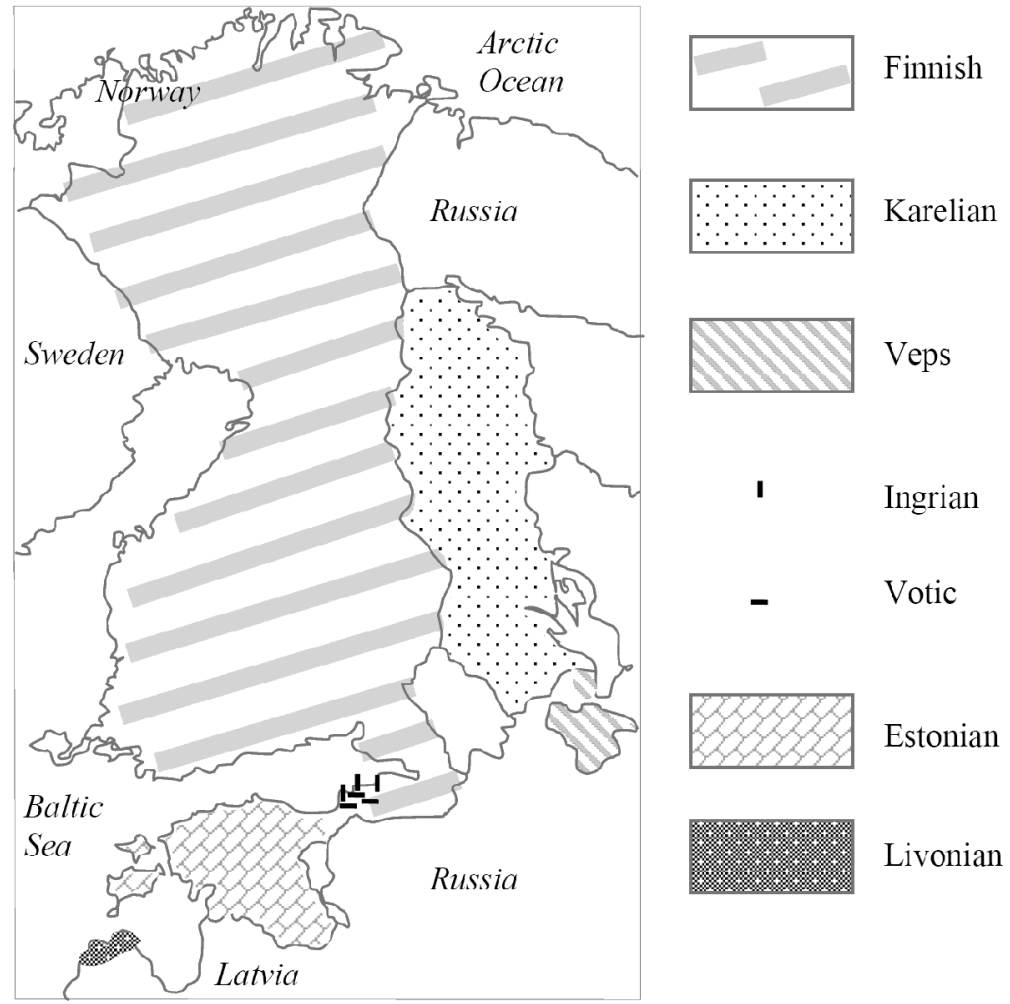

Map 1. Finnic languages. A full color version of this map can be found on $p . x x$

The main focus of the study is on semantics of Estonian color names. Only when critical from the semantic point of view, will morphological structure be briefly mentioned. Section 2 of the paper provides a survey of the system of color terms; Section 3 is dedicated to the semantic background of the color terms.

\section{Finnic color naming system: A semantic point of view}

Color term in the Finnic languages is quite an extensive notion, which includes names for chromatic as well as achromatic colors; names for basic as well as derived color categories; and terms for colors differing in lightness/darkness or other qualifiers. In Finnic dialects there are general terms for eight color senses that cannot be defined using other color terms (see Kerttula 2007; Koski 1983). Six of these eight denote - using the terms of Kay and McDaniel (1978:624ff.) - "primary basic color categories": white, black, red, blue, green, and yellow, while the remaining two denote secondary, or "derived categories": grey and brown. All other Finnic color names can be treated as partial synonyms of the general terms. So we can divide all Finnic color terms into eight synonym series. The database 
of Estonian color terms contains, for example, 349 different words for 'red', 252 words for 'grey.' Each group contains a few general terms (hyperonyms) for those categories (see Section 2.1) while the rest are hyponyms including, in turn, various lexical groups (see Section 2.2).

\subsection{Finnic hyperonyms for color categories}

In all Finnic languages 'white' is named by a noun with the stem val-: Est. valge; Fin. valkea, Kar. valkie, valkei, valged; Ingr. valkia; Vot. valkõa; Veps. vauged, vouged; Liv. vaalda (< Proto-Finnic valketa) (Suomen kielen etymologinen sanakirja [SKES], Toivonen et al. 1955-1981:1619-1621).

Words for 'black' have the stem musta-: Est. must, Fin., Ingr., Kar., Liv. musta, Veps., Vot. muss (a) (< Proto-Finnic musta) (SKES: 353-354).

For 'blue,' terms with the stem $\sin (n) i$ - are used in all Finnic languages: Est. sinine, Fin. sininen, Kar. sinine (Ludian šininee), Veps. sinine, sinine, sinīne, Vot. sinin, sinīn, Liv. si'nni (< Proto-Finnic sininen) (SKES: 1031-1032). It is noteworthy that in eastern Finnic territories a semantic shift has occurred: analogous to the two Russian [Rus.] terms for 'blue,' sinij and goluboj (cf. Paramei 2007), some Finnic dialects use two words whose semantic fields partly overlap (Koski 1983:164-165). The Karelians and Votes have come to refer to a lighter blue color by the Russian loanword goluboj (KKS, Vol. 1: 302; Makarov 1990:53; Vadja keele sõnaraamat [VKS], Adler \& Leppik 1990, Vol. 1:224), while the Vepsian equivalent for the Russian goluboj is an indigenous word taivazma, literally 'sky-blue' (Slovaŕ Vepskogo Jazyka [SVJ], Zajceva \& Mullonen 1972:558). Such usage is more similar to the Russian category partition than to that of the western Finnic cognates of Vepsian (e.g. Est. taevakarva).

'Brown' is referred to by an indigenous word ruskea in Finnish and Ingrian. The original meaning of ruskea was 'red' (Koski 1983: 82ff., 210; SKES: 881). The rest of the Finnic languages use relatively recent loans. The standard Estonian pruun and the dialectal variant pruum are Middle Low German loans. The same Middle Low German word brūn is the source of bruun in the South Estonian Leivu subdialect and the Livonian bruuni (both mediated by Latvian [Latv.]), as well as of the Finnish (p)ruuni, which is a Swedish (Sw.) loan (Mühlenbachs 1923-32, Vol. 1:341; Suomen sanojen alkuperä. Etymologinen sanakirja [SSA], Itkonen \& Kuloken 1992-2000, Vol. 3: 114; Vaba 1997:263-264). In Karelian, Votic, and Vepsian 'brown' is referred to by Russian loanwords koričnevoi or koričnevyi $<$ Rus. koričnevyj (KKS, Vol. 2:325; Koski 1983:225; VKS, Vol. 2:93).

We begin consideration of terms for the next four basic colors with the observation that for each of them two synonymous hyperonyms are used in Estonian dialects. These are: hall and hahk for 'grey'; punane and verev for 'red'; kollane and vahane for 'yellow'; roheline and haljass for 'green'; notably, the second member of each pair is of South Estonian origin. ${ }^{1}$

1. Up to the mid-19th century Estonian had two literary standards - North Estonian and South Estonian, of which modern literary Estonian is closer to the former one. 


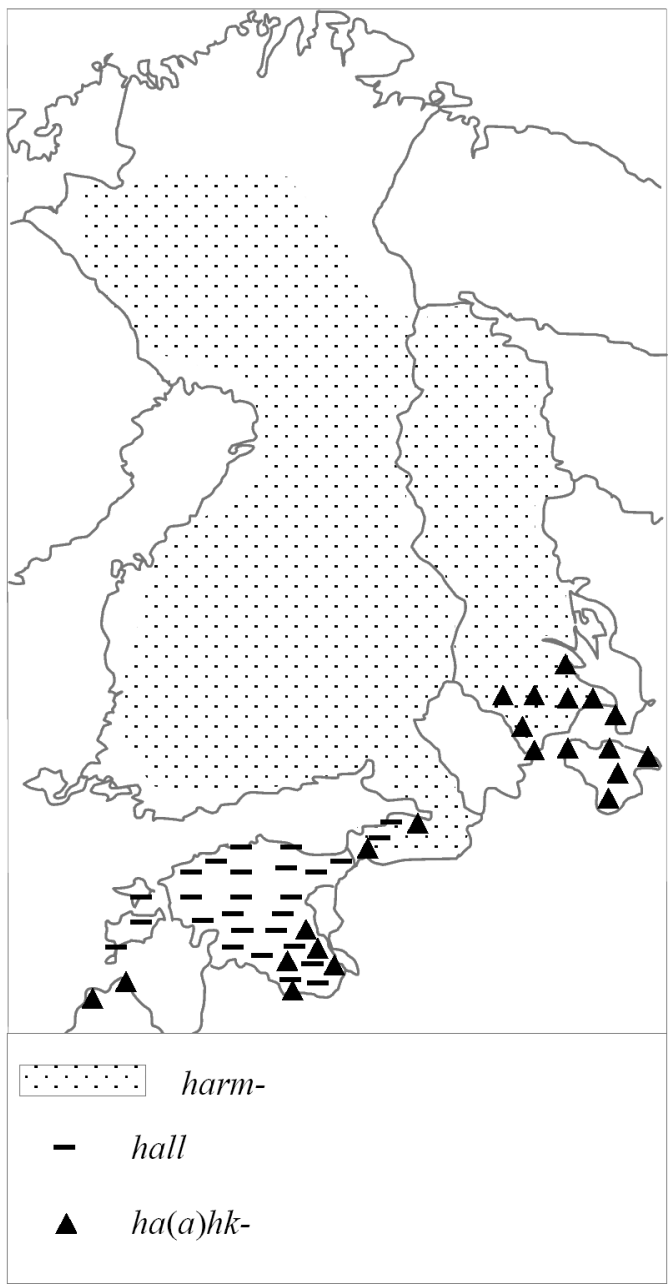

Map 2. Finnic terms for 'grey'

\subsection{1 'Grey'}

All Finnic terms for a neutral grey originate in one of the following three stems: harm-, (h)a(a)hk- and ( $h$ )all- (see Map 2).

Throughout the Estonian area hall (Genitive [Gen.] halli is a hyperonymous term for 'grey.' The word is a Baltic loan (Mägiste 1982/1983: 272; SKES:52). (H)alli is analogously common in the Votic area. In Finnish halli is normally used as a noun denoting 'a greyhaired person' or 'a grey animal,' in particular, in combination with 'dog' or 'ox.'

In South Estonian dialects we find hall and the adjective ( $h$ )ahk. In modern standard Estonian hahk [Gen. haha] means 'a vague grey like ashes' (Eesti kirjakeele seletussõnaraamat, Vol. 1: 387). A few South Estonian informants insisted on a distinction between the denotata of the two words: some considered hall lighter than ahk, while others darker. 
Further, some informants considered hall the finer word, to be referred solely to better or nicer things. The disagreement indicates that although the word is familiar enough, its meaning is not unambiguous. Most of the available material shows that in South-East Estonian dialects the semantic fields of the two lexemes totally coincide, but with hahk being older than hall. Earlier, hall is believed to have denoted the color of animal fur (Saareste 1924:267).

Beside South Estonian, ( $h) a(a) h k$-stem terms can be found in the Livonian, Votic and Karelian languages: West-Livonian a'agi; East-Livonian o'ogi; Votic aahka; Karelian Ludian huahk, hoahke; ${ }^{2}$ and Karelian Olonetsian haahkoi, hoahkoi. In the Olonetsian dialect of Karelian and in Votic those words denote a grey horse or sheep and its wool. In the same dialects we find, however, a parallel usage of broader terms: Votic harmaa and (h)alli, Karelian Olonetsian harmaa, harmoi. The form aatška has been suggested as the possible Finno-Ugric original of the ( $h) a(a) h k$-stem words (SKES: 45$)$. The word-initial $h$-can be secondary in Finno-Ugric languages (Mägiste 1982/1983:251). Association with the

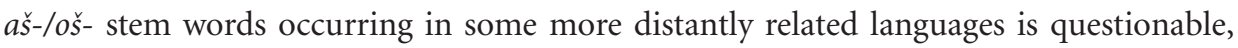
though (SSA, Vol.1: 124).

The harm- stem characteristic of Finland and Karelia is a Baltic loan (cf. Latv. sirms 'grey, silvery grey'; Lithuanian [Lith.] sirmas, širmas; širvas '(bluish) grey' < šarvas 'grey') (Fraenkel 1965:989; SKES:59). In South Estonian Võru dialect haŕm [Gen. haími] refers to a specific, slightly irregular grey as resulting from intermingled light and dark hair.

\subsection{2 'Red'}

According to Estonian orthological dictionaries (Kull \& Raiet 1976; Erelt et al. 1999) there are two co-existent hyperonyms for 'red' - punane and verev. As one can see in Map 3, the word verev has its own compact area of distribution in Southern Estonia.

The word verev is considered to be an indigenous South Estonian derivative $(<$ veri 'blood'), as there is no color term from this stem used in other Finnic languages, even though the noun veri, which is the derivational base of verev, is common throughout the Finno-Ugric area (Mägiste 1982/1983:3795). To cite a more distantly related language, an etymologically similar word is used to denote both blood and the red color in Mansi dialects, while the Hungarian vörös ( veres) 'red' (<vér 'blood') is analogous to the South Estonian verev (SKES: 1707). It has even been suggested that it was the original name for red in the Uralic languages (Sutrop 2002: 167).

In North Estonian dialects verev can be found to mean 'red' only in the vicinity of the South Estonian dialect area, along with several other linguistic features common in South Estonian. The sub-dialect of Viru-Nigula in Northeastern coastal dialect has the phrase vereva inime meaning 'a red-cheeked, ruddy person' (vereva $<$ veri 'blood'). Analogous usage of the lexeme in the sense of 'ruddy complexion' can be found in Finnish dialects (Oja 2001: 43).

2. Ludian and Olonecian are treated as Karelian dialect groups here. According to some existing classifications they are, though, defined as different languages. 


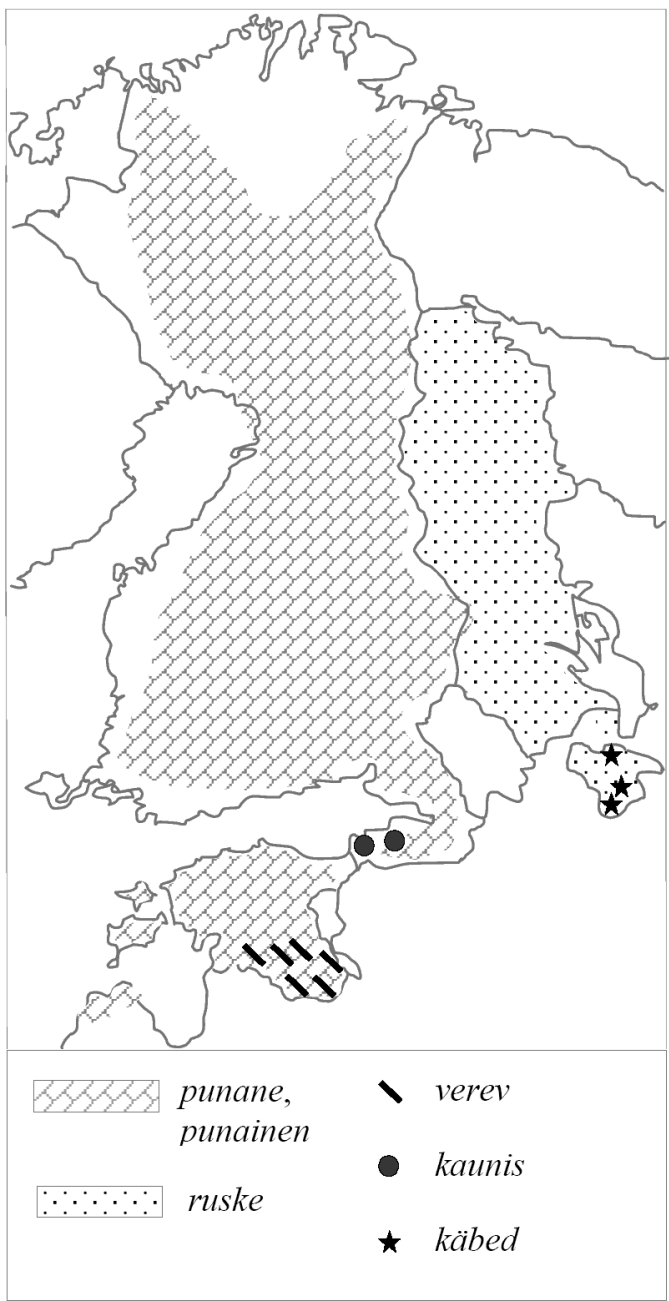

Map 3. Finnic terms for 'red'

The origin of puna is a Finno-Ugric term for 'animal hair, wool.' The color-denoting function of the word gradually advanced in the Proto-Finnic period and came to dominate upon the naturalization of the Baltic loan karva for hair (Koski 1983:64; Saareste 1924:266).

Both Estonian adjectives qualify for general reference to the red color of any substance and they both are also neutral, carrying no additional connotations. However, semantic fields of the two words are not quite identical. A closer study of the compound term helepunane 'bright red' and its word-for-word equivalent valssver $(r) e v$ used in Tartu dialect reveals that the former stands for an saturated bright red, whereas the latter refers to a pale reddish, pink or even whitish hue. This semantic difference may be referred back 
to the different origin of the words. Since the meaning of the adjective punane had been initially motivated by the brownish red color of animal fur, denoting the color of blood required adding of the attributive prefix hele 'light, bright.' By contrast, the adjective verev denotes the brightest possible red, so that any lighter hue implies red of a lower intensity and saturation. Although in dialects the adjective hele may also mean 'bright, radiant', the 'bright red' meaning of helepunane does not overlap literally with this connotation. In standard Estonian punane is the only chromatic color term whose quality of intense brightness is conveyed by the attributive part hele-.

Derivatives from puna are common names for 'red' in Finnish, as well as in Ingrian dialects - punane, punain, punnain - and the Livonian language - pu'nni. By contrast, in the eastern part of the Finnic area, in particular in Karelia and the Vepsian area, the notion is never conveyed by any puna- stem words. As for Votic, the word punain is known only in its Kukkusi sub-dialect; in the rest of the Votic dialects the color of blood is called kaunis, which means 'beautiful' in the Finnic languages. As a color term it is likely a translation loan from Russian, where krasnyj is the adjective for 'red,' also used to mean 'beautiful' originally (Saareste 1924:256). Another analogy can be observed in Vepsian, where käbed also means 'red' as well as 'beautiful' (SVJ: 259).

In addition to käbed the indigenous word rusked is used in Vepsian dialects. Derivatives of ruske- also denote the color of blood throughout Karelia. According to etymological dictionaries ruske- is a Proto-Finnic stem (Mägiste 1982/1983:2559; SKES:881-882; SSA, Vol. 3:110-111). Modern Finnish employs ruskea as a basic term for 'brown.' In addition there are many Finnic dialects using this adjective for a reddish brown or brownish red (e.g. ruske in Northern Estonia). Most often the usage implies the color of some reddish animal.

\subsection{3 'Yellow'}

The Estonian terms for 'yellow' are derived from either of the two stems: keld- and vaha-. The basic term of standard Estonian is kollane, but the dialects offer a wide variety of phonetic variants of the same stem: (1) strong grade (a) without syncope ( `kelda|ne; `kolda|ne; ‘ỗlda|ne, Gen. -se, (b) syncopated (`kolne, `koldne, Gen. `koltse); (2) weak grade (kolla|ne, Gen. -se; kõlla|ne Gen. -tse); (3) sound alternation ( kõldanõ, Gen. kõlladsõ). ${ }^{3}$

In the Finnic languages kelta- is a Baltic loan (cf. Lith. geltà 'yellow pigment,' geltas, geltónas; and Latv. dzęltãns, dzęltęns 'yellow' - SKES: 181). A color term from that stem occurs in all Finnic languages except Vepsian and Livonian. Reputedly, the originallyborrowed word was a noun - the name of a plant used to dye things yellow. Although a color term from that stem is absent in Vepsian, the language employs names of a yellow coloring plant (Rhus, sumach) that are derived from the stem kelta-: keud (in Kortlahti); küud (in Päžaŕ); keldezhein (in Sodjärvi) (SVJ:192, 258).

The same lexeme keldaańe is used in the Kuujärvi sub-dialect of Karelian Ludian dialect in the sense of 'bright green.' By comparison, in Western Estonia, in the island

3. The spread of the phonetic variants of Finnic words originating in the stem kelta- is demonstrated in the dialect atlases (Saareste 1955:Map 50; Oja 2007:272). 


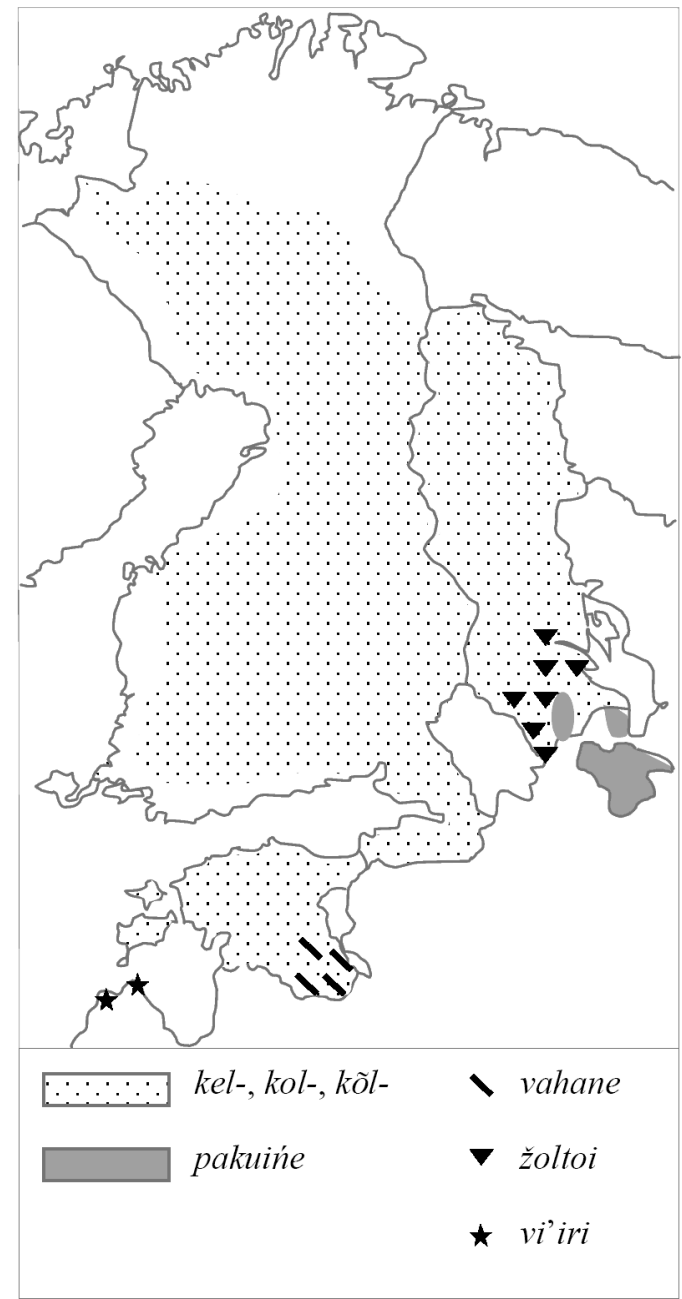

Map 4. Finnic terms for 'yellow'

of Hüumaa 'bright green' is referred to by using the compound term rohi|kollane or rohi|koldne (rohi 'grass').

The South Estonian adjectives for 'yellow' vahane and vahass are derived from the noun vaha 'wax,' which is indirect borrowing from Low German, mediated by Baltic languages (Mägiste 1982/1983:3631). For standard Estonian, vahane has been defined as 'covered with wax; containing wax; wax-colored' (Erelt et al. 1999:869). Wiedemann's Estonian-German dictionary provides vahane with the following German equivalents: wachsfarbig, schmutzig gelb 'wax-colored, soiled yellow' (1923: 1285). However, according to the IEL Dialect Archive material, there are several sub-dialects (see Map 4) in which the 
word refers to an altogether neutral yellow. In newer records of Tartu dialect vahane has been used for a lighter yellow hue, probably due to a literary influence.

In Vepsian 'yellow' is called pakuine (in dialects also pakuune, pakyine, pakyńe). The lexeme is also used in the Olonetsian and Ludian dialects of Karelian (Olonetsian: pakkuine; Ludian Kuujärvi: pakuine). Note that a 1907 record of the Uusikirkko subdialect of Finnish provided the noun pakkunen for denoting three-cleft bidens, Bidens tripartita (three-lobed beggar-ticks or trifid burr marigold), a plant that has been used for dyeing wool yellow (SKES: 470).

Along with the words with the stem kelta- and paku-, the Russian ( $<\check{z}$ ëltyj) loan žoltoi (in dialects also žoltuoi, žoltuo, žoltyi) is used in Karelian dialects. Finally, the Livonian word vi' iri 'yellow' originates from the same stem as the Finnish word vihreä 'green.'

\subsection{4 'Green'}

The Estonian general terms for 'green' come from two different roots: rohe- (the adjectives roheline, rohelene, rohelane, rohiline, rohilene, rohilane, roholine) and haljas (in dialects also haĺass, haillass) (see Map 5).

Possibly rohe- has been borrowed into the Finnic languages as a herb-denoting noun from Germanic sources (SSA, Vol. 3: 105). In Northern Estonia and Mulgi dialect the use of the adjective roheline is unrestricted, as it may refer to the color of plants as well as to color qualities of other objects. By contrast, in Võru dialect a rohi- derivative is hardly ever used to refer to natural plant color. There are also some reports of the Tartu dialect area stating that rohiline (rohilane) implies color of artifacts only. In the Setu sub-dialect spoken in the South-Eastern region of the Võru dialect area, rohilinõ has been sensed as alien to the local usage; to quote a Setu informant, "An Estonian says rohilinõ, but we say halass."

Derivatives of the $r(u)$ oh-stem can also be found among non-Estonian Finnic color terms for 'green'; for example, ruohoinen, rohoinen are used in the Ingrian dialects of Finnish, rohoin, rohhoin in Ingrian, rohoina, rohhoin( $\tilde{o})$ and roholiin in Votic. In addition, the Finnish and Karelian employ a number of compounds consisting of an herbdenoting noun as their first component and an adjective meaning "color of" as the last component. Some relevant examples are: Fin. ruohon|värinen (rohon-), ruohon|karvainen, ruoho(n)|päinen (-päiväinen); Fin. and Karelian Olonetsian heinän|karvainen (-karvaine, -karvaine, -karvane), heinän|päinen (heinäm|päińi), Fin. heinän|värinen, proper Karelian oraš|karvaine, and Karelian Ludian ozman|karvalline. The first component (oraš- and $o z m$-) of the last two compounds means 'the young green crops of autumn-sown corn.' Although lexically the compounds listed above are totally different, they have a similar semantic structure. Analogous color names made up of the same components (rohukarva, orasekarva) are known in Estonian dialects too, but those are used to refer to certain tones rather than to the focal color of the basic term.

The Baltic loan haljas (cf. Lith. žãlias 'green,' 'raw'; Latv. zalšs 'green' - SKES: 51; SSA, Vol. 1: 132) is used to denote the color of green plants to the south of the Gulf of Finland in Estonian, Votic and Livonian. In Estonian Vorru dialect, as well as in its vicinity in the area of Tartu dialect and apparently also in the southern part of the Eastern dialect area, 


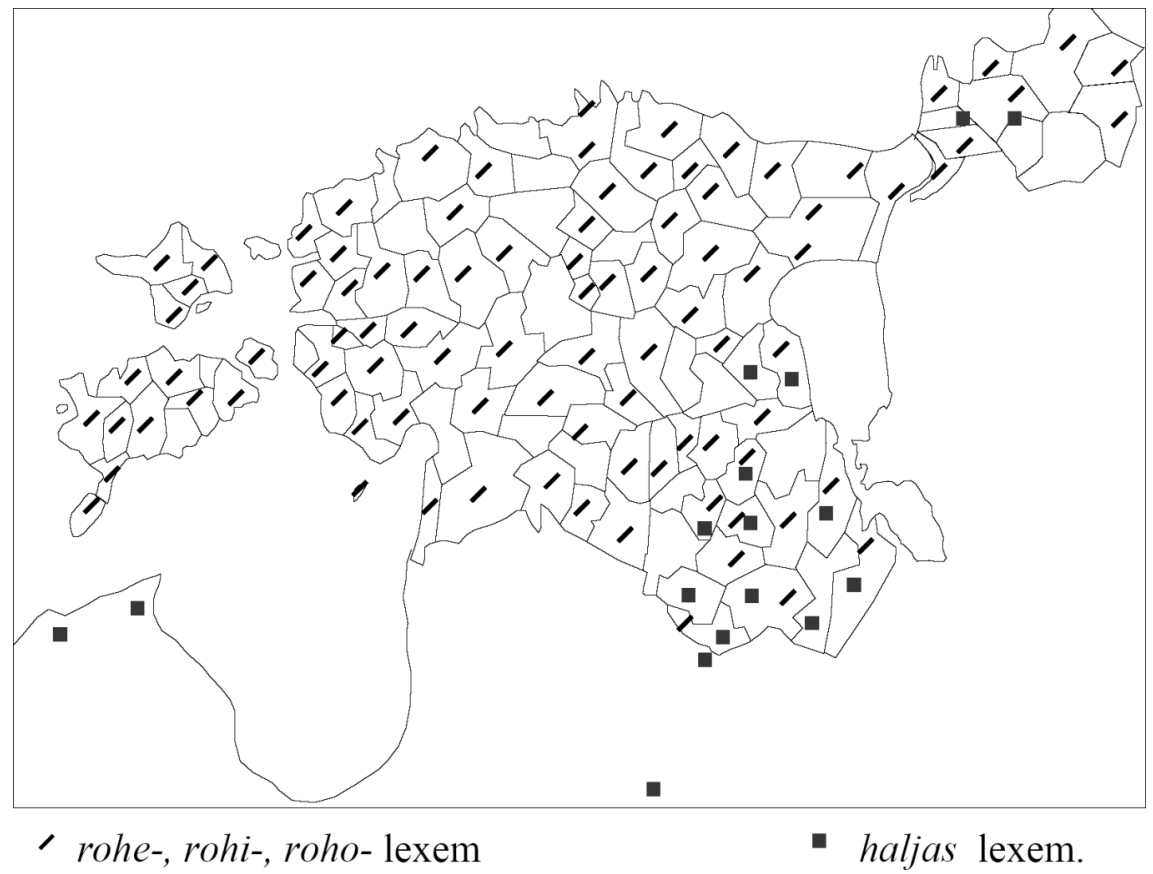

Map 5. General terms for 'green' in Southern Finnic dialects ${ }^{4}$

haljas was earlier the most general term for green used in the vernacular for denoting any green object.

Throughout the Estonian area the word haljas is used to refer to fresh green plants, such as grass, crop sprouts, forest, trees, bushes, leaves, meaning at the same time 'fresh, green and juicy.' In addition in some dialects $(h)$ aljas is used to denote the green color of dried hay. To keep on the safe side, the 'green' map shows haljas only in those dialects in which it is also used in non-herbal contexts. According to Koski (1983:133) it is possible that haljas has earlier served as a color term throughout the Estonian area without any connotative limitations. The same loan with the meaning of 'green' is also used in Votic (aĺjaz, allaz) and Livonian (oo'ĺaz, aaĺaz) (Kettunen 1938:267; VKS, Vol. 1: 98). In Livonian there is also another general term for 'green': mõltsi (East Livonian), miltsi, mültsi (West Livonian). As a noun the latter denotes a pigment, and as an adjective it mainly refers to dyed or painted objects, while designating plant color is a less frequent function (Kettunen 1938:221, 224).

A similar complication to that of the Estonian haljas arises with the Finnish words for 'green, verdant' vihanta, vihanto, vihelä and the Ingrian vihheerä, vihree. These, too, are associated, first and foremost, with the vitality of green plants. In the eastern regions of the Finnic area, i.e. in Karelian and Vepsian, we find derivatives of the viha- stem (vihand,

4. The Finnic words for 'green' are mapped by Oja (2001:55-57; 2007:278-281). 
$-a,-o,-e)$ serving as generic terms for 'green.' The same root lies at the base of adjectives for 'green' derived from the $v i h(e) r$-stem or its phonetic variants, which are widespread in Finnish dialects (for details see Oja 2001: 44-45, 55).

In the northern Finnic language group we find not only indigenous derivatives and very old loans but also some recent loans. In the eastern languages the źelon-stem is spread (< Rus. zelënyj), whereas in Finnish dialects $(k)$ rööni(nen), ryöni(nen) is used (< Sw. grön) (for the map see Koski 1983:146; Oja 2001:57, 2007:279). Those are used mainly when referring to pigments and painted or dyed objects, but there are also a few examples of their denoting plant color.

In Finnic languages the words for 'green' may also get mixed up with those for 'blue.' In Votic the compound rohosinin 'green-blue' has been recorded as a reference to the green color (in the files of the Votic Dictionary, IEL). In South Estonian, green peas have been described as allas 'green' with the explanation that "they aren't grey, they are blue" (Eesti murrete sõnaraamat, Vol. 1: 269). In some other cases 'green' and 'yellow' may also be referred to by nearly similar terms. As has been mentioned above, in Livonian vi' iri denotes 'yellow' (see Map 4), while 'bright green' is referred to by words meaning 'yellow' in the Estonian island of Hiiumaa (rohi|kollane, -koldne) and in Karelian Ludian dialect (keldaańe).

\subsection{Other color terms}

We cannot always pinpoint just one single hyperonym for each particular color name. Some terms function as hyponyms of several equal general terms. For example, Est. türkiis 'turquoise' is considered by some informants 'blue,' by other 'green'; in a similar way, one and the same animal may be denoted by terms for 'yellow,' 'red,' or 'brown.' The choice of a hyperonym depends on an individual system of color categories/terms, and on the speaker's partition of the color gamut, contingent on the motivation of the particular moment. In some cases, however, the hyperonym is ascribed to a mixed color following the principle of contrast, implying that just one hue-match is picked out in order to emphasize its difference from the other.

The fact that a derived color category may simultaneously belong to more than one basic color category is apparent in compounds consisting of two color terms (e.g. Est. punakaskollane 'reddish-yellow' mustjaspunane 'blackish-red,' sinkjasmust 'bluish-black,' sinakasvalge 'bluish-white, siniroheline 'bluish-green').

Alongside the generic terms, every Finnic language has a host of terms for various mixed colors and narrower color concepts. The most widespread ones in the standard Finnish are oranssi, violetti, turkoosi, beige. Modern standard Estonian makes definite use of such terms as roosa 'pink, oranž 'orange,' lilla 'purple,' beež 'beige,' kreem 'creamcolored.' In dialect usage, however, there are many other options to refer to those colors.

For 'purple, for example, we find not only lilla, but also violett or violetne, sinipunane 'blue-red,' punasinine 'red-blue,' lillasinine 'purple-blue,' anililla, liinapunane and liinasinine, viiulisinine and viiulipunane, lillepunane, kannikasinine, or kannikesesinine 'violet blue,' sirelikarva 'lilac,' marjanäoline 'berry-colored,' or even the terms for 'blue' or 'red' 
may be used. The Finnish terms for 'purple' include sinipunainen, punasininen, violetti, punavioletti, sinivioletti, violetinpunainen, violetinsininen, violetinvärinen, orvokinsininen 'violet blue.'

For 'orange' there are such words as punakaskollane 'reddish-yellow', kollakaspunane 'yellowish-red', tsinoober (< German [Germ.] Zinnober), ort $(<$ Germ. Orleans gelber Farbestoff 'annatto,' a particular cinnabar-red pigment, Wiedemann 1923:713), koosenikollane (< Germ Cochenille 'cochineal,' Wiedemann 1923:350), apelsinikollane 'orange yellow' (the first component < Germ. Apfelsine), põrkna verev 'carroty red,' ruuge, rebu in Estonian, and punakeltainen, kellanpunerva, oranssinvärinen, oranssinkeltainen, appelsiininkeltainen 'orange yellow' in Finnish (see Koski 1983:228ff.). None of those synonyms dominates to the same extent, though, as the terms considered in Section 2.2.1.

Although it is not easy to provide a brief survey of all words for mixed colors and for restricted groups of referents, as their number is large, these can still be analyzed in terms of mode of formation. From the thematic point of view three groups of words can be distinguished that considerably differ from the rest. These are (1) terms expressing partial hue content in a color; (2) words referring to secondary qualities of colors; (3) color terms with restricted reference.

\subsubsection{Color terms expressing partial hue content in a color}

In the Finnic languages, the partial, or less than maximum presence of a quality is usually expressed by adding a moderating suffix to the adjective. Although dictionaries report derivational color names only for Estonian, Finnish and Livonian, the same pattern is at work in other Finnic languages as well. For that purpose the Estonian language mainly employs compound suffixes -kas(s) or -kane/-kanõ (e.g. kõllakass, kõllakane 'yellowish') and -jas or -janel-janõ (e.g. mustjas, mustjanõ 'blackish'); in the Lutsi sub-dialect -lik (e.g. mustalik 'blackish', valgõlik 'whitish') (Oja 1997: 13). In Livonian we find the moderating suffix -liki, for example pu'nliki 'reddish,' mustliki 'blackish.' In Finnish the moderating suffix -hko (e.g. valkeahko 'whitish') or the compound suffixes -htava /-htävä (e.g. harmah-

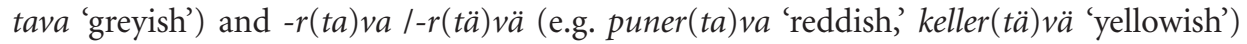
are attached to the noun stem (Koski 1983:304). Instead of a suffixed derivative, speakers may also choose a phrase, complementing the color word with a moderating adverb or a parameter word (a word meaning "color of") that functions as an adverb of emphasis. Thus, the Estonian expressions mustjas, mustakas, mustapoolne, mustavõitu, musta juhti ( vuhti), mustanatu, musta tümbä all denote the same semantic aspect 'blackish' (Oja, 1997: 16-17).

As a rule, such moderative color terms are rather vague with respect to the hue that they denote. The phrase hallikas riie 'greyish cloth' need not inform us of the real color of the cloth at all, since it may refer to impurity of a hue. Hence, a moderative term may bear a specifying function only if one is aware of which color category is implied. Moderative names may also be found as first components of compound color terms in which they qualify the hue in question, like in hallikas|sinine 'greyish-blue,' and punakas|kollane 'reddish-yellow.' 


\subsubsection{Terms referring to secondary (non-hue) qualities of a color}

Colors are often described by certain modifiers that refer to their lightness/darkness or saturation, for example Est. ere 'bright, vivid,' kahvatu 'pale,' hele 'light, bright, fair,' kirgas 'bright, radiant,' mahe 'soft, mild,' särav 'bright, brilliant, radiant,' tuhm 'dull,' tume 'dark.' In context, these may function as attributive components of agglutinative color terms, for example erksavärviline õis 'bright-colored flower'; heledad juuksed 'fair hair'; or in dialect usage: ilosa kirka karvaga riie 'a beautiful bright cloth'; tume-eliline lõng 'wool of a dark tone.' The most typical Finnic way to refer to light and dark colors as well as to colors of different degrees of vividness is to use a compound or word combination consisting of an adjectival attributive component and a color term.

In the Finnic languages the words for 'dark' fall into three semantic groups: adjectives (1) characterizing only (or mainly) the darkness of color (e.g. Est. tume, tunkel, pime); (2) expressing absence of brightness, in the first place, and darkness of color (e.g. Est. dial. tinnõ, tuhm); and (3) functioning both as separate color terms and with the meaning of 'dark' (Est. tõmmu, must).

Most of the Finnic adjectives marking lightness of a color are derivatives from either of the two stems, hel- or val-. Both may take on the suffix ${ }^{*}-k\left({ }^{*}-k k\right)$, for example Est. hele, elgas, elk; valus, vaalas, valss, valge, valkjas. Further, the meaning of the Estonian valge is associated with both luminosity as presence of light, and 'white.' Adjectives with the helstem may also refer to brightness (high intensity) or brilliance, for example Fin. heleä, helakka.

High saturation of color is characterized in Estonian by the adjectives ere, erk, kirgas 'bright, vivid,' täis 'full,' küps 'ripe,' while the adjectives for a low degree of saturation are hale, kahvatu, kahkjas, kahtjas, plass 'pale' (Oja 2001: 67-69).

The main semantic theme underlying the adjectives of lightness and darkness is the amount of light. The concept of light (as present in colors) is expressed by the same words as luminosity (Est. hele, helgas, valus) and the concept of dark is referred to as insufficiency of light (tinnõ, tuhm) or its absence (pime). Notably, along with being attributive of color, some of those adjectives are used to characterize sound or intensity of sensation (e.g. pain may be hele 'shrill' or tume 'dull'), cognitive abilities (such as hearing, vision and acumen), and even sharpness or bluntness of objects (e.g. of knife, axe, pencil, picket).

Adjectives of lightness or darkness, when added to the words denoting 'black' or 'white' as the two extremes of the achromatic scale, serve to emphasize the high or low degree of intensity or extremeness of the color. If the attributive part refers to the same half of the lightness scale as does the basic component, an intensifying effect is produced: hele 'light' + valge 'white' = 'extremely white'; tume 'dark' + must 'black' = 'deep black.' Conversely, the emphasis may be laid on the impurity of the color: tume + valge = 'not quite white', hele + must = 'not ideal black.'

The intensity of black and white is often referred to by adding adjectives for 'grey' or 'blue.' The former indicates poor saturation of a basic color (e.g. hallikasvalge 'greyishwhite,' hallikasmust 'greyish-black'), whereas the latter emphasizes its high degree of intensity ( sinakasvalge 'bluish-white', sinakasmust 'bluish-black'). In addition, high color intensity may always be expressed by reduplication of a color term, as in the Estonian 
dialect words šini-šinin 'extremely blue' and valivalge 'extremely white.' Although this formative pattern is particularly common in the marginal sub-dialect of Leivu (Mäger 1966; Niilus 1938), spoken in an Estonian enclave on the Latvian territory, the construction is not unfamiliar to standard Estonian.

\subsubsection{Color terms with restricted reference}

Most of the Estonian color terms are unrestricted in that they can refer to the color of any substance. Exceptional, as in many languages, are nouns and adjectives denoting the colors of animal coat or fur. Although Estonian dialects allow animal color to be discussed in terms of unrestricted reference (e.g. hall 'grey', must 'black', punane or verev 'red,' beež 'beige,' helepruun 'light brown,' pümmeverev 'dark red'), there are certain terms which are used only or mainly to differentiate between domestic animals (in most cases, horses and cattle) according to the color of their coat. Those words are kõrb, raudjas, ruske, tõmmu, paat, leet, pleek, lepp, võik, kimmel, haabjas, suitsik, hiir, kurõss, tuhk(ur), asu and their derivatives and compounds. There is no one-to-one correspondence between such Estonian and English terms. The meaning of the specific terms for animal color are set out, to a degree, in Table 1, enabling one to relate the name of an animal color to the corresponding color concepts.

The division is, however, rather conditional. Table 1 overlooks many nuances (e.g. pale, blackish, greenish, pinkish, bluish) and special features such as mixed-colored coat (as in kimmel) or a dark stripe along the back combined with lighter sides (as in hiir). The parentheses indicate partial presence of the color, usually expressed by moderative derivatives like kollakas 'yellowish,' beežikas 'kind of beige.'

Table 1. Semantics of Estonian special terms for animal color

\begin{tabular}{|c|c|c|c|c|c|c|c|}
\hline & brown & grey & beige & yellow & $\begin{array}{l}\text { light brown } \\
\text { or dark yellow }\end{array}$ & red & reddish-brown \\
\hline$k \tilde{r} r b$ & • & & & & • & $\bullet$ & • \\
\hline raudjas & $\bullet$ & $\bullet$ & & & & $\bullet$ & $\bullet$ \\
\hline ruske & $\bullet$ & & & & $\bullet$ & $\bullet$ & $\bullet$ \\
\hline tõmmu & $\bullet$ & $\bullet$ & & & & $\bullet$ & $\bullet$ \\
\hline paat & $\bullet$ & $\bullet$ & $\bullet$ & $(\bullet)$ & $\bullet$ & $\bullet$ & $\bullet$ \\
\hline leet & $\bullet$ & $\bullet$ & $\bullet$ & & $\bullet$ & & \\
\hline pleek & & & $\bullet$ & & • & $\bullet$ & \\
\hline lepp & & & $\bullet$ & $\bullet$ & - & & \\
\hline võik & & $\bullet$ & $\bullet$ & $\bullet$ & $\bullet$ & $\bullet$ & \\
\hline kimmel & $\bullet$ & $\bullet$ & $\bullet$ & $\bullet$ & & & \\
\hline haabjas & • & $\bullet$ & $(\bullet)$ & $(\bullet)$ & & & \\
\hline suitsik & $(\bullet)$ & - & & $(\bullet)$ & & & \\
\hline hiir & $(\bullet)$ & $\bullet$ & & $(\bullet)$ & & & \\
\hline kurõss & & • & $(\bullet)$ & & & & \\
\hline $\operatorname{tuhk}(u r)$ & & $\bullet$ & $(\bullet)$ & & & & \\
\hline asu & & $\bullet$ & & & & & \\
\hline
\end{tabular}


Some of them, for example raudjas, kõrb, hiir, belong almost exclusively to horse color. Although when speaking of horses one should also consider the color of the tail, mane and forelock, the Estonian color adjectives are mainly associated with the color of the coat. Along with denoting animal coat, the above terms may be used to refer to some other natural substances of inhomogeneous color, such as human hair, wool, plumage, or ripening grain.

\section{Semantic sources of the color vocabulary}

Discussing the semantic background or original motives of color-term formation, we cannot bypass two major groups of words: comparison-based color terms, and adjectives derived from the names of coloring substances. Among such, the Finnic material contains numerous borrowings. Even the list of basic color terms includes loanwords acquired in different periods (e.g. Est. pruun 'brown,' kollane 'yellow', hall 'grey'). Their use does not always reveal whether it was the color-denoting adjective that was originally borrowed, or a noun (denoting a basis of comparison or the name of a pigment) that has come into adjectival use only later. Like other color terms, the loans also include some that are used both as a noun and as an adjective, for example Est. kreem 'cream; cream-colored', purpur 'red cloth; deep (sometimes purplish) red,' türkiis 'turquoise.' Along with direct loans translation loans may also be encountered, for example Vot. kaunis 'red,' or Veps. käbed 'red' (see Section 2.1.2).

Not all these words have reliable etymologies to supply the naming motives. In the case of old words it is even hard to tell indigenous derivatives from (adapted) loans. Our studies have revealed, for example, that some Estonian words for animal color that bear a resemblance to denominal adjectives have actually been borrowed as adjectives from IndoEuropean languages (see Oja 2003, 2004). The foreign origin of terms for animal color may also account for the curious fact that cattle and horses are referred to by different color words (as they say in the Estonian Island dialect: hobu oli võik, lehm oli leet 'the [yellowish] horse was called võik, while the cow [of the same color] was leet').

\subsection{Comparison-inspired color terms}

The most frequent semantic motive detected in the background of the Finnic color terms is comparison. After all, any object of a color known to the communicators may serve as a basis of comparison. By this mode of reference one can describe not only the hue (e.g., Est. kirsikas 'cherry', rooste-karva 'rust-colored,' sambla-roheline 'green like moss'), but also the lightness/darkness, vividness and some other qualities of the color in question (e.g. Est. lumivalge 'snow-white,' hõbehall 'silvery grey,' sametmust 'velvety black'). Morphologically, a comparison-based color term may be either a compound word, a denominal suffixed adjective, or a noun used in adjectival function. 
As for the favorite bases of comparison, the Estonian color vocabulary is dominated by names of various natural objects, first of all plants, their flowers and fruits, but also animals. Many adjectives have also been derived from names of substances, including nonferrous metals such as gold, silver and copper (see Oja 1995). Dialect speakers often use such nouns as initial components of compounds ending in (1) the parameter word karva, värvi, meaning 'of color' (e.g. Est. kullakarva 'golden,' taevakarva 'color of the sky') or (2) a generic color term (e.g. türkiissinine 'turquoise', kuldkollane 'golden yellow') (see Oja 1998a).

In comparison-based Estonian compound terms the mode of compounding also has a semantic function. The color shared by the object and its basis of comparison is usually described by a compound with its first (attributive) component in the genitive case (e.g. kirsipunane 'dark red like cherry'), whereas nominative compounding emphasizes the high (or higher than usual) intensity level of the color expressed by the final (basic) component of the term (e.g. Est. läks näost kirsspunaseks 'blushed deeply'; literally 'his face got cherryred') (see Oja 1997:17-19). Saturation can be emphasized by comparison with an object or phenomenon well known for the intensity of its color (e.g. Est. must kui ronk 'black like a raven' or ronkmust 'raven-black'; punane kui veri 'red like blood' or veripunane 'bloodred').

Some attributive components used originally to denote the basis of comparison have gradually developed into words of intensity. In Estonian dialects, for example, we find tulivalge in the sense of 'extremely white,' tulimust meaning 'coal-black', tuliroheline kleit 'bright green dress,' while tuli means 'fire.'

The number of comparison-based color terms is practically unlimited in the Finnic languages. The existing patterns enable any speaker to make up new adjectives on the basis of analogy. Of course, for the meaning to cross over adequately the communicators have to share, on the one hand, the same implicit knowledge of the basis of comparison and, on the other hand, the local system of color naming.

\subsection{Color adjectives originating in the name of a coloring substance}

Many names of specific hues are based on nouns denoting a coloring substance, usually a dye. Some adjectives denoting certain colors originate in traditional plant dyes, some others in industrial dyes of more recent origin: for example madarane 'yellowish red dyed with the roots of northern bedstraw' (<madar 'bedstraw'); purpur (ne) 'purplish-red associated with cudbear'; košenill- or kooseni|punane 'cochineal red'; potisinine and potiroheline 'blue or green prepared in a pot with urine'; liina 'aniline red.'

The local meaning of a borrowed color name need not coincide with its original semantic background. For example, the word purpur 'purple-red, crimson' has been mediated into the Finnic languages by the Bible; in the Estonian dialect usage of this period it was used to refer to bright red cloth.

The word violett (< Germ. violett) reached Estonia as the name of a purplish-blue dyeing substance in the form resembling a piece of wood, and thus it came to be called viiulitepuu, or viiulipuu (< puu 'wood'). Most of the foreign color terms have undergone 
folk etymologisation resulting in phonetic similarity to one or another familiar word and in structural similarity to a comparison-based expression. In such a case, the color described need not be directly related to that of the substance in the position of the basis of comparison. Compare, for example, the standard Estonian violett or violetne for 'violet' to the dialect equivalents viiuldi värv, viiulite värv, viiulisinine, viiulipunane, viiulett, wherein viiul means 'wooden stick used as a spool' and 'string instrument.'

In Estonian dialects 'annatto' has been called ordenipunane literally 'order red,' ordenikollane 'order yellow, ordijaan, ort, while the Finnish dialect variants are orjaani, ordijaani, olijaani, (< Orleans). 'Claret,' called bordoopunane (< Bordeaux) in standard Estonian, sounds pordupunane literally 'brothel red' to a dialect speaker's ear (Koski 1983:237; Oja 2001: 149).

A commercial 'blue' was called neiblu-, neinpuu-sinine (Germ. neublau), and pronksi- ponksi- or pank|sinine (pronks 'bronze'; ponks 'candy'; pank 'clod'). With the passing of time, the origin of the word is dimmed in peoples' minds, which supports the process of the adjectival (color) sense gaining primacy.

The result of dyeing textiles, as we know, depends among other factors on the concentration of the dye. If the pigment is the same, the different tones produced by different concentrations of the solution bear the same name. Thus, potisinine 'pot-blue' may be light or dark, greyish or bright blue, while panksee has been described as 'pink,' 'sky-blue,' 'bright blue,' 'cornflower blue,' 'purple' and 'dark blue.'

There is no distinct boundary between comparison-based names and names motivated by the coloring substance, as the latter, too, can function as a basis of comparison. From the morphological point of view, some combinations of a substance name and a parameter word (like Est. ordikarva 'Orleans-colored,' viiuli värvi 'violet-colored') resemble comparison-based color terms rather than those originating in the name of a coloring substance. This may even give rise to homonymy, producing phonetically similar color names that denote different colors due to a difference in what is perceived to be their semantic background. The Estonian word lehekollane (leht 'leaf' + kollane 'yellow'), for example, refers either to a greenish-yellow color resulting in dyeing with leaves (in which case we have a compound motivated by the name of a pigment) or to the yellow of autumn leaves (the case of a comparison-based color compound).

\subsection{Semantic model}

When modeling color term semantics, one should consider both aspects discussed above: namely on one hand, the semantic categories expressed by the color terms, and on the other, the background motivation of the name-giving process. Since the presented model applies to a more or less synchronous situation, etymologies of stable adjectives (e.g. basic color terms) are not considered here. Naming motives for denominal color terms formed in the period in question are discussed. Distinct groups of terms are provided, including moderative color terms and compound terms containing reference to whatever secondary quality of the color. This model also covers the present-day Finnic color term formation. 
Since the Finnic color terms belong to an open system, a statistical diagram does not seem appropriate here. As for other possible graphs, their legibility would certainly suffer due to the complicated semantic relations to be modeled. This is why the following scheme of the Finnic color naming system is presented in text form, listing the main (not mutually exclusive) classes of terms and pointing out their mutual linguistic relations. The examples come from Estonian.

First is the general grouping $\mathrm{G}$ - terms for the most general color categories: words for red, green, blue, yellow, white, black, grey, brown.

The second grouping consists of all other color terms and is organized into three sections (Sp, Part, and Sec) as follows:

1. $\mathrm{Sp}$ - names for mixed tones and more specific color notions (Part - partial or limited hue content in a color):

(a) Color adjectives and their compounds:

$\mathrm{Sp}$ (oranž 'orange');

$\mathrm{Sp}=\mathrm{G}+\mathrm{G}$ (valge|hall 'white-grey');

$\mathrm{Sp}=$ Part $+\mathrm{G}$ (valkjas|hall 'whitish grey');

$\mathrm{Sp}=\mathrm{G}+\mathrm{Sp}$ (sinise| lilla 'blue-purple');

$\mathrm{Sp}=\mathrm{Sp}+\mathrm{G}$ (lilla| sinine 'purple-blue'); Sp = Part + Part (valkjas|kollakas 'whitishyellowish');

$\mathrm{Sp}=$ Part $+\mathrm{Sp}$ ( sinakas|lilla 'bluish-purple').

(b) Colors described by nouns:

(i) $\mathrm{NC}$ - noun of comparison ( $\mathrm{PW}$ - parameter word):

$\mathrm{Sp}=\mathrm{NC}$ (kuld 'gold');

$\mathrm{Sp}=\mathrm{NC}+\mathrm{PW}$ (kulla|karva 'golden');

$\mathrm{Sp}=\mathrm{NC}+\mathrm{G}$ (kuld|kollane 'golden yellow');

$\mathrm{Sp}=\mathrm{NC}+\mathrm{Sp}$ (kirsi|kõrb 'cherry-brown', sireli|lilla 'lilac').

(ii) $\mathrm{NP}$ - Noun of pigment:

$\mathrm{Sp}=\mathrm{NP}($ ort 'Orleans, annatto');

$\mathrm{Sp}=\mathrm{NP}+\mathrm{PW}$ (ordi|karva 'Orleans');

$\mathrm{Sp}=\mathrm{NP}+\mathrm{G}$ (madarapunane 'bedstraw red').

2. Part - words indicating partial hue content in a color (Mod - moderating suffix or word):

(a) Deadjectival derivatives:

Part $=\mathrm{G}+$ Mod (punakas 'reddish');

Part $=\mathrm{Sp}+\operatorname{Mod}$ (lillakas 'purplish').

(b) Denominal derivatives:

Part $=\mathrm{NC}+$ Mod (kirsikas 'almost like cherry').

3. Sec-terms indicating secondary qualities of a color:

(a) Secondary qualities are referred to by an adjectival part of a compound (Adj-adjectival component):

Sec = Adj + G ( tume|sinine 'dark blue,' ere|sinine 'bright blue,' hele|sinine 'light blue,' kahvatu|sinine 'pale blue,' mahe|sinine 'Nattier blue');

Sec = Adj + Mod + G (tumeda|poolne sinine 'darkish blue');

Sec $=$ Adj + Sp (tume|lilla 'dark purple, hele|lilla 'light purple, kärelilla 'bright purple,' kahvatu|lilla 'pale purple'); 


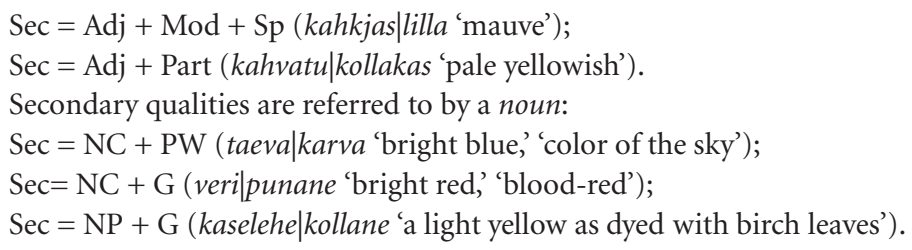

\section{Conclusion}

Based on the linguistic analysis of color terms in seven Finnic languages, we can describe these as covering eight basic color categories. In general, the semantic system of these color categories is rather similar across the languages considered. Some additional notes are worth mentioning.

The semantic field of the Estonian punane 'red' is more restricted than its Finnish cognate, since the lighter end of the former is subsumed under roosa 'pink' (cf. Fin. vaalean|punainen 'light' + 'red').

In the eastern Finnic languages (part of Karelian, Vepsian and Votic dialects) the terms for 'blue' have changed to mimic the Russian categorization of 'blue,' which is argued to have two basic terms with separate semantic fields.

The possible earlier membership of the Finnic 'red' and 'brown' in one meta-category is revealed by the use of the terms with the stem rusk-, which in different languages may mean either 'red' (Karelian), 'brown' (Finnish), or 'reddish-brown' (Estonian).

The earlier membership of the Finnic 'yellow' and 'green' in a common category is still indicated by (1) the Livonian vi' iri 'yellow' having the same stem and origin as the term for 'green' used in the northern Finnic group, (2) the Karelian Ludian term keldaane 'green' having the same stem as other Finnic languages use for 'yellow', and (3) the Estonian Hiiu-dialect term rohikollane (-koldne) referring to the color of green plants, yet including components for both 'green' and 'yellow.'

Although in Estonian the standard and dialect color terms are both formed according to the same pattern, one should pay attention to certain local semantic differences. In South East Estonia, for example, the meanings of the words haljas, verev and vahane are not quite the same as in standard Estonian. Across the Finnic area, semantic differences between terms derived from one and the same root are even greater. This is exemplified by the term haljas: in the southern Finnic language group the term denotes green plants, while it is not semantically identical throughout the area of its distribution.

A lexical comparison of the Finnic languages reveals various relationships between the cognates; in particular, oppositions between the Northern and Southern Finnic, or Eastern and Western Finnic vocabularies; the historical ties of the South Estonian dialects with the Eastern or Northern Finnic languages; contacts between the Finnish south-eastern dialects and the North Estonian dialects. The areal distribution of dialect color terms is often (1) eloquent about neighbor contacts and (2) etymologically suggestive. A considerable number of Finnic color words are indicative of Indo-European contacts, in particular 
with Germanic and Baltic languages. It is noteworthy that these contacts are revealed not only by the presence of loanwords, but also by similarity of underlying semantic categories.

Comparing the Estonian system of color term formation with that of the rest of the Finnic languages, we find a lot of traits in common, for example the basic structure of compound terms, moderative derivatives with adjectival suffixes, similar semantic background, restricted reference of some color terms. The Finnic color terms belong to an open system as the existing patterns enable any speaker to produce new compound words or derivatives without any need of explanation. Of course, proficiency in word creation requires competence in linguistic rules. The cognitive model presented in the present paper strives to describe, on the one hand, the semantic categories expressed by the Finnic color terms and, on the other hand, the semantic background of color term formation.

\section{Acknowledgment}

This study has been supported by the Estonian Science Foundation Grant No. 5967.

\section{References}

Adler, Elna \& Merle Leppik (Eds.) (1990-). Vadja keele sõnaraamat [Dictionary of the Votic language]. Tallinn: Eesti Keele Instituut.

Eesti kirjakeele seletussõnaraamat [Defining Dictionary of Standard Estonian]. (1988-). Tallinn: Valgus, Eesti Keele Sihtasutus

Eesti murrete sõnaraamat [Estonian dialect dictionary]. (1994-). Tallinn: Eesti Keele Instituut.

Erelt, Tiiu, Tiina Leemets, Sirje Mäearu, \& Maire Raadik (Eds.). Eesti keele sõnaraamat [Dictionary of Estonian language] Tallinn: Eesti Keele Sihtasutus.

Fraenkel, Ernst (1822/1965). Litauisches etymologisches Wörterbuch (Band 2). Göttingen: Vandenhoeck \& Ruprecht.

Itkonen, Erkki \& Ulla Maija Kulonen (Eds.) (1992-2000). Suomen sanojen alkuperä: Etymologinen sanakirja [The origin of Finnish words: Etymological dictionary]. Helsinki: Suomalaisen Kirjallisuuden Seura, Kotimaisten kielten tutkimuskeskus.

Kay, Paul \& Chad K. McDaniel (1978). The linguistic significance of the meanings of basic color terms. Language, 54, 610-646.

Kerttula, Seija (2007). Relative basicness of color terms: Modeling and measurement. In R E. MacLaury, G. V. Paramei, \& D. Dedrick (Eds.), Anthropology of color: Interdisciplinary multilevel modeling (151170). Amsterdam: John Benjamins.

Kettunen, Lauri (1938). Livisches Wörterbuch mit grammatischer Einleitung. Helsinki: SuomalaisUgrilainen Seura.

Koponen, Raija \& Pertti Virtaranta (Eds.) (1968-2005). Karjalan kielen sanakirja [Dictionary of the Karelian Language]. Helsinki: Suomalais-Ugrilainen Seura.

Koski, Mauno (1983). Värien nimitykset suomessa ja lähisukukielissä [The Names for colors in Finnish and closely related languages]. Helsinki: Suomalaisen Kirjallisuuden Seura.

Kull, Rein \& Erich Raiet (Eds.) (1976). Õigekeelsussõnaraamat [Orthological dictionary]. Tallinn: Valgus. 
Mäger, Mart (1966). Intensiivistavatest reduplikatiivsõnadest eesti murretes [Reduplicative words expressing intensity in Estonian dialects]. In Emakeele Seltsi Aastaraamat, annals 12 (91-107). Tallinn: Eesti Raamat.

Mägiste, Julius (1982-1983). Estnisches etymologisches Wörterbuch. Helsinki: Finnisch-Ugrische Gesellschaft.

Makarov, Grigori N. (1990). Slovaŕ karel'skogo jazyka (Livvikovskij dialekt) [Dictionary of the Karelian language (Olonetsian dialect)]. Petrozavodsk: Karelija.

Mühlenbachs, Karlis (1923-1932). Lettisch-deutsches Wörterbuch. Redigiert, ergänzt und fortgesetzt von J. Endzelin. Riga: Herausgegeben vom Lettischen Bildungsministerium.

Niilus, Valter (1938). Reduplikatiivsõnadest [About reduplicative words]. Eesti Keel, 17, 224-232.

Oja, Vilja (1995). Gold, silver and copper in Estonian colour names. Linguistica Uralica, 31, 178-187.

Oja, Vilja (1997). The Designation of Colours in Estonian Dialects. Tallinn: Tallinn Pedagogical University.

Oja, Vilja (1998a). Database and computer analysis of the Estonian colour adjectives. In B. Caron (Ed.), Proceedings of the 16th International Congress of Linguists [CD-ROM]. Oxford: Pergamon.

Oja, Vilja (1998b). Colour Terms in Dictionary and in Database. Tallinn: Institute of the Estonian Language.

Oja, Vilja (2001). Linguistic Studies of Estonian Colour Terminology. Tartu: Tartu University Press.

Oja, Vilja (2003). Hobu oli võik, lehm oli leet [Horse versus cow: Same color, different adjectives]. Keel ja Kirjandus, 2, 101-107, 159.

Oja, Vilja (2004). Some colour words with restricted reference. Latvijas Zinātnu Akadēmijas Vèstis. A daļa. Sociālās un humanitārās zinātnes, 5, 37-42.

Oja, Vilja (2007). Värien nimitykset; Harmaa; Keltainen; Punainen; Vihreä [Colour names; Grey; Yellow; Red; Green]. In Atlas Linguarum Fennicarum, ALFE 2 (263-284). Helsinki: Suomalaisen Kirjallisuuden Seura, Kotimaisten kielten tutkimuskeskus.

Paramei, Galina V. (2007). Russian 'blues': Controversies of basicness. In R. E. MacLaury, G. V. Paramei, \& D. Dedrick (Eds.), Anthropology of Color: Interdisciplinary Multilevel Modeling (75-106). Amsterdam: John Benjamins.

Saareste, Albert (1924). Leksikaalseist vahekordadest eesti murretes: I Analüüs [Lexical relations in Estonian dialects: I Analysis]. Tartu: Postimees.

Saareste, Andrus (1955). Petit atlas des parlers estoniens. Väike eesti murdeatlas [Small atlas of Estonian dialects]. Uppsala: Kungl. Gustav Adolfs Akademien.

Sutrop, Urmas (2002). The Vocabulary of sense perception in Estonian: Structure and history. Frankfurt am Main: Peter Lang.

Toivonen, Y. H., Erkki Itkonen, Aulis J. Joki, \& Reino Peltola (1955-1978). Suomen kielen etymologinen sanakirja. Helsinki: Suomalais-Ugrilainen Seura.

Vaba, Lembit (1997). Uurimusi läti-eesti keelesuhetest [Studies on the linguistic relations between Estonian and Latvian]. Tallinn/Tampere: Eesti Keele Sihtasutus, Tampereen Yliopisto.

Wiedemann, Ferdinand Johann (1923). Eesti-saksa sõnaraamat. Estnisch-deutsches Wörterbuch (Dritter, unveränderter Druck nach der zweiten, von Dr. Jakob Hurt redigierten Auflage). Tartu: Estnische Literaturgesellschaft.

Zajceva, Marija I. \& Marija I. Mullonen (1972). Slovaŕ vepskogo jazyka [Dictionary of the Vepsian Language]. Leningrad: Nauka. 



\title{
Color terms in ancient Egyptian and Coptic
}

\author{
Wolfgang Schenkel \\ Egyptological Institute, University of Tübingen, Germany
}

The examination of the ancient Egyptian-Coptic color terms, their grammatical identification, their range of usage, and their etymologies permit the following conclusions: There are four BCTs, all verbs, 'black,' 'white,' 'red' and 'green,' as traditionally translated. The focus of 'red' is in the red range and not in the middle of the red-with-yellow range. The focus of 'green' lies in the green range and not in the middle of the green-with-blue range nor in the pale green range. In the final, Coptic, stage, the partition of the red range into 'deep red' and 'light-red' must be assumed. The traditional written language allowed additional place for further verbal color terms, but these never entered the colloquial language as BCTs. Among these alternatives, the use of 'lapis lazuli-colored, blue' is strikingly common; that of 'charcoal-like, black' and 'golden' is less common. Egyptian did not develop a BCT for either blue or yellow. Basically Egyptian-Coptic remained at B\&K Stage IIIa.

\section{The ancient Egyptian and Coptic color terms in Egyptological discussion}

In a discussion published well before the fundamental work of Berlin and Kay (1969), I concluded that ancient Egyptian and Coptic had four widely used abstract color terms (Schenkel 1963): 'black' ( $k m m$ ), 'white' (hč ), 'red-with-yellow' (ťšr), and 'green-withblue' ( $w 3$ ç), all of which were verbs ('to be black, etc.). Based on morphological features, a word for 'yellow' was also postulated, but only in Coptic; etymological research has since revealed that this was actually another word for 'red' (Coptic mroš etc. < Egyptian $\left.m r \check{s} / m l^{\check{s}} /{ }^{*} m n \check{s}\right)$. All of the other color terms used in the comprehensive dictionary of the ancient Egyptian language (Erman \& Grapow 1926-1963) are concrete in the sense that they only refer to specific categories of objects, as blond is generally used for hair, beer and tobacco in some European languages, or they link the color with an object, as with the Egyptian word for the semi-precious stone lapis lazuli (hsbḅ̆) (noun), from which hasbç̣(.l? (adjective) 'lapis lazuli-like, blue' is derived.

A decisive impetus for my original project was content-related linguistics as represented by Leo Weisgerber, who had an influential role in Germany, far beyond the University of Bonn, where he was one of my teachers. Weisgerber stressed semantics - in contrast to the then-dominant structural linguistics, which stood in opposition to semantics (although the marginal Sapir-Whorf hypothesis enjoyed some support in the US). Strands 
of structural linguistics were, however, also included. One of the objectives was to organize the meanings of words through systems of opposition (semantic field theory), one of the principal examples of which were the color words, one of the few areas of vocabulary where the efforts were clearly successful, and my efforts must be understood in terms of this structural approach.

Content-related linguistics perceived the world as linguistically divided. Colors were not natural, but rather determined by language, and this, in turn, steers the behavior of humanity with regard to reality. The original essay also delved into the striking color substitutions in ancient Egyptian painting, which were documented but assumed to be without purpose. The specific cases were those where one and the same object was painted alternatively, sometimes red/sometimes yellow, or sometimes green/sometimes blue (Smith 1949; Williams 1932). The logical conclusion seemed to be that red \& yellow and green \& blue were not distinguished in language and thus that, although the Egyptians could distinguish the hues visually, the objects could be painted with different hues where the depiction did not depend upon the hue. In reality, of course, the link between the language-based denomination of the color to be depicted and the choice of a color cannot possibly be so simple. In any case, the choice of colors depended upon the materials available, which depended upon nature and technology. I would doubt the opposite contention, namely that the choice of colors depends exclusively upon the colors available (Blom-Böer 1994). The Egyptian artist would always have viewed the conceptual image as decisive, whereas the visual impression would have been less important. This can be illustrated by another example, neglect of the laws of perspective. An object in an ancient Egyptian image can be unfolded so that it can be simultaneously viewed from different angles, defying the principles of time and space. In the same fashion, the conception of a color was decisive in selection, meaning that the categorical classification must have matched a color term, which was mentally present. The issue of color substitutions merits a new study. I would advocate that my own statistical estimates of color substitutions in ancient Egyptian paintings should be replaced with more reliable data, but this must be left to our colleagues who are more competent in the study of painting. This is not the issue here, however, as we are concerned with color terms.

The Egyptological literature did not immediately seize upon my suggestion that a distinction be made between abstract and concrete color terms. Hermann (1969:362-373) even suggested that the idea of the Egyptians having only four abstract color terms was itself mistaken. He was unable to accept that 'red' could also be 'yellow' or that 'green' could also be 'blue,' or even that 'black' could also be 'gray.' He did, however, point out (as I did) that the Egyptian language permitted one to refer to yellow or blue by referring to an object or substance of the relevant color. He did not accept the concept of a categorical distinction between the abstract color words and other forms of color specification. Hermann also suggested that there was a significant problem in the discrepancy between the limited number of abstract color terms identified as opposed to the number of colors visible in Egyptian art. No less conciliatory was the response of Brunner-Traut (1977) who (like Hermann) composed a list of color terms (and substances) which was based on German, and not Egyptian, so that the equivalents are the German abstract terms, sup- 
plemented by references to substances (e.g. 'ochre colored' or 'lapis lazuli colored'). The linguistic approach thus met with no understanding.

The question of the meaning of the color terms was again taken up by Baines (1985), who was able to build on the theoretical structure established by Berlin and Kay (which would also have been possible for Brunner-Traut, had she not excluded theory from the outset). Baines concluded that Egyptian had four basic color terms (BCTs), 'black' ( $\mathrm{kmm}$ ),

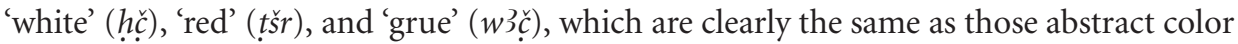
words (based on the terminology of an earlier school) presented in my 1963 paper. Baines was able to place this into a more universal linguistic system and thus to classify the ancient Egyptian language at Berlin and Kay Stage IIIa.

Unfortunately, the confusion between linguistics and pigments in the world of color was not eliminated from the Egyptological literature by Baines's use of Berlin and Kay. It remains a fact that the linguistic insights of Blom-Böer's (1994) work are not at the same level as her knowledge of pigments.

In his explanation of the Egyptian color terms, Warburton (2007) stresses the relationship with the real world, but also including Berlin and Kay and the subsequent discussion, including Baines. According to his version, the ancient Egyptian terms are really related to the colors of specific materials, namely precious stones, from which the terms would be etymologically derived. There would thus be at least initially no abstract color terms, but only concrete terms, so that, say, 'grue' ( $w 3 \dot{c})$ would thus originally at least mean 'malachite-like' or 'malachite-colored.' Clearly this explanation does not contradict Berlin and Kay: it is entirely reasonable to assume that in prehistoric times Egyptian stood at a lower level of development than the historical Stage IIIa. It is conceivable that the terms must have been derived from something, and minerals are thus a reasonable suggestion. It is, however, an entirely different matter when suggesting that the relationship between the color words and the colors of minerals continued into historical times. The extent to which the connection can be etymologically assumed remains to be discussed in the following paragraphs and in Section 4.

We will divide the matter into three issues:

1. The issue of color terms in general and particular usage. The discussion will be based upon the range of use dependent upon their roles as parts of speech, as a criterion for distinguishing BCTs and nonBCTs (Section 2);

2. The issue of the general meaning of the color terms and the determination of their specific focus (extension vs. intension) (Section 3);

3. The question of the etymologies, in Afroasiatic (Section 4).

Note that in the following discussion of ancient Egyptian matters, references to ancient Egyptian texts (requiring a knowledge of ancient Egyptian and familiarity with text editions) will be cited in a fashion familiar to Egyptologists. These Egyptological references are marked $\rightarrow$ and are not listed in the References (most of them can be found in Erman \& Grapow 1926-1963; Helck \& Westendorf 1992). 


\section{The basics: Parts of speech of the ancient Egyptian color terms}

\subsection{General notes on the parts of speech}

Color terms in Egyptian and Coptic are either verbs or adjectives. For example, tšr $r$ is a verb 'to be red,' while hsb čc(.l ?) is an adjective, 'relating to lapis lazuli, lapis lazuli-like, blue.' Verbs form participles, and thus adjectival verb forms. For example, ț̌r ' to be red' forms an active participle tšs 'being red,' and, simplified, an adjective, 'red.' Verbal color terms can thus appear in any syntactical context where nonparticipial adjectival color terms can be used. Nonparticipial adjectival color terms, however, can only be used in adjectival roles.

In Egyptian, all adjectives can be used attributively, and also generally as predicates. One can form the nominal phrases ir.t țšr.t 'red eye' and $i$ r.t h̆sbč.t 'lapis lazuli-like/blue eye' from the verb țšr 'to be red' and the adjective h $h s b c ̧$ (.l ? ) 'lapis lazuli-like, blue' (where the ' $t$ 'suffix is merely the feminine ending required to render the adjectives congruent with the ir.t 'eye', which is feminine). Adjectives can also be used as predicates, e.g., ț̌r ir.t 'the eye is red' or hhsbç(.l) ir.t 'the eye is lapis lazuli-like, blue.' The Egyptians particularly favored constructions linking an adjective and a defining substance, whereby grammarians are not in agreement as to whether the usage is adjectival or predicative: Hr.w tšr ir.ti 'Horus

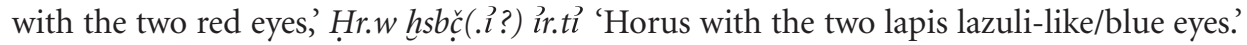
These can thus be understood as 'he who is red (blue) with respect to the eyes' (adjectival) or 'he whose eyes are red (blue)' (predicative). Verbal color terms can serve as adjectival predicates using adjectival participles, but they can also be used as verbal predicates, and thus in syntactic roles available only to verbs. Even if it is rare, verbal color terms can also be used in different tenses, which is impossible for adjectives. Summarizing, we can say that verbal color terms can be used more flexibly than nonparticipial adjectives, and thus it can hardly be accidental that the ancient Egyptian BCTs were verbs: $k m m, h \check{c}, t \check{s} r, w 3 \check{c}$, 'to be' 'black,' 'white,' 'red' and 'green,' respectively. Additional verbal color terms will follow, Subsection 2.3.

The parts of speech are also important for color terms since Egyptian-Coptic gradually lost the derived adjectival forms while retaining the category of the verb. In Coptic, the final stage, the adjectives are only preserved as relics. The paradigmatic link between the verbal color terms and the original derived participles and the possibility of using nonverbal color terms in predicative function was lost. Verbal color terms could no longer be used attributively (although adjectival relative clauses with verbal predicates could). The BCTs were thus all available as verbs (whereby individual words were replaced and a fifth BCT was added), but not the original adjectives. The verbal color terms were thus more stable in terms of the history of language.

The digitalized slip archive $(\rightarrow$ DZA) of the Egyptian-German Dictionary (Erman \& Grapow 1926-1963) of the Berlin-Brandenburg Academy of Sciences and Humanities [http.//aaew.bbaw.de/tla/] provides most of the important information beyond that of the dictionary (Erman \& Grapow 1926-1963). 


\subsection{Explicit statements about the colors of objects}

The abstract color terms appear as verbs in statements about the color of objects. Other indications of color, particularly with reference to minerals, are nonverbal. The situation is particularly clear in the schematic formulae of the so-called laboratory of the Ptolemaic temple at Edfu. From the prescriptions we shall - in the sense of the points discussed below Section 3 - seek those statements describing the colors of objects in the real world ( $\rightarrow$ Edfou II, 205ff.). The rules will be confirmed by additional references supplementing those from Edfu.

The following verbal BCTs are found:

$\mathrm{kmm}$ 'to be black';

$h \check{c}$ 'to be white';

ț̌sr 'to be red';

$m r s ̌$ ' to be (light-?)red' (for the meaning, see Section 3).

The word $w 3$ c ' to be green' is not found here, which can be explained as accidental, but perhaps also because the colors in the green-blue range are more precisely distinguished with references to minerals.

As minerals and other objects can be found:

$n b(. w)$ 'gold';

s3(.wi) 'two-thirds gold' (an alloy);

$n b . w n(. i) s p-3$ 'gold of the third quality';

$n b(. w) n f r n(. i) h 3$ s.t 'fine Desert Gold';

hrs.t 'carnelian' (which thus had a different color than the țšr 'red' found in the same textual context, possibly a yellow variety);

$n s ̌ m(. t)$ 'green feldspar' (casual reference, $\rightarrow$ Turin Cat. 1966 [love songs], rto. 2, 4, probably for green);

hssbç 'lapis lazuli';

čnh $n(. l) s f t$ 'the color of the wings of the sft-bird' (species undetermined).

In detail, the situation is as follows:

1. BCTs (verbs):

a. Clause with verbal predicate (Old Perfective):

$i w n=f$ 'its color':

$k m(. w)$ 'is black' ( $\rightarrow$ Edfou II, 208, 1), $k m(. w) n h t$ 'is dark black' ( $\rightarrow$ Edfou II, 207, 12-13; 207, 14-208, 1);

h.̣ (.w) nht 'is bright-white' ( $\rightarrow$ Edfou II, 207, 1);

tšrr.$w$ ) 'is red' ( $\rightarrow$ Edfou II, 205, 16; 206, 6; 206, 16; 207, 12-13; 207, 13), alongside

iwn $f p w$ : țšr 'that is its color: red (or: being red?)' ( $\rightarrow$ Edfou II, 205, 14);

$m r r^{\circ}(. w)$ 'is (light-?) red' ( $\rightarrow$ Edfou II 206, 1).

b. Attributive participle/adjective:

$h r(. t)(?)=w m$ 'their character consists of': 
iwn ťšr 'red color' (of mountains where gold is found) ( $\rightarrow$ Turin gold mine map [ASAE 49:340]).

2. Concrete color terms (colors of minerals):

a. Clause with nonverbal (adverbial) predicate; identification with mineral: iwn $=f m$ 'its color consists of':

$n b(. w)$ 'gold' ( $\rightarrow$ Edfou II, 205, 13; 205, 14; 206, 5; 206, 9);

hrs.t 'carnelian' ( $\rightarrow$ Edfou II, 206, 3);

liwn=sn $m$ 'its color consists of':

$s 3(. w i)$ 'two-thirds gold' (an alloy) ( $\rightarrow$ Edfou II, 217, 4, not in the context of a prescription);

also nonminerals as comparative material:

iwn $=f m i$ ' its color is like that of':

$i w n\langle n(. l)\rangle \stackrel{c}{c} n h\langle n(. \vec{l}) s f t\rangle$ 'the color of the wings of the sft-bird' ( $\rightarrow$ Edfou II 207, 7, corrected after 207, 11).

b. Indication of minerals in noun phrase:

'something is' $m \imath$ ' 'like':

$i w n n(. l) n b(. w)$ 'the color of gold' ( $\rightarrow$ Edfou II, 207, 8; 207, 10; presumably also 207, 8; $i w n\langle n(. l)\rangle n b(. w) h n^{c} k^{c}$ 'color of gold and $k^{C}$-mineral';

iwn n(.i) nšm(.t) 'the color of green feldspar' ( $\rightarrow$ Turin Cat. 1966 [love songs], rto. 2, 4);

iwn $n(. l) h$ hsbç 'the color of lapis lazuli' (of a lake) ( $\rightarrow$ Nauri-Decree [JEA 13, Pl. XL], 11);

'something is' $m$ 'consisting of':

$i w n n(. i) n b . w n(. i) s p-3$ 'the color of gold of the third quality' (of copper) ( $\rightarrow$ pHarris I, $78,4-5)$;

iwn $\langle n(. i) ?\rangle n b(. w) n f r n(. i) h 3$ s.t 'the color $\langle$ of $\rangle$ fine Desert Gold' (of copper) ( $\rightarrow$ pHarris $\mathrm{I}, 47,4)$;

$i w n\langle n(. l) ?\rangle n b(. w)$ 'the color $\langle$ of $\rangle$ gold' (of copper) ( $\rightarrow$ pHarris I, 6, 9);

here also a comparison with nonmineral:

iwn $\langle n(. i)\rangle$ čnh $n(. i) s f t$ 'the color of the wings of the sft-bird' ( $\rightarrow$ Edfou II, 207, 11).

\subsection{Color statements about objects}

In statements about the colors of objects, the BCTs generally appear as participles or adjectives, but occasionally in nonadjectival verbal forms, confirming their verbal character. Concrete color words and nonbasic color terms are also used as adjectives, as may be expected. There are, however, some cases opposing the basic rules. The usage was not consistent throughout the history of the ancient Egyptian language. For etymological reasons (more in Section 4) verbal usage cannot be assumed for the early period; the usage 
cannot be precisely defined. It must also be excluded for Coptic, the latest phase of the development of the language. The issue of those textual contexts where verbal forms appear is thus even more interesting: These are neologisms in the traditional written language, not the colloquial.

The following can be deduced from the statements about the color of objects:

1. BCTs (verbs, participles):

a. $k m m$ 'to be black':

The word is generally used as a participle or adjective, even as a predicate. There are, however, also references in which the word is used in a nonadjectival inflection at an epoch when the adjectival category was still existent:

"Keep (imperative form, meaning Aton) him (i.e., the king) here (i.e., among us) until

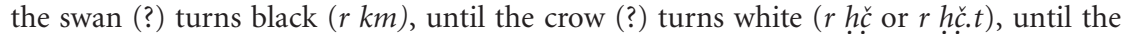
mountains stand up to go, and until the flood flows backwards, ..." ( $\rightarrow$ Amarna III, III, 6; XXIX, 10-13 (Synopsis: $\rightarrow$ Sandman, Texts from the Time of Akhenaten I, 9, 6-9)); 'so as to cause thee to be black ( $w n=t k m . t i)$ like Horus, and red (ț̌sr[.tỉ]) (like Seth).'

$(\rightarrow$ pChester Beatty VII, rto. 8,4$)$;

'he is red(brown?) (wn=ftšr $(. w))$, but not (entirely?) black $(n k m=f)$ ' ( $\rightarrow$ Edfou II, 208, 4);

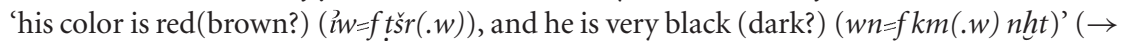
Edfou II, 207, 12-13).

Additional references are in Subsection 2.2 above.

In Coptic the verb was still used, being fully inflected (infinitive and qualitative, $\mathrm{kmom}$ etc.). The participle is preserved as a relic (kam etc.).

b. huç 'to be white,' later $w b h$ 'to be white':

The verbal character of the color word $h \grave{c}$ 'to be white' cannot be shown with equal clarity or even the same abundance as earlier. Aside from the specific meaning of a color word is the more general meaning 'to become bright' (meaning 'dawn'), and in later texts the meaning even appears as 'to shine' as the activity of the light of the sun. The verbal use is common in this general meaning, and can be seen in the reference just cited for black, and in Subsection 2.2 above.

Only the adjectival form of the word is preserved in Coptic (hat etc.). The verb is replaced by another, which only appeared in the course of the historical development of the language:

$w b h$ 'to be light, to shine'.

This only appears in the latest form of Egyptian using the original script (Demotic) as a color word for white and is then used in Coptic in place of hç ' to be white' (oubaš). The Coptic also used the adjectival relic of this verb (ouōbš).

As an adjective, and exclusively as an adjective, for white Coptic uses another neologism, ${ }^{*}$ Cnw 'beautiful' (?) > Coptic alaou 'white' which has not been discovered in any pre-Coptic stage of the language; even the origin of the word remains doubtful.

c. $t$ šr $^{\prime}$ 'to be red': 
The word is generally used as a participle/adjective, even as a predicate. There are, however, also references where the word is used in a nonadjectival inflection at an epoch where the adjectival category was still in use:

'But as for any future king who shall overthrow any of my plans, and just says: 'The lands are under my authority (anyway), and they are mine as they were his,' - (this will be) a bad thing in the opinion of the gods. See, he will be accused ( $m k t w=t w r w s b=f)$ in Heliopolis they (i.e., those of Heliopolis) are the Divine Tribunal ... they will defend (ir $=s n$ wšb) their property. They will become red ( $(. \grave{s} r=s n)$ as firebrands, so that they may burn up (snwh $=s n$ ) the bodies of those who fail to heed me; and they will consume ( $s t r$ sswn) whoever upsets my plans, to cast him (down) on the slaughtering-block of the Netherworld.' ( $\rightarrow$ KRI I, 69, 2-6, after $\rightarrow$ KRI Translations I, p. 59; although one could structure the text differently, it is a future verbal form: 'they will become red' or 'they will be red' of the suffix conjugation and not an adjective *'they are red.');

'his body is red ( $\underline{h} . t=f t \underline{s} r)$ like the color of gold' ( $\rightarrow$ Edfou II, 207, 8.).

Additional references have already been cited under $\mathrm{kmm}$ 'to be black':

'he is red(brown?) (wnn=f tšr $[. w])$, (but) not (entirely?) black ( $n k m=f)$ ' ( $\rightarrow$ Edfou II, 208, 4);

'his color is red(brown?) (iwn=ftšr [.w]), and he is very black ( $w n=f k m[. w] n h t)$ ' $(\rightarrow$ Edfou II, 207, 12-13).

Although partially restored, a reference cited above ('black like Horus,' 'red like Seth,' $\rightarrow$ pChester Beatty VII rto. 8, 4) is also highly indicative.

The verbal usage was also demonstrated in Subsection 2.2.

In Coptic the verb was in use, fully inflected (infinitive and qualitative tōršs, torš etc.), the participle surviving as a relic (tōrš etc.) (Crum 1939:432). In addition, a reduplicating verb (trošr (e) š) is more frequent in Coptic (Crum 1939:432), although unknown from Egyptian:

${ }_{t}^{*} t_{s ̌ r} r \check{r} r$, Coptic trošr (e)š 'to be very (?) red’ (for more detail, cf. Section 3 below).

An additional Coptic neologism is:

$m r \check{s} / m l_{\breve{s} /}^{*} m n n \check{s}$, Coptic mroš etc., and the like, Coptic 'to be (light-?)red' (for details, cf. Section 3 below).

This is already known occasionally from Roman Egyptian (Demotic) as an adjective, but it became a verbal color term (mroš); the adjective also survives in Coptic (mērš etc.).

d. $w 3$ ' to be green':

Although doubt may be thrown onto the verbal character of the color term, the word also means 'to be fresh, to prosper,' where the verbal character cannot be doubted. It is therefore difficult to isolate cases where the use of the color term as a verb can be claimed with certainty. On the other hand, logic would suggest that the verbal character of the color term can be assumed, based on the verbal character of the alternative meaning. 
The word is preserved in Coptic with the same range of meanings as in Egyptian (ouōt etc.).

2. Concrete/Nonbasic color terms unknown in colloquial Egyptian and in Coptic, which was closer to the colloquial:.

a. $\quad \check{c} c b$ (factitive) 'to render like charcoal, to blacken':

A verb $\grave{c} c b$ can be found in the literary language of the New Kingdom (Erman \& Grapow 1926-1963: V, 536, 4), but the verb is unknown in Coptic:

(label of a god) 'he who makes the body black (charcoal-like) with charcoal' (participle $\check{c}^{c} b$ with verbal actants) (magic spells in medical texts: $\rightarrow$ pHearst 11, 13; $\rightarrow$ med. pLondon 16,8)

b. hrs 'carnelian-like,' 'to be red':

The word, hrs 'carnelian-like,' 'to be red,' is found in Ptolemaic-Roman literary Egyptian. It is also used with respect to the eyes, metaphorically in the sense of 'being angry or furious' (like tšr 'to be red'), or 'gleaming, glittering.' It is not found in Coptic. Examples (Erman \& Grapow 1926-1963:III, 151,1-2; more references $\rightarrow$ DZA 27266750 ff.):

(caption above Horus of Edfu) 'whose two eyes are carnelian-like/red' ( $\rightarrow$ Edfou I 31, 8, participle hrs in a verbal relative construction);

with factitive meaning:

'I make my two eyes carnelian-like/red ( $h r s(. i)=i)$, I make my two eyes red like ins.li textile $($ ins $=i$ )' ( $\rightarrow$ Edfou VI, 75,7-8; also 178, 3).

This quote also includes an example of the word ins.l 'red,' known from Ptolemaic-Roman literary Egyptian (Erman \& Grapow 1926-1963:I, 100, 5), but not from Coptic.

c. mfk... 'turquoise-like,' 'to be green' (?):

The verb $m f k$ is known from the Ptolemaic-Roman literary language with the meaning 'to be glad, joyous,' strictly speaking, 'to shine' (Erman \& Grapow 1926-1963: II, 58, 3-5, but cf. also II, 58,2$)$, the meaning of which is derived from the glitter of faience, $m f k 3 . t(\hat{l}$ ? $)$ 'turquoise-like.' References where $m f k$ can be understood as a verb include the participle in most cases, and thus the adjectival usage, but this could easily be a nonparticipial adjective. It would thus follow that no specifically verbal color term $m f k$ can be identified (Erman \& Grapow 1926-1963:II, 57, 9-58, 1, and more detailed $\rightarrow$ DZA 23998820ff.). This does not, however, definitively exclude a verbal color word $m f k$ 'to be green.' In any case, such a word is not found in Coptic.

d. hsbč 'lapis lazuli-like, blue':

A verb $h s b t$ ( $<h s b c ̧$ ) 'lapis lazuli-like, blue' is found in literary Egyptian from the New Kingdom and the Ptolemaic-Roman period, but not in Coptic.

References from the New Kingdom (Erman \& Grapow 1926-1963:III, 334, 20):

'Nut (i.e., the personified heaven) is behind you (i.e., the sun-god, the sun), lapis lazulilike/blue (hsbt.ti).' ( $\rightarrow$ BD Naville, 15A, II 8);

'Nut enters lapis lazuli-like/blue (hsbt.t( $(?$ ? )) behind you.' ( $\rightarrow$ TT 65$)$;

'Your (i.e., the deceased to whom the text was directed) head is lapis lazuli-like (hsbt.y), 
your hair is blacker than the doors of any star when eclipsed. Your hair places (?, $\left.\check{s}^{c} m . w\right)$ lapis lazuli over your face' ( $\rightarrow$ BD Naville, 172, 12).

References of Ptolemaic-Roman date with factitive meaning (only verb and object):

(of the sun) 'who renders the fields lapis lazuli-like/blue (!) with his rays' ( $\rightarrow$ Edfou I, 106, 2-3, participle hsbt);

(of the sun) 'who renders his fields lapis lazuli-like/blue (!)' ( $\rightarrow$ Edfou I, 71, 11, participle $h s b t)$.

e. $\quad \check{c} f r r$ 'to be lapis lazuli-like, blue':

A verb čfrr 'to be lapis lazuli-like, blue' is known from the literary Egyptian of the Ptolemaic-Roman period (Erman \& Grapow 1926-1963:V, 300, 4), but not in Coptic:

'(fields) being lapis lazuli-like/blue (tfrr. $\{w\}(?) t(i$ ? $))$ with (the plant) color-of-heaven (i.e., blue blossoming flax)' ( $\rightarrow$ Edfou IV, 31, 109,10-11; $\rightarrow$ Dümichen, Geogr. Inschr. IV, 118), on 'flax' (cf. Meeks 1972:116).

\subsection{Summary}

Examining parts of speech that constitute color terms yields:

1. In all periods of the Egyptian language, four verbal color terms were in use, viz., 'to

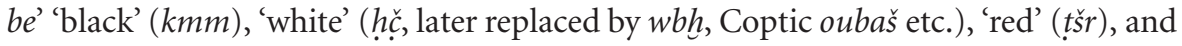
'green' $(w) \breve{c})$;

2. Beginning in the latest stages of the Egyptian language, the red range is partitioned into two verbal color terms (to be specified below, Section 3): red ( $t \grave{s} r$ ), more pre-

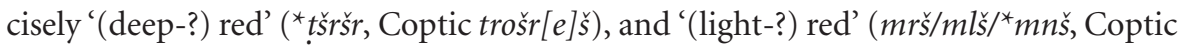
mroš etc.);

3. At various times, the traditional literary language also formed verbal color terms based upon materials, including '(dark-?) green,' and above all in the blue range. These latter are strikingly common. On the other hand, there are no terms which separate a yellow range from the red range.

\section{The identification of the meanings of the ancient Egyptian-Coptic color terms}

\subsection{Prolegomena}

The philologist studying the texts of a dead language approaches the meaning of a word initially through its use and its extension. Philology can establish the objects to which a color term is applied. The philologist's ability to reach the goal depends upon the sources. The absence of a native speaker means that further confirmation cannot be marshaled. One additional restriction under which the Egyptologist must work is that he can only turn to real objects for orientation whereas the texts deal largely with nonreal objects whose colors cannot be examined. It does not help him to know that the eye of the god Horus can be white under certain circumstances, but also green, nor that there is a Horus 
with red eyes and a Horus with lapis lazuli-like or blue eyes. With white and green it is not even possible to be certain that the colors are meant, rather than that the expressions perhaps refer to shining and flourishing. Nor is it possible to be certain about the color of real objects because it is not certain that a color aspect is the primary issue, and in the case of, say, textiles, we may have a recognizable color reference, but not the actual original color of the object, quite aside from the constant danger of an ancient error, where objects will have had different colors in reality.

In the earlier work (Schenkel 1963), the decisive criterion of the distinction for the purely abstract color words, the BCTs, was range of usage, the number of objects distinguished by a color word. It made no difference whether the color of an article could be empirically determined or not. The range of abstract color words was reached by two steps, establishing the translation equivalents in the German-Egyptian dictionary (extension) was followed by the projection of the semantic field of the abstract color terms onto the real world of color (the Ostwald Color Cone) in such a way that the abstract color terms thus covered the real world (intention).

Having recourse to the German Egyptian-German dictionary was legitimate because the definitions were inferred from the references, with the Egyptian usage in describing the world being given the primary role. This dictionary had the further advantage that discrepancies between the scope of the definition of a word and its translation equivalent were given a central role. Although certain dangers remained, the German Egyptian-German dictionary clearly established that the focus of the term for the warm (red-with-yellow) range lay in red, and the focus of the cool term in green. Since the main volumes of the German Egyptian-German dictionary were completed in 1931, there was no possibility of influence from Berlin and Kay. At the same time, projection onto the color cone distorts linguistic reality as far as it does not take into account the focus of the color words, but rather is a regression with respect to the earlier philological work.

Up to present, the discussion of the identification of color terms has neglected the distinction of whether (1) an object is designated with a color word or a derivative, or (2) is characterized using the color word. It is a designation when desert is described as the red (tšr.t). Such - presumably prehistoric - designations cannot be pursued, but they do not necessarily reveal the actual meaning which the color term has in the textual traditions of the historical period. It can be possible that the focus of the color word has shifted (see Section 4), and thus the desert differs from the focus of the color word known in the historical period (red). The real pertinent meaning of a color word is certain where an object is assigned a color, whether in the form of a predication or as an attribute.

\subsection{Extension of the Egyptian color words}

Note that only the specific references are cited here. References to the German EgyptianGerman Dictionary (Erman \& Grapow 1926-1931) can be found in Schenkel (1963: 140$143)$ and Brunner-Traut (1977: 118ff.). 


\subsubsection{Basic color terms}

1. $\mathrm{kmm}$ 'to be black/dark gray/brown':

Characterisation of real world objects:

Dark gray granite described as inr km 'black stone' (Erman \& Grapow 1926-1931:V, 123, 4; more references in $\rightarrow$ DZA 30595160 ff.; on granite, cf. Harris [1961:72-74]);

Flint;

The lizard $(\rightarrow$ pEbers 66,18$)$;

The sun-burnt harvester;

Designations of real world objects:

Km.t 'Egypt', the dark gr. arable land of the Nile Valley in contrast to țšr.t 'desert';

The black of the pupil in the eye.

2. $h \grave{c}$ ' to be white, light':

Characterizations of real world objects:

Limestone, described als inr hçç 'white, light stone';

Sandstone, described as inr hç $n(. l)$ rwč.t 'firm light stone'; in opposition to Warburton (2007) I understand $i n r$ ḥç n(.ỉ) rwčc.t to be 'white/light firm stone,' a stone which is light like limestone, but harder);

Milk;

Honey;

Bread ( $t$ ḥc 'white bread');

Teeth;

Designations of real world objects:

Silver;

ḥִ̆.w 'onions';

h.c.t 'the white of the eye' (in contrast to the 'black' pupil);

hic.t 'the white crown' (of the king).

3. tšr 'to be red/brown/yellow':

Characterizations of real world objects:

Carnelian/sard/chalcedony: Archaeologically discovered objects are flesh-red or reddish brown, in Middle Kingdom and New Kingdom also yellowish (Nicholson \& Shaw 2000:27). hrs.t ț̌r.t, traditionally translated as 'red carnelian' characterizes the darker variety (contrast to hrs.t h.c.t) (Harris 1961:121) and thus probably red to brownish (Nicholson \& Shaw 2000:27) sard (e.g. $\rightarrow$ pEbers 10,11);

Natron: ḩsmn țšr , traditionally translated as 'red natron,' "refers to red natron, so coloured by some impurity, presumably an iron compound" (Harris 1961:195);

Myrrh: yellow to brown bits;

The medical description of 'being red,' and the derived word form $t \grave{s} r$ 'reddening';

Sandstone: çw tšrr, Arabic al-Jabal al-ahmar, 'the red mountain' near Cairo, a sandstone ridge as an island in a limestone formation;

Mars, the red planet, personified as Hr.w ťšr, traditionally translated as 'the red Horus' (Erman \& Grapow 1926-1931:V, 489, 7);

Designations of real world objects: 
Blood, especially ț̌r.w 'blood';

The sandy desert in tšr.t, 'mountain land, desert, savannah (?)';

The flamingo: deduced from the hieroglyphic sign with the phonetic value tšr $r$

tšr.t 'the red crown' (of the king);

$m r \check{s} / m l \check{s} /{ }^{*} m n s ̌$, Coptic mroš etc. 'to be (light-?)red.'

The word is common in Coptic. The dictionaries vary in indicating the meaning between red and yellow, or intermediate tones, bright red and blond have also been used as translation equivalents. In determining the meaning in the recent literature, the etymological association with the Egyptian word, mnš.t, for a kind of ochre has played a major role. Whereas the earlier literature interpreted it as yellow ochre (also Schenkel 1963), red ochre is increasingly preferred (cf. Baines 1985).

The references in the Coptic Dictionary compel a definition in the red range, but a more precise determination is difficult since the Coptic translations of Greek do not seem to consistently distinguish mroš and the other words for red (tōrš etc., trošr(e)̌s) (Crum 1939: 183). This makes an Arabic translation which is not in the Coptic Dictionary more interesting. Jürgen Horn drew my attention to a Greek-Coptic-Arabic word list (Scale) where the Coptic adjective merš is translated into Arabic as 'šqar ( $\rightarrow$ Paris, BNF, Copte 44, fol. 66 vso., ll. 46 and 56) and thus something like 'fair-complexioned, blond, reddish' (among translations in Wehr 1979:562). Another Greek translation points in the same direction: zanthōr (read xanthón) ( $\rightarrow$ Paris, BNF, Copte 44, fol. 66 vso., 1. 56) which the Greek dictionary defines as "yellow, of various shades, freq. with a tinge of red, brown, auburn” (Liddell \& Scott 1940:1187b).

4. $w 3 \grave{c}$ ' to be green':

Characterizations of real objects:

Malachite and other green stones;

Green eye paint;

Plants, particularly papyrus;

Occasionally and not certain: the sky ( $\rightarrow$ Philae I, 160, 5-6);

Designations of real objects:

The sea called $w 3 \grave{c} w r$, 'The great green.'

\subsubsection{Concrete color words/NonBCTs}

For $i n s, m f k, h r s, \underline{c} c b$ in characterizations of nonreal objects, see Section 2.

1. čms 'red' or the like:

Characterization of real objects, as variant of țšr:

The medical finding of reddening;

Designation of real objects, as variant of ț̌r:

The Red Crown of the king;

Characterization, not real, but drawn from the real world: 
The backside of a baboon (cf. Edel 1956:74-76) whose ears are ț̌sr 'red.'

The hieroglyph used in the writing of the word represents the writing utensils. The color $\check{c} m s$ could thus be the red used in writing (as the Egyptians used black and red ink). Harris (1961:227) suggested, however, that "The word is derived from the flower or fruit of some tree, but cannot be identified precisely."

2. $\check{c}(w) r, w t r$ 'to be red' or the like:

Designation of real objects, as variant of $t \check{s} r$ :

'The red' as designation for blood (Erman \& Grapow 1926-1931:V, 386, 13).

3. hsbč (.l? ?) 'lapis lazuli-like, blue':

Designation for real objects:

Blue cloth;

The adjective $h s b \check{c}(. l$ ? $)$ 'lapis lazuli-like, blue' is derived from the word for the material, and is typically applied to nonreal objects, recalling the semi-precious stone or made of it, and thus means blue:

Eyes, hair, head, horns, or even the entire figure of gods;

Accidentally, also for blue: of the heavens, but also for the greening of the fields (by the sun whose rays render the fields $h s b \breve{c}(. l ?$ ? $))$, compare this with Section 2 above.

4. $\quad \check{f} f r r(. l$ ?) 'to be lapis lazuli-like, blue':

Designation of real objects, possibly less a designation than a metaphor:

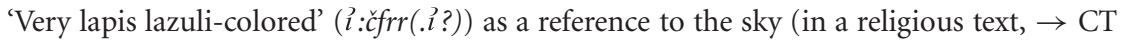
VII, 220b).

5. $n b . w(l ?$ ?) 'golden':

Designation of real objects, possibly less a designation than a metaphor:

'The Golden One' (?) as designation for the sun (Erman \& Grapow 1926-1931:II, 239, 8).

\subsection{Summary}

As the result of this investigation of the range of uses of the color terms, we can conclude:

1. Those words which were initially identified as abstract ( $k m m$ 'black'; $h c ̣$ c $w b h$ 'white'; tšr 'red'; and $w 3 \grave{c}$ c 'green') were the most widely used color terms;

2. The focus of $t \grave{s} r$ 'red' was in the red-range, and $w 3 \check{c}$ ' green' in the green-range, as traditional Egyptological interpretations imply, and not in the red-with-yellow or, respectively, green-with-blue ranges;

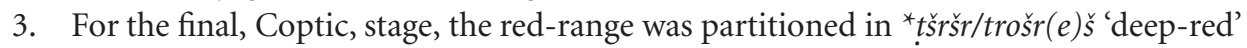
and $m r \check{s} / m l \check{s} /{ }^{*} m n \check{s} / m r o s ̌ ~ ' l i g h t-r e d ' ;$

4. All of the other, concrete terms have a restricted range of uses, largely restricted for nonreal objects;

5. In the final case, the prestigious blue-range is highly significant. 


\section{On the etymologies of the color terms}

Following the earlier Egyptological ideas adopted by Hermann (1969), the abstract color terms are derived from object-names: 'green' from papyrus-colored, 'red' from flamingocolored, 'white' from stone-mace-colored, 'black' from coal-colored. The origin of the principle is less the etymological link between the color terms and the object-names (which exists in at least two cases), so much as the hieroglyphic signs used as written symbols to write the color words: papyrus and green, flamingo and red, stone mace and white (coal for 'black' is an error). In contrast, deriving the abstract color words or BCTs from minerals, particularly semi-precious stones, as does Warburton (2007), is more adequate because such a link exists for the concrete color words or nonBCTs (Section 2 above).

In fact, however, in the case of the four existing abstract color words or BCTs, these are not new creations based on ancient Egyptian givens. All four (or most) can be identified as verbs in the Afroasiatic languages, and thus must have been verbs in the original Afroasiatic Ursprache. The following etymologies can be proposed:

1. $k m m$ 'to be black':

Related to Syrian and (Talmudic) Hebrew 'km 'to be black' (Takács 1999:219; also accepted in the critical review by Osing 2001:568);

2. hç ' to be white':

Related to Arabic șhw, Hebrew and Syrian șhy, șhh 'to be clear, light' (Osing 2001:579). The use as a color term would thus be an internal development in Egyptian, which would also explain the continued existence of the meaning 'to be light,' etc.;

3. tšs ' 'to be red':

Related to Semitic șhr 'to be reddish': Arabic (verb, stem XI) șhr 'to be white-red, yellowish', (adj.) 'aṣhar 'yellowish-red, desert-colored' ( $s a h r a \bar{a}$ 'desert,' 'Sahara'), Syrian aṣghar 'to blush' (Schneider 1997:208). This etymology is a brilliant example of the "New Comparative" school of Otto Rössler, rejected by the traditional comparative school because the phonetic changes are dismissed as impossible. However, should this be nevertheless correct, the ancient Egyptian designation of the desert (t.šr.t, 'the red (land)') would be derived from the same word as Arabic Sahara;

4. $w 3 c$ ' to be green':

Related to Semitic and Berber wrq 'to be green' (first Ember 1930:9; today generally accepted, cf. e.g. ["New Comparative" school] Rössler 1971:316; [traditional school] Vycichl 1983:238).

These etymologies permit the hypothesis that Egyptian was already at Stage IIIa in prehistoric times.

The specific philologically based translations of the color terms of languages other than Egyptian permit shifts of focus to be recognized. In historical times, the focus of tšr 'to be red' lay in the red range, but in the related Semitic languages it would appear to be closer to the lighter yellowish-red range. Whether Egyptian or Semitic preserved the earlier 
focus can only be concluded on the basis of a study of BCTs in Afroasiatic languages, which is beyond the competence of an Egyptologist.

Aside from the inherited BCTs, Coptic (the final stage of the Egyptian language) produced two neologisms: oubaš (etc.) 'to be white,' which replaced Egyptian huc 'to be

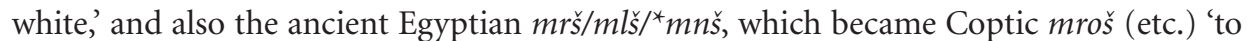
be (light-?) red.' The first is a shift whereby ancient Egyptian $w b h$ 'to be light' was replaced with Coptic oubaš (etc.) 'to be white'; the second is derived from the material red ochre (mnš.t).

The derivation of names for color terms in ancient Egyptian from object-names is demonstrable in some cases for NonBCTs (traditional written language): hssbç (.l? ?) '(to be) lapis lazuli-like,' čfrr(.l. ?) '(to be?) lapis lazuli-like, blue,' $m f k$ 3.ti 'turquoise-like, green/blue.' Other color indicators of this kind appear, e.g., $\stackrel{c}{c} b(. l$ ? ?) 'charcoal-like, black.' None of these NonBCTs which developed in Egyptian was maintained; none were used in Coptic.

The ancient NonBCTs in the red range $(\check{c} m s, \breve{c}(w) r)$ are probably of Afroasiatic origin, although a concrete etymology cannot be produced. Whether these were originally color terms or derived from objects will obviously depend upon discovery of the etymologies.

\section{Summary}

The examination of the ancient Egyptian-Coptic color terms and their grammatical identification, the range of usage, and their etymologies permit the following conclusions:

1. There are four BCTs, all verbs, 'to be' kmm 'black,' hç (later wbh) 'white,' ț̌rr 'red' and $w 3$ č 'green';

2. The focus of ț̌r 'red' is in the red range, as traditionally translated, and noted by Baines (1985:283), and not in the middle of the red-with-yellow range, as argued following the color cone approach by Schenkel (1963);

3. The focus of $w 3$ c 'green' lies, as traditionally translated, and noted by Baines (1985:283) in the green range, and not in the middle of the green-with-blue range as proposed by Schenkel (1963) on the basis of the color cone projection, nor in the pale green suggested by the Berlin and Kay hypothesis;

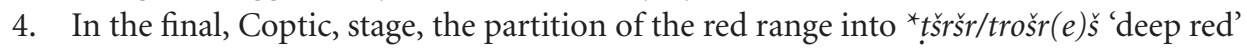

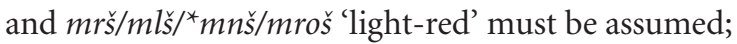

5. The traditional written language allowed additional place for further verbal color terms (i.e., BCTs), but these were not preserved in Coptic, and thus they never entered the colloquial language;

6. Among these alternatives, the use of $h s b c r(. l ?$ ? ) 'lapis lazuli-colored' is strikingly common; that of $c$ c $c m(. l$ ? ) 'charcoal-like, black', $n b w(. l$ ? ) 'golden' is less common;

7. The dominance of 'lapis lazuli-colored, blue' was inadequately treated by Schenkel (1963) and Baines (1985), due to their methodological premises (semantic field and B\&K hypothesis), although recognized by the empiricists (Hermann 1969; 
Brunner-Traut 1977), and recently explicitly formulated (Quirke 2001:187; Warburton 2007). However, these latter go too far when concluding that Egyptian developed "a term translatable as blue before it develops a term translatable as yellow" (Quirke 2001:187). Egyptian did not, in fact, develop a BCT for either blue or yellow;

8. Egyptian-Coptic remains at Berlin and Kay Stage IIIa. It must be affirmed that the red range was further partitioned in the final, Coptic, stage.

\section{Acknowledgments}

I would like to thank David Warburton, who translated this contribution from German into English and at the same time rendered it concise.

\section{References}

Baines, John (1985). Color terminology and color classification: Ancient Egyptian color terminology and polychromy. American Anthropologist, 87, 282-297.

Berlin, Brent \& Paul Kay (1969). Basic Color Terms: Their Universality and Evolution. Berkeley: University of California Press.

Blom-Böer, Ingrid (1994). Zusammensetzung altägyptischer Farbpigmente und ihre Herkunftslagerstätten in Zeit und Raum. Oudheidkundige Mededelingen, 74, 55-107.

Brunner-Traut, Emma (1977). Farben. In W. Helck \& W. Westendorf (Eds.), Lexikon der Ägyptologie (Vol. 2: 117-128). Wiesbaden: Harrassowitz.

Crum, Walter Ewing (1939). A Coptic Dictionary. Oxford: Clarendon.

Edel, Elmar (1956). Beiträge zum ägyptischen Lexikon III. Zeitschrift für ägyptische Sprache und Altertumskunde, 81, 68-76.

Erman, Adolf \& Hermann Grapow (1926-1963). Wörterbuch der aegyptischen Sprache. Leipzig: Hinrichs, Berlin: Akademie-Verlag.

Ember, Aaron (1930). Egypto-Semitic Studies. Leipzig: Asia Major.

Harris, John (1961). Lexicographical Studies in Ancient Egyptian Minerals. Berlin: Akademie-Verlag.

Helck, Wolfgang \& Wolfhart Westendorf (Eds.) (1992). Lexikon der Ägyptologie, Vol. 7. Wiesbaden: Harrassowitz.

Hermann, Alfred (1969). Farben. In T. Klauser (Ed.), Reallexikon für Antike und Christentum (Vol. 7: 358-447). Stuttgart: Hiersemann.

Liddell, Henry George \& Robert Scott (1940). A Greek-English Lexicon. Oxford: Clarendon.

Meeks, Dimitri (1972). iwn-n-pt $={ }^{\mathrm{S}}$ [auanmpe] = le lin. Revue d'égyptologie, 24, 116-119.

Nicholson, Paul T. \& Ian Shaw (2000). Ancient Egyptian Minerals and Technology. Cambridge: Cambridge University Press.

Osing, Jürgen (2001). Review of Takács (1999). Bibliotheca orientalis, 58, 565-581.

Quirke, Stephen (2001). Colour vocabularies in ancient Egyptian. In W. V. Davies (Ed.), Colour and Painting in Ancient Egypt (pp. 186-192). London: British Museum.

Rössler, Otto (1971). Das Ägyptische als semitische Sprache. In F. Altheim \& R. Stiehl (Eds.), Christentum am Roten Meer, Vol. 1 (pp. 263-325). Berlin \& New York: Walter de Gruyter. 
Schenkel, Wolfgang (1963). Die Farben in ägyptischer Kunst und Sprache. Zeitschrift für ägyptische Sprache und Altertumskunde, 88, 131-147.

Schneider, Thomas (1997). Beiträge zur sogenannten "Neueren Komparatistik". Lingua Aegyptia, 5, 189209.

Smith, W. Stevenson (1946). A History of Egyptian Sculpture and Painting in the Old Kingdom. Oxford: Oxford University Press.

Takács, Gábor (1999). Etymological Dictionary of Egyptian, Vol. 1. Leiden, Boston \& Köln: Brill.

Vycichl, Werner (1983). Dictionnaire étymologique de la langue copte. Leuven: Peeters.

Warburton, David A. (2007). Basic color term evolution in light of ancient evidence from the Near East. In R. E. MacLaury, G. V. Paramei, \& D. Dedrick (Eds.), Anthropology of Color: Interdisciplinary Multilevel Modeling (pp. 231-248). Amsterdam: John Benjamins.

Wehr, Hans (1979). A Dictionary of Modern Written Arabic (Arabic-English) (4th ed.). Wiesbaden: Harrassowitz.

Williams, Caroline Louise Ransom (1932). The Decoration of the Tomb of Per-nēb. The technique and the color conventions. New York: Metropolitan Museum of Art. 


\title{
Basic color term evolution in light of ancient evidence from the Near East
}

\author{
David A. Warburton \\ Department of Study of Religion, University of Aarhus, Denmark
}

\begin{abstract}
Ancient languages rely on concrete and specific meaning rather than abstraction, naming basic color categories differently than do the contemporary languages upon which Berlin and Kay base their universalist evolutionary theory. The ancient languages named red and blue with words transparently derived from precious stones; they applied these to more than one basic category; and they exchanged these traditions, and sometimes the terms. We find the evidence through interpretation of the same philological data used to support conclusions favorable to Berlin and Kay's thesis. Superficially, the validity of Berlin and Kay's thesis is thrown into doubt, as it would appear that it loses its "universal character" due to the neglect of the nuances visible in ancient languages: application of their hypothesis actually obscures the early evidence. Studying linguistic color expression of antiquity contributes to understanding color expression, thus improving overall insight into the evolution of color terminology.
\end{abstract}

\section{Issues}

Evidence from the earliest accessible languages provides a departure for any discussion of the evolution and distribution of color terms. Given the inherent difficulties of the ancient Near Eastern Languages, the issue has been misunderstood and neglected. We discuss such data as may be relevant to the mainstream theoretical discussion, warning against potential bias among presuppositions of Berlin and Kay's (1969) universal evolutionary order, restated by Kay and Maffi (1999) and reviewed by MacLaury (1999). The controversy centers on splitting of green-blue, especially on distinct terms designating blue, and is compounded by the issues of loanwords and levels of abstraction. The concept of an evolutionary sequence implies that words do not wander from language to language, but rather that languages evolve separately, and that abstraction comes naturally.

Our material from ancient Egypt implies that a vocabulary of seven or eight basic color terms persisted from the late third millennium BC. This complements evidence from languages of the second millennium, Akkadian and Mycenean Greek, which show that many color terms were known and that most were semantically concrete. We also find grounds to suspect that a great variety and exchange occurred among these terminologies: 
the Egyptian color vocabulary not only included two words for 'blue' but probably the ancestor of the Indo-European 'red' term; Akkadian and Hittite adopted as a color term an Egyptian nonchromatic natural object word; Mycenean acquired at least one color term from Akkadian. Although these words arguably had abstract meaning, they certainly were not used in the detached fashion of our modern experience. Our assumptions about abstraction may hinder understanding of color-term evolution. What we reconstruct of the exchanges provides an alternative route to this concept, one via loanwords. This alternative has implications for the professed autonomy of the evolutionary sequence, and the variety is particularly important for specific breaks in the blue and red ranges.

\section{Berlin and Kay (B\&K), basic color terms, and their order of acquisition}

The theoretical divide lies between pre- and post-B\&K. They offer a clear and coherent analysis of the spectrum that enables us to match terms to hues in natural languages; it suggests these equivalents are universal and hardwired. B\&K argue for eleven basic color terms that arise systematically through a predetermined increment of 'black,' 'white,' 'red,' 'green,' 'yellow,' 'blue,' and 'brown,' which is followed by naming 'purple,' 'gray,' 'pink,' and 'orange' in any sequence. This scheme contrasts with prior notions of a random system.

\section{Neurological and psychological arguments}

Jameson and Alvarado (2002, 2003) and Jameson et al. (2001) conclude that color terms name perception of brightness, saturation, and hue. They also confirm that perception and vocabulary need not match. It is obvious that the expression of perception is linguistically dependent, but the reality of perception in terms of brightness, saturation and hue is also confirmed. Based on Greek and Latin, Lyons (1999) argues that colors are not real, rather they "are the product of the lexical and grammatical structure of particular languages." Thus, he collides with the neurological research as well as with B\&K's anthropological theory that rests upon it: that remarkable regularity prevails in naming the spectrum. A link between perception and hardwiring of the partitions for color vocabulary is championed by adherents of the B\&K method (cf. MacLaury 1999).

Some authors are prepared to recognize the validity of the B\&K system, and they assume that certain languages present exceptions, for example, as Quirke (2001) suggests for Egyptian. Others find languages with features incompatible with B\&K's projections, as does Paramei (2007). Still others take such exceptions to annul B\&K's approach and argue in favor of a different system, as does Saunders (2007).

Our approach uses a sample of ancient evidence, suggesting that aspects of the B\&K methodology require reexamination. Accepting the link between perception and expression, we shall assume that color vocabulary reflects neurologically based perception beyond the influence of culture. Our objective is to argue for the integration of the ancient data into the modern debate, since the evidential and theoretical threads have failed hith- 
Table 1. Brunner-Traut's list of Egyptian color terms.

\begin{tabular}{|c|c|c|c|c|c|c|}
\hline black & white & red & yellow/orange/red & green & blue & variegated \\
\hline $\begin{array}{l}k m \\
\underline{d}^{c} b\end{array}$ & $h \underline{d}$ & $\begin{array}{c}d \check{s} r \\
\underline{t} m s \\
\underline{t r} \\
j m 3 w \\
j n s \\
j d m i t \\
m h n m t\end{array}$ & $\begin{array}{c}k \underline{t} \\
k n i t \\
n b\end{array}$ & $\begin{array}{c}w 3 \underline{d} \\
m f k 3 t\end{array}$ & $\begin{array}{l}h s b \underline{d} \\
\text { jrjtw }\end{array}$ & $s 3 b$ \\
\hline
\end{tabular}

Table 2. Schenkel's list of abstract Egyptian color words.

\begin{tabular}{lllll}
\hline red & black & white & green & variegated \\
\hline$d \check{r} r$ & $k m$ & $h \underline{d}$ & $w 3 \underline{d}$ & $s 3 b$ \\
\hline
\end{tabular}

erto to come together. In contention is the extent of the color vocabulary used in ancient Egypt, where neither theory nor its neglect have improved our understanding. A popular misconception assumes that color terminology in Egyptian and other early languages was extremely limited (e.g., Baines 1985; Landsberger 1967; Lyons 1999; Schenkel 1963). This has prevented the recognition of key elements relevant to the debate.

\section{Egyptian color terminology}

Scholars have taken two approaches to color vocabulary in ancient Egypt. The traditional method has been philological and unsystematic, with authors noting various words that appear to have been used as color-terms, listing them in accord with modern usage. Inevitably, many words are found, as for 'black,' 'white,' 'red,' 'yellow/orange/red,' 'green,' and 'blue.' Brunner-Traut (1977:118-120) lists some 17 terms for these colors and their shades, aside from a word for 'variegated,' as seen in Table 1.

The theoretical method is based on the B\&K system (Baines 1985) and pre-B\&K systems (Schenkel 1963). Schenkel examined the use of several terms to find five of them "abstract color words," those in Table 2.

Baines effectively recognized Schenkel's work, assuming the validity of Schenkel's identifications and the validity of the $\mathrm{B} \& \mathrm{~K}$ acquisition sequence; Baines transformed Schenkel's "abstract color words" into "basic color terms." This allowed him to argue that the absence of a word for yellow rendered usage of a word for blue impossible, and thus he classified the Egyptian color vocabulary at Stage III with a single term for green and blue (grue). He made a similar case for Akkadian, based on Landsberger's (1967) contributions. Whereas Baines assumed the validity of the B\&K system and thus excluded blue, Quirke (2001) argued for blue but suggested that ancient Egyptian departed from the B\&K system.

In narrowing the vocabularies, the difficulty is the presence or absence of a separate blue and its relation to yellow and green, because Stage I (black, white) and Stage 
II (add red) are rarely controversial. By applying this B\&K system of color terms and their evolutionary sequence, Baines (1985) obliged himself to deny blue in Egyptian because yellow is absent, a dilemma compounded by the likelihood of an ancient word for 'blue' (Quirke 2001; Warburton n.d.). Equally tortured is Baines's suggestion of Egyptian w3 $\underline{d}$ = 'grue.' This is usually understood as 'green,' but the B\&K system predestines that Baines interprets it as signifying 'green-blue'; whereas his Akkadian equivalent is warqu = 'grue,' which Landsberger (1967) glosses 'green-yellow.' Baines with B\&K's theory forces the color terminologies into a preconceptualized straightjacket. There is a fundamental problem.

Baines's prestige commands that his arguments against 'blue' be placed in context; it is unclear from his text that these involve peculiar twists. One of them is the implication that his survey covered the use of color per se. Like Schenkel (1963) before him, Baines restricts his discussion of colors in art to painting. Thus, he avoids the matter of jewellery, amulets, and other lapidary. He therefore implies that blue was unknown before "its introduction in the form of an artificial frit around ca. 2550 BC" (Baines 1985:286). But turquoise and lapis lazuli inlays and jewellery emerge from the fourth millennium BC, over a thousand years before $2550 \mathrm{BC}$ and hundreds of years before the earliest Egyptian writing. Further, the frit was in use from the beginning of Dynasty III (which Baines dates ca. 2650 BC). Contrary to Baines, blue cannot be denied "in the earliest material."

Baines also doubts that 'blue' had symbolic value, necessarily so as he denied that blue was in cultural usage. However, the terms 'lapis lazuli' and 'turquoise' were used with symbolic meanings (Warburton n.d.). One of the first attested uses of the blue artificial frit was for creation of an artificial sky in the subterranean tomb of king Djoser at the start of Dynasty III (Lehner 1997:92), which dates to before or around 2600 BC in every current chronological system, antedating the earliest complex texts written in Egyptian.

Conscious of the B\&K theory but drawing on both archaeological material while employing the random philological method, we (Warburton n.d.) propose an elaborate ancient Egyptian color vocabulary. See Table 3.

The analysis of Table 3 differs from those of Tables 1 and 2 in proposing two terms for blue and two for red plus a term for yellow. Our point of departure is the ancient material, not theory. From the usage, it would appear that conceptual terms designating colors might be anticipated, although hardly proven. We generally assume that it is necessary for additional references to supplement this preliminary suggestion. Although debatable, we do not employ a method that differs fundamentally from philological practice throughout Egyptology. Only our conclusions differ from those of other approaches, as is familiar in science. Therefore, based upon philological methods practiced for over a century, we offer that ancient Egyptian color terminology includes more words than previously proposed.

Table 3. Warburton's view of ancient Egyptian color vocabulary.

\begin{tabular}{cccccccc}
\hline black & white & bright red & light red & green & yellow & dark blue & light blue \\
\hline$k m$ & $h \underline{d}$ & $d s ̌ r$ & $r w \underline{d} w$ & $w 3 \underline{d}$ & $n b w$ & $h s b \underline{d}$ & $m f k 3 t$ \\
\hline
\end{tabular}




\section{Akkadian}

In what is now Syria and Iraq, Semitic Akkadian was widely spoken during the second and third millennia BC, another of the earliest Near Eastern languages. Landsberger (1967) discusses its color terminology, and finds words for only 'black,' 'white,' 'red-brown,' and 'green-yellow.' But here we examine the dictionaries (Black et al. 2000; von Soden 196581) to compile the terms in Table 4.

Table 4 displays almost 60 terms related to color. Some refer to specific items (e.g., takiltum is 'blue wool', damum is 'blood,' hence 'red wine'). Like ourselves, most observers have dismissed terms with such meanings as 'blood-red' and 'charcoal-black' as color words in Egyptian, and thus many terms of similar referents can be eliminated from Akkadian color naming. It is difficult to identify color nuances in Akkadian, even where a color term is recognized, since it requires (1) extensive work to identify the terms that maintain a color significance and (2) facing the challenges of relating this to color through the archaeological material from Mesopotamia, where neither the textual nor the archaeological material permits the quality of comparison between art and color vocabulary that is routine for ancient Egypt. Together with M. Casanova, we envisage examining this in the future.

Importantly, there are Akkadian words for 'blue': uqnu and khashmanum. Both are related to semi-precious stones, uqnu being derived from the word for lapis lazuli. In par-

Table 4. Akkadian color terms.

\begin{tabular}{|c|c|c|c|c|c|c|c|c|}
\hline $\begin{array}{l}\text { bright, } \\
\text { shining }\end{array}$ & $\begin{array}{l}\text { dark, } \\
\text { gloomy }\end{array}$ & $\begin{array}{l}\text { red } \\
\text { red-brown/ } \\
\text { red-purple }\end{array}$ & yellow & $\begin{array}{l}\text { blue/ } \\
\text { blue-green/ } \\
\text { blue-purple }\end{array}$ & white & $\begin{array}{l}\text { green/ } \\
\text { green-yellow }\end{array}$ & gray & black \\
\hline $\begin{array}{l}\text { ebb } \\
\text { helu } \\
\text { nabatum } \\
\text { nagalu } \\
\text { napardu } \\
\text { nawaru } \\
\text { setum } \\
\text { šalummu }\end{array}$ & $\begin{array}{l}\text { adaru } \\
\text { da'amu } \\
\text { ekelu } \\
\text { etu } \\
\text { hašu } \\
\text { pihatu } \\
\text { salmu }\end{array}$ & $\begin{array}{l}\text { argamanu } \\
\text { adammu } \\
\text { emeru } \\
\text { da'mum } \\
\text { kanašum } \\
\text { karanu } \\
\text { makru } \\
\text { marhallum } \\
\text { pelu } \\
\text { pendu } \\
\text { rašum } \\
\text { samum } \\
\text { sedum } \\
\text { siamum } \\
\text { sumu } \\
\text { sarpu } \\
\text { serpum } \\
\text { singabru } \\
\text { šippu } \\
\text { tabarrum }\end{array}$ & $\begin{array}{l}\text { azupirum } \\
\text { ešmare } \\
\text { sinkadru } \\
\text { šamsu } \\
\text { šipu } \\
\text { (w)araqum } \\
\text { warqum } \\
\text { wurqum }\end{array}$ & $\begin{array}{l}\text { burumu } \\
\text { khašmanum } \\
\text { hinziribu } \\
\text { qunu } \\
\text { takiltum } \\
\text { uqnu } \\
\text { zagiddurum }\end{array}$ & $\begin{array}{l}\text { litu } \\
\text { papparum } \\
\text { pesu } \\
\text { pusu }\end{array}$ & (w)araqum & šibtum & sallamu \\
\hline
\end{tabular}


allel usage of two 'blue' terms, one is known to designate 'dark blue (lapis lazuli)', which permits us to establish a contrast. Durand (1983:222-223) notes that khashmanum distinguishes a shade of 'blue,' "less intense than lapis lazuli". It is, however, rare that such cases are demonstrable; thus, nuances clear to the ancients remain obscure to us. This implies neither that the numerous terms conceal a complete list of eleven basic color terms nor that the Akkadian was imprecise in partitioning colors.

\section{Mycenean Greek, Homeric Greek, and classical Greek}

Approaches to the color vocabulary of ancient Greek have taken different routes. One is the traditional philological approach represented of Lyons (e.g., 1999), who linked Homeric and classical Greek terminologies and compared them with that of classical Latin. More recently, Blakolmer, Nosch, and others (personal communication) have undertaken analysis of color terms in the Linear B Mycenean texts of the second millennium BCE, and they have linked them with those of Homeric and classical Greek. We first turn to Lyons's analysis of the possibilities, which are based on familiar philological premises and whose data appear in Table 5.

Lyons records that Gladstone (1858) identified the Homeric adjectives of color in Table 6.

To these, we would add khloros and polios, bringing the terminology in classical Greek to 'white,' 'black,' 'yellow,' 'red,' 'blue,' 'purple,' 'scarlet,' 'green,' and 'gray.' Viewing this same data, Lyons (1999) indicates the deficiency of Greek with respect to blue by emphasizing that no single term seems suitable. The difficulties of the vague 'greens' and 'blues' (kuaneos, khloros, glaukos) remain but should not deflect attention from a possibly more important aspect in partitioning the spectrum: multiple 'reds' (eruthros, phoinikos, porphuros).

Blakolmer, Nosch, and colleagues take another approach by which they link Homeric Greek of the first millennium BC with the Mycenean Greek of the second half of the second

Table 5. Lyons' list of Homeric and Classical Greek color terms.

\begin{tabular}{|c|c|c|c|c|c|c|}
\hline white & black & grey & $\begin{array}{l}\text { yellow-orange- } \\
\text { brown }\end{array}$ & red & blue-purple & green \\
\hline $\begin{array}{l}\text { argos } \\
\text { leirioeis } \\
\text { leukos }\end{array}$ & $\begin{array}{l}\text { kelainos } \\
\text { melas } \\
\text { katakorês }\end{array}$ & $\begin{array}{l}\text { glaukos } \\
\text { polios } \\
\text { phaios }\end{array}$ & $\begin{array}{l}\text { aithôn } \\
\text { krokôtos } \\
\text { xanthos } \\
\text { xouthos } \\
\text { purrhos } \\
\text { sandarkinos }\end{array}$ & $\begin{array}{l}\text { daphoinos } \\
\text { eruthros } \\
\text { miltos } \\
\text { poprphureos } \\
\text { rhodoeis } \\
\text { phoinikoeis } \\
\text { oinops }\end{array}$ & $\begin{array}{l}\text { halourgos } \\
\text { kuaeneos } \\
\text { orphninos }\end{array}$ & $\begin{array}{l}\text { prasinos } \\
\text { khlôros } \\
\text { ôkhros }\end{array}$ \\
\hline
\end{tabular}

Table 6. Gladstone's list of Homeric Greek color adjectives as reported by Lyons.

\begin{tabular}{llllll}
\hline leukos melas & xanthos & eruthros & kuaneos & porphuros & phoinikoeis \\
\hline
\end{tabular}


Table 7. Mycenean Greek color terms of broadest use.

\begin{tabular}{llll}
\hline bright, white & white & red & purple \\
\hline $\begin{array}{lll}\text { re-u-ko } \\
(\text { leukos })\end{array}$ & $\begin{array}{l}\text { pa-ra-ko } \\
(\text { phalaros })\end{array}$ & $\begin{array}{l}\text { e-ru-to-ro } \\
(\text { erythros })\end{array}$ & $\begin{array}{l}\text { po-ni-ki-jo } \\
\text { (phoinikeos) }\end{array}$ \\
\hline
\end{tabular}

millennium BC, known from the texts in Linear B. Blakolmer (2000:226) tenders 39 words in Mycenean Greek as possibly related to color. As in Akkadian, many of the Mycenean words refer to textiles but not to other objects. These include terms which later name color irrespective of context: melas, leukos, phalaros, erythros, rhodeis, argos, polios, xanthos, phoinikos, porphuros, poikilos, and others. All color words used in classical Greek are traced to terms in Mycenean Greek, and all of those used in Mycenean Greek are contextualized to object categories, such as plants or textiles. Most of the words are related to specific articles, but some overlap color categories, as shown in Table 7.

It is extremely important that the words for 'black,' 'green,' 'blue,' and 'yellow' are not used in the Mycenean Linear B texts with reference to more than two categories; whereas two terms for 'white' and two for 'red' appear in Table 7. The evidence implies that many of the Mycenean color terms are directly linked to one or another type of material and that the idea of color as an abstracted property has not yet arisen.

In sum, different analytical and chronological approaches show that both Mycenean and classical Greek named red and blue with multiple terms, and that these languages did not reduce those parts of the spectrum to a single term. It further implies that not only the 'blue' problem but also the division of the red spectrum requires our attention.

\section{Summary of ancient evidence}

The ancient evidence manifests six points that require attention.

1. The written languages pose problems incomparable to those faced by anthropologists. These languages are those of the elites, and the sample is not random.

2. Verification usually is impossible. Only where chance preserves the physical traces of color represented in vocabulary are the nuances familiar to the ancients recognizable. In Egypt, tomb paintings preserve the colors and facilitate a link with the vocabulary. Many of the terms in Akkadian and Mycenean refer to textiles, which are rarely preserved and more rarely allow original colors to be identified.

3. Color words in written ancient languages had a meaning that differs from the fashion in which modern color words are employed. Dismissing any potential color significance in an ancient word requires proposing an alternative meaning.

4. Sources are dissimilar: administrative documents, poetry, and religious texts preserve different vocabularies and systems of symbolism. Different texts will reflect diverse purposes, as well as the thoughts of distinct classes. A term written into poetry by literati may not mean the same for an illiterate listener.

5. The written languages reveal a gamut of color terms. Some are more abstract than others, but many are closely linked to specific materials or objects. Virtually all essen- 
tial color terms for 'blue' across the ancient languages are linked to concrete objects. The significance of this may only become apparent when discussing loanwords. Any analysis of the evolution of color terminology must take account of both loanwords and partitions.

6. The ancient written languages demonstrate partitions in the blue and red spectrum which are not appreciated by the B\&K model, which assumes a single basic color term for 'red' and another for 'blue.'

\section{Evidence of Egyptian colors}

The ancient evidence has been handled philologically rather than analytically. The accepted meanings of words have been a matter of convention, and the methodology prevents separation of abstract color terms. We predicated our proposal upon the same philological methods as other scholars. There was no consensus, except that analysis of the terms was subjective. Discussion would digress to comparison of authorities, as we could only state that a color term meant what an authority stipulated. Outsiders could not evaluate.

Our hypothetical spectrum was based upon a systematic interpretation of a random sample of texts. In order to test the hypothesis, all possible sources of interference had to be removed. This was impossible because there was no way to verify the data: the sources could not speak for themselves. A survey of all ancient Egyptian was hindered by uneven preservation.

It was therefore decided that well preserved genres would be preferred. This meant text corpi such as the Book of the Dead and the Pyramid Texts were the only possible means. However, the Book of the Dead and the Pyramid Texts represented separate periods and two different genres, the former being private and from the second millennium, the latter royal from the third. Confusion could have arisen from the procedure of comparing these or incorporating them into one data set. The issue of concrete objects as opposed to abstract terms posed a further obstacle.

It was further decided that (1) every single spell of the Book of the Dead (Naville, 1886) be examined, and all possible terms be recorded. This corpus of private texts on the Netherworld would be supplemented with the contemporary sources available in two other collections: (2) the corpus of solar hymns in private tombs (Assmann 1983) and (3) the Amduat (Hornung 1987-1994), the royal texts relating to the Netherworld. The three genres should provide an adequate basis, and all three were contemporary, dating to the New Kingdom (ca. 1500-1100 BC). This would reduce superficial randomness in the sample (although skewing remains due to the unequal preservation) and afford it breadth while precluding incomparable material. Regular occurrence of color terms becomes highly probable, which favors such genres.

There was no chance of contradiction between our analysis and existing proposals, because we had agreed with the consensus, merely arguing that additional terms were color terms. The selection of words and texts would, thus, not be subjective even though it 
remained difficult. Many Egyptian words can appear as causatives of other words or with meanings that vary in degree of abstraction, or they may be homonyms of unrelated form.

The issue of concrete versus abstract was easily resolved, as one spell of the Book of the Dead contained a reference to 'green wings', wherein the color word was determined with the symbol signifying stones rather than the abstract determinative. Using the concrete measure of exclusion would rule out the word translated as 'green,' which could bias the data toward our interpretation. All such references would therefore be included. In another case, the text called for writing in 'lapis lazuli.' Since the Egyptians never used lapis lazuli as a pigment, this could only be an abstract reference. All such references would likewise be included.

It was also decided that well-known expressions employing familiar words (as for 'white crown,' 'black land,' 'great green') would be included. These inevitably draw on the recognized terms. This would preclude that the terms we added could assume disproportionate representation. However, all uses as causatives would be excluded, for example 'to cause to be green, white, red.' The sample should, then, be representative and available to any scholar, and the experiment should be repeatable by any.

\section{Results}

The first result of our examination of the texts appears in Figure 1. The distribution of the color terms in the texts is irregular. Comparative absence of 'black' is because the word used in the Amduat to oppose 'white (light)' is not 'black' $(\mathrm{km})$ but 'darkness' (kk.w). In the Pyramid Texts, the opposition of 'black' and 'white' is striking and repeated (e.g., Sethe 1908, I: 22-24), so that this reflects a specific style and genre rather than the use of those colors.

The second result is the philological finding that the ancient Egyptian word customarily translated 'green' and interpreted as 'grue' never applied to the sky, and that it occurred no more than the word for 'lapis lazuli.' Conversely, the words for 'lapis lazuli' and 'turquoise' were used with reference to the sky; turquoise with respect to the dawn sky and lapis lazuli invariably associated with the sky goddess Nut and with the night sky. Such references accord with neither the claim that the Egyptians did not separately name 'blue' nor the assertion that they used the word for 'green' to cover the blue spectrum.

The clear interpretation is that the Egyptians used two words for blue and that they did not understand the term widely translated as 'green' as covering blue. This conclusion is complemented by the Pyramid Texts (Sethe 1908, I:292) that oppose w3 $\underline{\text { d }}$ 'green' and $m f k 3 . t$ 'turquoise'; the Book of the Dead opposes w3 $\underline{d}$ ' 'green' and hsb $\underline{d}$ 'lapis lazuli blue ink' (Budge 1910, II:263, 1990:199; Naville 1886:pl. CXIII:9; Naville 1886:pl. CLXVII:16). The opposition of the traditional word for 'red' to 'dark blue (lapis lazuli)' with respect to eyes appears in the Book of the Dead (Spell 177).

Adjusting the data to exclude fixed expressions such a 'black land' (meaning Egypt), 'red lands' (foreign countries), 'great green' (sea), 'red crown' (Lower Egypt) and 'white crown' (Upper Egypt) provides a remarkable spectrum in which lapis lazuli follows gold 


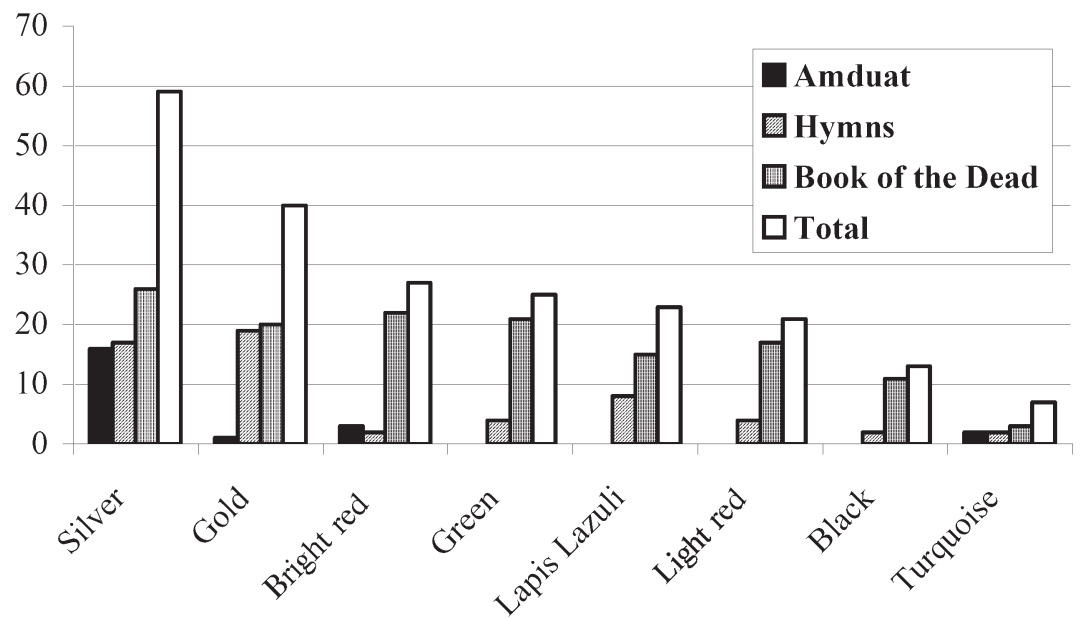

Figure 1. Distribution of color-term usage in three texts plus totals.

and silver when preceding 'green.' The sequence of 'gold,' 'silver,' and 'lapis' represents the sequence of values assigned to these articles in Egypt. That green precedes lapis lazuli is curious but corresponds to the value the Egyptians assigned to green stores. We had originally argued that names of colors were derived from precious metals and semiprecious stones, and this sequence seems to confirm it (Warburton n.d.).

This analysis is based on different genres of contemporary religious texts from the New Kingdom. From that period, there are also literary texts, administrative texts, and historical records. For comparison, we surveyed the Late Egyptian Stories (Gardiner 1973), which are coeval with the religious texts, seeking the same words discussed above, those from the second half of the second millennium BC. This genre can be further compared with the Middle Egyptian Stories (Blackman 1972) of the first half of the second millennium BC.

The literary material suggests that 'black' appears almost only because it was part of the word for Egypt, 'the Black Land.' The word for 'white' appears because it forms part of the word for dawn but otherwise only to name the metal silver or in terms composed of this sense, such as 'White Crown' and 'Treasury.' The term for 'green' appears only in the name for sea; 'black' and 'red' appear once each in the Middle Kingdom in geographical names. The word for 'gold' is used only for the metal, as the word for 'lapis lazuli' applies only to the mineral.

In the literary texts of the second half of the second millennium, not a single word serves as a color term. The term for 'green' does not appear; the word for 'sea' used in the concurrent dialect was not the earlier 'Great Green,' rather the Semitic loanword yam. Curiously, the 'green' meaning of 'grow, flourish' is also absent, this role being assumed by the alternative word for 'light red.' The ordinary word for 'bright red' fails to appear in any context. 


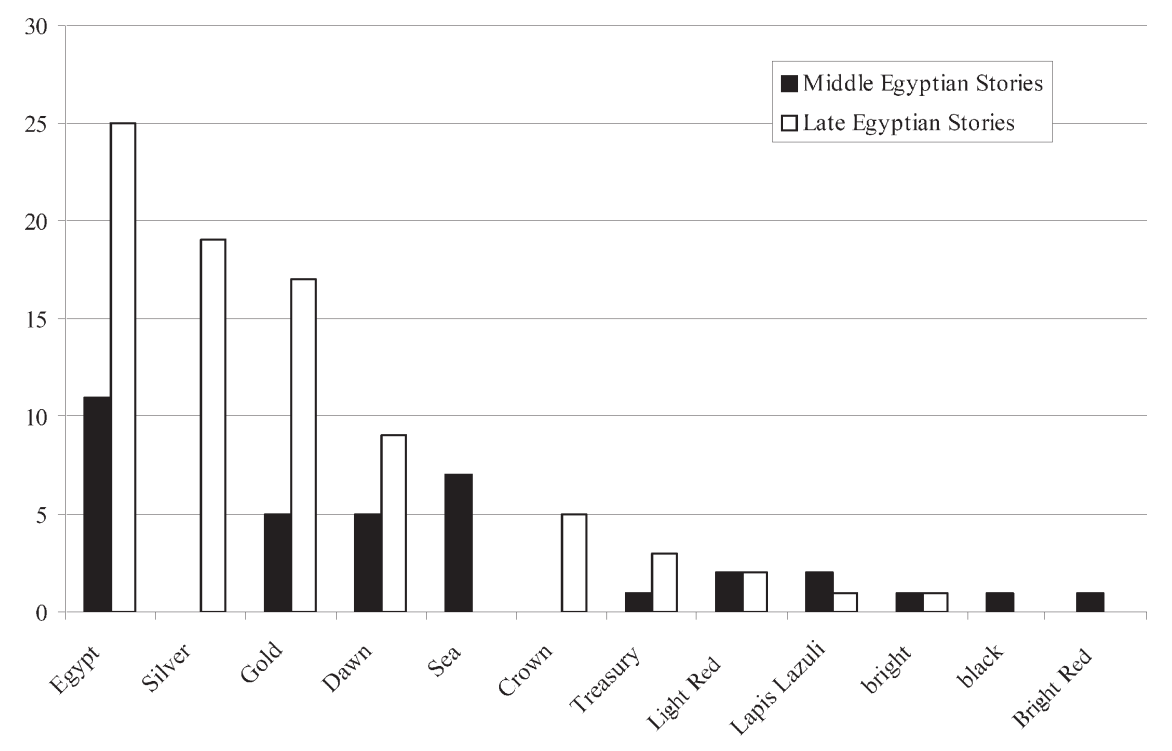

Figure 2. Color-term usage in Middle and Late Egyptian Stories.

In literary usage, colors did not constitute a category. Figure 2 compares the proportions at which their referents occur in Middle and Late Egyptian Stories.

\section{Hypotheses of color terminology}

The foregoing evidence will support our discussion of the color terminology in Egypt, whereby we shall extend our interpretation to more general claims based upon the significance and use of early loanwords. Our hypothesis asserts that the ancient Egyptian terms had meanings and that they were those that we propose. Otherwise, either an alternative interpretation must take its place or the very relevance of the evidence must be dismissed. We argue, further, that the meanings are linked to a system of color terms. Previous work has relied on simple and unsystematic philological methods, and our original hypothesis was developed using these. We have merely added a systematic presentation of data. Also, for its circular logic, earlier work precluded identification of blues, reds, and yellows because these concepts had not appeared in dictionaries. We advocate amending this lexicographic deficiency.

Mycenean Greek color terms abound across the red-yellow and blue-green segments of the spectrum. The color vocabulary of Akkadian likewise indicates a broad assortment of terms, as does the Egyptian. In the advanced Near Eastern civilizations of the second millennium $\mathrm{BC}$, languages named these gamuts with rich terminologies. 


\section{Evolutionary schemes}

The B\&K system postulates that individual languages evolve separately and that loanwords do not play a fundamental role in color vocabulary. But the premise of an evolutionary scheme must be seen in context. The ancient Egyptians lived a long time prior to the contemporary peoples who have preserved antiquated color schemes. These latter-day peoples do not live in technologically advanced societies, and they might employ simple schemes for designating colors (e.g., MacLaury 1999: 19-20). But such modern simplicity does not attest that ancient peoples used schemes of equivalent simplicity. Some ancient peoples were highly developed on a social plane, regardless of how their economies and technologies compare to those of the current world. The elites of ancient peoples used a variety of materials and colors in their art, and they communicated about them in a sophisticated fashion.

Modern languages may differ from ancient tongues in resources:

World and classical languages thus provide richer resources for communicative subtlety than do local languages in that the former frequently offer a larger variety of names for a given thing, depending on the level of abstraction at which the speaker wishes to place his description and the features of the denotatum or denotata to which he wishes to draw attention.

(Kay 1977:24)

But the level of social development in ancient Egypt implies that its elite had the support to cultivate language and thought. Their artifacts reveal unparalleled creativity. Their written languages may have been more precise than many modern colloquial tongues. However, ancient languages differ in accessibility from even the peasant languages within the current, rapidly changing, globalized milieu, which draw innovations from vast diffusion. The sophisticated languages of urban societies in antiquity developed to meet requirements of their communities, but their color terminology was still constrained by their conceptual means of expression. As precursors, these were inevitably radically different than those of the modern world; the terms, the partition of the spectrum, and the acquisition sequences need not represent or mirror earlier stages of modern color naming. However, the conceptual usage will have contributed to contemporary color naming through the development of abstraction and the related idea of color as a generic category.

\section{Evolution versus diffusion and loanwords}

The use of rhodon in Mycenean Greek and in Egyptian implies exchange, as does the Akkadian and Hittite use of khashmanum. One can see the same phenomenon with azur in French (from Arabic and Persian), sinij in Russian, and cafe in Mesoamerican dialects. A foreign word can be inserted into a language and used to designate a color. The word may not have a color meaning in its original language, or it may signify a different color. The phenomenon cannot be viewed in terms of local evolution.

Greeks and Egyptians were in contact with other peoples. The Akkadian word uqnu 'lapis lazuli,' was a kind of blue, eventually transformed into Greek kuaneos. The Egyptian 
term for lapis lazuli was used as a color word, as distinguished from the lighter blue of turquoise, which was also a color word. The Akkadian khashmanum likewise designates a lighter shade of blue than lapis lazuli (Durand 1983:222-223). We have argued that khashmanum is linked to the Egyptian word for 'amethyst' (Warburton n.d.), which was not used as a color-term in Egyptian. It is significant that Durand dismisses the possibility that khashmanum designates amethyst, since vases are made of the material. However, one could imagine the Egyptian word initially naming a specific stone but later transferred to the color spectrum, where it designated another stone of a similar hue - between dark blue and green. In Egyptian, the distinction between light blue and dark blue resides in the difference between 'lapis lazuli' and 'turquoise'; in Akkadian, the same difference was reflected in the words for 'lapis lazuli' and 'amethyst.' The significant difference lay in the nuance that the Akkadian word for the lighter shade was a loanword that did not have a color meaning in Egyptian and did not seem to have a concrete meaning in Akkadian. The complementary terms do not invariably designate comparable segments of the spectrum, and the meaning of the terms changes between languages. But the importance of this is that Egyptian, Akkadian, and Greek each names two shades of blue.

For decades, archaeological theory supposed that there was little contact between each ancient civilization and that each operated in a fashion comparable to the modern academic discipline associated with it. Therefore, the Aegean, the Levant, Anatolia, Mesopotamia, the Gulf, the Indus, and Egypt were separated into distinct spheres; interaction was viewed as insignificant, while local evolution was regarded as primary.

Today, the evidence in favor of interaction between the Aegean and the Near East is increasingly recognized. Since, at the beginning of the second millennium BC, the Indus civilization was in contact with Mesopotamia and Mesopotamia with the Aegean, the interaction spheres included almost the entire civilized world. This is a distinctly different approach than grudgingly conceding that certain elements may have been in contact. This contact involved the movement of colorful stones - lapis lazuli, turquoise, amethyst, rock crystal, jasper, carnelian, obsidian, and others. The names and colors of these stones moved.

The names of these stones were then transferred to textiles, which were also exported. Traces of these movements are found in modern languages. French azur comes from Persian lazuward 'lapis lazuli,' in turn from Arabic lazard, which is related to Arabic azraq 'blue' (Borg 1999b). The Greek kuaneos is from Akkadian uqnu, and likewise derived from the word for lapis lazuli. In all languages that use variants of the word 'purple,' the forms are traced to a textile dye whose name was used as a color term in Mycenean texts.

\section{Conclusions}

Color vocabulary in ancient languages

Mycenean Greek, Egyptian, and Akkadian used detailed vocabulary to partition the spectrum, and all used concrete objects to identify colors. Before the end of the second millen- 
nium BC, the Egyptians used eight basic color terms, which would imply a higher degree of compatibility with the B\&K system than Lyons (1999) assumes for Greek.

\section{Abstractness}

The majority of the Egyptian terms were based on concrete artifacts, a large proportion of them valuable metals (gold, silver) or semiprecious stones (turquoise, jasper, lapis lazuli). The same can be said of the color words in the Mycenean texts, as these are even more closely related to specific object categories and not abstract colors. And the same is true of Akkadian, in which we find a wide range of color terms but a low level of abstractness. We would, thus, argue that the condition of abstractness provides a barrier to the analysis of ancient languages in terms of the B\&K system; the notion slants interpretation toward a view through modern eyes.

\section{Evolution and diffusion}

One aspect of this problem is compounded by another: the loanwords. Among the Egyptian terms is $r w \underline{d}$, meaning 'pink' or 'rose' in Coptic and Demotic, and probably of similar meaning earlier. This term also appears in Arabic and Persian, quite aside from being the probable etymology of 'red' and 'rose' in English and other European languages. These ancient Egyptian terms, therefore, included one term, which may have been borrowed by Indo-European. It may also have been borrowed from Indo-European, but no Indo-European language was written before the first attested use of the word in Egyptian. Certainly, eruthros means 'red' in the Mycenean texts, where rhodeis is also preserved, both being of similar etymological and perceptual background. The word 'red' is best attested in the Indo-European vocabulary (Shields 1979). A diffusion from Egypt to Minoan and thus to Mycenean is reasonable.

The Egyptian color terms argued by us did not include a particular Egyptian term for a semiprecious stone ( $h s m n$ 'amethyst'), which was, nevertheless, borrowed as a basic color term by Semitic Akkadian, khashamnum.

This implies that shortly after the invention of writing, the Egyptians developed a highly complex lexicographical system. This also demonstrates a high degree of lexical interaction between languages and communities. We would argue that the $\mathrm{B} \& \mathrm{~K}$ principle of abstractness is more easily met when the term appears as a loanword in another language, after the basic concrete meaning is lost in transmission. The principle of abstractness is, thus, an obstacle to the objective study of the earliest languages because they could not borrow from their neighbors. The abstractness issue is facilitated by the introduction of loanwords, and the English word blue is such a loan, having been introduced from the continent to replace an earlier Old English term with a more concrete meaning (Casson 1997:230). To project these various points, it is useful to compare the situation with English, as adopted from Casson in Table 8.

Table 8 suggests that the color vocabulary of English draws on four words which originally meant 'to shine' and on one word 'to grow.' Such emphasizes luminosity, which 
Table 8. Indo-European and Old English color terms.

\begin{tabular}{lllll}
\hline & <Indo-Eur. & $<$ Skr. & $<$ O. Teut & $<$ Eg. \\
\hline White & kweit 'to shine' & & \\
Black & bloeg 'to shine' & & \\
Grey & gher 'to shine' & & & \\
Red & & rudhira & & \\
Green & & gro 'to grow' & \\
Yellow & gelwo 'to shine' & \\
Blue & & & loanword in English \\
Brown & & & not color term \\
Orange & & & loanword in English \\
Purple & & & loanword in English \\
Pink & & & loanword in English \\
\hline
\end{tabular}

Lyons (1999) has likewise attributed to Greek color naming. The only color words are all loanwords from other languages. The only real Old English color word with a significant hue nuance, 'red,' is probably an import into Indo-European from Egyptian. It is difficult to dismiss the ancient languages which contributed to the English vocabulary while claiming an evolutionary sequence: English did acquire blue after yellow and green, but only as a loanword. The same can be traced in French, wherein the 'blue' concept did not exist before the early second millennium $\mathrm{AD}$ and the words for 'blue' are loans from German and Arabic (Pastoureau 2002:26, 43ff.). The importance of exchange is, thus, underscored while the significance of evolution is cast in doubt. The fact that words move from language to language not only relativizes the significance of the evolutionary concept, but also emphasizes that it is not a sound approach to the language of color.

By breaking this association, we can introduce a word that covers a larger range of the spectrum. It is evident that words such as 'green jasper,' 'lapis lazuli' or 'turquoise' cannot be employed interchangeably to designate a broad spectral band. By introducing a loanword, this hindrance is avoided. The meaning of the original word is no longer clear, its concrete referent forgotten (e.g., amethyst, lapis lazuli, turquoise). The possibility of creating an abstract concept is now open, which facilitates emergence of words that will unite parts of the green-blue or the red-yellow gamuts. That was impossible when only the concrete reference was clear.

From same evolutionary view, access to the ancient languages is also blocked, however. B\&K argue that languages do not lose color words, but rather that once acquired, color words remain in the language. Assuming that this is valid, the B\&K approach will be obliged to deny that Egyptian, Akkadian, and Mycenean had several words for 'blue.' The condition of abstractness facilitates this procedure, dismissing the very languages which must lie at the origin of any evolutionary scheme for the acquisition of color words in the Near East. This problem will be compounded by another (see below). 


\section{Perception and partition}

We have interpreted Safuanova and Korzh's (2007) data as implying that Indo-Europeans were more sensitive to divisions in the red range of the spectrum than the blue. In any case, the evidence from the early written languages implies that the blue range and the red range were subdivided, and that the merging of the original expressions in 'red' and 'blue' in modern English is artificial and facilitated by use of loanwords in modern languages.

Next is the issue of partitions in blue. It is crucial that blue has contradicted claims regarding the universal character of the B\&K sequence and its division of the spectrum. The difficulty is familiar in Russian (Paramei 2007) and has been emphasized for both Greek (Lyons 1999) and Egyptian (Quirke 2001; Warburton n.d.). Lyons suggests that the problem be solved by arguing that colors reflected the "lexical and grammatical structure of particular languages" rather than a universal human system. We argue that the difficulty is neither in particular languages nor in the spectrum nor in blue, rather it is in that many languages divide blue with two words (in Russian, Egyptian, Greek, and Akkadian) or so divide green (Mandarin Chinese). Denying this principle disparages basic color terms, because admission of divided green and two blues does justice to the structure of language and to the fundamental ways of dividing the spectrum.

Terms in modern languages and the condition of abstractness are characteristic of one type of system. Understanding the ancient evidence is, thus, more difficult when the typological characteristics employed to analyze it are foreign to the ancient terms. This view on this issue is different from an approach that advocates cultural distinctions. Lyons argued that hue was less important in Greek and Latin than in modern languages. He concluded that it would be impossible to compile a list of B\&K basic color terms for ancient Greek, based on classical texts and Homer.

The evidence from the earliest Indo-European texts - the Mycenean Greek Linear B suggests a strong consciousness of the red range of the spectrum. Akkadian and Egyptian displayed a greater tendency to divide the blue range. We see this as significant in several different ways. On the one hand, it may imply an Indo-European tendency to be less concerned about blue, and on the other it should open consciousness to the possible breadth of the spectrum in red. A division of neither blue nor red is afforded by the B\&K system, even though the ancient evidence implies that these divisions were perceived and named. Subsequently, these categories may have simplified, with the result that the B\&K method obscures essential aspects of color-term development.

The ancient evidence demonstrates a sophisticated range of color vocabulary, which cannot be approximated on the basis of modern languages. The issue is not a matter of culture or perception, but of the way languages work in the past and present. The appearance of abstraction is as important an issue as partition, and both depend upon exchange and evolution. 


\section{References}

Assmann, Jan (1983). Sonnenhymnen in Thebanischen Gräbern. Mainz: von Zabern.

Aufrère, Sydney (1991). L’Univers minéral dans la pensée Égyptienne. Cairo: Institut français d'archéologie orientale.

Baines, John (1985). Color terminology and color classification: Ancient Egyptian color terminology and polychromy. American Anthropologist, 87, 282-297.

Berlin, Brent \& Paul Kay (1999). Basic color terms: Their universality and evolution (2nd. ed.). Stanford: CSLI Publications.

Black, Jeremy, Andrew George, \& Nicholas Postgate (Eds.) (2000). A concise dictionary of Akkadian. Wiesbaden: Harrassowitz.

Blackman, Aylward W. (1972). Middle-Egyptian stories. Brussels: Fondation Égyptologique Reine Élisabeth.

Blakolmer, Fritz (2000). Zum Charakter der frühägaischen Farben: Linear B und Homer. In F. Blakolmer (Ed.), Österreichische Forschungen zur Ägäischen Bronzezeit 1998: Akten der Tagung am Institut für Klassisiche Archäologie der Universität Wien 2.-3. Mai 1998 (225-239). Vienna: Wiener Forschungen zur Archäologie.

Borg, Alexander (Ed.) (1999a). The language of color in the Mediterranean. Stockholm: Almqvist \& Wiksel International.

Borg, Alexander (1999b). Prologue. In A. Borg (Ed.), The language of color in the Mediterranean (iii-x). Stockholm: Almqvist \& Wiksell International.

Borg, Alexander (Ed.) (n.d.). The language of color in the Mediterranean (2nd ed.).

Brunner-Traut, Emma (1977). Farben. Lexikon der Ägyptologie, II, 117-128.

Budge, Wallis (1910). The chapters of coming forth by day, Vol. 2. London: Kegan Paul.

Casson, Ronald W. (1997). Color shift: Evolution of English color terms from brightness to hue. In C. L. Hardin \& L. Maffi (Eds.), Color categories in thought and language (224-239). Cambridge: Cambridge University Press.

Durand, Jean-Marie (1983). Textes Administratifs des Salles 134 et 160 du Palais de Mari. Paris: Archives Royales de Mari 21.

Gardiner. Alan H. (1973). Late-Egyptian stories. Brussels: Fondation Égyptologique Reine Élisabeth.

Gladstone, William E. (1858). Studies on Homer and the Homeric age, Vol. 3. London: Oxford University Press.

Hardin, Clyde L. \& Luisa Maffi (Eds.) (1997). Color categories in thought and language. Cambridge: Cambridge University Press.

Hornung, Erik (1987-1994). Texte zum Amduat. Geneva: Ægyptiaca Helvetica.

Jameson, Kimberly A. \& Nancy Alvarado (2002). The use of modifying terms in the naming and categorization of color appearance. Journal of Cognition and Culture, 2, 53-80.

Jameson, Kimberly A. \& Nancy Alvarado (2003). Relational Correspondence between category exemplars and names. Philosophical Psychology, 16, 25-49.

Jameson, Kimberly A., Susan M. Highnote, \& Linda M. Wasserman (2001). Richer color experience for observers with multiple photopigment opsin genes. Psychonomic Bulletin \& Review, 8, 244-261.

Kay, Paul (1977). Language evolution and speech style. In B. G. Blount \& M. Sanchez (Eds.), Variability and change: Sociocultural dimensions of language change (21-33). New York: Academic Press.

Kay, Paul \& Luisa Maffi (1999). Color appearance and the emergence and evolution of basic color lexicons. American Anthropologist, 101, 743-760.

Landsberger, Benno (1967). Über Farben im Sumerisch-Akkadischen. Journal of Cuneiform Studies, 21, $139-173$. 
Lehner, Mark (1997). The complete pyramids. London: Thames \& Hudson.

Lyons, John (1999). The vocabulary of color with particular reference to ancient Greek and Classical Latin. In A. Borg (Ed.), The language of color in the Mediterranean (38-75). Stockholm: Almqvist \& Wiksell International.

MacLaury, Robert E. (1999). Basic color terms: Twenty-five years after. In A. Borg (Ed.), The language of color in the Mediterranean (1-37). Stockholm: Almqvist \& Wiksell International.

Naville, Eduard (1886). Das Aegyptische Todtenbuch der XVIII. bis XX. Dynastie. Berlin: Asher.

Paramei, Galina V. (2007). Russian 'blues': Controversies of basicness. In R. E. MacLaury, G. V. Paramei, \& D. Dedrick (Eds.), Anthropology of color: Interdisciplinary multilevel modeling (75-106). Amsterdam: John Benjamins.

Pastoureau, Michel (2002). Blue: The history of a color. Princeton, NJ: Princeton University Press.

Quirke, Stephen (2001). Color vocabularies in ancient Egyptian. In W. V. Davies (Ed.), Color and painting in ancient Egypt (186-192). London: British Museum.

Safuanova, Olga V. \& Nina N. Korzh (2007). Russian color names: Mapping into a perceptual color space. In R. E. MacLaury, G. V. Paramei, \& D. Dedrick (Eds.), Anthropology of color: Interdisciplinary multilevel modeling (55-74). Amsterdam: John Benjamins.

Saunders, Barbara A. (2007). Towards a new topology of color. In R. E. MacLaury, G. V. Paramei, \& D. Dedrick (Eds.), Anthropology of color: Interdisciplinary multilevel modeling (467-479). Amsterdam: John Benjamins.

Schenkel, Wolfgang (1963). Die Farben in ägyptischer Kunst und Sprache. Zeitschrift für Ägyptische Sprache, 88, 131-147.

Sethe, Kurt (1908). Die Altägyptischen Pyramidentexte. Leipzig: Hinrichs'sche.

Shields, Kenneth (1979). Indo-European basic colour terms. Canadian Journal of Linguistics, 24, 142-146. Soden, Wolfram von (1965-1981). Akkadisches Handwörterbuch. Wiesbaden: Harrassowitz.

Warburton, David A. (n.d.). Colors in ancient Egypt. In A. Borg (Ed.), The language of color in the Mediterranean (2nd ed.). 


\title{
Basic color terms from Proto-Semitic to Old Ethiopic
}

\author{
Maria Bulakh \\ Hamburg University, Germany
}

The paper presents a survey of the basic color terms of Old Ethiopic and attempts to reconstruct a typologically plausible transition from the Proto-Semitic basic color term system to the Old Ethiopic one. The immediate interpretation of etymological data results in a rather unlikely scheme of development: we have to suppose a transition from the Proto-Semitic system, which possesses at least four basic colour terms, toward four-member Old Ethiopic system; this transition would also involve replacement of the Proto-Semitic "yellow-with-green" colour category with a "green" category in Old Ethiopic without introduction of a new "yellow" category. An alternative reconstruction of the colour term evolution from Proto-Semitic to Old Ethiopic is tentatively suggested, according to which the Proto-Semitic basic colour terms are regarded as brightness terms.

\section{Introduction}

Old Ethiopic or Geez is an extinct language belonging to the Ethiosemitic branch of Semitic languages. Geez with Tigre and Tigrinya, modern languages spoken mostly in Eritrea, comprise the North Ethiosemitic subgroup. South Ethiosemitic languages include, among others, Amharic, the state language of Ethiopia.

The most important phases in the history of Geez are the Axumite period (from the 3 rd century to the end of the 1st millennium, when it was spoken) and the post-Axumite period (from the beginning of the 2nd millennium, when it was no longer commonly spoken but used as the language of religion and literature). An investigation on Geez color terms must consider the texts composed during the period of spoken Geez. However, the only native texts of the Axumite period are inscriptions, which contain too few color terms to reliably support research. All literary works in Axumite Geez are translations, mainly from Greek (e.g., the Bible in its Septuaginta version).

Therefore, the lexical data on the Geez basic color term (BCT) system can be trusted only to a degree. We never encounter spontaneous application of color terms but rather translation of color terms from a foreign language, which, on the one hand, provides better knowledge of the intended meaning of the Geez lexemes when we possess the original text but, on the other, exposes usage of Geez color terms to influence by the language of 
the original. The data are drawn from the dictionaries (Dillmann 1955; Kəflä Giyorgis \& Kidanä Wäld Kəfle 1948; Leslau 1987) and checked against their usage in texts of the Axumite period, while original Greek texts are consulted when possible. If we were to draw from the original Geez literature of the post-Axumite period, we might encounter BCTs prejudiced by its authors' native speech, Tigrinya or Amharic.

For each Geez color term, the question of its basic status was assessed with help of Berlin and Kay's criteria in Brenner's (1979:51ff.) modification, which adjusts them to requirements and restrictions of material in an ancient Semitic language. The first three criteria of Berlin and Kay become primary: (a) morphological simplicity [a simple lexeme in a Semitic language consists either only of a consonant-vocalic root or of a consonantal root plus vocalic pattern (cf. Brenner 1979:54)]; (b) nonhyponomy; (c) non-restricted usage. Criteria of frequency and of derivational productivity are added when needed (cf. Kerttula 2007).

The meaning of each Geez BCT is thoroughly investigated. The method of eliciting from native speakers the focus, range, and boundaries of a color category is inapplicable to an extinct language. Reconstructing the BCT system of an ancient language so as to project the equivalents requires an entirely different approach. Indirect information on a focus can be garnered from stock comparisons which match the best of the color to the object typically characterized by such, as with white as snow. Boundaries can be defined roughly by considering the gamut of objects whose color is designated by the $\mathrm{BCT}$, the glosses of sub-terms covered by it, and meanings of foreign color terms that translate it. Our description of each Geez BCT rests on: (1) the term and its translation according to the dictionaries; (2) arguments for its basic status, which include meeting the criteria of Berlin and Kay, frequency of usage, and number of derivatives; (3) data on its semantics, including its best example inferred from standard comparisons, and its range of reference based on usage in texts and on its Greek equivalents; (4) notes on the origin of the BCT. Lexeme meanings are quoted according to Leslau (1987); quotations from other dictionaries are added if they differ from Leslau's interpretation.

The Proto-Semitic BCT system reconstructed here consists of well-known ProtoSemitic roots with semantics of color, reflexes of which function in some daughter languages as BCTs. Further investigation might add roots to the system. However, such should not affect deeply the issues of the present, since our starting point is that the Proto-Semitic BCT system is of a stage not earlier than one composed of four basic categories.

Both in extinct Geez and reconstructed Proto-Semitic, the data are too scanty to provide the full information on color terms. Whenever direct evidence is wanting, the typological data (e.g., ethnographic results in MacLaury 1997, 2001) are consulted to identify the most plausible reconstruction among alternative paths in the evoluton from ProtoSemitic to Geez. The types empirically support the current, multilineal implicational sequences. 


\section{BCT system of Geez}

We obtain this Geez BCT system: s'āas dā (variants s'asadā, $\hat{s}^{\prime} a^{\varsigma} a d \bar{a}$ ) 'white', s'allim 'black',

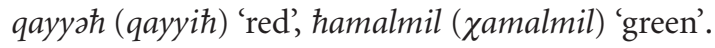

I. s'âa ${ }^{S} \bar{a}\left(s^{\prime} a^{S} a d \bar{a}, \hat{s}^{\prime} a^{S} a d \bar{a}\right)$ 'white, bright' (Dillmann 1955: 1307; Grébaut 1952:364; Leslau 1987: 542); 'white, clean' (Kəflä Giyorgis \& Kidanä Wäld Kəfle 1948:763). The term for white is well attested and meets all criteria of a BCT, being morphologically simple, not included in the meaning of another color term, and not restricted in application. It pos-

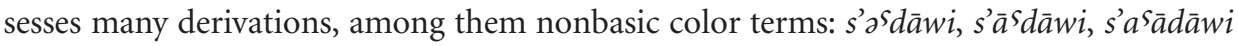
'white'; s'a'adsid 'whitish'; s'osdow 'white, bleached' (Leslau 1987:542).

The term is usually compared with snow, suggesting its best example, as, for instance, in the Book of Henoch 106:2 [wa-kona śogāhu ŝ̀ $a^{s} \bar{a} d \bar{a}$ kama ?ashatyā 'and his body was white like snow' (Knibb 1978, I:408; II:244)] or in Genesis 49:12, where the original comparison with halib 'milk' is substituted in one manuscript with barad 'snow'

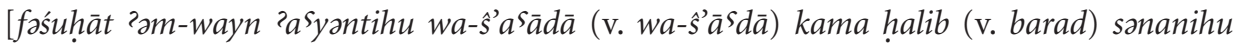
(Edele 1995:242f.)]. Such usage confirms that $s^{\prime} \bar{a} s d \bar{a}$ denoted a white category that assumed the purest focus. The usual translator's match of s'ā $s$ da to Greek leukós (sometimes leukóteros) corroborates this interpretation. Still, the choice of snow as the best example does not exclude the presence of a brightness component in the meaning. Snow is not only white but also bright: wa-yābarroh 'aklilātihomu kama barad 'their crowns glitter as snow' (Grébaut 1910:310f.) [however, usually such shining objects as sun or stars rather than snow are used to invoke the notion of brightness: wa-2asyontihomu baruh kama kok$a b a$ s'abā ' 'and their eyes (are) bright as the morning star' (Grébaut 1910:311)]. Because Geez $s^{\prime} \bar{a}{ }^{\varsigma} d \bar{a}$ matches also Greek lamprós 'bright, radiant' in Apocalypse 22:1 (Hofmann 1967:173), we may postulate that the semantics of brightness was incorporated into the meaning of the Geez term.

We find an interesting datum in the Book of Leviticus, which may enlarge our understanding of the reference range of $s^{\prime} \bar{a}{ }^{\varsigma} d \bar{a}$. In verses 13:30, 13:31, 13:32, 13:36, this term translates Greek lexemes denoting yellow hue: xanthós 'yellow' and its derived sub-term xanthizousōn (Boyd 1911:177f.). Existence of a white-with-yellow category on any stage after I is highly questionable (MacLaury 1997:60ff.). Yet translation of Greek xanthós by Geez $s^{\prime} \bar{a}{ }^{\varsigma} d \bar{a}$ suggests that the reference range of this term for white included at least the lightest hues of yellow. Probably the context opposition to s'allim 'black' in Leviticus 13: 29-37 provoked the interpretation of the Greek term as 'light yellow', 'almost white'.

Etymological evidence shows that the root $s^{\prime} s d(w)$ acquires connection with color only within the North Ethiosemitic branch of Ethiosemitic languages. Both in Tigre and Tigrinya the root functions as the basic term for 'white'. The cognates in South Ethiopic are not color terms but rather have a meaning related to whiteness, namely, 'be clean' (their phonetics suggest metathesis of laryngeal $\varsigma$ ). No cognates outside the Ethiosemitic languages have been found (see Bulakh 2006:738). 
II. s'allim 'black, dark, gloomy' (Dillmann 1955: 1259; Grébaut 1952:354; Kəflä Giyorgis \& Kidanä Wäld Kəfle 1948:756; Leslau 1987:556) is indisputably the basic term for black. It meets all Berlin and Kay's criteria: morphologically simple, not covered by another color term, describes various objects. It is well attested in texts and occurs as early as in the inscription DAE 11, lines 9-10: ... wa-s'alli [m] [sa]b?a qayyah ŝab? ... (Littmann 1913:33). While this passage allows different interpretations (Drewes 1962:98, 1980:39; Littmann 1913:33), it leaves no doubt as to the usage of the root s'lm to denote a color category, being paralleled by qayyą 'red'.

Its meaning is opposed to $s^{\prime} \bar{a} s d \bar{a}$ 'white', for instance, in the passage $k^{w}$ allāntāhu s'asadà wa-2albo mantani s'allim lāslehu (Hommel 1877:3) 'the whole of him is white and there is nothing black upon him'. The root s'm 'be darkened, be black' and related s'lmt 'be dark, grow dark' (Leslau 1987:556) produce multiple lexemes denoting facets of 'black, dark, obscure': s'alum 'dark, obscured'; mas'lam 'dark place, dark, obscure'; s'əlmut 'darkened, black, clouded over'; s'almutāwi 'dark, obscure' (Leslau 1987:556).

s'allim corresponds usually to Greek melás and derivatives melanós and melainós. Once it renders Greek psarós 'grey' in Zachariah 1:8 (Kramer 1898:28), which testifies to a meaning wider than "pure black", at least including dark grey.

It is usually defined as the color of soot, for example, in wa-qādāmāwi s'allim kama hamat (d'Abbadie 1960:74) 'and the first [mountain] is black as soot'. Since the choice of soot as an object possessing the prototypical dark maximum is normal for a "black" BCT, we surmise that focus of s'allim did not differ from the foci of black categories in most languages.

It is a difficult task to establish the reference range of this term, that is, to decide to what degree the dark parts of chromatic colors it covered. Since no separate BCT for the blue color is at hand, we must consider interpreting s'allim as black-with-blue. We have all the more reason to suspect such a scope, as its cognate in Tigre splits into two roots, one denoting black and the other blue: s'allim 'black, dark, empty' (Littmann \& Höfner 1956:632), tิtalma 'be blue, dark-colored' (Littmann \& Höfner 1956:622). Black-withblue in Geez would also conform the archaic usage of the black term to express the notion of blue in Amharic, for example, in the following explanation of Geez 'azraq 'blue', which we find in an Amharic-Geez lexicon: $t^{\prime} \partial q^{w} \partial r$, yänil aynät, t’əq ${ }^{w}$ ərätu sämay yämmimässəl 'black, the color of indigo, the blackness of which resembles [the color of] sky' (Kəflä Giyorgis \& Kidanä Wäld Kəfle 1948:430).

However, no Geez text usage suggests coverage of blue by s'allim. Geez denotes shades of blue with four nonbasic terms, an unusually high number in Geez for one hue:

1. 'azraq 'dark blue', borrowed from the Arabic BCT for blue, ?azraq- (Leslau 1987:53, who glosses the Geez term also as 'dark green', which, however, is not confirmed in the text);

2. darakano (variants darkano, darkāno) 'purple, hyacinth-colored, blue', borrowed from Greek dárkanos (Leslau 1987:143); the Greek term denotes a plant otherwise called eruthródanon and probably characterized by violet color (Frisk 1960:350);

3. samāyāwi 'sky-blue', derived from samāy 'sky' (Dillmann 1955:341);

4. $\quad k^{w} \partial h l i$ 'blue', derived from $k^{w} \partial h l$ 'antimony, dye for the eyelid' (Leslau 1987:279) [i.e. kohl]. 
Of these terms, only darakano is used frequently and is attested as early as in the Axumite period. However, even this term cannot be classified as BCT, since it is limited to clothes and textiles, and it regularly translates nonbasic Greek huákinthos. The other three color names are used extremely seldom and are not found in the Axumite texts ( $k^{w}$ ohli is not mentioned in Dillmann's lexicon and not found in the texts considered during the present investigation). Their origins are typical for nonbasic terms: $k^{w} \partial h l i$ and samāyāwi are object-derived; 'azraq is a loanword. Nothing speaks for considering any of the four terms to be a BCT.

However, no cover term for these secondary color names was found by this study. The blue hue seems always to be described with help of the nonbasic terms: wa-kona qaddus

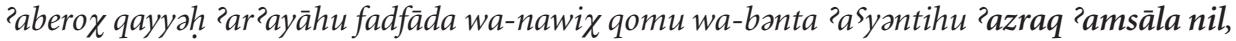
wa-qaddus 'atom kona nawixā qomu wa-bənta ?asyəntihu 'amsāla $k^{w}$ วhl wa-s'allim s'วḥmu (Guidi 1909:280) 'and Saint Aberoh was of a very red appearance and of tall stature, and the pupils of his eyes were as blue as indigo, and Saint Atom was tall and the pupils of his eyes were as antimony, and his beard was black'. It appears impossible to establish with the help of text evidence the hyperonym term for these "blue" senses. Thus, the question of boundaries of the s'allim category remains, and we shall have to address the typological data in order to explore it further.

The Geez BCT goes back to a Proto-Semitic root $\theta^{\prime} l m$, which denoted a black category with equally obscure boundaries (infra: Proto-Semitic BCT system II; Bulakh 2003:5f.).

III. qayyah, qayyih 'red' (Dillmann 1955:457; Grébaut 1952: 178; Kəflä Giyorgis \& Kidanä Wäld Kəfle 1948:790; Leslau 1987:456) is a well-attested color term meeting all criteria of Berlin and Kay. It is found as early as in Geez inscriptions paralleled by s'allim 'black' (supra: BCT system of Geez II). The term spawns many derivatives, among which is the nonbasic color term * qayāhyih 'reddish' (Leslau 1987:456).

The text evidence shows that this category was wide-ranging, probably including all of brown, pink, and orange (for the yellow see supra: BCT system of Geez I, s'a $\bar{a}^{\varsigma} d \bar{a}$ ). As for the best examples, the color of rose and of pomegranate are likely candidates: $w a-$ waŝ'? $a$ waldā za-bak ${ }^{w}$ orā wa-qayyạ (v. qayyih) $k^{w}$ allantu kama s'age radā in Genesis 25:25 (Edele 1995: 132), 'and her first son came out reddish all over as a flower of rose'; kama qiyāta romān qayyạ maläthiha (Grohmann 1919: 168), 'her cheeks are red as the redness of pomegranate. Geez qayyoh is used to render almost all Greek words of the red range: eruthrós, kókkinos, púrinos, puroeidês, purrákēs, purrós, purróteros.

Cognates of the root are BCTs for red in most Ethiosemitic languages. The root probably goes back to a Proto-Semitic nonbasic color term * $q y$ h with semantics of 'light-red' (see Bulakh 2006: 741f.).

IV. hamalmil, xamalmil 'green, verdant' (Dillmann 1955:69; Kəflä Giyorgis \& Kidanä Wäld Kəfle 1948:449; Leslau 1987:233). Although this color term is well-attested, questions arise about its status and meaning in Geez. First of all, it does not meet the criterion of morphological simplicity, critical for a BCT. Both synchronic and diachronic evidence speaks for its derivation from the noun haml 'vegetation' (Leslau 1987:232f.). The par- 
tial reduplication forms adjectives, especially nonbasic color terms (see Barth 1894:218). Thus, hamalmil can be viewed as an object-derived term meaning 'vegetable-like, of the color of vegetation'.

The criterion of general application also requires explanation. The term is used mostly as a qualifier for vegetables, grass and other plants. It combines the color semantics with the meaning of freshness, succulence. It is often contextually opposed not to color terms but rather to the adjective yabus 'dry', as for example: wa-mat't'awu kāl'anhi bis'omu ?abtorihomu 'anza manfaqon hamalmilāt wa-manfaqon yəbusāt 'and the other ones handed over their sticks, while half of them was green and half of them was dry' (d'Abbadie 1960:62f.). But this seemingly restricted distribution can be explained through the influence of Greek original texts; indeed, it corresponds exactly to the distribution and the semantics of Greek chlorós (cf. Lyons 1999:60). Conversely, we have one reliable datum testifying that the Geez term was applied to objects other than greenery, namely Genesis 2:12, which describes a gem: sanq ${ }^{w}$ xamalmil (Edele 1995:45). Thus, the usage of Geez hamalmil was not context-specific. Since no cover term for hamalmil is at hand, the criterion of nonhyponymy supports considering the term as basic. On the whole, it seems best to include the term into the BCT system of Geez as a name for green, in spite of its morphological and semantic connection with a plant name.

Although no stock comparisons involving hamalmil occur in the texts, we can surmise from its etymology and usage that its focus was the color of fresh grass and vegetation. We can also claim that its range covered all of "green", at least as supported by the absence of other names denoting green hues. This opinion is sustained in that the term renders not only the Greek BCT for 'green', chlorós, but also its rarer Greek sub-term prásinos 'light green, leek green' (in the foregoing passage of Genesis 2: 12).

Since no apparent BCT for yellow color is at hand, the question arises whether hamalmil included yellow hues as well as green. This suggestion is supported by the interpretation of Kidanä Wäld Kəfle, who explains the term as 'green, yellow' (Kəflä Giyorgis \& Kidanä Wäld Kəfle 1948:449). However, this Amharic lexicographer probably bases his statement on a Psalms passage 67:14: ba-hamalmāla warq (Devens 2001:6) 'in the green of the gold', which follows the Greek en chlōrótēti chrusíou. We have no assurance as to which hue was intended in the Greek version, and the usage of the Ethiopian term hamalmil in this context is uninformative, being explained by the influence of the Greek text. Since the Greek BCT for yellow, xanthós (cf. Lyons 1999:61f.) and its derived sub-term xanthízousōn are nowhere rendered into Geez by hamalmil (supra: BCT system of Geez I), we have no basis to include yellow hues within its green-focused range. Similarly, no text evidence suggests the usage of hamalmil as a cover term for blue hues (supra: BCT system of Geez II).

The noun haml 'vegetation' (Leslau 1987:233), from which the BCT is derived, has good cognates within the Ethiosemitic group but scarcely any in other Semitic languages (see Bulakh 2006: 742).

V. In order to build a reconstruction of the foci and boundaries of basic color categories in Geez, we must supplement the scant data provided by the texts of the Axumite period with 
typological material. We can safely postulate that the system consists of four BCTs. However, the four-category evolutionary stage provides the most possibilities for composite and primary BCT combinations across languages of the world, more than any other stage (see Berlin \& Kay 1969:4; Dedrick 1998:92; Kay 1975:261; Kay et al. 1997:33). Thus, we have to determine which four-category system is represented in Geez, at the same time attempting to solve two blatant problems that have emerged: first, the naming of yellow, which according to text usage is covered by the white category (even though such a distribution is typologically most improbable after stage II); second, the naming of blue, which is designated in the texts only by nonbasic color terms.

The category of white-with-yellow, which seems to be suggested by the text evidence, occurs extremely rarely at the four-category stage, if really at all, as such. While it is included in the typology suggested by Kay et al. (1991), it is rejected by most scholars in that it is missing in all other evolutionary sequences proposed until the present. Since the testimony of a sole text is not nearly enough to establish the relationship between yellow and white in Geez, it is best to suspect that the passage in Leviticus demonstrates only a partial inclusion of the yellow hues, presumably only of the light yellow, into the white category. As for the rest of the yellow part of the spectrum, it may pertain to the red category, to the green category or to both. The last solution suggests a division of yellow hues between white, red and green BCTs, plausibly an unstructured and variable encroachment from each direction.

As for the blue hues, they could be included either in the black or the green category, perhaps with variability. While the testimony of other Ethiosemitic languages seems to favor the first option, the typological plausibility of such a system is slight. While the reconstruction of a category blue-with-black for Geez is possible according to Kay et al. (1997:33, Fig. 2.4), such a category is rejected, for example, by MacLaury (1997:60f.).

Thus, the typologically plausible reconstruction of the Geez BCT system would consist of: (1) white (with a brightness component and, thus, extended to the lightest yellow hues); (2) black (possibly including the darkest blue); (3) red (possibly including a part of yellow); (4) green (possibly including mid-to-light blue; less certain is its partial inclusion of yellow hues). An example of a similar contemporary system is displayed by MacLaury (1997:61f., Fig. 2: 24a-b) for the Brazilian isolate language Múra Pirahá, which he classifies as stage IIIa and interprets as in transition from a brightness system.

\section{Proto-Semitic BCT system}

At present, four BCTs are securely reconstructed for Proto-Semitic: white (root ${ }^{*} l b n$ ), black (root $\left.{ }^{\star} \theta^{\prime} \mathrm{lm}\right)$, red $\left(\operatorname{root}^{\star}{ }^{2} \mathrm{dm}\right.$ ) and yellow-with-green with a brightness component $\left(\operatorname{root}^{*} w r q\right)$.

I. *lbn 'be white': Hebrew lābān 'white' (Koehler \& Baumgartner 1995:517); Phoenician lbn 'white' (Tomback 1978: 155); Judaic Aramaic lābān 'white' (Jastrow 1926:690); Mandaic lbina 'white, gleaming' (Drower \& Macuch 1963:229); Ugaritic lbn 'white' (Del 
Olmo Lete \& Sanmartín 2003:490); Arabic laban- 'milk' (Biberstein-Kazimirski 1860, II:962); Mehri awbōn 'white' (Johnstone 1987:251); Harsusi elbōn, elbenáyt / lében 'white' (Johnstone 1977:83); Jibbali lūn, līnát / lénáti 'white' (Johnstone 1981:159); Soqotri líbehon 'white' (Leslau 1997:228). This root is reconstructed by Del Olmo Lete and Sanmartín (2003:490, Ugaritic, Hebrew, Punic); Koehler and Baumgartner (1995:517, Hebrew, Phoenician, Ugaritic, Mandaic); Leslau (1938:228, Hebrew, Arabic, Mehri, Soqotri).

II. ${ }^{\star} \theta^{\prime}$ Im 'be black, dark': Akkadian s'almu 'black' from Old Akkadian (Gelb et al. 1962, XVI:77; von Soden 1965-1981:1078); Hebrew s'almāwät 'darkness' (< ${ }^{*}$ s'almūt, the attested form is due to the folk etymology that derived the word by compounding s' $\bar{e} l$ 'shadow' and māwät 'death') (Koehler \& Baumgartner 1995: 1029); Punic s'lm 'darkness' (Hoftijzer \& Jongeling 1995: 969); Ugaritic J’lmt 'darkness' (Del Olmo Lete \& Sanmartín 2003:1004); Arabic ð’alima 'be obscure, covered by darkness (said of dark night)' (Biberstein-Kazimirski 1860, II: 140); Sabaic ð’lm 'west' (Biella 1982:226); 'darkness; west; ?black' (Beeston et al. 1982:172); Geez s'allim 'black' (Leslau 1987:556); Tigrinya s'allim 'black' (Kane 2000:2541); ج̂t alamlam bälä 'be dim (light), glimmer' (Kane 2000:2493); Tigre s'allim 'black' (Littmann \& Höfner 1956:632); $\widehat{t \rho}$ alma 'be blue, darkcolored' (Littmann \& Höfner 1956:622); Amharic s'ällämä 'be dark' (Kane 1990:2250); t’ällämä 'become dark' (Kane 1990:2089); t̂ällämä 'get dark; cast a shadow' (Kane 1990:2200); Harari Tfilma 'darkness, dark' (Leslau 1963:51; Wagner 1983:280); Gafat s'älläma 'black' (Leslau 1956:235); Argobba t̂́ eläma 'be dark' (Leslau 1997: 197); Gurage: Selti, Wolane t’aläme 'be dark, be black' (Leslau 1979:618); Wolane t'em, Selti t'èm 'black (man, cattle, object)' (Leslau 1979:618); Muher, Masqan, Gogot, Wolane t’alim, Soddo t’alam 'soot of pots' (Leslau 1979:618); Muher, Masqan, Gogot ţällämä, Wolane $\widehat{t} \rho$ elämä,

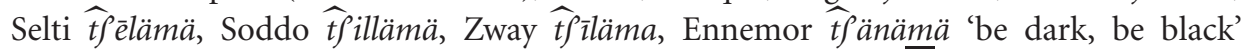
(Leslau 1979: 180); Mehri həð’láwm 'go dark' (Johnstone 1987:84); Harsusi méð’lem 'dark' (Johnstone 1977:30); Jibbali ð'éliũt 'darkness' (Johnstone 1981:49); Soqotri t'lm 'darken' (Leslau 1938:204).

The Sabaic root $\chi^{\prime} l m$ appears in the inscription R.E.S. 3945, line 15 (Ryckmans 1935:398) and is paralleled by hmrt, which may be translated as 'red' if equated with Arabic hmr 'be red' (Biberstein-Kazimirski 1860, I: 489; cf. Conti Rossini 1931:160; Drewes 1962:98). Besides, in Ethiopian Sabaic incriptions Abuna Garimā 1, line 5 (Schneider 1973:385), Abuna Garimā 2, lines 8-9 (Schneider 1973:387), and J. E. 1370, line 1 (Schneider 1961:61f.; Schneider 1973:388) the root s'lm is attested, contextually opposed to ? $d m$; while ' $d m$ may be the reflex of Proto-Semitic BCT for 'red', the interpretation of $s^{\prime} l m$ as 'black' is highly desirable; initial $s$ ' instead of $\chi$ ' is usually explained by Geez influence (cf. Drewes 1980: 15; Müller 1983:285; Schneider 1961:61f.; Schneider 1973:385ff.; for an alternative interpretation see Jamme 1964:347f.).

See reconstructions of this root by: Del Olmo Lete and Sanmartín (2003:1004, Ugaritic, Hebrew, Arabic, Geez); Koehler and Baumgartner (1995:1029, Ugaritic, Hebrew, Arabic, Tigre); Leslau (1938:204, Soqotri, Mehri, Arabic, Hebrew, Geez, Akkadian, Epigraphic South Arabian); Leslau (1963:51, Arabic, Geez, Tigre, Tigrinya, Amharic, Gafat, Argobba, Harari, Gurage); Leslau (1987:556, Arabic, Epigraphic South Arabian, 
Hebrew, Ugaritic, Akkadian, Geez, Tigre, Tigrinya, Amharic, Gurage); von Soden (19651981: 1076, Akkadian, Ugaritic, Hebrew, Geez).

III. ${ }^{*} 2 \mathrm{dm}$ 'be red': Akkadian adamātu 'dark red earth (used as a dye)' Old Babylonian (Gelb et al. 1964 I, part I:94f.; von Soden 1965-1981:10); cf. da'mu 'dark-colored, dark red' Standard Babylonian (Gelb et al. 1959 III:74; von Soden 1965-1981:158); Hebrew 'ādōm 'red' (Koehler \& Baumgartner 1995:15); post-Biblical Hebrew 'ādōm 'red' (Jastrow 1926: 15); Punic 'dm 'red?' (Hoftijzer \& Jongeling 1995: 14); Judaic Aramaic 'ădimmā 'red' (Jastrow 1926: 16); Ugaritic ' $d m$ 'become red, turn the colour red; put on red make up' (Del Olmo Lete \& Sanmartín 2003:17); Arabic 'dm 'be red, have a tanned skin'; 'ādam'brown, light' (Biberstein-Kazimirski 1860, I: 19); Ethiopian Sabaic 'dm 'red?' (Drewes 1980:39; supra: Proto-Semitic BCT system II); Geez ?addāmāwi 'red' (Leslau 1987:8).

See reconstructions of this root by: Cohen (1997:9, Akkadian, Ugaritic, Hebrew, Arabic, Geez 'adim 'red skin'); Del Olmo Lete and Sanmartín (2003:17, Ugaritic, Hebrew, Akkadian, Arabic, Geez); Koehler \& Baumgartner (1995: 15, Akkadian, Hebrew, Ugaritic, Arabic, Geez); Leslau (1987: 8, Akkadian, Hebrew, Ugaritic, Arabic, Geez).

IV. * wrq 'be yellow-green, bright': Akkadian arqu (warqu, barqu, irqu) 'yellow, green' from Old Akkadian, Old Babylonian (Gelb et al. 1968 I, part II:300; von Soden 19651981: 1471); Hebrew yaraqraq 'yellowish-green, pale' (Koehler \& Baumgartner 1995: 441); post-Biblical Hebrew yārōq 'light-colored, yellow or greenish' (Jastrow 1926:595); Old Aramaic, Official Aramaic yrq 'verdure; vegetables' (Hoftijzer \& Jongeling 1995:471); Judaic Aramaic yaraq 'be light-colored, pale, green, yellow' (Jastrow 1926:597); Jewish Palestinian Aramaic yrq 'green, yellow' (Sokoloff 1992:245); Samaritan Aramaic yrq 'green' (Tal 2000:361); Syriac yūrāqā(?) 'green, yellow' (Brockelmann 1928:309); Mandaic $y u$ raqa 'yellow, green, pallid' (Drower \& Macuch 1963: 191); Ugaritic yrq 'greenish yellow (a metal/gold)' (Del Olmo Lete \& Sanmartín 2003: 982); Arabic waraq- 'foliage' (BibersteinKazimirski 1860, II: 1522); Sabaic wrq 'vegetable crops; vegetable plot' [Beeston et al. 1982: 162; this meaning is, though, disputed by Kogan and Korotayev (2004:112)]; Harari wårīq 'green; yellow' (Leslau 1963:161).

Geez waraqriq 'of golden color' (Leslau 1987:618), in spite of Leslau, does not derive from warq 'gold, gold coin' but goes back to the Proto-Semitic BCT ${ }^{\star}$ wrq. It appears as early as in the Axumite period, in "Physiologus", as an epithet of a dove. The passage alludes undoubtedly to Psalm 68:14 where the dove's wings are described as covered with the yellow (or greenish, or bright) of the gold - yaraqraq ḩārūs', and the Hebrew color term corresponds to the Geez adjective, not only phonologically but also morphologically, using the same reduplication of final radicals (cf. Hommel 1877:xxvi). Thus, on the one hand, one can suspect a semantic similarity between the Geez and the Hebrew terms. On the other, Geez waraqriq should reflect the meaning of the corresponding term of the Greek version, poikilai 'spotted, many-colored' (Hommel 1877:80). Overall, interpretation of the Geez term as 'bright' seems the most plausible, allowing deviations into the meaning 'yellow, bright as gold', on the one hand, and 'variegated, spotted', on the other. On the semantic development of 'bright' > 'spotted' see, for example, Cotton (1950); 
the same shift may be suspected in South Ethiosemitic cognates of the root: Amharic warqa 'coat of bovines which is spotted with red and white' (Kane 1990:1506), Chaha, Ezha, Enemor, Gyeto, Muher aräq $q^{w} \ddot{t}$, Gyeto, Muher aräq $q^{w} \ddot{a}$ 'spotted color (black and white)' (Leslau 1979:90). The meaning 'bright, shining' of Geez waraqriq is confirmed by Tigrinya and Amharic cognates of the same partly reduplicated pattern: Tigrinya wərəqraq bälä 'shine, sparkle, glitter' (Kane 2000:1729); Amharic täwräqürräqä 'be dazzling, shine, gleam, sparkle' (Kane 1990: 1506). The brightness or lightness component in the semantics of the root should be reconstructed to the Proto-Semitic stage. It is reflected in derivatives with the meaning of 'gold' or 'silver' in Epigraphic South Arabian (cf. Beeston et al. 1982: 162 for Sabaic; Ricks 1989:56, Qatabanian) and in Ethiosemitic (cf. Kane 1990: 1504 for Amharic; Kane 2000:1728, Tigrinya; Leslau 1979:664, Gurage; Leslau 1987:618, Geez; Leslau, 1997:226, Argobba). We find evidence for the component of 'lightness, paleness' in post-Biblical Hebrew (Jastrow 1926:595), Judaic Aramaic (Jastrow 1926:597), Syriac (Brockelmann 1928:309f.), Mandaic (Drower \& Macuch 1963:191), Akkadian (Gelb et al. 1968, I (part II):300). Thus, the Proto-Semitic BCT * wrq was either a hue term denoting yellow-with-green and having an additional brightness component, or mainly if not solely a brightness term spreading over at least the yellow and green parts of the spectrum. This Proto-Semitic BCT has correspondences in Egyptian and Berber (cf. Schenkel 2007).

See reconstructions of this root by: Cohen (1997:632ff., Akkadian, Hebrew, Old Aramaic, Judaic Palestinian Aramaic, Syriac, Mandaic, New Syriac, Arabic, Sabaic, Harari); Del Olmo Lete and Sanmartín (2003:982, Hebrew, Eblaite, Akkadian, Ugaritic, Epigraphic South Arabian, Arabic, Geez); Koehler and Baumgartner (1995:440, Hebrew, Judaic Aramaic, Syriac, Mandaic, Old Aramaic, Ugaritic, Akkadian, Epigraphic South Arabian, Arabic, Geez); Leslau (1987:618, Geez, Arabic, Epigraphic South Arabian, Hebrew, Judaic Aramaic, Syriac, Mandaic, Ugaritic, Akkadian); see also Bulakh (2003:8ff.).

V. If we were to reconstruct for Proto-Semitic a system of hue terms, we could define it as corresponding to stage IIIc according to Kay (1975:261) [the same as Stage IIIY/G in Kay et al. (1997:33) or stage IIIe in Dedrick (1998:92)], that is, the stage comprising terms for white, red, yellow-with-green and black-with-blue. Note that while Kay et al. mention "three yellow/green languages at Stage III with W, R, Y/G, and Bk/Bu" (1997:54, Footnote 19), this stage is missing in the scheme proposed by Kay and Maffi (1999:761). Likewise, MacLaury (2001:1240ff.), basing his typology of yellow-with-green on the Pacific Northwest data (almost the only yellow-with-green categories attested with Munsell chips), postulates his stage III' as the earliest, which incorporates, however, five BCTs: white, black, warm, cool, yellow-with-green; the system descends from his stage-III" fourcategory brightness naming. Plainly, the plausibility of the reconstructed Proto-Semitic BCT system would depend on yet undiscovered and undescribed exact information on the yellow-with-green color naming at a four-category stage. The presence of a safely reconstructed term that at least covered yellow and green hues testifies that Proto-Semitic BCT system included this category at a stage not earlier than of four-categories.

Another possibility must be considered, namely, that the Proto-Semitic BCT system represented not hue but rather brightness categories (cf. MacLaury 2001:1236). We have 
Table 1. Lexical replacement in the course of evolution from Proto-Semitic to Old Ethiopic

\begin{tabular}{|c|c|}
\hline Proto-Semitic & Old Ethiopic (Geez) \\
\hline *lbn 'be white' & 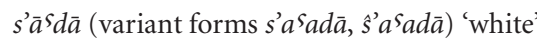 \\
\hline${ }^{*} \theta^{\prime} l m$ 'be black' & s'allim 'black' \\
\hline${ }^{*} 2 \mathrm{dm}$ 'be red' & qayyah, qayyih 'red' \\
\hline${ }^{*}$ wrq 'be yellow-green' & hamalmil, xamalmil 'green' \\
\hline
\end{tabular}

seen the undeniable evidence of the brightness meaning for the root wrq. Similarly, the semantics of 'darkness, dullness' can be postulated for ${ }^{*} \theta^{\prime} \mathrm{lm}$. Besides, several lesser brightness terms are safely reconstructed for the Proto-Semitic. For example, reflexes of the root ${ }^{\star}$ brh (cf. Brockelmann 1928:61; Cohen 1997:49; Koehler \& Baumgartner 1995:111; Leslau 1987:103) are attested almost in all Semitic languages. They express both brightness and lightness. A reflex of ${ }^{\star} z r q$ (cf. Brockelmann 1928:207; Cohen 1997:802; von Soden 1981:1515), having lost the meaning of brightness - which, however, is attested in Mandaic and earliest stages of Arabic - transformed into BCT for blue in modern Arabic.

Since brightness systems remain practically unexplored, on the one hand, and since the reconstruction of the BCT system of Proto-Semitic is incomplete, on the other, we shall refrain from classifying the system of Proto-Semitic in terms of the typology of brightness systems but shall keep open the possibility of treating the Proto-Semitic as favoring brightness instead of hue.

\section{From Proto-Semitic to Geez}

Both Proto-Semitic and Geez BCT systems consist of four members, which establishes the rough semantic correspondences which are given in the Table 1.

In the transition from Proto-Semitic to Geez, only the black category preserved its name in the BCT system: Geez s'allim is a simple reflex of the Proto-Semitic BCT ${ }^{*} \theta^{\prime} l m$. The three other members of the system were renamed in Geez, the old terms either disappearing or becoming nonbasic color terms. For the white category, Proto-Semitic BCT ${ }^{*} l b n$ is not attested in Geez, being replaced by the term $s^{\prime} \bar{a}^{s} d \bar{a}$ of unknown origin. A reflex of the Proto-Semitic ${ }^{*} 2 d m$ 'be red' occurs in Geez as nonbasic 'addāmāwi 'red' (supra: Proto-Semitic BCT System III), while a reflex of Proto-Semitic nonbasic color term * qy h 'light-red' is employed to name the Geez red category (see Bulakh 2006:740f.). The Proto-Semitic *wrq 'yellow-with-green' transforms in Geez into a lesser brightness term waraqriq. The place of this brightness or yellow-with-green color term is partially occupied in Geez by an object-derived BCT for 'green', hamalmil (variant xamalmil), not the renaming of a category but a term innovated for a new category.

Now we consider the transition from the Proto-Semitic to Geez BCT system in terms of typology of BCT evolution. If we assume the change occurred between systems of exclusively hue naming, then we shall encounter great difficulties in adapting the empirical data to the typologically based evolutionary sequences of BCTs. As we have seen, 
the Geez BCT system is reconstructed as having four categories, and probably belongs to stage IIIa (cf. Kay 1975:26, white, black, red-with-yellow and green-with-blue categories). According to the evolutionary BCT hue sequences suggested until now, we should expect of Proto-Semitic either stage IIIa (which supposes that Geez inherited the ProtoSemitic categorization of color practically unaltered) or stage II [which would enable us to reconstruct a development stage II > stage IIIa according to Berlin and Kay (1969:4)]. However, the presence of yellow-with-green in the BCT system of Proto-Semitic forces us to reject both classifications of the system, as stage II and as stage IIIa (vis-à-vis the typology of yellow-with-green systems in MacLaury 2001:1240ff.). We must suppose that the Proto-Semitic system of BCTs, instead of developing into stage IV, has retracted its yellowwith-green category into green without adding a new BCT for yellow. Such a transition is typologically unparalleled, unlike any stage throughout the various evolutionary BCT sequences.

However, the problem of typological plausibility is solved as soon as we adopt our second interpretation of Proto-Semitic data, namely, the reconstruction of a brightness system for Proto-Semitic. According to MacLaury (2001:1232, Fig. 90: 3), a brightness system of stage III" can transform either into a common stage IIIa hue system or into a III' yellow-with-green hue system. The former development has probably taken place in Geez, while the latter sequence is to be supposed for the Semitic languages where yellowwith-green category is attested, as with Akkadian (cf. Bulakh 2003:8).

Thus, in reconstructing the Proto-Semitic BCT system, brightness terms should receive special attention, while the role of the brightness component in the hue terms of the Semitic languages is to be reconsidered as well. Because the diachronic data usually present an incomplete picture of a BCT system, the usage of ethnographic material provides support for its reconstruction by way of filling gaps in the historical data. Finally, thorough ethnographic investigation of current brightness systems is a vital prerequisite for reconstruction of the Proto-Semitic BCT system.

\section{Acknowledgments}

This study is partly based on a presentation delivered at the 15th International Conference of Ethiopian Studies, Hamburg, July 20-25, 2003. The work was supported by the Russian Foundation Basic Research grant No. 03-06-08133a. I am deeply grateful to the late Robert E. MacLaury for his comments and discussions on various parts of the manuscript. My sincere thanks go to my teacher Leonid E. Kogan for his comment on this paper as well as for his constant help and support. The generous help of Denis A. Nosnitsyn in the course of investigation is highly appreciated.

\section{References}


Barth, Jacob (1894). Die Nominalbildung in den Semitischen Sprachen. Leipzig: Hinrichs.

Beeston, Alfred Felix Landon, Mahmud SAli Ghul, Walter W. Müller \& Jacques Ryckmans (1982). Sabaic Dictionary. Louvain-la-Neuve: Peeters.

Berlin, Brent \& Paul Kay (1969). Basic Color Terms: Their Universality and Evolution. Berkeley \& Los Angeles: Univ. of California Press.

Biberstein-Kazimirski, Albin de (1860). Dictionnaire arabe-français. Paris: Maisonneuve.

Biella, Joan Copeland (1982). Dictionary of Old South Arabic: Sabaean Dialect. Chico: Scholars Press.

Boyd, James Oscar (1911). The Octateuch in Ethiopic, Part II: Exodus and Leviticus. Leyden: Brill.

Brenner, Athalya (1979). Colour terms in the Old Testament. Doctoral dissertation. Manchester: University of Manchester.

Brockelmann, Carl (1928). Lexicon Syriacum. Halle: Max Niemeyer.

Bulakh, Maria (2003). Etymological notes on the Akkadian color terms. In Orientalia. Studia Semitica (3-17). Moscow: Russian State University for the Humanities.

Bulakh, Maria (2006). Basic colour terms in Geez: Synchronic and diachronic aspects. In S. Uhlig (Ed.), Proceedings of the XVth International Conference of Ethiopian Studies (737-745). Hamburg July 20-25, 2003. Wiesbaden: Harrassowitz.

Cohen, David (1997). Dictionnaire des Racines Sémitiques ou attestées dans les langues sémitiques. Leuven: Peeters.

Conti Rossini, Carlo (1931). Chrestomathia Arabica Meridionalis Epigraphica edita et glossario instructa. Roma: Istituto per l'Oriente.

Cotton, Gérard (1950). Un Équation sémantique: 'mouvement rapide' = 'lueur, eclat'. Les études classiques, $18,436-441$.

Dedrick, Don (1998). Naming the Rainbow. Colour Language, Colour Science, and Culture. DordrechtBoston-London: Kluwer Academic Publishers.

Del Olmo Lete, Gregorio \& Joaquín Sanmartín (2003). A Dictionary of the Ugaritic Language in the Alphabetic Tradition. 2 vols. Leiden-Boston: Brill.

Devens, Monica (2001). A Concordance to Psalms in the Ethiopic Version. Wiesbaden: Harrassowitz.

Dillmann, Augustus (1955). Lexicon Linguae Aethiopicae cum indice latino. New York: Friedrich Ungar.

Drewes, Abraham Johannes (1962). Inscriptions de l'Éthiopie Antique. Leiden: Brill.

Drewes, Abraham Johannes (1980). The lexicon of Ethiopian Sabaean. Raydãn, 3, 35-54.

Drower, Ethel Stefana \& Rudolf Macuch (1963). A Mandaic Dictionary. Oxford: Oxford University Press.

Edele, Blain Alan (1995). A Critical Edition of Genesis in Ethiopic. Doctoral dissertation, Duke University. Ann Arbor: University Microfilms International.

Frisk, Hjalmar (1960). Griechisches Etymologisches Wörterbuch. Heidelberg: Carl Winter-Universitätsverlag.

Gelb, Ignace J., Benno Landsberger, A. Leo Oppenheim \& Erica Reiner (Eds.) (1956-). The Assyrian Dictionary of the Oriental Institute of the University of Chicago. Chicago-Glückstadt: the Oriental Institute-J. J. Augustin Verlagsbuchhandlung.

Grébaut, Sylvain (1910). Littérature Éthiopienne Pseudo-Clémentine. Revue de l'Orient Chrétien, 15, 198214.

Grébaut, Sylvain (1952). Supplément au Lexicon Linguae Aethiopicae de August Dillmann (1865) et Édition du Lexique de Juste d'Urbin (1850-1855). Paris: Imrimerie Nationale.

Grohmann, Adolf (1919). Aethiopische Marienhymnen. Leipzig: Teubner.

Guidi, Ignazio (1909). Le Synaxaire Éthiopien. Les Mois De Sanê, Hamlê et Nahasê, II: Mois de Hamlê. Paris: Firmin-Didot.

Hofmann, Josef (1967). Die Äthiopische Übersetzung der Johannes-Apokalypse. Louvain: Secrétariat du Corpus SCO. 
Hoftijzer, Jacob \& Karel Jongeling (1995). Dictionary of the North-West Semitic Inscriptions. Leiden-New York-Köln: Brill.

Hommel, Fritz (1877). Die Aethiopische Uebersetzung des Physiologus. Leipzig: Hinrichs'sche Buchhandlung.

Jamme, Albert (1964). Annales d'Ethiopie, IV (Boekbesprekingen). Bibliotheca Orientalis, 21, N 5/6, 346349.

Jastrow, Marcus (1926). A Dictionary of the Targumim, the Talmud Babli and Yerushalmi, and the Midrashic Literature. New York-Berlin: Verlag Choreb.

Johnstone, Thomas (1977). Harsūsi Lexicon. Oxford: Oxford University Press.

Johnstone, Thomas (1981). Jibbāli Lexicon. Oxford: Oxford University Press.

Johnstone, Thomas (1987). Mehri Lexicon. London: School of Oriental and African Studies, University of London.

Kane, Thomas Leiper (1990). Amharic-English Dictionary. Wiesbaden: Harrassowitz.

Kane, Thomas Leiper (2000). Tigrinya-English Dictionary. 2 vols. Springfield: Dunwoody Press.

Kay, Paul (1975). Synchronic variability and diachronic change in basic color terms. Language in Society, 4, 257-270.

Kay, Paul, Brent Berlin, Luisa Maffi \& William R. Merrifield (1997). Color naming across languages. In C. L. Hardin \& L. Maffi (Eds.), Color Categories in Thought and Language (21-56). Cambridge: Cambridge University Press.

Kay, Paul, Brent Berlin \& William R. Merrifield (1991). Biocultural implications of systems of color naming. Journal of Linguistic Anthropology, 1, 12-25.

Kay, Paul \& Luisa Maffi (1999). Color appearance and the emergence and evolution of basic color lexicons. American Anthropologist, 101, 743-760.

Kerttula, Seija (2007). Relative basicness of color terms: Modeling and measurement. In R. E. MacLaury, G. V. Paramei \& D. Dedrick (Eds.), Anthropology of Color: Interdisciplinary Multilevel Modeling (151170). Amsterdam: John Benjamins.

Knibb, Michael A. (1978). The Ethiopic Book of Enoch. 2 vols. Oxford: Clarendon Press.

Koehler, Ludwig \& Walter Baumgartner, revised by W. Baumgartner (1995). The Hebrew and Aramaic Lexicon of the Old Testament. Leiden-New York-Köln: Brill.

Kogan, Leonid \& Andrey Korotayev (2004). Animals and beyond. A new work on Epigraphic South Arabian realia. A review article of Alexander Sima. Tiere, Pflanzen, Steine und Metalle in den altsüdarabischen Inschriften. Wiesbaden, Harrasowitz. 2000. Wiener Zeitschrift für die Kunde des Morgenlandes, 94, 95-118.

Kramer, Friedrich Oswald (1898). Die Äthiopische Übersetzung des Zacharias. Leipzig: Ackermann \& Glaser.

Kəflä Giyorgis \& Kidanä Wäld Kəfle (1948). Mas’hafa sawāsəw wagass wamazgaba qālāt hadis. Addis Abäba: Artistik Matmäya Bet.

Leslau, Wolf (1938). Lexique Soqotri. Paris: Librairie C. Klincksieck.

Leslau, Wolf (1956). Étude descriptive et comparative du Gafat (Éthiopien méridional). Paris: Librairie C. Klincksieck.

Leslau, Wolf (1963). Etymological Dictionary of Harari. Berkeley-Los Angeles: University of California Press.

Leslau, Wolf (1979). Etymological Dictionary of Gurage. Vol. III. Wiesbaden: Harrassowitz.

Leslau, Wolf (1987). Comparative Dictionary of Gesez (Classical Ethiopic). Wiesbaden: Harrassowitz.

Leslau, Wolf (1997). Ethiopic Documents: Argobba: Grammar and Dictionary. Wiesbaden: Harrassowitz.

Littmann, Enno (Ed.) (1913). Deutsche Aksum-Expedition. Sabäische, griechische und altabessinische Inschriften. Vol. IV. Berlin: Reimer.

Littmann, Enno \& Maria Höfner (1956). Wörterbuch der Tigrē-Sprache. Wiesbaden: Franz Steiner. 
Lyons, John (1999). Color in Ancient Greek and Latin. In A. Borg (Ed.), The Language of Color in the Mediterranean. An Anthology on Linguistic and Ethnographic Aspects of Color Terms (38-75). Stockholm: Almqvist \& Wiksell International.

MacLaury, Robert E. (1997). Color and Cognition in Mesoamerica: Constructing Categories as Vantages. Austin: University of Texas Press.

MacLaury, Robert E. (2001). Color terms. In M. Haspelmath, E. König, W. Oesterreicher \& W. Raible (Eds.), Language Typology and Language Universals: An International Handbook, Vol. 2 (1227-1252). Berlin-New York: Walter de Gruyter.

Müller, Walter W. (1983). Äthiopische Marginalglossen zum Sabäischen Wörterbuch. In S. Segert \& A. J. E. Bodrogligeti (Eds.), Ethiopian Studies Dedicated to Wolf Leslau (275-285). Wiesbaden: Harrassowitz.

Ricks, Stephen David (1989). Lexicon of Inscriptional Qatabanian. Rome: Editrice Pontificio Istituto Biblico.

Ryckmans, Gonzague (Ed.) (1935). Répertoire d'Épigraphie Sémitique. Vol. VI. Paris: Imprimerie Nationale.

Schenkel, Wolfgang (2007). Color terms in ancient Egyptian and Coptic. In R. E. MacLaury, G. V. Paramei \& D. Dedrick (Eds.), Anthropology of Color: Interdisciplinary Multilevel Modeling (211-228). Amsterdam: John Benjamins.

Schneider, Roger (1961). Inscriptions D’Enda Č'erqos. Annales d'Éthiopie, 4, 61-66.

Schneider, Roger (1973). Deux inscriptions Sudarabiques du Tigré. Bibliotheca Orientalis, 30, N. 5/6, 38589.

Sokoloff, Michael (1992). A Dictionary of Jewish Palestinian Aramaic of the Byzantine Period. Ramat-Gan: Bar Ilan University Press.

Tal, Abraham (2000). A Dictionary of Samaritan Aramaic. 2 vols. Leiden-Boston-Köln: Brill.

Tomback, Richard S. (1978). A Comparative Semitic Lexicon of the Phoenician and Punic Languages. Missoula: Scholars Press.

von Soden, Wolfram (1965-1985). Akkadisches Handwörterbuch. 3 vols. Wiesbaden: Harrassowitz.

Wagner, Ewald (1983). Harari-Texte in Arabischer Schrift. Wiesbaden: Franz Steiner. 



\title{
Towards a history and typology of color categorization in colloquial Arabic
}

\author{
Alexander Borg \\ Ben-Gurion University of the Negev, Israel
}

The word stock of Old Arabic attested in the Pre-Islamic literature displays by far the most elaborately lexified color paradigm attested within the Semitic language family. The present article surveys color usage in the modern Arabic colloquials (both Eastern and Western) from a diachronic angle and suggests that a qualitative evolutionary shift from brightness to hue categories coincided roughly with the sedentarization of Arabicspeaking nomads which brought them in contact with basic color systems of sedentary communities influenced by the dyer's palette. The Old Arabic five-term color system (abyad 'white, bright', aswad 'black, dark', ahmar 'red, light brown', axdar 'green, blue, black', and asfar 'yellow'; Fischer 1965) paralleled in ancient Hebrew and Aramaic lies at the basis of most modern colloquial color paradigms. Whereas urban Arabic vernaculars spoken, for instance, in Cairo, Beirut, Jerusalem, etc., display systems approximating the Berlin/Kay eleven-term paradigm, co-territorial semi-nomadic communities tend to retain archaic color paradigms with fewer basic categories alongside a rich non-basic nomenclature encoding predominantly ecological hues.

\section{Preliminaries}

The Arabic Sprachraum is roughly co-extensive with a geographical continuum stretching from the Arabian peninsula and the lands adjoining the Fertile Crescent to Morocco and from S.E. Anatolia to Sudan. ${ }^{1}$ Research on contemporary Arabic vernaculars spoken across this geographical continuum has tended to restrict its concern to a brand of linguistics largely modelled on the theory and practice of dialect research in the West. A more anthropologically oriented approach to the study of the Arabic vernaculars entailing the execution of systematic ethnographic (alongside conventional linguistic) fieldwork would

1. The following abbreviations have been resorted to in this article: Akkad - Akkadian; Alep - Aleppo; Ar-Arabic; Aram - Aramaic; Assyr - Assyrian; Bahr - Bahrein; Bed - Bedouin; Cai - Cairene; Cypr - Cypriot; Dam - Damascene; Egyp - Egyptian; Gk - Greek; Heb - Hebrew; Kurd - Kurdish; Leb - Lebanese; Malt - Maltese; Mand Mandaic; Mor - Moroccan; Palest - Palestinian; Pers - Persian; Skt - Sanskrit; Syr - Syrian; Tang - Tangiers; Tk Turkish; Ugar - Ugaritic; Yem - Yemeni. 
seem to be called for to do justice to the differential cultural patterns underlying language use across this vast territory (and, not infrequently, within the same dialect region) ${ }^{2}$ and to create adequate lexicographic documentation.

Relic areas of Arabic speech outside the pale of the Arab countries - in S.E. Anatolia, Cyprus, Malta, Central Africa, Afghanistan, and Uzbekistan - each with its own sociohistorical and cultural profile, also merit attention in this regard since they can potentially yield the diachronist valuable chronological checkpoints for dating evolutionary processes in the color paradigms of mainstream Arabic vernaculars by providing the relevant data for contrasting the center with the periphery.

The composition and historical developent of color categories constitute a salient linguistic topic in the lexical semantics of both literary and vernacular Arabic. Color terms identify and differentiate surface qualities in the visual world, and the "inhomogeneities of these surface qualities underlie the perception of contour on which the distinguishing form rests" (Bornstein 1975:774). It may therefore be symptomatic of a principle of iconicity in the morpholexical subsystem of Arabic grammar, determining the correlation of form and meaning, that attributes of both shape and color in this language are encoded by the same canonic form: the scheme $a \mathrm{CCaC}$ : 'abyad 'white,' 'ahmar 'red,' 'axlaq 'smooth,' 'a'wağ 'crooked,' etc. '

A conspicuous trait of the Arabic color system is its high degree of grammaticalization rendering it - like that of classical Latin - "un domaine sémantique de très forte cohérence" (Mignot 1969:295). In effect, from the global perspective of the Semitic language family, the highly elaborate formal and semantic treatment accorded to color terms in Arabic constitutes a striking if not unique typological trait (see $\$ 2$ ).

Systematic diachronic study of color categorization in colloquial Arabic is bedevilled by two major obstacles. Firstly, as noted in Stewart (1991:119),

There exists a vast number of textual sources from which we may derive knowledge of color terms and the history of their development in the various Arabic dialects, yet efforts to do so have been and will continue to be severely limited by the state of Arabic lexicography.

Secondly, not only are large dialect areas in the Arabic-speaking world still unmapped, but lexicographers of dialectal Arabic have tended, in many cases, to content themselves

2. In the Palestinian dialect area, for instance, urban vernaculars (spoken in Jerusalem, Haifa, etc.) display the eleven basic categories of the B\&K paradigm, while the Negev Bedouin colloquial shows only the first six (cf. Borg 1999).

3. In traditional Arabic grammar, the $a C C a C$ scheme encodes adjectives denoting colors and defects (Wright 1896-1898:143). A grammatically more refined definition takes cognizance of the fact that Arabic possesses several other canonic shapes for ordinary adjectives and that what distinguishes the $a C C a C$ set from other adjectival subclasses is the fact that they refer to striking or highly marked traits. It is significant, from this viewpoint, that this morpholexical scheme also encodes the elative form of ordinary adjectives: atwal 'taller,' asggar 'smaller,' etc. (cf. Wehr 1953). Color terms on the $a C C a C$ scheme can also convey an elative meaning; observe abyad 'white' $\Rightarrow$

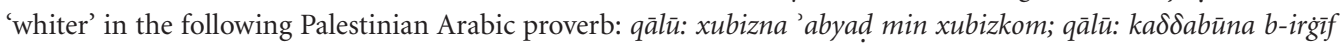
(Thilo \& Kampffmeyer 1933:166) 'Our bread is whiter than yours - Prove it!' [my transcr. and transl., AB]. 
with noting literal equivalents of the Arabic terms without attempting to specify actual meanings and patterns of usage in sufficient detail for serious scientific research.

On the positive side, not only is Arabic the best known and most elaborately recorded of the Semitic languages but, it also displays a prolix word stock lexifying chromatic concepts in meticulous fashion "as if the smallest detail, the most minute nuance, was deemed to require a nomenclature sui generis" (Morabia 1986:698).

In another context, Wierzbicka (2001:21) has expressed the view that

Exploring the lexicon in a systematic and methodical way we can discover how 'ordinary people' (in contrast to experts and scientists) conceptualize the world; and we can learn to discern the line which separates language-related every-day-knowledge from the languageindependent specialists' knowledge.

Given the well-known sociolinguistic factor of diglossia characterizing the language situation in the Arab countries, the dualistic distinction here made between popular and learned representations of the external world - incidentally, evoking Whorf's modality of 'habitual thought' (1941:134ff.) - is highly suggestive with regard to color categorization in Arabic inasmuch as it foregrounds:

(a) the acute problem of discriminating between popular and learned uses of color terms, for instance, in literary Arabic reference works, the indigenous dictionaries being panchronic and cumulative in nature, i.e., often record learned historical usage as synchronic actuality;

(b) the closely related vexed issue, arising in linguistic fieldwork, whereby an informant's knowledge of the literary Arabic color system tends to obscure, for the researcher, cognitive aspects of genuine native competence in the vernacular register.

Wierzbicka's aforecited observation about discovering how ordinary people - in contrast to experts - conceptualize the world, is pointedly illustrated in Behnstedt and Woidich (1987:15), where the authors state in relation to the color term 'ahmar 'red' in the Arabic dialects of Upper Egypt:

Die Farben "rot" und "braun" gehen für unsere Auffassung manchmal durcheinander; starker Tee ist z.B. nach Meinung der Oberägypter "rot."

This anomaly disappears once a basic macro-red category - fairly common in colloquial Arabic - is set up for this Egyptian Arabic vernacular, comprising, as in Old Arabic, roughly the hue range from a reddish sunset sky to the light brown of camel's hair (cf. Fischer 1965:334f.), as opposed to the standardized category denoting focal red in Modern Literary Arabic, modeled largely under the impact of European languages (cf. V. Monteil 1960:219).

\section{Formal traits of Arabic color terms}

Classical Arabic utilizes the aforementioned morpholexical matrix $a C C a C$ (f. $C a C C \bar{a}$, pl. $\mathrm{Cu} C \mathrm{C}$ ) for adjectives and the schemes $\mathrm{CaC} \bar{a} \mathrm{C}$ and $i C C a C C a$ for nominal and verbal lex- 
ifications of color, respectively (cf. Borg 1999:216); ${ }^{4}$ thus the root $s f r$ 'yellow' yields the following terms:

'așfar, f. șafrā', pl. șufr 'yellow' | șafār 'yellow color, pallor' | iṣfarra 'turn yellow, become pale.'

Color nomenclature probably constituted, from an early stage, a well-articulated and highly evolved semantic and formal subsystem in Old Arabic, and variants of the morpholexical paradigms exemplified above occur across the Arabic dialect area among both sedentary and nomadic speakers. Bedouin dialects - known for their archaic tendencies here also continue an Old Arabic diminutive scheme aCayCiC lexifying approximation to a basic color focus (Hess 1920:75; Borg 1999 passim): Negev Bed 'așfar 'yellow' $\Rightarrow$ 'aṣêfir (dim.) 'yellowish'; non-basic color terms lack this morphological option. Nonbasic color terms on the $a C C a C$ scheme generally lack verbal equivalents, possibly on account of their low referential salience:

Dam 'ašhal 'having dark grey eyes' | 'abraš 'grey, albino' | 'aš ar 'blond' | 'adham 'black (horse),' etc. (Cowell 1964:130)

Native Arabic color terms (basic and nonbasic) are subject to tight morphosemantic constraints, and loanwords are easily identified by their deviance from the inherited canonic shapes and grammatically invariant stems:

Ar 'urğuwān 'purple' < Syriac 'argəwānā Late Heb/Aram 'argawān 'purple (garment)' (II Chr 2: 6) Akkad argamannu (Sokoloff 1990:73) | Ar burtuqā̄i 'orange (color)'s

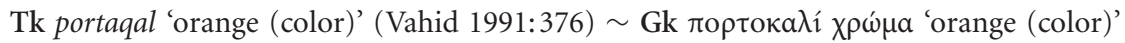
(Magaziz 1995: 161), etc.

Morphological productivity here emerges as a formal trait of historical interest as in the case of Cairene, "an eleven-term stage VII language, similar to modern English or French" (Stewart 1999:106). Whereas the first six terms in the Berlin \& Kay sequence are fully inflected for gender and number in this vernacular, the remaining five - being later acquisitions - are not. Furthermore, only the first five terms in the B\&K sequence (i.e., 'abyad 'white,' 'iswid 'black,' 'ahmar 'red,' 'axdar 'green,' 'asfar 'yellow,' 'azra' 'blue') display verbal equivalents: byadd 'become white,' swadd 'become black,' hmarr 'turn red, blush,' etc.

As already noted, the grammatically evolved nature of the five-term basic color paradign of Old Arabic reflects its early systemic ontogeny. Observe, in this connection, the striking formal analogy with the behavior of English basic color terms where synchronic generation of color verbs is restricted to three lexemes representing the two earliest and semantically most salient developmental stages in the $\mathrm{B} \& \mathrm{~K}$ sequence: white $\Rightarrow$ whiten,

4. In vernacular Arabic, these schemes undergo slight phonological changes varying from one dialect area to the next (mainly elision of short vowels in unstressed open syllables).

5. On the likely origin of this color term, see Warburton (1999). 
black $\Rightarrow$ blacken, red $\Rightarrow$ redden; the terms green, yellow, etc. not being amenable to this derivational process. ${ }^{6}$

The subsequent integration in the Arabic color paradigm of canonic shapes untypical of the native morphemic inventory intimate, on the one hand, the impact of foreign languages (cf. banafsaği, lellaki < Pers) on the Arabic color system and, on the other, the waning derivational productivity of the adjectival scheme $\mathrm{aCCaC}$, even with regard to native roots encoding the later evolutionary stages of the basic color paradigm yielding, e.g., Pal Ar zahri 'pink' and ramādi 'grey' rather than the morphologically possible forms *azhar and *'armad.

Equally striking are pragmatic traits typifying certain expressions conveying color comparisons; observe the minimal syntactic encoding implemented in such expressions in Maltese and Negev Bedouin Arabic:

Malt 〈abjad silg〉 'pure white' [white + snow $=$ as white as snow] | <iswed faham ` 'jet black' [black + coal $=$ as black as coal $] \mid\langle a \hbar m a r$ nar $\rangle$ 'very red' $[$ red + fire $=$ as red as fire $] \mid$ 〈isfar mewt ' 'very pale' [yellow + death = deathly pale] | Negev Bed 'abya laban 'pure white' [white + milk $=$ as white as milk] | 'ahámar damm 'blood red' [red + blood $=$ as red as blood], etc.

Incidentally, the evolution and objectification of Arabic color concepts also yielded a sophisticated nomenclature encoding discourse about color:

To express the concept "color", Arabic uses the general term lawn which, besides this precise sense, also denotes "shade", "aspect", "type", "dish (of food)", 8 etc. In addition to this general term, we also have the following words: $b \bar{u} s$ (stressing a notion of brightness, of clear color), sabīb (liquid color or tincture, also applied to the object which it colors), suhna (applied to the color of the complexion), layt, najr, xutba (applied to a dirty color, etc.). (Morabia 1986:699)

\section{The Old Arabic stage}

The earliest diachronic stage of the Arabic color lexicon - as reflected in the language of Pre-Islamic poetry (6th-7th century A.D.) - has been studiously researched in the Humboldtian paradigm of 'language and culture' by the Arabist and orientalist Wolfdietrich Fischer whose classic philological and ethnographic study, Farb- und Formbezeichnungen in der Sprache der altarabischen Dichtung (Wiesbaden 1965) - a rare event on this scale in

6. The other morphological process possible in English color terms - suffixation of -ish - is not revealing in this respect since it appears with most basic color terms. On the use of morphological criteria in the analysis and historical reconstruction of color paradigms, see Corbett and Morgan (1988:36-42).

7. On the vitality of the $a \mathrm{CCaC}$ scheme at various diachronic stages in the evolution of Arabic, see Fischer (1965: 176f.).

8. Cf. Syriac gawnā 'colour, complexion, appearance, sort, kind' (J. Payne Smith 1903:65) < Pers gûn 'colour, species.' [A.B.] 
Arabic and Semitic linguistics - may well be one of the most comprehensive and authoritative investigations of color semantics to date dealing with a specific language. ${ }^{9}$ Fischer reconstructed for Pre-Islamic Arabic a five-term basic color system comprising:

'abyad 'hell leuchtend, weiß' | 'aswad 'schwarz, dunkel, finster' | 'ạ̣mar 'rot, braun' | 'axdar 'dunkelfarbig, blau, grün' | 'așfar 'alle Farbtöne vom hellsten Gelb und Beige bis Orange und gelblich Dunkelbraun', (op. cit., pp. 243, 273, 335, 306, 358).

Significantly, the very same five basic categories typified Biblical and the later Mishnaic Hebrew:

lābān 'white' | šāhōr 'black' | 'ādōm 'red' | yārōo 'green' | șāhōb 'yellow, bright'.

(Brenner 1982 passim).

It is reasonable to attribute systemic convergence between these two offshoots of Semitic in the lexical domain of color to a cultural synthesis reached in the ancient Near East. If on the mark, this observation suggests inter alia that the Pre-Islamic color system as reconstructed by Fischer was not the exclusive product of a purely nomadic Weltanschauung but, more plausibly, the outcome of interaction of semi-nomadic pastoralists with a sedentary culture, subsequently encoded in an inter-tribal koiné and crystallized in Arabic belletristic discourse. ${ }^{10}$

In Fischer's terms, the Old Arabic color system segmented the chromatic spectrum (i.e., excluding BLACK and WHITE) into three zones: (i) a dark category corresponding to GRUE; (ii) a RED category extending from red to light brown; and (iii) a YELLOW category comprising bright yellow and orange shading into dark brown:

Im eigentlichen Bereich der Farbigkeit werden nur drei Grundfarben unterschieden: 1. Grün-Blau = Dunkelfarbigkeit (axḍar), 2. Rot-Braun (ạ̣mar), 3. Gelb-Braun (aşfar). Die qualitative Differenzierung der Farben ist also viel geringer ausgebildet als diejenige, die in den uns geläufigen Farbwerten rot, gelb, grün, blau, violett und braun ihren Ausdruck hat. Die Feststellung, daß die dem System der altarabischen Farbwörter zugrundeliegende Farbauffassung nur sehr grobe und summarische Unterscheidungen trifft, wird noch dadurch unterstrichen, daß das Altarabische mit einer einzigen Ausnahme auch innerhalb der einzelnen Grundfarben keine qualitativen Farbtöne oder Farbnuancen differenziert. Farbwörter wie 'purpurrot, karminrot' oder 'gelbgrün, türkisgrün', die einzelne Farbwerte innerhalb der Grundfarben charakterisieren, haben im Altarabisch keine Entsprechungen. (Fischer 1965:381)

9. Fischer's paradigmatic work is inexplicably omitted in the bibliographies of both editions of B\&K's Basic Color Terms. For recent general bibliographical data on linguistic aspects of color, see MacLaury (1997: Ch. 2 and 539591) and Grossmann (1988:320-366).

10. The point at issue has a broad diachronic significance beyond the lexical semantics of Arabic itself and extends to the prehistory of pastoralism among Semitic peoples and its impact on their languages (cf. Zohar 1992). As hinted in Borg (1999), if one adopts the notion of a world view as a system of cultural adaptation, the domination of a color system by a pastoralist-ethnic Weltanschauung determined by ecological considerations could be argued as a fairly strong case of Whorfian linguistic relativity. 
Of special significance to the developmental aspect of the Old Arabic color system is its domination by brightness contrasts rather than hue distinctions:

Diejenige Farbbezeichnungen, die den generellen Farbwörtern axdar, ahmar, așfar untergeordnet sind und die einzelne Farbtöne bezeichnen, differenzieren allein den Helligkeitsgrad der Grundfarbe, nicht den Farbwert (loc. cit.). [emphasis added] ${ }^{11}$

As will be seen below, the role played by brightness in the apperception of chromatic stimuli among the ancient Arabs is a crucial cognitive factor in a diachronic survey of color categorization since it (a) yields a clear contrast with the color systems of modern sedentary speakers who tend to have a more evolved, hue-oriented color paradigm, and (b) underscores an element of continuity with the color apperception of certain modern Bedouin communities who also emphasize brightness at the expense of hue contrasts. ${ }^{12}$ This factor strongly suggests that a decisive determinant of change in the evolution of the Arabic basic color paradigm has been the sedentarization of nomads, which brought them in contact with the saturated focal colors of the dyer's palette. ${ }^{13}$

\section{Substratal residue of Aramaic color terms in Eastern vernacular Arabic}

Further insight into the early development of the Old Arabic color system can be gleaned by examining residual traces, specifically within levantine Arabic, of an Aramaic lexical substrate encoding chromatic meaning patterns. Significantly, for the systemic aspect of the basic color paradigm, the terms in question hark back to the roots $h w r$ 'white,'s šhr 'black,' smq 'red,' and yrq 'green,' which are known to have lexified basic color categories, for instance, in Syriac:

Syriac (J. Payne Smith 1903): hwwar 'be or become white', hewōrō 'white, silvery, clear, bright' (p. 134) | šhar 'become black, sooty' (p. 572) | (R. Payne Smith 1897): smeq 'be red, turn red' (p. 1633) | yōrūqō 'cinereus colore [ash-colored]' (p. 381).

A residue of these Aramaic lexemes in Syrian, Lebanese, and Palestinian Arabic is exemplified in Figure 1. ${ }^{14}$

11. Cf. also Ducatez and Ducatez (1980).

12. This is presumably the gist of Lawrence's impressionistic statement that Bedouin "have little color feeling" (1926:117).

13. A useful evolutionary parallel worth noting here is that of the Japanese basic colour system which, before its exposure to Western dyeing technology in the 19th century, comprised: white, black, orange, turquoise, and yellow (McNeill 1972), the reason being that the main natural dyestuffs available to the Japanese yielded orange, turquoise and yellow.

14. Though, areally speaking, these modern Arabic vernaculars define the western reaches of a linguistic region formerly pertaining to the Aramaic heartland (which also included Mesopotamia and Anatolia), in actual fact, the periphery of its diffusional outreach in the East extended to Arabia, cf. Yemeni reflexes of Aram $h w r$ 


\section{$h-w-r$}

Palest Ar hōr 'Pappelholz' (Dalman 1964:VI, 123), 'Schafleder (weiß); Leder (gelb, rot), hawar/huwar 'Kalkstein (mehlig, gelblich-weiss)' (Bauer 1957:169, 191), huwar 'weißer Senonstein' (Dalman 1964:VII, 115); hawwar 'écrire au tableau noir avec de la craie,' hawwāra 'terre blanche, marne', hawwāre 'bâton de craie' (Denizeau 1960:128) | Alep ḥōr 'peuplier,' ha/uwwāra 'pierre blanche et friable comme la craie ou le blanc d'Espagne, employée comme blocage dans les murs des maisons' (Barthélemy 193554: 183) | Egyp Ar hū r 'poplars, kidskin,' hawar 'intense contrast between the white of the eye and the darkness of the iris (regarded as a sign of beauty)' (Badawi \& Hinds 1986:231)| Yem hawwar 'dye with indigo,' hōr 'indigo of Yemen' (Piamenta 1990:112) || Class Ar hawira 'have its white and its black strongly delineated,' ihwwarra 'be intensely white' (Hava 1982:148) || Bibl Aram hwar 'weiss sein' (Gesenius \& Buhl 1915:219), hiwwwār 'white' (Daniel 7, 9), ḥür 'white' (Esther 1, 6; 8, 15) || Syriac hewārā 'white, silvery, clear, bright,' hwar 'the white poplar' (J. Payne Smith 1903:134).

$$
\check{s}-\underline{h}-r
$$

Palest Ar šaḥhar 'schwärzen,' šaḥar/šaḥhar/šahwwar 'Rušen,' šḥār/šuhbār 'Schwärze des Kessels' (Bauer 1957:249, 269), mešhara 'Kohlenmeiler' (Dalman 1964:IV, 9) | Alep šahhar 'noircir, mâchurer de suie; au Fig. dénigrer', šạ̣war 'noircir,' šaḥūr 'merle' < Syriac šahrūrā 'id.' (Barthélemy 1935-54:380, 381)| Dam šahwar 'blacken, smoke' (Stowasser \& Ani 1964:23) | Leb ya šihhāri 'Oh weh! Oh Unglūck' (Jiha 1964: 180), šhār 'noir, noir de fumée' (Denizeau 1960:271) || Syriac šhar 'become black, sooty' (J. Payne Smith 1903:572) || Aram šāhōr 'schwarz, (bei Feigen) blau; (bei Wein) rot', šhōrīm 'schwarze Gewänder' (Dalman 1938:419) || Heb šăhōr 'schwarz', ṣ̌̂̄or 'Ruß' (Gesenius \& Buhl 1915: 819) || Class Ar siḥr 'witchcraft' (Hava 1982:311).

$s-m-q$

Palest Ar samaqa 'rote weiche Erde' (Bauer 1957:97; Canaan 1916:165) | Mand s(u)maq(a), sumqa

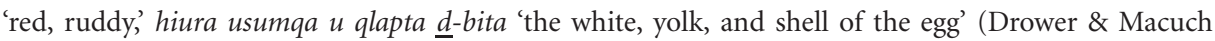
1963: 142, 322) || Class Ar summāq 'Sumach, Rhus coraria or its berry, ... intensely red' (E.W. Lane 1430) || Aram su(m)māqā 'hellrot (vom Wein)' (Dalman 1938:294) || Syriac smaq/smeq 'be red' (J. Payne Smith 1903:381).

$$
y-r-q
$$

Palest Ar rayaqān/yaraqān 'Gelbsucht' (Bauer 126)| Cai yara'ān/yaraqān 'jaundice' (Badawi \& Hinds 1986:962) || Syriac yarqōnō 'livor, ikterus' (Nöldeke 1910:309; Gesenius \& Buhl 1915:320), yarūqō 'egg yolk' (Manna 1975:317) || Heb yarāqōn 'pallor' (Jer 30,6) || Akkad yarqānu 'a certain type of garden herb,' arāqu 'grow pale,' arqu 'yellow, green' || Ugar yrq 'greenish yellow (a metal/gold), yrx b 'lyh yrq 'if the moon in its ascension is (: appears) greenish yellow' || Ethiop warq 'gold' (Hoftijzer \& Jongeling 471; del Olmo \& Sanmartín 2003: 982) || Class Ar waraq 'leaves, foliage,' riqa 'verdant plant,' waraqa 'become luxuriant,' 'ìrāq (vn) 'become yellow (grapes)' (Hava 1982:864).

Figure 1. Substratal reflexes of Aramaic color terms in Eastern Arabic vernaculars.

As can be inferred from Figure 1, these Arabic derivates of ancient Aramaic lexemes now relegated to the semantic periphery of the color domain - denote mostly natural hues (e.g., of soil types) or convey meanings only marginally related to color.

'white' (Piamenta 1990:112). For more detail on Aramaisms at the periphery of the Arabic Sprachraum, see Borg (2004:41f.). 
The transfer of color terms from ancient Aramaic ${ }^{15}$ to Pre-Islamic Arabic is not without theoretical interest to the historian of color. The Aramaic WHITE category was lexified with the root $h w r$ :

Syriac ḥewārā 'albus, candidus' (J. Payne Smith 1879:1230) || JAram ḥăwar 'be white, shine; glisten; be pale; be bright, etc.' (M. Jastrow 1886:438-39)

The denotation of brightness takes on a significantly different form in the Aramaic loan hawira 'to have its white and black strongly delineated (eye)' (Hava 1982:148) ${ }^{16}$ where the focus is not so much on radiant whiteness as on the éclat produced by the juxtaposition of BLACK and WHITE. For a likely real life source of this Arabic meaning, note OA ahwar 'mit besonders stark hervortretendem Weiss (vom Gazellenauge)' (Fischer 1965: 115). The diffuse semantic pattern conveyed by OA hawira - emphasizing the contrast between 'bright, white' and 'dark, black' rather than one specific category - is a significant formal indicator of an early stage in the development of the five-term basic color system of the Arabic color system since "broad categories of brightness merge with the hue sequence at any of Stages I through V" (MacLaury 1997:46) of the B\&K evolutionary model (Berlin \& Kay 1969: 17f.). Fischer (1965:50f.) has insightfully outlined an analogous development for Ar 'azraq, initially encoding a brightness category, later becoming a basic term for BLUE.

Residual traces of this brightness category survive right across the Arabic dialect area, for instance, in Southern Arabia, where the denotations of the root hwr strikingly range over both light and dark semantic values: ${ }^{17}$

Yem hū r 'purity' | hūri 'of fair complexion' | hōr 'indigo of Yemen' | huwār 'indigo used for dyeing black clothes' | hawwar 'dye with indigo' (Piamenta 1990:112) || Mehri hōower 'noir'. (Landberg 1920:510)

These data invite interpretation in the light of MacLaury's (1992) seminal paper postulating a shift from brightness to hue in the evolution of color systems. Significantly, several Arabic dialects exemplified in Figure 1 - for instance, Palestinian - retain highly archaic residual categorizations of brightness/whiteness such as the term hawar/huwar 'Kalkstein (mehlig, gelblich-weiss); gelb, rot (Leder)' (Bauer 1957:169, 191, 253), denoting a lightwarm category as at Stage I in the B\&K sequence. MacLaury (1997:53-54) has pertinently

15. Derivates of the roots $h w r, s m q$, and $y r q$ show up in both ancient and modern varieties of Aramaic, for instance, in the contemporary vernacular commonly called Țuroyo spoken in Țur 'Abdin (S.E. Turkey): heworo 'white,' smoqo 'red,' yaroqo 'green.' The root šhr 'black' may well be a survival from Common Semitic since it figures as a basic term in Biblical Hebrew (Brenner 1982 passim), Akkadian, and Syriac and also survives in the Eastern Aramaic language called Mandaic (see Fig. 2) spoken until recently in Ahwaz (S. Iran). Mandaic exists in classical and vernacular varieties; the former is the liturgical language of the Gnostic Mandaean sect (cf. Drower \& Macuch 1963).

16. This is the origin of the Old Arabic term hüriyya 'houri, nymph of paradise' in Muslim belief.

17. On the etymology of the root $h w r$, also attested in Biblical Hebrew, note Gradwohl (1963:58): "Neben lābān tritt die Wurzel auf. Im Aramäischen ist sie sehr geläufig und konnte daher aus diesem Bereich entlehnt worden sein." 
noted that a brightness category is not "constrained by universal hue perception of fixed elemental points" and can therefore fluctuate more widely than a hue category.

The source of šhr 'black' cited in Fig. 1 can also be derived from an earlier brightness category; the ancient term was semantically linked with Aram šah̆ărā 'das Morgengraue' (Levy 1924:IV, 536) cognate with Ar sahar 'daybreak,' suhira 'beginning of dawn' (Hava 1982:311). The proposed early chronology of the roots ḩwr 'white,' šhr 'black,' and smq 'red' within Eastern Arabic find independent support in the realm of toponomastics where these reflexes of Aramaic root morphemes frequently encode placename components, for instance, in Lebanon:

Baḥwarte 'Ort der Weisspappel' < ${ }^{\star} b \bar{e}(t)$ ḥwartā $\mid$ Bšaḥhāra 'Ort der Schwärze oder dgl.' $<{ }^{*} b \bar{e}(t)$ šahhārā; Wādi šahrūr 'Wādi der (Schwarz-)Drossel' | Smiqta < smaqtā 'roter Färbstoff der Sumach-Wurzel'. (Wild 1973:184, 136, 305, 42)

The lexeme yaraqān 'jaundice' (= yellow) is of historical interest inasmuch as its root yrq is ordinarily associated with basic 'green,' for instance, in the modern Aramaic languages: Turoyo yaroqo 'green' as opposed to ša ' $u \theta 0$ 'yellow.' ${ }^{\prime 18}$ This usage in modern Arabic already attested in Biblical Hebrew (yarāqōn 'pallor'; Jer 30, 6) - clearly continues an early evolutionary stage (i.e., III in the B\&K sequence) of the Aramaic color system, at which point both yellow and green were assigned the same categorial designation as in modern Mandaic yrq 'become green, pale', iarqa 'herbs, green salad' (Drower \& Macuch 1963: 440, 187).

\section{From natural to artificial colors in vernacular Arabic}

Mainstream vernaculars in the contemporary Arabic Sprachraum lend themselves to a three-fold general classification into nomadic, rural, and urban varieties. In effect, this division correlates roughly with three stages in an evolutionary process in which Bedouin vernaculars generally exemplify the most conservative linguistic type and the urban vernaculars the most evolved. The earliest diachronic split within the Arabic dialect family separates the nomadic from the sedentary vernaculars.

Assuming that the language and culture of contemporary Arab nomads continue inherited traditions harking back to ancient Arabia, it is reasonable to infer that the demonstrably archaic color systems in their dialects can yield valuable insights into formal and typological aspects of the Proto-Arabic color paradigm, in essence, enhancing the applicability of methods of internal reconstruction.

Color can represent a remarkably salient visual dimension in desert and steppe landscapes. The expressive chromatic language employed, for instance, by Western travelers to

18. Cf. also yaruqá 'green' vs. zahirá 'yellow' in the Jewish Aramaic vernacular of Arbil (N. Iraq) (own observ.). Significantly, the second term is cognate with Biblical Aram zhr 'glänzen' (Gesenius/Buhl 1905: 194), Syriac zahrō 'brightness, brilliancy, flashing ( $d$-nūrō 'of a fire')' (J. Payne Smith 1903:111), and Galilean Aram zahrā 'the moon' (Sokoloff 1990: 172). 
the Arab East, such as Charles M. Doughty, Thomas E. Lawrence, Robert E. Cheesman, and Alois Musil in their descriptions of physical features of desert ecology is, to say the least, striking. ${ }^{19}$

Particularly imposing to the observer is the cohesive visual impact - conveying an impression of color harmony with the environment - produced by the protective mimicking of certain species of desert fauna:

In a desert journey such as that to Jabrin the mind is naturally exercised in trying to find a reason for the fact that almost every specimen of bird, beast, reptile, and insect is arrayed in beautiful shades of pale cream and buffs. (Cheesman 1926:309) 20

The underlying theme of Borg (1999: 121) - based on fieldwork among settled tribesmen of the 'Azāzmih in the Central Negev - was the impact exercized by the Bedouin's ecology on their apperception and categorization of color. Nativist theories of language acquisition often underpin the search for universal explanations of interlinguistic convergence noted in much literature on color systems (cf. Deacon 1997:116) and it is easy to succumb to the illusion

that different languages refer to the same human reality and that people can assume they know what this reality is like. (Lee 1984:115)

It would, in this respect, be revealing to survey the typologies of color systems pertaining to nomadic groups outside the Arabic-speaking world to determine (a) the extent to which their categories are modeled on their peculiar ecologies, and (b) how they come to terms with the artificial hues of co-territorial sedentary cultures. ${ }^{21}$

Particularly striking, in this regard, is the fact that native Arabic nomenclature for physical features in a desert landscape also frequently refers directly or obliquely to ecological hues; the semantic structure of this profuse inventory of Arabic folk-terms for natural colors has yet to be addressed. As already noted in the previous section, some words lexify

19. Lawrence is especially sensitive to ecological colors: "They rose on each hand regularly, precipices a thousand feet in height, of granite-brown and dark porphyry-coloured black, with pink stains ..." (p. 86) | "The walls [of Kharma valley] were of bare red and black rock ... They made the freshness of the trees and grass seem luxurious" (p. 90)| "a reef of coral crag” (p. 108) | "a surface of slate-blue a brown-red rock" (p. 140)| "great grey striated piles of volcanic rock, reddish coloured” (1926:179). Note also Musil (1926:153): “The northern and southern slopes were as if painted a dark blue. In the deeps gaps between the various knolls and cones there were already displayed dense violet-colored veils of mist, above which a golden-yellow firmament was arched."

20. Note also the observation in Doughty (1888 I:395): "When we were come upon the main lava-field ... a startled troop of gazelles scudded before us; here they are ... nearly of the colour of basalt; gazelles are white in the sand plains."

21. In Borg (1999) I proposed as a research objective the hypothesis that semantic categories encoded in the vernaculars of Arab nomads represent cultural rather than simply areal isoglosses setting Bedouin apart from sedentary Arabs. Co-territorial urban Palestinian speakers, for instance, have a considerably more evolved basic color system comprising the maximal set of eleven color categories posited in the B\&K model (see below). The point of this remark is that: "speakers of a single language could represent different stages of Berlin and Kay's sequence" and that "variation presages evolutionary change" (MacLaury 1997:23). 
diffuse, chromatic apperceptions associated with a relatively early evolutionary stage of color systems; others are simply covert context-bound color terms:

Groom (1983): burșa 'white or distinctly different patches in the sands where nothing grows' (p. 64) | burqah/abraq/barqa') 'an area of rugged ground with stones, sand and earth, mainly white or dust-colored' | 'abla ' 'a rock, especially a white rock, a white hill; a strip of land with white stones' | ag்ar 'dust-colored with an ashen or red hue' (p. 34); a'far 'dust-colored with an ashen or red hue' (p. 34) | așbah 'black, inclining to red' (p. 47); 'a rock, especially a white rock, a white hill' (p. 66) | hāl 'black mud or soil' (p. 102), etc. ${ }^{22}$

The developmental stage reflected here in retrospect saw the proliferation of lexemes combining allusions to color with light effects yielding inter alia an extensive set of color terms denoting irregular or dotted surfaces evidently perceived by the ancient Arabs as light contrasts (e.g., burșa and burqah). Such terms were fairly common in Old Arabic:

Fischer (1965): Class Ar ablaq 'weiß (blitzende) Flecken auf dunklem Grund aufweisend' (p. 83) | abqa' 'weiß gefleckt' | arqat 'gepunktet, getüpfelt (Antilope, Ziegenbock, Schlange)' (pp. 99-100) | arbad 'gefleckt, gepunktet' < abrad Heb bārōd (p. 100) | ğawn 'einfarbig im Gegensatz zu gefleckt' (p. 32)| ahamm 'teilweise schwarz gefärbt' (p. 115).

As indicated in Borg (1999: 134), semantically diffuse designations in Old Arabic referred to complex stimuli only partially defined by hue:, e.g., axtab 'dunkelfarbig (blau, grün) und hellfarbig gefärbt' (Fischer 1965:321) which could apply equally well to the juxtaposed yellow and green hues of a colocynth as to a combination of light and dark plumage (Fischer op.cit. : 240, 323, 381).

The circumstance that the lexification of basic and nonbasic color categories among Bedouin routinely utilizes the same terms for the hues of terrain and of animal coats abga $\theta$ 'staubgrau (Berge, Wolf, Hase, etc.),' ahamm 'rote Erdfarben, Farbe von Kamelen'; Hess 1920:76-77), etc. - suggests that the aforementioned perception of natural color as a principle of visual cohesiveness plays a cognitive role in these nomads' categorization of color.

The extensive inventory of nonbasic colors generally reported among Bedouin in various parts of the Arab world witnesses to their functional salience in these nomads' naturalistic world-view since "culturally important objects and concepts tend to be lexicalized more abundantly ..." (Wierzbicka 1997). Color systems modeled on the ecology have been reported for other Naturvölker, such as the Zulu (Bryant 1949) and the Mursi cattle herders of Ethiopia (Turton 1980:320), among others. MacLaury (1997:333) noted

22. References to ecological colors also frequently occur in Arabic toponyms recorded, for instance, in Musil (1908, I) (my transl.): Qașr al-Azraq 'Black Castle' (p. 18); Rās al-Uhaymer 'Reddish Headland' (p. 193); 'Ayn Bēda 'White Water Source' (p. 319), Nuqrat al-Bēda' 'White Crater' (II, 18), etc.; and in Dussaud (1927): Nahr al-Abyad 'White River' (p. 157); Nahr al-Aswad 'Black River' (p. 436), etc. Color terms (most often white, black, and red) here encode spatial representation which, in Durkheim's conception "consists essentially in a primary co-ordination of the data of sensuous experience (1961:23)." Parallels occur in other languages: White Springs, White River, Black Hills, Red Lake, Redwater, etc. (Times Atlas, Index), Blancheroche, Blanruz 'ruisseau blanc,' Rouge-Fosse, Rouges-Terres, Noires-Fosses, etc. (Rohlfs 1971:63-64). 
that "as small societies join the orbit of worldly ones, the context vocabulary depletes." Context-restricted terms for ecological colors are generally lost by urban speakers of Arabic; a residual set of such terms can sometimes be observed among rural sedentaries (fallāhīn) who still practice pastoralism. An interview of a Lebanese shepherd conducted by the noted Arabic dialectologist and cleric Mgr. Michel Feghali in the early decades of the 20 th century yielded the following exchange:

Connais-tu chaque chèvre par son nom, car je vois que tu as un grand troupeau? - Mais exactement! Nous appelons: qaț̌a, celle qui a les oreilles coupées; sakka, celle qui a les oreilles longues come le doigt; $b$ dèn, celle dont les oreilles mesurent un empan; grabiyye ou 'bayde, celle qui est complétement noire; hmème, celle qui est toute blanche; malha, la chèvre qui a les oreilles blanches; ' atra, celle qui les a rouges; kahla, celle dont les oreilles et les yeux sont rouges; mzannra, celle qui est tachée de blanc et de noir; șaḅa, dont le milieu du front est taché de blanc; najma, celle qui a, à la tête, une petite tache blanche; xazma, celle qui a des poils blancs sur le nez; zahra enfin, celle dont le bout de la queue est blanc. (Feghali 1935:181-82)

The color categories of modern urban Arabic vernaculars have evolved considerably beyond the Old Arabic stage; most if not all of them have acquired new categories and often attain or approximate the maximal set of eleven terms representing Stage VII in B\&K's evolutionary sequence. Figure 2 exemplifies the color systems of the Arabic colloquials spoken in Cairo, Jerusalem, Damascus, and Mecca:

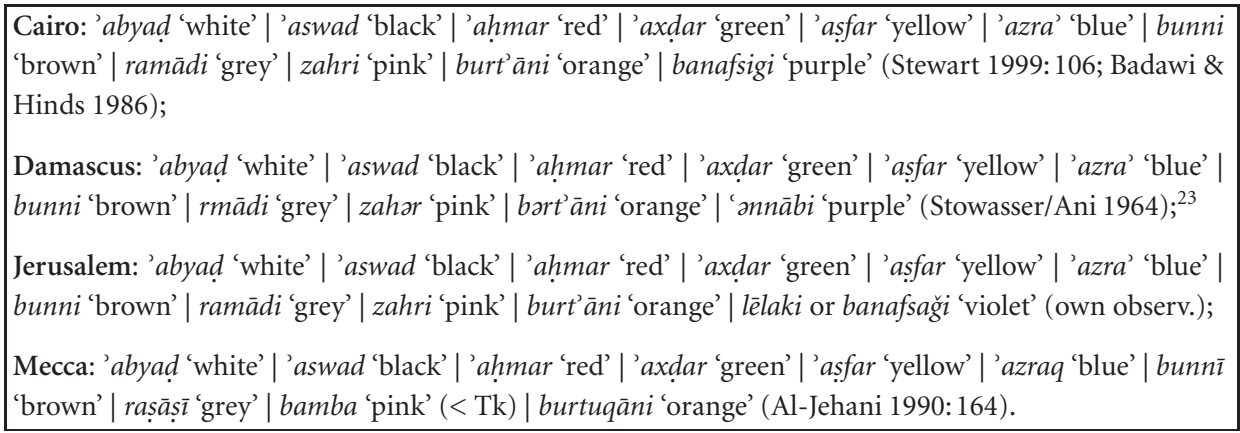

Figure 2. Basic color systems in some urban Arabic vernaculars.

The functional relationships of color categories and their boundaries within a particular system can be quite flexible; thus, Cairene GREEN has retained distinct elements of light blue (i.e. green-blue, pale blue; Badawi \& Hinds 1986:254) notwithstanding the subsequent emergence of a fullfledged BLUE category which can also shade into lighter hues:

23. As in many other dialects, the black category her has a hyponym 'asmar whose meaning is restricted to skin color: bášarto samra, tamām matəl 'abū 'he has a dark complexion just like his father' (Stowasser/Ani 1964:60; Cowell 1964:130). 
Cai 'azra' 'blue; grey (horse), dove grey' (op. cit., 1986:369). Apparently, the acquisition of new categories need not immediately entail retreating boundaries of earlier ones. ${ }^{24}$

\section{Arabic color terms and material culture}

The foregoing sections have addressed formal systemic aspects of the Arabic color paradigm. As pointed out in Conklin's (1973) review of Berlin \& Kay (1969), an account of the temporal development of color categorization should at some point address the inevitable impact of artificial colors in the emergence of perceptual foci of color systems (p. 935).

The situation in ancient Arabia vis-à-vis the production and use of colored fabrics remains unclear:

Detailed descriptions of Arab clothing in Antiquity with regard to color, pattern, and fabric, are, alas, woefully lacking. The Bedouin have into modern times always shown a preference for dark garments. And there is reason to believe that this was true at least as far back as late Antiquity. The Babylonian Talmud cites the dark garments of an Arab as an example of a blue-dark color it is trying to define with precision (Tract., Niddah, 20a).

(Stillman 2000:8)

What is certain is that the Middle Ages witnessed the efflorescence in the Arab urban scene of a rich array of color stimuli generated by the dyer's art. Goitein (1983:174-6) characterized this development as a veritable "color-intoxication" produced by medieval dyers treating animal and plant fibres according to traditional formulae and methods long practiced by the settled peoples of the Near East using locally available or imported plant, animal, and mineral substances. Dye plants included madder root (Ar fuwwa Heb puwwa $=$ Rubia tinctorum) and insect cochineal (qirmiz = Coccus ilicis) for reds and pinks, turmeric; (kurkum = Curcuma longa) for orange; oak bark, pomegranate and vine leaves for shades of brown and yellow; indigo (Ar $n \bar{\imath} l<$ Skt $n \bar{i} l a=$ Indigofera tinctoria) for various shades of blue and green, etc.

It can therefore be plausibly hypothesized that the shift within the Arabic color system from brightness to hue contrasts occurred after the language came in contact with the sedentary cultures of the Near East and their color technologies relating to clothand carpet-weaving, painting, glass-blowing, etc. Some idea of the range of color concepts entailed in the realm of fabrics can be obtained from Goitein (loc. cit., passim) which mentions:

24. An interesting subcase of this shifting boundary occurs in the poetic language of the Sinai Bedouin evoking blood red for dark brown in references to the hue of freshly made coffee (Bailey 1991:308). This usage is presumably a covert reference to wine (forbidden to Muslims) as the 'blood' of the vine. It recalls an identical simile in Iṣfahāni’s Kitāb al-Aghān̄̄ IV, 16: "And it was their most famous men who boasted of wine drinking, preferably when the wine was 'red like the blood fof a slaughtered animal ...' The imagery of blood in relation to wine is much older and also occurs, for instance, in biblical language (Gen 49:11-12; Deut. 32:14). 
various shades of white (glittering snow-color, pale white, pearl color, cloud color [= white fluffy cumulus]), pomegranate red, crimson, vermilion, sumac, purple, musk-color [= reddish brown], sand-color, yellow, orange, amber, wax, saffron, safflower, honey color, brownish pink (eye of the partridge), asparagus color, emerald green, pistachio, sky blue, cloud blue, indigo, turquoise, blackish blue, lead-color bluish gray, pepper gray, etc.

The precise linguistic details of terminological and semantic shifts in the Arabic chromatic lexicon linked to dyeing technology will probably only emerge with some clarity after the production of a dictionary of Middle Arabic - the subclassical literary variety of Arabic used outside the realm of belles lettres in the Middle Ages and later.

The manufacture of colored fabrics involved complex socio-economic interaction concomitant with a well-defined division of labour, for instance, in the production of oriental rugs. ${ }^{25}$

The societal factor entailed in the production of colored fabrics introduced into color discourse a 'normative' dimension vis-à-vis the emergence of saturated focal colors alongside (or replacing) the inherited nomenclature for the mostly unsaturated nonfocal hues of the ecology which found no expression in the textile industry. Of special significance for the linguistic observer here is the factor of progressive institutionalization affecting color concepts via the impact of official nomenclature as it emerged from the day-to-day practice of the dyers' profession for as noted in Goitein (1983:173):

Language had to be inventive to create conventional terms for identifying a color precisely.

The production and export of cotton, linen, and silk in various centers of the region no doubt ensured the widespread standardization of color foci and nomenclature particularly since, as noted in Mez (1909:461) for the Delta in 10th-11th century Egypt, the cloth industry was carried on in the home with women spinning the linen and men weaving it:

The clothier's art was more elaborated than any other, and interior decoration consisted entirely in colored hangings for the rooms (op. cit., 459).

The central importance of the dyeing industry in different parts of the Arab world survived into the modern period and, in Egypt, for instance:

until the 1930s dyeworks abounded in all the major cities and towns, usually linked to weaving establishments. They proliferated above all in Cairo. From the fifteenth to the nineteenth century (when it became absorbed by the expanding city) Cairo's Nile port of Bulaq, an important commercial entrepôt, greatly developed its industries, cloth dyeing among them. (Balfour-Paul 1997: 101)

The impact of colored products of material culture on the Arabic color system can be exemplified by the nonbasic Arabic term șin̄i 'Chinese' and its cognates in several Middle Eastern languages. A.Lane (1957:21) stated:

25. On the carpet industry in medieval Cairo, see Mez (1909:459 and 465). 
The debt of the Islamic potters to the Chinese is sufficiently indicated by the fact that they habitually gave the name sini 'china' to the wares of fine artistic quality which they made themselves.

The word eventually became a term for 'blue' and yielded a series of cognate color terms in several Near Eastern languages:

Iraqi Ar șini ‘schwarz' (Weißbach 1930:338) | Negev Bed șini ‘blue, grey’ (Borg 1999) | Anatolian Ar šìni 'grün' | Neo-Aram (Ṭuroyo) šīn 'blau' (Jastrow 1990:180) Arbil šìn 'blue' (own observ.) < Kurd šin 'blue'. (Blau 1965:224) ${ }^{26}$

The context for the shift 'Chinese' > 'blue' in the meaning of vernacular Ar șini was provided by the dissemination of blue-on-white ceramics originally imported from China. From the etymon șini the Arabic neologism șiniyya/șeninya/șayniyya was coined, now meaning simply 'a large, round metal plate with raised brim, especially one made of copper, used as baking tin, serving tray and table top'(cf. Wehr 1979:623); interestingly, the plural form of this word (sawānī) retains something of the original meaning: 'chinaware, porcelain vessels' (loc. cit.).

The intrusion of European - for instance, English - textiles in the early eighteenth century and, later, the adoption of aniline dyes in the second half of the nineteenth, tended effectively to put an end to local traditions and methods in the production and coloring of fabrics and no doubt contributed to a gradual semantic alignment of the Arabic color paradigm with Western norms.

Cootner (1990:34) cites Matisse's view that "the Orientals had made color a means of expression." In a study of Arabic chromatic concepts, the linguistic observer adverts in particular to the objectification of color and of talk about color, above all in the domain of clothing, since the color of fabrics in the medieval period often mediated - as it still does (cf. Lindisfarne-Tapper \& Ingham 1997:62) - a value system referring to social and/or religious semiotics:

Under the Abbasid caliphate black became the fashion for the highest classes, although not for clothing with tiraz inscriptions, apart from an interlude when green, which presumably was dyed with indigo and yellow, was the favourite color. All those below the most elevated ranks in society were forbidden to wear black but could choose other colours within the bounds of propriety. (Balfour-Paul 1997:15)

Under Islam, non-Muslims were often subject to the so-called laws of differentiation (ghiyār) ensuring that Christians, Jews, and others did not pass for Muslims. In the Mamluk period, blue was enjoined on Christians, yellow on Jews, and red on Samaritans (op. cit., 84). ${ }^{27}$

26. The closely similar form and meaning of Russian sinij 'dark blue' is intriguing.

27. This obligation came into force for the first time under Harun al-Rashid (766-809) (Philby 1933:63). 
Among Muslims the preferred color for men is traditionally white, allegedly in conformity with the Prophetic injunction: "the best of your clothes are white" (Stillman 2000:23): ${ }^{28}$

The true Egyptian style was white uncoloured linen.... In the 10th century it was unfashionable for a man to appear in an attire of many colours ...

(Mez 1909:460)

Above all, red was to be strictly avoided (Margoliouth 1914:159). The ascetic spirit dictating avoidance of ostentatious colors appears to have remained a stable feature of male dress under Islam. Von Oppenheim (1899:141-2) noted the same cult of simplicity in dress also among the Druze:

Die Tracht der heutigen Drusen besteht aus einem Hemd (qamīs ) und bauschigen Beinkleidern (libās), über dem Hemd wird eine kurze, armellose Schwarz und weiß oder rot gestreifte Abaje getragen vielfach auch ein langes hemdartiges Gewand (qumbāz) das verschieden gefärbt ist... Das Turbantuch ist immer weiß.

The social semiotics of color in traditional Arab dress has survived until the present time. Weir (1989: 15), for instance, notes:

Up to 1949 , and to an extent still today, styles of dress reflected the major social divisions of Palestinian society. Male and female costume differed primarily according to whether the wearer was a town dweller, villager or Bedouin, and secondarily according to the region they came from.

The design and color of men's headdress (küfyya) may indicate the wearer's tribal or regional affiliation (Lewis 1995:5). Women's clothing, on the other hand, can be very colorful; the interpretation of the social meanings of color in Arab women's apparel has been insightfully studied in Abu-Lughod (1986:134-142).

\section{Observations on some basic color categories in vernacular Arabic}

The preceding sections have addressed general socio-cultural issues relating to the evolution of Arabic color categories; the following remarks relate to diachronic aspects of the first six categories in the B\&K sequence; in the interests of brevity, I will present my comments in note form.

(i) The Old Arabic opposition abyad 'white': aswad 'black' entailed both brightness and hue contrasts without differentiation; thus Fischer (1965:243) glosses these terms 'hell, leuchtend, weiß' and 'schwarz, dunkel, finster', respectively. The brightness/translucence value of the root byd 'white' in Old Arabic is exemplified in the following expressions:

28. On the color white in early Islam, Margoliouth (1914:140) stated: "The colour white as the colour of graveclothes, is also at this time connected with mourning and asceticism. By a man's coarse white raiment it was possible to guess that he was an ascetic, though he might be either a Muslim ascetic or a Christian monk." 
bayāe al-nahār 'daylight (lit. the whiteness of day)' | al-bayḍā' 'silver, wheat, Sun, paper [lit. the white one f.] | al-xayt al-abyad 'first gleam of light [lit. the white thread]' | al-abyadān 'milk and water' [lit. 'the two white ones']. (Hava 1982:53)

For the term abyad in the 'Utaybah Bedouin vernacular of Central Arabia, Hess (1920:76) records only the straightforward meaning 'weiß (Kleider, Baumwolle, frische Milch, Schafe, Pferde)' without any reference to a brightness value. Since this dialect retains the adjective 'awdah 'white,' the typically Bedouin term for whiteness in camels - cognate with the noun OA wadah 'gleam of dawn; moon,' wädih 'white (camel), bright (star); strahlend weiß’ (Hava 1982:875-76; Fischer 1965:10), it is conceivable that the absence of brightness values in Hess's treatment stems from his field methodology, which, as already noted, seems to have entailed principally the use of the Code de Couleurs (Klincksieck \& Valette 1908). From the Arabian dialect area, Goitein (1934:153) cites the Yemenite proverb min bayāọak tkuzz iš-šams "Mit deiner Helligkeit blendest du die Sonne," where the term for whiteness clearly refers to brightness. A similar use of this lexeme also occurs among the Negev Bedouin (attributing whiteness to the sun). Sedentary Arabic dialects do not appear to retain this archaic semantic pattern byd = 'bright.'

Color symbolism restricts use of the term aswad 'black' which is taboo in several parts of the Arab world; among Bedouin, in particular, the root swd is generally associated with the honor ethic and is studiously avoided in speech (cf. Borg 1999: 140-41), often through the use of euphemistic substitutes. Marçais (1906) has studied this trait in Algerian Arabic:

byād «la blancheur» est à Tlemcen le nom habituel du charbon dont la couleur est noire et considérée funeste. byā d chez les ruraux de Mascara désigne «la suie» ... et encore à Tlemcen un cosmétique noir, employé par les femmes, et qui est à base de noix de galle. ${ }^{29}$

Of some interest, from the systemic angle, is the ethnographic detail noted in Hartmann (1899:85) to the effect that Libyan Bedouin here traditionally resorted to the euphemistic use of 'axdar 'green,' which, in its darker shade, overlaps with the BLACK category.

(ii) OA 'ahmar, a cognate of Akkad emēru 'gerötet sein,' Heb hōmer 'clay', and Ugar hmmr, falls into Fischer's heller Farbbereich of yellow and red and ordinarily denotes a hue range from red to light brown with a focus in blood-red (1965:334f.). The term 'ahmar appears in connection with bloodstained swords, red skies at sunset, red wine, bay camels, reddish sand, and ripe dates. Since Old Arabic lacked a term for brown, 'ahmar could also denote the hue of dark brown objects (details from Fischer, loc. cit.).

A macro-red category ranging over pink, orange, purple, and light brown occurs in several contemporary Arabic vernaculars. Interestingly, though speakers of Arabic dialects often recognize the color of blood as the best example of red, unsaturated redness in the coloration of animal pelts, terrain, and other natural colors still figures prominently in this category:

29. For a more detailed study on euphemistic usage in Algerian Arabic relating to the color 'black', see also W. Marçais (1955). 
Cai 'ạ̣mar 'red, reddish,' aḥmar šafāyif 'lipstick,' husạan aḥmar 'a bay horse,' laḥma hamra 'lean meat,' naḥ̄s aḥmar 'copper' (Badawi \& Hinds 1986:224) | Mor Ar hmar 'rouge; fauve; bis; roux, blond (individu); bai (chameau); basané, bronze (visage); qui a la peau cuivrée (personne); d'un jaune grisâtre' (de Premare et al. III, 218) | Upper Egyp (alDāxila) hamrä' 'hellbraun (Tier)' (Behnstedt \& Woidich 1994:94) | Leb 'ard il-hamra 'rote Erde, lehmhaltige Ackererde, die lange die Feuchtigkeit hält'; mžaddra hamra 'Gericht aus Linsen und Burgul; rot bzw. braun in Olivenöl gebratene Zwiebel' (Jiha 1964:104, Fn. 1, 7) | Dēr iz-Zōr (Syria) 'ahmar mhammas, mhammar mqammar 'knusprigbraun' (Jastrow 1981:448, Fn. 14) | Siirt (S.E. Turkey): šawt lē ləššèr dībyaỵ, alhənța-mana tahmarr 'wo sich die Gerste weißlich färbt, wird der Weizen goldgelb' (op. cit., 299)| Oman yahmarr 'to become brown (of roasting meat)' (Brockett 1985:85)| Chad mara hamra (lit. a red woman) 'une femme au teint clair [cuivré]' (Pommerol 1999:70) | E. Arabia 'ahmar 'red, reddish, pink, fair-skinned; brown' (Holes 2001:128)| Cypr Ar xmarra l-im'all 'the wheat has turned golden brown (i.e., is ripe)' (own observ.).

Thus the interdialectal treatment meted out to RED furnishes a striking example of the point discussed in $\$ 4$ above: the matter of categorial boundaries. Some Arabic dialects have developed a stable brown category (cf. Dam kastanāwi 'chestnut brown', banni 'dark brown,' 'asali 'honey-colored,' 'asmar 'dark (complexion), brown (hair)'; Stowasser \& Ani 1964:30); nonetheless, traces of macro-red persist; note the use of hmr 'red' in Dam hammar al-lahme mnīh 'Brown the meat well' (loc. cit.). Cairene actually displays a degree of free variation between RED and BROWN: ahmar/bunni 'bay (horse)' (Badawi \& Hinds 1986: 107). ${ }^{30}$

Data on the red category are available for the Maltese and Cypriot varieties of marginal Arabic. Given its long history of contact with European languages (principally, Italian and English), Maltese has here understandably retained only marginal instances of contextbound terms designating redness; references to red fall almost entirely within its focal area, e.g., ‘ahmar qrolli ' 'coral red,' etc. Nonetheless, the foreign provenience of terms for pink (rōza), orange (oranğo), light brown (kannella) ${ }^{31}$ suggests that the red category in earlier forms of the language embraced this entire domain, as in certain rural parts of Europe before the onset of industrially produced colors. Observe, in this respect, the following comment on French rural usage in the first half of the 19th century:

Il est à remarquer que les paysans ne reconnaissent que le rouge, dont le domain pour eux embrasse le rose et l'orange, et toutes les nuances comprises entre ces couleurs; - le jaune, mais seulement certaines nuances; quand il est pâle, ils l'appellent blanc; quand il est un peu foncé, c'est du rouge; etc. (Karr 1851:107-108)

Cypriot Arabic appears, on the other hand, to have retained a genuine macro-red category extending to light brown:

30. The color term bunni 'brown' and its dialectal cognates derive from bunn 'coffee-beans.'

31. Cf. It cannella 'albero delle Lauracee dalla corteccia aromatica (Cinnamomum zeylanicum)' (Zingarelli 1970:262). 
pakra xampra 'reddish brown cow', žeže xampra 'reddish brown hen,' xmarra xops xost il-forn 'the bread turned brown in the oven'. (Borg 2002:85)

Cypriot Greek being the second language of Cypriot Arabic speakers, it is interesting to note, in this connection, that a macro-red category was noted for this variety of Greek in Rivers (1901:68-69; cited in Berlin \& Kay 1969:28).

(iii) The Old Arabic lexeme 'axdar denoting "die den ganzen Farbbereich umfassende Grundfarbe, sowohl 'grün' als auch 'blau,' but also shading into grey ${ }^{32}$ and black (Fischer 1965:306) has been almost certainly inherited from general Semitic since cognates with closely related meanings occur in Akkadian, Aramaic, Phoenician, and Hebrew (Zammit 2002:162).

The Old Arabic GRUE category constituted a semantic pattern common to other ancient Near Eastern languages: Sumerian, Akkadian, Ancient Egyptian, and Hebrew (Baines 1985:284). ${ }^{33}$ Stewart (1999: 107) states:

The term 'azraq/zarqa $\bar{a}$ ' came already in Classical Arabic to designate 'blue,' which was isolated from 'axdar 'green.' There are of course many indications that 'axdar meant both blue and green in the medieval period; such indications also remain, to varying degrees, in the modern dialects. In Mauritanian Hassaniyya Arabic, for example, there is no distinct term for blue and blue objects, such as the sky, are termed 'axdar. Al-Amīn (1953:30) reports that the common people in Egypt often use 'axdar as a euphemism for the color blue, avoiding ' azra' because they associate blue with bad fortune, as opposed to green, an auspicious color, and 'axdar is still used occasionally to denote 'light blue'. (p. 254)

A GRUE category has also been reported for Upper Egypt (Rivers 1901), Sudan (Reichmuth 1981), Chad (de Pommerol 1999:120), the Sinai Peninsula (Borg, own observ.), Jewish Yemeni ('axઈ̧ar 'green, navy-blue, greenish-dark colour'; Piamenta 1990:130), Mauritanian Arabic (i.e., Hassāniyya), where green runs into violet (Pierret 1948, cited in Roth 1986:31), and probably also occurs in S. E. Anatolia.

The blue/green range of the Old Arabic category axdar, shading into grey and black, finds close parallels in the Arabic vernaculars of modern urban Egypt, Yemen, and rural Morocco:

Cai 'axdar 'greenish-blue, pale blue, green-grey, and greyish brown' (Badawi \& Hinds

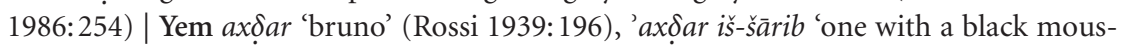

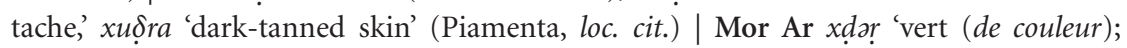
verdoyant (sol, jardin); [rur.] brun très foncé, à robe brune, bai brun (chameau, âne, mulet)' (de Premare et al. IV, 93) | Chad axadar 'vert, brun foncé (teint de peau); frais, cru, immature' (Pommerol 1999:208) | Sudan axadar zara'i 'grün', axadar labani 'hellblau,' axadar 'ein dunkles Braun (Hautton)' (Reichmuth 1981:56) | 'Anēzih Bed axdar 'white (horse)' (Burckhart 1830:121) | Andalusi Ar ‘axdar〉 'green; reddish-black (horse)'. (Corriente 1997:159)

32. Cf. Syriac yōrūqō 'pale, ashy, livid' cognate with yarōqō 'green, yellow' (J. Payne Smith 1903: 197).

33. The GRUE category also appears to be pan-dialectal trait in Berber, suggesting continuity with proto-Berber (cf. Basset 1896 passim). 
As can be inferred from the aforecited dialectal data, dark hues encoded by the lexeme axdar in contemporary Arabic dialects refer mainly to the colors of the human skin and of animal pelts. Bender (1983) has convincingly suggested that, in the history of color in Arabic, the GREEN category emerged from an earlier term for 'macro-black'; the sources cited in E. W. Lane's definition of OA xudra 'greenness' bear out this view:

colour between black and white; an ashy or dark dust colour; a tawny, brownish colour; blackish hue inclining to green, intense blackness, etc. (p. 755)

The range of meanings conveyed by GREEN in the dialects cited above renders this Arabic color category a distinctly exotic one for a modern observer and concretizes Fischer's statement about Old Arabic:

Die scheinbare Mehrdeutigkeit aller Farbbezeichnungen des dunklen Bereichs macht bei der Interpretation einzelner Kontextstellen erhebliche Schwierigkeiten ... (1965:305)

Old Arabic GREEN also conveyed the notions 'soft, or tender; fresh or juicy' (E.W. Lane, loc. cit.). De Haas's (1954) study of the lexical coding of the semantic spectrum of moisture in Classical Arabic here oddly overlooked the relevance of the green category and the plausible semantic interface between the phonologically close forms OA $x d r$ 'green' and $x d l$ 'be moist, wet.' OA axdar, meaning 'fresh' rather than 'green,' is exemplified in the twelfth Sura of the Qur'ān (45-46), where the seven green ears of corn are contrasted with the seven wilted ones and the color term xudr 'green (pl.)' is opposed to yābisāt 'dry (f. pl.)' which is not a color term. ${ }^{34}$

The semantic association of greenness with moisture and succulence is highly developed in modern Arabic vernaculars spoken both by Bedouin and by certain traditionally sedentary populations:

Cai 'axdar 'fresh, not salted or cured (of fish); damp, moist': il-hidūm lissa xadra 'the clothes are still damp', ǧild 'axdar 'half-tanned leather' (Badawi \& Hinds 1986:254) | Alep ğabne xadra 'fromage frais' (Barthélemy 1935-54:207) | Palest Ar 'axdar rabī' 'junges Frühlings' (Bauer 1957: 143), damm 'axdar [lit., green blood; A.B.] 'frisches Blut' (Fischer 1965:306, fn. 3) | Tang $x$ dar "vert; pas cuit, en parlant du pain, des legumes, de la viande; ainsi à Tlemcen, Nedroma, Alger; ... à Constantine et chez la plupart des ruraux, on emploie ce mot en parlant de la viande ... mais non en parlant du pain" (Marçais 1911:281) | Dathīna $x d r$ 'être vert et ensuite avoir de l'eau (terrain)'; axdar 'mouillé, humide': $\theta a w b \bar{\imath}$ axdar 'mon vêtement est mouillé' (Landberg 1920:606) | Upper Egyp tū axdar 'ungebrannte Lehmziegel' (as opposed to tūu 'ahamar 'gebrannte Lehmziegel' (Behnstedt \& Woidich 1994:292) 35 $^{35}$ Yem 'ax $\widehat{\delta} a r$ 'damp, moist, humid, wet' (Piamenta 1990:130) | Gulf Ar: fi šay 'axdar fi šay yābis 'there's wet and there's dry (manure)' (Holes 2001:152) | Iraqi

34. Cf. Harold Conklin's celebrated (1955) study of color categories in Hanunóo, a language of the Philippines, highlighting the salience in this language of nonchromatic meanings of green conveying notions of freshness and succulence of plant material.

35. In the Arabic dialect of Upper Egypt, the 'greenness' metaphor extends to young offspring of certain fowl wizz 'axdar 'Gänsejunges', batt 'axdar 'Küken (Ente)' (loc. cit., 292). 
Ar axadar 'frisch (Straußenmist)' (Weißbach 1930: 129) | Syr Ar xadī ' 'fresh dung of cows'. (Hava 1982:173)

The same meaning pattern occurs in the dialect of the Negev Bedouin as in the following gloss of 'ax $\oint a r$ by a native speaker:

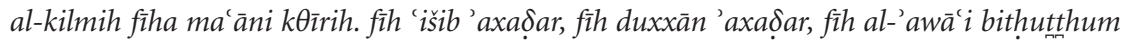
fil-mā u tg்assilhum, fìh 'indak kbāš u widdak tnazzilhum aṣ-șūf, widdak tg்assilhum l-ikbāšal-ikbāš xuß̣ur.

The word [green] has several meanings. Grass is green, smoke is green, and clothes [too], when you immerse them in water to wash them. [Or] you may have sheep to be sheared and you might want to bathe them - the sheep are green (i.e., when wet).

Though often considered a specially exotic trait since Conklin's aforementioned paper, this semantic pattern finds analogous parallels in a number of European languages:

grüne Häute 'undressed skins' | grüne Hering 'fresh herring' | green meat 'not cured, unprocessed' | green cheese 'unreife Käse' (Betteridge 1978: 1083, 280) | green mortar (freshly set but not completely hardened) | green bricks or pottery (not fired) (RHD, 620).

(iv) Concrete notions of yellow ('asfar) in Old Arabic drew mainly on natural hues (of sand; horse and camel coats; ivory; early morning skies; singed hair; butter, etc.), and for specific shades of natural yellow, the language had specific hyponyms (cf. Fischer 1965:365f.). The Arabic dialects continue this trait:

Upper Egyp 'ardı șafra (lit. yellow earth) 'sandiger Boden' (Behnstedt \& Woidich 1994:4) Mor Ar şfār 'devenir jaune; devenir blond / pale/livide,' șār 'jaune d'œuf', ş̧frāwi 'de teint jaune; bilieux, de temperament bilieux; coléreux, irascible, nerveux,' sfầri 'isabelle clair (robe de cheval),' sfōrēyt aš-šams 'moment qui précède de peu le coucher du soleil,' şaffêr 'jaunisse, ictère' (de Premare et al., VIII, 72-73) | Leb ṣafrat 'avoir le visage jaune, jaunir (céréales),' bu șuffayr 'orange sauvage' (Denizeau 1960:308) | Palest Ar șufur 'Geld' (Schmidt/Kahle I, 70, 80) | Iraqi Ar işfarr woğha ușār mïl in-nūmāya '... wurde sein Gesicht gelb wie eine Zitrone' (Weißbach 1930:74) | 'Anēzih Bed asfar 'dark grey (horse)' (Burckhart 1830:121) | E. Sudan asfar 'light pinkish, fawn' (Acland 1932:148) | Yem 〈așfar〉 'fair complexion, light brown,' șaffar 'become pale, yellowish', sifr 'brass'. (Piamenta II, 283)

Yellow exemplifies a peculiar oddity in the designation of camel colors in Old Arabic and in contemporary Bedouin Arabic; in both varieties of the language, 'asfar is rendered yellow or black "because a black camel always had an intermixture of yellow, with some yellow hairs coming through" (E. W. Lane 1699). This color-naming trait typifies a tendency among Arabic speakers to focus on a striking point of detail.

The Old Arabic term 'asfar also conveyed the quality of reflectance, for instance, in gold (al-ṣafrä' 'yellow f.') as opposed to silver (al-bayḍa ' 'white f.'). Maltese, while adopting focal yellow (isfar), here retains intimations of a brightness value in the expression isfar lellux 'golden yellow' deriving from 〈lellux〉 'marigold'; note, in particular, the cognate verb 〈lellex〉 'shine, glitter' ( $<$ Berber). 
In his survey of ethnographic and linguistic materials relating to North African Arabic, Breteau (1999:68) commented on the relatively rare references to yellow in the sources examined and its generally negative associations. In their study of Russian color terms, Corbett and Morgan (1988:44) also observed a low incidence for this color paralleled by closely comparable statistics for some other languages specified in Hays et al. (1972:1112): English, Castilian Spanish, French, German, Rumanian, Japanese, and Hebrew.

(v) The most common terms for BLUE in the Arabic dialects are cognates of Old Arabic 'azraq designating usually dark blue:

Palest Ar 'azraq 'blau' (Bauer 1957:62) | Dam 'azraq 'blue' (Stowasser \& Ani 1964:25) | Cai 'azra' 'blue; grey (horse), dove grey', 'azra' șini 'china blue' (Badawi \& Hinds 369) | Upper Egyp 'ardı zar'a 'dunkle Erde' (Behnstedt \& Woidich 1994:4) ${ }^{36}$ | Leb zaytūn 'azraq 'olives pas mûres, encore vertes' (Feghali 1935: 155, 1) | Dathīna 'azraq 'bleu, mais ce thème implique aussi l’idée de gris ou de noir; Grauschimmel'. (Landberg 1942:1836)

Fischer (1965:48) has shown that this term did not originally allude to chroma but encoded the glittering or gleaming of a sharp point, star, etc., in other words, a combination of brightness and movement:

Die ermittelte Bedeutung 'blinkend, glitzernd, schillernd' hat sich als primäre Benennung an allen altarabischen Belegstellen bestätigt. Es kann noch das Bezeichnungsmerkmal hinzugefügt werden, daß nur von solchen Dingen gesagt wird, an denen das Glitzern und Schillern punktartig, wie bei Sternen, Lanzenspitzen und Augen. (loc. cit., 54)

Closely related meanings associated with brightness rather than hue have been noted for the Jewish Yemeni Arabic cognates zāriq 'sparks (of fire),' zärigah 'sunray(s) ... radiating through a window' (Piamenta I, 199); also noteworthy, in this regard, are the glosses 'multicolour, shining' for 'azraq (loc. cit.) and the Lebanese verbal derivation zarqat 'prendre des couleurs' (Denizeau 1960:218) which does not refer to a specific color.

The aforementioned reference to the sensation of a flashing movement associated with 'sparkling, glimmering' in the meaning of Old Arabic azraq is also highly suggestive since numerous dialectal Arabic terms with the root $z$ rq convey closely relevant semantic patterns referring to movement:

Yem zaraq 'to throw' (Piamenta 1990:199) | Dam zārūqa 'pompe, seringue' (Denizeau 1960:218) | Palest Ar zaraq 'se glisser ('ala)' (Schmidt/Kahle II: 76, 6) | Leb zaraq 'jaillir avec force d'un orifice,' zarzaq 'tomber de haut ou descendre dans une conduite depuis un endroit en faisant du bruit (eau),' zarrāqa 'roseau creux muni d'un piston; on le remplit d'eau et, en appuyant sur le piston, on asperge (jeu d'enfant)' (Denizeau 1960:218) | Malt zerzaq 'slide down' | E. Arabia zarrag/k 'cause to slide' (Holes 2001:221) | Dathīna zarraq 'throw' (Landberg 1942:1835) | Cai zarra' 'slip, pass s'thing covertly' (Badawi \& Hinds 1986:369) | Iraqi Ar zirag 'to dash, hurry, go quickly' (Woodhead/ Beene 1967:203) ||

36. This recalls ancient Egyptian $k m(t)$ "le pays noir" (la brune foncée, la contrée noire comme un tas de charbon) ... désignation de l'Egypte en tant que terre cultivable, par opposition à dehrit : les régions rouges ou fauves' qui était le nom des deserts sablonneux encadrant à l'est et à l'ouest le pays noir..." (Gauthier 1925-31); cf. Hobbs (1989 passim). 
Class Ar mizrāq 'short spear, javelin' (Hava 1982:288), etc. || Aram zraq 'to throw, sprinkle' (Sokoloff 1990: 182) || Syriac zraq 'to scatter, sprinkle, disperse' (J. Payne Smith 1903:121) || Assyr zarāqu 'streuen, sprengen' (Gesenius \& Buhl 1915:208).

Fischer (loc. cit.) insightfully pointed out the closely parallel semantic development of Ancient Greek $\gamma \lambda \alpha \cup \kappa \varsigma^{\prime}$ "originally without any notion of colour, gleaming; later, of color, blueish green or grey, of the olive, ... elder, grapes, vine leaves, frequently of the eye, light blue, grey, etc." (Liddell \& Scott 350-1). ${ }^{37}$

True to type, from the standpoint of B\&K's theoretical model, BLUE as a color term appears to have been a late development in the Arabic color system. This is a remarkable fact when it is recalled that the use of blue dyes extracted from indigo has had a continuous history going back, at the very least, to the second millennium B.C. ${ }^{38}$ (cf. Balfour-Paul 1997 passim) and that the color blue has always enjoyed a potent symbolic objectification in the Arab world. ${ }^{39}$

A salient cultural and dialectal boundary here occurs with Maltese, which has objectified light blue (čelest $i<$ It celeste) alongside blu $<$ Eng blue / It blu, yielding a twelve-term color system.

\section{Synopsis and conclusion}

The Arabic dialect family presents the diachronist with a rich stock of comparative data rendering possible the reconstruction of empirically based evolutionary hypotheses relating to semantic fields in the modern vernaculars. The developmental pathway of the Arabic color system etched in the preceding sections corresponds roughly to a time span vis-à-vis the native Arabic data, at least - of about a millennium and a half. The residual Aramaizing nomenclature examined in $\$ 4$ projects further back in time to the first

37. The putative influence of Greek on the Old Arabic color system conceivably through the mediation of Western Aramaic cannot be ruled out with regard to the emergence of the blue category lexified by Ar. 'azraq when it is recalled that "at the beginning of the eighth century, ... liberal education at Constantinople collapsed as a result of the instability of imperial rule ... Paradoxically, the tradition of Greek letters was meanwhile kept alive in Arab-dominated Syria and Palestine" (Mango 2002:214).

38. Tyrian purple (Jensen 1965) was lexified in various Semitic languages (for details, see Powels 1999).

39. The same source (pp. 156-57) notes: "Blue in Islam was often considered beautiful, mysterious and lucky, but equally it was sometimes regarded as so inauspicious that a person would even say 'green' when he actually meant blue, in order to avoid saying such an ill-omened word. Blue eyes like those of Christians, are considered sinister by a Muslim. Goitein encapsulated these attitudinal contradictions when he described the colour blue as being for the Arabs of the Middle Ages, 'a kind of homeopathic repellent'; in other words, as blue, especially its lighter shades, is a pleasing colour, wearing it might have attracted the 'evil eye'; children and pregnant wives therefore who wore blue clothes need protection afforded by blue beads and other amulets whose function was to deflect the 'eye.' ... Blue paper was used for warrants of execution in those countries where indigo had associations with death and mourning, notably Egypt and Syria." Cf. also Shinar (1999 passim). 
millennium B.C. ${ }^{40}$ Given the as yet relatively undeveloped state of the art in the study of color categorization in Semitic, extrapolations relating to this considerable time span can at this stage be only tentative. ${ }^{41}$ The present essay has suggested that the emergence of fullfledged basic color paradigms in the urban Arabic colloquials along the lines postulated by Berlin and Kay represents primarily the outcome of ecological factors concomitant with the transfer of spoken Arabic from a nomadic, tribal context to a sedentary urban one. Thorbecke (1871:5) stated:

... mit dem Heraustreten aus Arabien liess das völlig veränderte Leben den grossen Theil des Wortschatzes, der sich auf die Zustände in der Wüste bezog, ausser Gebrauch kommen oder wenigstens in seiner strengen Bedeutung vergessen, und gerade hier hatte der so scharf die Erscheinungsformen der belebten und unbelebten Natur bis ins Einzelste beobachtende Sinn des Beduinen einen bewunderswerthen und charakteristischen Reichtum geschaffen.

Recent research has provided a more nuanced picture of the sociohistorical background pertaining to the early cultural history of Arabic:

The conception that until Muslim Arab expansion the Arabs lived mainly in the Peninsula and the term was confined to camel nomads must be discarded. In fact the Arabs inhabited all the regions lying to the north of the Peninsula proper, Syria and Palestine, Mesopotamia and Babylonia, even parts of western Persia, intermingled with the Aramaic-speaking peoples of these regions.... Even the linguistic definition is inadequate: not only were there great differences in the spoken Arabic of the different groups, as the inscriptions they left behind reveal, but the settled and semi-settled Arabs in Syria and Mesopotamia spoke Aramaic, the universal Semitic language of the age.

(Trimingham 1979:1)

Thorbecke's viewpoint invites comparative research into nomadic and sedentary color systems on the lines of the preceding remarks. The available field data on Bedouin color systems (Hess 1920; Roth 1983; Borg 1999) bear out the view that these paradigms do indeed retain typologically authentic Old Arabic traits (e.g., a small set of basic categories alongside a fairly extensive inventory of nonbasic terms encoding mostly nonsaturated natural hues, brightness oriented foci, etc.).

Trimingham postulates a more complex scenario emphasizing continuous interaction with other Near Eastern languages of wider communication, e.g., Aramaic. The data to hand intimates, for instance, that contact of Bedouin vernaculars with Aramaic transpired also in Arabia itself. Interestingly, Socin (1901) records for the 'Utaybah Bedouin the nonbasic color term for 'yellow' ša'wā' 'goldfarbig (von Kamel)' < Syriac ša' $\bar{u} \theta \bar{a}$ 'wax; flavus'

40. The earliest written attestations in Aramaic hark back to the 9th century B.C. (Lipinsky 1997:61).

41. Given the salience attained by the domain of color in the research paradigm of linguistic anthropology (Grossmann 1988:320-366), and the fact that Semitic - documented from the third millennium B.C. - has "the longest history of any recorded language" (Zohar 1992: 165), a historical and comparative assessment of the color lexicon current in the textual materials available in Akkadian, Aramaic, Hebrew, Arabic, etc. in the light of color systems in modern offshoots of Semitic would seem to represent a vital contribution to the historical typology of color. 
(J. Payne Smith 588; Brockelmann 1928:792). ${ }^{42}$ A globalizing trend induced by hellenistic Greek may possibly be responsible for the parallel evolutionary paths towards a blue category selected by Old Arabic azraq and Gk $\gamma \lambda$ גuкóc as portrayed in Fischer (1965:50f.). As already noted, at a later stage, Persian and Turkish color terms infiltrated several modern vernaculars of Eastern Arabic: banafsaği 'violet,' lēlaki 'lilac, purple' < Pers banafša, nīlak 'bluish' (Steingass 1892), bamba 'pink' < Tk penbe, etc.

Exactly how the cognition of color categorization interrelates with societal factors must remain a moot point at this juncture. The Negev Bedouin, for instance, have probably been drinking coffee out of blue-on-white ceramic cups for over three hundred years; the term sīniy 'dark blue' in their vernacular has nonetheless remained a non-basic term and the BLUE category in Negev Arabic derives from a lineally inherited Old Arabic term for 'grey': ašhab. Robert MacLaury neatly summed up the problem in the adage that languages acquire new categories when they are ready for them (p.c.).

The historical approach to color paradigms has lately come under some criticism:

First, because, if it were correct, this would be one of the few instances in which linguistic development proceeds unidirectionally from simplicity to complexity. Second, because it readily suggested to some the now-taboo late-nineteenth-century picture of an evolutionary culture chain, with Papua New Guineans at the bottom ... and sophisticated situated Europeans comfortably and properly at the top.

(Hardin \& Maffi 1997:5)

This is surely a retrogressive viewpoint since the disciplinary implications of comparative study on color systems, for instance, in a Near Eastern context, transcend the limits of color research per se and throw light on the history of ideas in a Near Eastern cultural milieu. Whereas the first reservation articulated here must remain vacuous without at least an attempt at a working definition of systemic 'complexity' vs. 'simplicity', the second impresses the present author as a blatant concession to political correctness since experience in the field has repeatedly shown that color systems with few basic terms are not of necessity cognitively simple if taken in their totality. Thus scholars studying 'primitive' classifications of natural colors among Naturvölker, such as pastoralists, are not infrequently baffled by the sophisticated skills required to use contextualized terms correctly (Turton 1980:326; Ingham 1990:74; Borg 1999:138). Very apposite, in this connection, is an assessment by the Swedish linguist Bertil Malmberg of Wilhelm von Humboldt's views on linguistic evolution - however one visualizes this process of change (see Dedrick 1998: 80f.):

Les différences entre les langues ... n'impliquent pour lui aucune base de classement de leur niveau de civilisation.

(Malmberg 1991)

Humboldt's attitude is no doubt implicitly shared by all serious cultural anthropologists.

42. This color term is, however, basic in Syriac where its integration marked the split of the older category yarūq $\bar{a}$ 'green, yellow' (Audo 1896:440-1); cf. yarōqā 'egg yolk' (Manna 1975:317). 


\section{References}

Abu-Lughod, Lila (1986). Veiled Sentiments. Berkeley/Los Angeles: University of California Press.

Acland, P.B.E. (1932). Notes on the camel in Eastern Sudan. In Sudan Notes and Records XV: 119-149.

Al-Amin, Ahmad (1953). Qāmūs al-'ādāt wa al-taqālīd wa al-ta'āà̄r al-mișriyyah. Cairo: Maṭa' at lajnat al-ta'lif wa al-tarjama wa al-našr.

Audo, Thomas (1897). Dictionnaire de la langue chaldéenne. Mosul [repr. 1985]: Glane (Holland).

Badawi, El-Said \& Hinds, Martin (1986). A Dictionary of Egyptian Arabic, Arabic-English. Beirut: Librairie du Liban.

Baines, John (1985). Color terminology and color classification: Egyptian color terminology and polychromy. American Anthropology 87: 282-97.

Balfour-Paul, Jenny (1997). Indigo in the Arab World. Richmond: Curzon.

Barthélemy, Adrien (1935-1954). Dictionnaire arabe-français (dialectes de Syrie, Alep, Damas, Liban, Jerusalem). Paris: Paul Geuthner.

Basset, André (1896). Les noms de métaux et de couleur en berbère. Mémoires de la Société de linguistique de Paris, 9: 58-92.

Bauer, Leonhard (1933). Deutsch-arabisches Wörterbuch der Umgangssprache in Palästina und im Libanon, [zweite Auflage unter Mitwirkung von Anton Spitaler, 1957]. Wiesbaden: Harrassowitz.

Behnstedt, Peter \& Manfred Woidich (1987). Die Dialekte der Gegend von Șa'dah (Nord-Jemen). Wiesbaden: Harrassowitz.

Behnstedt, Peter (1993). Glossar der jemenitischen Dialektwörter in Eduard Glasers Tagebüchern (II, III, VI, VIII, X). Wien: Verlag der Österreichischen Akademie der Wissensschaften.

Behnstedt, Peter \& Manfred Woidich (1994). Die ägyptisch-arabischen Dialekte, vols 3 and 4. Glossar: Arabisch-Deutsch. Wiesbaden: Dr. Ludwig Reichert.

Bender, M. L. (1983). Color term encoding in a special lexical domain: Sudanese Arabic skin colors. Anthropological Linguistics 25:19-27.

Berlin, Brent \& Kay, Paul (1969). Basic Color Terms: Their Universality and Evolution. Berkeley: University of California Press.

Blau, Joyce (1965). Kurdish-English-French Dictionary. Brussels: Centre pour l'étude des problèmes du monde musulman contemporain.

Borg, Alexander (1985). Cypriot Arabic. Stuttgart: Franz Steiner.

Borg, Alexander (1999). The color categories of the Negev Bedouin. In Borg, A. (ed.), The Language of Color in the Mediterranean (pp. 121-147). Stockholm: Almkvist/Wiksell.

Borg, Alexander (2004). A Comparative Glossary of Cypriot Maronite Arabic (Arabic-English). Leiden: Brill. Bornstein. Marc H. (1975). The influence of visual perception on culture. American Anthropologist 77: 774-798.

Brenner, Athalya (1982). Color Terms in the Old Testament. Sheffield: Academic Press.

Breteau, Claude H. (1999). Note sur așfar. Dictions du Prisme: Littérature Orale Arabo-Berbère 16-17: $67-77$.

Brockelmann, Carl (1928). Lexicon Syriacum, editio secunda aucta et emendata. Halle: Max Niemeyer.

Brockett, A. A. (1985). The Spoken Arabic of Khābūra on the Bātina of Oman. Manchester: University Press.

Bryant, Alfred T. (1949). The Zulu People: As They Were before the White Man Came. Pietermaritzburg: Shuter and Shooter.

Canaan, T. (1916). Die Wintersaat in Palästina. Zeitschrift der Deutschen Morgenländischen Gesellschaft 70: $164-178$.

Cheesman, Robert Ernest (1926). In Unknown Arabia. London: Macmillan.

Conklin, Harold. C. (1955). Hanunóo color categories. Southwestern Journal of Anthropology 11: 339-44. 
Conklin, Harold. C. (1973). Color categorization: review of Berlin B. and Kay, P. (1969). American Anthropologist 75: 931-942.

Cootner, Cathryn M. (1990). Anatolian Kilims. London: Philip Wilson for Sotheby's Publications.

Corbett, Greville \& Gerry Morgan (1988). Colour terms in Russian: reflections of typological constraints in a single language. Journal of Linguistics 24: 31-64.

Corriente, Federico (1997). A Dictionary of Andalusi Arabic. Leiden: Brill.

Cowell, Mark W. (1964). A Reference Grammar of Syrian Arabic. Washington, D.C.: Georgetown University.

Dalman, Gustaf (1964 [1928-1939]). Arbeit und Sitte in Palästina, 8 vols. Hildesheim: Olms.

Deacon, Terence, W. (1997). The Symbolic Species. The Co-evolution of Language and the Brain. New York: W.W. Norton.

Dedrick, Don (1998). Naming the Rainbow. Dordrecht: Kluwer Academic Publishers.

De Haas, W. P. (1954). The Spectrum of Moisture in Arabic. 'S-Gravenhage: H.H.L.Smits.

Denizeau, Claude (1960). Dictionnaire des parlers arabes de Syrie, Liban, et Palestine. Paris: G.-P. Maisonneuve.

Doughty, Charles M. (1888). Travels in Arabia Deserta. London: Jonathan Cape.

Dozy, Reinhart P. A. (1881). Supplément aux Dictionnaires Arabes, (2 vols.). Leiden, [reprint., 1968, Beirut: Librairie du Liban].

Drower, Ethel Stefana \& Macuch, Rudolf (1963). A Mandaic Dictionary. Oxford: OUP.

Ducatez, Guy \& Jacky Ducatez (1980). Formation des dénominations de couleur et de luminosité en arabe classique et pre-classique: Essai de périodisation selon une approche linguistique et anthropologique. Peuples Méditerranéens 10: 139-172.

Durkheim, Emile (1965). The Elementary Forms of the Religious Life, trans. from French by J.W. Swain. New York. Free Press [= Formes élémentaires de la vie religieuse: le système totémique en Australie (1925). Paris: Alcan.]

Dussaud, René (1927). Topographie historique de la Syrie antique et mediévale. Paris: Paul Geuthner.

Feghali, Michel (1935). Contes, légendes, coutumes populaires du Liban et de Syrie. Paris: Librairie d'Amerique et d'Orient.

Fischer, Wolfdietrich (1965). Farb- und Formbezeichnungen in der Sprache der altarabischen Dichtung. Wiesbaden: Harrassowitz.

Gauthier, Henri (1925-1931). Dictionnaire des noms géographiques contenus dans les textes hieroglyphiques, 7 vols. [reprint., 1975]. Osnabrück: Otto Zeller Verlag.

Gesenius, Wilhelm \& Frants Buhl (1915). Hebräisches und Aramäisches Handwörterbuch über das Alte Testament; unveränderter Neudruck [1954] der 17. Auflage. Berlin: Springer-Verlag.

Goitein, S. D. (1967). A Mediterranean Society, vol. IV. Berkeley: University of California.

Goldziher, Ignaz (1889). Muhammedanische Studien, = Muslim Studies (ed., S.M. Stern, 1967. Albany: State University of New York Press.

Gradwohl, Roland (1963). Die Farben im Alten Testament. Eine terminologische Studie. Berlin: Alfred Töpelmann.

Grossmann, Maria (1988). Colori e Lessico. Tübingen: Gunter Narr.

Hardin, C. L. \& Luisa Maffi (1997). Introduction. In Hardin \& Maffi (ed.), Color Categories in Thought and Language. Cambridge: CUP.

Hartmann, Martin (1899). Lieder der libyschen Wüste. Leipzig: F.A. Brockhaus.

Hava, J. G. (1982). Alfaraid Arabic-English Dictionary, 5th ed. Beirut: Dar al-Mashriq.

Hays, D. G., E. Margolis, R. Naroll \& D. R. Perkins (1972). Color term salience. American Anthropologist 74: 1107-1121.

Hess, Johann Jacob (1920). Die Farbbezeichnungen bei innerarabischen Beduinenstämmen. Der Islam 10: $4-86$. 
Hobbs, John Joseph (1989). Bedouin Life in the Egyptian Wilderness. Austin: University of Texas Press. Hoftijzer, J. \& K. Jongeling (1995). Dictionary of the North-West Semitic Inscriptions, 2 vols. Brill: Leiden. Holes, Clive (2001). Dialect, Culture, and Society in Eastern Arabia. Leiden: Brill.

Ingham, Bruce (1990). Camel terminology among the Āl Murrah bedouins. Zeitschrift für arabische Linguistik 22: 67-78.

Jastrow, Marcus (1903). A Dictionary of the Targumim, the Talmud Babli and Yerushalmi, and the Midrashic Literature, 2 vols [reprint.] Jerusalem.

Jastrow, Otto (1981). Die mesopotamisch-arabischen Qaltu-Dialekte, 2. Band. Wiesbaden: Harrassowitz. Jastrow, Otto (1990). Lehrbuch der Turoyo-Sprache. Wiesbaden: Harrassowitz. Jaussen, Antonin (1908). Coutumes des arabes au pays de Moab. [repr. 1948] Paris: J. Gabalda.

Al-Jehani, Nasir M. (1990). Color terms in Mecca: A sociolinguistic perspective. Anthropological Linguistics 32: $163-174$.

Jensen, L. B. (1965). Royal purple of Tyre. Journal of Near Eastern Studies XXII: 104-118. Jiha, Michel (1964). Der arabische Dialekt von Bišmizzīn. Beirut: Franz Steiner Verlag.

Kalfon Stillman, Yedida (ed. Norman Stillman) (2000). Arab Dress from the Dawn of Islam to Modern Times: A Short Study. Leiden: Brill.

Karr, Alphonse Jean-Baptiste (1851). Voyage autour de mon jardin. Paris: Calmann Levy.

Klincksieck, Paul \& Th. Valette (1908). Code des couleurs à l'usage des naturalistes, commerçants et industriels. Paris.

de Landberg, Carlo, Comte (1920). Glossaire Dathīnois. Vol. 3, publié par K.V. Zetterstéen. Leiden: Brill. Lane, Arthur (1957). Later Islamic Pottery (Persia, Syria, Egypt, Turkey). London: Faber/Faber.

Lane, Edward William (1863-1893). An Arabic-English Lexicon. London: Williams/Norgate.

Lawrence, Thomas Edward (1935 [1926]). Seven Pillars of Wisdom. New York: Jonathan Cape.

Levi-Strauss, Claude (1964). Structural analysis in linguistics and in anthropology. In Hymes, Dell, Language in Culture and Society. New York: Harper/ Row: 40-53.

Lewis, Bernard (1995). The Middle East. London: Weidenfeld/Nicholson.

Lindisfarne-Tapper, Nancy \& Bruce Ingham (1997). Languages of Dress in the Middle East. Curzon: SOAS. Lipinsky, Edward (1997). Semitic Languages: Outline of a Semitic Grammar. Leuven: Peters.

Loubignac, Victorien (1952). Textes arabes de Zaër (transcription, traduction, notes et lexique). Paris: Librairie orientaliste et Americaine.

Lucy, John A. (1997). The linguistics of 'color.' In Hardin \& Maffi (ed.), Color Categories in Thought and Language (pp. 321-346). Cambridge: CUP.

MacLaury, Robert E. (1992). From brightness to hue: An explanatory model of color-category evolution. Current Anthropology 33: 137-186.

MacLaury, Robert E. (1997). Color and Cognition in Mesoamerica. Austin: University of Texas Press.

Magazis, George A. (1995). Word Master: English - Greek, Greek - English Dictionary. Efstathiadis Group.

Malmberg, Bertil (1991). Histoire de la linguistique de Sumer à Saussure. Paris: Presses Universitaires de France.

McNeill, Noriko B. (1972). Colour and colour technology. Journal of Linguistics 8: 21-32.

Mango, Cyril (2002). The revival of learning. In Mango, C. (ed.). In The Oxford History of Byzantium (pp. 214-229). Oxford: OUP.

Manna, Jacques Eugéne (1975 [1900]). Vocabulaire chaldéen-arabe, 2nd ed. Beirut: Babel Center Publications.

Marçais, William (1906). L'euphémisme et l'antiphrase dans les dialectes d'Algerie. In Orientalische Studien Theodor Nöldeke zum siebzigsten Geburtstag (2. März 1906) gewidmet von Freunden und Schülern (C. Bezold ed.), vol. I (pp. 425-438). Leipzig: Alfred Töpelmann.

Marçais, William (1910). Textes arabes de Tanger. Paris: Imprimerie nationale. 
Marçais, William (1955). Nouvelles observations sur l'euphemisme dans les parlers arabes maghrébins. In Mélanges Isidore Lévy. Bruxelles: Ed. de l'Institut: 331-91.

Margoliouth, D. S. (1914). The Early Development of Mohammedanism. London: Williams/ Norgate.

Mez, Adam (1909). The Renaissance of Islam, [trans. 1937] London: Luzac and Co.

Mignot, Xavier (1969). Les verbes dénominatifs Latin. Paris: C. Klincksieck.

Monteil, Charles (1927). Le coton chez les Noirs. Paris: Larose.

Monteil, Vincent (1960). L'Arabe moderne. Paris: C. Klincksieck.

Morabia, Alberto (1986). Lawn. In Encyclopedia of Islam V: 698-707.

Musil, Alois (1907-1908). Arabia Petraea, 4 vols. Wien: [reprint. 1989] Hildesheim: G. Olms.

Musil, Alois (1926). The Northern Ḥeḡāz: A Topographical Itinerary. New York: Geographical Society of New York.

Musil, Alois (1928). The Manners and Customs of the Rwala Bedouins. New York: American Geographical Society.

Nöldeke, Theodor (1910). Neue Beiträge zur semitischen Sprachwissenschaft. Strassburg: K.J. Trübner.

del Olmo Lete, Gregorio \& Joaquin Sanmartín (2003). A Dictionary of the Ugaritic Language in the Alphabetic Tradition, 2 vols. (trans. by Wilfred G.E. Watson). Leiden: Brill.

von Oppenheim, Max Freiherr (1899). Vom Mittelmeer zum persichen Golf (erster Band). Berlin: Dietrich Reimer.

Payne Smith, J. (1903). A Compendious Syriac Dictionary founded upon the Thesaurus Syriacus of R. Payne Smith, D.D. Oxford: Clarendon Press.

Payne Smith, R. (1897). Thesaurus Syriacus. Oxford: Clarendon Press.

Penny, Lee (1984). The Whorf Theory Complex: A Critical Reconstruction. Amsterdam: John Benjamins. Philby, Harry St.John Bridger (1933). Harun al Rashid. London. P. Davies.

Pierret, R. (1948). Etude du dialecte maure des régions sahariennes et sahéliennes de l'Afrique occidentale française. Paris: Imprimerie Nationale.

Pommerol, Patrice Julien (1999). Dictionnaire arabe tchadien-français. Editions Karthala. Paris.

Powels, Sylvia (1999). The historical and cultural background of two non-basic terms for 'blue' in the Arabic dialect of the Negev Bedouin. In Borg (pp. 152-166).

de Premare et alii. (1993-1999). Dictionnaire Arabe-Français, 12 vols. Paris: L’Harmattan.

Reichmuth, Stefan (1981). Die Farbbezeichnungen in sudanesisch-arabischen Dialekten. Zeitschrift fur arabische Linguistik, Heft 6: 57-66.

RHD - Random House Dictionary. New York.

Rivers, W. H. R. (1901). The colour vision of the natives of Upper Egypt. Journal Asiatique: 229-247.

Rohlfs, Gerhard (1971). Romanische Sprachgeographie. Munich: Beck.

Ross, Heather Colyer (1981). The Art of Arabian Costume; a Saudi Arabian Profile. Fribourg: Arabesque.

Rossi, Ettore (1939). L'arabo parlato a Șan'a $\bar{a}$. Roma: Istituto per l'Oriente.

Roth, Arlette (1986). Introduction à l'étude des systèmes de désignation de la couleur dans les parlers arabes du Maghreb. Littérature Orale Arabo-Berbère 16-17: 21-65.

Schmidt, Hans \& Paul Kahle (1918-1930). Volkserzählung aus Palästina, 2 vols. Forschungen zur Religion und Literatur des alten und neuen Testaments, 17 und 47. Göttingen: Vandenhoeck/Ruprecht.

Siggel, Alfred (1950). Arabisches-deutsches Wörterbuch der Stoffe. Berlin: Akademie-Verlag.

Shinar, Pessach (1999. Quelques observations sur le rôle de la couleur bleue dans le Maghreb traditionnel. In Borg, A. (ed.), The Language of Color in the Mediterranean (pp. 175-199). Stockholm: University of Stockholm.

Smith, A. H. (1956). English Place-Name Elements, (2 vols). Cambridge: Cambridge University Press.

Socin, Albert (1901). Diwan aus Centralarabien. Leipzig: B.G. Teubner.

von Soden, Wolfram (1965). Akkadisches Handwörterbuch unter Benutzung des lexikalischen Nachlasses von Bruno Meissner (1869-1947). Wiesbaden: Harrassowitz. 
Sokoloff, Michael (1990). A Dictionary of Jewish Palestinian Aramaic, [reprint. 1992]. Ramat Gan: Bar Ilan University Press.

Solayman, Abd el-Majyd \& H. Charles S.J. (1972). Le parler arabe de la voile et la vie maritime sur la côte Syro-Libanaise. Beyrouth.

Spiro, Socrates (1897). An English-Arabic Dictionary of the Modern and Colloquial Arabic of Egypt, [reprint., 1974] Beirut: Librairie du Liban.

Steingass, Francis Joseph (1892). A Comprehensive Persian-English Dictionary [2nd imp., 1930]. London: Crosby Lockwood.

Stowasser, Karl \& Moukhtar Ani (1964). A Dictionary of Syrian Arabic. Washington, D.C.: Georgetown University Press.

Swadesh, Morris (1964). Linguistics as an instrument of prehistory. In Hymes, Dell (ed.): Language in Culture and Society: A Reader in Linguistics and Anthropology. New York: Harper/ Row.

Tauzin, Aline (1986). Des couleurs et des voiles. Dictions du Prisme (Littérature Orale Arabo-Berbère 16-17 (1985-1986): 79-100.

Thilo, Martin \& Georg Kampffmeyer (1933). 5000 arabische Sprichwörter aus Palästina. Berlin: de Gruyter. Thorbecke, H. (ed.). (1871). Kitāb durrat al-ġawwāṣ fì awhām al-xawāṣṣ. Leipzig: F.C.Vogel.

Thomas, Bertram (1936). Arabia Felix: Across the Empty Quarter. London: Jonathan Cape.

Tilke, Max (1923). Orientalische Kostüme in Schnitt und Farbe. Berlin: Ernst Wasmuth.

Times Atlas of the World, vol. V (1957). London: The Americas.

Trimingham, Spencer (1979). Christianity Among the Arabs in Pre-Islamic Times. London: Longman.

Vahid, A. (1991 [1924]). An English-Turkish Dictionary. Beirut. Librairie du Liban.

Vocke, Sybille \& Wolfram Waldner (1982). Der Wortschatz des Anatolischen Arabisch. Erlangen.

Watson, Janet (1992). A lexicon of Cairene horse terminology. Journal of Semitic Studies XXXVII/2: 247303.

Wehr, Hans (1953). Der arabische Elativ. In Abhandlungen der Geistes- und sozialwissenschaftlichen Klasse: Jahrgang 1952 - NR.7. Akademie der Wissenschaften und der Literatur. Wiesbaden.

Wehr, Hans (1979). A Dictionary of Modern Written Arabic. J. Milton Cowan ed.). Wiesbaden: Harrassowitz.

Weir, Shelagh (1989). Palestinian Costume. London: British Museum.

Weißbach, F. H. (1930). Beiträge zur Kunde des Irak-Arabischen. Leipzig: J.C. Hinrichssche Buchhandlung. Whorf, Benjamin Lee (1941). The relation of habitual thought and behavior to language. In Spier, Leslie (ed.), Language, Culture, and Personality. Essays in Memory of Edward Sapir. Menasha Wisconsin: Sapir Memorial Fund.

Wierzbicka, Anna (1995). Dictionaries vs. encyclopaedias: How to draw a line. In Davis, Philip W. (ed.), Alternative Linguistics (pp. 289-315). Amsterdam: John Benjamins.

Wierzbicka, Anna (1997). Understanding Cultures Through their Words: English, Russian, Polish, German, and Japanese. Oxford: OUP.

Wierzbicka, Anna (2001. Lingua Mentalis: The conceptual system of the human mind. Humboldt Kosmos 78: 20-21.

Wild, Stefan (1973). Libanesische Ortsnamen: Typologie und Deutung. Beirut: Franz Steiner.

Wright, William (1896-1898). A Grammar of the Arabic Language, 3rd ed. Cambridge: CUP.

Zammit, Martin. R. (2002). A Comparative Lexical Study of Quranic Arabic. Leiden: Brill.

Zingarelli, Nicola (1970). Vocabolario della lingua italiana, 10a ed. Milano: Zanichelli.

Zohar, Mattanyah (1992). Pastoralism and the Spread of Semitic languages. In O. Bar-Yosef and A. Khazanov (eds.), Pastoralism in the Levant (pp. 165-180). Wisconsin: Prehistory Press. 



\title{
Japanese color terms, from $400 \mathrm{CE}$ to the present
}

\section{Literature, orthography, and language contact in light of current cognitive theory}

\author{
James M. Stanlaw \\ Department of Sociology and Anthropology, Illinois State University, USA
}

The aim of this chapter is to describe the history of Japanese color naming and relate it to current models in color nomenclature research. Though Japanese was among the twenty languages most carefully examined in the original Berlin and Kay (1969) investigations thirty years ago, the complexity and history of its color-term nomenclature system have yet to be fully explored. I will analyze evidence from the first classical texts that reveals how this vocabulary might have operated 1500 years ago, and present a possible evolutionary sequence of Japanese color terminology from circa $400 \mathrm{CE}$ to the present. I will examine this sequence in light of the Berlin and Kay standard model, and show how it fails to account for certain data. Other explanations, such as those offered by MacLaury (2001), give more robust accounts, and elucidate much in a very succinct fashion. Nonetheless, there appear to be aspects of the Japanese color sequence that defy formal modeling.

\section{Introduction}

The aim of this chapter is to describe the history of Japanese color naming and relate it to current models in color nomenclature research. Though Japanese was among the twenty languages most carefully examined in the original Berlin and Kay (1969) investigations thirty years ago, the complexity and history of its color-term nomenclature system have yet to be fully explored. I will analyze evidence from the first classical texts that reveals how this vocabulary might have operated 1500 years ago, and present a possible evolutionary sequence of Japanese color terminology from circa $400 \mathrm{CE}$ to the present. I will examine this sequence in light of the Berlin and Kay standard model, and show how it fails to account for certain data. Other explanations, such as those offered by MacLaury (2001), give more robust accounts, and elucidate much in a very succinct fashion. Nonetheless, there appear to be aspects of the Japanese color sequence that defy formal modeling.

Besides presenting further data for formal models to explore, the Japanese case offers special advantages. Some have challenged the universalist claims of current models of color nomenclature, arguing that the world's present-day color vocabulary is due to West- 
ern contact. Japan, however, intentionally isolated itself until the mid-nineteenth century, leaving a color vocabulary that developed with little outside influence. Also, in spite of periods of long isolation, Japan has also at certain times been quick to borrow foreign items (e.g. writing from China, English from the West). An examination of color vocabulary in such a venue is of theoretical interest.

I will begin by briefly discussing the texts used as sources of data, while making some general remarks about special problems of Japanese color nomenclature. ${ }^{1}$

\section{Early texts and data sources}

Most of what we know about early Japanese color nomenclature comes from written sources, though these were not always in pure Japanese. The Japanese did not have their own writing system until contact with the Asian mainland began when Chinese travelers, missionaries, and government representatives visited the islands of Kyushu and Honshu in the fifth and sixth centuries. Different Chinese teachers often spoke different Chinese dialects - which were, in fact, often mutually unintelligible languages - so Japanese people were learning a variety readings for a single ideograph. Thus, even today, most SinoJapanese characters have at least two pronunciations. The reading of a character based on one of the Chinese pronunciations is called an on-yomi reading. The reading of a character that is based on an indigenous Japanese pronunciation is called kun-yomi. Table 1 gives these readings, plus other information that we will return to later, for twenty Japanese colors. $^{2}$

The earliest Japanese texts that we know of were written in the first half of the eighth century. Of these, three are the largest and most important, and I have used each as a source for Japanese color data. The Kojiki (Kanda \& Oota 1962), literally the "Record of Ancient Matters," is Japan's oldest book and was written using pure Chinese prose for the

1. Some English discussions of Japanese color terms can be found in Haarmann (1989: 190-218), Hinds (1974), McNeill (1972), Stanlaw (1987, 1997a, 2003), Tanaka and Koike (1982), and Uchikawa and Boynton (1987). Though not examined at length in the text, Japanese discussions on color psychology and aesthetics include Chijiwara (1987), Hidaka (2003), Kawamoto (1978), Kobayashi (1974, 1981, 1984, 1990a, 1990b), Matsuoka (1999), Ooyama (1994), Shibugawa and Takahashi (1983), Takimoto and Fujisawa (1977), and Yamanouchi (2001). This is an area that seriously demands further investigation in English.

Some approximations of the Japanese colors discussed in the text can be found at the following websites:

http://geocities.co.jp/HeartLand-Icho/9109/irome.html (HeartLand-Icho)

http://tenka1.jp/ karasu_2/jcolor_list/htm (Wa-shoku Risuto);

Of course, the colors vary somewhat from monitor to monitor. They are also in Japanese. For those requiring more accuracy, an accessible book with accurate individual colors swabs there is Nihon Shikisai Kenkyuujo (1984), which is kind of a smaller, Japanese version, of the Munsell Book of Color.

An interesting film showing classical dyeing processes for a dozen traditional colors is BG Japan/ Nikei Eeijoo (c. 2000).

2. The Hepburn system has been used to romanize Japanese words (with repetition of the letter used to represent phonemic vowel length). 
Table 1.

\begin{tabular}{|c|c|c|c|c|c|c|}
\hline Color category & Orthography & kun-yomi reading & on-yomi reading & Munsell value & Freq. & Year \\
\hline WHITE & 白色 & shiro-iro & haku-shoku & N 9.5 & 298 & 712 \\
\hline BLACK & 黒色 & kuro-iro & koku-shoku & N 1.5 & 302 & 720 \\
\hline RED & 赤色 & aka-iro & seki-shoku & 5R $4 / 14$ & 510 & 712 \\
\hline YELLOW & 黄色 & ki-iro & oo-shoku & $5 Y 8 / 14$ & 1101 & 720 \\
\hline BLUE & 青色 & ao-iro & sei-shoku & 10B $4 / 14$ & 394 & 712 \\
\hline GREEN & 緑色 & midori-iro & ryoku-shoku & $2.5 \mathrm{G} 6.5 / 10$ & 1170 & 720 \\
\hline BROWN & 茶色 & cha-iro & kas-shoku & 5YR 3.5/4 & 871 & 1158 \\
\hline PURPLE & 紫色 & murasaki-iro & shi-shoku & $7.5 \mathrm{P} 5 / 12$ & 1524 & 759 \\
\hline PINK & 桃色 & momo-iro & - & $2.5 \mathrm{R} 6.5 / 8$ & 1611 & 759 \\
\hline ORANGE & 橙色 & daidai-iro & - & 5YR $6.5 / 13$ & $2135+$ & 1905 \\
\hline GREY & 鼠色 & nezumi-iro & - & N 5.5 & $2135+$ & 934 \\
\hline GREY & 灰色 & hai-iro & - & N 5 & 1659 & 1818 \\
\hline yellow-green & 黄緑色 & ki-midori-iro & oo-ryoku & $2.5 \mathrm{GY} 7.5 / 11$ & - & 1862 \\
\hline indigo & 藍色 & $a i$-iro & ran-shoku & $2 \mathrm{~PB} 2.5 / 4$ & 2077 & 927 \\
\hline crimson & 紅色 & beni-iro & koo-shoku & $3 \mathrm{R} 4 / 14$ & 804 & 759 \\
\hline dark blue & 紺色 & kon-iro & - & $6 \mathrm{~PB} 3 / 5$ & 1609 & 990 \\
\hline purple (ELW) & パープル & paаpuru & - & 7.5P 5/12 & - & 1872 \\
\hline pink (ELW) & ピンク & pinku & - & $2.5 \mathrm{R} 7 / 7$ & - & 1884 \\
\hline orange (ELW) & オレンジ & orenji & - & 5YR $6.5 / 13$ & - & 1860 \\
\hline grey (ELW) & グレー & guree & - & N 5 & - & 1864 \\
\hline
\end{tabular}

Notes to Table 1:

Data is taken from Halbern (1990), Nagata (2002), and Arakawa (1977), and compared with tallies given in Kokuritsu Kokugo Kenkyuujoo $(1965,1971,1972,1973,1974)$. In this table are the twenty most important color terms discussed in the text (Color category) given with their Sino-Japanese characters (Orthography). Lexemes claimed to be basic color terms in the standard Berlin \& Kay model are given in CAPS (and note there are two proposed terms for the category GREY). At the bottom are four English loanword color terms (designated by ELW in parenthesis) that I have argued (Stanlaw 1987, 1997) are currently in the process of replacing their native Japanese equivalents. Munsell values are those designated by the Japan Industrial Standards (JIS) agency. Under Freq. (frequency) ranks for characters range from 1 (highest) to 2135 for all the Jooyoo kanji (the 1,945 common usage characters officially used in print and taught in school) and some names. Thus, the character for WHITE is the 298th most frequent of all Japanese characters (the 298th most frequent in terms of tokens of the 2,135 types). Note that characters for daidai and nezumi are now relatively rare (and are not among the 2,135 most frequently cited types). This is indicated as "2135+." All native terms are cited with the (often optional) -iro (suffix). Kun-yomi reading is the Japanese-based reading of a character and on-yomi reading is the Chinese based reading. Not every color character has a Chinese reading, nor, obviously, do English loanwords (which are written in the katakana syllabry). Under Year is the first appearance in written form of a color term/character as near as can be determined. All dates are CE. Generally the year 712 means that the term was found in the Kojiki; terms cited as 720 are from the Nihon-shoki, and terms cited as 759 are from the Man'yoo-shuu.

introduction and a complex rebus-like mixture of spoken Japanese and Chinese characters for the stories and poems. It was compiled in 712 CE. The Nihon-shoki or Nihongi (Sakamoto et al. 1967) - "The Japan Chronicles" - is the other classic history, and was completed in 720. It is written in kambun, that is, a genuine version of the Chinese lan- 
guage (using Chinese characters) written by Japanese. These two histories are the sacred books of the indigenous pre-Buddhist Shinto religion and are a nationalist repository of Japanese myths and legends. ${ }^{3}$

The third, and most important book for our purposes, is the Man'yoo-shuu (Kojima et al. 1971-1975) - "The Collection of 10,000 Leaves." It is the first book written in the Japanese vernacular, and is a collection of 4,539 poems (depending on the count) compiled in 759 at the earliest. They were transcribed in a complex orthography using borrowed Chinese characters for both their phonetic as well as semantic value. As different scribes and authors used different characters for each function, reading the Man'yoo-shuu in its original form today is extremely difficult even for scholars. ${ }^{4}$

However, there are at least three reasons why the Man'yoo-shuu is unsurpassed for providing a window on what the ancient Japanese language must have been like. First, at least some of the poems in the collection depict a ritualized verbal art form - probably sung, as the word for both poem and song in Japanese is the same (uta) - that might have gone back to $300 \mathrm{CE}$ or earlier, when a recognizable Japanese polity first began to take shape. Second, the Man'yoo-shuu is a representative work, showing many different styles of verbal expression (Levy 1981:4). Finally, while the "study of Chinese and writing in Chinese were done by only a small number of people, namely the court nobles and priests," the Man'yoo-shuu "contains poems from a broader segment of society" as can be seen by the many annoynous authors (Habein 1984: 15).

Most poems in the Man'yoo-shuu are in classic tanka form of 31 syllables written in five lines of 5/7/5/7/7 syllables each. The following two poems, besides being noted for their vivid color imagery, are among the most famous. To set the context, Princess Nukata was forced to marry the Emperor Tenchi, but was previously married to his brother Crown Prince Temmu, with whom she is still in love. At a hunt organized by Tenchi, where she will pass by Temmu, she wrote:

（1）あかねさす紫野行き標行き野守は見ずや君が袖振る

$\begin{array}{ll}\text { akane sasu } & \begin{array}{l}\text { As you, my love, stand in front } \\ \text { of the red setting sun }\end{array} \\ \text { shime-no yuki } & \text { and wave to me } \\ \text { nomori was mizu ya } & \text { in the purple field, } \\ \text { kimi ga sode furu } & \text { the guard is watching us (20) }\end{array}$

(End numbers refer to Man'yoo-shuu poem numbers.).

3. English translations of parts of the Kojiki can be found in Philippi (1968), and for the Nihon-shoki in Aston (1896/1972).

4. The indexes and citations in Shin-hen Kokka Taikan (1983a, 1983b, 1984a, 1984b) are priceless resources for examining poetry of the period, saving researchers literally weeks of work. English translations of parts of the Man'yoo-shuu can be found in Levy (1981) and Nippon Gakujutsu Shinookai (1965). 
Temmu replied:

(2) 紫のにほへる妹を憎くあらば 人妻ゆ㤩に我恋ひめやも murasaki no nioeru imo o Though you are now another's wife, nikuku araba hitozuma yue ni ware koimeya mo could I keep from being in love with you - you as beautiful as this purple grass if I blamed you? (21)

Note that according to the standard Berlin and Kay model, the colors like purple (紫 murasaki) and terms like akane (茜 a kind of red) are unexpected at this Berlin and Kay stage in Japanese color development. We will return to them shortly.

\section{The evolution of color terms in Japan}

Table 2 gives a possible sequence of the evolution of Japanese color development, circa $400 \mathrm{CE}$ to the present. This is a speculative exercise, of course, because examining within historical texts the characters used today to write color names in Japanese is no guarantee that (1) these were the only color names, or color categories, present at any point in time (with others simply not being written down), or (2) the referents and denotations were the same as they are now. There is little that can be done about the first problem. The second is to be expected, but at least some conjectures can be made by careful readings and comparisons.

Another difficulty in determining the basic color terms in early Japan concerns what exactly a color term in the Japanese language is. Is it the spoken morpheme or the Sino-

Table 2. The possible evolution of Japanese color terms.*

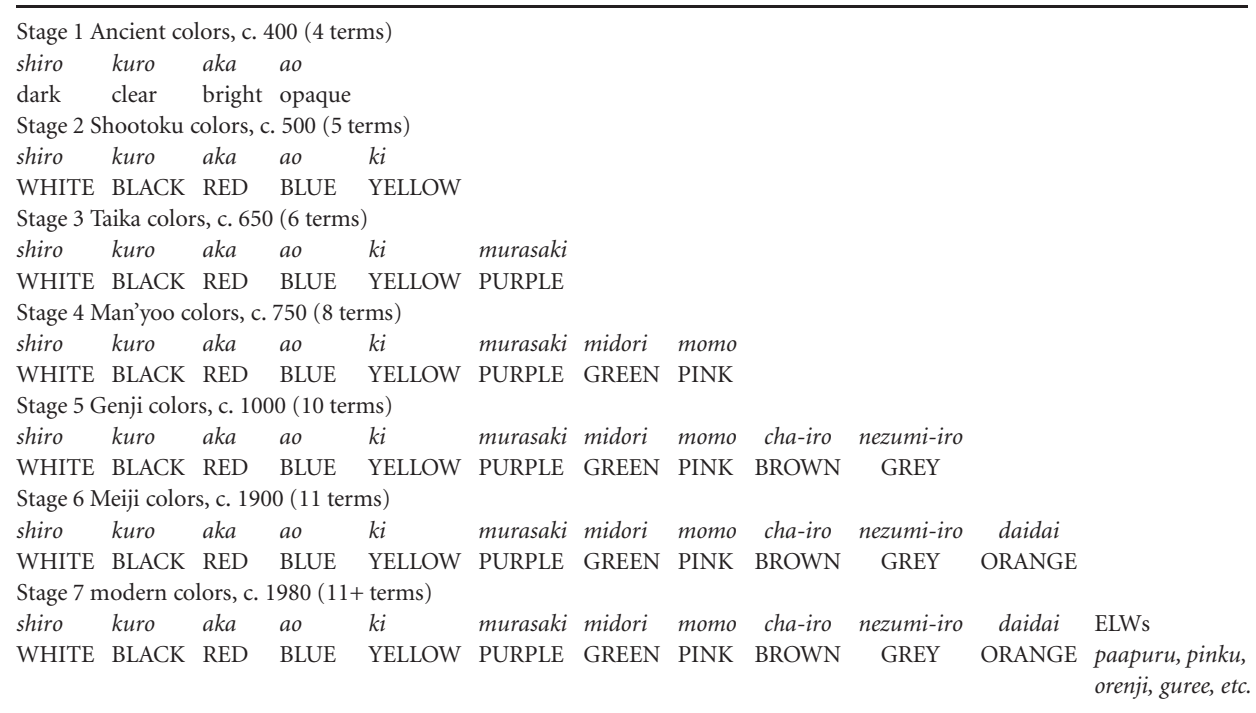

* Note: these are not the stages in the Berlin and Kay model (which use roman numerals) 
Japanese character? There are some color terms written in classical texts with characters that no one today knows how to pronounce. However, we know what the term might theoretically denote by the semanticality of the characters. Also, as almost every modern Sino-Japanese character has at least two readings - some based on the indigenous Japanese ways of pronouncing the concept that the character represents, others based on borrowed Chinese pronunciations - there is another problem: What is the real basic color term - the native Japanese term (the so-called "Yamato kotoba," 'authentic Japanese word'), or the borrowed Chinese character and its reading? ${ }^{5}$ In the twenty-first century, with a vocabulary of English loanwords that is often as high as ten percent (including a complete set of English color terms), resolving the issues stemming from language contact is a familiar predicament for Japanese.

These problems aside, it is very hard to know with certainty what the basic color term inventory was for ancient Japanese. Even color as a cognitive concept may have been different from the notion of a referent-detached abstract sense practiced in Japan today. Presumably environmental and plant stimuli were the initial resources for naming abstract colors (e.g., white being associated with pearls - shira-tama - or black being associated with the color of the swamp or the marsh - numa). ${ }^{6}$ Apparently ancient Japanese divided colors into three broad categories (Nakae 2003): (1) 植物の色 shoku-butsu no iro (the colors of plants), (2) 動物の 色 doobutsu no iro (the color of animals), and (3) 自然の色 shizen no iro (the colors of nature). An example of this latter type would be yoake (夜明け) or the color of dawn. Thus, tactile, or multi-sensual, ties to the natural universe were important in color term nomenclature in early Japan.

\section{Japanese stage 1 (4 terms): Ancient colors, circa $400 \mathrm{CE}$}

Satake (n.d.) claims that the earliest Japanese had concepts of three abstract colors - white, black, and red - but it is hard to know when a fourth color concept developed, or what it was. One of blue, purple, or yellow is the likely candidate, and Kunihiro (citing Shibata in MacLaury 1997:342) argues for a composite blue-green-yellow term, which he reconstructs as ${ }^{\star} a w a$ or ${ }^{\star}$ awo (one inference being made from the way this term was written in syllabary in the eighth century). It seems that in the early texts there were at least four color concepts, if not actual categories; however, their labels were not clear color terms. According to the Kojiki, these were brightness, darkness, clearness, and opacity, as shown in Table 3.

However, as seen in the table, when these characters were used as color terms they had different pronunciations: they were pronounced in what were apparently the names

5. All suspected or proposed basic color terms in the standard BKM model are written in capitals.

6. To complicate matters even further, some informants say that there are some differences in precision in how color terms are used, depending on the reading of the character/character compound. For example, the term ao (青 'blue') by itself might be thought to be the most precise (e.g., “It is blue”). Addition of the iro suffix (青色 aoiro) makes it more general (e.g., "It is a blue"). Reading these same two characters in on-yomi Chinese fashion (i.e., sei-shoku) can make things sound even more general or academic (e.g., "It has blueness"). These interpretations, however, are somewhat idiosyncratic. 
Table 3. Four incipient color notions or categories in ancient Japanese.

\begin{tabular}{lcccc}
\hline Category & Brightness & Darkness & Clearness & Opaque/foggy/dim \\
\hline character & 明 & 暗 & 顕 & 菠 \\
usual pronunciation of the character & $a k a r u i$ & an & ken, arawa $(r e r u)$ & $b a k u$ \\
pronunciation as a color & $a k a$ & kuro & shiro & $a o$ \\
associated color term character & 赤 & 黒 & 白 & 青 \\
\hline
\end{tabular}

of indigenous color terms in ancient Japanese (aka, kuro, shiro, and ao). But these four spoken words also had four other Sino-Japanese characters associated with them (shown in the bottom row of the table). These were the characters the Chinese presumably used to write the red, black, white, and blue colors in their language. When these characters were borrowed, the Yamato Japanese words for these four basic colors were also applied to them. Apparently, these four hue-characters replaced those which signified brightness, darkness, clearness, or dimness.

\section{Japanese stage 2 (5 terms): Shootoku colors, circa 500 CE}

Ancient Chinese philosophers apprehended reality via 'five elements' (gogyoo 五行) the key cosmological symbols of wood, fire, earth, metal, and water - which interacted with the opposing ying and yang forces (in 陰 and yoo 陽). This system was readily adopted all over East Asia and Prince Shootoku (574-622), the first unifier of Japan, canonized it by $600 \mathrm{CE}$. Each element, however, had an associated cardinal direction, season, planet, virtue, taste, sense, color (sometimes called the "official" colors), and much else. In Japanese, these five colors were shiro 'white,' kuro 'black,' aka 'red,' ao 'blue,' and ki 'yellow.' The top of Table 4 shows these colors and some other associations (the bottom of the table will be discussed shortly).

\section{Japanese stage 3 (6 terms): Taika colors, circa 650 CE}

The third stage of Japanese color term evolution saw the introduction of a sixth category, purple (murasaki). The character for murasaki (紫) certainly had been extant for hundreds of years in China, and was brought to Japan with the other myriad characters. In fact, it was mentioned in the Confucian Analects - a book that came to Japan as Rongo (論語), one of the important books of Chinese learning at the time. Purple became the national color, and synonymous with the imperial court, after the decree by the Empress Suiki in 647 (during the so-called Taika Reforms - discussed in the Nihon-shoki - which unified the incipient Japanese state). Certain purple hues became reserved for the aristocracy and commoners were forbidden to wear them; violators were subject to harsh penalties.

As shown in Table 4, the Chinese Confucian philosophical system of specialized elements - with associated colors - was adopted in Japan early on. However, modifications were made, and an additional virtue and color was added (as shown at the bottom of the table). The virtue - toku - referred to a particular kind of goodness or moral perspective, a sense of humanity or compassion often said to only be obtained by the most empathetic of 
Table 4. The traditional East Asian five elements and colors, c. 600 CE.

\begin{tabular}{llllll}
\hline 5 gyoo & 木 & 火 & 土 & 金 & 水 \\
(natural & wood & fire & earth & metal & water \\
elements) & $k i$ & $k a j i$ & tsuchi & kin & mizu \\
5 shoku & 青 & 赤 & 黄 & 白 & 黒 \\
(colors) & blue & red & yellow & white & black \\
& $a o$ & $a k a$ & $k i$ & shiro & kuro \\
5 hoo & 東 & 南 & 中央 & 西 & 北 \\
(directions) & East & South & center & West & North \\
& higashi & minani & chuuoo & nishi & kita \\
5 ji & 春 & 夏 & 土用 & 秋 & 冬 \\
(seasons) & spring & summer & hottest of summer & fall & winter \\
& haru & natsu & doyoo & aki & fuyu \\
5 joo & 仁 & 礼 & 信 & 義 & 智 \\
(virtues) & benevolence & politeness & fidelity & justice & wisdom \\
& jin & rei & shin & gi & chi \\
\hline
\end{tabular}

Note: There was one other position that was higher of than jin (仁) in the 'virtues' category. This was toku (徳 'morality' or 'virtue'), and the associated color was purple (murasaki 紫). Thus, if these were added to the above chart, the 5 virtues and 5 colors rows would look respectively as follows:

$\begin{array}{llllll}\text { 徳 } & \text { 仁 } & \text { 礼 } & \text { 信 } & \text { 義 } & \text { 智 } \\ \text { morality } & \text { benevolence } & \text { politeness } & \text { fidelity } & \text { justice } & \text { wisdom } \\ \text { toku } & \text { jin } & \text { rei } & \text { shin } & g i & \text { chi } \\ \text { 紫 } & \text { 青 } & \text { 赤 } & \text { 黄 } & \text { 白 } & \text { 黒 } \\ \text { purple } & \text { blue } & \text { red } & \text { yellow } & \text { white } & \text { black } \\ \text { murasaki } & \text { ao } & \text { aka } & k i & \text { shiro } & \text { kuro }\end{array}$

monks. The associated color was purple, and certain shades were reserved for the robes of the Buddhist clergy. Both these elements were placed at the head of their respective rows.

\section{Japanese stage 4 (8 terms): Man'yoo colors, circa 750 CE}

The fourth, and most interesting, stage of Japanese color term evolution came around the time the Man'yoo-shuu was compiled. There were apparently eight full-fledged linguistic color terms. Terms for green and pink were added to the six terms of the Taika colors (white, black, red, blue, yellow, and purple). As this is the stage that I will discuss in most detail, I will give examples of each color term from poems in the Man'yoo-shuu:

shiro/shira: WHITE

（3）白波の 浜松が枝の手向けくさ幾代までにか年の経ぬらむ shira-nami no

hama-matsu ga e no

tamuke-kusa

ikuyo made ni ka

toshi no kenuramu
I wonder how many generations

is that branch of pine

on the beach,

where white waves

come and go? (34) 


\section{kuro: BLACK}

（4）おほかたは誰が見むとかもぬばたまの我が黒髪を靡けて居らむ ohokataha

ta ga mimu to kamo

nubatama no

waga kuro-kamio

nabikete oramu

With my beautiful black hair

like a little cuckoo bird,

I am flowing in the wind

thinking that

someone would see me. (2532)

aka: RED

（5）赤絹の 純裏の衣長く欲り 我が思ふ君が見えぬころかも

aka-kinu no

hitoura no koromo

These days

nagaku hori

when I have not seen my love,

aga omou kimiga

with whom I wish to keep our relationship,

mienu koro kamo

are as long as

a nobleman's red clothes. ${ }^{7}(2972)$

$a o:$ BLUE

（6）青山を横ぎる雲のいちしろく我れと笑まして人に知らゆな ao-yama o As obvious as a white cloud

yokogiru kumo no

crossing over the blue mountain,

ichi shiro ku

please do not tell anyone

ware to emashite

hito ni shira yuna

that I have such a smile

for you. (688)

\section{ki/momichi: YELLOW}

（7）黄葉に 置く白露の 色端/色葉にも 出でじと思へば 言の繁けく

momichi-ba ni

oku shiratsuyu no

Like white dew

iroha nimo

on yellow leaves,

ideji to omoe-ba

we are trying

koto no shigekeku

not to be seen.

Still, people are talking about us very much. (2307)

murasaki: PURPLE

（8）紫の 帯の結びも 解きも見ずもとなや妹に 恋ひ渡りなむ

murasaki no

obi no musubi mo

toki mo mizu

moto-naya imo ni

koi watari namu
Even though

I have not yet untied

the purple sash around her waist,

I miss this girl

very much. (2974)

7. Note: the sleeves of a nobleman's dress are twice as long as regular sleeves, and have red lining. 
midori: GREEN

（9）春は萌え夏は緑に紅の綵色（まだら/しみいろ）に見ゆる秋の山かも haru wa moe In the spring natsu wa midori ni kurenai no the yellowish leaves are just sprouting, and madara ni miyuru aki no yama kamo in summer they are fresh and green. In fall they are very crimson but in the winter less red is seen in the mountains. ${ }^{8}$ (2177)

momo/toki: PINK

（10）桃花染めの 浅らの衣浅らかに toki some no 思ひて妹に 逢はむものかも asara no koromo asaraka ni Seeing the cloth omoit) imo ni softly colored pink, awanu monokamo like that of the crested ibis, I wonder if I might meet the girl. (2970)

Japanese stages 5 to 7: Genji colors, Meiji colors, and modern colors During the Heian Period, (794-1185), one of Japan's most fruitful literary times, the 'brown' (cha-iro) and 'grey' (nezumi-iro) terms appeared. These are both found in the Tale of Genji (c. 1000), Japan's greatest literary masterpiece and the world's first novel, as well as in other Heian works (Kondo 2002). ${ }^{9}$ As far as can be told from written texts, little further change took place in Japan's color vocabulary until the Meiji Period (1868-1912), when the 'orange' (daidai) term developed. In the late twentieth century, extensive borrowing has provided an English loanword for each native Japanese color term. The native terms for pink, orange, and grey have become almost archaic, and I have argued (Stanlaw 1987) that English loanwords are replacing them.

\section{The BKM standard model}

The standard model of color nomenclature was first proposed in 1969 by Brent Berlin and Paul Kay $(1969,1991)$. I will call this the BKM model (the M standing for any or all of several important collaborators: William Merrifield, Luisa Maffi, Robert MacLaury, Chad McDaniel). Though facing several methodological and philosophical challenges over the past thirty years, it is still the starting point for any cross-cultural comparisons of color vocabulary.

In short, the BKM model posits that humans name the whole color space, and do so using a dozen basic color terms at most. Color terminology systems develop in a predicable

8. Note: 萌黄色 moegi iro means 'greenish yellow'; 綵色 madara/shimi-iro means 'spotted'; 紅 kurenai means 'crimson.'

9. There are now three major English translations of the Tale of Genji. Seidensticker (1976) is most often cited. 
evolutionary order by breaking up large composite categories eventually into six smaller presumably biologically based - primaries (i.e., the LIGHT-WARM category splitting into WHITE and WARM, with WARM splitting into RED and YELLOW; the DARK-COOL category splitting into BLACK and COOL, with COOL splitting into BLUE and GREEN). Additional terms add on in less predicable ways, but still are constrained by human psychological, biological, and linguistic universals. Though not fully investigated as yet, it is assumed that these additional remaining "derived" color categories develop out of some sort of set intersections of the primaries (i.e., BROWN being derived from YELLOW with BLACK, PURPLE from RED with BLUE, ORANGE from RED and YELLOW, and PINK from RED with WHITE). The achromatic GREY color is a wild card).

The BKM model has gone through several refinements, the most important revisions being in 1975 (Kay \& McDaniel 1978) 1991-1997 (Kay et al. 1991a; Kay et al. 1997), and 1999 (Kay \& Maffi 1999). Table 5 gives a summary of both its current status, as well as some of the historical developments. It is clear that most of the points of contention have been in the growth of stage III and stage IV systems. Seven paths or trajectories have been proposed. Paths A, B, C, D, and E are now the accepted trajectories hypothesized by the current BKM model (e.g., Kay \& Maffi 1999:749), though I have listed the proposals chronologically (from 1975 to 1999) in the table.

Path A and Path B are essentially versions of the model initially first proposed in the 1969 (i.e. at stage III the next color to develop is GREEN followed by YELLOW, or YELLOW followed by GREEN) with the added 1975 insight that these are terms are really composite macro colors (that is, BLUE often means the green, blue, or even black colors). Paths C, E, X and Y were added in the early 1990s when further analysis of the World Color Survey Data (Kay et al. 1991b) revealed many cases that could not be accounted for by the 1975 model. Further analysis (e.g., MacLaury 1997; Kay et al. 1997), however, has shown that no language followed Paths X or Y, while a new Path D was proposed to account for a few languages where the relatively rare YELLOW-GREEN-BLUE split into YELLOW and COOL categories.

In Table 5, the "Main Line" (Kay \& Maffi 1999:749-751) evolutionary trajectory of the BKM model is Path A. If we infer from the World Color Survey data, more than 80 percent of the world's languages would follow some version of Path A. The explanation for this trajectory simply says we should apply three principles in order until all the primaries are reached: first partition the total color space into two (the macro-white and macro-black categories); second split WHITE off from WARM if possible, or BLACK off from COOL; then, if possible, derive RED.

While this model is parsimonious and elegant, the other paths in Table 5 suggest that there are either (1) exceptions to the motivations above, or (2) other parameters that must be operating to account for extant data.

An example of an exception would be Paths B and C in the table, where RED is split off from YELLOW before the expected splitting of BLACK and COOL. This seems to occur about 10 percent of the time (Kay \& Maffi 1999:751). The remaining Paths D and E show a violation of a different kind. Here we find the presence of a YELLOW-GREENBLUE composite category. The BKM model explains this as follows: in some - albeit rare - 
Table 5. Some possible BKM evolutionary sequences of color terms, c.1975 to c. 2000.

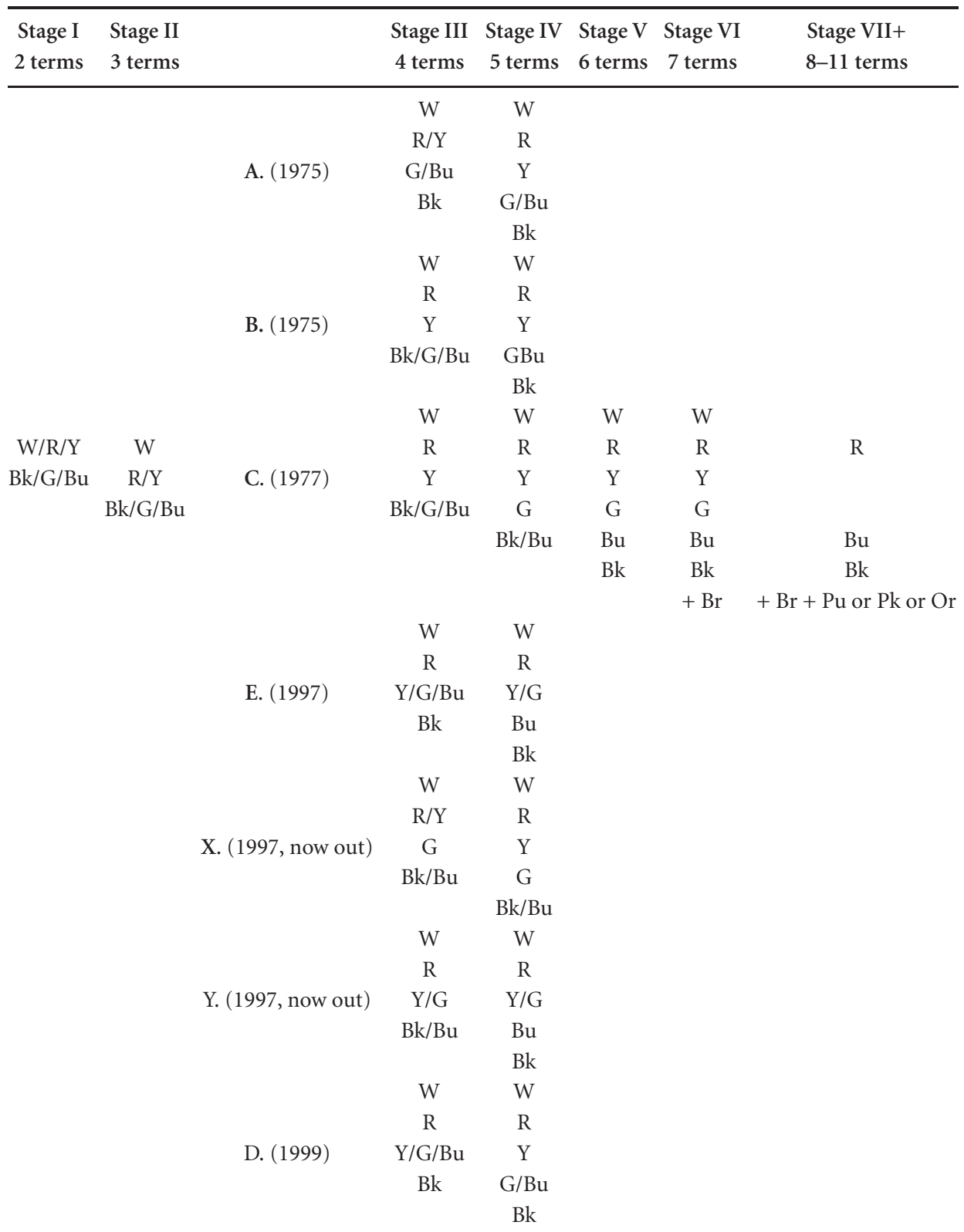

Note: Labels A through E are those cited in Kay and Maffi (1999:749). My own labels X and Y are earlier paths (Kay, Berlin, Maffi, \& Merrifield 1999:32-33), now rejected. Years in parentheses are the times of first proposals. The composite categories are often named as follows: LIGHT-WARM=W/R/Y (White, Red, Yellow); DARK-COOL=Bk/G/Bu (Black, Green, Blue); WARM=R/Y (Red, Yellow); COOL=G/Bu (Green, Blue) 
cases, a language does not fully partition color space at the earliest stages. In other words, there may be terms for WHITE, BLACK, and RED, but these are not broadly inclusive (for example, BLACK would not include the green, blue, or other dark hues). This would leave much of color space unnamed. If a language later applied partitioning - in essence, naming what was unnamed before - the leftover yellows, blues, and greens would now be labeled, initially, as a single category. The YELLOW-GREEN-BLUE composite category could split into YELLOW and COOL (Path D) or YELLOW-GREEN and BLUE (Path E, but apparently having only one case).

\section{Explaining the evolutionary sequence of Japanese color terms}

How does the Japanese data relate to the BKM standard model? As seen in Table 2, the ancient colors of $400 \mathrm{CE}$ and the Shootoku colors of $500 \mathrm{CE}$ follow the mainline trajectory (Path A of Table 5). The first major discrepancy comes with the Taika colors when the murasaki PURPLE term appears. Besides PURPLE, in the Man'yoo colors of $750 \mathrm{CE}$ we also unexpectedly find momo (PINK); midori (GREEN) also appears. The Genji colors (c. $1000 \mathrm{CE}$ ) of medieval Japan finds the appearance of both BROWN (cha-iro) and GREY (nezumi-iro), and ORANGE (daidai) appears in the Meiji period (1868-1911). These last stages are consistent with the standard model. Finally, in the late twentieth century, we find the appearance of English loanword synonyms for every basic Japanese color. The BKM standard model has little to say, in a predictive sense, about language contact.

The point of interest, obviously, is how the Man'yoo colors relate to the BKM stage IV to stage VI transitions. How might the Japanese data be explained? The explanatory mechanism of the BKM model discussed previously (first, partition; then WHITE-WARM or BLACK-COOL splits; then RED) cannot account for this data, even if the RED exceptions (Paths B and C) and YELLOW-GREEN-BLUE exceptions (Paths D and E) are considered. One problem is that neither PURPLE nor PINK should appear before BROWN. More importantly, as seen in Table 6, is why the PURPLE murasaki term should not appear here. For the Taika colors (at Japanese stage 3), PURPLE theoretically develops out of a mixing of RED and BLUE. However, the Japanese COOL term (ao) has not yet split into its component parts. Even at the stage of the Man'yoo colors (Table 6, stage 4), there seems to be incomplete splitting of the COOL category. The relationship between the ao and midori terms and the GREEN and BLUE - and even at times, BLACK - color categories are unclear (and will be discussed shortly).

Either some other mechanisms are operating, or another model needs to be employed, for the early Japanese color data. Both these possibilities will now be considered at the stage of the Man'yoo colors.

\section{The Man'yoo colors terms, circa 759 CE}

Let us summarize what we know about the eight Man'yoo colors terms found in Table 2. The WHITE (shiro or shira) term seems straightforward, though BLACK (kuro) sometimes overlaps with ao (BLUE) and/or midori (GREEN). Aka (RED), while apparently 
Table 6. Early evolution of Japanese colors, composites and derived colors. ${ }^{\star}$

\begin{tabular}{|c|c|c|c|c|c|c|c|c|}
\hline \multirow{3}{*}{ Stage 1, c. 400 Ancient colors } & \multirow{3}{*}{$\begin{array}{c}\text { Light } \\
\text { White } \\
\downarrow\end{array}$} & \multirow{2}{*}{$\begin{array}{l}\text { Dark } \\
\text { Black }\end{array}$} & \multicolumn{4}{|c|}{ Warm } & \multicolumn{2}{|c|}{ Cool } \\
\hline & & & & $\mathrm{Red} / \mathrm{Ye}$ & low & & Greel & Blue \\
\hline & & $\downarrow$ & & $\swarrow$ & $\searrow$ & & & \\
\hline Stage 2, c. 500 Shootoku colors & $\begin{array}{c}\text { White } \\
\downarrow\end{array}$ & $\begin{array}{c}\text { Black } \\
\downarrow\end{array}$ & & $\begin{array}{c}\text { Yellow } \\
\downarrow\end{array}$ & $\begin{array}{c}\text { Red } \\
\downarrow\end{array}$ & & Grees & Blue \\
\hline Stage 3, c. 650 & White & Black & & Yellow & Red & Purple & Greel & Blue \\
\hline Taika colors & $\downarrow$ & & & & $\downarrow$ & $\downarrow$ & $\downarrow$ & $\downarrow$ \\
\hline Stage 4, c. 750 Man'yoo Colors & White & Black & Pink & Yellow & Red & Purple & Green & Blue \\
\hline
\end{tabular}

* These are not the stages in the Berlin and Kay model (which use roman numerals).

basic, competes with several other frequent and salient red terms (e.g., see akane in Example 1 and kurenai in Example 9). PINK (momo or toki) appears to name only a restricted very light range of pink connected to the hues of significant flowers such as cherry blossoms. The character for the YELLOW category (黄) has several spoken forms (ki momichi, or even kariyasu), and its range floats around, usually including modern BROWNs and sometimes interacting with the BLUE and GREEN terms. It is possible that the character appeared before the $k i$ reading. The importance of the murasaki term is still noted today, but was an extremely important color in early Japan. It was the color of the nobility and apparently had common light (nise-murasaki) and dark (hon-muraski) forms which might have almost been basic color categories themselves. The ao (BLUE) term is apparently the COOL term, referring to blue and green, and even sometimes black and grey colors. Its Sino-Japanese character (青) was one of the five “official colors" of East Asia. However, in texts where actual vernacular Japanese was used midori (GREEN) is cocontemporaneous with ao. For example, we find both ao-sora 'blue sky' and midori-sora 'green sky' in Japanese poems. This at least suggests that in the spoken language ao and midori both waffled in labeling the COOL category, and might not have completely split apart until much later.

\section{Alternative Explanations}

An alternative to the standard BKM model is MacLaury's cognitive-dynamic multidimensional model $(1997,2001)$. MacLaury based his theory on the analysis of the 110 languages in the World Color Survey along with about 120 languages from his Mesoamerican Color Survey and Pacific Northwest Color Survey. While acknowledging the primacy of focusing on hue in color-naming in most languages, MacLaury argues that there are several other dimensions, or sets of categories, that are involved in the development of color vocabulary. The most important of these are early derived-hues, color terms based on brightness, and color terms based on desaturation (while the others are color terms based on hue by-passing or hue-refraction, and a special yellow-with-green category). ${ }^{10}$ There are rules

10. For a further in-depth discussion on the brightness dimension see MacLaury (1992) (and my comments, Stanlaw 1992). 
which allow color terms to transform into the each other at these different levels, allowing for some rather complex and variable typological sequences of color term evolution (see MacLaury 2001:1123-1234).

MacLaury begins by postulating that when processing a pair of perceptual stimuli, a person can either pay more attention to their similarities, or differences. For example, the first terms found in the BKM evolutionary sequence are due to people in a language/culture focusing on the maximal similarities and differences to, first, brightness, and then hue. WHITE and BLACK split, thus, because maximal attention is paid to differences in brightness, etc. Besides MacLaury, several others have made similar suggestions (e.g., Witkowski \& Brown 1977:55; Stanlaw 1987:203), but MacLaury's great insight (1995, 1997) was (1) to show how such perceptual processes operate in other dimensions (such as with desaturated colors), and (2) develop a formal theory of how people use physical spatial analogies to anchor their mental perceptions. He demonstrated several new semantic color relations using this approach. Under the name Vantage Theory he has generalized this extended theory of categorization to other domains (e.g., MacLaury 2002).

\section{COOL, Green and Blue Colors}

Several of the apparent inconsistencies of the early Japanese color system can be explained by this alternative interpretation. First, the COOL category has always been an intriguing problem. It is often assumed that ao names this category. Even today, as Table 7 shows, the character used to write Japanese ao (青) can refer to several different hues - green, blue, black, and grey - in both Japanese and Chinese. Thus, historically, it is probable that ao was as equally broad.

Uemura and Yamazaki (1950:50) believe that the many green hues were generally referred to as ao in the earliest writings, such as in ao-yama 'green (lit. 'blue') mountains' or ao-jinsoo 'green (lit. 'blue') grass' in the Kojiki. In the Kojiki is also found the phrase

Table 7. Examples of the usage of the ao-term (青) in Japanese and Chinese

\begin{tabular}{|c|c|c|c|}
\hline Referent & Character & Japanese & Chinese \\
\hline green hills & 青山 & ao-yama & qīng-shān \\
\hline green frog & 青蛙 & ao-gaeru & qing-wā \\
\hline green bamboo & 青竹 & ao-dake & qīng-zhú \\
\hline green peas/beans & 青豆 & ao-mame & qing-dòu \\
\hline blue sky & 青天 & sei-ten & qīng-tiän \\
\hline blue sky & 青空 & ao-zora & \\
\hline black (Artic) fox & 青狐 & ao-gitsune & qing-hú \\
\hline black ox & 青牛 & & qing-niú \\
\hline black earth & 青丹 & $a o-n i$ & \\
\hline black horse & 青馬 & ao-uma & \\
\hline grey horse & 青馬 & ao-uma & qīng-mă \\
\hline pale, pallid, bluish grey (Chinese), bluish white (Japanese) & 青白 & ao-jiro & qing-bái \\
\hline
\end{tabular}


"sonogiri no ao ki mi-keshi" 'the kingfisher's blue dress' referring to his green wings. This term is certainly fluid, at times labeling everything from black foxes, to grey horses, to blue skys, and green beans. The Manyoo-shuu explicitly even says that ao was a white color with a bit of grey added (hai-iro ga katta shiro-iro). Thus, it appears that ao is the exemplar COOL term.

As seen from the Man'yoo-shuu poem mentioned in Example 9, midori no doubt named green hues. Uemura and Yamazaki (1950:50) claim that the first appearance of the character for midori is in the Nihon-shoki in the section "Midori-No" 'green fields' in the chapter on the Keikoo emperor. They go on to state that "In the Manyoo-shu, midori (緑 'green') is also called the 'color of duck's wings.' However, in general, this color [of duck's wings] was called ao (青 'blue').”

Does this mean, then, that midori was a contained within $a o$ ? Perhaps not. In terms of written Japanese - not Chinese - texts, midori (GREEN) and ao (BLUE) appear together. Some philologists (Nakae 2003:64-65) claim that the origin of the midori green term can be traced back to the earlier term mizu 'water,' and indeed can be derived from it through consonant assimilation. Also, "sometimes, the midori-iro green color (緑) was used as one of the five official color terms, and sometimes it meant ao (青 blue) and even ai (藍 dark blue)" (Uemura \& Yamazaki 1950:50). And, as we have seen, the sky could at times be both BLUE (ao-sora) and GREEN (midori-sora). These examples would suggest that while ao may have often been more broad, midori was near synonymous with it: we can find cases of green hues being called $a o$ and blues being called midori.

The mechanisms of the BKM model do not lead themselves to easily interpreting such a finding (i.e., two labels closely naming one category). At best, they can suggest that perhaps the category is labeled in free variation, or perhaps is splitting (e.g., Berlin \& Kay 1969:42-3 for the Japanese case), but how this splitting might be taking place is left unexplained. MacLaury, however, calls such a phenomena coextension, a situation where two terms label the same category but with distinct stresses (1997:111). Thus, the question "Was there an ancient Japanese COOL category, and if so, was it labeled by ao or midori?" is a bit more subtle than at first glance. Why do the same poets use different terms at different times? While we do not have the ability to check these labels with ethnographic protocols and color term elicitation tools (such as mapping exercises) - and we cannot be certain if we are dealing with only hue or brightness categories - we can at least speculate on what might be happening.

MacLaury (1997:292) argues that in COOL categories, often neither green-hues nor blue-hues by themselves shape a category as much as a does the manner in which their relation is constructed, perceived, or thought about. In the case of Japanese, we first notice that the blue hues tend to be brighter in Munsell values than the green hues. Thus, at least some of the time, the ao term might be naming brightness - rather than hue - vis-à-vis midori. It also has a sense of priority, being one of the five official colors of Asia, as well as carrying feelings of youth, inexperience, and freshness. A definition, then, of ao would include all these things, besides just its hue. In American English, for example, a fire engine is still called red even if it is actually more brown or orange or yellowish. Context, situa- 
tion, and the nature of the categories themselves give individuals great variety in selecting a color term. Such processes can be explained by vantages.

\section{Early Derived Categories: PURPLE and PINK}

Among the Man'yoo colors are murasaki and momo, two terms unexpected at this point in the general BKM model. Derived basic terms name colors that are perceptually most distinct from each other (PURPLE being maximally far from both RED and BLUE; PINK far from RED and WHITE). MacLaury (2001:1228-1236) argues that one (or a few at most) of the derived basic color terms can commonly appear at stage III or stage IV of Table 5 (the expected order being BROWN, PURPLE, PINK, ORANGE, or GREY).

The early appearance of murasaki (PURPLE) among the Taika colors coincides with the composite COOL category (ao) and precedes BROWN by many centuries. Among the Man'yoo colors, momo (PINK) appears - also centuries before BROWN - at around the same time we see evidence of the ao-midori COOL coextension split.

There are probably no formal mechanisms to predict or explain these ancient Japanese color term sequences. Instead, we must examine other factors. For example, consider this part of a Manyoo'shuu poem:

$$
\begin{aligned}
& \text {... 紫草のにほへる ... } \\
& \text {... murasaki kusa no nioeru ... } \\
& \text {... your lover's smell of the purple grass... }
\end{aligned}
$$

Notice that "blue grass" or "green grass" is not used here (cf. Examples 1 and 2). While this not definitive evidence for the possible cognitive primacy of Man'yoo PURPLE over COOL, or BLUE or GREEN, this allusion does show the importance of murasaki. Purple, being the most virtuous of colors at the time, took on great prestige and value.

As purple was so attractive and significant a color, it became rather important for everyone to be able to discern just what kind of purple was acceptable for everyday wear and what kind was off limits to the common person. Over the course of history of the dyeing process, then, the purple colors apparently became divided into the two groups fuka-murasaki (深紫 'deep purple') and asa-murasaki (浅 紫 'light purple'), which were important secondary, if not quasi-basic, color terms themselves. Table 8 depicts the growth the evolution of these two purple shades.

Nagasaki claims (2001:72, 258-260) that eventually one shade of fuka murasaki ('deepish purple,' or the color often known as royal purple in English, or 5P 3/7 in Munsell notation) became the exemplar purple color. This most-purple of all the purples - and its neighboring shades - came to be known as hon-murasaki 'primary, original, purple.' But for all practical purposes, these colors were simply called murasaki, and they were reserved for the court and monks. They were kin-jiki (禁色 'forbidden colors') for the common folk. The fact that this was the title of novelist Yukio Mishima's 1968 masterpiece shows how such notions can linger.

The meta-chromatic nature of this color term, then, affected color choice among users in various indeterminate ways. It also directed the possible development of the Japanese color nomenclature system. We will probably never be able to know for certain the status 
Table 8. The split of the purple colors in classical Japan.

\begin{tabular}{cc}
\hline & Purple \\
\hline light purplish hues & dark purplish hues \\
$\downarrow$ & $\downarrow$ \\
asa-murasaki & fuka-murasaki \\
浅紫 & 深紫 \\
light-purple & deep-purple \\
$\downarrow$ & $\downarrow$ \\
asa-iro & koki-mursaki/koki-iro \\
浅色 & 深紫深色 \\
the lights (purples) & $\downarrow$ \\
$\downarrow$ & deep-purple; the darks (purples) \\
aka-murasaki & kuro-murasaki \\
赤紫 & 黒紫 \\
reddish-purple & blackish-purple \\
$\downarrow$ & $\downarrow$ \\
nise-murasaki & hon-murasaki \\
似せ紫 & 本紫 \\
ordinary purple & primary purple \\
$\downarrow$ & $\downarrow$ \\
“fake” or imitation purple & "real” purple \\
\hline
\end{tabular}

of purple terms in Table 8 - were they secondary color terms on their way to being basic? but it is certain that their presence was an influence.

The term for PINK (momo) is also interesting, appearing so early in the history of Japanese color term development. Its range seems to have been rather restricted, generally referring to only very light hues (mostly used for the seasonal shades of various plants and flowers depicted in diaries and verse). It is not clear how this term interacted with RED $(a k a)$. As metioned, there were a half dozen other common red-terms that were competitors with $a k a$ for basic status. The development of PINK might have grown out of this contest. Whether or not it was really only a secondary color term, it took on great import because of its poetic connotations and frequent use in verse.

\section{Conclusion}

We saw that the BKM standard model has difficulty accounting for much of the early Japanese color term data. It is for these reasons that some question the efficacy of using a formal standard model of color nomenclature. Indeed, even though the Berlin and Kay/World Color Survey/Mesoamerican Color Survey project seems theoretically and empirically sound, there are nevertheless some who pose philosophical challenges (e.g., Saunders 1992, 2000; Saunders \& van Brakel 1988, 1997, 2002) or ask some pointed ques- 
tions (e.g., Davis 2000; Gage 1999). ${ }^{11}$ Besides finding anomalous cases, some critics think it is too convenient that the evolutionary color term sequence is as parsimonious as it is. They believe that the eleven or twelve so-called basic color terms are either ad hoc at best or presumptions at worst, and represent little more than how Westerners or those living in industrial societies might see the world. At the same time, formalists argue that ethnographic or historical undertakings are flawed because "Color sensations have multitudes of names, and being totally subjective, we shall never determine whether the color sensation of one observer is the precise equivalent of the color sensation of another observer, even when both use the same descriptor" (Cohen 2001:6).

I think the Japanese data do not necessary contradict the standard model so much as enrich it. What we are seeing is a very complex series of cognitive and social interactions taking place, where color categories, ranges, connotations, and denotations are in a state of flux. Here, I am not referring to informant indeterminacy in color term elicitation tasks, or changes taking place in a color term system due, say, to language contact. Such things have been well documented in the literature (see MacLaury 1997:249-251 for some meticulous analyses). I am talking more broadly about the nature of the color world in general, and its color nomenclature. Color terms do not spontaneously generate, nor are their definitions fixed. These are emergent and negotiated, just as the rest of the semantic system in a language/culture.

There is one other reason why Japanese is an important case-study in color nomenclature theory. Saunders and van Brakel have argued that much of the world's present-day color term vocabulary is due to contact with the West (especially of the imperial or colonial kind). We have here, however, some sense of how the Japanese color nomenclature system developed 1500 years ago before contact with the West, and it seems that many of these cautions are unwarranted. Also - though not discussed here - we can also see in the Japanese situation what happens when a country not just tolerates, but encourages, language and culture contact.

In ancient times in Japan, color was often synonymous with dyeing. But because dyeing was expensive, color - like writing and other things borrowed from mainland Asia was a high-class preoccupation. The lower classes and the farmers, by far the vast majority of the population, did not have the knowledge or the opportunity to make new colors. Their clothes were basically colorless drab whites or plain browns. High class men in classical and medieval Japan wore extremely loud and bright clothes, very much in contrast to the prescribed black, blue, or (on a daring day) brown suits worn by Japanese businessmen today. Typical colors of the upper crust male wardrobe were beni (紅 'crimson red'), koobai (紅梅 'light pink' or 'plum blossom red'), sakura (桜 'cherry blossom red'), haji-momiji (はじ紅葉 'colors of the start of Fall') (Yoshioka 2002:111). This indicates that color was a marker of social class, and not of gender as in today's Japan and the West.

11. These debates - in what some of the participants have called a docudrama - have been rather tumultuous. For some reactions to these criticisms see Hardin and Maffi (2000), Kay (2000), Kay and Berlin (1997), and Stanlaw (1993). 
Thus, we must remember that to early Japanese, color was as much a social product as a biological response.

The Japanese color terms we have examined here expand and contract, depending on their age; some have even disappeared. Their referents and ranges at times overlap, sometimes separate, and sometimes morph into things new or unexpected. There are social and cultural exigencies, and contingencies, to every cross-linguistic universal. The historical Japanese case presented here demonstrates a few of them, but there are many more to uncover.

\section{Acknowledgments}

I thank several people for their help in preparing this manuscript. My friend and anthropologist-colleague Masanori Yoshida of Nihon University helped me obtain Japanese color sources, films, and books. Without his generous assistance I could not have attempted this philological exercise. My friend Roger Thomas, a specialist on Japanese poetry, provided many rare sources, and was a patient sounding board (who never told me what a literary fool I was). Robert MacLaury, as always, has given me much direction and insight into how to handle this wealth of (often conflicting) material. I am surprised how he can sometimes take my own data - which I had previously thought mute - and make it say things I never imagined. Finally, without Nobuko Adachi's knowledge and patience this paper would have been half as rich and half as accurate. Once more, I am profoundly in her debt.

\section{References}

Arakawa, Soobee (1977). Kadokawa gairaigo jiten [Kadokawa loanword dictionary] (2nd. ed.). Tokyo: Kadogawa Shoten.

Aston, W. G. (1896/1972). Nihongi: Chronicles of Japan from the earliest times to A.D. 697. Tokyo: Charles E. Tuttle Company.

Berlin, Brent \& Paul Kay (1969). Basic color terms: Their universality and evolution. Berkeley: University of California Press.

Berlin, Brent \& Paul Kay (1991) Basic color terms: Their universality and evolution (2nd ed.). Berkeley: University of California Press.

BG Japan (Nikei Eeijoo, producers) (c.2000) Koto no juni-shoku [Twelve basic colors of the old capital]. Nikei Eeijoo.

Chijiwa, Hideaki (1987). Color harmony: A guide to creative color combinations. Rockport, MA: Rockport Publishers/Cincinnati, OH: North Light Books.

Cohen, Josef (2001). Visual color and color mixture. Urbana: University of Illinois Press.

Davis, Steven (Ed.) (2000). Color perception: Philosophical, psychological, artistic, and computational perspectives. New York: Oxford University Press.

Gage, John (1999). Color and meaning: Art, science, and symbolism. Berkeley: University of California Press. Haarman, Harald (1989). Symbolic values of foreign language use. Berlin: Mouton de Gruyter. 
Habein, Yaeko Sato (1984). The history of the Japanese written language. Tokyo: University of Tokyo Press. Hardin, C. L., \& Luisa Maffi (Eds.) (1997). Color categories in thought and language. Cambridge: Cambridge University Press.

Hardin, C. L. \& Luisa Maffi (2000). Color categories: The docudrama. American Anthropologist, 102, 321.

HeartLand-Icho (2003). Japanese colors available at http: //geocities .co.jp/Heartland-Icho/9109/irome. html

Hidaka, Kaoru (2003). Nihon bujutsu no kotoba annai [A guide to the words of Japanese art]. Tokyo: Shoogakukan.

Hinds, John (1974). Make mine BURAKU. Language research, 10, 92-108.

Itoo, Haku (1985/2002). Man'yoo-shuu (2 vols.). Tokyo: Kadokawa.

Kanda, Hideo \& Yoshimaro Oota (1962). Nihon koten zensho: Kojiki [Collected Japanese Classics: The Kojiki] (2 vols.). Tokyo: Asahi Shimbun-sha.

Kawamoto, Shigeo (1978). Kotoba no shikisai [Words of coloration]. Tokyo: Iwanami-shinsho.

Kay, Paul (2000). In defense of color categories in thought and language: A response to B. A. C. Saunders's review. American Anthropologist, 102, 321-323.

Kay, Paul \& Brent Berlin (1997). There are non-trivial constraints of color categorization. Behavioral and Brain Sciences, 20, 196-202.

Kay, Paul, Brent Berlin, \& William Merrifield (1991a). Bicultural implications of systems of color naming. Journal of Linguistic Anthropology, 1, 12-25.

Kay, Paul, Brent Berlin, \& William Merrifield (1991b). World color survey. San Antonio: Summer Institute of Linguistics.

Kay, Paul, Brent Berlin, Luisa Maffi, \& William Merrifield (1997). Color naming across languages. In C. Hardin and L. Maffi (Eds.), Color categories in thought and language (21-56). Cambridge: Cambridge University Press.

Kay, Paul \& Luisa Maffi (1999). Color appearance and the emergence of basic color lexicons. American Anthropologist, 101, 743-760.

Kay, Paul \& Chad McDaniel (1978). The linguistic significance of the meanings of basic color terms. Language, 54, 610-646.

Kelly, Kenneth, \& Deane Judd (1976). Color: Universal language and dictionary of names. Washington, DC: U.S. Department of Commerce.

Kobayashi, Shigenobu (1974). Nihon-jin no kokoro to iro [The heart of Japan and color]. Tokyo: Kodansha International.

Kobayashi, Shigenobu (1984). A book of colors. Tokyo: Kodansha International.

Kobayashi, Shigenobu (1981). The aim and method of the color image scale. Color Research \& Application, 6, 93-107.

Kobayashi, Shigenobu (1990a). Karaa-imeeji Sukeeru [Color image scale]. Tokyo: Kodansha.

Kobayashi, Shigenobu (1990b). Color image scale. Tokyo: Kodansha International.

Kojima, Noriyuki, Masatoshi Kinoshita, \& Akihiro Satake (1971-1975). Nihon koten bungaku zenshuu: Man'yoo-shuu [The collection of Japanese literary classics: The Man'yoo-shuu] (Vols. 1-4). Tokyo: Shoogakukan.

Kokuritsu Kokugo Kenkyuujo (Japanese National Language Research Institute) (1964). Gendai zasshi kyuujusshu no yooji yoogo [The vocabulary and Chinese characters of ninety magazines of today]. Tokyo: Shuuei Shuppan.

Kokuritsu Kokugo Kenkyuujo (Japanese National Language Research Institute) (1971). Denki-keisanki ni yoru shimbun no goi choosa [Studies on the vocabulary of modern newspapers] (Vol. 1). Tokyo: Shuuei Shuppan. 
Kokuritsu Kokugo Kenkyuujo (Japanese National Language Research Institute) (1972). Denki-keisanki ni yoru shimbun no goi choosa [Studies on the vocabulary of modern newspapers] (Vol. 2). Tokyo: Shuuei Shuppan.

Kokuritsu Kokugo Kenkyuujo (Japanese National Language Research Institute) (1973). Denki-keisanki ni yoru shimbun no goi choosa [Studies on the vocabulary of modern newspapers] (Vol. 3). Tokyo: Shuuei Shuppan.

Kokuritsu Kokugo Kenkyuujo (Japanese National Language Research Institute) (1974). Denki-keisanki ni yoru shimbun no goi choosa [Studies on the vocabulary of modern newspapers] (Vol. 4). Tokyo: Shuuei Shuppan.

Kondo, Tomie (2002). Fukusoo de tanoshimu Genji monogatari [Appreciating the clothing of the 'Tale of Genji']. Tokyo: PHP Kenkyuujo.

Levy, Ian Hideo (1981). Man'yooshuu (Vol. 1). Tokyo: University of Tokyo Press.

McNeill, Noriko (1972). Colour and colour terminology. Journal of Linguistics, 8, 21-33.

MacLaury, Robert (1992). From brightness to hue: An explanatory model of color-category evolution. Current Anthropology, 33, 137-186.

MacLaury, Robert (1995). Vantage theory. In J. Taylor and R. MacLaury (Eds.), Language and the cognitive construal of the world (231-276). Berlin: Mouton de Gruyter.

MacLaury, Robert (1997). Color and cognition in Mesoamerica: Constructing categories as vantages. Austin: University of Texas Press.

MacLaury, Robert (2001). Color terms. In M. Haspelmath et al. (Eds.), Language typology and universals: An international handbook (1227-1251). Berlin: Mouton de Gruyter.

MacLaury, Robert (Ed.) (2002). Vantage theory: Applications in linguistic cognition [Special issue]. Language Sciences, 24(5/6).

Masuda, Koh (Ed.) (1974). Kenkyusha's new Japanese-English dictionary. Tokyo: Kenkyusha.

Matsuoka, Takeshi (1999). Shikisai to shinri omoshiro jiten (shite yaku tatsu) [Interesting dictionary of hue and mental state (which are useful to know)] (rev. ed.). Tokyo: Mikasa-shoboo.

Nagasaki, Seiki (1983). Nihon dentoo shikisai-koo [A study of Japanese traditional colors]. Tokyo: Kyotoshoin.

Nagasaki, Seiki (2001). Nihon no dentoo shoku: Sono iro to shiki-shoo [Japanese traditional colors: Their color names and color tones]. Tokyo: Seigensha.

Nagata, Yasuhiro (2002). Iro no techoo: Nohon no go-hyaku-shoku [Notes on color: 500 Japanese colors]. Tokyo: Shookagukan.

Nakae, Katsumi (2003). Iro no namae de yomi toku Nihon-shi [Understanding Japanese history through color names]. Tokyo: Seishun-shuppan.

Nihon Shikisai Kenkyuujo (Japan Color Research Institute) (Eds.) (1984). Shikimei shooji-ten, kaiteiban [Concise manual of color names] (rev. ed.). Tokyo: Nihon Shiki Kenjigyoo Kabushikigaisha.

Nippon Gakujutsu Shinkookai (Japan Society for the Promotion of Scientific Research) (1965). The Man'yooshu. New York: Columbia University Press.

Ooyama, Tadasu (1994). Shikisai shinrigaku nyuumon [Introduction to color psychology]. Tokyo: Chuuoo Kooron-shinsha.

Ozaki, Saeko (2003). Onna o migaku chishiki-gaku [The science of how color develops womanhood]. Tokyo: Riyon-sha.

Philippi, Donald (Trans.) 1968). Kojiki. Princeton: Princeton University Press.

Sakamoto, Taroo, Saburoo Ienaga, Mitsusada Inoue \& Susumu Oono (Eds.) (1967). Nihon Koten Bungaku Taikei: Nihon-shoki [The great collection of Japanese literary classics: The Nihon-shoki] (2 vols.). Tokyo: Iwanami Shoten.

Satake, Akihiro (n.d.). [The character of color terms in ancient Japanese]. Files of Robert MacLaury (331343). 
Saunders, Barbara A. C. (1992). The invention of basic color terms. Utrecht: ISOR (Interdisciplinary Social Science Research Institute).

Saunders, Barbara A. C. (2000). Reply to Hardin \& Maffi \& Kay. American Anthropologist, 102, 323.

Saunders, Barbara A. C. \& Jaap van Brakel (1988). Re-evaluating basic color terms. Cultural Dynamics, 1, 359-378.

Saunders, Barbara A. C. \& Jaap van Brakel (1997). Are there non-trivial constraints on color categorization? Behavioral and Brain Sciences, 20, 167-212.

Saunders, Barbara A. C. \& Jaap van Brakel (Eds.) (2002). Theories, technologies, instrumentalities of color: Anthropological and historiographic perspectives. Lanham, MD: University Press of America.

Seidensticker, Edward (Trans.) (1976). The Tale of Genji by Murasaki Shikibu. New York: Alfred A. Knopf.

Shibugawa, Ikuyoshi \& Yumi Takahashi (1983) Hai-shok jiten [Color scheme dictionary]. Tokyo: Kazan Shoboo Shin-sha.

Shin-hen Kokka Taikan (The National Literary Collection, New Edition) (1983a). Chokusenshuu-hen: Kashuu [Imperial collections: Poems] (Vol. 1). Tokyo: Kadokawa.

Shin-hen Kokka Taikan (The National Literary Collection, New Edition) (1983b). Chokusenshuu-hen: Sakuin [Imperial collections: Index] (Vol. 2). Tokyo: Kadokawa.

Shin-hen Kokka Taikan (The National Literary Collection, New Edition) (1984a). Shisenshuu-hen: Kashuu [Private collections: Poems] (Vol. 1). Tokyo: Kadokawa.

Shin-hen Kokka Taikan (The National Literary Collection, New Edition) (1984b). Shisenshuu-hen: Sakuin [Private collections: Index] (Vol. 2). Tokyo: Kadokawa.

Shinmura, Izuru (Ed.) (1998). Koojien [Comprehensive Japanese dictionary] (5th ed.). Tokyo: Iwanami Shoten.

Stanlaw, James (1987). Colors, culture, and contact: English loanwords and problems of color nomenclature in modern Japanese. Doctoral dissertation, University of Illinois.

Stanlaw, James (1992a). Commentary on MacLaury's "From brightness to hue: An explanatory model of color-category evolution." Current Anthropology, 33, 167-168.

Stanlaw, James (1992b). Make mine BURAK's: Shedding light on brightness in Japanese-English color terminology. Paper presented at the 91st annual meeting of the American Anthroplogical Association, San Francisco, December 2-7.

Stanlaw, James (1993). Review of The Invention of Basic Color Terms by Barbara Ann Saunders. American Anthropologist, 95, 183-185.

Stanlaw, James (1997a). Two observations on culture contact and the Japanese color nomenclature system. In C. L. Hardin \& L. Maffi (Eds.), Color categories in thought and language (240-260). Cambridge: Cambridge University Press

Stanlaw, James (1997b). Making light of keeping color categories in the dark: Some arguments against the notion of trivial constraints in color nomenclature of Saunders and van Brakel. Behavioral and Brain Sciences, 20, 209-209.

Stanlaw, James (2004). Japanese and English language and culture contact. Hong Kong: Hong Kong University Press.

Takimoto, Takao \& Hideaki Fujisawa (1977). Nyuumon-shikisai shinri-gaku (Introduction to color psychology). Tokyo: Dai-nihon Tosho.

Tanaka, Ikko \& Kazuko Koike (1982). Japan color. Tokyo: Libro Port Co., Ltd.

Uchikawa, Keiji \& Robert M. Boynton (1987). Categorical color perception of Japanese observers: Comparisons with that of Americans. Vision Research, 27, 1825-1833.

Uemura, Rokuro and Yamazaki, Katuhiro (Eds.) (1950). (Kaitei) Nihon shikimei takian [(Revised) Japanese color term encyclopedia]. Kyoto: Yootokusha.

Wa-shoku Risuto (和色リ スト) (2003). Wa-shoku list of Japanese color terms. available at http:// tenka1.jp/ karasu_2/jcolor_list.htm 
Yamanouchi, Nobuko (2001). Karaa [but using the characters for 色彩] serabii [Color therapy]. Tokyo: KK Rongu Seraazu.

Yoskioka, Sachio (2002). Nihon no iro o someru [Dyeing Japanese colors]. Tokyo: Iwanami-shinsho. 


\title{
Color terms in Colonia Tovar, an Alemannisch Enclave in Venezuela ${ }^{1}$
}

\author{
Albert C. Heinrich \\ Corvallis, Oregon, USA
}

The Tovarese are located in a mountain valley some 40 kilometers west of Caracas. They are an ethnically distinct, endogamous group numbering some 3,000, mostly engaged in small-scale farming. Until about 1964 they were fairly well isolated from the rest of Venezuela, but since then - with the advent of a hard surfaced road - they are rapidly becoming integrated. Most speak Spanish; some older adults speak Modern Standard German (Schriftsdeutsch), and some of the middle-aged read it; and almost all speak the local form of Alemannisch. This is known both as dialekt and as baadiš, and, though many consider it as somehow inferior, they nonetheless regard it to be their own, proper ethnic-group-identifying language. It is the color terminology of this language that is under consideration.

1. "Color in Colonia Tovar: a Preliminary Report on the Color Terminology of an Alemannish Speaking Enclave in the Mountains of Northern Venezuela" was prepared in 1974 by Albert C. Heinrich, Ph.D., Department of Anthropology, University of Calgary, who sent duplicate copies to Brent Berlin and Paul Kay at the Language Behavior Research Laboratory, Department of Anthropology, University of California, Berkeley. They filed it with what was to become the manuscript collection of the World Color Survey, 1976. Dr. Heinrich's paper does not appear on the program of "Studies in the Domain of Color, I-II," a symposium coordinated by Nancy P. Hickerson at the 73rd Annual Meeting of the American Anthropological Association, Mexico, D.F., 1974, at which Dr. Heinrich was scheduled as a discussant. (Thanks to Stacy Lathrup of the AAA for pages 61 and 70 of the Preliminary Program, compiled July 1974.) Dr. Heinrich says he submitted his paper to that symposium, which is why the original includes references to twenty slides and not to figures; but he was unable to attend and never presented it elsewhere (personal communication, February 14, 2003). Dr. Hickerson (personal communication, February 17, 2003) offers, "I honestly can't remember what transpired" but recalls the program was full and Dr. Heinrich might have agreed to present his slides with his discussion. His paper lay dormant in the files until he permitted its publication here with his approval of editorial adjustments for printing. (R. E. MacLaury transferred the second copy to his files in 1986.) Both originals remain on file, and Dr. Hickerson retains a third original. Neither Dr. Heinrich's slides nor his diagrams of data that the slides depict are in the files, or were exhaustively sought in deep storage at the home of his son in Corvallis, the only other place they might be found. Dr. Heinrich had deposited his many academic records there about twenty years earlier, perhaps in 1983. - Editor. 


\section{Introduction}

The people under discussion are located in a mountain valley some 40 kilometers west of Caracas. They are an ethnically distinct, endogamous group numbering some 3,000, mostly engaged in small-scale farming. Until about 20 years ago [about 1964] ${ }^{2}$ they were fairly well isolated from the rest of Venezuela, but since then - with the advent of a hard surfaced road - they are rapidly becoming integrated. Most speak Spanish; some older adults speak Modern Standard German (Schriftsdeutsch), and some of the middle-aged read it; and almost all speak the local form of Alemannisch. This is known both as dialekt and as baadiš, and, though many consider it as somehow inferior, they nonetheless regard it to be their own, proper ethnic-group-identifying language. It is the color terminology of this language that is under consideration.

The speech form reference to Baden harks back to the homeland of the original settlers who came to Colonia Tovar early in the 1840s, which homeland was Schwarzwald, more specifically the Kaiserstuhl area of Germany. These people appear to have been mostly nonliterate rural people, fairly homogeneous in speech and culture. In the 1850s, a smaller contingent, mostly from Hessen, immigrated to the Colony, but these seem to have had little influence on the speech of the Tovarese. The present day dialekt is said, by those who have had an opportunity to make the comparison, to be very similar to the present-day Kaiserstuhl form of Alemannisch, but to have retained some older features now lost in the home area. I have not had opportunity to check this, but I did observe that there is one aspect of the color system and its lexemes (see brün, below) that is different from modern German and English, and which could be archaic.

The original purpose of the visit to Colonia Tovar - financed by a short-term grant from the University of Calgary - was mainly to explore the possibilities: whether these people would in fact be amenable to fieldwork; whether it would be worth the time and money to do fieldwork there, and what would be needed to carry on a study. The answers to the first two questions very positively were affirmative and encouraging, so much so that I even had time to do a bit of fieldwork after I got done with the orientation. I actually succeeded in interviewing six people fairly extensively during the three weeks I was able to spend in the colony. This is not a very large sample, nonetheless I feel that it yielded enough information to give a preliminary report what will be of value to those interested in the ethnosemantics of color.

2. Editor. 


\section{Data}

Five persons spontaneously (in absence of visual stimuli) gave six color terms - which I label their "firm", or primary colors. ${ }^{3}$ These were: grian 'green,' ro:t 'red,' bloi 'blue', ga:l 'yellow', šwats 'black,' and vi:s 'white,' usually given in that order. When prompted for more terms, most of them - with some hesitation - gave brün. When prompted more, most informants gave terms for Orange, Brown, Grey, and Purple in rapid succession. Most made reference to most of these added domains, but all did so hesitantly and tentatively, some even stating that they were not sure whether these really were proper color terms. The various terms employed were: hálrot/gálrot/aróse/naránalonóto for Orange; ĉokolá:t/kaksi/maróy/braun for Brown; ašəfá:rb/ašrí:k/gri:s/groi/grau/hálšwats for Grey/ and muráu/violét for Purple. These I refer to as secondary, or nonprimalry, colors for this system. The Tovarense appear to currently be in the process of adding these color categories to their system but have not yet standardized the (apparently borrowed) terminology.

\section{Primary Colors}

The primary colors conform to the postulated Stage V of the Berlin and Kay formulation, but their domains are more like that of a fully developed Stage VII type, that is, of somewhat narrower ranges. ${ }^{4}$ I have sorted out the individual colors and their domains as follows.

Red has the domain that we would expect. It is more restricted than the domain of Red is among the Central Canadian Eskimos (Heinrich 1972, 1974), who do not have Purple as a named color category;

Yellow also has a smaller range than Eskimo Yellow, but it has the same sorts of leftdownward and right-downward extensions of Yellowish;

Green (along with Blue) is not as firm a color category as we, intuitively, would expect, but it is a Tovarense category. As is the case with those Central Eskimos who are bi-morphemic for Green/Blue (but not for those who are mono-morphemic for these two colors), there is some overlap of the domains of Yellow, Green and Blue - at least the peripheral areas are not well separated;

Blue, in addition to the complication with Green mentioned above, has an "adhesion" with brün, and with Grey and Black. The very dark Blues are sometimes called Black or Blackish; sometimes they are called brün, and sometimes informants (conversely) tentatively call Greyish chips bloi. This seems to be connected with the phenomenon or phenomena related to brün (see below);

3. They were simply asked to recite the color terms that readily and immediately came to mind, "What color names do you know?"

4. Data gathering here consisted of having informants go through the Munsell Books of Color and name those chips they recognized and then my going over the Books of Color again and asking for terms for areas (usually unsaturated) that they had passed by during the first going-over. 
Black is generally restricted to the very darkest of the achromatic chips, except that the Light Value 2 BG, B and PB chips were called Black, and some Light Value $2 \mathrm{R}$ chips were called Blackish. The latter was also observed among Canadian Eskimos, and the tendency to have a Black "upward bulge" in the lowered Light Value region of the BG, B and PB range was also very marked among them.

White is restricted almost entirely to the very lightest of the achromatic chips, except that there is a tendency to call Light Value 9 BG, B and R chips White or Whitish.

$\underline{B r u ̈ n}$ is apparently something different from šwats, but it occurs in much the same place where the "upward bulge" or black occurs, and its domain appears to be larger. It appears to go further upward into the higher Light Value ranges and seems to encompass what we popularly call "powder blue," and this apparently results in some terminological confusion with both Blue and Grey. Another source of confusion lies in the fact that the (apparent) modern German cognate of brün, braun, refers to something else, to Brown. Though used interchangeably to some extent, statistical analyses of the responses (report on file with the University of Calgary Grants Office) show that (for this sample at least) brün is more frequently used to designate medium to dark unsaturated Blues, and braun is more frequently used to designate Munsell chips that we would call Brown or Brownish in English. Brün apparently was a name for a color category of the original system of the Tovarense, but a category which is now on its way out.

\section{Secondary Colors}

The colors that now seem to be becoming firmly entrenched, possibly through acculturation, all bear borrowed names. Name borrowing, and apparently category borrowing, has been from both Modern Standard German and from Venezuelan Spanish, with the latter apparently predominant and becoming more so. Informants differ with one another, and even with themselves from one time to another, as to (1) how many secondary colors there are, and (2) what the proper terms are. But, with the partial exception of Brown, the domains of the colors that are named show a remarkable consistency, as do the focal chips. This quality, it should be noted, holds for most colors, regardless of whether primary, archaic or secondary.

Orange focuses on the high chroma Yellowish - Reds (10R5/16, 10R6/16, 2.5R6/16, 5YR6/16, 7.5YR7/16). Terms used to identify this color are hálrot 'light red', gálrot 'yellow red,' narána 'orange,' onóto 'paprika, ${ }^{5}$ and eróšz (not identified). The range of the domain for Orange is very restricted.

Brown is rendered by cokolá:t (chocolate), marón (chestnut), kaksi (cocoa), braun (from modern German), and sometimes by brün. Most browns are found where English brown occurs, among the darker saturated YR chips, but there were hints of the unsaturated purplish sort of brown found among Canadian Eskimos. Eskimo actually has two

5. Ultimately derived from urucú, Bixa orellana. 
allomorphs for 'brown,' qayuq and qatcuixtuq, and this may relate to the etiology of the present bi-modal distribution of Eskimo 'brown.'

Grey is rendered by ašafárb/ašri:k 'ash-colored/ashey', gri:s (from Spanish), grau (from modern German), hálšwats 'light black,' and groi. The latter term would conform to the Tovarense Schriftsdeutsch-au/-oi correspondence (cf. bloi/blau). This is not necessarily proof of great age. The term could have arisen quite recently by analogy. Grey takes in most of the low Chroma, medium Light Value chromatic chips as well as the medium Light Value achromatics. Infrequently bloi is used to designate chips that are unmistakably grey.

Purple is usually rendered by muráu (from Spanish) and less frequently by violét. Sometimes marón and muráu are confused and transposed, but statistical analysis clearly demonstrates that the correct usage is the former for brown and the latter for purple.

\section{Propositions}

In preparation for further research, I advance the following:

1. There are certain Munsell chips that appear to universally attract the attention of humans, at least of those that speak Eskimo, Alemannisch or English. These very often are those chips that have higher Chroma values.

2. Brown seems to be an exception to the above. What we, in English, recognize as brown seems to be composed of two things - saturated (but not maximum Chroma) low Light Value YR, and unsaturated, low Light Value P.

3. The focal points of any chromatic color category except brown, regardless of terminology, will always be restricted to a certain few high Chroma chips. These show remarkable consistency from one informant to another and for the same informant over time.

4. The nonfocal portions of any chromatic color domain are always of lower Chroma value. These do not show the stability and consistency of the foci.

5. There is a great deal of inter- and intra-informant variability in the matter of which and how many color percepts the perceiver employs. Percepts in this sense are the intra personal analogs of physically identifiable constant focal points and domains. This is not variability in perception (except in cases of visual deficiency), but in the model he happens to be using at that time.

6. The Tovarense apparently did not have grey, orange, purple, and brown as named percepts when they came to settle in Venezuela, but they apparently had brün.

7. Brün, cognate with both 'brown' and 'brunette,' apparently had in common with brown that it was unsaturated and/or nonmaximal Chroma, but differed from it in that it was located on different hues, that is, it was focused on the (present-day) upward bulge of Black. Brün had in common with grey that it, too, encompassed many low-chroma colors. Modern Tovarense brün is probably a survival of an older Alemannisch color category that is now being taken over by grey and blue. 
8. Grey, orange, purple, and brown apparently were not percepts of the original settlers of Colonia Tovar. These are now being added, in the places where, because of psychophysical factors, these colors are usually found.

9. As a general trend (data not given here) the primary color terms tend to be nonallomorphic for the foci and then to have a proliferation of modified terms for the nonfocal portions of each domain, whereas the secondary color terms tend to have a variety of allomorphs for the foci but to have a very restricted total domain, for which modified terms have not yet developed.

\section{References}

Heinrich, Albert C. (1972). A non-European system of color classification. Anthropological Linguistics 14, 220-227.

Heinrich, Albert C. (1973). Systematics of Canadian Eskimo color terminology. Unpublished report, Canada Council.

Heinrich, Albert C. (1974). Color classification of some Central Canadian Eskimos. Arctic Anthropology $11,68-72$.

Heinrich, Albert C. (1974). Color in Colonia Tovar: A preliminary report on the color terminology of an Alemannisch speaking enclave in the mountains of northern Venezuela. Unpublished manuscript, University of Calgary.

Heinrich, Albert C. (1977). Some notes on Central Eskimo color terminology. In William C. McCormack \& Stephen Wurm (Eds.) Language and thought: Anthropological issues (45-59). The Hague: Mouton.

Heinrich, Albert C. (1978). Changing anthropological perspectives on color-naming behavior. Journal of Psychological Anthropology 1, 341-363.

Heinrich, Albert C. (1981). The colors of Kannada. South Asian Anthropologist 2, 37-47.

Heinrich, Albert C. (n.d.). Ethnosemantics of color: Some preliminary observations on color naming behavior in three societies. Unpublished manuscript. 


\title{
Mien (Yao) color terms
}

\author{
Theraphan L-Thongkum \\ Department of Linguistics, Chulalongkorn University, Bangkok, Thailand
}

The aims of this research project are to investigate color terms, the formation of color terms and the evolution of basic color terms in the Mien language, and the attitudes towards colors of the Mien, living in northern Thailand. The data on Mien colors were collected by interviewing 48 female informants, 20-30 years old, in six villages in the provinces of Chiangrai, Phayao, Nan and Lampang, northern Thailand. Two hundred and twenty one color chips cut from a color chart used in the printing business were used for data elicitation. The research results are as follows: there are six basic color terms in the Mien language, $p \varepsilon^{221}$ WHITE, $c i a^{345}$ BLACK, $s i^{345}$ RED, $j a \eta^{31}$ YELLOW, $m \varepsilon^{33}$ GREEN and $b u a^{454}$ BLUE. The Mien language is thus in Berlin and Kay's Stage V of color term evolution. It is on its way to reach the sixth stage; that is, the word lua PURPLE is becoming a new basic color term. This finding contradicts the theory that the seventh basic color term in Stage VI should be BROWN.

\section{Introduction $^{1}$}

During the Ming and Qing dynasties of China, there was a saying that "There is no mountain without the Yao" (The Yao nationality, 1990: 8). The Yao ethnic group is regarded as one of the 56 "nationalities" of China. The Yao are an indigenous people of southern China. They are scattered in 140 counties in the provinces of Guangxi (2.13 millions, or $60 \%$ of the Yao population in China), Hunan, Yunnan, Guangdong, Guizhou, and Jiangxi. ${ }^{2}$ About 200 years ago, the Yao began to migrate to Vietnam, Laos and Burma, from southern China, and then to Thailand.

In the past, the Yao lived in the Huanghe or 'Yellow River' basin. Later on, during the reigns of Emperor Huangdi and Emperor Yanti, they started to migrate southwards.

1. The main content of this paper is from the author's research report written as a pamphlet in Thai (L-Thongkum 1992). The project on Mien (Yao) Color Terms is a sub-project of the major project "A Comparison of ThailandYao and Guangxi-Yao" funded by the Toyota Foundation (Tokyo) from 1988 to 1990.

2. On the basis of language and clothing, the "Miao nationality" of Hainan Island is not Miao but a sub-group of Landian Yao or Kim Mun who migrated from Guangxi Province to Hainan Island four hundred years ago. At present, they are scattered in Baoting, Qiongzhong and Baisha counties. (This information is from the author's field notes collected in Hainan in May 1992.) 
A great migration took place in the Qin and Han dynasties. In the Ming dynasty, a large number of Yao people were suppressed or killed. Consequently, they moved to live in the mountains and jungles of Guangxi, Guangdong and Yunnan. Around 1819, they migrated further south to the northern areas of Southeast Asia where there were high mountains and jungles. Later, their peaceful life in the highlands was ended by the liberation wars in China and Indo-China. Some of the wealthy and powerful Yao who did not want to join the communist regimes were forced by the circumstances to leave their home and migrate to the United States of America, France and Canada (Huang Yu 1990: 10-11).

Based on linguistic evidence, the Yao nationality of China can be classified into four major sub-groups: Mien (Iu Mien), Kim Mun, Byau Min and Yau Min (Pan Chengqian 1991: 47-48). The Yao (about 35,000) living in northern Thailand belong to the Mien sub-group. They are scattered in the provinces of Chiangmai, Chiangrai, Phayao, Nan, Lampang, Kamphaengphet and Sukhothai (Tribal Social Welfare Department 1986).

\section{Procedure}

The data on Mien color terms were collected by interviewing 48 female informants, 2030 years old, in six villages (eight informants per village) in the provinces of Chiangrai, Phayao, Nan and Lampang where the majority of the Mien live. The six villages are shown in Table 1.

The six villages were chosen as the research sites on seven criteria:

1. population not less than two hundred;

2. located in the provinces where a large number of the Mien lives;

3. at least a dirt road;

4. lodging available for about one week;

5. safe, clean, and free of narcotics;

6. villagers harbor no hostility toward outsiders;

7. authorities cooperate and take responsibility for security.

The field surveys were conducted from October 1988 to March 1989, and the data on Mien color terms were collected from May to June 1989. The research team comprised the author and two research assistants who were graduate students in the Linguistics Department at Chulalongkorn University.

Table 1. Villages from which data were collected

\begin{tabular}{llll}
\hline Village & Sub-district & District & Province \\
\hline Kiwtam & Pongtao & Ngaw & Lampang \\
Paklang & Silalaeng & Pua & Nan \\
Maipangkha & Phachangnoi & Pong & Phayao \\
Huaimaesay & Maeyaw & Mueng & Chiangrai \\
Khunmaebong-mai & Pongnoi & Maechan & Chiangrai \\
\hline
\end{tabular}


Two hundred and twenty color chips cut from a standard color chart used in the printing business were used for data elicitation. Each color chip was pasted on a $2 \mathrm{in} . \times 2.5 \mathrm{in}$. $(5 \mathrm{~cm} \times 6.25 \mathrm{~cm})$ piece of white cardboard. One blank-cardboard representing white color was added; altogether there were 221 color chips. The numbers 1-221 were written on the upper right corner of the cards. The order of these 221 cards could be matched with the order of the color chips on the Graf-G Process Color Chart of Dainippon Ink and Chemicals, Incorporated (DIC), so that the data elicited from each informant could be compared and plotted on the diagram. Underneath each color chip, a hue, for example M100 or pure magenta, was specified. ${ }^{3}$

The color chips cut from the Graf-G Process Color Chart were used for practical reasons: ${ }^{4}$

1. Five hundred copies of the color chart, which would accompany the five hundred copies of the published research report could be ordered from an agent of DIC in Bangkok;

2. The price was reasonable;

3. Only one copy of the Munsell Color Chart, which could be used as standardized color stimuli, was available in the departmental library;

4. When the Munsell Color Chart was used during the field surveys, the unsophisticated Mien informants were unable to cope with the 329 color chips, which were equally spaced with even degrees of brightness and at maximum saturation. To avoid predictable problems, less complex color stimuli were used to suit the circumstance.

Besides the 221 color cards, two types of worksheet were designed, one for recording the data on Mien color terms (see Figure 1 in Appendix 1) and another for recording the attitudes of the Mien towards colors. For each informant, one worksheet of the first type was used; and for each research location, five worksheets of the second type were used. Approximately three hours were spent for an interview with one informant. The data were collected only in the morning from 9 a.m. to 12 noon and were carefully checked in the afternoon. The interviews were conducted in the shady areas outside the informants' houses.

At each field site, after making appointments with the eight selected informants, the two research assistants $\left(\mathrm{RA}_{1}\right.$ and $\left.\mathrm{RA}_{2}\right)$ followed the same procedure when they interviewed each of the informants. The procedure consists of five steps. The first four steps were repeated 221 times. If something went wrong, sooner or later the mistake could be detected.

3. The codes for colors comprise four Roman letters: $\mathrm{Y}$ (yellow), M (magenta), C (cyan) and BL (black), and six sets of Arabic numbers: 10, 20, 40,60, 80 and 100. These numbers indicate the degrees of brightness and saturation; for examples, $\mathrm{Y} 100=$ yellow, M100 = magenta, C100 = cyan, BL100 = black, Y100 M100 = red, Y100 C100= green, M100 C100 = dark blue, M80 C60 = purple, Y60 M60 BL40 = brown, Y60 M60 = orange and so on. Since there is no color chip and no code for white, the codes for pink (magenta + white) and grey (black + white) are M40 and BL40, respectively.

4. The Graf-G Process Color Chart has some weak points. There are too many hues of green color, and the color chips no. 21 (Y100 M100) 'bright red' and no.101 (Y100 C100) 'bright green' are repeated twice as no. 26 (Y100 M100) and no. 106 (Y100 C100) in order to obtain a symmetrical color chart of $220(11 \times 20)$ color chips. 
When $\mathrm{RA}_{2}$ suspected that the color word given by the informant was not a basic color term, he would ask for the meaning of each part of the color word. After resting for a while, the second half of the interview (the fifth step) was begun. The five steps were:

1. The two research assistants tried to create a friendly atmosphere, by chatting with the informant and also explained what was supposed to be done when they were playing the "card games."

2. The necessary information was filled in the worksheet used for recording the data on color terms elicited from each informant, such as the informant's name and age, village name, date, and so on.

3. After shuffling the 221 color cards, $\mathrm{RA}_{1}$ picked up the cards one by one, read out the number of the color chip, showed it to the informant, and asked the question in Mien "What is this color?" She also said something else to make the informant feel at ease and eager to continue playing the card games with her.

4. $\mathrm{RA}_{2}$ searched for the number read out by $\mathrm{RA}_{1}$ on the worksheet (Figure 1) and transcribed the color term in IPA phonetic symbols.

5. The informant was asked to divide the 221 color chips into five groups based on her personal attitudes: very beautiful, beautiful, plain, ugly, and very ugly. For each color group, $\mathrm{RA}_{1}$ read out the number on every card, and $\mathrm{RA}_{2}$ wrote the number down on the worksheet.

\section{Results}

Since the aims are to investigate the system of basic color terms - that is, formation of color terms, evolution of basic color terms in the Mien Yao language, and attitudes of the Mien towards colors - results will be presented in that order.

\section{Color terms and color categories}

Berlin and Kay (1969) say that although different languages encode in their vocabularies different numbers of basic color categories, a total universal inventory of eleven basic color categories are WHITE, BLACK, RED, YELLOW, GREEN, BLUE, BROWN, PURPLE, PINK, ORANGE and GREY. Kay (1975) adjusts the concept of universality of color categories by adding one more color category, i.e., GRUE (green + blue) which later becomes two separate categories, green and blue. Kay and McDaniel adapt the previous views and propose their theory of fuzzy sets. According to this theory, there are three types of basic color categories: primary basic color categories (Stage V), composite basic color categories (Stage I-Stage IV), and derived basic color categories (Stage VI-Stage VII).

5. The author usually sat near them but pretended that there was no interest in the on-going activity, in order to reduce the stress and strain of the informants as well as of the two research assistants. Notes and remarks were recorded for data checking in the afternoon sessions. 
The Mien basic color terms are determined by the characteristics suggested by Berlin and Kay (1969), Hickerson (1971), and Crawford (1982). It can be concluded that the Mien language contains six basic color terms: $p \varepsilon^{221}$ 'WHITE,' cia ${ }^{345}$ 'BLACK,' $\mathrm{il}^{245}$ 'RED,' $j a \eta^{31}$ 'YELLOW', $m \varepsilon \eta^{33}$ 'GREEN' and $b u a^{454}$ 'BLUE.' ${ }^{6}$ These six basic color terms encode the six primary basic color categories.

The 48 Mien informants classified the 221 color chips into six basic color categories of which the details are given below (See Figure 2 in Appendix 2.):

1. The category $p \varepsilon^{321}$ 'WHITE' consists of only one hue, i.e., no. 221.

2. The category cia ${ }^{45}$ 'BLACK' consists of nine dark hues. No. 16 (BL100) can be regarded as the representative of this color category, since $100 \%$ of the informants classified the color chip no. 16 as $\mathrm{cia}^{345}$.

3. The category $s i i^{i 45}$ 'RED' consists of twelve warm hues. The most salient hue of the $s i^{i 45}$ category is no. 6 (M100).

4. The category $j a \eta^{31}$ 'YELLOW' consists of twelve bright warm hues as the $s i^{i 45}$ category. The color chip no. 1 (Y100) represents this color category.

5. The category $m \varepsilon \eta^{33}$ 'GREEN' consists of thirty-nine cool hues. The color chip no. $101 / 106$ (Y100 C100) is the best representative of this color category.

6. The category bua $a^{454}$ 'BLUE' consists of seven bright cool hues. The color chip no. 11 (C100) represents this color category.

It is noticeable that the Mien informants seem to perceive the hues of $m \varepsilon \eta^{33}$ 'GREEN' very well. But the Graf-G Process Color Chart contains a lot of green chips.

\section{Color term formation}

Regarding color term formation, besides the six basic color terms presented in the previous section, when the Mien want to coin some color terms in order to describe some particular colors with which they are less familiar, three devices have been in use:

1. Device 1: Basic color term + basic color term, such as $j a \eta^{31} s^{i 45}$ 'yellowish orange (yellow + red),' $s i^{221} \mathrm{ja \eta}^{31}$ 'reddish orange (red + yellow)';

2. Device 2: Basic color term + adjective, such as $m \varepsilon \eta^{31}$ bjaan $^{33}$ 'very pale green (green + pale),' $m \varepsilon \eta^{31}$ siam $^{33}$ 'bright green (green + clear),' $m \varepsilon \eta^{31} k_{w a t}{ }^{45}$ 'subtle green (green + dirty),' $m \varepsilon \eta^{31} l u n^{35}$ 'pale green (green + young)';

3. Device 3: Comparison with the colors of natural objects, such as $j a \eta^{31} \mathrm{gaj}^{454}$ 'brownish yellow (yellow + excrement),' si ${ }^{221}$ dzaam ${ }^{454}$ 'scarlet (red + blood), and so on.

The second device is the most common. Some adjectives are more frequently used as attributes than the others; for examples, $l u n^{35}$ 'young, immature,' $k o^{35}$ 'old, mature,' $h_{m u a \eta} 232$ 'dark,' kwaj 454 'ordinary', bjaan 33 'pale, tasteless,' niam ${ }^{232}$ 'raw, green (of fruit),' $l o^{35}$ 'unclear, old (of things),' gaaj ${ }^{33}$ 'dry,' kwat ${ }^{45}$ 'dirty', bəw ${ }^{22}$ 'clear, clean (of water)', lu $u^{22}$

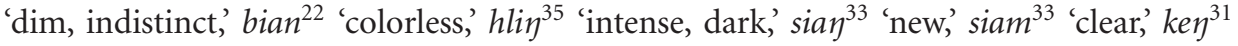

6. Due to the tone sandhi rules, i.e., $45>21,33$ and $454>31$, then, $c i a^{345}$ 'BLACK', $m \varepsilon \eta^{33}$ 'GREEN' and $b u a^{454}$ 'BLUE' always become $c i a^{221}, s i^{221}, m \varepsilon \eta^{31}$ and $b u a^{31}$. 
'true,' cian $^{33}$ 'real,' hmat ${ }^{45}$ 'intensifier used for black', $t e^{35}$ paa $^{454}$ 'a little bit,' waaj 22 'ugly', biaw ${ }^{35}$ 'blurred, vague.'

\section{Basic color term evolution}

The Mien language possesses the color system which comprises six basic color terms: $p \varepsilon^{221}$ 'WHITE', cia ${ }^{245}$ 'BLACK,' si $i^{345}$ 'RED,' jay ${ }^{31}$ 'YELLOW', $m \varepsilon \eta^{33}$ 'GREEN' and bua 454 'BLUE.' This finding supports very well Berlin and Kay's claim on the universality of basic color term evolution, i.e., there are six basic color terms in their Stage V.

However, there is another important finding in Mien. From the interviews, the author discovered that the Mien language spoken in northern Thailand is on its way to containing a new basic color term. 'PURPLE' is becoming the 7th basic color term, not 'BROWN', in Stage VI of the basic color term evolution, as proposed by Berlin and Kay (1969), Kay (1975), and Kay and McDaniel (1978).

The Mien informants did not recognize the brown color. When the brown color chips were shown to them during the interviews, their first response was "I do not know." After thinking very hard, they came up with inconsistent answers. For example, they responded to the color chip no. 42 (Y100 M80 BL60), which is a shade of brown, as $s i^{245}(39.58 \%)$, cia $^{345}(33.33 \%)$, bua $^{454}(14.58 \%)$, lua $a^{221}(8.33 \%)$, and $j a \eta^{31}(4.17 \%)$.

It looks as if the color term lua $a^{221}$ 'PURPLE' in Mien would be the 7th basic color term. For examples, the color chip no. 63 (M100 C60), reddish purple was identified as bua ${ }^{454}$ (56.25\%), $l u a^{221}(37.50 \%)$, and $s i^{245}(6.25 \%)$; and the color chip no. 61 (M100 C100), bluish purple, was identified as bua $a^{454}(60.42 \%), l u a^{221}(37.50 \%)$, and $s i^{245}(2.08 \%)$. This phenomenon reflects the fact that $l u a^{221}$ is regarded as a separate color category by some of the Mien informants (about 38\%). Like the other Mien basic color terms, it can have an attribute and also could be an attribute of the other basic color terms; for examples, lua ${ }^{221}$ lun $^{35}$ 'pale purple,' lua ${ }^{321} \mathrm{ko}^{35}$ 'dark purple,' lua ${ }^{321}$ bian $^{35}$ 'faded purple,' lua ${ }^{321}$ bjaan $^{33}$

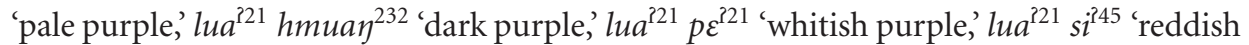
purple,' lua $a^{321}$ bua $^{454}$ 'bluish purple', lua ${ }^{321}$ cia $^{345}$ 'blackish purple', $p \varepsilon^{221} l u a^{321}$ 'purplish white,' si ${ }^{i 21} l u a^{221}$ 'purplish red,' bua ${ }^{31} l u a^{221}$ 'purplish blue.'

Moreover, the Hmong language, belonging also to the Hmong-Mien (Miao-Yao) language family, has seven basic color terms: dau ${ }^{35}$ 'WHITE', $d u^{35}$ 'BLACK, lia ${ }^{55}$ 'RED,' $d a^{51}$ 'YELLOW', d $3 u a^{55}$ 'GREEN,' sia ${ }^{35}$ 'BLUE,' and $t$ sho ${ }^{33}$ 'GREY.' The seventh basic color term is 'GREY' not 'BROWN' as proposed by Berlin and Kay.

Since 'BROWN' is not the seventh basic color term in either Mien or Hmong, even though the two languages belong to the same language family, perhaps it is too early to postulate a definite evolutionary sequence after Stage V of the color category evolution. Using the same research methodology, more data on unwritten languages should be collected. Then, a more definite answer on the universality of basic color terms and of basic color categories can be made. It is worthwhile to point out that most of the word lists used for investigating the color vocabulary in unwritten languages spoken by the ethnic

7. Data on White Hmong basic color terms are from the author's field notes. 
minorities of Southeast Asia include only five color items, namely 'white,' 'black,' 'red,' 'yellow', and 'green.' The rest of the color words seem to be ignored, especially due to the fact that they are rather useless for a comparative historical study. A typological study of color systems in world languages spoken by unsophisticated peoples should be formally conducted. When more solid evidence is obtained, then, we will certainly know what are the basic color categories in Stage VI of the evolution. Five more stages of the evolution after Stage V, Stage VI-Stage X, might be possible. The author would like to hypothesize that 'BROWN,' 'GREY,' or 'PURPLE' can have an equal chance of becoming the seventh basic color term in the sixth stage, not only 'BROWN' as previously postulated. So far, the findings obtained from a brief survey of the recent basic-color term studies, especially of the Southeast Asian languages, do not contradict the author's hypothesis.

\section{Attitudes towards colors}

The research result indicated that most of the hues on the Graf-G Process Color Chart were considered very ugly or ugly by the Mien informants, due to the fact that it contains a lot of pale and subtle hues which are very hard to identify. When the five scales of attitudes: very beautiful, beautiful, plain, ugly, and very ugly, were reduced to three scales of interpretation: positive, neutral and negative, to meet the criterion that at least 80 percent of the informants must share the same opinion, the results can be summarized as follows:

1. In general, vivid and dark colors were regarded as positive, while subtle and pale colors were regarded as negative.

2. Among the 20 positive hues, six hues were regarded as very beautiful. The degrees of beauty can be ranged from the most beautiful to the less beautiful ones: no. 29 (Y40 M100), no. 11 (C100), no. 30 (Y20 M100), no. 12 (C80), no. 26 (Y100 M100), and no. 70 (M20 C100). For the Mien, shocking pink (magenta), bright red (scarlet), and bright blue (cyan) seem to be their favorite colors. The colors of the cotton yarns used for their famous embroideries also reconfirm this finding. See examples in ButlerDiaz (1981).

3. Among the 22 negative hues, six hues were considered very ugly. The following order indicates the degrees of ugliness ranged from the ugliest to the less ugly ones: no. 54 (Y20 M20 BL20), no. 191 (Y20 M10 C10), no. 207 (Y40 M40 C40), no. 190 (Y20 M20 C10), no. 171 (Y10 M20 C20), and no. 211 (Y10 M20 C10). All of these are subtle brownish colors.

4. The rest of the hues including black and white were neutral. This means that the Mien have neither positive nor negative attitude towards most of the colors, about 179, represented by the hues on the Graf-G Process Color Chart.

\section{Conclusion}

The Mien (Yao) language contains six basic color terms: $p \varepsilon^{221}$ 'WHITE,' cia ${ }^{345}$ 'BLACK,' $s i^{245}$ 'RED,' $j a \eta^{31}$ 'YELLOW' $m \varepsilon \eta^{33}$ 'GREEN' and $b u a^{454}$ 'BLUE.' Regarding color term 
formation, three devices have been in use: basic color term (head) + basic color term (attribute), basic color term (head) + adjective (attribute), and comparison with the colors of natural objects. The second device is the most common. Regarding the evolution of basic color term, lua $a^{321}$ 'PURPLE' is becoming the seventh basic color term. This contradicts the theory proposed by Berlin and Kay that the seventh basic color category in Stage VI of the basic color category evolution is 'BROWN.' The color systems of Mien and Hmong, languages in the Hmong-Mien language family, suggest that the seventh basic color term (Stage VI) is 'PURPLE' or 'GREY.' After Stage V, the concept of universality does not seem to work well. Therefore, a reanalysis of the sets of derived basic color categories after Stage $\mathrm{V}$ should be done when more solid data are available. Regarding the attitudes towards colors of the Mien, vivid and dark colors are more preferable than subtle and pale colors. To the Mien, shocking pink (magenta), bright red (scarlet) and bright blue (cyan) are the most beautiful colors.

\section{References}

Berlin, Brent \& Paul Kay (1969). Basic color terms: Their universality and evolution. Berkeley: University of California Press.

Butler-Diaz, Jacqueline 1981). Yao design of northern Thailand. Bangkok: The Siam Society.

Crawford, T. D. (1982). Defining "basic color terms." Anthropological Linguistics, 24, 338-343.

Hickerson, Nancy P. (1971). Review of "Basic Color Terms." International Journal of American Linguistics, $37,257-270$.

Huang Yu (1990). Formation, distribution and development of the Yao nationality. In The Yao nationality. Nanning: The Nationality Affairs Commission of Guangxi Zhuang Autonomous Region.

Kay, Paul (1975). Synchronic variability and diachronic change in basic color terms. Journal of Language in Society, 4, 257-270.

Kay, Paul \& Chad K. McDaniel (1978). The linguistic significance of the meanings of basic color terms. Language, 54, 610-646.

L-Thongkum, Theraphan (1992). Khamriak si nai phasa Yao (Mien) [Color Terms in Yao]. Bangkok: Rongphim, Chulalongkorn University.

Nationality Affairs Commission of Guangxi Zhuang Autonomous Region (1990). The Yao nationality. Nanning: Guangxi.

Pan Chengqian (1991). Yao dialectology. In Jacques Lemoine \& Chiao Chien (Eds.), The Yao of South China: Recent international studies (47-70). Paris: Pangu, Editions de l'A.F.E.Y.

Tribal Social Welfare Department (1986). Tribal village names. Bangkok: Ministry of Interiors. 


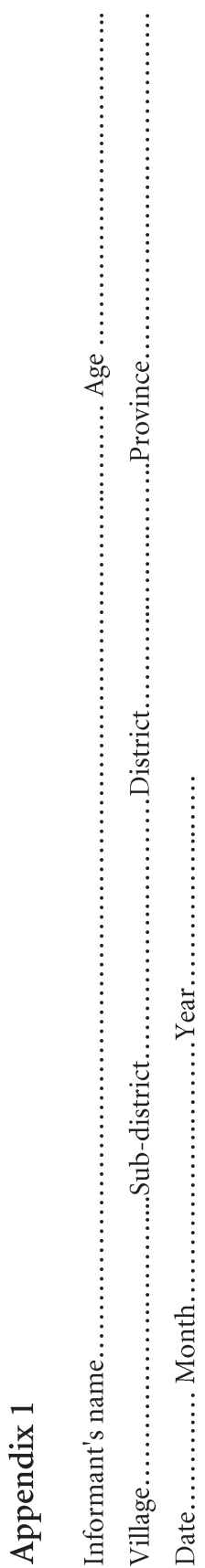

\begin{tabular}{|c|c|c|c|c|c|c|c|c|c|c|c|}
\hline$\stackrel{i}{ }$ & 아 & 8 & $\infty$ & 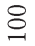 & తి & 요 & $\stackrel{8}{0}$ & $\stackrel{\triangleright}{\stackrel{\infty}{二}}$ & 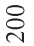 & तి & \\
\hline$\curvearrowright$ & $\dot{m}$ & in & হ & ন & $\stackrel{\partial}{\exists}$ & $\stackrel{\vec{m}}{-}$ & 点 & $\stackrel{\curvearrowright}{\curvearrowright}$ & ळ & $\stackrel{\vec{\sim}}{\sigma}$ & $\overrightarrow{\widetilde{N}}$ \\
\hline$\stackrel{\infty}{-}$ & $\stackrel{\infty}{m}$ & $\stackrel{\infty}{i n}$ & $\stackrel{\infty}{\curvearrowright}$ & $\infty$ & $\stackrel{\infty}{=}$ & $\stackrel{\infty}{\sim}$ & $\begin{array}{l}\infty \\
\stackrel{\sim}{\sim}\end{array}$ & $\stackrel{\infty}{=}$ & $\stackrel{2}{\leftrightharpoons}$ & $\stackrel{\infty}{\vec{\sim}}$ & \\
\hline
\end{tabular}

$\therefore \hat{m}$ 向

$\because$ 문

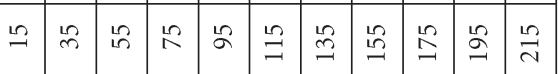

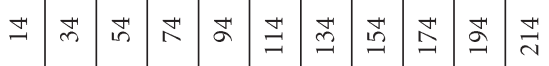

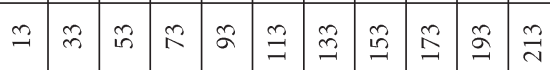

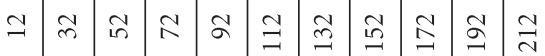

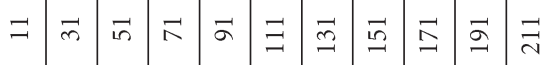

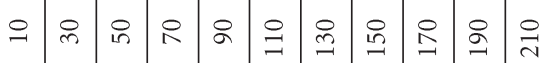

๙

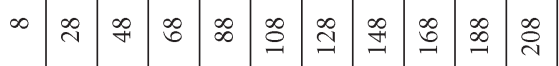

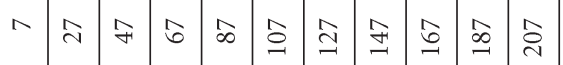

•

的

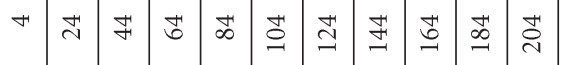

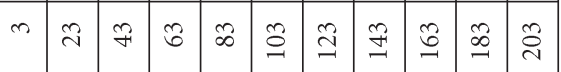

\begin{tabular}{|c|c|c|c|c|c|c|c|c|c|c|}
\hline$N$ & $\approx$ & $\mathcal{F}$ & గ్ర & $\infty$ & 으 & $\widetilde{\Xi}$ & $\mathcal{I}$ & $\widetilde{\sigma}$ & $\underset{\sim}{\infty}$ & రి \\
\hline-1 & $\vec{\sim}$ & $F$ & $\vec{\sigma}$ & $\bar{\infty}$ & 흐 & $\vec{\beth}$ & $\exists$ & $\vec{\sigma}$ & $\vec{\infty}$ & $\overrightarrow{\mathrm{d}}$ \\
\hline
\end{tabular}

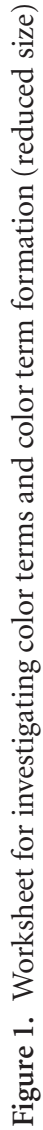




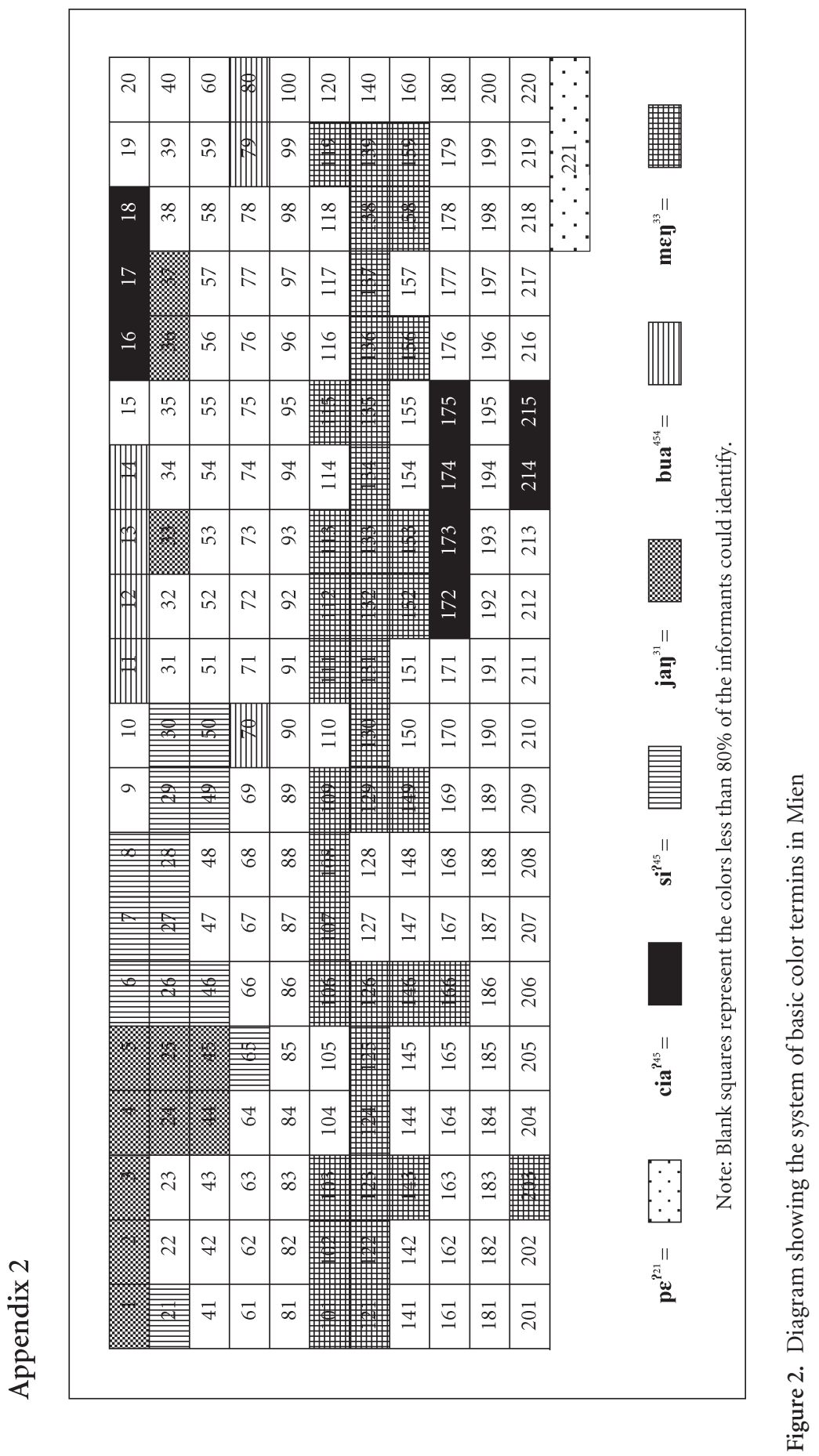




\section{Color Semiosis}

The studies in this Part emphasize the use of basic color terms in action within cultures, including the culture of the nation state. The authors consider the different ways in which color conceptualization is adaptive within the social context 



\title{
The semiosis of Swedish car color names
}

\author{
Descriptive and amplifying functions
}

\author{
Gunnar Bergh \\ Department of Humanities, Mid-Sweden University, Sweden
}

This article deals with the different semiotic functions of complex car color names in Swedish. Based on a body of 150 authentic name types, collected from both newspapers and manufacturers' catalogues, the study looks into the properties of these names from both a quantitative and qualitative perspective, discussing aspects such as morphological structure, information value, and status thinking as they apply to the data. The results of the analysis demonstrate that such color terminology is used to serve both descriptive and amplifying purposes, indicating that there is a complex interaction between physical and cultural aspects in the domain of car color semiosis. As a corollary of the patterns derived, it is argued that the car industry often seems to prefer connotation to denotation as its main color naming strategy.

\section{Introduction}

Buying a new car is often a long and complex process. Apart from familiarizing oneself with the wide range of makes and models, different technical solutions offered, and the financial side of the matter, it is also necessary to decide on an appropriate color of the vehicle. In the early days this was not a problem. At that time cars were black and nothing else: that was the case with, for example, Henry Ford's classic creation, the Model T-Ford, and the same simplicity applied for some time to the paint of many other early makes, such as the Volvo. Thus, when people were in the process of acquiring a new car, no choice of color was offered: the cars were black by default, reflecting largely the contemporary notion of nobility and elegance. Subsequently, when the winds of fashion veered somewhat, the odd grey car appeared on the market, and later also such painted in solid blue, red, and green. But the range of available colors was still limited. And, above all, the color names were simple and easy to understand.

Today the situation is different. Anyone who visits a Swedish car dealer with the aim of buying a car painted in, say, an ordinary solid blue is likely to face problems. The reason is, simply, that there are no such cars on the market anymore. Rather, the present day fleet of vehicles is coated in paint of a more sophisticated kind, accompanied by an equally rich and varied color terminology. Accordingly, the descendants of the solid blue car are 
now referred to in terms of, for example, aquablåpärlemo 'aqua blue mother-of-pearl', keramikblå 'ceramics blue,' mingblåpärl 'Ming blue pearl,' pariserblåmetallic 'Parisian blue metallic', polarhavsblå 'polar sea blue' or windsorblåmetallic 'Windsor blue metallic' - an internationally founded color assortment which is able to take the sting out of even the fashion industry.

This development towards an increasingly complex terminology accentuates several interesting questions in the field of color semiosis: What are the general properties of Swedish car color schemes today? Why have the old solid color names been replaced by longer and more sophisticated variants? What type of information is conveyed by the current type of terminology? How can the physical and cultural functions of the color names be integrated into a representative semiotic model?

\section{Possible and impossible car colors}

In order to be able to answer the above questions, it is necessary to perform some market research and try to find out how the car industry has capitalized on the combination of surface colors and verbal denominations. The most convenient way of doing this, arguably, is to go through a collection of (used) car advertisement columns in newspapers, where such terminology is regularly displayed, or to consult the primary source of these names - different sales catalogues and brochures issued by the car manufacturers/dealers themselves.

The present investigation draws on both these types of material. Thus, the advertisement material used includes the July 1996 issues of the Swedish newspaper GöteborgsPosten, while the sales material consists of a random collection of catalogues and brochures featuring the most common makes and models from the same year. From this dual material, the first instance of each type of complex Swedish color name available - that is, a name including a basic color term plus a prefixed modifier and, optionally, a suffixed modifier, for example flamröd 'flame red' and olivgrönmetallic 'olive green metallic' - was manually excerpted and categorized according to its relationship to the set comprising nine of 11 basic color terms (Berlin \& Kay 1969) and the non-basic but frequently used term for 'beige' (henceforth for brevity termed as basic). Table 1 summarizes the numbers of complex derivatives within each group of color names (without indication of their frequency of occurrence).

Assessing the quoted figures, we can see that there are no less than 150 complex color names in the material, distributed over ten basic color terms. Clearly, in their richness these names represent a considerable (nominal) color shade variation, particularly the upper half of the list; yet, they do not constitute a perceptual problem, since the human eye is able to distinguish millions of different color nuances (e.g. Halsey \& Chapanis 1951). What is problematic, rather, is the cognitive task of relating the rich assortment of color names available to the different shades that we are able to perceive.

From the above statistics it is obvious, furthermore, that it is the basic colors of the spectrum that are dominant. Paint names with a blå 'blue' basis top-rank the frequency 
Table 1. Number of complex derivatives as a function of Swedish basic color terms and 'beige.'

\begin{tabular}{lc}
\hline Basic color term & Derivatives \\
\hline blå 'blue' & 48 \\
grön 'green' & 30 \\
röd 'red' & 28 \\
grå 'grey' & 16 \\
svart 'black' & 11 \\
vit 'white' & 7 \\
violett/lila 'purple' & 4 \\
gul 'yellow' & 3 \\
beige 'beige' & 2 \\
brun 'brown' & 1 \\
Total & 150 \\
\hline
\end{tabular}

list, followed by such featuring grön 'green' and röd 'red,' whose noted nuance variation in their turn is more than twice as extensive as that of the grey scale of colors grå 'grey', svart 'black,' and vit 'white.' At the bottom end of the list, we find more glaring color variants with gul 'yellow' and violett's/lila 'purple,' together with such that more modestly express themselves in beige 'beige' and brun 'brown,' all of which occur in only a handful of linguistic forms. Incidentally, it can thus be established that the present type of vocabulary reflects fairly well the essence of Berlin and Kay's (1969) institutionalized typology of basic color terms, although, as expected, there is no straightforward relationship between the seven inherent stages and the numbers in Table 1.

\section{Status thinking}

However, even if the explanation in terms of human special visual abilities has a certain attraction to it, it is more likely that the main cause of the phenomenon is to be found in the cognitive realm. One important factor in this context, arguably, is color symbolism. There is reason to believe, for example, that the colors blue, green and red for many people have connotations of being elegant, tasteful, subdued or active, and that they therefore have a potential of giving a certain cachet to cars dressed in them. This view finds support in the fact that there are rather many complex paint names that imply such properties in combination with these basic color terms, such as diplomatbla 'diplomat blue,' kungsblå 'royal blue, oxfordgrön 'Oxford green,' classicgrön 'classic green,' arenaröd 'arena red' and imperieröd 'imperial red.' Further support comes from selected charts of color associations featuring people's reactions to these colors: classified as objective impressions, the concepts of subduing and sober are said to be triggered by blue, quieting and refreshing by green, and passionate and active by red (e.g., Birren 1950: 143). Conversely, one might assume that garish colors such as yellow, orange, and purple tend to make a slightly simpler or more vulgar impression, wherefore they do not suit prestigious vehicles equally well. 
According to Birren's quoted association chart, these colors would invoke concepts such as cheerful for yellow, jovial for orange, and mystic for purple.

Indirect support for the above contention may also be gleaned from the fact that other lines of business, with priorities other than those of the car industry, make use of partly different color shades. One case in point is the fashion industry. As noted in shop windows and on the catwalk, this trade has a predilection for show and ostentation, and accordingly it flourishes with conspicuous colors and names, which find it difficult to adhere to cars, such as laxrosa 'salmon pink, krämgul 'cream yellow', and syrénlila 'lilac violet.'

In addition, the low frequency of yellow and orange cars in Sweden may be partially explained by the fact that the prosaic official cars of the Post Office Administration and Swedish Telecom used to be painted in these colors respectively, a situation which is likely to have diminished the status of these colors even further in the context under interest.

So far, then, it seems clear that there is a certain connection between car colors and the notion of status in general. We may now develop this argument further by considering more specifically various color names used, and in particular their length and complexity. Thus, it is a commonplace observation that certain car makes and models are more prestigious and status-laden than others. While the price label is obviously influential, it is also a question of car quality and design properties. In particular, it seems reasonable claim that makes such as Mercedes, Porsche, and Volvo come with a higher status value than, say, Fiat, Opel, and Skoda. Similarly, with respect to the color name domain, most people would probably agree that there is a better ring to long and complex names compared to short and simple ones, for example, tropikgrönmetallic 'tropic green metallic' versus just grön 'green.' ${ }^{1}$ On this account, it lies near at hand to draw the conclusion that a car painted in a color termed with a long, sophisticated name appears more exclusive than one painted in a color with a short, plain name. In other words, a Volvo equipped with the color tag tropikgrönmetallic would seem to be more appealing than one with just the color tag grön.

On the basis of these observations, one might wonder whether it is generally the case that cars with a high status value come with a paint expressed by a long and remarkable name. The answer seems to be both yes and no. Swedish sales catalogues indicate that up to the 1990s there was a certain trend to single out high-status cars, not least those of German production, by means of sophisticated names, for example diamantbla 'diamond blue' and calypsoröd 'calypso red.' In recent years, however, manufacturers of low-status cars have tried to bridge the prestige-image gap by introducing sophisticated paint names for their own makes. This mongrelized practice has led to the present situation where it is possible to buy, say, a Volkswagen coated in mysticblåpärlemo 'mystery blue mother-ofpearl' or a Skoda appearing in romanticröd 'romantic red.' It is not very likely, though, that this measure has boosted the status value of corresponding car makes to any great extent,

1. This observation, incidentally, may be related to Zipf's law, postulating that there is an inverse relationship between the length of a word and its frequency (e.g. Crystal 1987:87), which warrants giving longer names a touch of exclusiveness. 
since virtually all current makes maintain an equally advanced color scheme terminology. The exception has simply become the rule.

\section{Structure and information value}

The next question to consider is how these eye- and ear-catching color names have been formed and what type of information they are intended to convey to the consumer. If we try to break them down morphologically, it turns out that they usually consist of a basic color term which functions as the word pivot, and that they are accompanied by verbal paraphernalia in the form of various descriptive elements, prefixed as well as suffixed.

The suffixed modifiers involved are typically associated with notions of brilliance and luster, and take on two different forms - metallic and pärlemor 'mother-of-pearl.' The former element is a direct loan from English, a circumstance which in itself is usually sufficient to give a product a higher status score in Sweden (e.g. Chrystal 1988), while the latter is a translation loan from (low) German Perlmutter, in its turn derived from the Latin expression mater perlorum 'mother of pearls.' Semantically both suffixed elements have the effect of adding a luster component to the basic color name, while pragmatically they seemingly inform consumers that the paint in question is more refined or exquisite than corresponding solid ones.

The prefixed modifiers of the color names are more remarkable. Here we find an abundance of variants, many of which indicate a relationship to a natural object or environment designated by the corresponding basic color term; that is, they act as prototypical reference points (cf. Wierzbicka 1996:160-161). The following list provides a sample of possibilities:
citrongul 'lemon yellow';
havsblå 'sea blue';
himmelsbla 'sky blue';
jadegrön 'jade green';
lavasvart 'lava black';
polarvit 'polar white';
rubinröd 'ruby red';
skogsgrön 'forest green';
vinröd 'wine red'.

As we can see, many of these color names involve designations of natural phenomena, precious stones, fruit, and such like whereby the prefixed element carries a metaphorical relation to the basic color term; that is, the nuance in question is expressed through the color of the object referred to, such as 'yellow as a lemon,' 'blue as the sea' or 'red as a ruby.'

2. Occasionally the English variant (mother-of-) pearl is also employed, without any spelling modification, as in aquablåpearl 'aqua blue pearl.' 
There are, however, also prefixed modifiers of a non-metaphorical nature. Such elements either describe luster or intensity of the color, as in blanksvart 'glossy black,' mellanblå 'medium blue,' and klarröd 'bright red,' or involve other colors as modifiers, as in blåsvart 'bluish black,' indigoblå 'indigo blue', and magentaröd 'magenta red.' For the reader of car advertisements and sales catalogues, this type of description is both natural and helpful, since the prefixed elements give concrete, physical information about the specific color shade in question. Yet, in this connection, we need to note that the car industry does not seem to approve of all natural phenomena with a clear color reference: for example, in the collected material, there was not a single instance of such natural combinations as bländvit 'dazzling white,' roströd 'rust red,' slemgrön 'slime green,' smutsgul 'dirty yellow, or kolsvart 'pitch black.' Given the negative connotations involved, there is no need for an explanation.

\section{Amplifying functions}

Thus far the situation in the Swedish car color world is fairly clear-cut. However, it becomes slightly more complicated when we bring up color names in which prefixed modifiers no longer express a natural connection with the basic color term, at least not from a Swedish perspective. The following examples illustrate the point:

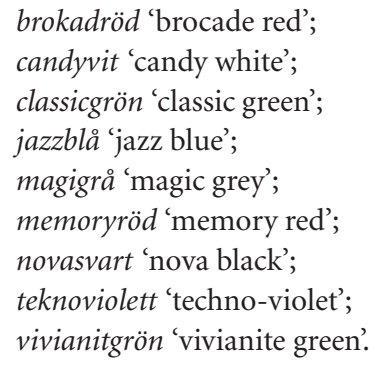

Clearly, these color names raise questions: for example, what is the connection between jazz music and the color blue (blues, though, would be a possibility), between memory functions and the color red, or between the mineral vivianite and the color green (cf. the Swedish synonym blåjord 'blue earth')? Apparently, there is no obvious connection at all. For the consumer, this means that in these prefixed elements there is hardly any information on physical appearance; semantically speaking they do not contribute anything to the interpretation of how the primary color is shaded off. Equally confusing is the situation where one and the same prefixed element - often with reference to a particular gem is employed to modify two or more basic colors. Consider the word onyx, for example, which is used to refer to a semiprecious stone with black and white layers. The compound name onyxsvart 'onyx black' is thus something which follows from the nature of the stone, but what about onyxgrön 'onyx green'? An opposite case is represented by the modifier smaragd. This term refers to an attractive green gem, whose natural color paves the way 
for the expression smaragdgrön 'emerald green'; strangely enough, however, it also occurs in the term smaragdsvart 'emerald black.'

How, then, are we to explain the usage of the above prefixed modifiers when they exhibit no direct or metaphorical relation to the basic color term in question? Is there any other function to take into account here, for example one of amplification or reinforcement, much in the same way as there are amplifying prefixes in general? Given the present data, there is a lot to be said for that option. What such modifying elements typically do, although not exclusively, is to arouse general positive associations, primarily by referring to cultural phenomena of a certain attraction or cachet. Thus, these elements form part of expressions which allude to, for example, deities and famous people, such as isisröd 'Isis red,' minervablå 'Minerva blue', and chagallblå 'Chagall blue,' to precious stones and minerals, such as ametistgrå 'amethyst grey,' diamantsvart 'diamond black', and turmalingrön 'tourmaline green,' to renowned places, such as casablancavit 'Casablanca white,' madeiraviolett 'Madeira purple', and panamabrun 'Panama brown,' or which are borrowed from a prestigious language, notably English, such as electronicgrön 'electronic green', flashröd 'flash red', and mysticbla 'mystery blue.' In this way, a status-boosting effect is achieved for the color in question, which the car industry apparently deems more important than the concrete information value of the color name itself. In short, connotation seems to override denotation in these cases.

\section{Semantic fields}

It may be of interest, furthermore, to find out if the pre-modifying parts of the color names - no matter if they have a descriptive or amplifying function - show any general pattern with regard to their semantic origin. For this purpose, six informal semantic fields were established, over which the prefixed elements of the present 150 color names were distributed, as shown in Table 2.

As could be expected, the frequency data show that, on the whole, general natural phenomena are significant in the formation of complex color terminology. What is remarkable, however, is the fact that no less than a third of the prefixed modifiers originate from such limited semantic fields as precious stones and minerals and geographical denominations. It is understandable, one might argue, that the geological category is well represented here, since elements of this kind often provide both color information (i.e. precious stones/minerals have color) and a status-boosting effect (i.e. they are coveted commodities); in other words, they have the potential of functioning as both descriptive

3. Admittedly, there are indirect connections between some of these prefixed modifiers and their respective color terms. In the case of deities and famous people, for example, the compounds isisröd and minervabla probably reflect the fact that Isis and Minerva were often painted with these colors; similarly, one might argue that the term Chagallbla is basically due to the particular saturated blue that Chagall used in many of his paintings. Yet, naturally enough, the crucial point is to what extent the general car consumer can be said to be aware of such indirect connections. 
Table 2. Distribution of prefixed elements as a function of six different semantic fields.

\begin{tabular}{lc}
\hline Prefixed element & Distribution \\
\hline General natural phenomena & 46 \\
Cultural manifestations and abstractions & 29 \\
Precious stones and minerals & 27 \\
Geographical denominations & 22 \\
Light and color concepts & 18 \\
People and professional life & 8 \\
Total & 150 \\
\hline
\end{tabular}

and amplifying elements. With regard to the geographical names, only the latter function is applicable; under normal circumstances they can only function as cultural amplifiers. It is also through this fact that they mark their distinctive character. When similar proper names are converted into appellatives, for example falukorv 'Falun sausage' and manchestertyg 'Manchester fabric (i.e. corduroy),' there is usually an element of origin present: that is, an important part of the meaning is 'sausage from Falun' and 'fabric from Manchester.' This semantic relationship does not, however, apply to the current color names of cars. In these cases, such as bostongrön 'Boston green,' dakargul 'Dakar yellow', and toscanaröd 'Tuscany red,' we are instead faced with a show of strength with sociolinguistic overtones, where positive associations to certain places are used more or less randomly to give commercial potency to car color schemes.

\section{Conclusion}

To sum up this colorful discussion, we may note that the car industry has been rather ingenious in recent years. Apart from bringing the car into the computer age, developing its interior into a high-technological product, designers have spent a great deal of effort on its exterior, not least when it comes to the superficial aesthetic impression. One manifestation of this is the current availability of several types of paint, some of which bring special brilliance and luster effects in terms of metallic and mother-of-pearl coats. Another, more important one is the host of subtle color shades that have come to the fore, offered under various more or less transparent and logical names. The analysis of the present data has shown that this terminology serves both descriptive and, in the first row, amplifying purposes, thus indicating that there is a complex interaction between physical and cultural aspects in the domain of car color semiosis. Yet, it is of course difficult to tell whether this aesthetic-cum-verbal development is to the benefit or detriment of today's car consumer. But with the Model T-Ford and its consistently black color in mind, one point is perfectly clear: if not better, things were at least much simpler in the old days. 


\section{References}

Berlin, Brent \& Paul Kay (1969). Basic color terms. Their universality and evolution. Berkeley: University of California Press.

Birren, Faber (1950). Color psychology and color therapy. Secaucus, NJ: Citadel Press.

Chrystal, Judith-Ann (1988). Engelskan i svensk dagspress. Solna: Esselte Studium.

Crystal, David (1987). The Cambridge encyclopedia of language. Cambridge: Cambridge University Press.

Halsey, Rita \& Alphonse Chapanis (1951). On the number of absolutely identifiable spectral hues. Journal of the Optical Society of America, 41, 1057-1058.

Wierzbicka, Anna (1996). Semantics: Primes and universals. Oxford: Oxford University Press. 



\title{
Colors and emotions in English
}

\author{
Anders Steinvall \\ Department of Modern Languages, Umeå University, Sweden
}

This paper presents a corpus study of collocational patterns of English color and emotion words. A collection of 50 color terms and 135 emotion terms served as the starting point for the search of patterns. The obtained patterns are described and analysed with regard to salience from two different points of view: For each emotion category we ask which color categories are most salient, and for each color category we ask which emotion categories are most salient. The analysis of the underlying motivation for salient color-emotion collocations demonstrates that the commonest motivations are based in metaphorical models linked to the body. This indicates the importance of experiential and embodied models for creating associations involving color terms.

\section{Introduction}

That colors and emotions are somehow linked has long been known. For instance, in color therapy various colors are believed to make people calm or happy (e.g. Birren 1978). Boyatzis and Varghese (1994) and Hemphill (1996) show that people frequently associate colors with emotions. D'Andrade and Egan (1974), on the other hand, show that color qualities of brightness and saturation may foster emotional associations.

From a linguistic point of view, etymology can evidence a link between color and

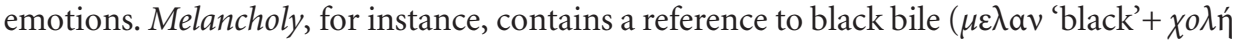
'bile'), which indirectly connects blackness and depression. Moreover, many expressions in English combine colors and emotions, for example, green with envy, and occasionally a color term has come to stand for an emotion without an overt tie, as in to feel blue (possibly motivated by Romanticist emotional loading of the color blue, cf. Pastoureau 2001: 140). Despite such well-known relations, it would appear that no study has systematically described collocations of color terms and emotion terms.

Here I attempt to remedy this lack. My aim is to analyze more closely the coloremotion collocations found in a large English corpus. Where a pattern emerges, its motivation will be discussed within the framework of cognitive and cultural models (e.g. Holland \& Quinn 1987; Lakoff 1987). Using the general corpus picture as a starting-point, I shall explore the collocations of emotion and color categories from two perspectives, investigating which color categories are salient from the viewpoint of emotions and vice versa. Finally, the results will be discussed and compared with those of other studies. 


\section{Method and material}

Studies of colors and emotions have usually been carried out by psychologists who apply a method by which they elicit reaction out of context - subjects are asked to react to color chips (e.g. Boyatzis \& Varghese 1994). A common query concerns how closely the procedure reflects the way we normally associate colors with emotions. However, a different methodology whereby a broad context is taken into account may possibly yield other results. Exploration of a large language corpus might effectively provide such a context.

In the present study one extensive corpus is used - the Bank of English (BoE), which is owned by Collins COBUILD, a division of HarperCollins Publishers, and housed at the University of Birmingham. Perhaps best characterized as a monitor corpus (cf. Sinclair 1991:24-26), it is one of the largest computerized corpora of English, comprising approximately 450 million words in 2003: British (71.6\%), American (17.1\%), Australian (7.8\%), Canadian (3.5\%) texts. It contains many subcorpora of text genres. Yet the corpus could be considered unbalanced because most subcorpora represent written language, among which newspapers dominate.

The color terms used in the present study are the 50 used by Steinvall (2002). They were chosen on the basis of three studies (Battig \& Montague 1969; Brown 1972; Taft \& Sivik 1997) to represent salient Anglo-American color terms (for details see Steinvall 2002). ${ }^{1}$

The emotion terms were chosen in similar fashion; they are the 135 that Shaver et al. (1987) use in a hierarchical cluster analysis. Their procedure should guarantee the salience. Although they list only nouns, included in the present study are adjectival forms because emotions are frequently ascribed to people in this way. In what follows, the nominal form will be used as a cover term to include the whole semantic field of the nominal and adjectival concept; for example, irritation represents irritated, irritating, and irritable.

The collocations of color and emotion terms were collected from the BoE. The amount of context taken into account for the search was a span of 5:5; to be registered the color word appears within five words on either side of the emotion word. This span is more generous than that used by most corpus researchers: Sinclair (1991), for instance, ignores collocations outside 4:4. With colors and emotions, there is often wider distance between their terms, as in (1) below. It would, however, be virtually impossible to include great spans such as the following:

(1) Although she did her best to be her usual tranquil self, Adrienne could see that she was excited. Her brown eyes were alight with eagerness and her cheeks pink. (BoE: brbooks)

Even with the small span of 5:5, the material needs refinement since many proximations do not represent proper collocations of color and emotion, as in (2).

1. The 50 terms are: amber, aqua(marine), azure, beige, black, blue, brown, carmine, charcoal (grey), chartreuse, cream, crimson, emerald, fawn, fuchsia, gold, golden, green, grey/gray, indigo, lavender, lemon, lilac, lime, magenta, maroon, mauve, mustard, navy (blue), olive, orange, peach, pink, plum, puce, purple, red, rose, rust, scarlet, silver, tan, tangerine, teal, turquoise, ultramarine, vermilion, violet, white, yellow. 
(2) Wearing his customary dark cords and jumper (Farrow said he had a phobia about bright colors, such as pink), he discusses his love of New York, his admiration for his former girlfriend Diane Keaton and his disillusionment with analysis. (BoE: brnews)

In (2), no direct connection pertains between pink and love. Collocations are recognized when both color and emotion refer to the same object and are linked through causation (red with anger) or some other way, as when they qualify one another (black depression, gloomy grey). Some connections between the color word and emotion are difficult to judge, as when the emotion term is polysemous. In (3), irritation does not have an emotional meaning, which can easily be overlooked. Such examples are excluded.

(3) They were also receiving facials, and Debbie couldn't help but notice that all of their faces looked red and irritated, ... (BoE: usbooks)

Another difficulty arises when an emotion term and color refer to an object other than a human being but describe it in an anthropomorphic way, as in a cheerful pink color. Such collocations suggest motivation for the connection between the color and the emotion, albeit distinct in different cases. Generally I accept them, maintaining a generous attitude toward borderline data.

\section{Results}

The collocation of 135 emotion terms and 50 color terms composes a 6750-cell matrix. Of the 135, 30 score $\varnothing$ and 65 score 5 or lower. To highlight essential patterns, only the 40 that score 6 or higher are analyzed in detail. ${ }^{2}$ They are categorized in accordance with the six basic emotion categories set up by Shaver et al. - LOVE, JOY, SURPRISE, ANGER, SADNESS, FEAR - so as to identify their patterns and also treat them individually. The six basic

2. The emotion terms scoring six or higher are (in order of frequency): envy (161), anger (147), cheerfulness (98), rage (94), depression (64), embarrassment (64), gloom (57), fury (51), passion (36), despair (35), happiness (29), excitement (28), fear (28), misery (28), love (27), shame (23), jealousy (21), shock (20), sadness (19), hate(red) (13), hope (13), joy (13), worry (13), frustration (12), grief (11), pleasure (11), sorrow (11), anxiety (10), panic (10), horror (9), nervousness (8), tenseness (8), agitation (7), defeat (7), hopelessness (7), irritation (7), jolliness (7), fright (6), optimism (6), thrill (6).Emotion terms that score five or lower are (in order of frequency): ferocity (5), loneliness (5), longing (5), pride (5), resentment (5), terror (5), unhappiness (5), annoyance (4), caring (4), contentment (4), enthusiasm (4), exhilaration (4), glumness (4), guilt (4), humiliation (4), hurt (4), agony (3), bliss (3), delight (3), desire (3), distress (3), eagerness (3), joviality (3), outrage (3), spite (3), adoration (2), affection (2), apprehension (2), bitterness (2), compassion (2), dismay (2), glee (2), hostility (2), lust (2), sympathy (2), torment (2), alarm (1), amazement (1), amusement (1), anguish (1), arousal (1), astonishment (1), attraction (1), dejection (1), disappointment (1), disgust (1), ecstasy (1), enjoyment (1), euphoria (1), gladness (1), grumpiness (1), isolation (1), loathing (1), pity (1), regret (1), rejection (1), relief (1), remorse (1), sentimentality (1), suffering (1), surprise (1), triumph $(1)$, vengefulness (1), wrath (1), zeal (1), aggravation (0), alienation (0), contempt (0), dislike (0), displeasure (0), dread (0), elation ( 0$)$, enthrallment (0), exasperation (0), fondness (0), gaiety (0), grouchiness $(0)$, homesickness $(0)$, hysteria (0), infatuation (0), insecurity (0), insult (0), jubilation (0), liking (0), melancholy (0), mortification (0), neglect $(0)$, rapture $(0)$, revulsion $(0)$, satisfaction $(0)$, scorn $(0)$, tenderness $(0)$, uneasiness $(0)$, woe $(0)$, zest $(0)$. 
Table 1. Number of collocations between basic emotion categories and basic color categories in the Bank of English corpus.

\begin{tabular}{|c|c|c|c|c|c|c|c|c|c|c|c|c|}
\hline & 岂 & 惫 & 国 & 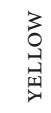 & 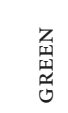 & 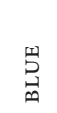 & $\begin{array}{l}z \\
z \\
0 \\
o \\
\text { a }\end{array}$ & 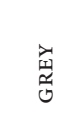 & 惫 & 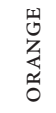 & 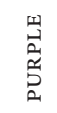 & त्ञ \\
\hline LOVE & 1 & 4 & 25 & 2 & 1 & 0 & 0 & 0 & 21 & 2 & 7 & 63 \\
\hline JOY & 1 & 30 & 44 & 44 & 12 & 28 & 1 & 1 & 30 & 17 & 3 & 211 \\
\hline SURPRISE & 0 & 0 & 0 & 0 & 0 & 0 & 0 & 0 & 0 & 0 & 0 & 0 \\
\hline ANGER & 25 & 62 & 192 & 2 & 177 & 2 & 7 & 4 & 8 & 3 & 31 & 513 \\
\hline SADNESS & 117 & 8 & 69 & 4 & 11 & 19 & 6 & 75 & 6 & 1 & 10 & 326 \\
\hline FEAR & 11 & 54 & 16 & 2 & 0 & 2 & 0 & 20 & 4 & 0 & 3 & 112 \\
\hline Total & 155 & 158 & 346 & 54 & 201 & 51 & 14 & 100 & 69 & 23 & 54 & 1225 \\
\hline
\end{tabular}

emotion categories were divided into a set of subcategories by Shaver et al. Thus, LOVE comprises AFFECTION, LUST and LONGING; JOY includes CHEERFULNESS, ZEST, CONTENTMENT, PRIDE, OPTIMISM, ENTHRALLMENT and RELIEF; ANGER IRRITATION, EXASPERATION, RAGE, Disgust, ENVy and TORMENT. SADNESS is divided into SUFFERING, SADNESS, DisAPPOINTMENT, SHAME, NEGLECT and SYMPATHY, and finally FEAR into HORROR and NERvousness. Each of these subcategories is then codified by a number of terms, e.g. Lust includes the terms arousal, desire, lust, passion and infatuation in Shaver et al.'s hierarchy. In accordance with linguistic tradition, concepts, mental categories (and subcategories), are here represented in small caps (e.g. LOVE) whereas terms are given in italics (e.g. love).

In similar fashion, each of the 50 color terms is grouped into one of the 11 basic color categories of English - WHITE, BLACK, RED, YELLOW, GREEN, BLUE, PINK, ORANGE, PURPLE, BROWN, GREY (Berlin \& Kay 1969). For assigning the 50 color terms (e.g. red, crimson, chartreuse etc.) to the basic categories, I use Kerttula's (2002) classification, which is based on definitions in the OED. This reduces the matrix to 66 cells $(6 \times 11)$, as depicted in Table 1 . The reduction of the cells described above does not imply a necessary link between basic emotions and basic color terms. It merely serves as a tool for identifying potentially interesting areas for further analysis. As the analysis below shows, the patterns and the underlying motivations are considerably more complex than a simple correlation of basic terms would suggest.

Conspicuous is the 0 score of SURPRISE. Shaver et al. (1987) point out that its status as a basic emotion category is doubtful; although sometimes described as basic, it usually scores lower in listing tasks than other candidates for basic emotion-term status (e.g. Fehr \& Russel 1984). And perhaps the nature of SURPRISE fails to invite color collocation.

Chi-square analysis was used to determine whether the variation in collocations fails to exceed chance. The null hypothesis is that the collocation of emotion categories with color categories is independent of color and emotion, which the analysis rejects $\left(\chi^{2}>\right.$ $1138.85, p<0.001)$. Thus, English speakers maintain a system of preference by which they link colors and emotions, at least as suggested by the BoE data.

Detailed analysis of the patterns follows, wherein two perspectives pertain: for each emotion category, we may ask which color category is most salient or, for each color cate- 
Table 2. Salience of basic color categories in reference to basic emotion categories as percentage of total collocations with each emotion category.

\begin{tabular}{|c|c|c|c|c|c|c|c|c|c|c|c|c|}
\hline & 冚 & 焉 & 密 & $\begin{array}{l}3 \\
0 \\
\exists \\
\text { ज્ર }\end{array}$ & $\begin{array}{l}\text { Z } \\
\text { 㱏 } \\
\text { 嵌 }\end{array}$ & 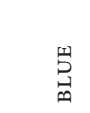 & $\begin{array}{l}z \\
z \\
z \\
0 \\
\text { of }\end{array}$ & 离 & $\underset{z}{\stackrel{z}{z}}$ & $\begin{array}{l}\text { 떼 } \\
\text { Z } \\
\mathbb{3} \\
\text { ه }\end{array}$ & 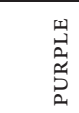 & 胥 \\
\hline LOVE & 1.59 & 6.35 & 39.68 & 3.17 & 1.59 & 0.00 & 0.00 & 0.00 & 33.33 & 3.17 & 11.11 & 100 \\
\hline JOY & 0.47 & 14.22 & 20.85 & 20.85 & 5.69 & 13.27 & 0.47 & 0.47 & 14.22 & 8.06 & 1.42 & 100 \\
\hline SURPRISE & 0.00 & 0.00 & 0.00 & 0.00 & 0.00 & 0.00 & 0.00 & 0.00 & 0.00 & 0.00 & 0.00 & 0 \\
\hline ANGER & 4.87 & 12.09 & 37.43 & 0.39 & 34.50 & 0.39 & 1.36 & 0.78 & 1.56 & 0.58 & 6.04 & 100 \\
\hline SADNESS & 35.89 & 2.45 & 21.17 & 1.23 & 3.37 & 5.83 & 1.84 & 23.01 & 1.84 & 0.31 & 3.07 & 100 \\
\hline FEAR & 9.82 & 48.21 & 14.29 & 1.79 & 0.00 & 1.79 & 0.00 & 17.86 & 3.57 & 0.00 & 2.68 & 100 \\
\hline
\end{tabular}

gory, we may ask which emotion category is most salient. Our answers need not coincide with numbers in Table 1. For example, the color category that most frequently co-occurs with LOVE is RED. But LOVE is not the emotion category that most co-occurs with RED. Rather, it is ANGER, which suggests that the term love may generate the association of red, whereas the term red mostly generates the association of anger.

\section{Emotion categories as points of reference}

For each emotion category, we ask which color categories are most salient: 'What color or colors does emotion X call to mind?' Every collocation is analyzed by counting individual emotion lexemes, and its model is described. In Table 2, the distribution of color categories in reference to emotion categories is expressed in percentages: the higher the percentage, the more salient a color category for the corresponding emotion category. Color categories collocating at $30 \%$ or more of all instances are highlighted. It is conspicuous that positive emotions (JOY and LOVE) frequently co-occur with warm, light colors.

\section{LOVE}

In the LOVE category, two color categories have almost equal strength - PINK and RED. Of the 16 terms belonging to LOVE, only love (27 instances) and passion (36) collocate more often than five times with color terms. In the case of love, pink has most at 19 out of 27. The result is skewed, however. Of the 19 collocations 16 are from a book on new-age color therapy, and constitute meta-level description rather than ordinary collocation, as in (4).

(4) I know this is not easy to do, but working with the pink ray of love will help you. (BoE: brbooks)

So the collocation figure for love and pink in ordinary usage is only three. With passion, a different picture emerges. Red is the most frequent collocation (21), whereas purple co-occurs six times. Co-occurrence of red and passion is often explained by a metaphorical model of heat (cf. Kövecses 2000): PAssion Is HEAT. Red-hot passion occurs most. However, there are several direct associations, as in (5), for which we detect no motivation.

(5) Finally, red is the color of passion so get going on your nails and lips. (BoE: sunnow) 
JoY

The Joy category manifests a more evenly distributed pattern of collocations. Table 2 shows that five color categories collocate at between $13.27 \%$ and $20.85 \%$. In contrast to most other emotional categories, there is no centre of gravity constituting one color category. Nine emotion terms score higher than five: cheerfulness, jolliness, joy, happiness, excitement, thrill, pleasure, hope and optimism. They belong to four subclusters in Shaver et al's hierarchy. Cheerfulness, jolliness, joy and happiness belong to that of CHEERfulness. Of these, cheerfulness (98) is most frequent. We find, however, a pattern in which the adjective cheerful is used to qualify the color term, as in (6).

(6) The cheerful sky blue exterior walls and sunny yellow front door give an almost Mediterranean feel to this English suburban setting. (BoE: brmags)

Cheerful seems to indicate brightness as opposed to gloomy, dark shades of the same color. This pattern co-occurs with a collection of different color terms such that no centre is identified: yellow (24), blue (21), red (15), orange (10) and white (10). This kind of construction is mostly used in reference to interior decorations, clothes and flowers, which points to an indirect link of the color reflecting a feeling in the language user.

Happiness is similar to cheerfulness in lacking a centre of association. Fourteen different color terms collocate with happiness but only yellow scores above five. Although no specific pattern stands out, most of the 14 belong to the warm color categories, YELLOW, RED, PINK, ORANGe. However, the terms blue and green also collocate. With joy, the same emerges, as with many terms and no singular pattern of motivation. But here all color terms are of warm meaning, which also fits the other observations regarding CHEERFULNESS.

Jolliness is different from other terms belonging to CHEerfulNess; it only collocates with red while maintaining one structure and motivation. There are only seven instances, but their homogeneity indicates a sole model of facial color, as in (7).

(7) This is surely why, when we imagine pre-Industrial Revolution Britain, jolly, red-faced squires and jolly, red-faced peasants spring to mind - people who hunted or worked the land in all seasons, before they were forced into factories, mines or offices. (BoE: indy)

This connection seems old, as suggested in (8).

(8) 1696 PHILLIPS, Rubicund Blood-red. Said of a jolly red countenance colored with Wine (OED: rubicund).

The other emotion terms belong to either of three subclusters. Excitement and thrill belong to one; they have different patterns that overlap. Excitement co-occurs more with color terms (28), commonly collocating with pink, red and white. Pink and red are motivated by facial color, as in (9), white in the phrase white-knuckle excitement.

(9) "You saw a little Cub Scout with a gold tooth?" he asked, his face pink with excitement. (BoE: brbooks)

The association of excitement, as with pleasure, is modeled on a heat metaphor whereby facial color reflects heat. This model will be discussed in detail below. However, the model 
does not work with jolliness: JolLiness Is HEAT(?). Thrill co-occurs with color terms only six times, three of which are in the phrase white-knuckle thrills, where we can see overlap between excitement and thrill.

Hope and optimism belong to the same subcluster, optimism, in the taxonomy of Shaver et al., but there is very little in collocational pattern to indicate it. With optimism, collocations are too few (six) to define a pattern. Two patterns emerge for hope: a direct link between green and hope is pointed out in three quotations, but the most frequent collocation is between white and hope in white hope. The motivation behind this phrase hales from the domain of boxing. The OED affords: "a white boxer who might beat Jack Johnson, the first Black to be world heavyweight champion (1908-15); hence, a person who, or a thing which, it is hoped will achieve much or on whom or which hopes are centered." In the BoE, white hope is not used in reference to boxing but only in its extended sense, as in (10). Although the original motivation may still be alive to some, others could create new models to legitimize the connection between white and hope.

(10) What started at the beginning of the decade as China's white hope for economic development thus turned into a potential victim of political divisions within the party leadership. (BoE: brbooks)

The last term in the Joy category, pleasure, has a strong connection to pink, instantiated in particular in pink with pleasure (11) below.

(11) ... he was usually so shy, ill-at-ease and constrained that it was no kindness to address him; but this afternoon he was bright pink with pleasure, .... (BoE: brbooks)

This connection is based on facial color, suggesting that cheeks turn pink (or red) when people experience pleasure. Again the metaphorical model of heat pertains: PLEASURE Is HEAT. The collocation of pink and pleasure may also be reinforced by the alliteration, and possibly by in the pink referring to health or wellbeing. It should be noted that the phrase in the pink does not derive from the color sense but from the flower sense. Here separate motivations merge in a folk model to reinforce a semantic association.

\section{ANGER}

ANGER is the category that collocates most often with color terms: $41.9 \%$ (513/1225, cf. Table 1) of emotion-color collocations involve ANGER. Table 1 shows that collocations form a bipolar pattern wherein RED and GREEN dominate. ANGER contains 29 emotion terms that Shaver et al. divide into six subclusters. The nine terms with a score higher than five belong to four clusters.

The RAGE subcluster collocates most with color terms: Anger (147 instances), rage (94), fury (51), hate (13). Apart from hate, two models appear to motivate their patterns: facial color and the metaphor ANGER IS HEAT (cf. Kövecses 2000), as in (12) and (13).

(12) Red-faced with anger and puffing out his cheeks he charged up to the wicket for a second time. (BoE: brbooks)

(13) He felt a sudden surge of white hot anger. (BoE: brbooks) 
The two are specific to one general idea of anger as heat. Rage is usually regarded as the stronger emotion (cf. e.g. Strongman 1996:36), and it collocates more with darker shades (purple and puce) than does anger. We can explain this pattern with Kövecses's proposal of ANGER IS A HOT FLUID IN A CONTAINER; increased heat will increase pressure, while facial color will darken. We have a scale by which pink with anger implies low pressure, whereas black or purple with anger or with rage means high pressure, in turn, enhancing anger, as in (14).

(14) He came to think of Schwarzkopf as a volcano - at times nearly dormant but for a small hiss of steam, at other times erupting with molten rage. His temper built progressively: the voice climbing to a bellow, the complexion flushing from pink to red to purple. (BoE: usbooks)

White with anger/rage/fury is also based on facial color. However, the heat and pressure models are inapplicable. In common with white-knuckle expressions, it is difficult to find a folk model that goes beyond mere description.

The model of heat is also present in the phrases red-hot and white-hot anger/fury/rage. The color terms refer to heated metals so that white entails the strongest emotion. The close link between anger and red is visible in angry red color, which mainly refers to skin, and to which the heat model does not apply.

There is overlap between anger/fury/rage and hate. In both, a heat metaphor pertains. Thus we find phrases such as white-hot and red-hot hatred, as in (15).

(15) The world in front of my eyes turned red, but it didn't match the white heat of the hatred that threatened to explode from within me. (BoE: oznews)

However, in (16) another collocation links black and hate.

(16) Every cubic centimeter of atmosphere above my shoulders and head was heaped with last straws. Black hatred clogged my very blood. (BoE: usbooks)

A plausible model for expressions of this kind is the antonymic relation to LOvE. Love is warmth and light (cf. LOVE IS FIRE), and hate should be associated with the opposite coldness, darkness, or blackness. In this way, hatred is close to sadness.

In the subcluster of IRRITATION, irritation and agitation show more than five collocations. Both have the same kind of pattern as do anger, fury and rage, in that red is most frequent and refers to facial color. Frustration, belonging to the subcluster of EXAsPERATION, has the same motivation as anger - facial color. This motivation is predominant for ANGER. However, this is not so with envy and jealousy, which belong to the subcluster Envy. They are strongly associated with green (154 out of 161 and 19 out of 21 instances, respectively), which might seem to rule out a link to facial colors. Niemeier (1998: 133) suggests, however, that the motivation could be facial color: "Probably the greenish, unhealthy-looking color of the face which may appear when one is feeling unwell ..., is taken as a metonymic reference." Historical records point to a tie between sickness and greenness and paleness:

(17) 1661 FELTHAM Resolves II. lvi, [Envy] the green sickness of the soul... impallids all the body to an Hectique leanness. (OED: impallid) 
The collocation of green and pale is frequent in the OED material, for example in the quotes under green (A3a).

(18) $c 1300$ Havelok $470 \mathrm{Al}$-so he wolde with hem leyke, pat weren for hunger grene and bleike. (OED: green A.3.a)

But another motivation is possible (Steinvall 2002). Geeraerts and Grondelaers (1995) observe that ancient and mediaeval humoral pathology leaves traces in English vocabulary, even though we are unaware of them as in opaque catarrh and rheumatism. With envy and jealousy, evidence for history in humors is inconclusive. There is, however, a link to jaundice, which might tie the complex to humoral theory.

(19) 1629 SYMMER Spir. Posie I. i. 8 Envie hath the yellow Iaundies. (OED: jaundice, n, 3.)

Osgood et al. (1975:327) find that the association between envy and yellow abounds in European languages, for example, German gelb vor Neid. Perhaps models have merged in green with envy. Synchronically the sickness motivation is strongest.

\section{SADNESS}

Shaver et al. divide SADNESS into six subclusters, in which 11 terms have more than five collocations with color terms. Black, grey and red dominate.

SADNESS, as the subcluster, has most associations with color terms, wherein eight terms collocate: depression, despair, hopelessness, gloom, sadness, grief, sorrow, and misery. Black is the commonest color collocation (103 instances, 43\%), followed by grey (73; $31 \%)$. At best other terms associate weakly.

In depression and despair, black is predominant (44 out of 64 and 34 out of 35 instances, respectively). With both, the color term modifies the emotion term, as in (20). We are not concerned with type modification, suggesting that there also exists red despair, but with description and reinforcement.

(20) I left it shortly before dawn the next day, totally exhausted, in a mood of black despair. (BoE: sunnow)

(21) When Kathleen fell pregnant with Rebecca, they were over the moon, and she hoped that would relieve his black fits of depression. (BoE: sunnow)

In (21), we see a construction that occurs frequently with both terms. The pattern varies with many nouns: black blanket of ..., black sea of ..., black cloud of ..., black hole of ... etc. The motivation builds on absent light, which is frequently given some embodiment or manifestation, as in cloud or blanket. Kikuchi and Lichtenberk (1983) note that the association between sadness and blackness is widespread and cross-cultural. Given our universal experience of the importance of light, this need not be surprising. The collocation of sadness and black may also have been motivated by humoral theory, wherein overproduction of black bile caused sadness and melancholy (Geeraerts \& Grondelaers 1995: 156).

Gloom and misery collocate most with grey. In gloom, many of the collocations are of the type in (21), where gloom refers to absence of light rather than emotion. But to many native speakers both senses are activated; that is, the senses seem to be so related that it 
would not be useful to attempt a distinction, as in (22). We saw such vagueness among the senses of cheerful.

(22) It was deeply depressing. In fact, all in all it was a day of shame at Wembley from start to finish. The gloomy, grey clouds that hung menacingly over the great old stadium before kick-off were clearly a portent of what was about to be played out before us. (BoE: sunnow)

The same emerges with misery, wherein the most frequent collocation is between grey and miserable and where the described object is not a human being. This pattern is also detectable with sad, where we find the emotion term modifying a color term.

(23) The Gilt-head is grey-black, with sad yellow bands on its face, and can grow to $30-40 \mathrm{~cm}$ $12-16$ in. (BoE: brbooks)

There is an alternative explanation to (23). The OED (sad, A.8a) indicates that sad developed a color sense 'dark, deep' independently of the emotion sense, although the latter influenced the color sense later. Accordingly, the sense of sad in (23) may have a diachronic motivation apart from emotion.

The frequent collocation of SADNESS and RED may seem surprising. However, the connection is natural; in Shaver et al.'s analysis, the subcluster of NEGLECT belonged to SADNESS. Under NEGLECT, terms that collocate with RED are embarrassment (52 instances) and shame (12 instances), the motivation facial color. As with ANGER, increased darkness suggests increased emotion. Precise terms like crimson, scarlet and puce also imply a higher degree of the emotion, as in (24).

(24) I realized with amusement it was the schoolteacher speaking from the jury box. She turned scarlet from embarrassment, once she realized what she had done. (BoE: usbooks)

Shame also collocates with black (eight instances). The motivation appears to be dirt, as in (25).

(25) He had just two years of formal education, was ignored by relatives ashamed of his German father and was taunted when he found a factory job. "We were a shame, a black spot on Norwegian society," Mr Hansen, 59, said. (BoE: times)

\section{FEAR}

FEAR has only two subclusters, and its pattern reveals that FEAR collocates most with white, almost 50\%. Most of those are motivated by facial color, which (26) illustrates. However, grey occurs, as in (27), and, to a lesser extent, red.

(26) Brewster staggered back, his face white with fear. (BoE: strathy)

(27) He was dressed in his sailing gear, sweater, blazer and cotton twill trousers, but it was the anxiety in his face which made him look crumpled and worn. He looked grey with worry. (BoE: brbooks)

Interesting is black cloud of, in (28), which we earlier saw in connection with sADNEss. It suggests that emotion can be modeled as weather; sunshine represents good mood, clouds the unpleasant. The weather metaphor also implies that emotions are beyond control. 
Table 3. Salience of basic emotion categories in reference to basic color categories in percentage of instances.

\begin{tabular}{|c|c|c|c|c|c|c|c|}
\hline & 崩 & 흐 & 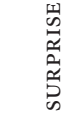 & 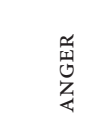 & 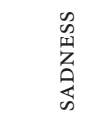 & 茎 & 氶 \\
\hline BLACK & 0.65 & 0.65 & 0.00 & 16.13 & 75.48 & 7.10 & 100 \\
\hline WHITE & 2.53 & 18.99 & 0.00 & 39.24 & 5.06 & 34.18 & 100 \\
\hline RED & 7.23 & 12.72 & 0.00 & 55.49 & 19.94 & 4.62 & 100 \\
\hline YELLOW & 3.70 & 81.48 & 0.00 & 3.70 & 7.41 & 3.70 & 100 \\
\hline GREEN & 0.50 & 5.97 & 0.00 & 88.06 & 5.47 & 0.00 & 100 \\
\hline BLUE & 0.00 & 54.90 & 0.00 & 3.92 & 37.25 & 3.92 & 100 \\
\hline BROWN & 0.00 & 7.14 & 0.00 & 50.00 & 42.86 & 0.00 & 100 \\
\hline GREY & 0.00 & 1.00 & 0.00 & 4.00 & 75.00 & 20.00 & 100 \\
\hline PINK & 30.43 & 43.48 & 0.00 & 11.59 & 8.70 & 5.80 & 100 \\
\hline ORANGE & 8.70 & 73.91 & 0.00 & 13.04 & 4.35 & 0.00 & 100 \\
\hline PURPLE & 12.96 & 5.56 & 0.00 & 57.41 & 18.52 & 5.56 & 100 \\
\hline
\end{tabular}

(28) We were busy being tourists, you see, and trying to forget the black cloud of fear that had overwhelmed us the week before. (BoE:brmags)

\section{Summary}

We have seen that positive emotion categories, LOVE and Joy, collocate with bright and warm colors. With Joy, however, it is difficult to identify a cultural model that might explain the association, although light would seem to be one possibility. A model of light will be discussed below. With ANGER, SADNESS and FEAR, the patterns are obvious, in particular a motivation of facial color.

\section{Color categories as points of reference}

In the previous section we considered the material from the angle of emotion categories. But here our approach is reversed. For each color category we shall ask what emotional category is most salient: What emotion(s) does this color make you think of? Table 3 displays the salience of emotion categories for color categories in terms of explanatory percentage among instances. Emotion categories that account for more than thirty percent of the instances are highlighted.

Table 3 reveals that WHITE, BLUE, BROWN and PINK are bipolar; two emotion categories represent more than $30 \%$ of the collocations. Other color categories have one centre. YELLOW, BLUE, PINK, and ORANGE primarily collocate with positive emotions (LOVE and JOY). The surprising occurrence of BLUE in this context will be addressed in detail. Color terms with the same salient centre are treated together.

\section{BLACK and GREY}

The most frequent collocation of BLACK is SADNESS, which is to be expected. Black is the color of death and mourning in European and American cultures, and this should be re- 
flected in language. The GREY category is also associated with SADNESS. The link appears to be metaphoric of weather: emotional gloom is described as grey weather and grey weather is called gloomy; as in (29) for the latter. The double direction of nature metaphors is observed by Taylor and Mbense (1998: 212).

(29) The days merged in an oppressive grey gloom. The sun seemed never to shine .... (BoE: brbooks).

\section{White}

White shows a bipolar pattern with two emotional categories of strong associations ANGER and FEAR. In both, collocation is motivated by facial color. Although very different, both fear and anger produce a white face. However, no singular folk model that comprises anger and fear appears to motivate the collocations. In addition we must here recognize two perspectives: from the point of view of WHITE, ANGER is salient, but from the view of ANGER, WHITE is not salient. This underscores the importance of perspective in modeling.

\section{RED, PURPLE and BROWN}

RED is prominent in many emotion categories; it scores higher than $20 \%$ in all categories except FEAR (Table 2). However, Table 3 shows that ANGER is by far the most frequent collocation from the point of view of RED. The common motivation for this collocation is facial color, followed by heat, which are linked in the EмотіON IS HEAT metaphor model. Red dominates completely but scarlet and crimson occur so as to emphasize the impression of the emotion. PURPLE and BROWN are part of the system, indicating a higher degree of either anger or embarrassment. Noteworthy is that, in contrast to other categories, it is not the basic color term, brown, but a non-basic term, puce, that dominates the statistic in BRown. Kerttula (2002:200), following the OED, categorizes puce as BRown, but many dictionaries define the term as a shade of purple (cf. Steinvall 2002:260). As with purple, the use of puce is hyperbolical. Interestingly enough, puce is mostly used in reference to facial color, which means that it rarely designates its decontextualized meaning.

\section{YELLOW and ORANGE}

Both the YELLOW and ORANGE categories collocate most with JOY, seldom with another emotion category. However, the association appears to be weak in that the emotion terms usually qualify the color term (as in cheerful yellow) in description of inanimate objects or flowers. Thus, no instance suggests a connection between emotion and color in the context of the human body. Although yellow accounts for most collocations, a few instances of golden (eight) collocate with Joy.

\section{GREen}

GREEN is closely linked to the ANGER category, precisely to envy and jealousy. Models of this association are considered above. Within the GREEN category, we find marginal usage of emerald and chartreuse for emphasis and humor, as in (30). This corroborates the observation above that specific terms emphasize emotions. 
(30) It's followed by the magnificently overblown Morning Glory, which will have Bon Jovi chartreuse with envy. (BoE: guard)

\section{BLUE}

Perhaps our most surprising result is the pattern of BLUE most collocating with JOY. BLUE collocates with SADNESs frequently, but the stronger position of JoY is striking. How can this be explained given the notionally strong association between blue and melancholy? Moreover, it is remarkable that one color category should collocate with categories of opposite emotion. Pastoureau (2001:140) mentions that the Romanticist conception of blue was associated with melancholy as well as love, pure poetry and ideal life. However, whereas it is possible to see a connection between these concepts, the opposition of Joy and SADNESS would seem to be much greater. The answer to the paradox appears to be twofold.

Firstly, it could be precisely the strong connection between blue and sADNEss that produces the low score of sadness. Based on collocations, we assume that the two terms should be mentioned close together in text. However, if the association is strong, then it would be tautological to mention them together. The same can be detected with puce and livid, which are now polysemous, encompassing a sense of 'anger' which makes it unnecessary to mention the emotion. Secondly, as with yelLow, collocation with terms in the Joy category is based on a pattern where the emotion terms qualify the color term, as in a cheerful blue color to describe objects in interior decoration, and the like. Thus the collocation does not indicate a direct link between the color and the emotion.

\section{PINK}

PINk collocates most with LOVE and JOy. As developed in the discussion of LOVE above, the frequent collocation with LOVE is due to discussion of color therapy and may be disregarded. With Joy, the association is based on facial color, as in pink with pleasure. It would seem likely that other associations of pink, such as in the pink and tickled pink, reinforce the pattern. Pink facial color further indicates mild anger and embarrassment. Accordingly, there is risk of ambiguity when the emotion is not spelt out. On the whole it appears that the link to positive feelings is stronger.

\section{Discussion}

The collocation study of color terms and emotion terms in the Bank of English corpus reveals that three models of association are particularly strong. All models are formed on an experiential basis and point at least partly to the embodied nature of conceptualization and language, because most associations are with the body. This could be viewed as a natural consequence of our bodily experiences of emotions.

The strongest source of motivation is facial color. With color terms such as white, pink, red, purple, blue, black, and perhaps even green in description of facial color, we convey emotions such as anger, embarrassment, pleasure, fear and envy. Notably the categories of ANGER and EMBARRASSMENT find motivation in the metaphorical cognitive model of 
EMOTION IS HEAT whence we infer that darker facial color reflects increased heat and a stronger emotion. The metaphor surfaces concretely in phrases such as red-hot anger and white-hot hate. Kövecses (1995: 187-190) demonstrated the cross-cultural character of the heat model in the context of ANGER. Taylor and Mbense (1998) showed that the association between anger and redness exists in Zulu too, but they derived it from a blood model.

The body also serves as the basis for a metaphorical model, creating the bridge between black and saDNEss and green and ENVY. Here the model for understanding the emotion is (NEGATIVE) EMOTION IS AN ILLNESs, which is associated with skin color or bodily fluids according to the old model of humoral pathology (Geeraerts \& Grondelaers 1995: 155-159). The OED does not proffer a conclusive result of the underlying view, but (31) points to the association with illness:

\section{(31) Envie hath the yellow Iaundies. (= 19 above)}

Another collocational pattern which has general significance is that based on a metaphorical model derived from opposition between light and dark. Kövesces (2000:39) claims that this model applies to happiness and sadness only, but it seems that the opposites of love and hate could be included. The collocation pattern for hate, in particular, appears to be linked to this model. D'Andrade and Egan (1974) found that colors and emotion were more closely linked to the dimensions of saturation and lightness rather than hue. Perhaps this tendency is modeled on weather phenomena such as clouds and sunlight. Many instances involving black and grey refer directly to this model, as in (32) and (33) below; (34) illustrates a historical reference.

(32) A black cloud of depression and despair hung heavy over anyone who loves English football in Marseilles yesterday.(BoE: sunnow)

(33) My spiritual gloom was mirrored by perpetual grey skies. (BoE: brmags)

(34) 1839 F. BARHAM tr. Grotius' Adamus Exul v. 47 Deadly rage, And the black hurricane of thick despair Urge on the unshunnable doom. (OED: unshunnable)

Similarly, the surprising connection between blue and joy turns out to be modeled on the brightness of blue, as in (35).

(35) Now the cream walls are covered with roses and creeping plants, offset by cheerful blue shutters and doors.

Generally, the linguistic material correlates with associations of colors reported in the literature. A comparison with cross-cultural connotations reported by Osgood et al. (1975:323-326) reveals few differences, the main one being their weak association between fear and whiteness. The similarities could be based on shared experiential and metaphorical models. Kövecses (2004) points out that there are some metaphorical models, for example THE ANGRY PERSON IS A PRESSURIZED CONTAINER, which may be close to universal. However, this is not to say that one would expect universal collocation patterns for colors and emotions. As is also mentioned by Kövecses (2004), there is considerable metaphor variation both with regard to cross-cultural and within-culture dimensions. Alternative models and motivations could no doubt be the basis for collocation patterns 
diverging from those found here. Moreover, evidence of alternative categorizations of color and emotions further complicates a quest for universal patterns.

In sum, the aim of this study has been to describe and analyze color-emotion collocations found in English. Three metaphorical models (EMOTION IS HEAT, EMOTION IS ILLNESS, and EMOTION IS A WEATHER PHENOMENON) account for most collocations of color terms and emotion terms in the study. This finding indicates the importance of experiential and embodied models for creating associations between color terms, a cornerstone of my analysis.

\section{Acknowledgment}

In writing this paper, my work has benefited greatly from the constructive comments from Galina Paramei and the late Robert MacLaury on previous versions of it. All remaining flaws are, of course, mine.

\section{References}

Battig, William F. \& William E. Montague (1969). Category norms for verbal items in 65 categories: A replication and extension of the Connecticut category norms. Journal of Experimental Psychology, 80, $1-46$.

Berlin, Brent \& Paul Kay (1969). Basic Color Terms: Their Universality and Evolution. Berkeley: University of California Press.

Birren, Faber (1978). Color and Human Response. New York: Van Nostrand Reinhold.

Boyatzis, Chris J. \& Reenu Varghese (1994). Children's emotional associations with colors. The Journal of Genetic Psychology, 155, 77-85.

Brown, W. P. (1972). Studies in word listing: Some norms and their reliability. Irish Journal of Psychology, $1,117-159$.

D’Andrade, Roy G. \& M. Egan (1974). The colors of emotion. American Ethnologist, 1, 49-63.

Fehr, Beverley \& James A. Russell (1984). Concept of emotion viewed from a prototype perspective. Journal of Experimental Psychology: General, 113, 464-486.

Geeraerts, Dirk \& Stefan Grondelaers (1995). Looking back at anger: Cultural traditions and metaphorical patterns. In J. Taylor \& R. E. MacLaury (Eds.), Language and the Cognitive Construal of the World (pp. 153-179). New York: Mouton de Gruyter.

Hemphill, Michael (1996). A note on adults' color-emotion associations. The Journal of Genetic Psychology, $157,275-280$.

Holland, Dorothy \& Naomi Quinn (Eds.) (1987). Cultural Models in Language and Thought. Cambridge: Cambridge University Press.

Kerttula, Seija (2002). English Colour Terms: Etymology, Chronology, and Relative Basicness. [Mémoires de la Société Néophilologique de Helsinki, 60]. Helsinki: Société Néophilologique.

Kikuchi, Atsuko \& Frantisek Lichtenberk (1983). Semantic extension in the color lexicon. Studies in Language, 7, 25-64. 
Kövecses, Zoltán (1995). Anger: Its language, conceptualization, and physiology in the light of crosscultural evidence. In J. Taylor \& R. E. MacLaury (Eds.), Language and the Cognitive Construal of the World (pp. 181-196). New York: Mouton de Gruyter.

Kövecses, Zoltán (2000). Metaphor and Emotion: Language, Culture, and Body in Human Feeling. Cambridge: Cambridge University Press.

Kövecses, Zoltán (2004). Introduction: Cultural variation in metaphor. European Journal of English Studies, 8, 263-274.

Lakoff, George (1987). Women, Fire, and Dangerous Things: What Categories Reveal about the Mind. Chicago: University of Chicago Press.

Niemeier, Susanne (1998). Colorless green ideas metonymise furiously. In F. Ungerer (Ed.), Kognitive Lexicologie und Syntax (pp. 119-146). Rostock: Universität Rostock.

Osgood, Charles E., William H. May \& Murray S. Miron (1975). Cross-Cultural Universals of Affective Meaning. Chicago: University of Illinois Press.

Oxford English Dictionary, The. Online edition. Available at http://www.oed.com/ Oxford: Oxford University Press. [OED]

Pastoureau, Michel (2001). Blue: The History of a Color. Translated by Markus I. Cruse. Princeton: Princeton University Press.

Shaver, Phillip, Judith Schwartz, Donald Kirson \& Cary O'Connor (1987). Emotion knowledge: Further exploration of a prototype approach. Journal of Personality and Social Psychology, 52, 1061-1086.

Sinclair, John (1991). Corpus, Concordance, Collocation. Oxford: Oxford University Press.

Steinvall, Anders (2002). English Color Terms in Context. Umeå University: Skrifter från moderna språk 3.

Strongman, Kenneth T. (1996). The Psychology of Emotion: Theories of Emotion in Perspective. 4th ed. New York: John Wiley \& Sons.

Taft, Charles \& Lars Sivik (1997). Salient color terms in four languages. Scandinavian Journal of Psychology, $38,29-34$.

Taylor, John R. \& Thandi G. Mbense (1998). Red dogs and rotten meakies: How Zulu talk about anger. In A. Athanasiadou \& E. Tabakowska (Eds.), Speaking of Emotions: Conceptualisation and Expression (pp. 181-196). New York: Mouton de Gruyter. 


\title{
Linguistic construal of colors
}

\section{The case of Russian}

\author{
Ekaterina V. Rakhilina \\ All-Russian Institute for Scientific and Technical Information, Russian Academy \\ of Sciences, Moscow, Russia
}

The paper provides a specific approach to the semantics of color, which differs from the most widespread "psycholinguistic" account - in that it privileges linguistic behavior of color terms. The main object of this investigation is linguistic combinability of color-term adjectives with regards to nouns of "colored" objects. We argue that coexistence of two or more color terms related to the same physical fragment of the color gamut, but whose use is motivated by the need for denoting colors of either natural or artifact objects, is one of the basic grounds for systemic variability in the term meaning.

\section{Introduction}

Research on colors undertaken by linguists usually calls upon psycholinguistic studies, which establish a relation between certain color terms, or mental concepts of colors, and standard arrays of color stimuli. Accordingly, a native speaker is asked whether a given color term can be used for referring to one or another hue. Evidence from a representative sample of native speakers allows one to delineate the focal hue as well as the limits for linguistic use of the color term in question.

This methodology, with some variations, has been used in studies by Berlin and Kay (1969), Frumkina (1984), Frumkina and Mikhejev (1996), MacLaury (1997, 2001), Moss et al. (1990), Vasilevich $(1987,2003)$ and many others.

The present study approaches the semantics of colors in a different way. The main object of our investigation is linguistic combinability of color term adjectives with regards to nouns of colored objects, in the way that combinability is represented in attributive constructions that are taken from Russian language corpora or elicited from native speakers.

This syntactically based view of color semantics can, we conjecture, considerably modify and advance currently available theories of color term meaning. Our research methodology has its theoretical foundations primarily in Wierzbicka's (1990) cognitive hypothesis, which rather treats color terms, than as abstract entities, as related to objects of the physical world that are culturally salient, and hence reify the meaning of a given color. The reification hypothesis seems to us insightful; also, it encounters additional sup- 
port in an argument related to language acquisition. For a child, possible and impossible linguistic combinations with the names of important objects in the world around - rather than templates of color samples - are the most likely source of information about the semantics of color. Wierzbicka's theory does not specify, however, the mechanism of linkage between a culturally rooted color and a physical object; nor does it discuss in great detail the methodology of studying languages of different types.

Our study can be viewed as a further elaboration of the cognitive hypothesis that complements the Wierzbicka theory. Although, as will be demonstrated below, some results of our linguistic experiments do not fit very well in Wierzbicka's original scheme.

The present linguistic study can be of interest, however, independently of the initial theoretical framework, since it sheds light on unsettled questions with which the domain of color terms is fraught, despite a long history of investigation. These questions are related, to our mind, primarily to the significative aspect of color terms rather than to the denotative one. It is well established that languages have a system of basic color terms and that historical development of languages involves the incorporation of an increasing number of basic color terms (Berlin \& Kay 1969; Kay \& Maffi 1999). The question that is not answered, however, is "What are the exact mechanisms of this process?" That is, how does a given color term enter or leave the basic group and how does the linguistic system of color terms evolve? It is stated that, within a specific basic color category, subranges of the color may be represented in a language as coexisting dominant and recessive varieties (MacLaury 1997), but the mechanisms ruling their coexistence and interplay remain largely unexplored.

To our mind, the combinability of color terms with the names of objects can serve as a mirror to capture complex linguistic dynamics, and thus may help to answer the questions addressed above.

\section{Attributive and predicative constructions}

Before proceeding to examine the data, we would like to specify what kind of adjectival constructions is chosen for our analysis and what the grounds are for this choice.

The crux is that color adjectives can be used in two different types of constructions referred to as attributive and predicative. The difference between these is syntactical. (In Russian it is partly also morphological, since Russian adjectives have a set of so-called short forms, which occur only in the predicative function.) More important, however, is that the difference between the two types of constructions is rooted in their semantics. This issue was first raised by Bolinger (1967): his analysis of pre- and postpositional uses of English adjectives showed that preposed (attributive) adjectives tend to denote permanent object properties, whereas postposed (predicative) adjectives are predominantly related to transient object properties. In addition, in attributive constructions the nouns are semantically modified by the adjectives: the adjective modulates the original meaning inherent in the noun. By contrast, in predicative constructions the adjective ascribes the noun a completely new characteristic. 
For English, it is argued that different semantic groups of adjectives tend towards use in either the attributive or the predicative construction. For example, only the main reason is grammatical - unlike ${ }^{\star}$ the reason is main. (Here and in the following, the * marks grammatically impossible expressions). By contrast, both following expressions are correct, where the man is ready is opposed to the ready man. According to Quirk et al. (1972:263), English adjectives tending towards the predicative position (such as well, faint, ill) are semantically closer to verbs or adverbs (in particular, with regards to temporal relations), because they denote transient characteristics rather than permanent ones. By contrast, typical perfect participles with only attributive uses are most likely to denote (visible) traces left that provide an object with permanent characteristics, for example $a$ bruised cheek versus ${ }^{*}$ a scratched head or labeled goods versus ${ }^{*}$ sent goods (see Bhat 1994; Bolinger 1967).

This implies that attributive and predicative constructions are opposed semantically. Summarizing the differences listed above, we can state that attributive constructions presuppose semantic concordance between the adjective and the noun, whereas predicative constructions impose onto the noun an external incidental characteristic. Given this, there is a need not only to distinguish these two types of constructions while describing adjectives, but also to decide which one has priority of status over the other. Bolinger (1967), Dik (1989) and many other linguists gave priority to the attributive construction. The primary status of the attributive construction is advocated in Bhat (1994: 104ff.) on a wide typological data. Bhat demonstrates, in particular, that adjectives in the predicative position tend to lose their individual properties, which opposes them, among others, to verbs.

For the color-term domain, these inferences motivated us to exclude predicative contexts in favor of attributive ones. In fact, we are concerned not with adjectives as such, but with the properties of names of objects revealed by means of those adjectives. Such semantic effect is explicable, however, only for the case of noun-dominated adjectives concordant with the nouns semantically. It is this type of construction that provides numerous nontrivial combinatorial restrictions, which can further be used as a powerful tool for semantic description of both adjectives and nouns. By comparison, predicative contexts turn out to be, in most cases, much less restrictive with regards to their combinability and hence, from the point of view of semantic analysis, less promising.

\section{Combinatorial restrictions}

Let us assume that in the real world each physical object has a color (or colors). In principle, the color can be always rendered by linguistic means, even if in some cases somewhat clumsily or vaguely (in expressions like it was half-blue and half-brown; with small red spots throughout; and pale stains). In this (and only in this) sense one can speak of free ascription of a color and absence of any combinatorial restrictions. However, if linguistic units are meant, such as names of objects $(N)$ and names of colors $\left(A d j_{\mathrm{CoL}}\right)$, and if these units are part of an attributive construction, the situation changes crucially. Recall that the attributive construction reveals semantic characteristics of the object name, which are 
built into its semantic structure. Color is one such characteristic; therefore an $A d j_{\mathrm{col}}+N$ construction is possible only when it is supported by the semantics of the noun $N$, namely when there is a certain semantic congruence between the color adjective $A d j_{\text {coL }}$ and $N$. Further, the semantic structure of $N$ may imply certain determined concepts in relation to the color of its referent, thereby yielding numerous (as will be shown below) contextual constraints on adjectival use.

It is noteworthy that not any noun $N$ is possible (or, at least, natural) in the construction of the type $A d j_{\mathrm{CoL}}+N$; consider, for example, nouns such as skripka 'fiddle,' šljuz 'sluice,' rubl' 'rouble,' and ulitka 'snail.' Those objects do have a specific color, which, of course, could be named, though not by means of an attributive construction.

We should therefore define the conditions at which color, as a feature, appears of marked relevance for an object. We argue that the attributive construction enables the named color to distinguish the object under consideration from other similar objects. This distinctive function of color names is especially prominent in the cases when the objects are available (concentrated) in large quantities, so that their color serves as an additional distinctive feature and therefore becomes linguistically relevant, which we see in such regular combinations as zelënoe plat'e 'green dress,' žëltaja stena 'yellow wall,' krasnoe pjatno 'red spot,' and the like.

It is obvious that for the nouns that denote objects with a constant color (e.g., coal, blood, lime), the feature of color is not distinctive and, thus, not relevant in the attributive construction. It follows that a plausible interpretation of expressions like 'čërnyj ugol' 'black coal' or 'belaja izvest' 'white lime' requires a strong pragmatic context, which should establish the relevance of the constant color. (Here and below, the symbol ? marks an awkward or problematic expression.) Such context may, for example, oppose in an expressive way this color to the color of a counterpart object, as in: “Čërnyj kot, ves' v beloj izvesti èto, ja vam skažu, zrelišče! [A black cat, all covered with white lime - that is a jolly spectacle, I would say!]"

From a lexicographic point of view, the constant color is a permanent property, inherent in the lexemes describing natural objects, and hence, not worth naming. It should be noted that for artifacts, too, color does not always appear distinctive. In the linguistic group of artifacts there are, however, quite a few objects used with achromatic color names; for example, gvozd' 'nail', molotok 'hammer,' mina '(mining) mine,' rel's 'rail,' and vilka 'fork.' In a sense, these, too, are objects with a constant color (i.e. that of metal), but its communicative salience is low to the extent that in Russian it is even not lexicalized, lacking specific color term. As can be seen, constant color occurs many more commonly than one might expect, a fact that considerably restricts the use of $A d j_{\mathrm{col}}+N$ constructions.

It should be emphasized that what we call "the relevant feature" is established for the case of constant-color adjectives, as well as for other qualitative adjectives according to the same basic principle. Indeed, objects with a constant color are distinguished due to the fact that they do not change that feature during the whole period of their existence; that is, speakers ascribe no significance to their color, since it is invariable. For Russians, when they say Naden' čistuju rubašku 'Put on a clean shirt' or Posteli cistuju skatert' 'Lay 
a clean cloth, an opposition between clean shirts/cloths and dirty ones is implied and, further, that one and the same shirt/cloth can be both clean and dirty during its existence. The sentences like 'Naden' čistuju šubu/galstuk 'Put on a clean sheepskin/cravat' sound more problematic. This paradoxical asymmetry is accounted for by the fact that, from the linguistic point of view, the objects like coats and ties have a constant feature of being 'clean' and, hence, this feature is irrelevant to them, just as in the case of constant colors.

\section{Unconstrained versus conventional color terms}

As noted above, the least restricted linguistic performance with regards to the color-name gamut is characteristic of artifacts: in modern surroundings, the color of clothes, furniture, buildings, utensils and similar is provided by a paint or dye the object was processed with and, hence, varies practically without limits. By contrast, constraints in the use of color adjectives emerge, when an adjective acquires a specifying function. In these cases what one deals with is actually a phraseological unit rather than a free combination. For example, čërnyj xleb 'black bread' and belyj xleb 'white bread' apparently denote two different sorts of victuals (rye-bread and wheat bread, respectively) and not two variants of one bread sort opposed by their color. The same is true for Russian equivalents of collocations like 'white skin,' 'red wine,' 'white flag,' or 'Red Cross.' It is noteworthy that in many such collocations the choice of the named color is in great degree conventional: for example, white wine is known to be limpid (like water) rather than really white.

Along with the two extremes of color-adjective usage - unconstrained as opposed to conventional - there exist many intermediate cases.

Let us consider Russian names of animals combined with color adjectives. Two groups of such combinations can be clearly delineated. One of them consists of constant collocations (with implied nuance of meaning often rooted in Russian folklore) like ryžaja lisa '(brownish) red fox,' čërnyj voron 'black raven,' buryj medved' '(dust) brown bear,' seryj/buryj volk 'grey/(dust) brown wolf,' zelënaja ljaguška 'green frog,' rozovyj porosënok 'pink piglet.' These collocations are almost identical to phraseological units: they are firm and do not undergo any variations. Although nothing prevents, for example, a real living fox (unlike its fairy-tale counterpart) from having different colors, the term ryžaja '(brownish) red' would invariably be applied to foxes by speakers of Russian. In this collocation group, the choice of a certain color adjective is, of course, still reasoned but semantic conditions may be very subtle and complex.

By contrast with these collocations, in Russian a large number (actually an overwhelming majority) of the recorded names of animals participate in no way in attributive constructions with color adjectives, as if these animals do not possess any color. For example, attributive constructions cannot be used for referring to the color of a deer, elk,

1. The translations are given according to Frumkina and Mikhejev's (1996:86) list of English equivalents for Russian color terms, where ryžij means '(brownish) red, ginger' and buryj 'dust brown'. 
kangaroo, ostrich, badger, sable, nightingale, or hippopotamus, though these animals are familiar to Russian speakers, who can easily imagine and describe their natural color, if needed - but not within an attributive construction. These nouns behave in accordance with the constraints formulated above: they denote species with a constant color, which, from the linguistic point of view, is irrelevant and, hence, does not call for an attributive construction. This implies that the names of animals considerably enlarge the domain of linguistically colorless objects. Against this background, units like čërnyj voron 'black raven' or zelënaja ljaguška 'green frog,' that name animals of a constant color, appear to be exceptions; see below for a possible explanation related to some general semantic properties of color terms.

By comparison with the "colorless" species, a small group of names of animals do exhibit color variation, which therefore can be expressed within an attributive construction; cf. phrases such as belyj/čërnyj/ryžij kot (pës) 'white/black/(brownish) red cat (dog).' The problem is, though, that kot 'cat' allows also the adjective seryj 'grey' to be included in this list, whereas the combinations 'seryj pës or 'seraja sobaka 'grey dog,' seem problematic in Russian, despite the fact that canine species of grey color occur in nature; consider also a possible seren'kij kozlik '(little) grey kid' versus a highly problematic ?seraja korova 'grey cow.' Thus it becomes evident that the variation of color in these examples is not as free as one might expect, since the choice of color is likely to approach the conventional pattern.

As a further example, in Russian 'mice' can be accompanied by adjectives 'grey' and 'white', but serye myši 'grey mice' are not exactly grey - the collocation actually implies a darker color. Its precise subtlety of meaning is mirrored by a counterpart expression myšinyj cvet, 'mouse color' (= 'dun, mouse grey color,' according to Frumkina \& Mikhejev 1996: 86). Phrases like Na nej bylo seroe pal'to 'She wore a grey coat' and Na nej bylo pal'to myšinogo cveta 'She wore a mouse grey coat' differ not only with regards to the actual color of the clothing, but foremost in their connotations.

In Russian the problem of specifying the color of an object can, in principle, be solved by linguistic means, since the language disposes of variety of terms for tinges of certain colors (e.g., pepel'nyj 'ashen' as an attribute of hair rather than seryj 'grey'). In the language, however, even non-grey mice appear to be grey, whereas grey dogs are denied any color at all. A linguistic image of the animal world, as reflected in attributive constructions, looks as follows: there exist prototypical (conventional) colors of prototypical animals that do not imply direct relation to denotata in the real world - rather, the concepts of these animals are conventional, being part of the semantic structure of the corresponding lexemes. It is worth noting that the domain of conventional colors appears to be strongly restricted as compared to the named chromatic gamut. In Russian, color terms that frequently function as conventional are belyj 'white,' čërnyj 'black,' ryžij '(brownish) red' and krasnyj 'red,' zelënyj 'green,' žëltyj 'yellow'; less often are sinij 'dark blue' and goluboj 'light blue.' In contrast, color terms such as fioletovyj 'purple,' oranževyj 'orange,' koričnevyj 'brown' are never used in this role, a fact that may be explained by the rather late emergence of the latter in the Russian lexicon (at least the first two terms are not attested before the 17th century; Bakhilina 1975:208, 240, 247); as well as by the structure inherent to these adjectives that semantically and formally are derived. 
Interestingly, the Russian terms for conventional colors comprise all six primary basic color terms, but also two color terms of old origin: non-basic ryžij ' (brownish) red' derived from the Proto-Slavonic roots rud-/rus-/ryd- (Comrie \& Corbett 1993:111) and goluboj 'light blue,' which emerged in Russian as early as the 13th century (Bakhilina 1975:36) and is argued to be a basic color term, too (e.g., Corbett 1989; for a review see also Paramei 1999, 2005). Usually it takes a long time for a color term to become conventional. "New" color terms enter the linguistic system gradually by spreading initially in the domain of natural objects and only later gaining access to conventional patterns of use. In addition, they reveal a strong connotative relation to both natural objects and cultural stereotypes and, within a culture under consideration, apparently elaborate on certain parts of the color gamut (cf. Mikhailova 1994).

As examples of color terms with the conventional color status we regard below the meaning and combinability of Russian adjectives for 'grey,' 'green,' and 'yellow.' Beyond these, we consider the process of spreading of a relatively new color term taking as an example koričnevyj 'brown.'

\section{Seryj 'grey'}

The Academy Dictionary of Russian (Evgen'eva 1980:Vol. 4, 83) defines the meaning of seryj as follows: "The color of ashes obtained by mixing black and white." To our mind, this definition is incomplete with regards to modern semantics of seryj. The authors of The Academy Dictionary approach the linguistic problem by defining denotata of the term, while identifying seryj (with no obvious prototype) with a semantically close denominal adjective pepel'nyj 'ashen, ash-grey,' whose relation to the prototype is quite transparent. Yet in Russian the combinability of pepel'nyj is first, very restricted, and second, completely different from that of seryj. On the one hand, pepel'nyj cannot be substituted by seryj in its key context, to wit, pepel'nyel'serye volosy 'ash-grey/"grey hair.' On the other hand, the context most typical for seryj excludes pepel'nyj; compare with: serajal'pepel'naja bumaga/ten'/pyl' 'grey/*ash-grey paper/shadow/dust.' Both adjectives are possible only in the more trivial area of artifact colors. In contrast, the combinability of seryj in Russian is remarkably selective: apart from the contexts mentioned, seryj is possible mostly with certain names of animals (most often volk 'wolf'), as well as with names of garments. In Old Russian, according to Bakhilina (1975:39), the combinability of seryj was no less restricted: as attested by old chronicles, it was used to refer exclusively to the color of wool or monks' garments.

We hypothesize that Russian seryj is primarily related to the concept of 'low visibility', to being hardly perceptible. This meaning would explain its apparent negative connotations (grey color is undoubtedly a "bad" one in Russian folk semantics) and the suggestion of being "featureless" and "blurred", which shows up, for example, in the proverb nočju vse koški sery 'at night all cats are grey' or phraseological unit seryj kardinal 'power broker' (literally 'grey cardinal'). Incidentally, all grey animals are likely to be imperceptible and furtive, as mice, hares and wolves; compare this with a frequent collocation serye teni 'grey shadows.' This would further explain, why seryj is usually incompatible in Russian with 
names of signs or symbols (cf. ?seryj znak/flag/strelka 'grey sign/flag/arrow' etc.), since a sign is designed for being visible and salient. Similarly, a combination like ?? serye černila 'grey ink' sounds odd. This strong semantic loading of seryj excludes its combinability with many other nouns as well, such as 'serye cvety/napitki 'grey flowers/drinks' and the like. $^{2}$

\section{Zelënyj 'green' and žëltyj 'yellow'}

In general, the combinatorial properties of zelënyj 'green' and žëltyj 'yellow' have much in common: for both, there exists a strong nominal domain - semantically homogeneous and most frequently used - related to living vegetation. In the case of zelënyj, the focal denotatum is the color of young and growing grass and leaves. Note, that Russian zelënyj (as well as its Ukrainian cognate) can never apply to mown grass, though still green and fresh, because semantically this color term is strictly associated with the idea of growth (for details see Rakhilina 2000; Yavorska 1999). By contrast, žëltyj 'yellow' figures primarily as the color of fading. The vegetation domain appears the most probable candidate for prototypical adjectival uses in Russian, but is suggested for other languages as well.

This analysis agrees well with Wierzbicka's (1990) view of 'green.' The treatment of the 'yellow' prototype as 'sun' by Wierzbicka and some of her followers, for example Tokarski (1995, 1997), is more problematic: in Russian, the names of natural objects with apparently positive connotations (such as solnce 'sun,' zoloto 'gold,' volosy 'hair,' xleb 'bread,' and kaša 'kasha, gruel') seem to avoid this color adjective. This fact alone argues powerfully against 'sun' as a possible prototype for Russian žëltyj.

Another point is related to possible sources of negative connotations. For žëltyj, a negative component can be easily detected in the prototypical contexts of fading vegetation as such. For zelënyj, however, the prototypical contexts are definitely neutral, whereas a negative assessment appears only, when the adjective applies to growing fruits, yielding the meaning 'not ripe, premature'; compare this with a figurative zelënyj junec 'greenhorn.'

In still another sense, both žëltyj and zelënyj tend to be used for describing a sickly complexion and unpleasant discharge: žëltyj for describing sickly or aggressive eyes (especially those of animals), zelënyj for describing spoilt food and most reptiles and insects (which in Russian are ascribed pragmatically negative connotations).

An interesting hypothesis explaining a similar behavior of the gloss for 'green' in Ukrainian (and applicable equally well to Russian) is suggested by Yavorska (1999). This author relies heavily on the history of the word (which, incidentally, goes back to the same Indo-European root as the term for 'yellow') and argues that the unusual behavior of 'green' can be accounted for in the light of the old Indo-European opposition of living with non-living, since 'green' was used to denote both poles of it. From the historical point of view, this explanation is plausible. Moreover, given that 'green' and 'yellow' have

2. Borodina and Gak (1979), who provide a detailed study of French color terms (with special attention to the history and causes of French loans for 'white,' 'brown,' and 'grey' from Germanic languages), suggest that Germanic 'grey' (English grey, German grau) is related to the color of human life's decline - unlike its semantics in Russian. 
a common origin, it must hold for 'yellow' as well, which also describes the color of living (albeit fading) vegetation.

One may speculate that the common semantic origin and similar syntactic behavior of 'green' and 'yellow' in Russian and Ukrainian - as well as in other Slavic languages (Tokarski 1997; Waszakowa 1997) and, probably, other Indo-European languages - are rooted in an ancestral 'macro'- color category, grellow including both 'green' and 'yellow', an analogue of the 'macro'- color category grue covering 'green' and 'blue' (Berlin \& Kay 1969).

In search of an attributive meanings of zelënyj and žëltyj (or the concept of a prototype, according to Wierzbicka 1990), along with their common reference to vegetation, still other factors that affect both these color terms should be taken into account. Indeed, Russian zelënyj 'green' and especially žëltyj 'yellow' are similar in their opposition to the colors that describe human skin in its natural states, such as krasnyj 'red,' belyj 'white,' as well as rozovyj 'pink' and even zolotoj 'golden' (linguistically and etymologically the latter has the same origin as zelënyj and žëltyj). This attributive proximity of zelënyj and žëltyj in their opposition to the set of natural human colors, by virtue of anthropocentric character of the world image, is supposed to render to both a certain negative connotation.

We do not think, though, that the negative connotation is inherent to the semantics of zelënyj and žëltyj, since there exists a large class of their combinations with names of artifacts, in which both adjectives are emotionally neutral, for example, zelënyj/žëltyj sviter/dom/zabor/abažur 'green/yellow pullover/house/fence/lamp-shade.' Yet to incorporate all attributive and combinatorial peculiarities of zelënyj and žëltyj, an exhaustive description of their semantic meaning should convey the idea of opposition to the natural color of a healthy human body.

\section{Koričnevyj 'brown' and related terms}

In modern Russian koričnevyj, the term considered by Berlin and Kay (1969) as basic for 'brown,' is still not used for referring to color of natural objects, but primarily denotes the color of a paint or dye applied to artifacts. Specifically, koričnevyj is not used when one refers to the hides of animals like cows, cats, bears (although, from a physical point of view, all these can, of course, be described in terms of a brown-like color). Also, the following combinations sound highly problematic: 'koričnevaja zemlja/palka 'brown soil/stick,' ?koričnevyj stvol dereva 'brown tree trunk.' In contrast, their artifact-related counterparts are quite acceptable: koričnevaja kraska/kryša/židkost' 'brown paint/roof/liquid,' koričnevye šnurki/botinki 'brown laces/shoes.'

In Russian, natural objects of brown color are described with the help of the following adjectives, depending on the exact tinge: buryj '(dust) brown,' tëmnyj 'dark,' čërnyj 'black,' or ryžij '(brownish) red, sorrel'; for example, buraja zemlja '(dust) brown soil,' buryj medved' '(dust) brown bear,' ryžij kot '(brownish) red cat', tëmnye stvoly derev'ev 'dark trunks of trees.' In particular, the adjective buryj '(dust) brown' is used only for denoting colors of natural objects from the brown gamut and is not at all applied to artifacts: consider such impossible expressions as ${ }^{\star}$ my kupili buroe pianino 'we have bought a (dust) 
brown piano,' `segodnja ja, požaluj, nadenu burye botinki 'maybe, today I will put on (dust) brown shoes. $^{3}$

Linguistic behavior similar to that of buryj is also characteristic for ryžij '(brownish) red': consider the following impossible expressions * ryžij dom '(brownish) red house,' ?ryžaja čaška '(brownish) red cup,' *daj mne ryžuju knigu 'give me the '(brownish) red book.' The same is also true for the adjective pegij 'piebald, skewbald,' which is, though, corroborated by its lexicographic treatment by Evgen'eva (1980:Vol. 3, 37): in contrast to buryj and ryžij, as her analysis explicitly states, pegij is used only for denoting colors of animals. Notice also that a broader synonym of pegij, pjatnistyj 'spotted, dappled' also seems to be restricted to describing colors of natural objects (or, as the only possible extension, their imitations): for example, pjatnistaja škura 'spotted pelt', pjatnistye stvoly derev'ev 'spotted trunks of trees,' soldaty odety $v$ pjatnistuju formu 'soldiers are clothed in a spotted uniform.' By comparison, the following combinations are impossible: ${ }^{\star}$ pjatnistyj serviz 'spotted (dinner) service,' 'pjatnistyj divan/plašč 'spotted sofa/raincoat.' In these latter cases one is expected to use an expression like $v$ gorošek 'polka-dotted' or $s$ razvodami 'with free design' which, in turn, are not applicable to natural objects (for example, ${ }^{\star}$ leopard $v$ gorošek 'polka-dotted leopard' sounds ridiculous because it makes one think of an inanimate sofa rather than a living beast of prey).

Highly probably, the adjectives bagrovyj 'crimson' and lazurnyj 'azure' belong to the same class of nature-oriented color terms. Their use is gradually shrinking in modern Russian (Vasilevich 2003:53-54): bagrovoe lico/nebo 'crimson face/sky', bagrovyj nos/sinjak 'crimson nose/bruise,' as opposed to impossible ?bagrovye oboi/steny/mašiny/knigi 'crimson wallpaper/walls/cars/books'; similarly, lazurnoe more/nebo 'azure sea/sky' are opposed to *lazurnyj karandaš 'azure pencil' and the like.

Apparently, "artificial" color terms like koričnevyj do not have a proper basis for semantic description of the corresponding color, since they do not have any match to natural objects with an easily identifiable color. In contrast, "natural" color terms like buryj or ryžij are readily analyzable in this way. It is, however, noteworthy that in modern Russian the latter two adjectives are very infrequent: according to results of a psycholinguistic experiment, in which Russian native speakers were requested to rapidly list color names, buryj was ranked 108; by comparison, tëmno-koričnevyj 'dark brown' was assigned rank 31, svetlo-koričnevyj 'light brown' rank 70, and bledno-koričnevyj 'pale brown' rank 85 (Morgan \& Corbett 1989: 129-132).

The peculiar Russian lexical representation in the domain of 'brown' betrays an ongoing naming change in this part of the gamut: buryj and ryžij are becoming gradually obsolescent, giving way to the term koričnevyj that has emerged much later (Corbett \& Davies 1995:329). Indirect evidence of the expansion of koričnevyj can be inferred from emergence of derivatives (for example, koričnevatyj 'brownish') and its frequent use with brightness and saturation qualifiers, like tëmnyj 'dark' and svetlyj 'light.' By contrast, for

3. This attributive differentiation between the two Russian terms for 'brown' has a loose analogue in French, which possesses marron for describing artifacts of brown color as compared to brun with broader referential use (Lyons 1997:205-207). 
buryj and ryžij such modifiers, albeit possible, are very rare - tëmno-buryj 'dark brown' and tëmno-ryžij 'dark (brownish) red, auburn' are attested, but 'bledno-buryj 'pale (dust) brown,' ?bledno-ryžij 'pale (brownish) red,' 'svetlo-buryj 'light (dust) brown' and 'svetlo ryžij 'light (brownish) red' are all hardly acceptable.

According to Berlin and Kay (1969:6-7), derivational productivity constitutes a subsidiary, fifth criterion for basicness of a color term reflecting distributional potential of the term (see also Corbett \& Morgan 1988; Dixon 1982; Morgan \& Corbett 1989). Based, in particular, on the derivational productivity of Russian counterparts for 'brown, koričnevyj was assumed by Berlin and Kay to have status of a basic color term. We consider, however, that the election of koričnevyj as the basic color term corresponding to brown is premature. If one takes into account the semantic aspect, it turns out that the conceptual content corresponding to English brown is not entirely occupied by Russian koričnevyj.

Data from contemporary Russian corpora show a slow ongoing expansion of koričnevyj into the domain of natural objects (such as clay, sunburned or old man's skin, faded grass or leaves etc.). Examples available from the Bank of Russian National Corpus, which illustrate the use of koričnevyj for describing natural brown, originate mostly from texts, whose authors tend towards vernacular or colloquial style. For example:

(1) Ja razvernula bumažku, v nej byli zavërnuty dva koričnevyx oreška. (V. Belov) "I unfolded the paper; in it, there were two brown nuts."

(2) Ruki ležali na kolenjax - koričnevye, suxie, v užasnyx morščinax. (V. Shukshin) "The hands rested on the lap, brown, dry, with awful creases."

(3) Tëmnye koričnevye stvoly okružajut vas v ètom lesu. (V. Soloukhin) "In this forest, you are surrounded with dark brown trunks."

(4) Grant tem vremenem nalil mne iz termosa bol’šuju kružku tëploj koričnevoj burdy, kotoruju zaokeancy počemu-to nazyvajut "kofe." (Ju. Poljakov)

"Meanwhile, from his thermos Grant poured me a big mug of lukewarm brown wish-wash that for some reason the overseers name 'coffee."

The example of the term koričnevyj and color adjectives with similar functioning corroborates, again, the assertion that defining the natural-language system of color terms only in terms of light characteristics proves to be strained in many aspects - above all, reasoning of this kind does not take into account the internal, culturally stipulated structure of the system and the way color terms are used by speakers of the language under consideration. We argue that a native speaker does not proceed from an assumption that the spectrum is partitioned into disjoint fragments, each of which is associated with a certain color term if this were the case, there would be no language-internal difference, for example, between naming the color of earth and that of shoes or naming the color of hide and that of cups. The fact that such differences not only do exist but, moreover, are inherent to and deeply integrated in the semantics of linguistic units proves that one uses the color term system in another way. It might function with recourse to concepts of certain prototypes, or reference objects associated with each color term, as suggested by Wierzbicka (1990; for further development see also for Russian: Rakhilina 1995, 2000; for Ukrainian: Yavorska 1999; for Polish: Grzegorczykowa \& Waszakowa 1999; Tokarski 1995, 1997; Waszakowa 1997, 1999). 


\section{Conclusions}

Below we summarize our main findings on combinability of color adjectives as an indicator of their inherent semantics in Russian.

1. The combinability of color adjectives with object names in attributive constructions is essentially nontrivial. It reflects complex linguistic adaptation of a color term by the culture in question. We therefore argue that Berlin and Kay's third criterion - that basic color terms should be psychologically salient and in common use - needs an additional linguistic qualification.

2. The patterns of attributive combinability indicate that basic color terms can characterize natural objects that belong to the living environment of speakers of the language; thereby the basic color terms become conventional and acquire language-inherent connotations. This is possible due to the culturally-specific concept behind a prototype color, which stipulates combinatorial properties of the corresponding color term. In Russian, this mechanism can be illustrated, for example, by seryj 'grey' related to the concept of 'imperceptibility,' by žëltyj 'yellow' related to the concept of 'fading,' and by zelënyj 'green' related to the concept of 'the living and dead.' Due to culturally specific concepts, a stable area of focal uses for basic color terms is established in everyday language. Further, it is the concept that primarily accounts for semantic and syntactic cross-linguistic differences in focal uses of terms for one and the same physical color.

3. A color term emerging in a language has to follow a long path before it becomes basic. In Russian, brand-new color terms combine solely with the affix whose gloss is cveta 'color of', while escaping combinability in the form of adjectives with ordinary object names.

For example, with respect to a novel adjective kirpičnyj 'brick-red,' one can only say mašina/lico kirpičnogo cveta 'car/face of brick-red color,' but not ${ }^{\star}$ kirpičnaja mašina/kirpičnoe lico 'brick-red car/face' (the asterisked phrase would be acceptable in the sense 'made of brick', which in these cases is obviously pragmatically odd).

Similarly, there exists an expression černil'nogo cveta 'color of ink, ink-colored,' but the adjective černil'nyj 'inky' alone can hardly be used as a color term. The data from modern Russian corpora indicate, though, that černil'nyj is at a more advanced stage than kirpičnyj: whereas no single example of kirpičnyj 'of brick' alone as a color term has been found, we can observe sporadic use of černil'nyj alone, although the construction černil'nogo cveta predominates in color-describing contexts. For example:

(5) Ja zalez v kusty eževiki i stal est' spelye, černil'nogo cveta jagody. (F. Iskander)

"I crept into blackberry bushes and started to eat ripe, ink-colored berries."

In comparison, however, some rare examples of the "true" adjectival use of černil'nyj are also available, such as černil'nye tuči 'inky clouds' or černil'nyj asfal't 'inky asphalt':

(6) $<\ldots>$ zarosli, ele prosmatrivalsja svetlyj v nix proem, na fone sovsem černil'nogo neba. (An. Pristavkin) 
" $<\ldots>$ the shrubbery, ... there loomed in it a light aperture against a background of totally inky sky."

4. At the next stage of a color term's evolution, it combines unconstrained with names of artifacts; however, combinations with names of natural objects remain prohibited or very restricted. Full incorporation of a color term into the existing linguistic system, being a long process, is characterized by competition between a new, "strong" color term and an old one that is gradually replaced within the domain of artifacts. As a result, a coexistence of the two emerges, wherein the old color term is contrasted to the new term by way of their scopes: the old term persists in the domain of natural objects, whereas the new one invades the domain of artifacts.

This evolution is exemplified by the Russian old buryj '(dust) brown,' ryžij '(brownish) red' or bagrovyj 'crimson' and their "new” counterparts koričnevyj 'brown,' oranževyj 'orange,' and fioletovyj 'purple,' respectively. The adjectives from the latter group were borrowed in the 17th century, and despite gaining basic status, still remain at a stage of restricted combinability.

5. The analysis above provides ground to predict that in future development, certain new color terms would completely replace their predecessors to occupy the place of the latter within the color-term system. We suppose that among the new supplanting color terms there would be primarily those that have direct counterparts among the old color terms, like koričnevyj. In comparison, those color terms that do not have immediate competitors in the old system (like fioletovyj) are likely to repeat the path of Russian goluboj 'light blue': once a niche in the domain of natural objects has been conquered, these terms have a good chance to enrich the system by means of colorterm differentiation (cf. a review of Paramei 2005).

There is general agreement that the seminal work of Berlin and Kay (1969) made a break-through in the systematic description of color terms. From the current linguistic perspective it is, however, evident that the framework proposed in their theory is too tight to cope with patterns that actually determine the use of color terms within each specific language. Being aware of the problem, investigators in a host of subsequent studies are endeavoring to amend this conception (e.g. Jameson 2005; Roberson 2005).

An important step on this way was marked by the Vantage Theory of MacLaury (1997, 2001) with its emphasis on the cognitive grounds for the extent of color terms undergoing social activity pressure. Another view, which underlines variability of the color-term structure, is grounded in the role of cultural system of references (e.g. Dedrick 1998; Kay \& Maffi 1999).

Our investigation carried out by means of linguistic analysis - unlike those in cultural anthropology named above - converges with their conclusions and elaborates on variability of color term meaning. We argue that coexistence of two or more color terms related to the same physical portion of the color gamut, but whose use is driven by the need to denote colors of either natural or artifact objects, is one of the basic grounds for systemic variability in term meaning. It may also generate the vantage effect in psycholinguistic studies, such as those of Frumkina (1984) or MacLaury (1997), since native speakers, while ascrib- 
ing color terms to color samples, implicitly confront the uncertainty of whether the term should refer to color of natural or non-natural origin.

Further, we propose an alternative explanation for the relative stability of focal uses that the basic color terms have across languages; specifically, the focal uses usually reflect a pragmatically motivated relationship between the color term and the color of culturally salient natural objects.

\section{References}

Bakhilina, Natalja B. (1975). Istorija cvetooboznačenij v russkom jazyke [The history of color terms in the Russian language]. Moscow: Nauka.

Berlin, Brent \& Paul Kay (1969). Basic color terms: Their universality and evolution. Berkeley: University of California Press.

Bhat, Darbhe Narayana Shankara (1994). The adjectival category: Criteria for differentiation and identification. Amsterdam \& Philadelphia: John Benjamins.

Bolinger, Dwight (1967). Adjectives in English: Attribution and predication. Lingua, 18, 1-34.

Borodina, Melitina A. \& Vladimir G. Gak (1979). K tipologii i metodike istoriko-semantičeskix issledovanij [On the typology and methodology of historico-semantic studies]. Leningrad: Nauka.

Comrie, Bernard \& Greville G. Corbett (Eds.) (1993). The Slavonic languages. London \& New York: Routledge.

Corbett, Greville G. \& Ian R. Davies (1995). Linguistic and behavioural measures for ranking basic colour terms. Studies in Language, 19, 301-357.

Corbett, Greville G. \& Gerry Morgan (1988). Colour terms in Russian: Reflections of typological constraints in a single language. Journal of Linguistics, 24, 31-64.

Dedrick, Don (1998). Naming the rainbow: Colour language, colour science, and culture. Dordrecht/ Boston/London: Kluwer Academic Publishers.

Dik, Simon C. (1989). The theory of functional grammar. Part: 1 The structure of the clause. Dordrecht: Foris.

Dixon, Robert M. W. (1982). Where have all the adjectives gone? And other essays in semantics and syntax. Berlin: Mouton.

Evgen'eva, Anastasija P. (Ed.) (1980). Slovar' russkogo jazyka v 4-x tomax [Dictionary of Russian in 4 volumes]. Moscow: Russkij jazyk.

Frumkina, Revekka M. (1984). Cvet, smysl, sxodstvo: Aspekty psixolingvističeskogo analiza [Color, meaning, similarity: Aspects of a psycholinguistic analysis]. Moscow: Nauka.

Frumkina, Revekka M. \& Alexei V. Mikhejev (1996). Meaning and categorization. New York: Nova Science.

Grzegorczykowa, Renata \& Krystyna Waszakowa (Eds.) (1999). Studia z semantyki porównawczej. Nazwy barw. Nazwy wymiarów. Predykaty mentalne [Studies on comparative semantics. Color terms. Measure terms. Mental predicates.] (Part I). Warsaw: University of Warsaw.

Jameson, Kimberly A. (2005). On the role of culture in color naming. Cross-Cultural Research, 39, 88-106. Kay, Paul \& Luisa Maffi (1999). Color appearance and the emergence and evolution of basic color lexicons. American Anthropologist, 101, 743-760.

Lyons, John (1997). Colour in language. In T. Lamb \& J. Bourriau (Eds.), Colour: Art and science (194224). Cambridge: Cambridge University Press.

MacLaury, Robert E. (1997). Color and cognition in Mesoamerica: Constructing categories as vantages. Austin: University of Texas Press. 
MacLaury, Robert E. (2001). Color terms. In M. Haspelmath, E. König, W. Oesterreicher, \& W. Raible (Eds.), Language typology and language universals (Vol. 2: 1227-1251). Berlin/New York: Walter de Gruyter.

Mikhailova, Tatjana A. (1994). “Krasnyj” v irlandskom jazyke: ponjatie i sposoby ego vyraženija ['Red' in the Irish language: The concept and means of its expression]. Voprosy jazykoznanija, No. 6, 118-128.

Morgan, Gerry \& Greville G. Corbett (1989). Russian colour term salience. Russian Linguistics, 13, 125141.

Moss, Anthony, Ian Davies, Greville Corbett, \& Glynis Laws (1990). Mapping Russian basic colour terms using behavioural measures. Lingua, 82, 313-332.

Paramei, Galina V. (1999). One basic or two? A rhapsody in blue. Behavioral and Brain Sciences, 22, 967.

Paramei, Galina V. (2005). Singing the Russian blues: An argument for culturally basic color terms. CrossCultural Research, 39, 10-38.

Quirk, Randolph, Sidney Greenbaum, Geoffrey Leech, \& Jan Svartvik (1972). A grammar of contemporary English. London: Longman.

Rakhilina, Ekaterina V. (1995). O cvetnom i bescvetnom [On the colored and colorless]. Rusistika segodnja, 1, 50-69.

Rakhilina, Ekaterina V. (2000). Kognitivnyj analiz predmetnyx imën: semantika i sočetaemost' [Cognitive analysis of object names: Semantics and combinability]. Moscow: Russkie slovari.

Roberson, Debi (2005). Color categories are culturally diverse in cognition as well as in language. CrossCultural Research, 39, 56-71.

Tokarski, Ryszard (1995). Semantyka barw we współczesnej polszczyźnie [Semantics of colors in modern Polish]. Lublin: University of Lublin.

Tokarski, Ryszard (1997). Regularny i nieregularny rozwój konotacji semantycznych nazw barw [Regular and non-regular development of semantic connotations of color terms]. In B. Nilsson \& E. Teodorowicz-Hellman (Eds.), Nazwy barw i wymiarów [Color and measure Terms] (63-74). Stockholm: University of Stockholm.

Vasilevich, Alexander P. (1987). Issledovanie leksiki v psixolingvističeskom èksperimente: Na materiale cvetooboznačenija $v$ jazykax raznyx sistem [An investigation of nomenclature lexicon in a psycholinguistic experiment: On material of color terms in languages of different systems]. Moscow: Nauka.

Vasilevich, Alexander P. (2003). Jazykovaja kartina mira cveta: metody issledovanija i prikladnye aspekty [Linguistic picture of the world of color: Methodology of investigation and applied aspects]. Postdoctoral dissertation. Moscow: Institute of Linguistics.

Waszakowa, Krystyna (1997). Dotychczasowe badania w zakresie nazw barw w poszczególnych językach a perspektywa ich całościowego opisu porównawczego [Contemporary studies in the domain of color terms in different languages and a perspective of their integral comparative description]. In B. Nilsson \& E. Teodorowicz-Hellman (Eds.), Nazwy barw i wymiarów [Color and measure Terms] (87-96). Stockholm: University of Stockholm.

Waszakowa, Krystyna (1999). Struktura znaczeniowa podstawowych nazw barw: założenia opisu porównawczego [Semantic structure of basic color terms: Fundamentals of a comparative description]. In R. Grzegorczykowa \& K. Waszakowa (Eds.), Studia z semantyki porównawczej. Nazwy barw. Nazwy wymiarów. Predykaty mentalne [Studies on comparative semantics. Color terms. Measure terms. Mental predicates] (Part I: 59-73). Warsaw: University of Warsaw.

Wierzbicka, Anna (1990). The meaning of color terms: Semantics, culture, and cognition. Cognitive Linguistics, 1, 99-150.

Yavorska, Galina M. (1999). Movni koncepty koljoru (do problemy katehoryzacii) [Linguistic concepts of color (on the problem of categorization)]. Movoznavstvo, No. 2/3, 42-50. 



\title{
Color words in painting descriptions
}

\section{Some linguistic evidence for entity-like conceptualization}

\author{
Alena V. Anishchanka \\ Philology Department, Yanka Kupala State University of Grodno, Belarus
}

The chapter contains linguistic analysis of a sample of 100 catalogue entries written for three American museums to describe individual paintings. The aim of the study is to investigate the use of color words by art critics and painters in American English. Results of the analysis indicate an extensive use of nominalization in the color lexicon of discourse studied - along with attributive function of color words, which perform as modifiers of nouns and signal color as a property of an entity. Notably, both basic and non-basic color terms tend to be used as nouns. The nominalized color words exhibit the full range of morphological, semantic, and syntactic features of nouns. We suggest that this kind of verbalization of color concepts in painting descriptions reflects a certain model of understanding color as a domain of human experience, in particular, categorizing color as a thing-like manipulable entity.

\section{Introduction}

The analysis of color language use has become an indispensable part of current understanding of color categorization - and very often its starting point. Researchers in the fields of anthropology, psychology, psycholinguistics, and cognitive science traditionally approach the problem of color semantics by mapping color terms on standard arrays of color samples (e.g. Berlin \& Kay 1969; MacLaury 1997). This methodology is in accord with the basic assumption that language reveals underlying conceptual constructs, that words and grammatical structures signal a speaker's construal of a certain cognitive domain. Depending on the conceptualizer's viewpoint and the manner in which s/he mentally scans a situation (fragment of the real world), there emerge alternative ways for its cognitive construal and, accordingly, alternative ways to speak about it (Casad 1995; Taylor 1995).

In recent years an increasing number of voices have been raised pointing out that psycholinguistic and anthropological research on color semantics are focused predominantly on denotation of color terms. This latter is modeled by the three dimensions (qualities) 
of color - hue, brightness/lightness, and saturation, usually in terms of the Munsell Color Solid or similar color models. In this vein, the meaning of color words is understood in terms of their reference, or capacity to label certain referents represented by color stimuli (for a criticism see Lucy 1997:322-323; Rakhilina 2007; Sahlins 1976:8-9). Lyons (1999:50) stresses that the prevalent methodology favors context-independent reference of color terms, rather than their use for description of colored entities and substances, that is, an attributive use of color terms.

We believe, however, that semantics of a color term is not restricted to its referent; rather, an additional component of the color term's meaning is signaled by its lexical category and word form. These latter situate the color concept in the discourse and construe its interrelationships with other concepts in the language. From this it follows that a better understanding of how color is conceptualized and spoken about can only be achieved through an analysis of discourse structures that convey color concepts.

Lucy suggests two factors that contribute together to the semantic meaning value of linguistic categories; and we argue that these should be given attention in the analysis of color semantics:

The first is the category's characteristic referential range, that is, its routine use to pick out or differentially signal certain referents across a wide range of contexts... . The second factor is the category's formal distributional potential, that is, its position within the available categories in the language with which it contrasts and combines. (1997:322)

The formal distributional potential and interaction of color categories with other categories in the language are our main interest here. These are revealed, in particular, by morphological studies of color terms and their derivational structure (see, e.g., Corbett \& Morgan 1988). Of special interest is the interaction of color semantics and entity concepts, which one encounters in denominal derivation of color terms. Within this derivational pattern, a color property emancipates from an object with which it is commonly associated, and emerges as an independent color concept, while relegating the notion of the object to the background (MacLaury 1997:186-187; Steinvall 2002:142-149; Vasilevich 1987:40-52).

To study distributional potential of color terms, an original approach is proposed by Rakhilina (1995) that focuses on discourse properties of color concepts. She analyzes the linguistic combinability of color adjectives with nouns, which denote colored objects, while drawing special attention to extralinguistic and cognitive factors that impose combinatorial restrictions on the adjectival use of color terms (Rakhilina 2007).

Of further interest are studies of the functions of color terms in a discourse. These allow one to investigate color as an essential part of a virtual world created in the text, as well as the role that the color component plays in discourse of various authors and in texts of different types and styles. With such purpose, an analytical approach was elaborated by Sereda (1984), who models the interaction of major lexical groups in a short story color being one of them - and traces their role in the narrative development. Her central notion is that of a lexical-thematic group, a group of lexical units used within the text interchangeably to convey related semantic content. The notion allows one to describe the 
color domain as a "decorative" element of an overall semantic unity created in the story and interwoven with other lexical groups, thereby revealing its relation with other story elements (Sereda 1984:15).

The present study focuses on a verbal expression of color conceptualization in descriptions of painting. By analyzing the way artists, art critics, and art historians speak about color, we seek for linguistic evidence of alternative ways of conceptualizing color.

The possibility of this alternative color conceptualization in the context of painting description has been prompted by the following poem by Wassily Kandinsky, the renowned Russian artist and theorist of art:

Blue, blue rose up, rose up, and fell.

Sharp, thin whistled and pressed in, but could not pick through.

There was a droning from every corner.

Fat brown hung seemingly for all eternity.

Seemingly. Seemingly. (1911/1994:226)

C. A. Riley (1995:144) comments that "abstract meditations of this kind are always susceptible to a number of interpretations," and suggests his own, with the perspective of an art historian.

A linguist, in comparison, would infer from Kandinsky's passage much more of the artist's use of color words than just reference to blue, brown, or green. One notes in particular that colors here act - move and feel ("rise up," "fall," "drone"); they become characters in a "story" narrated on a canvas (one is "fat," the other is "thin" and "sharp"); and they are self-sustained entities, quasi-living beings capable of their own expression.

Such an animated conceptualizing of color is far from being unique to painting in the 20th century; one finds a similar case in modern literature and music. For example, C. A. Riley comments on the use of color words by James Joyce in Ulysses: "They [colors] are active characters, or states through which Bloom and Stephen travel, just as they represented characters to Schoenberg or Kandinsky" (1995:230).

In the theory and history of art, the concept of an emancipated color is formulated in terms of a competition between color and line, the attempts to liberate color from its descriptive function (C. A. Riley 1995; B. Riley 1997; Walch \& Hope 1995). This view motivates B. Riley to distinguish two modes of artists' conceptualization of color-perceptual and pictorial. The former is a natural and common mode of seeing and understanding color as color of something, namely a property of a substance. In contrast, pictorial conceptualization is inherent in picture making, where color is conceived as itself, independent of the carrying substance:

For painters, colour is not only all those things which we all see but also, most extraordinarily, the pigments spread out on the palette, and there, quite uniquely, they are simply and solely colour... So the painter has two quite distinct systems of colour to deal with one provided by nature, the other required by art - perceptual colour and pictorial colour. (1997:31-32) 


\section{Purposes and method of the study}

The scope of the present study is the semantics of English color terms beyond their referential characteristics, in particular their distributional potential, context contingency, and discourse constraints. We seek linguistic evidence for the dual - perceptual versus pictorial - (or possibly multiple) conceptualization of color conveyed by descriptions of paintings.

To pursue this goal, we analyze a sample corpus of catalog entries written by art critics for three American museums: Columbus Museum of Art (Agee \& Baur 1989), Munson-Williams-Proctor Institute (Schweizer 1989), and Helen Frankenthaler retrospective (Carmean 1989). The catalogue entries describe individual paintings by artists of different artistic traditions, in particular: Hudson River School (F. Church, S. Gifford, M. Head); Impressionism (J. Sargent, J. Whistler); Post-Impressionism (M. Prendergast); Synchromism (S. Macdonald-Wright); Precisionism (P. Dickinson, Ch. Sheeler); Cubism (L. Feininger); Color-Field school (H. Frankenthaler, K. Noland); and Abstract Expressionism (W. de Kooning, B. Tomlin).

Each text is a description of a painting, its content, and artistic methods from the critic's perspective. Most of the texts contain, in addition, the artist's comments on the meaning of the painting, motives and history of its creation, and her/his attitudes to elements of the work, in particular to color.

We make no specific distinction as to the difference in the color conceptualization by art critics versus artists, since the studied phenomenon (entity-like conceptualization of color) is found equally in the critics' texts as well as in the quotations of the artists.

The present study focuses on the construal of the situation where color property is ascribed to a certain entity. The analysis is based on the color-term's minimal syntactic context sufficient to ascertain its lexical and grammatical categories. In most cases, the required syntactic minimum is provided by a noun phrase (NP), which designates an entity and its properties (including the color property). In some cases, however, semantics of a color term can be analyzed adequately only at the sentence level, since, when substantivized, color words become part of the sentence proposition.

\section{Color words as adjectives}

The analysis of the corpus shows that the most commonly used way to verbalize the semantic relationship between an entity and its color property (41.69\% of all tokens) is in the form of adjectival head modifier construction $\left(A_{C} N\right)$, which is also the most common construction in any discourse. The $A_{C} N$ construction (highlighted by italics) is exemplified as follows:

(1) In the painting, orange, purple-red, and white rectangles at the top and a large white rectangle below float on a pink field, their indefinite edges making them seem to emerge from and fade into the field (Schweizer 1989: 186). 
He depicts recognizable components - a bowl of oranges, a goblet holding an orange flower, and two bananas on a table in front of rich blue curtains - using devices introduced by Cézanne and the Cubists (Agee \& Baur 1989: 108).

Here the head noun $(N)$ names the entity, the carrier of the color-property. The modifier position is taken by a color word, which is an adjective $\left(A_{C}\right)$. Characteristic of this type of construction is that the object, in this situation regarded as the carrier (Downing \& Locke 2002:131), dominates in the construal of the color-and-object relationship and serves as its focus.

In such constructions, the object name is the syntactic and semantic center of the noun phrase; it possesses all morpho-syntactic markers of nouns. As the noun phrase heads, the object names may be singular or plural, may take determiners, adjectives, and demonstratives. In (1) it is the noun form that carries additional grammatical meanings of singularity and plurality, definiteness and indefiniteness, which are associated with the entity and manifested through the single or plural noun form and the use of articles and determiners. Also, in the semantic structure of the sentence the role of the noun phrase is conditioned foremost by the semantic structure of the noun.

In $A_{C} N$ constructions the color meaning - color seme, or denotatum of color properis expressed in the adjectival form, which takes the semantically and syntactically dependent position of the modifier. Hence, color is conceptualized as an objective property of an entity, subordinate to and inseparable from it, a property that does not exist without its carrier, but is only conceived of as an attribute of the entity.

This semantic interpretation is in straightforward accord with the traditional semantic description given to the basic lexical classes of noun, verb, or adjective, whereby nouns describe entities, verbs actions, and adjectives properties (for a discussion see also Taylor 1995: 183-197; Wierzbicka 1986: 353-356).

The nature of the color-and-object relation is constrained by semantics of both components of the NP - a noun and an adjective; by the combinability of the noun that designates the object and of the color adjective; and by the context, in which the object is given the color property (see Rakhilina 2007).

Within the NPs, the color property can be designated with varying degree of categorical specificity. Particularly, in the analyzed type of texts, basic color terms (BCTs, Berlin \& Kay 1969) dominate. Along with the BCTs, we find non-basic monolexemic and compound color terms. Further, to express shades and mixtures of colors, both basic and non-basic color terms may take on derived forms - as indicated by the following examples.

\section{Non-basic monolexemic color adjectives:}

(2) Against this dense verdant screen-like setting ... is arrayed a band of figures in the foreground (Schweizer 1989: 100).

The "hanging downward" character of this mauve field looks toward the "banner" paintings of the next years (Carmean 1989:46). 
2. Derived adjectives with suffix -ish describing insufficient quality:

(3) Here, the left side of the canvas is filled with a trio of elongated blackish shapes (Carmean 1989:26).

A deeper, reddish light can be seen through the windows (Schweizer 1989:105).

3. Compound adjectives with two color-adjective stems:

(4) Columns of grey-green and white-grey smoke rise in parallel rows (Schweizer 1989:105). As Frankenthaler notes, "The gray-green border was painted with a small detailing brush in order to adhere to and not overlap the exact yellow edge" (Carmean 1989:42).

4. Compound adjectives with a basic-color stem combined with an achromatic modifier:

(5) Brushed over a mauve ground color, the brilliant white plaster facades seem to merge with the atmosphere (Agee \& Baur 1989:30).

Of particular note is the way a wide light brown stroke near center left locks into the black configuration (Schweizer 1989:201).

5. Compound adjectives with a noun and a color-adjective stem:

(6) “'Grey Fireworks," wrote Patricia Johnson in 1982, "is a dove gray slip of background upon which bursts of color are applied" (Carmean 1989:84).

Characteristic of his work during the 1930s is the silhouetting of brown, rust-red, or gray forms against a clear blue sky (Schweizer 1989:152).

6. Compound adjectives with the component -colored:

(7) Even the woman's cream-colored flesh is modeled with yellow, blue, violet, and green (Agee \& Baur 1989: 134).

Vertical salmon-colored bands punctuate and ground the canvas (Carmean 1989:88).

7. Denominal words where the noun-stem implies characteristic color of an object:

(8) The charcoal lines were original guideposts that eventually became unnecessary. (Carmean 1989: 13).

Like his earlier figurative watercolors, such as "The Circus," "Bowl of Oranges" is a graceful composition with curving graphite lines and fluid design (Agee \& Baur 1989: 108).

In (6)-(8), denominal color words are derived from the names of objects which have typical coloration. Such color words can be seen as retaining in their semantic structure an implied reference to the object, varying in its salience, best represented as a scale (Steinvall 2002: 145-149).

The nature of the object which is attributed a certain color property, and the grammatical features of the noun that designates the object, also affect color conceptualization (e.g. Rakhilina 2007). From this perspective, one can distinguish different types of semantic groups of nouns. In the studied discourse corpus, we distinguish three groups of $A_{C} N$ combinations, which differ in semantic properties of head nouns.

1. Combinations of color adjectives with nouns designating physical objects (natural, artificial, and living). For example: 
(9) The whole painting escapes the threatened boredom of a monochrome by grace of the yellow hat with its flick of ribbon (Schweizer 1989: 142).

The contrast between the apartment buildings and the small white house below them is indicative of the city's continual growth (Schweizer 1989:119).

Combinations of this type are used to describe the objects of the pictorial world that represent the objects in the world out there, serving as models and inspiration to an artist. Three-dimensional objects designated by the nouns of this group are projected onto the pictorial reality and their use potentially is not limited to the context of painting. Such constructions are found predominantly in descriptions of realist paintings, whereby the language means are intended to verbalize a pictorial world isomorphic to the reality.

2. Combinations of color adjectives with nouns naming non-representative elements of the pictorial reality, for example:

(10) Moreover, it is significant that few white areas in "The Bridge" are opaque (Schweizer 1989:201).

Other lines scattered throughout the blue field serve as small animated accents (Agee \& Baur 1989: 150).

The green rectangle at the lower left, for example, is echoed by the white (painted) triangle in the center (Carmean 1989:32).

The quotations in (10) indicate that meaning of this type of nouns is mainly that of the shape and space - the few features of the real world which are left in abstract art. The entities designated by these nouns are two-dimensional elements of pictorial reality created by the artist on the canvas, which are inherently connected with the domain of painting.

3. Combinations of color words with nouns designating aspects of paint (colorant) application, where color property is not that of an object but of the applied paint (coloring agent):

(11) a. Black drips and runs, as well as more substantially painted black details, are visible beneath the white underpainting (Schweizer 1989:201).

$\langle\ldots\rangle$ both used pale linear configurations on a darker ground (often called "white writing") (Schweizer 1989:161).

b. Frankenthaler's thin layering of white film echoes the underpainting and glazing found in the Titian (Carmean 1989:78).

Elsewhere, thinly brushed orange and blue pigment over a pale cream primer give the effect of a watery atmosphere (Agee \& Baur 1989:132).

Entities designated by nouns like in (11a \& b) can be viewed as belonging to the threedimensional physical world, as well as to the two-dimensional pictorial reality. They give another perspective of a painting as an artifact (canvas and paints put together). The dynamic aspect of paint application is indicated by deverbal nature of the nouns like drips, runs, writing in (11a).

Finally, in several cases we encounter combinations of color adjectives with a noun naming a real object embedded in pictorial reality of an abstract painting. In these cases 
the abstract two-dimensional elements receive an interpretation based on their similarity to objects of the real world:

(12) This blue "lake" sits on passages of green and yellow and is bordered by nearly closed brown rectangle (Carmean 1989:28).

The largest shape in "Yellow Caterpillar" is the long yellow passage at the top for which the work is named (Carmean 1989:30).

Thus, the yellow passage in the painting is interpreted as a yellow caterpillar, and in the same vein the blue shape becomes a lake.

\section{Color words as nouns}

One of the most striking features of the studied discourse is an abundant use of substantivized color terms, which function as nouns and exhibit a set of semantic and syntactic characteristics different from those of adjectival color terms.

There is some disagreement as to the basic categorical nature of color words. On the one hand, Lyons (1999) suggests that color is probably not a real property (see also Taylor 1995: 183). On the other hand, in his seminal work on adjective types Dixon (1977:29-31) assigns color words to the class of prototypical adjectives. In the present study we follow the view that notionally color words express properties and that they are adjectives, which can be substantivized, as in (13):

(13) She recalls: "This picture existed for quite a while without the other, outer green" (Carmean 1989:38).

The passage from day to night is conveyed by the interaction of reds and blues in the sky (Agee \& Baur 1989:84).

Notably, in the analyzed discourse sample, substantivized color adjectives constitute 58.3\% of all tokens. The syntactic structure can formally be represented as $(X) N_{C}(X)$, where $N_{C}$ is the substantivized color adjective that represents the color denotatum (color seme) and serves as the headword of the noun phrase and its semantic and grammatical center. The $(X)$ indicates various dependent elements (determiners, adjectives, and prepositional phrases), which modify the color concept designated by the headword.

The headword position of the color word in these constructions can be attributed to the conceptualization that essentially differs from that described in the previous section. Substantivization of the color term supposes construal of color as a self-sufficient entity, independent of the carrier, which is possible due to the reifying nature of human thinking. Following Frawley we understand entities in a broad sense as "relatively stable and atemporal discourse, ontological, and conceptual phenomena" (1992:68), which underlie the formal class of nouns.

Substantivized use of color words in the painting descriptions can be best explained in terms of the theory of discourse-based features of lexical categories by Hopper and Thompson (1984). The authors consider nounhood as a manifestation of entity-like cat- 
egorization, which is inferred from the form's ability to introduce a participant of the discourse. Namely, to the extent that a linguistic form is carrying out this prototypical function, it is coded as a noun and manifests the full possible range of nominal trappings conventional in the language (Hopper \& Thompson 1984:707-712). Accordingly, when color concepts are construed as the discourse participants, that is, the things most significant in the situation and spoken about, color words tend to surface as nouns.

Indeed, the following examples illustrate that the substantivized color word manifests the full range of morpho-syntactic markers typical of a noun.

1. May be singular or plural:

(14) Yellows and blues are mixed to an extraordinary range, from the sunlit and shadowed green of the wall to the dark emerald window shade, to a gamut of saturated greens and pale or deep blues in the vertical strips of window molding (Agee \& Baur 1989:134).

Indeed, close observation reveals that the horizontal strata are still present and are clearly made of blues of different pigments, most likely nine distinct blues in all (Carmean 1989:36).

Example (14) reveals the relevance of the bound/unbound distinction for the entity-like conceptualization of color, which motivates mass and count use of color words. Whereas prototypically color words are mass nouns, they can be pluralized (and counted) under special semantic constraints involving the notion 'kind of, or 'portion of. The context imposes a reinterpretation of color, which notionally is unbounded (hence unpluralizable and uncountable), as something bounded (a kind of, a stroke of) (Kay 1999:79-80).

2. May take determiners, adjectives, and demonstratives:

(15) The rich warm colors, most intense near the center of the painting, project strongly from the receding blues in the drapery (Agee \& Baur 1989:108).

This blue, in turn, becomes a further tone, as a wash over the white at the lower center of the work (Carmean 1989:74).

Once conceptualized as an entity, color acquires semantic features typical of a noun. For instance, within one of the basic semantic relations of possessor-possessed, color can take on the possessed role, as is illustrated by catalogue samples:

(16) $<\ldots>$ her dark and lighter browns at the right accord with Manet's copper pot (Carmean 1989:80).

Substantivized color words acquire the ability to take substantive positions in a sentence and function as arguments of the predicates becoming part of the sentence proposition.

Colors conceptualized as entities are typically found in the propositions that describe material processes (processes of “doing”) (Downing \& Locke 2002:114) involving manipulation of objects.

Most commonly color appears in the position of the grammatical object that describes color as an entity handled by an artist and can be ascribed the thematic role of a patient (Downing \& Locke 2002: 115; Frawley 1992:210): 
(17) a. "I looked at the painting for a long time, then added the orange, the darker blue, and the black" (Carmean 1989:64).

This along with a comparable layering of blacks and the use of brown creates a sharply felt planar tension (Schweizer 1989:201).

b. And in turn the blue enlivens the orange (Agee \& Baur 1989:94).

This kind of conceptualization is found in two contexts: (i) when the process of painting and the creation of a picture is described, as in (17a); and (ii) when the content of the picture is commented on (17b). In both cases, it is the paint, as it comes out of the tube and is applied to the canvas, which is the carrier of the color property. However, in the context of painting description the situation is reinterpreted: the artist or the critic speak about the paint, as if it were color itself that was applied.

As an element of the pictorial reality, color is even more liberated from the carrier and more entity-like, whereby it can function as a sentence subject:

(18) The white created an overall, controlled, autonomous shape (Carmean 1989:74). $<\ldots>$ the deep pink at the bottom jumps over the greenish line (Carmean 1989:86).

In this position color words receive the thematic role of an actor affecting the situation (Frawley 1992:203; cf. Downing \& Locke 2002:114-115).

Further, in the description of a material process, color can be conceived of as an instrument:

(19) "What got to me in the Titian," Frankenthaler says of this picture, "is how much color he created using essentially black and white..." (Carmean 1989:78).

His palette is arranged around warm blue-grays, siennas, and umbers heightened with pink (Agee \& Baur 1989:134).

Color as an entity appears in several kinds of relational processes (processes of "being " or "becoming"), in which a participant is characterized or identified, or situated circumstantially (Downing \& Locke 2002: 131).

For instance, in the attributive relational propositions, where a certain property is ascribed to the participant, color can function as a carrier (attributant) of the property:

(20) The right-side orange, by contrast, seems more solid, like a banner suspended from the upper edge (Carmean 1989:52).

That pink was a vital, last-minute gesture (Carmean 1989:86).

Another characteristic feature of the color conceptualized as an entity is its precise localization in the space of the pictorial world, for which various lexical means and syntactic structures are used.

1. Prepositional phrases:

(21) Sequences of analogous colors, such as the blue-greens and blue-violets in the upper left of the canvas, are gentle in their visual energy (Agee \& Baur 1989:94).

The brilliant greens and yellows of the park below the tenements are vitalized by the highly saturated reds on the billboards (Schweizer 1989:119). 


\section{Adjectives in attributive position:}

(22) A similar kind of opening can be found in "Nature Abhors a Vacuum," at the upper left profile of the green, or in the small opening in the topmost yellow (Carmean 1989: 62). The exterior composition is direct and cool - in contrast to the glow of the interior oranges (Carmean 1989:44).

\section{Predicates:}

(23) She continues: "Arden does not make reference to an ideal or spiritual place, and - with the pink surrounded by the greens - you can conceive of it as an enclosed place" (Carmean 1989:32).

In fact the green is laid on over the black mass (Agee \& Baur 1989:22).

These constructions are typical of contexts where the content of the painting is described. They allow color entities to be located in the pictorial world, in a similar way as real objects are located in the physical world.

\section{'Of'-constructions}

In a number of contexts, color concept is designated with the help of the of-constructions. These can be interpreted as cases of substantivized use of color, since the structure consists of two nouns: the head noun, which is the semantic and syntactic center of the phrase, and the prepositional phrase, which serves to modify the head noun and is semantically and syntactically dependent on it.

The sample corpus provides examples of color words used in both the head and the modifier positions. Accordingly, one can distinguish two types of constructions that differ with regard to the conceptualization of the color property itself and the ways in which it is ascribed to an entity.

1. Of-constructions $N$ of $N_{C}$, in which the color seme is signaled by the substantivized color word $N_{C}$ functioning as a syntactically dependent element, whereas the headword $N$ is a noun that names the entity whose color property is described. For example:

(24) In this diffuse light, one spots the short, quick strokes of yellow marking an early spring leafing (Schweizer 1989:91).

Because border colors are either analogous (yellow, pink, ivory) or complementary (lavender, green, gray-blue) to the dominant field of orange, visual unity is maintained (Agee \& Baur 1989: 152).

Here the color word is substantivized and acquires some of the morpho-syntactic markers of a noun (for example, it can have its own modifier as in "the band of rigid grey"), but it still takes on the dependent syntactic position. Thus, this structure is similar to the $A_{C} N$ in the way it signals the dependency of the color property on the entity. 
Characteristically the head nouns in these constructions designate the two-dimensional elements of the pictorial world and are related to the contents of a painting; compare with (10).

2. Of-constructions $N_{C}$ of $N$, in which the color seme is expressed by the substantivized color adjective $N_{C}$ functioning as the headword:

(25) For example, the dominant blues of the sky and water are intensified by small areas of complementary orange interspersed throughout the composition (Agee \& Baur 1989:94).

The title "Sesame" carries a double reference: one is to the ocher color of sesame seeds not unlike the way in which Tangerine refers to the orange of the fruit - while the other is to the magical command first given by Ali Baba (Carmean 1989:54).

This type of construction includes nouns that visibly describe objects of the physical world out there. However, the carriers of the color property take on the dependent syntactic position and become the ground, whereas the focus is apparently shifted from the objects to their color.

The two types of of- constructions are syntactically different. Whereas Type 1 could be transformed into an $A_{C} N$ phrase (yellow strokes, orange field), Type 2 corresponds to a genitive (the fruit's orange) (Steinvall, personal communication).

\section{Conclusions}

The analysis of linguistic constructions involving color terms in the painting descriptions indicates that semantic meaning of color may greatly extend beyond its denotatum. This analysis reveals alternative ways of conceptualizing color - as a property of a physical entity, but also as a virtual entity per se. In a noun phrase, these ways of conceptualizing the color are signaled by the different semantic and syntactic positions and functions that the color word takes on. In the analyzed sample of the catalogue texts, we find prototypical noun phrases, those where a color adjective names the color property and lends it dependent syntactic and semantic status. However, along with these, there is another, dominating type of constructions containing color words, where a color adjective is substantivized and acquires the morpho-syntactic properties of a noun. Within these, the color concept can be pluralized and become the carrier of other properties; it can receive localization in space and build up a relationship to other participants of the discourse. Conceptually, in such constructions color words designate color as a manipulable or animated entity, which undergoes the actions of actors or becomes an actor itself.

Four basic types of constructions containing color words can be distinguished, which signal different degrees of dependency between the entity and the color property:

Type 1. $A_{C} N$ (e.g. blue line): color is conveyed by an adjective, conceptualized as an object's property, and thus it is syntactically and semantically dependent;

Type 2. $N$ of $N_{C}$ (e.g. horizon of blue): color word is substantivized but it takes the dependent position of a modifier to the noun naming the entity; 
Table 1. Types of color attribution to objects signaled by different linguistic constructions. $\mathrm{N}$ - prototypical noun; $\mathrm{A}_{\mathrm{C}}$ - adjectival color word; $\mathrm{N}_{\mathrm{C}}$ - substantivized color word. Exemplified by minimal noun phrases with the color word blue.

\begin{tabular}{lllll}
\hline & Type 1 & Type 2 & Type 3 & Type 4 \\
\hline Perceptual mode & $A_{C} N$ & & $N_{C}$ of $N$ \\
& blue sky & & the blue of the sky & \\
Pictorial mode & $A_{C} N$ & Nof $N_{C}$ & & $N_{C}$ \\
& blue line & horizon of blue & & the left blue \\
\hline
\end{tabular}

Type 3. $N_{C}$ of $N$ (e.g. the blue of the sky): color is conceptualized as an entity; this is signaled by substantivization of the color word, which is semantically dominant due to its position as a headword;

Type 4. $N_{C}$ (e.g. the left blue): color word is substantivized, whereby color is conceptualized as an independent entity and argument in the discourse.

There also appears a tendency to differentiate two types of objects whose color is described, which affect color conceptualization: (i) three-dimensional objects of the world "out there" projected onto the painting; and (ii) two-dimensional elements of the pictorial reality created by the artist. These two kinds of objects arouse the two modes of considering color - perceptual and pictorial, in terms suggested by B. Riley (1997) - and are signaled by different types of syntactic constructions, as summarized in Table 1 .

Type 1 constructions convey both perceptual and pictorial modes of color conceptualization and imply color as a dependent property of an entity.

Type 3 constructions signal color conceptualized primarily in the perceptual mode as liberated from a physical entity. In these constructions the color word is substantivized and appears in the focus position, whereas the physical entity is relegated to the modifier position.

In comparison, the pictorial mode of color conceptualization is signaled by Type 2 and Type 4 constructions: The substantivized color word either keeps the modifier position within the of-construction, so that the object is still the focus (Type 2), or, alternatively, it designates an independent virtual entity (Type 4).

Our linguistic analysis demonstrates that conceptualizing color within a pictorial reality promotes its complete liberation from an object. This conclusion is in agreement with the view that in the context of the painting, color can be conceptualized by itself, independent of objects (B. Riley 1997). This mode of conceptualization may be rooted in artists' and critics' experience in handling employed paints, as well as in their expertise in perceptual properties and subjective impression of color - as the instrument for creating pictorial reality.

It is also conceivable that the purpose of the analyzed discourse - descriptions of painting- and the incentives behind such discourse might be an additional factor that imposes entity-like color conceptualization. For color, as the immediate object of painting discussion, it is natural to be topicalized and reified in the discourse. 


\section{Acknowledgments}

I am deeply grateful to Galina V. Paramei, Anders Steinvall, Liudmila M. Sereda, David L. Bimler, and an anonymous reviewer for their valuable comments on earlier versions of this paper.

\section{References}

Agee, William C. \& John I. H. Baur (1989). The American Collections: Columbus Museum of Art. Columbus: Columbus Museum of Art.

Berlin, Brent \& Paul Kay (1969). Basic Color Terms: Their Universality and Evolution. Berkeley: University of California Press.

Carmean, E. A. Jr. (1989). Helen Frankenthaler: A Paintings Retrospective. New York: Harry N. Abrams.

Casad, Eugene (1995). Seeing it in more than one way. In J. R. Taylor \& R. E. MacLaury (Eds.), Language and the Cognitive Construal of the World (23-49). Berlin \& New York: Mouton de Gruyter.

Corbett, Greville G. \& Gerry Morgan (1988). Colour terms in Russian: Reflections of typological constraints in a single language. Journal of Linguistics, 24, 31-64.

Dixon, Robert M.W. (1977). Where have all the adjectives gone? Studies in Language, 1, 19-80.

Downing, Angela \& Philip Locke (2002). A University Course in English Grammar. New York: Routledge.

Frawley, William (1992). Linguistic Semantics. New Jersey: Lawrence Erlbaum Associates.

Hopper, Paul \& Sandra A. Thompson (1984). The discourse basis for lexical categories in universal grammar. Language, 60, 703-752.

Kandinsky, Wassily (1994). Complete Writings on Art. [Original: Der Blaue Reiter Almanach (1911).] K. C. Lindsay \& P. Vergo (Eds.). New York: Da Capo Press.

Kay, Paul (1999). The emergence of basic color lexicons hypothesis: A comment on The vocabulary of colour with particular reference to ancient Greek and classical Latin. In A. Borg (Ed.), The Language of Color in the Mediterranean (76-90). Stockholm: Almqvist \& Wiksell International.

Lucy, John A. (1997). The linguistics of "color". In C. L. Hardin \& L. Maffi (Eds.), Color Categories in Thought and Language (320-347). Cambridge: Cambridge University Press.

Lyons, John. (1999). The vocabulary of color with particular reference to ancient Greek and classical Latin. In A. Borg (Ed.), The Language of Color in the Mediterranean (38-75). Stockholm: Almqvist \& Wiksell International.

MacLaury, Robert E. (1997). Color and Cognition in Mesoamerica: Constructing Categories as Vantages. Austin: University of Texas Press.

Rakhilina, Ekaterina V. (1995). O cvetnom i bezcvetnom [On the colored and colorless]. Rusistika segodnja, 1, 50-69.

Rakhilina, Ekaterina V. (2007). Linguistic construal of colors: The case Russian. In R. E. MacLaury, G. V. Paramei, \& D. Dedrick (Eds.), Anthropology of Color: Interdisciplinary Multilevel Modeling (363-378). Amsterdam: John Benjamins.

Riley, Bridget (1997). Colour for the painter. In T. Lamb \& J. Bourriau (Eds.), Colour: Art \& Science (3164). Cambridge: Cambridge University Press.

Riley, Charles A. (1995). Color Codes: Modern Theories of Color in Philosophy, Painting and Architecture, Literature, Music, and Psychology. Hanover, NH: University Press of New England.

Sahlins, Marshall (1976). Colors and cultures. Semiotica, 16, 1-22. 
Schweizer, Paul D. (Ed.) (1989). Masterworks of American Art from the Munson-Williams-Proctor Institute. New York: Harry N. Abrams.

Sereda, Liudmila M. (1984). Funkcionirovanije leksiko-tematičeskix grupp v strukture anglijskogo korotkogo rasskaza [Functioning of Lexical-Thematic Groups in the Structure of an English Short Story]. Ph.D. diss. Minsk: State Pedagogical Institute of Foreign Languages.

Steinvall, Anders (2002). English Color Terms in Context. Ph.D. diss. Umeå University: Skrifter från moderna språk 3 .

Taylor, John R. (1995). Linguistic Categorization: Prototypes in Linguistic Theory. Oxford: Oxford University Press.

Vasilevich, Alexander P. (1987). Issledovanie leksiki v psixolingvističeskom èksperimente: Na materiale cvetooboznačenija $v$ jazykax raznyx sistem [An Investigation of Nomenclature Lexicon in a Psycholinguistic Experiment: On Material of Color Terms in Languages of Different Systems]. Moscow: Nauka.

Walch, Margaret \& Augustine Hope (1995). Living Colors: The Definitive Guide to Color Palettes through the Ages. San Francisco: Chronicle Books.

Wierzbicka, Anna (1986). What's in a noun? (Or: how do nouns differ in meaning from adjectives?) Studies in Language, 10, 353-389. 



\title{
Metaphors as cognitive models in Halkomelem color adjectives
}

\author{
Brent D. Galloway \\ First Nations University of Canada
}

Halkomelem is a Central Salish language of the Pacific Northwest with dialects of Upriver, Downriver, and Island. Color terms are adjectival verbs in Upriver Halkomelem, used syntactically and cognitively as both verbs and adjectives. Fieldwork with Munsell chips reveals that speakers of Upriver Halkomelem use a variety of strategies to name shades of color. Galloway 1992 reported on the use of aspect inflection to name the shades; thus color term roots are inflected for stative (be red), inceptive (get red, turn red), and these can be combined with continuative aspect (being red, getting/tuming red, getting in a state of red) and even with diminutives (a little red, getting/tuming a little red). There is one augmentative affix but adverbs like 'very' and 'real' are preposed by certain speakers to express some shades. Color adjectives, with or without adverbs and affixes, may be used sentence-initially as verbs or may be preposed to nouns within noun phrases. It seems, then, that different speakers use distinct metaphorical cognitive models in creating these terms. All speakers share the metaphorical model that COLORS ARE PROCESSES. This cognitive model allows further metaphors to be applied, for example, INCREASING SATURATION \& BRIGHTNESS ARE CONTINUATIVES. Another metaphorical model is seen with the diminutive: DECREASING SATURATION \& BRIGHTNESS ARE DIMINISHING IN SIZE OR AMOUNT. These last two mental models are coherent with each other and, therefore, can be used together. There are also adjectives meaning 'light' and 'dark' which some speakers use with color terms, and some use two basic color terms together to express variations in hue (like blue-green in English).

Investigation continues into whether there are submodels of modifiers used to express variations in saturation independently from those in brightness. In Upriver Halkomelem, thinking of color in terms of culturally sanctioned metaphors is a convention that exceeds mere application of linguistic form to unprocessed color perception. Communicative subtlety depends on them.

\section{Introduction}

Halkomelem is an endangered Central Salish language of the Pacific Northwest (B.C. and Washington) with dialects of Upriver, Downriver, and Island Halkomelem. Each dialect had subdialects, and for Upriver and Island some of these remain. Gerdts (1977) described 
some of the subdialect differences. Island Halkomelem was and is spoken on Vancouver Island from Malahat to Nanoose. Downriver Halkomelem was spoken in the downriver end of the Fraser Valley, from Vancouver to Matsqui; the last first language speaker of the Matsqui subdialect died June 2004, but several moderately fluent speakers of the Musqueam subdialect remain in Vancouver. Upriver Halkomelem was and is spoken between Abbotsford and Yale, B.C. and by a few speakers in Whatcom Co., Washington, members of the Nooksack Tribe there.

There are probably less than ten elders left who spoke Upriver Halkomelem as their first language; the same is true of Downriver Halkomelem, but the situation is somewhat better for Island Halkomelem with perhaps 25 such speakers left. Fortunately, for all three dialects, English-speaking tribal members have been actively trying to learn and revive the language with the help of five or six linguists and the remaining fluent elders, and there are now some moderately fluent speakers of each dialect. A group of about eight students just finished a three-year language immersion course in Upriver Halkomelem, and there is a similar number of graduates from an earlier three-year sequence of courses given through Simon Fraser University extensions. Several years of classes and sophisticated computer lessons have also been developed and given in Washington for the Nooksack Tribe. And similar efforts are underway for Musqueam and Cowichan.

The dialects are sufficiently different that separate full grammars have been written for them (Leslie 1979, for Island Halkomelem; Suttles 2004, for Downriver Halkomelem, particularly Musqueam; and Galloway 1993, for Upriver Halkomelem, including Chilliwack, Scowlitz, Chehalis, Tait, Pilaltxw, Nicomen, and Sumas subdialects). In addition there are also separate dictionaries either done or being completed for each, also based on original fieldwork (Gerdts, Compton, Edwards, \& Ulrich 1997; Hukari 1996; Suttles n.d. in preparation).

\section{The verbal nature of Halkomelem color terms}

Color terms are adjectival verbs in Upriver Halkomelem, used syntactically and cognitively as both verbs and adjectives (Galloway 1993:339). Fieldwork with Munsell color chips by MacLaury and Galloway in 1987 revealed that speakers of Upriver Halkomelem use a variety of strategies to name shades of color (MacLaury \& Galloway 1988; Galloway 1992, 1993; this latter includes the Munsell array in pocket). For example, aspect inflection/derivation is used to name many shades; thus color term roots are inflected for stative (be red), inceptive (get red, turn red), and these can be combined with continuative aspect (being red, getting/turning red, getting in a state of red) and even with diminutives (be a little red, getting/turning a little red)(Galloway 1992). These include:

(1) $\{c-\}$ or $\{s-\}$ stative prefixes: $\left\{c=k^{w} \hat{\imath} m\right\} /$ 'be red, red, reddish-brown, copper-colored'/. The equals sign shows derivational affixes, the dash inflectional affixes, square brackets within a root shows infixing; multiple glosses within slashes and single quotes are allosemes, variant meanings of a morpheme, word, or idiom that are semantically similar and predictable from the semantic context. (See Galloway 1993:460-473 for a thorough discussion of this 
notation and semantic analysis; in the same source, 473-637, I apply the method in detail to Upriver Halkomelem.)

(2) Inceptive $\{=a l\} /$ 'inceptive, get, turn, become'/, as in $\left\{k^{w} i \cdot m=\partial l\right\} /$ 'got red, became red, gone red'/; $\left\{c=k^{w} i m=a l\right\} /$ be red, get red, go red, become red'/;

(3) Combined with continuative aspect (being red, getting/turning red, getting in a state of red) $\left\{s=k^{w} \hat{\imath}\left[=C_{1} \partial=\right] m=a l\right\} /$ '[be getting red, be going red]'/ with $\{s=\}$ 'stative,' infixed reduplication $\left\{=C_{1} \partial=\right\}$ 'continuative, and $\{=\partial l\}$ 'inceptive'; $\left\{k^{w} \hat{\imath}\left[-C_{1} \partial-\right] m=\partial l\right\}$ ['getting red'] (square brackets and single quotes surround a single alloseme); $\left\{c=k^{w} \hat{\imath}\left[=C_{1} \partial=\right] m\right\}$ ['being red']; $\left\{c=k^{w} i\left[-C_{1} \partial^{-}\right] m=a l\right\}$, ['reddish'], further from the focus of $\left\{c=k^{w} i m\right\}$ than $\left\{c k^{w} \hat{i} k^{w} \partial m\right\}$ is, $\left\{c k^{w} \hat{i} k^{w} \partial m a l\right\}$ is usually furthest of all from the focus (literally it means something like 'going/coming/getting to have red';

(4) Combined with diminutives (be a little red, getting/turning a little red), for example, $\left\{s=C_{1} \partial=k^{w} \hat{\imath}\left[=C_{1} \partial=\right] m=\partial l\right\} \sim\left\{c=C_{1} \partial=k^{w} \hat{\imath}\left[=C_{1} \partial=\right] m=\partial l\right\}$ both /'be getting/going a little red'/, ['the very very lightest reds (actually more white tinged with pink, but not named as varieties of white or pink'].

There is an augmentative infix (vowel lengthening), $\left\{c=k^{w} i\left[={ }^{*}=\right] m\right\}$ ['be extra specially red'], ( $\{c=\}$ /'have, get, stative with colors'/, $\{==\} /$ '(very emphatic), really'/, allosemes ['especially characteristic shades of red and, perhaps occasionally, surprise at a newlyglimpsed shade']. And adverbs like $\{c$ 'ćc'al $\}$ 'very' and $\{w a l\}$ 'real' are preposed by certain speakers to express some shades. There are adjectives meaning 'light' $\left(\left\{s-t \varepsilon\left[-C_{1} \partial-\right] w\right\} /\right.$

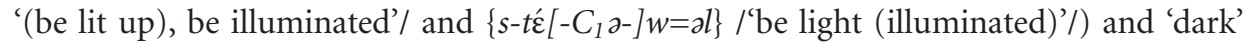
( $\{\theta \dot{\varepsilon} t\} /$ '(be) dark (as at night)'/ and $\{s=\bar{\lambda} i-\bar{\lambda} a s=\partial l\} / /$ '(be) a dark color (of clothes, complexions, etc.), dark gray, dark brown'/) which some speakers use before other color terms. The last of these is also a color term in itself.

Some speakers also use two color terms together to express variations in hue (like blue-green in English). For example, the following color terms were used by Agnes Kelly before $\left\{c=k^{w} i m\right\}$ 'red' to specify shades of that color: $\left\{c=x^{w} i k^{\prime w}\right\} /$ 'gray'/, $\left\{c q^{\prime} i x / /\right.$ 'black'/, $\left\{c q^{\prime w}\left\{x^{w}\right\} /\right.$ 'brown'/, $\left\{q^{\prime w}\left\{\left[-C_{1} \partial-\right] x^{w} \partial l\right\} /\right.$ 'brownish-black'/, and $\{$ si $i \bar{\lambda}$ asal $\}$ (as seen).

Color adjectives, with or without adverbs and affixes, may be used sentence-initially as verbs or may be preposed to nouns within noun phrases (Galloway 1993:338-339). For example, from my nearly complete Upriver Halkomelem dictionary (Galloway, in preparation):

(5) $\left\{c=k^{w} i ́ m\right.$ ta (músmas, $q^{w}$ amc=áls)\}

is-red the (cow, cranberry)

The (cow, cranberry) is red.

(6) $\left\{C_{1} \partial C_{2}-l a ́ m=a T-c-x^{w} m a ́ k^{w}\right.$ yac $=k^{w} i ́ m l$ lć $\left.^{w} w k^{w}\right\}$

pl.-fold-purposeful-indicative-you all the(pl.) red my belongings

/'Fold all my red clothes.'/

(7) $\left\{l a c^{\prime} \partial c=k^{w} i ́ m\right.$ téla $\}$

one red money

/'one penny'/ 


\section{The metaphors involved}

When a second color term or the word for 'light' or 'dark' is used to modify another color term, we have binomial expressions with both members from the same domain, that of LIGHT or its subdomain COLOR, and there is no metaphorical expression required. When the augmentative infix or adverbs meaning 'very' or 'real' are used as modifiers, there doesn't seem to be any metaphor involved. However, when aspect inflections or diminutive inflections are applied to the color term roots, these can produce metaphorical senses (metaphorical allosemes) in which the colors referred to are not actually changing or smaller in size but are different shades instead. Semantically we have aspect or diminutive derivation rather than inflection.

There are also literal allosemes in many of these cases, where something is changing, literally, 'getting red' or where something red is small, 'small red things,' for example. These latter allosemes are not metaphorical, while the former set are. Which alloseme is intended, for each morpheme and for each word, is predictable from the semantic context, and by that I mean usually the meaning of the morpheme, word, or sentence the color term is applied to or found within; sometimes the semantic context is found in sentences preceding or following the utterance in question; sometimes the semantic context is in part pragmatic, for example body language, prior information shared by both speaker and hearer, cultural context, or visual context (Galloway 1993:460-477).

It seems that what produces these two sets of allosemes for these color term words is actually the fact that the aspect and diminutive morphemes each have two allosemes, one that shows change or size and the second that shows saturation and brightness. When these morphemes are attached to color term roots they can have either alloseme, but the aspect and diminutive morphemes have the process and size allosemes in most other environments and only have the saturation and brightness allosemes in the semantic context of color term morphemes (when affixed to them derivationally). The resultant words then have their process and size allosemes in semantic contexts where process and size allosemes are most likely, and have their saturation and brightness allosemes where the semantic context/environment excludes color change or size specification, where shade meanings are more likely. In ambiguous contexts where either process or shade specification is possible, then the allosemes of the color term words are in free variation (remain ambiguous), unless one alloseme is much more frequent than the other in general; then the most frequent one may be taken to be the most likely meaning intended. This I call the "Most Likely Alloseme" principle, and it has been demonstrated as a natural function of the brain.

(Galloway 2000, 2001)

In looking at the results from my fieldwork with Rob MacLaury and half a dozen different speakers of Upriver Halkomelem, it seems that some speakers use more metaphorical expressions, others use more literal expressions, and some use a mix of both. This may vary depending on whom they are talking with, but in our fieldwork, they were each interviewed individually by MacLaury and me, so the hearer was a constant. This allows us to see the most salient metaphorical or non-metaphorical vantages used by each speaker (for 
Vantage Theory see MacLaury 1997). In addition, working with a larger group of speakers a few years earlier, I obtained lists of words to which some of the color terms would be applied. They provide contexts which reveal different literal allosemes (shades in this case) of each of the basic color terms. Since these are not metaphorical they will be vantages discussed in a following section.

It seems, then, that speakers use distinct cognitive models (Lakoff 1987) in creating these terms. All speakers share the non-metaphorical model that COLORS ARE PROCESSES. This cognitive model allows for the literal allosemes where colors are changing and also allows metaphors to be applied, for example, INCREASING SATURATION \& BRIGHTNESS IS CONTINUATIVE. Another metaphorical model is seen with the diminutive: DECREASING SATURATION \& BRIGHTNESS IS DIMINISHING IN SIZE OR AMOUNT. These last two mental models are coherent with each other and, therefore, can be used together. Some examples follow (from my Upriver Halkomelem dictionary, April 2004 draft).

\section{INCREASING SATURATION \& BRIGHTNESS IS CONTINUATIVE}

(8) $<$ tskwikwem $>,\left\{c=k^{w} \hat{i}\left[=C_{1} \partial=\right] m\right\}$ LT ['[being red'], ( $<t s=>$ have, get, stative with colors), $(<=\mathrm{R} 1=>$ continuative), ASM ['it can be a darker shade, or if preceded by $<\mathrm{tu}>$ it can be a lighter shade of red'], ASM ['tskwimel is further from the focus of tskwim than tskwíkwem is, and tskwikwemel is usually furthest of all from the focus (literally it means something like 'going/coming/getting to have red')'] (LT means these allosemes belong in the domain of LIGHT, ASM means comment on allosemes; words in angle brackets are in the official Upriver Halkomelem orthography, used by the Stó:lō nation).

(9) <skwikwemel>, $\left\{s=k^{w} \hat{i}\left[=C_{1} \partial=\right] m=a l\right\}$ LT /'be getting red, be going red'/, ( $<s=>$ stative), ( $<=\mathrm{R} 1=>$ continuative), ( $<=e l \sim=$ =il $>$ go, come, get, become, inceptive), attested by $\mathrm{AH}$, $\mathrm{NP}\left(\mathrm{R} 1\right.$ is reduplication type 1 , i.e., $\left.\left\{=C_{1} \partial=\right\}\right)$

(10) $\left\langle p^{\prime}{ }^{\prime} p^{\prime} e q ' e l>,\left\{C_{1} \dot{\varepsilon}=p^{\prime} \partial q^{\prime}=\partial l\right\}\right.$ LT /'get whiter, getting white'/, ASM [occurs almost alternately with $p^{\prime}$ ' $p$ ' $e q$ ' within the same areas (as shades of white tinged with orange, yellow or blue) so no difference in meaning between the two can really be distinguished], $(<\mathrm{R} 7=>$ comparative or emphatic, (sometimes) continuative), this type of reduplication was not attested in color term words by any of the others we interviewed], $(<=e l \sim=$ il $>$ go, come, get, become), attested by EB.

(11) $<q^{\prime} \hat{\imath} q^{\prime} e \underline{x} e l>,\left\{q^{\prime} \hat{\imath}\left[=C_{1} \partial=\right] x=\partial l\right\}$, ['getting black'], ( $<=\mathrm{R} 1=>$ continuative), $(<=e l \sim=\hat{l}>$ go, come, get, become), attested by EB, NP in Galloway 1993.

(12) <tsq'íq'exel $>,\left\{c=q\right.$ ' $\left.\left[=C_{1} \partial=\right] x .=\partial l\right\}$, LT ['be getting black'], $(<t s=>$ have, get, stative with colors), ( $<=\mathrm{R} 1=>$ continuative), ( $<=e l \sim=$ il $>$ go, come, get, become), attested by EB, NP.

(13) <sq'íq'exel $>,\left\{s=q\right.$ ' $\left.\left[=C_{1} \partial=\right] x .=\partial l\right\}$, LT ['be getting black'], $(<s=>$ stative $),(<=\mathrm{R} 1=>$ continuative), (<=el $\sim=$ =il $>$ go, come, get, become), attested by NP.

(14) <qwóqweyel $\sim$ qwóqwiyel $>,\left\{q^{w} \varepsilon\left[-A a ́ C_{1}\right.\right.$ - $\left.] y=a l\right\}$ LT /'getting yellow, turning yellow, turning green'/, (<ó-ablaut plus -R1-> continuative), (<=el> go, come, get, become), phonology: ablaut, reduplication, attested by NP, AC, AD.

(15) <sqwóqweyel $\sim$ sqwóqwiyel $>,\left\{s=q^{w} \varepsilon\left[=A a ́ C_{1} \partial=\right] y=\partial l\right\}$, LT /'be yellowish, be tan'/, literally $/$ '(stopped) in the state of becoming yellow' $/,(<s=>$ stative), $(<$ ó-ablaut plus $=\mathrm{R} 1=>$ continuative (here derivational)), (<=el> go, come, become, get), phonology: reduplication, 
ablaut, attested by AC (1971 1009 on trip to see Agnes Murphy in Laidlaw, AC's halfsister), MV (root-digging trip 197804 10), AH (Deming 197802 22, 1987), SJ esp. (Deming 197901 24), NP (1987), TG (1987), for example: <sqwóqwiyel te (músmes., stiqíw., sqwíqwemey., chékels.) $>,\left\{s=q^{w} \varepsilon\left[=A a ́ C_{1} \partial=\right] y=\partial\right.$ ta (mús $=C_{1} \partial C_{2}, s=t i q i ́ w, s=C_{1} \hat{i}=q^{w} \partial m=\varepsilon y$, cákals)\} /'The (cow, horse, puppy, chicken) is tan. (also) The chicken is yellow.'/, attested by SJ esp. (Deming 19790503 ).

(16) <tsqwóqwiyel>, $\left\{c=q^{w} \varepsilon\left[=A a ́ C_{1} \partial=\right] y=a l\right\}$ LT /'stative/be getting yellow, stative/be getting green'/, (semological comment: the get/have meaning for $t s=$ seems less likely than the stative meaning since $=e l$ already means get/become/go/come, also in most cases $t s=$ terms with $=e l$ label shades intermingled with those labelled by $s=$ stative terms with $=e l$ or label shades closer to the focus of color than those labelled with $s=$ plus $=e l$ terms $),(<t s=>$ have, get, stative with colors), (<ó-ablaut plus =R1=> continuative), ( $<=e l>$ go, come, get, become), attested by TG, NP, AH.

$<t s q w o ́ q w e y>,\left\{c=q^{w} \dot{\varepsilon}\left[=A a ́ C_{1} \partial=\right] y\right\}$ LT ['having/getting/being in a state of yellow or green'], ASM ['it can be a darker shade or if preceded by /tu/ it can be a lighter shade of yellow/green'], $(<t s=>$ have, get, stative with colors $),(<=\mathrm{R} 1=>$ continuative $)$, attested by TG.

(18) $<q w^{\prime} i q w^{\prime} e x w>,\left\{q^{\prime w} i\left[=C_{1} \partial=\right] x^{w}\right\}$ LT ['(brownish)'], ASM ['circled the following colors on the Berlin and Kay (1969) color chart (where rows are B-I top-bottom, columns left-right are 1-40): H7, H8, H9'], (<=R1=> continuative), phonology: reduplication, attested by AK (1979 0124$)$.

(19) <tsqw'iqw'exw $>,\left\{c=q^{\prime w} i\left[=C_{1} \partial=\right] x^{w}\right\}$ LT ['brown'], ASM ['it can be a darker shade or if preceded by /tu/ it can be a lighter shade of gray/brown'] (TG also uses this root for brown; the other speakers use the root for brown and label gray with forms from $\left.\left./ x^{w} \imath k^{\prime w} /\right)^{\prime}\right],(<t s=>$ stative with color terms), ( $<=\mathrm{R} 1=>$ diminutive or continuative), phonology: reduplication, attested by EB (mentioned while digging for cedar roots 197804 10), TG (1987).

(20) <qw'íqw'exwel $>,\left\{q^{\prime w} \hat{\imath}\left[=C_{1} \partial=\right] x^{w}=a l\right\}$, LT ['getting brown'], (<=R1=>continuative), $(<=e l$ $\sim=$ il > go, come, get, become), attested by AK, NP.

(21) <tsqw'íqw'exwel>, $\left\{c=q^{\prime w} i\left[=C_{1} \partial=\right] x^{w}=\partial l\right\}$, LT ['be getting brown'], $(<t s=>$ have, get, stative with colors), $(<=\mathrm{R} 1=>$ continuative $),(<=e l \sim=i l>$ go, come, get, become), attested by NP.

(22) <sqw'iqw'exwel $>,\left\{s=q^{\prime w} \hat{\imath}\left[=C_{1} \partial=\right] x^{w}=\partial l\right\}$, LT ['be getting brown'], $(<s=>$ stative $),(<=\mathrm{R} 1=>$ continuative), (<=el $\sim=$ il > go, come, get, become), attested by NP.

(23) <sqw'íqw'exwelomex $>,\left\{s=q^{\prime w} i\left[=C_{1} \partial=\right] x^{w}=\partial l=a ́ m a x y\right.$, LT /'looks a state of going brown, be getting brown-looking'/, $(<\mathrm{s}=>$ stative $),(<=\mathrm{R} 1=>$ continuative $),(<=e l \sim=i l>$ go, come, get, become), (<=ómex > looks, -looking, in color), attested by NP.

$<x w i x w e k w$ ' $>,\left\{x^{w} \hat{i}\left[=C_{1} \partial=\right] k^{\prime w}\right\}$ LT ['being gray'], (<=R1=> continuative), attested by NP. $<$ tsxwixwekw'>, $\left\{c=x^{w} i\left[=C_{1} \partial=\right] k^{\prime w}\right\}$ LT ['be getting gray'], ( $<t s=>$ have, get, stative with colors), $(<=\mathrm{R} 1=>$ continuative), attested by TG.

$<x w i k w^{\prime} e l>,\left\{x^{w} i k^{\prime w}=a l\right\}$ LT /'be faded (of clothes), (get/become) faded, (go or get or become gray)'/, (<=el> go, come, get, become), attested by CT (1976 06 08), for example: $<$ la xwíkw'el te kopú.>, \{le (or la) $x^{w} \hat{\imath} k^{\prime w}=$ al to kapú\} /'The coat is faded.'/, attested by CT.

$<x$ wixwekw'el>, $\left\{x^{w} i\left[=C_{1} \partial=\right] k^{\prime w}=\partial l\right\}$ LT /'(grayish, getting gray)'/, semantic environment ['not of hair'], literally /'going/getting/ becoming gray'/, $<=\mathrm{R} 1=>$ continuative), phonology: reduplication, ASM ['squares circled on the Munsell color chart of Berlin and Kay 
(1969) (rows top to bottom $=$ B-I, columns left to right $=1-40$, side column of white to black $=0$ ); NP circled the following squares for xwixwekw'el: H12, H13, H14'], contrast <xólemthet> 'turn gray (of hair)', attested by NP (Elders Group 197902 07, 1987).

$<$ tsxwixwekw'el $>,\left\{c=x^{w} \hat{\imath}\left[=C_{1} \partial=\right] k^{\prime w}=\partial l\right\}$ LT ['grayish'], semantic environment ['not of hair'], literally /'be getting/becoming gray'/, $(<t s=\sim c h=>$ have, stative with colors), attested by JL (1979 0508$)$.

\section{DECREASING SATURATION \& BRIGHTNESS IS DIMINISHING IN SIZE OR AMOUNT}

(29) <skwekwíkwemel>, $\left\{s=C_{1} \partial=k^{w} \hat{\imath}\left[=C_{1} \partial=\right] m=a l\right\}$ LT ['be getting/going a little red'], ASM ['the very very lightest reds (actually more white tinged with pink, but not named as varieties of p'éq' 'white' or qálq 'rose')'], ( $<s=>$ stative), ( $<\mathrm{R} 5=>$ diminutive), $(<=\mathrm{R} 1=>$ continuative), ( $<=e l \sim=i l>$ go, come, get, become, inceptive), double reduplication, attested by NP. $<$ tskwekwikwemel $>,\left\{c=C_{1} \partial=k^{w} \hat{i}\left[=C_{1} \partial=\right] m=a l\right\}$ LT ['be getting/going a little red'], ASM ['the very very lightest reds (actually more white tinged with pink, but not named as varieties of p'éq' or qálq)'], ( $<t s=>$ have, get, stative with colors), ( $<\mathrm{R} 5=>$ diminutive), ( $<=\mathrm{R} 1=>$ continuative), $(<=e l \sim=$ il $>$ go, come, get, become)

$<$ tsmimeth'>, $\left\{c=C_{1} i=m \partial \theta\right.$ ' $\}$ LT ['be a little blue'], (semological comment: the diminutive indicates all the lighter shades of blue here but also most of the greenish and lavender margins within blue as well; this extensive coverage is either cause or effect of the fact that the speaker uses no other modifiers with this root-no inceptive, continuative, etc. $),(<t s=>$ have, get, stative with colors), ( $<\mathrm{R} 4=>$ diminutive), attested by EB.

$<p^{\prime} i p^{\prime} e q$ '> $\left\{C_{1}\right.$ í=p'aq'\} LT ['a little white'], ASM ['occurs almost alternately with p'áp'eq'el within the same areas (as shades of white tinged with orange, yellow or blue) so no difference in meaning can really be distinguished between the two forms'], $(<\mathrm{R} 4=>$ diminutive $)$, attested by $\mathrm{EB}$

$<p^{\prime} i p^{\prime} e q$ 'el $>,\left\{C_{1} \hat{i}_{=} p^{\prime} \partial q^{\prime}=a l\right\}$, LT ['get(ing)/go(ing)/ becom(ing) a little white'], (semological comment: commonest for shades of white tinged with green or pink $),(<\mathrm{R} 4=>$ diminutive), (<=el $\sim=$ il $>$ go, come, get, become), attested by EB

(33) <tsp'íp'eq'el>, $\left\{c=C_{1} \hat{\imath}=p^{\prime} \partial q^{\prime}=\partial l\right\}$, LT ['be getting a little white'], (semological comment: occurs only once on EB's labelling of the Munsell chart as white tinged with lavender or light lavender (perhaps since nowhere else does the root p'éq' allow the prefix $t s=)),(<t s=>$ have, get, stative with colors), ( $<\mathrm{R} 4=>$ diminutive), ( $<=e l \sim=i l>$ go, come, get, become), attested by EB

(34) <tsqwíqweyel $>,\left\{c=C_{1} i=q^{w} \varepsilon y=a l\right\}$ LT ['have/get/be in a state of going a little yellow or green'], (semological comment: this term labels almost all the margins of tsqwáy (from green's margins with blue, to yellow's margins with orange and pink and white), it also includes some moderately light green close to the focus of tsqwá: $y$, which, for EB of Chehalis, is green (as it is for AH of Sumas/Matsqui subdialect)), $(<t s=>$ have, get, stative with colors), ( $<\mathrm{R} 4=>$ diminutive), ( $<=e l \sim=$ il $>$ go, come, get, become), phonology: vowel-reduction to schwa following a stressed prefix, attested by EB.

(35) <qwiqwóyáls $\sim$ qwiqwóyéls $\sim$ qwiqwòyàls $>,\left\{C_{1} i=q^{w} \dot{\varepsilon} y=a ́[=M 2=] l s\right\} \mathrm{EB} /$ 'orange (fruit), especially mandarin orange (the fruit), also domestic orange, (also orange (color))', ['especially Citrus sinensis, also Citrus aurantium'], LT ['orange (color)'], literally /'little yellow fruit'/, ASM ['the mandarin orange may have been the first to reach the Stó:lo people from the orient and especially from the Chinese who came in great numbers to 
work building the two railroads along the Fraser River in the 1860s and 1870s; the fruit was probably named before the color since $=$ óls is /'fruit'/ here rather than $=e l s$ structured activity verbalizer'], ASM ['the following color terms are used by AK before qwiqwóyáls to specify shades of the color: xwixwekw', tsqw'ixw, tsqw'iqw'exwel, stl'itl'esel'], $(<\mathrm{R} 4=>$ diminutive), ( $<=\mathrm{M} 2=>$ metathesis type 2 (of non-adjacent vowels), derivational), $<=$ óls $>$ fruit, spherical object, phonology: metathesis, reduplication, updrifting, possible vowelreduction, syntactic analysis: nominal, adjective/adjectival verb, attested by AC, LJ, EP, NP, AK, TG and RP, EL, AK (1987), EB (1987), NP (1987), AH (1987), charts by Rob McLaury, ASM ['squares circled on the Munsell color chart of Berlin and Kay (1969) (rows top to bottom $=\mathrm{B}-\mathrm{I}$, columns left to right $=1-40$, side column of white to black $=0$; individual speakers circled the following squares for qwiqwóyáls, NP (1979 02 07): C7, C8, C9; AK (1/24/79): C6, C7, C8, C9; TG (with RP) (1979 01 24): C6, C7, C8, C9, D6, D7, D8, D9; EL (1979 02 20): D4, D5, D6; Lawrence James (1979 02 07): D38; EP (1979 02 07) (Katzie dial. of Downriver Halkomelem): C9'].

\section{Exemplars as semantic contexts for color allosemes}

(36) <tskwi:m te (músmes, qwemchó:ls, qwelíyes, sth'íweq', qéléx, sxewál, sqá:le, s'ó:ytheqw, qá:lq, elíle, swáyél, schi:ya, t’qwém, qwe’óp, pqwá:y, témelh, xpá:y, sqáwth, sp'áq'em $\rangle,\left\{c=k^{w} i\right.$ 'm to

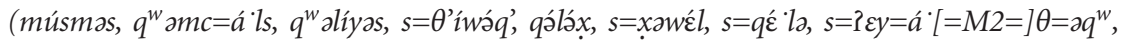

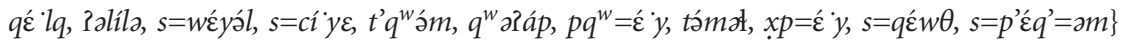
/'The (cow, cranberry, red-flowering currant, red elderberry, fish eggs, fox, red huckleberry, raspberry, rose, salmonberry, sky $\sim$ day, strawberry, thimbleberry, crabapple $\sim$ apple, rotten bark, red ochre soil or paint (iron oxide), red cedar, potato (domestic or wild varieties), flower) is red.'/, attested by Elders Group, TG, SJ, NP, <tskwi:m siyáth'qels $>$, $\left\{c=k^{w} \hat{i}\right.$ 'm $\left.s=h \partial=y_{\varepsilon} \varepsilon \theta^{\prime} q=\partial l s\right\}$ /'red paint'/, attested by DM 196412 04, <lemlémetchxw mékw'

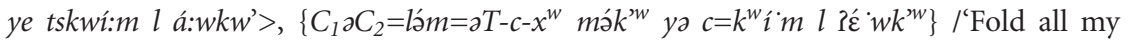
red clothes.'/, attested by Elders Group, $\left\langle\right.$ tskwí:m tále $>,\left\{c=k^{w} i ́ m\right.$ téla $\}$ /'penny'/, literally /'red money'/, attested by Elders Group, <léts'e tskwím tále>, \{lác’a c=kwím téla\} /'one penny'/, <isále tskwí:m tále $>,\left\{\right.$ iriséla c $=k^{w} i$ ím téla $/$ 'two pennies'/, attested by Elders Group, $<$ tskwí:m shúkwe>, $\left\{c=k^{w} i\right.$ 'm šúk $k^{w}$ o\} /'brown sugar'/.

Similar sets can be cited for each of the basic color terms, accounting for most of their color allosemes.

\section{Conclusion}

Investigation continues into whether there are submodels of modifiers used to express variations in saturation independently from those in brightness. Further study of the allosemes of stative and inceptive aspect may also reveal further refinements. One alloseme of inceptive is '-ish' (as in "reddish", for example); this is not limited to color contexts. There are some nice minimal sets for these in the data above.

The cognitive systems underlying these complex color term sets are all covert, but showing the metaphorical nature of those presented here helps to show the most pre- 
dominant systems of MacLaury vantages used. An additional nice case of vantages is the contrast between the alloseme ['copper (color)'] of $<t s k w i$ : $m>\left\{c-k^{w i} \mid m\right\} /$ 'red'/ and the allosemes ['copper (color)'] and ['copper (metal)'] of $<$ qwiyqwiy $>\left\{q^{w} i y=C_{1} \partial y\right\}$ based on the root $\left\{q^{w} \dot{\varepsilon} y\right\} /$ 'yellow, green'/. I believe these may show different vantages on 'copper color' the former from the vantage of the reddish elements in copper and the latter from the vantage of the yellowish elements of copper. In Upriver Halkomelem, thinking of color in terms of culturally sanctioned metaphors and vantages is a convention that exceeds mere application of linguistic form to unprocessed color perception. Communicative subtlety and accuracy depend on them.

\section{References}

Galloway, Brent D. (1992). Aspects of color in Halkomelem. Paper given in a session "Light on Color Ethnography" at the 91st Annual Meeting of the American Anthropological Association, San Francisco.

Galloway, Brent D. (1993). A grammar of Upriver Halkomelem. Berkeley: University of California Press.

Galloway, Brent D. (2000). Cognitive semantics in Halkomelem. Paper given the 39th conference on American Indian languages at the American Anthropological Society annual meetings, San Francisco.

Galloway, Brent D. (2001). Integrated cognitive semantics applied to Halkomelem. Paper given at the 36th international conference on Salishan and neighboring languages. In University of British Columbia Working Papers in Linguistics (Vol. 6: 91-111).

Galloway, Brent D. (in preparation). A dictionary of Upriver Halkomelem. Unpublished manuscript.

Gerdts, Donna B. (1977). A dialect survey of Halkomelem Salish. Unpublished master's thesis. University of British Columbia.

Gerdts, Donna B., Brian Compton, Leonard Edwards, \& Charles Ulrich (1997). Hul'q'umin'um' words: An English-to-Hul'q'umin'um' and Hul'q'umin'um'-to-English dictionary. Prepared for the Chemainus, Nanaimo and Nanoose First Nations \& Nanaimo School District No. 68.

Hukari, Thomas (Ed.) \& Ruby Peter (Assoc. ed.) (1996). Hul'qumi'num' dictionary. Duncan, B.C.: Cowichan Tribes.

Lakoff, George (1987). Women, fire, and dangerous things. Chicago: University of Chicago Press.

Leslie, Adrian R. (1979). A grammar of the Cowichan dialect of Halkomelem Salish. Unpublished doctoral dissertation. University of Victoria.

MacLaury, Robert E. (1997). Color and cognition in Mesoamerica: Constructing categories as vantages. Austin: University of Texas Press.

MacLaury, Robert E. \& Brent D. Galloway (1988). Color categorization and color qualifiers in Upriver Halkomelem, Samish, Lushootseed, Nooksack, and Yakima. In Working papers of the 23rd international conference on Salishan and neighboring languages (166-199). Eugene: University of Oregon.

Suttles, Wayne (2004). A reference grammar of the Musqueam dialect of the Halkomelem Language. Vancouver: University of British Columbia Press.

Suttles, Wayne (n.d.). A dictionary of the Musqueam dialect of the Halkomelem language. Unpublished manu-

script. 



\title{
Prototypical and stereotypical color in Slavic languages
}

\section{Models based on folklore}

\author{
Lyudmila Popovic \\ Filoloski fakultet, University of Belgrade, Beograd, Serbia
}

\begin{abstract}
Cognitive theory of worldview in language specifies speakers' ability to isolate a prototype as the construct of interpretative reality and to use a stereotype as an associative prototype, a process undertaken by certain Slavic cultures. Linguistic conceptualization of basic color categories provides data to explore criteria for assigning prototypes as well as the reasons to encode stereotypes. Data derive from nineteenth century Russian, Ukrainian, and Serbian folklore. Therein, color prototypes are radiant and positive, nonprototypes not radiant and secondary, for which they come to symbolize negative stereotypes. Each color concept harbors a duality of both semantic potentials, which appear to descend from like oppositions in ancient ancestral languages. The data provide background in time depth for comparison with outcomes of associative experiments and with results from psycholinguistic research in contemporary Russian and Ukrainian.
\end{abstract}

\section{Introduction}

Cognitive linguistics embraces worldview as part of its primary design. This helps us to understand how cognitive structures are reflected in language and how their formation is influenced by sociophysical reality (Lakoff 1987). Here we resume the well-known triad language, thought, and reality, such that we may find a model of the way such structures interpenetrate. Language sign structure means simultaneous representation of terms and conception; reality structure includes mutual relations between appearance, denotative space, meaning, and spacetime; whereas, the timing fragment of reality to which this appearance belongs is enigmatic, individual, and resistant to inquiry that is purely linguistic. Meanwhile, thought structure implies mentalized perceptions and processes by which information is conceptualized. They proceed by generalizing, typologizing, inducing, deducing, analogizing, and other operations.

Such conceptualization criteria for the apprehension of reality provokes special interest in the structure of thought. The important desideratum for choosing criteria of linguistic conceptualization is that reality can be either perceived or not perceived through 
experience of the speaker. If a referent and its denotative space represent part of individual experience, the speaker formats psychological experience according to his point of view, idiosyncratic or shared. The individual shapes the denotative space of that phenomenon, bringing a typology into the scope of subjective identification according to criteria of good versus bad.

The way conceptualization is modeled can be characterized as subjectivizing the concrete denotative space, which is obtained by embracing in a certain context not only spaciotemporal characteristics but correlated experience of the speaker, which is thusly incorporates into their view of the world. Each fragment of reality is identified with such a context. Depending on place, the phenomenon can be identified as good and bad at the same time, that is, in the so-called cognitive frame.

According to van Dijk (1981:217), this is about theoretical and cognitive abstraction of different views of the world as concepts organized in a speaker's conscience. For example, the notion of fire could be identified according to a criterion of good before a warm hearth or accordingly bad in a context of getting burned. The identification process is uncontrolled; we might say it is reduced to a level of mental reflexes, as could be easily realized by even a small child. We can only say "hot" to the child who has already burned their hand; the child will immediately withdraw their hand because fire is identified according to a criterion of bad, which is reduced to the reflex initiated by the spoken stimulus.

If that part reality is not located in subjective denotative space, that is, if it does not present part of the speaker's experience, they use a stereotype, which could be defined on the psychological level as someone else's experience that is linked to the fragment of extralinguistic reality. Depending on context, the phenomenon can be identified as good and bad simultaneously. In language and sign, the relation between authentic and stereotypic valuation of a referent could be seen through potential separation of a basic concept and the formation of an appearance paradigm, while the stereotype alone is based on established syntagmatic relations.

Language stereotypes are mostly epithets, formulas, idioms, phraseologies, proverbs, statements, and the like (Putnam 1975; Rosch 1975, 1978; Bartmiński 1985, 1988;). Actually, they are about a speaker's option to either separate a prototype as a concept of interpretative reality or use a stereotype as an associative prototype and as characteristic of a particular appearance in a specific culture.

In this case, we are interested in color conceptualization in language, the criteria of separation of a prototype for basic color, as well as the reasons of using language stereotypes in functions of color terms. An intensive search for color universals, initiated by Berlin and Kay (1969), shows that a color concept is not only nonuniversal but that its role is restricted in human communication. What represents universals is the difference between day, when a human can see, and night when he cannot see (Wierzbicka 1996:232); the contrast motivated many a researcher to found color typology on the opposition of light versus dark. This division is said to dominate color conception in the majority of languages around the world (ibid.); the divide suggests a reason for psychological assessment of colors in human conscience, that is, a positive relation with light colors based on 
waking perception versus a negative relation with dark colors associated with nocturnal darkness (Ivic 1995:62).

In this dichotomy, we find yet a second important color universal concerning denotative space, meaning, background, phoneme, and natural ambiance, in which, most of all, we notice the situation of certain artifactual correlates of color. Such denotative space represents a base, which is modeled and characterizable as the speaker subjectivizing the space concretely, that is, evaluating color according to criteria of good versus bad, yet this prototype is based on personal experience. For example, some women like black color to emphasize their slim figure or blond hair and for the same do not like a white color; while some men do not like a certain green, because it reminds them of a military uniform. But, the same color in a distinct situation might be psychologically evaluated differently, for example, a woman could associate black stockings with a funeral, white ones with a wedding dress, and so on.

Subjective denotative space for psychological evaluation of colors is formed in childhood, when the child is trying to determine a world by its psychological coordinates and, in this way, to select a place in it. Using colored pens, the child always favors one of them, although he cannot articulate his relation to the colors. As time goes by, however, the subjective denotative space of colors is cast out and replaced by a stereotypic picture, one based on an image of the usual color of some thing. In this way, red color is equated with fire or blood, although a person from a temperate climate might more often come in contact with the light red color of the poppy flower or observe all nuances of red at sunset.

The foregoing examples refer to an inference that during a person's separation from a prototype based on personal experience, an individual takes their first step from the beautiful versus the ugly; but as they adopt a stereotype, they are guided by the typical. This approach to prototypicality matches Rosch's assertion that "by prototype of categories we have generally meant the clearest cases of category membership defined operationally by people's judgments of goodness of membership in the category" (1978:36).

Frameworks of other principles regarding prototype separation emerge in:

1. Linguistics as a model of ideal subject certified in language (Wierzbicka 1985:81);

2. Philosophy of language as a first example of a research category (Putnam 1975, 1989);

3. Psycholinguistics and philosophy of language as experience connected with an object or situation (Lakoff 1987).

These principles provide grounds for thinking about the ideal example of color or about its bad type starting from the subjective assessment, while the stereotype has lost its primary motivation and function of affirming the criteria of a typical example as adjusted to the cultural climate.

The language of folklore give us the ideal corpus for researching the relations between prototypical and stereotypical ways of color conceptualization because, on the one hand, folklore conserves elements of primary subjective color evaluation starting from the prototype, which confirms the role of color as a symbol, while on the other hand, in folklore color already functions as a stereotype, which will store everlasting traces in its linguistic tradition (as we shall see later from etymologies). 
It is clear that a song becomes a symbol if its lyrics refer to something evanescent or cryptic. Of special interest to color symbolic research is color nomination in the language of folklore. By its architecture, folklore produces conditions for origination and realization of esthetic meaning. Further, it represents a genre in which emotive contexts produce a psychological semantic plan of color terms, dislocates their nominative meanings, and sometimes represses them by a generally symbolic interpretation. Color terms ingress into folklore as a prototype, which is based on psychological apprehension about good color as a positive symbol and about bad color as a symbol of evil.

Considering that this is about visual perception, a complex positive appraisal of beautiful color reminds us of the Greek concept kaloskagatos, the synthesis of beautiful and good. As time goes by, a color term loses its symbolic meaning as an emotive prototype and dissimulates into a stereotype, as is represented by so-called dead epithets.

After the analysis is conducted on the semantic potentiality of color terms in Serbian, Ukrainian, and Russian folklore, it will be found that symbolic interpretations based on the prototypical conceptualization of colors present the most dominant part of color-term semantics in that traditional expression. They represent the sphere that is communal for all three systems (Serbian, Ukrainian, and Russian); that is, they represent the kernel around which other meanings spread, while the semantics includes the stereotypical associations, as with white $=$ color of milk, black $=$ color of coal, and many others.

\section{Color terms in folklore}

We can illustrate the foregoing thesis by componentially analyzing semantic potentials of color terms that occur frequently in elaborated corpuses of Russian, Ukrainian and Serbian folklore (Franko 1966; Karadzhic 1977; Kirijevskij 1986;). From these, about 4000 examples of color terms are excerpted (fully presented in Popovic 1991a). Analytic results are seen in Table 1, where + and - mark the presence or absence of lexicosemantic features in selected texts.

The greater part of the semantic potentiality of analyzed color terms are their symbolic interpretations, which in most cases correspond in all three corpuses, as shown in Table 1. For example, in the semantic structure of the term for white we note these meanings, which are basic to the function of attributes appropriate to white as a symbol 'bright' (Russ. бельй день, Ukr. білий день, Serb. бели дан); 'light' (Russ. бельь, кудри, Ukr. білий світ, Serb. бели свет); 'clean' (Russ. бель ручки, Ukr. біле ложе, Serb. бијело тело); 'beautiful, healthy' (Russ. бел жених, Ukr. біла яко ягода, Serb. бела снашица); 'solemn, festal' (Russ. бел платок, Ukr. біле вбрання, Serb. беле даре); 'pale, sickly' (Russ. om бела лииа больна); 'virgin' (Russ. невестушка белая, Serb. бијело голубиће); 'magical' (Serb. бела вила); and 'unfriendly' (Ukr. білий враг). On close scrutiny and disregarding lexicosemantic variation, we see that these color terms have a specific and special aesthetic bipolarity. Each semantic interpretation reduces to one of two principlespositive or negative color experience. Such opposition underpins symbolic assignment. For terms designating white, the positive pole includes 'clear,' 'light,' 'clean,' 'beautiful,' 
Table 1. Color terms in Slavic folklore

\begin{tabular}{|c|c|c|c|c|}
\hline \multirow[t]{2}{*}{ Color term } & \multirow[t]{2}{*}{ Lexical-semantic variant } & \multicolumn{3}{|c|}{$\begin{array}{l}\text { Presentation of lexical-semantic } \\
\text { variant in folklore corpus }\end{array}$} \\
\hline & & Russian & Ukrainian & Serbian \\
\hline 1. 'white' & bright, shining & + & + & + \\
\hline Russ. БЕЛЫЙ & color of milk & + & + & + \\
\hline Ukr. БІЛИЙ & light & + & + & + \\
\hline \multirow[t]{8}{*}{ Serb. БEO } & clean & + & + & + \\
\hline & beautiful, healthy & + & + & + \\
\hline & solemn, festal & + & + & - \\
\hline & pale, sickly & + & + & + \\
\hline & innocent & + & - & + \\
\hline & clear & + & - & - \\
\hline & magical & - & - & + \\
\hline & unfriendly & - & + & - \\
\hline 2. 'black' & dark, shining & + & + & + \\
\hline Russ. ЧЕРНЫЙ & dirty & - & + & - \\
\hline Ukr. ЧОРНИЙ & heavy & - & - & + \\
\hline \multirow[t]{2}{*}{ Serb. ЦРН } & shameful & - & - & + \\
\hline & rainy & - & + & - \\
\hline 3. 'ruddy' & shade of red & - & - & + \\
\hline Russ. РУМЯНЫЙ & ruddy color of the face & + & + & + \\
\hline Ukr. РУМ’ЯНИЙ & beautiful, shining & + & + & + \\
\hline \multirow[t]{2}{*}{ Serb. PУMEH } & healthy & + & + & + \\
\hline & ripe & - & - & + \\
\hline 4. 'blue' & sea color & + & + & + \\
\hline Russ. СИНИЙ & dark & - & + & + \\
\hline Ukr. СИНІЙ & pale & + & + & - \\
\hline Serb. СИњ & shining & + & + & + \\
\hline 5. 'golden' & shining & & + & \\
\hline Russ. ЗОЛОТОЙ & made of gold & + & + & + \\
\hline Ukr. ЗОЛОТиЙ & dear, darling & - & + & + \\
\hline \multirow[t]{4}{*}{ Serb. ЗЛАТАН } & beautiful & + & + & + \\
\hline & happy & - & + & + \\
\hline & ceremonious & + & - & + \\
\hline & magic & - & - & + \\
\hline
\end{tabular}

'healthy,' 'solemn,' 'festive,' and 'virgin,' and the negative pole includes 'pale,' 'sickly,' 'magical', and 'unfriendly.' For terms naming black, the positive is 'beautiful' or 'solemn,' the negative 'dark,' 'ill-omened,' 'magical,' 'heavy,' 'shameful,' and more.

With such division, we can polarize semantic potentials of different color terms to form two lines of appropriate opposites, each term matched to another in a pair. The two are formed by independent aesthetic principles, that is, according to prototypical good/bad color, which include their nominative meanings. So the positive principle separates 'light,' 'radiant,' 'clean,' 'solemn,' 'festive,' 'dear,' 'good,' 'beautiful,' and 'agile.' The named variants belong to a context of semantic potentiality for these color terms: 'white' 
(Russ. бельй, Ukr. білий, Serb. Бео), 'golden' (Russ. золотой, Ukr. золотий, Serb. златан), 'ruddy' (Russ. румяний, Ukr. рум'яный, Serb. Румен), and 'black' (Russ. чернысй, Ukr. чорний, Serb. ирн) as well as for many others in the three corpuses. Their adequate symbolic interpretation fosters appropriate aesthetic synonymy, which explains the functioning of syntax common to folklore, such as the Serbian девојка бела и румена, Ukrainian сив-зелен соко, Russian сив бел сокол. It also explains color contamination, as Ivic (1995:96) interprets for gray and green in his "About a Green Horse." The second separable synonym line is negative: 'dark,' 'pale,' 'unhealthy,' 'dirty,' 'shameful,' 'sinister,' 'magical,' 'unfriendly,' and so forth. Starting from the semantic potential of the color terms 'black' (Russ. черныцй, Ukr. чорний, Serb. ирн), 'blue' (Russ. синий, Ukr. синій, Serb. сиғь and 'white' (Russ. белььй, Ukr. білий, Serb. беo) and others, we can restore an isofunctional relation plus their nominative meanings (complete listing in Popovic 1991a, reduced here to the terms of Table 1).

Certain synonym and antonym relations, through their variants, include color terms. Exemplified syntax, such as the Russian мильй бел да румян, is based on aesthetic parallel functionality of such color terms as those connected with semantic variants of 'beautiful' and 'healthy'. According to this principle, color terms could be contra-functional if the variants constituting their semantic potential are opposite and if they form an antonym pair. In this way the Serbian бео, румен 'white, ruddy' and модар, зелен 'blue, green' function as aesthetic antonyms, as in Serbian folksongs at weddings we hear that the girl before marriage was white and ruddy, бела и румена, but after the marriage she becomes blue and green, модра и зелена (Karadzhic 1977:7-59).

Opposition between these color terms is based on antonymy of beautiful versus ugly, healthy versus unhealthy, which constitute a certain semantic potentiality. Assigning of the lexicosemantic variants concretely connects each with only one color term, as Serbian бела functions in the meaning of 'beautiful' and модра in 'ugly.' These are symbolic, vibrant meanings based on appreciative experience of the color. Such meanings could not be transmitted in another way, say, by paraphrase, because this would eliminate the characteristic symbolic parallelism with its formulated mysticism (Eco 1995:97).

In the semantic structure of color terms, sometimes is found enanthiosemy (Greek

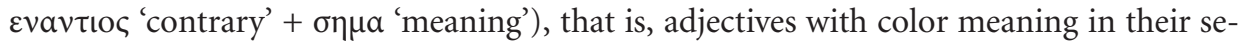
mantic potentiality but also with semantic features of different aesthetic facets - positive or negative. For example, the Ukrainian color term чорний 'black' further means 'dirty, ordinary', as in чорна сорочечка 'dirty shirt'. But the Ukranian чорний further means 'new, clean, solemn,' as in я би його шанувала, в чорні шати убирала 'I would honor him, dress him in a black (solemn) suit.' A Russian term for color has meanings of 'beautiful, shiny, solemn' (сине глазоньки, синь кафтан), but also 'dark,' 'pale,' 'unhappy' (синеньки, худеньки; сине тучи). Such variants show that one color is simultaneously composed of diametrically opposite aesthetics within one national conscience. By what means does the conscious beholder accomplish these complex semantics, and how might they pertain to the attested prototypicality? As suggested by analyzing semantic fields of color terms, a synchronic and diachronic basis of conceptualization repeatedly emerges as 
the "intensive" criterion, the maximum expression of a definite concept in the base of one color prototype.

\section{Radiance in prototypicality}

Most of the basic color terms are developed from a meaning connected to 'shine' or 'flash.' We might suppose this persists in the nuances of color prototypes, at least in a repetitive appreciation for qualities of reflected light, as with shinning or glittering. In support are the etymologies of most primary color terms, for example. 'White' (Russ. бельй, Ukr. бiлиц̆, Serb. бeo) and others were developed from Indo-European ${ }^{\star} b h \bar{a}$ 'to radiate, to shine, to glare' (Shanskij 1963:vol. 1, 88; ESUM 1982:Vol. 1 196; Skok 1971:Vol. 1, 152). 'Blue' (Serb. син, Russ. синий, Ukr. синій) from the Indo-European base ${ }^{*}$ skei- (Preobrazhenskij 1959:Vol. 2, 287; Skok 1973:Vol. 3, 239), which is connected to glare and radiance. The adjectives 'yellow,' 'green,' and 'golden' (Russ. жёлтый, зелёный, золотой, Ukr. жовтий зелений, золотий, Serb. жут, зелен, златан) were developed from the Indo-European root ${ }^{*}$ ghel-, ${ }^{*}$ ghol- (ancient Slavic Russ.L ${ }^{\star}$ g-llt-), also terms of radiance (ESUM 1985: Vol. 2, 275; Skok 1973:Vol. 3, 648). 'Ruddy' (Russ. румяный, Ukr. рум'яний, Serb. румен), which is etymologically primary in the semantic field of red color, owes its frequent use in folk texts to later 'red' (Russ. красныцй, Ukr. червоний, Serb. ирвен), which emerged from the Ancient Slavic root ${ }^{*}$ roud-, also connected to radiance (Skok 1973: Vol. 3, 171; compare in Serbian 'the dawn is blushing' зора ce pyдu or the Ukrainian term for 'shiny red hair' pyдuй). The investigators of Ancient Slavic roots ascribe the Indo-European base ${ }^{\star}$ roudh- with the same meaning (Herne 1954:58).

The maximal manifestation of radiant characteristics during conceptualization of a prototype serve as criteria for certain color assortments in the positive aesthetic system.

Therefore, in folklore which as a genre differs by positive aesthetic homogeny of texts for example, wedding texts - the frequency of color terms such as 'white,' 'ruddy', and 'golden' reinforces the impression of a shiny, sunny, bright, and joyous atmosphere.

The prototypical bad colors are marked by absence of radiance. Concepts that allude to loss of basic color quality we can nominally mark radiance-, the opposite of radiance+. Loss of the primal quality - the radiance - predominates in the transformation of typical, natural color to atypical, unnatural color. This is the point at which the prototypical and stereotypical conceptualizations come together. The positive color prototype concept is based on a manifestation of the radiance characteristic of color, while the stereotypic content derives from its loss. This is a critical quality of objects or appearances that differ by natural color, such as skin, hair, and other bodily semblance. These referents do not comprise artifacts, that is, products of human activity (to be discussed later). Those that connote radiance are semantically primary, while those conveying its loss are semantically secondary.

The thesis is illustrated by transformation of skin color. The prototypes of human skin from our temperate European climate are based on a concept of white or ruddy color, for example, white (mortuary white, unhealthy white - pale, without radiance) or red (with- 
out radiance) that refers to hardships of health such as high blood pressure (cf. Serb. uрвен у лицу [color nominated by situation, without radiance] and Ukr. червоні щічки 'ruddy cheeks') and often in portraits of women, wherein red color is semantically primary and high in radiance). Typical skin color of various black races, as based on positive prototype valuation, is bright black and sometimes dark blue (in Serbian folk songs Aрапин мор 'black Arab,' in Russian эфиопь сини' 'black Ethiopians'), while dark blue without radiance is reserved for skin color negative in prototype of people from our temperate climes, taking an example from Russian folklore синеньки, худеньки 'blue skin, skinny.'

Determined by situation, atypical color is marked in opposition to what is semantically primary; therefore absence of color radiance is marked in reference to its normal presence, which means that the negative color prototype is secondary - the reverse of the positive primary. The prototype of positively perceived color, that is, radiance, resides at the base of the stereotype, a typical sample of its sort.

Ivanov and Toporov (1965) reshuffle typologies of the primary archaic system of abstract classifications. They isolate within the framework of this semantic system a dichotomy of white versus black, not as an opposition to visually based feelings but as contrasting halves of the world - black as dark and white as light but removed from any background of dark. Parallel to this opposition, they segregate a further contrast, reshuffled as red versus black, and they caution on "functional identity of white and red who pose the reverse to black" (201). Their conclusion, on the one hand, illustrates the present claim about isofunctionality of color with a positive radiance+ concept, white and red, while, on the other, it simplifies the approach to perception and conceptualization of colors, which could be reduced only on one principle - positive or negative. Such an approach contradicts these authors, because they are confronted in the same research with the following: at different realms within the boundaries a singular opposition, the relation of color as marked versus unmarked can change (193).

Starting with the research on color symbolism in folklore, we can add that this relation can change in the framework of the kind of semantic system that incorporates enanthiosemy in color terms, optional and mutually exclusive features. Accepting the aesthetic bipolarity of each color as potentially positive or negative, we further presume the archaic system of radiant-nonradiant according to the dichotomy of semiotic color oppositions. In this case, there are such oppositions as these: radiant white-non-radiant white; radiant white-non-radiant black; radiant black-non-radiant white; radiant black-nonradiant black; and radiant red-non-radiant black. The quality of radiance is marked on the term for black color in the same manner that absence of radiance is marked on the term for white color. Such is confirmed by their etymologies and physical attributes: each color has dual aesthetic-semantic structure in the process of development and perfection of reality conceptualization in human conscience.

Our research deals with the phenomenon that describes the dualistic system of the color concepts radiant-non radiant, such as etymological data and narrated facts regarding color terms in the oldest linguistically accessible civilizations. For example, in one sentence from the Upanishads we can find traces of such an archaic system: "Red color of flame is a color of fire, white color of fire is color of water, black color of fire is color of the 
land" (Birren 1978: 19). Researchers project that this phrase is mystic and they interpret it in the fabric of their preconceptions (Wierzbicka 1996:253), but we can find in this example confirmation for the symbolic isofunctionality of colors, that which is based on the positive concept of prototypical colors as radiant and shiny. In such a context, phrases can be decoded: radiant red color is a color of fire, radiant white color is a color of water, radiant black color is a color of land. That is, the three basic colors are connected according to a positive appraisal, and they are assigned as attributes to essential, known elements. From that, we can further deduce that the three primary colors in the same aesthetic-symbolic system should be received also in accord with the negative principle in another semantic context, that is, white, black and red should be comprehended as negatively founded on loss of the basic quality - on absence of radiance.

\section{Contemporary primitives}

A parallel to our dualistic archaic system of radiant-nonradiant prevails in color semantics of some primitive nations. Universalist color-term researches using the so-called table of colors (catalogue of all color nuances) became popular with Berlin and Kay (1969). But, sometimes, researchers are confronted with contradictions during sample identification by questionnaires, because color terms which circulate in some tribes do not mach with any from familiar models. Such was confronted by Jones and Meehan (1978), who describe two basic color terms: gungaltja and gungundja in the Anbarra language of an Australian aboriginal band. It turns out that gungaltja does not correspond to the Munsell table, but the aboriginal interviewee managed to demonstrate its meaning on shiny foil that lay across a bench in their tent. Later he sorted among the pigmented chips gungaltja as only about 10 percent, while he identified the other 90 percent as from the contrasting gungundja class. In this modern archaic opposition, people perceive on the basis of radiant-nonradiant, the analogue of our proposal from archaic Slavic and Indo-European reconstruction.

Acceptance of dualistic conceptualization for each color of which roots we find in archaic semantic systems addresses questions confronting color universalists. There are difficulties connected to earlier attempts to divide colors according to light versus dark. Researchers strove to reduce all colors on this principle, which meant that speakers of any language that uses only two basic color terms obligatorily apply one term to 'light,' the other to 'dark.' But, the model ambiguously sanctions assortment of red colors in one of the classes, because researchers do not agree on this question (Turner 1966; Rosch 1972; Conklin 1973). Further, sorting of red color among the light stimuli inspired perfection of this model to admit cold versus warm; that is, the words distinguish light-warm versus dark-cold (Rosch 1972), as well as light versus dark-warm colors as in Anbarra (Jones \& Meehan 1978). Yet this model also could be even further discussed, because the dichotomy of warm versus cold color is not natural for many ethnic populations.

Trying to solve this problem, Wierzbicka (1996:267-276) proposes a macro concept model, that is, of macro white, macro black and macro red color, in which imperfection 
pervaded this one-sided concept of each individual color. She mentions the connection of macro white with radiance and brilliance; she also suggests that in languages of primitive tribes that use only two color terms, such lexemes actually are not color nominations, rather "generalized description of a reality aspect, as well as visual sensation according to it" (272). But flaws of her conception are seen in that each color is a one-sided observation sorted into one class of macro white or macro black or macro red. Rather, the dualistic conception based on opposition of radiant-nonradiant gives us grounds for each color assortment in both classes simultaneously, thus conforming to the radiance concept. Perception of radiance as present or absent is surely connected to the exterior color environment: shortage of sunlight causes absence of radiance as well as so-called dark or cold colors; conversely, plenitude of sunlight causes light and warm colors with radiance and brilliance identification. But it still remains that each color can be both light and dark, cold and warm, which depends on its positive or negative prototype actualization in recipient conscience.

\section{National worldviews}

Considering each color as one-sided, only as one principle symbol - positive or negative even in the reference of one national worldview - sooner or later will clash with subsequent demand of an account. For example, in research on color symbolism in Slavic medicine, Radenkovic (1989:141) connects each color with only one aesthetic pole. Yet confronting the problem of dual symbolic meaning of certain color terms, researchers confirm the aesthetic ambivalence of red and grey, which they explain by their medial locality in color systems. The need to explain certain meanings which do not fit a one-sided system testifies to the aesthetic ambivalence of colors. Thus, it is difficult to start only from a positive symbol to explain meanings of 'sickly', 'unfriendly.' or 'magical,' which are imposed on terms for white in Russian and Serbian folklore, or on black as a materialization of evil; whereas, black symbolizes 'beautiful' and 'solemn' in Ukrainian folklore (Table 1).

As a further example, the primary color term 'golden' (Russ. золотой, Ukr. золотий, Serb. златан) is marked exclusively by positive symbolism, which is compatible with the criterion of perceiving radiance+, but according to the etymological analyses, color terms combining 'green' with 'yellow' stem from the Indo-European root ${ }^{\star}$ ghel- in whose evolution we notice two tendencies. First, a semantic line from Indo-European ${ }^{\star} g h e l$ - 'to radiance, to light' can be followed in ancient Irish glõr 'shine', glõra 'to shine,' in the ancient Greek $\chi \lambda$ želtas 'golden,' želvé 'red mushroom, field mushroom,' in Lithuanian zèlte 'gold,' and in Slavic languages derivatives of the base ${ }^{\star} z o l t o-$. Second, and in contrast, is the conspicuous phonetic-product identity of examples meaning 'gall, gall-bladder, irritability, anger, rage, poison,' which also are traced to Indo-European ${ }^{\star}$ ghel- (compare ancient Greek

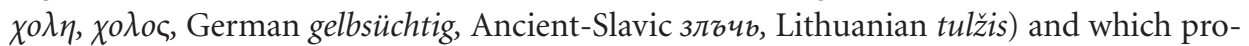
vide convenient facts about the reality of opposite semantic model (e.g., Nechipurenko 1989: 13). Evolution of opposite meanings from one Indo-European root is obviously con- 
nected with color perception according to the criterion radiance+. In this way we have the pulsing of red color in the words from the first semantic group, and in the second the perception of yellow color of physiological liquid which is muddy and dispossessed of shininess. Nechipurenko (1989:11) tries to explain this contamination by changing the color of denotative grass from green to yellow and, later, to red, although it is not clear why he chooses only grass if the primary meaning of ${ }^{\star}$ ghel-is reconstructable as 'to shine, to light').

The positive conception of bright green and the negative green without brightness can be also confirmed by examples in different languages. Welsh preserves the color term gwyrrd, which nowadays approximates English green. But Wierzbicka associates its meaning with radiant green grass after the rain: "In this case, it isn't so much vegetation that is taken for reference, as much the prototypical situation.... The whole scenario is reproduced in consciousness by living, natural verdure" (1996:255). On the contrary, some things that English speakers consider green are consigned to dark blue by Welsh speakers (Hjelmslev 1953:53), that is, they interpenetrate blue with green and grey. Regarding negative symbolism of green, Cherepanova (1983:66) interprets green as the functional synonym for black and blue according to this syntax, as in the Russian синиц̆, чёрныц̆, зелёный чёрт 'dark blue, black, green devil.'

In Slavic folklore texts, blue is, as well as yellow, often marked with negative symbolism. We presume pre-Slavic Serb. ${ }^{\star}$ синь arises from Indo-European ${ }^{\star}$ skei-, which is interpreted as a concept that has synthesized within itself two opposite meanings - 'light' and 'shadow', for there are examples from ancient Indian chaya 'shadow' and Gothic skeinan 'radiance' (Dzivak 1973:56).

Taking a diachronic overview, if both aesthetic poles for one color term in one language system are equally strong, they have conserved both symbolic meanings (e.g., cuнuй in Russian folklore). If one of the aesthetic meanings has overcome the other (Serb. curb), the symbolism may become one-sided, for example, a dominant negative color perception gradually can be overridden by others of positive persuasion, as in Serbian negative nлав was repressed by positive Serbian curb.

Following the notion of aesthetic bipolarity, we can explain unusual color-term polisemy, which is akin to enanthiosemy. For example, in the semantic composition of Serbian плав are the meanings (1) 'color of radiant sky'; (2) 'dark, closed'; (3) 'light yellow' (Rechnik 1967-1976: Vol. 4, 444). Color of the radiant sky and the nuance of radiant yellow are connected by the basic concept of radiance, which is opposite the sense of 'closed, dark.'

In some north Russian provinces of the nineteenth century, the color term голубой was used for marking gray with radiance, with blue, and with yellow (Dal' 1903:914), which could be sorted into aesthetic synonyms on as basis of radiance+ criteria.

In Russian and Serbian folklore are examples of functional synonymy among color terms. In Russian: бел-лазоревыц платок 'white, light blue wrap', сив-зелен соко ' greу, green falcon,' модра-зелена свила 'dark blue, green silk'; in Serbian, жут-бео кою 'уеllow, white horse,' This synonymy directly connects gray and green, livid and green, yellow and white and, thus, testifies about different color appraisals starting from the same aesthetic 
criteria. For example, the Serbian модра-зелена марама 'dark blue, green wrap' and девојка модра и зелена 'dark blue, green girl' are different in the nominal situation; actual terms for 'livid' and 'green' (Serb. модар и зелен), which are not usual attributes for skin color in modern comprehension (ancient Indian häri- and ancient Greek $\chi \lambda$ $о \rho \circ \varsigma$ signified 'yellow,' 'green,' but also 'pale') are based on criterial radiance-, while radiance+ lies in the base of the first example. In this case, we find oppositions of radiant green versus green minus radiance, radiant green versus livid minus radiance, radiant livid versus livid minus radiance, radiant livid versus green minus radiance.

Green and grey contamination abounds in Serbian folklore. Aesthetic attributes show isofunctionality in the following examples: зелен кон 'green horse,' зелен топ 'green gun,' зелена пушка 'green cannon,' зелен соко 'green falcon,' сив соко 'grey falcon,' витезови сиви 'grey knights,' and зелена му сабла и рука 'green is his sword as his arm.' In each of these epithets with equal success could be found attributes of the Serbian cjajar 'radiant' (further means 'great'). In one case this pertains to the radiance of plumage or hair, in another to the radiance of metal, but in both cases dominate denotata of positive perception, connected to background, sound, and lyrics, that is, for universal exhilaration which follows a scene in folk song. As Ivic (1995:97) observes, "In our [Serbian] folk poetrу зеленко is not only a hero's friend in his warlike struggles, he is also a parade horse, appropriate appearance on ceremonies of mighty and rich.”

Grey of hair without radiance also could be replaced by green, as in the Serbian зелен во 'green ox,' but while it is usual to talk about a horse's radiant hair, the radiant hair of an ox, dog, or even a wolf is marked. As Ivic finds, "Our Greenish Зељьв is green as a wolf, but when he sheds hair he is sallow" (1995:96). Opposition is based on radiant hair color before shedding.

\section{Prototype versus stereotype}

Color terms name attributes of skin or hair as assigned both by the situation and by semantically coherent definitions of artifact color. Such calls forth the issue about prototype and stereotype conceptualization of the same colors. Loss of radiance by a denotata whose color is usually radiant is a logical reason for marking its negative perception. But, during positive or negative perception of artifact color, radiant quality does not serve as a basic perceptual component in a prototype, rather it provides one element of stereotypically radiant color as beautiful, rich, or luxuriant. Compare the mass culture stereotypical tendency of, so to say, "trashy" works of art - shiny leather, brilliant clothes, costume jewels, metallic car paint, and tinsel Christmas ornamentation.

A concept of radiance that is directly evident during formation of certain color prototypes, construction of meaning, or shaping of related personal experience, does not have to be an obligatory element of stereotypical language conception of those colors. Rather, the radiance concept ingresses in a stereotype through the appropriate prototype, which in turn dominates. The role of folk texts in stereotype formation is crucial, whereupon the stereotypes come to dominate in the view of the world cast by modern language. The 
color terms of folklore, with their prototypes based on the presence or absence of radiance and that so condition positive or negative symbolism, are perpetuated in contemporary language according to the principle of the typical and ideal example, that is, a stereotype.

We shall try to confirm our thesis using data from a questionnaire about the Ukrainian and Russian social pictures of people. Psycholinguistic experimental results are, as is the questionnaire itself, from Terekhova (1997). The experiment questioned 300 Ukrainians and 283 Russians. It obliquely investigated psycholinguistic characteristics of bodily reception in Ukrainian and Russian contemporary language. But, as seen from its results, it could have as well been conducted in the nineteenth century, because the most frequent associations were based on traditional comprehensions of ideals for male and female beauty, and these were taken from folklore. For example, black color of hair was chosen by 68 Ukrainians but the blonde by only 31. In sentences with key words of bodily reference, for which were expected appropriate color terms to be written, the black color of hair in the pictured Ukrainian womanly image was chosen by all subjects on their questionnaires; but on 49 Russian questionnaires blond hair was chosen, while on only 11 Russian questionnaires was selected black hair (48-49). These results absolutely correspond with those of color terms researches based on nineteenth century Ukrainian and Russian folklore (Popovic 1991a). In those Ukrainian folk texts, gradation of the beauty ideal is directly connected with hair color. Thus in the Ukrainian song Ой у полі три криниченьки [There were three wells in the field] we read these verses: Любив козак три дівчиноньки: / чорнявую та білявую, / третю руду препоганую. // Що чорнявую від душі люблю, / до білявої залиияюся, / а з рудою препоганою / піду навік розпрощаюся [Once a Cossack loved three girls: / a black-haired, a fair-haired, the third was red-haired. // The black-haired I love with all my heart, / the fair-haired only a little / and the redhaired one / I will leave forever]. The brunette is in first place, blonde in second, and red-headed women have the role of comical, ugly characters. In Russian texts, the ideal of female beauty is obligatorily blonde. That many contemporary women in Ukraine choose exactly a nuance of blond or red color when they want to change their natural hair color testifies to how much this linguistic stereotype does not match reality. Here the linguistic stereotype does not correspond to the behavioral stereotype, which suggests that the linguistic stereotype actually is not constitutive of a worldview. Rather, it is often connected to syntagmatic associations, which are purely linguistic, that is, based on quotation.

For other bodily traits, as with skin color, most respondents in each culture chose white (80 in each). Brown, suntanned skin color, is less popular according to frequency of linguistic stereotype (totaling 30 respondents in each group). From these data arise a question about motivations of the sun's popularity and of desired vacations on southern seas, from which women obligatorily return sun-tanned.

Cheek color presents a special section in the questionnaire. According to its results, the ideal of Ukrainian beauty is connected to red color of cheeks (98 responses), less choose ruddy color (81), and rose takes third place (53). In Russian, associative portraited cheeks are first of all ruddy (83), next red (79), and then rose (41). It is interesting that in Serbia the red color of cheeks first of all associates with a sanitary hardship uрвен у тииу, while Serbian color term румен took over functions of whole palettes for cheek skin color. The 
popularity of red color as a beautiful cheek attribute is equated with the red kalina (a bush with small red berries, Viburnum opulus), which has a role in Ukrainian folklore. The association is of a beautiful girl - red kalina in repose as a folk symbol - which is based on perception of radiant red fruits. We can infer that the radiant concept appears as a basic component of red color perception in the folk prototype, and from folklore as a linguistic stereotype the concept enters contemporary language, loses its primary motivation, and becomes mystical. The motivation of Russian kalina symbolism has a different nature as a basic prototype concept served as fruit of bitter taste. From this point is derived the semantics of a basic plan transferred to the denoted referent: kalina is, first of all, a symbol of lovelorn separation and ruinous marriage (Djachenko 1997:69; Avtamonov 1902:50). The concept of red radiant color as the prototype of healthy complexion in Russian folklore is connected to another symbol, альй изветок 'light red colored flower' that of love and the beloved (Djachenko 1997:70).

In Ukrainian associative portraits, puce color dominates the eyes (93), followed by light blue (56), dark blue (37), green (28), sky blue (25), black (22), and gray (17), while in Russian portraits light blue (71) prevails over brown (59), green (34) and dark blue (22). As these data show, no larger variations from folk epithets pertain to this bodily feature.

\section{Conclusion}

When we embrace all examples and facts as singular manifestations of a whole appearance, we can conclude that the micro system of color terms is grounded in recipient conscience as a complex of aesthetic evaluation. In this, we can isolate prototypic elements, based on personal experience and in accord with a primary reception in a denotative context of the basic radiant quality and stereotypic elements - an associative prototype taken from someone else's experience - which is, in first instance, collective experience as a component of national worldview reflected, among much else, in folk texts.

\section{References}

Avtamonov, Jakov (1902). Символика растений в великорусских песнях [Plant symbolism in Russian songs]. Журнал министерства народного просвещения, С.-.Петербургъ, 344, 234288.

Bartmiński, Jerzy (1985). Stereotyp jako przedmiot lingwistyki [Stereotype as a subject of linguistics]. Z problemów frazeologii polskiej i slowiańskej, 3, 25-53.

Bartmiński, Jerzy (1988). Slownik ludowych stereotypów językowych. Zalożenia ogólne [Dictionary of folk linguistic stereotypes: General elements]. Etnolingwistyka, 1, 11-34.

Bartmiński, Jerzy (Ed.) (1996-1999). Slownik stereotypów i symboli ludowych [Dictionary of folk stereotypes and symbols] (Vols. 1-2). Lublin: Wydawnictwo Uniwersytetu Marii Curie-Skłodowskiej.

Berlin, Brent \& Paul Kay (1969). Basic color terms: Their universality and evolution. Berkeley: University of California Press.

Birren, Faber (1978). Color and human response. New York: Van Nostrand Reinhold. 
Cherepanova, Olga A. (1983). Мифологическая тексика народов русского севера [Mythological vocabulary of Russian northern folk nations]. Leningrad: Ленинградский государственный университет.

Conklin, Harold G. (1973). Color categorization. Review of Berlin \& Kay 1969. American Anthropologist, 75, 931-942.

Dal', Vladimir I. (1903-1909). Толковый словарь живого великорусскаго языка [Defining dictionary of the living Russian language] (4 Vols.). St.-Petersburg.

Djachenko, L. M. (1997). Фольклорна символіка як засіб відображення національного світобачення [National worldview and its reflection in folklore). Мовознавство, 23, 67-71.

Dzivak, O. I. (1973). 3 історії назв кольорів [From the history of color terms]. Українська мова $i$ література в школі, 9, 56-62.

Eco, Umberto (1995). Simbol. Belgrade: Narodna Knjiga/Alfa.

ESUM (1982-1980). Етимологічний словник української мови в $7 m$ [Etymological dictionary of the Ukrainian language] (7 Vols). Kiev: Наукова думка.

Franko, Ivan J. (1966). Народні пісні в записах I. Я.Франка [Ukrainian folk songs, transcribed by I. Ja. Franko]. Lviv: Каменяр.

Herne, Alf A. Gunnar (1954). Die slavischen Farbenbenennungen: Eine semasiologisch-etymologsische Untersuchung. Uppsala: Almqvist \& Wiksells.

Hjelmslev, Louis (1953). Prolegomena to a theory of language [Memoir 7]. International Journal of American Linguistics.

Ivanov, Vjacheslav V. \& Vladimir N. Toporov (1965). Славянские языковые моделирующие семиотические системы. (Древний период) [Slavic linguistic modeling of semiotic systems (ancient period)]. Moscow: Институт славяноведения.

Ivic, Milka (1995). O zelenom konju. Novi lingvistički ogledi [About a green horse: The new linguistic essays]. Belgrade: Biblioteka XX vek.

Jones, Rhys \& Betty Meehan (1978). Anbarra concept of colour. In L. R. Hiatt (Ed.), Australian aboriginal concepts (20-29). Canberra: Australian Institute of Aboriginal Studies.

Kirijevskij, Pjotr V. (1986). Собрание русских народных песен П. В. Кириевского в записях П. И. Якушкина 1-2 [The P. V. Kirijevski collection of Russian folk songs, transcribed by P. I. Jakushkin] (Vols. 1-2). Leningrad: Наука.

Karadzhic, Vuk St. (1977). Српске народне пјесме [Serbian folk songs]. Belgrade: Нолит.

Lakoff, George (1987). Women, fire, and dangerous things: What categories reveal about the mind. Chicago: University of Chicago Press.

Lovmjański, Henryk (1996). Religija Slovena (Slavic religion). Belgrade: Slovograf.

Nechipurenko, Nina G. (1989). Этимологический анализ тексики иветообозначения (на материале латинских производных от и.-е. * ghel-) [Etymological analysis of color terms (based on Latin derivatives of Indo-European ${ }^{\star}$ ghel-)]. Doctoral Dissertation. Moscow: State University.

Popovic, Lyudmila (1991a). Семантички потенцијал назива за боје у украјинском, руском и српском језику (на материјалу фолклора 19. века) [Semantic potentiality of color terms in Ukrainian, Russian, and Serbian languages (based on the 19th-century folklore)]. Doctoral dissertation. University of Belgrade.

Popovic, Lyudmila (1991b). Семантика назива за боје у руском, украјинском и српском фолклору [The semantics of color terms in Russian, Ukrainian and Serbian Folklore]. 36орник Матище српске за славистику, 41, 149-155.

Popovic, Lyudmila (1992). О семантици назива за црвену боју у украјинском, руском и српском фолклору [On the semantics of the term for red color in Ukrainian, Russian and Serbian folklore]. Расковник. Часопис за книжевност и културу, 18, 69-70, 91-102. 
Preobrazhenski, Alexandr G. (1959). Этимологический словарь русского языка 1-2 [Etymological dictionary of the Russian language] (Vols. 1-2). Moscow: Гос. Изд-во иностр. и нац. словарей.

Putnam, Hilary (1975). Mind, language and reality: Philosophical papers 2. Cambridge: Cambridge University Press.

Radenkovic, Ljubinko (1989). Символика цвета в славянских заговорах [The symbolism of colour in Slavic folk incantations]. In N. I. Tolstoj (Ed.), Славянский и балканский фольклор: Реконструкиия древней славянской духовной культуры: источники и материаль (pp. 122-148). Моscow: Наука.

Rechnik (1967-1976). Српскохрватског књижевног језика 1-6. [Dictionary of the Serbocroatian language] (Vols. 1-6). Novi Sad-Zagreb: Матица Српска-Матица Хрватска.

Rosch, Eleanor H. (1975). Cognitive representations of semantic categories. Journal of Experimental Psychology: General 104, 192-233.

Rosch, Eleanor H. (1972). Probabilities, sampling and ethnographic method: The case of Dani colour names, Man, 7, 448-466.

Rosch Eleanor H. (1978). Principles of categorization. In E. Rosch \& B. B. Lloyd (Eds.), Cognition and categorization (27-48). Hillsdale: Lawrence Erlbaum.

Shanskij, Nikolaj M. (Ed.) (1963). Этимологический словарь русского языка [Etymological dictionary of the Russian language]. Mоscow: Московский государственный университет.

Skok, Petar (1971-1973). Etimološki rječnik hrvatskoga ili srpskoga jezika 1-4 [Etymological dictionary of the Croatian or Serbian language] (Vols. 1-4). Novi Sad-Zagreb: Matica Srpska-Matica Hrvatska.

Terekhova, D. I. (1997). Асоціативній портрет українця та росіянина [An associative portrait of a Ukrainian and a Russian]. Мовознавство, 6, 43-50.

Turner, Victor W. (1966). Colour classification in Ndembu ritual. In M.Banton (Ed.), Anthropological approaches to the study of religion (48-87). London: Tavistock.

Van Dijk, Teun A. (1981). Studies in the pragmatics of discourse. In Context and cognition: Knowledge frames and speech act comprehension (215-241). The Hague: Mouton.

Vasmer, Max (1964-1973). Этимологический словарь русского языка. Пер. с нем. и дополнения О. Н. Трубачева 1-4 [Etymological dictionary of the Russian language, translated from German by O. N. Trubachev] (Vols. 1-4). Moscow: Прогресc. (Original work published 1951)

Wierzbicka, Anna (1985). Lexicography and conceptual analysis. Ann Arbor: Karoma.

Wierzbicka, Anna (1996). Язык. Культура. Познание [Language, culture and cognition]. Moscow: Русские словари. [Original: 1992. Oxford: Oxford University Press.] 


\title{
Color terms in fashion
}

\author{
Dessislava Stoeva-Holm \\ Department of Modern Languages, Uppsala University, Sweden
}

The object of this study is an analysis of the lexical means employed in the naming of colors, focusing especially on the form, function, and meaning of these color terms in the German language. Fashion magazines were used as source material for the research. The choice of magazines was determined by the high frequency of color terms. The basic color terms proved to be the most frequently exploited lexical means to describe one color, although it had been hypothetically assumed that there would be a quantitative preponderance of word formation structures, syntactic groups, and transferred designations. The decisive factor in the high frequency of basic color terms is their ability to function in a variety of ways. Not only as they describe a particular color of a garment, they are also increasingly used to express prediction and color trends, which is achieved by nominalization. But the goal has not always been a denotative correctness - rather the conveying of positive connotation.

\section{Introduction}

We live in a colored world. What we perceive within the range of colors are those that are visible to the human eye. Wittgenstein (1950/1977:5) wrote "White water which is pure is as inconceivable as clear milk." Things are mentally conceived to be colored. Water may appear blue or brown or grey or green, and air cannot be seen since it is transparent, or pure or clear in Wittgenstein's language, but it is spoken of as being blue in the phrase "blue air," and especially so when we speak of "the blue sky." Convention and norms make us see things as having the colors ascribed to them.

This study deals with the question "How do human beings cope with color in words and text?" The central aim of this study is a linguistic one, ${ }^{1}$ that is to filter out the linguistic side of color naming or: what are a person's linguistic means and instruments for speaking of color or colors? Of course, this involves above all making reference to a color vocabulary and reflecting on what kind of words are used to denote color, what are their grammatical properties, what do such words serve for apart from the narrow color designation, where

1. It is not possible to exhaust colorimetric, philosophical, anthropological, psychological, or semiotic issues in this study, but we may point out that color is difficult to discuss without an interdisciplinary approach. 
do emotive issues become recognizable or relevant, and what creative power is inherent in color names?

To refer to color or express what a person relates to color is part of this human attempt at expressing in words, sentences, and texts what a person sees and what they want their readers to see. Speaking of text, one would expect, on account of the pervading presence and importance of color in human perception, that color references are frequent in writing or, in other words, that color words or color modifiers are constituents of written texts with a high degree of frequency of occurrence.

If we look at fashion, and at fashion magazines in particular, we see changes in color and shape taking place every season and every year. We may expect a commensurate innovation and abundance of color terms in this area. Moreover, inventiveness and deviation from the ordinary and everyday seem to be essential. Since colors can be of paramount importance for the sale of products, the naming of seasonal colors contributes to financial success.

The following example from Burda, the German fashion magazine, illustrates how color words are used, and from it we are able to get an impression of the variety and the position of color words in this particular discourse:

(1) Einen wesentlichen Anteil am saisontypischen Ausdruck der Modelle haben die Farben. Sie sind im Frühjahr und Sommer hell, klar und kräftig, aber auch abgemischt, leicht verblaßt wirkend, entsprechend den Einsatzmöglichkeiten. So beleben z.B. die abgemischten Mitteltöne (Tabak, Sand, Puder, Orchidee, Karamel u.ä.) als modischste Farbgruppe die Kollektion entscheidend. Sie werden als Unis für alle Sortimente eingesetzt und prägen durch die Kombination auch mit anderen Farbgruppen das sommerliche Bild. Kräftige Pastelle (Geranie, Vergißmeinnicht, Forsythie, Lindgrün) findet man vor allem in der Kleidung für Damen und Jugendliche. Intensive Töne (Türkis, Lagune, Baccara) gelten als Effektfarben, sie setzen die modischen Tupfer. Die tiefen, satten Töne, wie Graubraun, Thymian, Eberesche, Pacific, Rehbraun, Pflaumenblau, Terrakotta und Marine zählen zu den sogenannten Summerdarks, die als Unis in sommerlichen Qualitäten auftauchen und sich besonders als Kombinationspartner für die abgemischten Mitteltöne eignen. ${ }^{2}$

2. Colors play an essential part as for the formation of fashion related expressions with special reference to the season wearing. During spring and summer the colors are light and bright as well as rich and full, but also of different mixed assortments, with more or less pale color, corresponding to the actual need of usage of possible fashion clothings. In this respect, the mixed and half-tinted colors (tobacco, sand, powder, orchid, caramel and the like) most decisively have an animating effect as the most stylish and elegant category of color shades. They unify all assortments of clothings in a standardizing way and, by means of their combination, also with other categories of colors, also characterize the summer season. Rich and full pastel colors (geranium, forget-me-not, forsythia, and lime) are first and foremost to be found in the fashion for women and younger persons. Intense tones (turquoise, lagoon, baccara) are commonly regarded as color shades with special effects; they fundamentally define the fashionable spot patterns. In the same way, the deep, dark, and full colors, such as greyish brown, thyme, rowan, pacific blue, fawn-color, plum blue, terracotta, and navy blue are by and large reckoned among the so-called summer darks. These turn up as single-colored summer-type fabrics and are especially suited to be combined with the mixed and half-tinted color variations.] 
Judging by the appearance, we may now ask whether the list of 20 color designations found in the example could easily be increased with every successive issue of Burda, when the fashion magazine informs its readers of the coming new colors for autumn, winter, spring, and summer, is utterly entropic or whether it can be structured according to a categorical system. Furthermore, we may ask if certain color terms which are used for fashion escape classification altogether, possibly on account of their being neologisms or ad-hoc formations.

To get the answers we may use an onomasiological approach which focuses on the task of color terms in fashion magazines and can be defined as follows. Color terms are used to:

name a single color or shade and to meet the requirement of gradation;

name multiple colors on garments;

name color and other specifications like the design/material/gloss of the garment.

\section{Motivation and value of information}

The onomasiological approach is closely connected with the question of the motivation of words, word formations, and syntactic groups. Motivation is mostly considered in connection with compounds and can be investigated by using word formation paraphrases to define the semantic content. These paraphrases should not have additional semantic markers. Such markers would indicate a big discrepancy between the meaning of the motivation and the meaning of the lexeme.

If we discuss the motivation of words, word formations, and syntactic groups we put the issue of lexeme structure, and thus the analytical aspect, in the foreground. The possibilities and the prediction of new words, word formations, and syntactic groups (in other words the synthetic aspect) are less in focus. Kastovsky connects analysis and synthesis with the terms Empirie and Theorie in the following stating:

Ausgangspunkt ist jeweils die Sammlung, Analyse und Klassifizierung von beobachtbaren Fakten mit dem Ziel, eventuell vorhandene Gesetzmässigkeiten aufzudecken. Der zweite, synthetische Schritt besteht in der Formulierung vermuteter Gesetzmässigkeiten als Hypothese, d.h. als eine Theorie, die die Natur der beobachteten Daten erklären und Vorhersagen über die beobachteten Fakten hinaus ermöglichen soll. Anhand dieser Vorhersagen wird die Theorie durch den Vergleich mit weiteren Daten auf ihre Stichhaltigkeit überprüft. $(1982: 12)^{3}$

The analytical principle is represented by American structuralism, and the synthetic by generative transformation grammar, which always presupposes an analysis. Among German word formation theories Fleischer and Barz (1992) represent the analytical-

3. TThe starting point is the compilation, analysis, and classification of observable facts with the main goal to uncover possible regularities. The second and synthetic step consists of the formulation of assumed regularities as a hypothesis, i.e. as a theoretical approach, which will explain the nature of the observed data as well as enable certain predictions beyond the observed facts. On the basis of these predictions, the theory is to be closer examined regarding its validity by means of a comparison with further data.] 
structuralistic aspect with its emphasis on the functional-semasiological question (What does a word or a word formation element perform?). In contrast, Olsen represents the synthetic-generative aspect. She declares her aim to be not the explanation of all synchronic data within the field of word formation, but the Isolation and Description of that certain part of the language system, which enables the productive formation and composition of new words and expressions (1986:17).

In this study all color terms in the fashion magazines Burda (1984) and Illustrirte Zeitung (1883) have been examined with the intention to observe relations of motivation, which contribute to the structuring of vocabulary and lead to the creation of new words. Therefore we do not concentrate the analysis only on basic color terms (BCTs) and frequently used non-basic CTs like schwarz 'black,' weiss 'white,' rot 'red,' blau 'blue,' grün 'green,' gelb 'yellow, braun 'brown,' rosa 'pink,' lila 'lilac,' beige, violett 'purple,' and grau 'grey', but also on word formations and syntactic groups containing a color term. Here we can find the importance of motivation in language, which only affects part of the language reality and which has to be distinguished from other characteristics in the language system, for example the tendency to idiomaticity.

To the word formation discipline belong not only the processes of word forming but also the results of these processes, the word formations, if we realize them as such. From a diachronic perspective, word formations are a result of processes in the past. This may lead to distinguishing two aspects of synchronic reflection: "den prozessuellen, der auf die Neubildung und Wiedergabe der Bildung der Wörter eingestellt ist, und den struktur-funktionellen, der auf die Struktur der Wortgebilde und deren Funktion zielt. ... Der eigentlichen Wortbildung im prozessuellen Sinne wird somit die, Wortgebildetheit', die Wortbildungsstruktur gegenübergestellt" (Dokulil 1968:205). ${ }^{4}$ The structural-functional aspect can be interpreted as analytical and the processual as synthetic. Dokulil's statement about the significance of Wortgebildetheit is central to this study.

Motivation is used on synchronic language with reference to semantics. There are different sorts of motivation in language; in this study morphological and semantic motivations (the possibilities of making the meaning of a word formation accessible from its constituents) are in focus. Word formations with suffixes are motivated by a base lexeme as in rötlich 'reddish' or by the base itself as in sandfarben 'sand colored.' In the last example the suffix -farben makes it clear that the color is in focus and not the consistency or other characteristics of sand. The stages of morphological and semantic motivation differ. In idioms, for instance, we cannot access the meaning from its different parts.

The relevance of motivation in the language system is pointed out by Wurzel (1984). Its importance can be considered in the orientation of the language systems towards increasing the potential of motivation by restoring lost motivation relations and creating new ones. It is, however, something of a problem to speak about an autonomous language

4. [the processual aspect, which is focused on the new formation and reproduction of words, and the structuralfunctional aspect, which aims at the structure of word creations as well as their function. In a processual sense, the lexematization of words or the word formation structure is consequently set opposed to word formation proper (Wortgebildetheit).] 
system which aspires to motivation. The language speakers are responsible for the formation of the system, and changes in this system are a mutual process between the system and the needs of the speakers. Speakers' reflections on the metalanguage level can be the mediating element.

Gauger (1970) and Rettig (1981) stress the morphosemantic relevance to the individual language speaker, which is apparent from the metalanguage reflection. Rettig (1981:76) points out that words are not per se motivated in the language system but only in the speaker's consciousness as expressed in the metalanguage reflection about language. He refers to Gauger, who focuses on the speaker's consciousness as the center of language processing. Gauger assumes that the speaker possesses metalinguistic knowledge. With the help of this knowledge he discovers the transparency of the language. The difference between a transparent and a non-transparent word is that a transparent word in contrast to a non-transparent, opaque one does not exist by virtue of its own, but through one or several other words in the language. When looking at a transparent word, the eye of the language beholder does not get stuck - it sees through this word and catches another or even more words (Gauger 1970: 13).

In addition to metalinguistic knowledge we also have to consider the etymological competence of the language speaker, which tends to motivate words synchronically. Etymological competence is not diachronic but oriented towards the present language condition: Consequently, this etymological competence represents a synchronic etymology, the aim of which is to describe systematically the multitude of word families within the vocabulary as well as the wealth of different interpretations attached to a word in certain semantic context (Augst 1975:177). The result of such etymological competence we can discover in the creativity of the language speaker to use lexical means by combining and modifying them.

It is important to accentuate the transparency of words (morphologic-semantic motivation) in German compared with English (see Fill 1980). In German we have a higher level of word-transparency and in English greater syntactic transparency, where English tends to express the relations between the words in syntactic groups.

Polenz (1979:28) emphasizes that in communication the motivation of words is irrelevant. He points out that to assume that unmotivated words have a less transparent meaning than motivated ones is off the track. We will not argue against this statement, but it is conceivable that under certain circumstances motivation is a factor that facilitates the understanding of a word and can also create structures for the language speaker in certain parts of the vocabulary. In a concrete situation motivation can give the recipient an additional chance to understand an entity which is not available as a pre-formed complex in the hearer's or speaker's mind. In this situation the motivation can function as an equalization of the differences between speaker and hearer or writer and reader.

In general, the language system should be treated as an open system, as the language speaker's collected, collective consciousness, which is more than the individual consciousness. Most of its structures are common to all speakers; some structures are looked upon differently by the speakers. This is the case when structures are not relevant in the parole to the motivation of lexemes. 
Motivation should be assumed in the language system as a structural (semantic and formal) relation between elements. In detail these structures can be formed differently in the speakers' minds and motivation relations may also differ. Motivation relations postulated through metalanguage reflection indicate that structures do exist in language. Therefore we chose a way between Wurzel's postulates, on the one hand, and Gauger's and Rettig's, on the other.

We think that the pattern of morphological and semantic motivation may differ among language speakers, because motivation generally appears to them as a structural relation between the constituents and can hence be looked upon differently. Motivated words and word formations make patterns available for new formations of words.

The morphological semantic analyses of 154 types from the fashion magazine Burda (1984) and 78 types from the Illustrirte Zeitung (1883) lead to the statement that:

1. New and well known colors and hues and especially a single color are named by

a. BCTs: rot 'red,' blau 'blue,' schwarz 'black';

b. BCTs combined:

i. Color terms containing noun and BCT: rubinrot 'ruby red', himmelblau 'sky blue';

ii. Color terms containing adjective and BCT: hellblau 'light blue,' reingrau 'pure grey';

iii. Color terms containing BCT and BCT: rotbraun 'reddish brown,' blaugrün 'blue-green';

c. Color term and noun: Sandtöne 'sand shades', Blautöne 'blue shades';

d. Syntactic groups: aktuelles Grau 'up-to-date grey,' modisches Jadegrün 'fashionable jade-green';

e. Transferred designations: Vergissmeinnicht 'forget-me-not', Burgunder 'burgundy';

f. Derived color terms:

i. Derived color adjectives with the suffix -lich: grünlich 'greenish', rötlich 'reddish';

ii. Derived color adjectives with the suffix -farben or -farbig: beigefarben 'beige colored,' crémefarbig 'creamy.'

2. Multiple colors in combination are named by:

a. Copulative adjective and noun compounds: schwarz-weiß 'black-white,' MarineGelb 'navy-yellow';

b. Determinative noun compounds: Rot-Schilf-Kombination 'red-reed combination';

c. Syntactic groups: schöne Grau-Rot-Farbigkeit 'beautiful grey-red coloring.'

3. Color and other specification like the design/material/gloss of the garment are named by using lexical forms as follows:

a. Determinative compounds from BCT and adjective/participle: graumeliert 'mixed grey'; 
b. Syntactic groups: grau meliert 'pepper and salt', glänzendes Schwarz 'glittering black';

c. Determinative noun compounds: Marine-Melangen 'navy mélange';

d. Transferred designations: Marengo 'marengo.'

\section{Color terms for naming a single color}

From the classification of the used lexical possibilities we can find different varieties of naming color: from BCTs and combined color terms to non-motivated but expressive words: rot 'red,' kirschrot 'cherry red,' Baccara 'baccara.'

After their function, color terms could be systematized by lexical means which:

1. Express one color without precision (BCT);

2. Are used for the precision of a single color (combined color terms, attributive groups, and transferred designations);

3. Express a certain level of vagueness/uncertainty in a single color (derived color adjectives with the suffix -lich '-ish').

\section{Basic color terms}

Studies conducted by linguists like Oksaar (1961), Ortner (1981), Klaus (1989), and Römer (1976) indicate a limited use of BCTs in fashion. This hypothesis, however, could not be confirmed, although it seems to be natural to use more suggestive and figurative color terms in fashion. BCTs occur frequently and are typical of German fashion magazines despite their mostly denotative and less often connotative characteristics. The high frequency (57\% from all excerpts which designate one color in 1983 and 60\% in 1984) can be traced back to their certain function and to their special position among the other color terms in fashion magazines.

Every BCT can mediate neutral information about the color of a garment:

(2) Schulterpasse und wieder etwas mehr Weite im Schnitt sind der Clou dieser roten Popelinjacke [Shoulder-strap and more volume in the cut are the essence of this red poplin-jacket].

BCT covers a large spectrum of the color, it seems to overlap and includes all shades of this color:

(3) Alle Farben enden mit Weiß - Winterweiß, Schneeweiß, Wollweiß usw. [All the colors end with white - winter-white, snow-white, wool-white, and so on].

It is interesting to note that in fashion, within the Benneton chain in particular, 'white' or a modified 'white,' such as 'off-white,' is replaced by the following color terms: 'banana,' 'swan,' 'albatross,' 'stone,' 'ecru,' 'parchment,' 'cream,' 'buttermilk,' 'wool-white,' 'winterwhite,' 'summer-white' (see Cencig 1990). 
BCTs are able to express tendencies in fashion:

(4) Weiß beherrscht die Sommerszene [White rules the summer scenery].

With the improvement of printing techniques, where every shade is visible to the reader, the exact linguistic naming of special hues and shades has become more difficult. This results in the special liking for BCTs if the aim is not to denote a special shade. The simultaneous existence of pictures and color terms in magazines involves the establishment of a mutual relation between these matters and shade precision appears redundant.

As adjectives BCTs can be used attributively as well as predicatively. Attributive usage is preferred when the material or the design of the garment is in focus and not the color itself. In this case the reader's attention can be attracted to something other than color:

(5) Das zweiteilige Kleid ist aus lila Georgette [The two-piece dress is made from lilac georgette].

Among morphological aspects we can establish that color terms are easily convertible into nouns. In this way they can serve to foreground in fashion. The possibility of nominalizing BCTs guarantees making the color (das Blau) one of the most important parts of the statement/predicate and to put it in focus, which often includes anaphoric processes:

(6) Dieses Blau sollten Sie sich merken - Sie werden's noch oft bewundern [You should remember this blue - you will admire it furthermore].

It also creates the premise for achieving personification of the color:

(7) Blau bringt Farbe in den Winter [Blue paints the winter].

From the examples we are able to see that the sentences have an informative function combined with an evaluative aspect. They do not only inform the reader about the new colors of the season but they also use the strategy to influence the reader. The reader has to get the impression of objectiveness in the reports. But the objective representation can be combined with an advertising element. BCTs can create this symbiosis because the recipient is very familiar with them and can relate new information about color design to her already existing knowledge about colors in general. ${ }^{5}$

\section{Word formations}

The restriction to a small number of terms causes difficulties when a color designation with BCTs is not satisfactory. This is the case when reference is made to marginal areas of hues or color segments, when certain shades of a hue have, by convention, acquired a well marked and psychologically relevant status as is the case with Flieder 'lilac,' Azur 'azure,' Türkis 'turquoise,' or when Value or Chroma make a writer feel that the use of a

5. BCTs are the oldest color terms. They have a certain place in human language and the acquisition of them takes place in childhood. 
BCT which subsumes all tints and tones of a particular hue under a representation of the hue which is more or less the focal color is unsatisfactory.

The problem is solved by modifying the color names by a color-modifying element which is in some way internally related to Value and Chroma, both of which contribute to the appearance of a hue. Color term modification is, of course, common in ordinary language use. In German, we can distinguish between determinative compounds where a color name is freely added to a noun, for instance in schokoladenbraun 'chocolate brown,' and where a color word is modified by an ordinary language modifier such as dunkel'dark', hell- 'light,' rein- 'pure,' or modified by another color word (orange-). ${ }^{6}$

In the first case, there is a comparison with a comparable typical color of an object: olivengrün is grün wie Oliven 'green as olives'; Sandfarbe is Farbe wie, die Farbe des Sandes 'colored like the color of sand'. In the second case the modifier has the function of a value characteristic or connotation element: reingrau is reines Grau 'pure/clear grey'; hellgrün is helles Grün 'light green.' In the third case the combinations of color terms express a special hue: graubraun is graues Braun 'greyish brown.'

The nouns in the determinative compounds can come from plants (tomatenrot 'tomato red'), flowers (sonnenblumengelb 'sunflower yellow'), metals and minerals (silbergrau 'silver-grey'), human artifacts (weinrot 'vine red'), fauna (lachsrosa 'salmon pink'), natural phenomena (lagunenblau 'lagoon blue'), and miscellaneous subjects (marineblau 'navy blue'). Color terms tend to form clusters, and elements of word formation can be understood as semantic or lexical fields. This nomenclature is, as such, also an example of how ordinary language can be extended so as to serve as a special language. Ordinary language is, by its nature, less systematic and more open in the choice of modifying morphemes. In the case of using ordinary nouns as modifiers in determinative compounds the modifier may even be so much in the foreground of visual perception or mental color awareness that the name of the object functioning as modifier takes over the function of the color word as such. Salience of color in objects favors the generation of color terms because of existing motivation and transparency. This may be the case with reseda 'mignonette' from resedagrün 'mignonette green' or türkis 'turquoise' from türkisblau 'turquioise blue.'

But also derivations with the modifying suffix -lich and syntactic groups can explain the designation of marginal areas or segments of color. The suffix -lich indicates a certain level of vagueness; for example, rötlich - der roten Farbe ähnlich - 'similar to red color.' German can form color adjectives with the ending -lich, comparable to English -ish, but this formation is restricted to the BCTs rot, blau, gelb, grün, braun, weiss, and schwarz. English color names ending in $-y$ such as creamy, peachy have no equivalents in German. An etymologically related cremig would refer to the viscosity of a liquid substance, not to its color.

6. In contrast to English, any color in German can be modified by hell and dunkel. Thus, where in German one could use such a modified CT as dunkelrosa, a phrase such as dark pink would be unusual. Instead expressions would be used like intense pink or very pink. 
It is clear that these constructions are not only preferred to specifying the color hue, but also in cases when there is a need of compressing the verbalization of the garment's color and when to awaken the reader's interest through unique, metaphorical and positively connoted determinants. This is possible because of the existing and recognizable motivation in these word formations.

\section{Syntactic groups}

Syntactic groups as a lexical possibility to express color, used specifically for the depiction of nuances, have hardly been taken into account in German linguistic color-term research. The possibilities of these lexical means for color distinction are revealed by the creativity of choice among adjectives or participles, which show either a denotative-valuating (dunkles Blau 'dark blue') or a connotative-valuating character (aktuelles Grau 'up-to-date grey'). Another factor is the possibility of double differentiation and valuation of color if a compound color term is used to designate the color (aktuelles Lodengrün 'up-to-date woolen coat green'). The productivity of syntactic groups can be seen in the possibility of two-way differentiation. Consequently, differentiation can be achieved via comparison with the positively connoted color bearer Loden 'rough woolen waterproof cloth' and via pointing out the topicality of this special color hue by using the attribute aktuell 'up-to-date.'

To use a color term in a syntactic group can be seen as an attempt to take advantage of the psychophysical reactions in color perception. Research in color psychology aims has shown that certain colors evoke the sensation of warmth or cold in the observer (see Marx 1973:62). Blau 'blue' is often referred to as a cold color, whereas rot 'red' is considered to be a warm color with textiles. However, psychophysical reactions do not need to be restricted to the sensation of warmth and cold: the sensation of weight, richness, or freshness can also determine the observer's reaction to a color: leichtes Rosa 'light pink', reines Blau 'pure blue,' transparentes Gelb 'transparent yellow.' Although black and white are achromatic or neutral colors they are not treated or used differently in fashion magazines from chromatic colors. Language users take little notice of color physics and do not distinguish between colors and noncolors; to them black is a dark color, white a light or brilliant color. The group of neutral colors also comprises grey.

Beyond the above-mentioned possibilities we find in the magazines expressions such as: fröhliches Gelb 'cheery yellow' and aufregendes Rot 'exciting red.' The last example is interesting insofar as it reveals the extremely susceptible borderline between modification and color symbolism. This is achievable through personification. In the expression fröhliches Gelb 'cheery yellow' the writer's imagination or creative power has a wider scope for using color term modifications belonging to people's individual norms or even such that are innovations. They manage to express in the modifiers not only a visual perception but also emotional reactions and, as the case may be, a metaphorical or symbolic implication. Moreover, color terms acquire, in the course of this process, a new semiotic function: they become signs with a meaning besides that of hue.

In comparison with determinative word formations from adjectives and color terms such as dunkelgrün 'dark green' we can see that the constituents of the compounds have to 
be semantically and morphologically compatible. Restrictions of this kind which regulate the use of constituents in attributive groups do not exist (see aktuelles Grau- ${ }^{\star}$ aktuellgrau 'up-to-date grey'). Accordingly, we speak of aktuelles Grau, but not *aktuellgrau, because the compounds require a relation between the constituents which can be described as semantically-modifying and in which the determinate has an informative function to express lightness and saturation (two components which are characteristic of color) and not the time dimension. Furthermore, morphologically words ending in -ell cannot appear as first constituents in German compounds (see Fleischer \& Barz 1992:121). When we wish to focus on connotative aspects we can only modify BCTs with attributive elements (klassisches Blau- ${ }^{\star}$ klassischblau 'classical blue'). This restriction can be applied to the determinative compounds consisting of a noun and a color term.

\section{Transferred designations}

The technique of modification with a transferred designation in whose semantic content the generic hue is thematically a dominant color is typical of fashion. The thematic name comprises a whole set of different or related shades, Value and Chroma of colors. Thus Sahara is no longer the color name of a specific shade of color as it is listed in a dictionary; Sahara can be understood as a thematic color name comprising all possible colors or shades of colors that can be related to the Sahara Desert in a person's imagination. Similarly, Frühling 'Spring' is the thematic color name under which can be subsumed or associated with such colors or shades as green, yellow, white, pink and so on.

The use of transferred designations apparently focuses on the precision of the color shade. These designations are transferred from different spheres. We can point out that the domains of plants and material (especially noble metals and precious stones) are the most exploited ones. This can lead our reasoning in two directions. Either we can think of the transferred designations as a form of ellipsis where the BCT is left open and the noun activates two of the semantic components: color and positive connotation linked to conventions. Or the metaphoric nature of color designations explains why it is possible, on the one hand, to use almost any name of an object as a color name and why, on the other hand, certain lexemes cannot be used as color names. The property required for a metaphoric process is that the two lexemes participating in the metaphoric process have a semantic feature in common. Where color is concerned this requirement excludes all abstract terms since they have no semantic feature (+COLOR). Nevertheless there exist abstract terms with no semantic feature (+COLOR) like Baccara. The motivation of these transferred designations is derivable from the context in which they appear.

The metaphoric process is very obvious in German where usually the reference to the non-figurative usage of the lexeme is indicated by the suffix -farben/-farbig '-colored': eine crémefarbene Bluse 'a creamy-colored blouse,' ein kirschfarbenes Hemd 'a cherry-colored shirt.' The comparison with an object as regards the property of color is retained and somehow remains recognizable. The function of -farben/-farbig '-colored' in the word formations can be said identify the color term as an adjective. On the syntactic level this guarantees the attributive use of the designations. 
However, transferred designations do not always reach precision, because the designated phenomenon may occur with a number of different colors or may not allow associating to a concrete color at all. There are, for example, about 20,000 species of orchids with different colors, so it is not obvious which color is meant by the color term Orchidee 'Orchid.' It is evident that the aspiration towards harmony often works against accuracy in designations. Even in a field where novelty, originality, and possibly deviance (from the ordinary or obvious) are essential and at the strongest, the creative possibilities are governed by some constraints which can ban totally deviant, fancy color names. It seems that some semantic relationship or associative link or at least the possibility of implication regarding either the name or the object providing the color component must be recognizable. As has been mentioned, two contradictory tendencies appear in this part of the vocabulary:

1. An aspiration towards shortness and unequivocality/unambiguity;

2. An aspiration towards sounding and fancy designations.

The material studied from German magazines shows that the hypothesis of an increasing use of transferred designations can be proved. Over a period of 100 years their share in color designation tripled compared to all designations, which is related to the decrease of determinative compounds and not to BCTs.

\section{Diachronic observations}

The results from the diachronic analysis point to some interesting conclusions. Color naming seems to be arbitrary and open-ended. Terms for shades of color occur with reference to objects or by a willful act of naming. It is the language speaker who tends to motivate color words synchronically; it is the speaker's consciousness in the metalanguage reflection about color designations and their etymological competence that seem to be important in the naming process.

In modern German the morphological possibilities of color terms are on the whole restricted to morphological changes of existing color terms rather than the creation of color terms from word stems or etyma. This means that when color terms are needed to designate a color without traditional color naming, the name of an object whose color is salient in some way or other or which can be associated with the color serves to function as a color name.

Nevertheless it turns out (from percentage calculations covering a period of 100 years) that BCTs are exploited more than non-basic terms to describe singular colors in fashion, contrary to the appealing thought that there might emerge a preponderance of word formations, syntactic classes, and metaphors pertaining to exotic color shades. What is decisive is that BCTs exhibit the ability to function in diverse ways. Not only do they describe particular color garments, but they also express predictions and describe color trends, which, among various means, is achieved by nominalization. At the same time, an analysis of word-formation structures, syntactic groups, and transferred designations reveals that the goal in fashion has not always been denotative correctness cum precise 
rendering of color. Rather, a primary concern is to convey positive connotations. In fashion, lexemes are used not so much for having a definable semantic content but rather to function as lexematic markers for specific colors to be remembered by the reader or the user. Despite the productivity of determinative compounds we can observe a decrease of them, especially compounds of the BCT + BCT type like blaugrün 'blueish green' and from the adjective + BCT type like hellgrün 'light green.' Compounds of the noun + BCT type like maisgelb 'corn yellow' are also decreasing but not to the same extent as the types mentioned before. This was not the expected result, because of the metaphorical power and positive connotations they convey.

\section{Color terms used to describe multiple colors in combination}

Few linguists have dealt with color terms used to describe multiple colors in combination. For this, it is above all copulative compounds that are used, with individual terms appearing in deliberate order. From the analysis of the appearance of color terms in the description of multiple colors we can conclude that the focus on and adherence to conventions have created linguistic patterns. The conventionalized Schwarz-Weiss 'Black-White' constitutes a pattern for other color terms that include the elements schwarz 'black' or weiss 'white.' This means that if schwarz or weiss appears in a copulative compound it takes the intended place: schwarz as the first component in compounds like schwarzgelb 'yellow-black,' or schwarz-rot 'red-black' and weiss as the second component, as in rot-weiß 'red-white,' braun-weiß 'brown-white', and blau-weiß 'blue-white'. In copulative compounds the achromatic color term grau 'grey' appears only as second component.

The arrangements within the compounds of chromatic color terms show regularities that corroborate universalistic research with respect to the evolutionary stages of basic color terms: secondary BCTs (like rosa 'pink', violett 'purple,' orange, beige), which were lexicalized later, are placed as the first compound and that makes the attributive use of the copulative compound (beige-braun 'beige-brown') possible. ${ }^{7}$ The regularities of ap-

7. Berlin and Kay (1969:6) claim that BCTs are generally "monolexemic, that their significations are not included in that of any other color term and their application is not restricted to a narrow class of object." The list comprises eleven terms: white, black, red, yellow, green, blue, brown, purple, pink, orange, grey, which were lexicalized at different stages in history and can be summarized as follows:

a. All languages contain terms for white and black;

b. If a language contains three terms, then it contains a term for red;

c. If a language contains four terms, then it contains a term for either green or yellow (but not both);

d. If a language contains five terms, then it contains terms for both green and yellow;

e. If a language contains six terms, then it contains a term for blue;

f. If a language contains seven terms, then it contains a term for brown;

g. If a language contains eight or more terms, then it contains a term for purple, pink, orange, grey, or some combination of these. 
pearance could also be seen from the rest of the investigated material and they evoke the assumption that the arrangement of the components in the copulative compounds used in fashion magazines shows tendencies of conventionalization (gelb-rot, blau-rot, blau-gelb) because the possibility of inflecting the components can be excluded as a criterion.

A diachronic comparison finds that the naming of multiple colors shows a tendency for greater variation in the lexical means. Determinative compounds (Schwarz-WeißKontrast 'black-white contrast') and syntactic groups (Grün in Grün 'green in green') are especially productive.

\section{Color terms used to describe color and other specifications}

The lexical means to describe color and another quality like design, material, brilliance or condition are interesting insofar as there are elements of intersection between color and other specifications. They thus illustrate a complex state of affairs. The communicative aspect of these word formations we can find in the close integration of color and other categories which results from the need of a connection between:

1. The color and the material categories (grauleinen 'grey linen');

2. The color category and the design/condition category of immense importance (rotweiß-schwarz-gestreift 'red-white-black striped,' dunkelblau meliert 'mixed with dark blue,' rotgrundig 'red grounded,' Terrakotta 'Terracotta');

3. The color and brilliance categories (schwarzglänzend 'glitterring black').

We may note that color terms which describe color and other specifications are of limited use. The very restricted occurrence of these word formations in color naming and some other parameters appears to contrast with the naming potential that their dual transmission of information possesses.

A diachronic comparison shows that the naming of colors is not characterized by the development of new models, but rather through:

1. A changed usage level of the existing lexeme possibilities;

2. The creative recombining of the existing possibilities.

\section{Color - Color terms - Context}

According to Zollinger (1980) a human being can - at the most - remember one hundred different colors, i.e. hues and shades of hues differing in brightness or saturation. An examination of six issues of Burda in the year 1984 produced a list of 154 different colors or color designations, which in Zollinger's view is more than a reader of fashion magazines can remember.

Color terms can therefore, in certain contexts, be given a specific meaning. They then acquire a significance and act as signals, which means that we have to consider physio- 
logical and psychological aspects as well (apart from their designative function). ${ }^{8}$ If we consider color terms in their capacity as color designations as the primary coding of a set of morphemes, we can consider color terms in their function as signals to be a secondary coding of the same morphemes. Whereas the primary coding of color terms must be understood as an act of naming, the secondary coding is either a willful act carried out by an individual (although the individual may be anonymous as in fashion magazines) or is suggested by the conventions of a society or culture. Consequently, within the sphere of secondary coding, we can distinguish between intended (secondary) coding and intuitive (secondary) coding. In fashion magazines the intended (secondary) coding relies on the fact that some colors, generally basic or focal colors, are easily perceived, recognized and remembered. This is definitely the case with such colors as schwarz 'black' and weiss 'white,' rot 'red,' gelb 'yellow,' or grün 'green.' Such colors are then, in German as well as in similar societies, given a semiotic quality, i.e. they function as signs in order to effect a certain behaviour. ${ }^{9}$ Special use is made of this signal function of colors in fashion, though here with a clear psychological intention: black may signal elegance, blue may allude to style, good taste, and so on. This special use clearly occurs in sentences where the color term has been personified:

(8) Klassisch die Farben Schwarz, Weiss, Rot, sehr englisch die Stoffe Tweed und Flausch [Classical are the colors black, white, red; very English are the fabrics tweed and pilot-cloth].

(9) Als Alternative bieten sich die Klassiker Rot oder Blau an [The classic red or blue are offered as alternatives].

But intuitive (secondary) coding of colors is also used in fashion, where the coding refers to the acquisition of meaning without a knowledge of the act of giving significance to a specific color. This type of coding is not far from and not even discernible from association families, for instance when weiss occurs as a sign or signal of 'purity', 'innocence,' or 'chastity' as in the dress of the bride. What is typical of fashion magazines is that they aspire to activate only positive connotations. The symbolic force of black as the symbol of evil is consequently avoided.

In many instances color names may serve as nothing more than to designate a color without any further implication, yet in most cases they serve as pure description. In fashion magazines descriptions are rarely mere color indications, where color terms occur in order to modify a noun or rather to foreground the color of an object. In the text, color words, apart from designating the colors of objects actively, must contribute to the creation of a picture in the reader's mind.

A color term assumes a definite function, and it must help to create what can be termed an atmosphere, i.e. a certain uniqueness of scene, and to give certain objects more prominence, and help to evoke color in the mind of the reader.

8. Minsky (1985:56) defines signals as "acts whose consequences are not inherent in their own character but have merely been assigned to them."

9. The best known examples of this type of coding are the traffic lights. Red is not only a warning 'danger ahead' but a clear order 'stop.' 
The predominant use of BCTs enables the reader to interpret the color indication, so that she can decide what interpretation she wants to give to a default value, whether it is a focal color or whether it is a shade for which a more specific color name could have been offered.

These tendencies are borne out with reference to the growth of fashion reports over a period of 100 years. The proliferation of pictures in magazines has been accompanied by the elaboration of color terms in slogans and texts. Thus, the function of terms is not only bound to naming processes but also given to aesthetic aims and ever-unfolding subtleties of impression. From a syntactic perspective, sentences have become much shorter during the 100-year period, mostly serving as slogans and presentations:

(10) Neu: Blau und Lachs [New: blue and salmon].

The longer sentences offer more detailed information about tendencies in fashion for the season:

(11) Die neuen Farben sind weich und zart, ohne fahl zu sein, es gibt keinen harten, sich vordrängenden Ton in der neuen Farbenscala, in der wir auch erfahren, daß es nicht nur Binsenweisheiten sondern auch ein "Binsengrün" gibt, neben einem schmeichlerischen "Absynth"- und "Benedektinergrün".

[The new colors are soft, gentle and smooth, without being pale; this recent scale of shades has no austere or harsh tint to it. Also, we not only experience the usual platitudes regarding the coloring, but also a "truistic" green shade like rush, apart from a flattering verdant with an "absinth like" and "benedictine" tone, respectively.]

Since the last decades of the 20th century very short syntactic forms have been used where the shortage of the expression can hardly be more minimized. This may express the changing position of the text part in fashion magazines as an instrument of information transmitting, which is correlated to the dominance of the picture. This can also be the result of the writer's changing information intention, which can be subsumed in the transition from the informative character of the texts to a consumer-friendly and advisory character at the end of the 20th century. Color terms have thus evolved from a peripheral import to the center stage in fashion literature, where they are used as designative, emphasized, and connotative elements:

(12) Grau mit Türkis: Sehen Sie selbst den leuchtenden Erfolg [Grey with turquoise: it is easy to see the shining effect].

As regards pragmatic aspects, the tendency to normalize and personify colors can be mentioned, which has come into prominence in the last few decades:

(13) Weiß beherrscht die Sommerszene [White dominates the summer scenery].

Furthermore, especially strong evaluating lexemes (aktuell 'up-to-date'):

(14) Besonders aktuell sind Weiß, Gelb, Grau und Blautöne wie Matisse, Lagune und Salbei [White, yellow, and blue shades like Matisse, lagoon and sage are extremely up-to-date]. 
or connotative lexemes (Liebling 'Favorite'):

(15) Brauntöne sind die Lieblinge der Saison [Brown shades belong to the season's favourites].

are used in connection with color designations. With these representation techniques the color is stylized and aesthetisized. The texts are easy and stereotypic for the purpose of creating parts which can be easily memorized and which intensify the message. In these texts the denotative accuracy is not the most important part, due to the short life of modern colors. Such needs are no longer emphasized and instead the texts concentrate on transmitting emotive experiences. Fashion magazines may have served as examples of how specific demands, economic strategies in this case, help to produce an abundance of color names. Many of these names are intended to last only for a short time. Bennett (1988) classifies them under the heading "Ephemera." ${ }^{10}$ A characteristic sort of synonymity can also be observed where a hue or shade of hue bears more than one name. This sort of synonymity may depend on the object. It is not precision and propriety; it is figurativeness, connotations, values, and expressivity that count.

\section{Conclusion}

In the beginning of this investigation it seemed that discussing color designations in fashion implies a fundamental difference from discussing color names in ordinary everyday language. Whereas such names in everyday speech have a long history and have arisen from so-called natural colors of objects (such as plants, flowers, fruits) the colors of textiles are the result of dying processes developed by humans, where a large variety of forms and structures can be observed. Moreover, such dying processes have been undertaken with specific intentions. It was the objective of this contribution to analyze and, if possible, to categorize the underlying principles of naming and the semantic and semiotic implications with the motivation aspect in focus. It was also shown that these processes make use of inherent color-term properties in general, as we have to reckon with the faculty of natural and constructed naming, acting as distinguisher and valuator and involving descriptive, associative, or suggestive functions, to mention just a few of the properties which come into play with textiles. It was also demonstrated that these processes cannot take place without the language speaker's consciousness, expressed in metalanguage reflection about color designations, and their etymological competence as an important aspect of the naming process. Against this background we can see that the speaker tends to motivate color words synchronically.

10. Cencig (1990:12,3) points out the following: “The strategy is self-revealing: yesteryear's colors should appear visibly dated, obsolete, passé. Very rarely are old color terms resorted to, and if at all, then only after a safe interval of some ten years or so. It remains to be seen how long the trendsetters or 'profashionals' (as one fashion magazine extemporizes) will succeed in creating some thirty new shades each season, together with the corresponding color terms." 
Furthermore, it must be remembered that fashion is an object of economic activity. This implies principles, methods, and strategies of marketing, which in turn imply human behavior in general in such areas as advertising, selling, and buying. One must also keep in mind that textiles are fundamental elements in the culture and lifestyle of human communities. Thus, in addition to designation, semantic implications, psychology, and marketing, cultural factors add to the complexity of the color naming of textiles. Color terms occur more frequently in the language of the fashion magazines of today. They have become a focal part of the texts and their suggestive and symbolic function can be activated in the fashion magazines.

These considerations could give rise to the hypothesis that there is a frequent use of non-basic color terms. But such a hypothesis could not be proved. Of course, there are different word formations and a large variety of structures among the color terms in fashion magazines. Nevertheless, BCTs remain the most frequently used lexical means of designating color, also from a diachronic perspective. Even though this study was intended to investigate color terms exclusively at the linguistic level, related to the analytical aspect of word formation theory, the description of Wortgebildetheit (Dokulil 1968), this was not possible without reference to many other spheres like color perception, psychology, and semiotics. A study of color terms will therefore always be in some way or other an interdisciplinary study.

\section{References}

Augst, G. (1975). Untersuchungen zum Morpheminventar der deutschen Gegenwartssprache. Tübingen: Narr.

Bennett, T. J. A. (1988). Aspects of English color collocations and idioms. Heidelberg: Winter.

Berlin, B. \& P. Kay (1969). Basic color terms: Their universality and evolution. Berkeley: University of California Press.

Burda Moden (1984). Offenburg.

Cencig, E. (1990). United colors: Colour terms in contemporary fashion trends. Fachsprache, 12, 3-4.

Dokulil, M. (1968). Zur Theorie der Wortbildung. Wissenschaftliche Zeitschrift der Karl-Marx-Universität Leipzig. Gesellschafts- und Sprachwissenschaften Reihe, 17, 203-211.

Fill, A. (1980). Wortdurchsichtigkeit im Englischen. Eine nichtgenerative Studie morphosemantischer Strukturen. Mit einer kontrastiven Untersuchung der Rolle durchsichtiger Wörter im Englischen und Deutschen der Gegenwart. Innsbruck: IBS Verlag.

Fleischer, W. \& I. Barz. (1992). Wortbildung der deutschen Gegenwartssprache (2nd ed.). Tübingen: Niemeyer.

Gauger, H.-M. (1970). Wort und Sprache. Sprachwissenschaftliche Grundfragen. Tübingen: Niemeyer.

Illustrirte Zeitung. (1883). Wöchentliche Nachrichten über alle Zustände und Persönlichkeiten der Gegenwart. Leipzig.

Kastovsky, D. (1982). Wortbildung und Semantik. Düsseldorf: Pädagogischer Verlag Schwann-Bagel.

Klaus, H. (1989). Beobachtungen zu den Modefarbenwörtern in der deutschen Gegenwartssprache. Zeitschrift für germanistische Linguistik, 17, 22-57.

Marx, E. (1973). The contrasts of color. New York.

Minsky, M. (1985). The society of mind. New York: Simon and Schuster. 
Oksaar, E. (1961). Über die Farbbezeichnungen im Deutsch der Gegenwart. Muttersprache, 71, 207-220.

Olsen, S. (1986). Wortbildung im Deutschen. Eine Einführung in die Theorie der Wortstruktur. Stuttgart: Kröner.

Ortner, H. (1981). Wortschatz der Mode. Düsseldorf: Schwann.

Polenz, P. (1967/1979). Fremdwort und Lehnwort sprachwissenschaftlich betrachtet. In Braun (Ed.), Fremdwort-Diskussion (9-31). München: Fink.

Rettig, W. (1981). Sprachliche Motivation. Zeichenrelationen von Lautform und Bedeutung am Beispiel französischer Lexikoneinheiten. Frankfurt a.M.: Lang.

Römer, R. (1976). Die Sprache der Anzeigenwerbung (2nd ed.). Düsseldorf: Schwann.

Stoeva-Holm, D. (1996). Farbbezeichnungen in deutschen Modetexten. Eine morphologisch-semantische Untersuchung. Uppsala: Universitetsförlag Almqvist \& Wiksell.

Wittgenstein, L. (1977). Remarks on color (McAlister and Schättle, Trans.). Oxford: Blackwell.

Wurzel, W. U. (1984). Zur Dialektik im Sprachsystem: Widerspruch-Motiviertheit-Sprachveränderung. Deutsch als Fremdsprache, 21, 202-211.

Zollinger, H. (1980, January 16). Farben erkennen und benennen. Neue Zürcher Zeitung, Sektion Forschung und Technik, 53. 



\title{
To have color and to have no color \\ The coloring of the face in the Czech linguistic picture of the world
}

\author{
Irena Vaňková \\ Faculty of Arts and Philosophy, Charles University, Prague, Czech Republic
}

The semantic analysis of color vocabulary provides insight into models of perception and thinking. Color and coloring play an important role in the way phenomena of human perception and reflection are conceptualized. Various linguistic and cultural connotations related to colors in specific contexts are explored and a semantic analysis of the role of color in these contexts is given, with reference to Czech color words.

\section{Colors and colorfulness in the Czech linguistic picture of the world}

Semantic analysis of color vocabulary provides insight into models of perception and thinking, about the way some phenomena related to human perception and reflection are conceptualized - and also about various linguistic and cultural connotations related to colors in different contexts.

\section{Semiotics of colorful and colorless}

First, let us take a look not at words, but at pictures. A girl has drawn "Bad Luck and Good Luck". She drew them as two female figures. One of them is only black and white; she has a black dress, black hair, her complexion is colorless, and the figure is holding a black cat. For her second drawing, the girl has used a whole range of colors: the complexion of the figure is painted orange, her hair yellow, her eyes blue, her lips and cheeks red, and the figure holds a green four-leaf clover in her hand.

It would hardly be difficult to decide which of the figures is Bad Luck and which Good Luck. We always perceive the "colorful" as positive: colorfulness is associated with light, beauty, life, wealth, good luck, joy, diversity of full life. The non-colorful - characterized often as "colorless", grey, black-and-white; and pale when a human being is concerned - represents the other extreme: gloom, ugliness, a poor spiritual and physical life, the oppressive weight of everyday life, poverty, sadness, boredom, lethargy, disgust, and monotony. 
As far as clothes are concerned, the role of the opposition colorful versus non-colorful played a different role for our ancestors than it does for us today. The possibilities of and access to dyed fabrics used to be quite limited. In the medieval Czech lands, the most common fabric used to be home-made cloth of grey color that was called šeré 'gloomy, dim (grey)' or šer 'grey cloth.' Rare fabrics, at first only imported from abroad, gaily colored mainly red - were accessible only to the rich. Šarlatový 'scarlet', purpurový 'crimson', or nachový 'purple' dress was a privilege of the highest walks of society. The opposition 'scarlet' and 'gloomy, grey' characterizing a social opposition originates here: One poem from the fourteenth century says “Jedinét' smrt rovně měř́, bez peněz každému měrí, tak v šarlatu jako $v$ šeri [Only death measures equally, measures to everybody without money, equally to both the scarlet and the grey]" (Němec-Horálek 1986:122)

\section{Colorful and colorless in language}

The fact that connotations of colorfulness in Czech are only associated with positive emotions is seen also in a layer of the meaning of the adjective barevný, 'colorful', and its synonyms. If we want to capture the meaning of the adjective barevný, 'colorful', we should ask the simple question: "Does everything really have a color?"

Similar questions are discussed by the Russian scholar Rakhilina (1995) in her article "On the colored and colorless". What color is, for example, an ostrich, a badger, a kangaroo, or a nightingale? Or wood or metal? The author writes that in Russian (and we can add that not only in Russian) names of these animals fall into the group of colorless nouns. According to her, these objects have a stable, so called fixed color. The color, typical of the whole kind, is not a distinguishing element, it is not communicatively relevant and therefore it does not exist in language. Many things are thus linguistically non-colorful.

\section{Colorfulness as a color markedness}

Barevný 'colorful' in Czech has (see Appendix I) besides a purely relational meaning barevný odstin 'a shade of color' - especially the meaning "to be of other color than black, white, black-and-white", as the dictionaries write, and even here we add "or another color than is usual for or typical of the object", barevný means marked by color. We have barevné sklo 'colored (i.e. stained) glass' in contrast to clear; barevný papír 'colored (i.e. non-white) paper', barevné dřevo and barevné kovy 'colored wood' and 'colored (i.e. nonferrous) metals' in opposition to those that look natural or normal; barevné prádlo 'colored (i.e. not white) linen', and also barevné obyvatelstvo 'colored inhabitants' - in contrast to white (a classic example of white-man's ethnocentrism). A separate group is formed by phrases like barevná televize, fotografie, film 'color TV, photography, film' in opposition to black-and-white, in the past the "normal" option, and originally, the only possible one.

Jungmann's dictionary adds to our account examples from older Czech (from the beginning to the 1830's). The entry barevny' 'colorful' here starts with evidence from old medical books dobře barevný člověk 'person with good color', with the explanation červený 'red' and continues: barevný = zhněda červený, braunroth 'colorful = brownish-red'. 
Barevné vlasy, obočí, tj. tmavé, nebledé 'colorful hair, e.g. dark, not pale'. Barevné pivo 'colorful beer' (today we describe such beer as černé, 'black' - and in reality it is dark brown). And another important bit of information pointing to the meaning of the word barevný 'colorful', as it has already been discussed: V rostlinářství barevný (coloratus) slove oud, který jinou než zelenou má barvu 'In botany, such a limb is called colorful that has other than green color. Here again the concept of color as markedness is confirmed.

Jungmann mentions also the idiom barevný prítel 'colored/colorful friend' explained as "not true, just for show, Scheinfreund", which resembles the meaning of the noun barva 'color' stated in Appendix I under number 6, but in Jungmann's dictionary, it is much more strongly associated with falsehood, appearance, fallacy, pretension. Komenský 'Comenius' is cited twice: Pravdy barvu šalbám světa dáti 'to give the color of truth to the deceptions of world' and pěknou barvou mrzkost hříchu zastíral 'he was covering the meanness of sin with a nice color', from other frequent Old Czech examples let us mention víc barvy než pravdy 'more of color than of truth' or často pod ovčí barvou tají se vlci loupežni 'there are often robbing wolves hidden beneath the color of sheep'. This meaning of deceit and pretension (or better, covering up - covering with a color, painting over) seems to be common in older Czech. Today we encounter it rather exceptionally, especially through idioms connected with the colors of playing cards: priznat barvu 'to confess the color' - to show one's cards), muset s barvou ven 'to have to come out with the color' - to have to show one's cards), that is in their primary meaning, to show or reveal ones cards, and in their figurative meaning, to reveal unwillingly some important information.

In contemporary Czech, the adjective barevny 'colorful' is used in more meanings: jolly, having various, many colors (barevná mozaika 'colorful mosaic', barevné korálky 'colorful beads'; see Appendix I). From these we move toward figurative meanings with distinctly positive connotations: heterogeneous, interesting, happy, causing joy.

\section{Prototype of the colorless: non-colorful colors}

Být bez barvy 'to have no color', bezbarvý 'colorless', bledý 'pale' - especially about people, human complexion), vybledly' 'pale, pallid', in figurative meanings also jednotvárný 'monotonous', nezajimavý 'not interesting', obyčejný 'common, usual', všední 'everyday', nevýrazný 'inexpressive, unidentifiable', nemastný neslany' 'wishy-washy' belong among the opposite expressions (in the sense of non-colorfulness) in various contexts.

In opposition to colorfulness - and in figurative meanings, too - is especially the prototype of non-colorful: grey and greyness, in specific cases black-and-whiteness, and in some cases also black. We could say that the physically non-colorful (achromatic!) colors black, white and grey are in the position of not-colors also in language.

The definition of the basic meaning of the adjective šedy 'grey' in SSČ contains the description "to have the inexpressive, nondistinct color of ash", and in figurative meanings the adjectives "not gay, sad" (šedý podzimní den 'grey autumn day'), "not interesting, inexpressive, monotonous, boring” (šedý život 'grey life', šedá realita 'grey reality', šedá fráze 'grey phrase'). The nouns šed', šedo, šedost (all denoting 'greyness'): šedost a prưmérnost literatur, 'greyness and mediocrity of literature'; unavujicí šedo jednotvárného života 'tiring 
greyness of monotonous life'; and the adverb šedě 'greyly' (fádně, šedě zpracovaný námèt 'theme handled in a dull, grey way' - the opposite is barvit 'in a colorful way') are all similarly characterized. The same is true of the variant šedivý and its derivatives.

Prototype of colorfulness: red (color, beauty, body, blood, fire)

If the prototype of the non-colorful and colorless is grey, the prototype of colorfulness is the color red. Red is understood as the color of colors, color par excellence.

Not many know today that in the old times clothes were dyed using a special kind of worm, červec nachový: červily se 'they were wormed' (were made red using the worms) and then they were črvené 'became wormed' (i.e. became red). ${ }^{1}$ The meaning of this participial adjective was transferred from the meaning 'dyed red' to red color in general (Rejzek 2001).

It is interesting that with Slavs (in old Czech too), the root kras- meant not only beauty, but also red color (as in Russian today: krasnyj 'red') and also color in general. The old common Slavonic krasa is said to mean originally probably "gloss, redness, color of fire" (Rejzek 2001). Kraslice 'decorated Easter egg' the main (and magical) requisite of Slavonic spring feasts is believed to originate from the times when, for Czechs, krásný meant 'red' and at the same time 'dyed', 'colorful' and also 'beautiful'.

The adjectives červený 'red' and krásný 'beautiful' hint at the meaning of color in general and its relationship to beauty: what is red is beautiful, and also - what is colorful is beautiful.

Red connotes life - energy, warmth, body, emotions, passions, love, sexuality, but also aggression, war, injury, bloodshed. We perceive it through two prototypes, blood and fire. The fact that this color is most closely connected with our body (and also subjectivity and emotionality) is well demonstrated by idioms. Idioms containing red tend to express bodily symptoms of states or emotions - and at the same time they imply the presence of energy in the form of fire and blood that in the moments of great excitement rushes to one's face. We say vzplál hněvem 'he burned/exploded with anger', zahořela ruměncem 'she blushed/burned with a blush', byl rudý až po uši, jako by ho krví polil 'he was red to his ears as if poured on with blood'.

Also the prototypes themselves - fire (ignition of flame, process of burning, heat or glow) and blood (bleeding, injury, meat) often participate in the conceptualization of human emotions and passions. This is evident in fiction and especially in poetry.

1. The name of the worm červec and the Czech word for red červený are etymologically related; nachový means purple. 


\section{Color of face: good and bad color}

For example, in Czech (and in other languages), we say that somebody was zimou celý modrý 'whole blue with cold', vzteky zelený 'green with anger', bilý jako stèna 'white as a wall', or rudý jako rak 'red as a crayfish'. We rarely realize that criteria for this blue, green, white or red color are quite debatable; in reality, they are hues that only very distantly resemble the colors mentioned (only a very little child might think that vzteky zeleny' 'green with anger' means 'to have the color of fresh grass'). We also do not realize that the blue, green, white or red color applies synecdochically only to one's face (cf. Waszakowa 1999). And thirdly, when we think about the real, normal color of the human face, when the person is neither white, nor green, blue or red or purple, we find out that, in fact, we are not able to give an unambiguous answer. When drawing, even children hesitate over which color they should choose for the human body and face. We have the adjective télovy' fleshcolored' used to denote, for example, the color of stockings or underwear, but we never talk about télová pastelka 'flesh-colored marker' because it does not exist, and children have to be satisfied with red, pink or orange.

But what color is a human being if s/he is neither red, nor pale? If there is no deviation from the normal state? We find out that in language a person has in fact no color. In Czech, we do not have a neutral expression denoting the normal color of human complexion. Human beings (or rather their complexion) rank among those objects that are colorless in language (Rakhilina 1995; and cf. above). Various types of coloring are talked about only when they are communicatively relevant, i.e. different from the normal state. Then there is available a whole spectrum of linguistic means of various intensity, expressivity and stylistic value.

This semantic sphere, specifically connected with the usage of color terms, is our theme now. The question is how Czechs talk about the color of human complexion. But we have in mind neither different expressions for colors of skin as part of differences between races (lidé všech barev pleti 'people of all skin colors'), nor the denotation of color of hair, eyebrows, etc. as in blondýnka a bruneta 'blonde and dark-haired woman' or albin 'albino'. We are interested in those expressions where the color of face (and its changes - začervenat se 'to blush', zblednout 'to become pale', zezelenat 'to become green') is a symptom of certain human characteristics or human mental states such as emotions, etc.

We interpret the color of faces (perceived specifically in language) as a result of bodily and/or mental states and experience, and it is for us usually part of their conceptualization (e.g. becoming red is connected with anger, but also with embarrassment; paleness in general implies illness or physical and/or mental discomfort, a yellow face - more serious illness and so on).

At the same time the question is how one of the aspects of human bodily existence is reflected in the Czech linguistic picture of the world, how semantic oppositions connected with the color of human complexion are engaged in the wider frame of human experience of the world, how they are perceived, mutually connected and evaluated by language and culture. In this respect, the Czech situation will probably be, in many aspects, similar to that in Polish or Russian and maybe even in German and English. But it will be different 
in languages spoken primarily not by white men, but by people of other skin colors. Can a person with black complexion 'become red' (blush)? Are there - and if yes then what? equivalents of 'becoming red' (blushing) in Chinese or Vietnamese, too? It is not our task to find a solution to this question here, we merely want to hint that the middle-European basis of the linguistic picture of the world is so self-evident for us that we rarely perceive it as culturally specific.

\section{Opposition 'red'-'pale'}

Let us focus on the fourth meaning of the word barva mentioned in Appendix A ("natural coloring of cheeks, complexion'). In Czech, rich vocabulary and phraseology suggesting interesting connections are related to this semantic field.

Barva 'color' is without doubt connected with blood (see also the meaning from huntsman's slang listed under number 9, "blood of hunted game or hunting dogs", and there is no doubt about the fact that red is the color meant here). Red color, especially in connection with face and bodily existence - and life - is also one of the marginal meanings of the adjective živy' 'live, living. Today this meaning probably does not survive even in dialects, but in PS we find such evidence from nineteenth century fiction: oči méla od pláče celé živé 'here eyes were all live (i.e. red) from crying', utřela šátečkem své živé rty 'she wiped her live (i.e. red) lips with a scarf', děvčata měly ... přes hlavu živé šátky 'the girls ... wore live (i.e. bright) scarves over their heads.'

When we say that somebody nemá (žádnou) barvu 'has no color' or that he or she has bad or unhealthy color, it means that the complexion of the face signals illness by its color. Such a person is most often (and least markedly) said to be bledy' 'pale'. A higher intensity of paleness (appearance of illness) is expressed by the adjectives bily' 'white', zelený 'green', in special cases - and with even higher intensity - also žlutý (nažloutlý) 'yellow (yellowish)', šedý, šedivý (popelavý) 'grey (ashy)', and especially modrý (sinalý) 'blue (pallid)'. Whole clusters of synonymically-hyponymical expressions (including idioms) with various connotations and collocational validity are related to these basic concepts (examples are given in Appendix B in the right half of the table).

In Appendix B, to the left of "normal coloring", is shown how Czechs conceptualize the state "to have (healthy, nice) color". The opposite of bledost 'paleness' - non-color is color, namely červená 'red'. The basic adjective can be modified by word formation (červeňoučký, red with positive emotional connotations), it can function in various transformations (mit červené tváře 'to have red cheeks'), its meaning can be expressed and enriched also idiomatically (je jako rưže 'she is like a rose'). Here idioms usually do not mean only (healthy) color, but they are related to the whole appearance of the person usually a girl or a young woman, her (healthy) physical beauty, attractiveness and freshness (děvče jako růže, malina, jahoda, karafiát 'girl like a rose, raspberry, strawberry, carnation'). Pink often has a similar meaning to red. As Tokarski writes about pink in Polish it is in fact less intensive red (Tokarski 1995:183), with tuned down activity and energy, connoting especially young girls' (or possibly children's) freshness and innocence: babies, children and very young girls have pink complexion in Czech. 
The prologue to Grandmother by B. Němcová starts with words: Dávno, dávno již tomu ... co jsem zulíbala to bledé líce, plné vrásků [It is long, long time ago ... I kissed that pale cheek full of wrinkles]. Very young Barunka is, in opposition to bledá tvár stařenky 'pale face of the old woman' (98), shown as čerstvá, růžová jako poupátečko 'fresh, pink like a little flower bud' (133), her grandmother utírá slzičky z rưžového líce 'wipes tears from her pink cheek' (197). Children podávají svoje růžová lícka babičce k políbení 'give their pink cheeks to grandmother to kiss' (12). Miss Hortensie, suffering from unhappy love, is characterized by the grandmother several times as bled'oučká, bled'ounká, skoro prìhledná 'very pale, almost transparent', but after the positive change in her life, her color is changed too: tvářinky jí rozkvetly jako růžové lístečky 'her little cheeks blossomed like little pink leaves' (176), byla den ode dne růžovějši: babička mínila, že jí připadá milostslečna ne jako růžička, ale jako jabloně květ zarưžověly' 'day by day she was pinker: the grandmother thought that the noble miss seemed to her to be not like a little rose, but like a pinkish blossom of an apple tree' (183). Kristla is děvče jako karafiát, čerstvá, čiperná 'a girl like a carnation, fresh, bright' (26): but when her lover is taken to the army, she has zmènèná tvář 'changed face' (149), she becomes bledé děvče 'a pale girl', she is usoužená, uplakaná, bledá jako stěna 'careworn, tearful, white as a wall' (166) during Jakub's recruitment. Also unhappy insane Viktorka bývala vždy bledá, oči jí svitily jako dva uhly, černé vlasy méla $v z ̌ d y$ rozcuchány 'used to be always pale, her eyes glowed as two pieces of coal, she had her black hair always unkempt' (20) in spite of the fact that she used to have krásná tvárička a byla děvče jako malina 'beautiful face and she used to be a girl like a raspberry' (52) (Němcová 1971).

So far we move within the frame of the oppositions health/happiness/beauty/being young/life versus illness/suffering/ugliness/old age/death, and connotations connected with red are always distinctly positive within this frame. Later we will see that it is not always so in conceptualization of (temporary) psycho-physical states and their changes (vzteky zrudl jako krocan 'he went red with anger as a turkey'). But pink never has negative connotations - except in the idiom být růžový jako prasátko 'to be pink like a pig', which is mocking and pejorative.

When we say about somebody that he zbledl (zbělel), že by se v něm krve nedořezal 'became pale (white) that one could not cut blood in him' or that he was bledý (bilý), jako by v nèm ani krve nebylo 'pale (white) as if there was no blood in him', and on the contrary krev (se mu) vhrnula do tvárí 'when blood rushed to one's cheeks', or when he zưstal, jako by ho krví polil 'stayed as if one poured him with blood' (went red as a beetroot), it is well recognizable what forms the basis of the opposition 'red'-'pale' (the expression bily' 'white' is less common than bledy' 'pale' and semantically stronger). Changes in the coloring of face are caused by circulation of blood in the human body. During strong physical or mental stimulus, blood either runs to one's face and one becomes red, blushes (zrudne, zardí se, zčervená, začervená se) - or on the contrary it suddenly leaves the face and one becomes 'pale' zbledne, or becomes 'green' zezelená, 'white' zbělá, 'grey' zšedne, 'blue' zmodrá, or 'pallid' zesiná - and also 'yellow' zežloutne.

As Wierzbicka (1996) shows for English and Tokarski (1995) for Polish, it is true also in Czech that the prototypical bearer of red is blood. In Czech, the adjective červená func- 
tioning as a noun can be used directly with the meaning 'blood', for example teče mu z nosu červená 'red (i.e. blood) runs from his nose'. Blood in this context connotes life, animality, health - and also warmth and energy, as well as the second prototype of red - fire (e.g. zapálila se studem 'she flared up with shyness', byla v jednom ohni 'she was all in fire', tváre jí hořely 'her cheeks burned').

On the contrary, bledost malé zdraví ukazuje 'paleness shows little health', states Jungmann in his dictionary. And let us add - also little energy, coldness, little life. SSJČ describes the meaning of the adjective bledy' 'pale' negatively - through lack of color. Bledý 'pale' means nemající prrirozeně sytou, výraznou barvu 'not having naturally rich, pronounced color': the basic sphere of usage is connected with a human being, his or her face etc. In the definition of other, figurative meanings, the synonyms "not pronounced, dim, vague" (e.g. bledé světlo 'pale light') and colloquially also "bad" (je to s ním bledé 'it is pale with him', i.e. his odds are slight, vypadá to bledě 'it looks pale', i.e. poor, miserable).

\section{Green}

Both red and green colors are closely connected with elementary human experience with the world. As was already said, also in Czech, red is modelled through it prototypical relation to blood and fire.

Green is - contrary to animal red - primarily the color of plants, vegetative life. If green is used in connection with a human being, it always has a negative connotation - it is a higher degree of paleness, a more distinct opposition to positive red. Být zelený 'to be green' means to look bad, unhealthy, tired; it also often signals (contrary to, for example, yellow) momentary indisposition.

It is said about green that it is healthy for the eyes, connotes freshness and joy - but only in connection with the world of plants. A healthy plant is green (the opposite is dry, yellow, yellowish, brown), but a healthy human being is red. The coldness of green (in relation to vegetation functioning as freshness) stands in opposition to the warmth and animality of the world of animals represented by red. In relation to the human body, green always means illness, even closeness of death and decay (with connotations of mould and rotting). Mrtev je bưh a morem páchne jeho zelené tělo 'dead is the god and his green body stinks with plague' is a verse by one Czech decadent poet (Jiř́ Karásek ze Lvovic 1995; about green in more detail see Vaňková 2003).

The humorous idiom mit zdravou lesní barvu 'to have the healthy color of woods' is built upon the paradox of healthy color (which is usually red) and the woods - an environment traditionally perceived as healthy and at the same time connected with green. If a person's complexion is of this shade of health, the person definitely does not look healthy.

Beings that are non-human - but that resemble human beings and have a face - are often depicted as green, especially water goblins or water nymphs that often have not only green hair, but also a green complexion. We also imagine, for example, inhabitants of Mars (zelené mužǐcky 'green little men') as being green. Green demons, green phantoms, and green faces often appear in horror films: green in combination with a partly human appearance probably inspires horror because a complexion of this color looks 
like the complexion of a dead person or of a creature in transition between two forms of existence - alive yet not alive.

\section{Yellow}

Apart from being (and probably not only in Czech) the color of sun and light, a joyful, gay color of spring flowers, yellow shows another face, too - it is the so-called "color of change for the worse" (Vaňková 2003). In autumn, the bright green grass and leaves turn old, dry, yellowish, and it is similar with aging that changes a young, pink complexion into a pale face with wrinkles and a yellowish shade.

Žlutá, yellow (nažloutlá, zažloutlá - yellowish) is associated not only with old age, but also with illness - usually a more serious and longer illness and even death. ${ }^{2}$ Another important field connected with acomplexion changed to yellow are negative emotions jealousy, envy and hatred.

In all Slavic languages, the adjective žlutý 'yellow' is etymologically related to the adjective zeleny 'green', and with the noun zlato 'gold' (cf. common Indo-European * ghel), but also with the noun žluč 'bile, gall' (cf. also German Galle and English gall and Greek chólos, cholé - and from here also cholerik 'choleric'). Gall is a yellow-green liquid and bitter secretion of the liver that can be in case of illness secreted into the blood and cause a yellowish color of complexion. But in Czech, gall is connected with anger, too: žlučovitý 'rancorous' denotes an unpleasant, hateful person whose body is flooded with gall. In the fairy tale Bad Mother, B. Němcová writes "Děvče bylo denně hezčí a hezčí, a paní Kačenky bílá plet' žlučí žloutla jako podmásníček [the girl was day by day more beautiful, and the white complexion of Mrs Kačenka was by gall made more and more yellow like buttermilk]" (Němcová 1950:111).

Blue

The opposition of blue and red is formed above all by the fact that one is the most typical cold color (blue) and the other one the most typical warm color (red). Red means life and stands in opposition to blue, a color suggesting the sky, air, cold, emotional distance (Tokarski 1995: 130) and spirituality.

In association with the human body, blue also connotes physical discomfort, and, even more strongly than green, danger to life. A person is modry 'blue' when he or she lacks oxygen or when he or she is too cold for a long time (modrý, promodraly zimou 'blue, all blue because of cold'). Bluish-violet coloring of the skin and mucous membrane (cyanosis) is caused by insufficient oxygenation of blood. In general we can say that it is an extreme pole of paleness, even an attribute of death (e.g. in the idiom tuhla / chladla mu v žilách krev 'his blood was getting stiff / cold in his veins').

2. The Czech name of the illness žloutenka 'jaundice' and the word for yellow color žlutý are etymologically related. 
The adjective modry 'blue' motivates the noun modrina 'bruise', a (usually dark blue) blood contusion. When we say about somebody that byl cely modry' 'he was all blue', it can mean not only suffering from cold or lack of air, but also beaten, strongly bruised, full of bruises after some accident or physical attack.

\section{Short time psychophysical states and changes of coloring}

Paleness/red coloring can be connected with the overall psycho-physical type of a person, his habitus (Vaňková 2000), it is also connected with state of health (both physical and mental), degree of tiredness, age etc. In such cases the color of the face can be manipulated or at least corrected, to a certain degree. An actor uses make-up to create the appearance (and type) of the role he or she is supposed to play. Cosmetics serve women in similar way: one can make up oneself decadently white or on the contrary can choose a make-up that is blood and milk or we can at least try to change pale old-age into pink youth. In such cases we can mask ourselves, feint or stylize ourselves into desired appearances just as actors change costumes.

But changes of coloring of facial complexion seem to be (at least on the evidence of the testimony of language) often momentary, an uncontrolled reaction of a person to some stimulus, either physiologic (heat, fever: zrudl jako rak 'he became red as a crayfish' i.e. like beetroot; cold: zmodral zimou 'he became blue with cold'; lack of oxygen: ditě se dusilo, až celé zmodralo 'the child was suffocating so much that he or she became blue') or under the influence of strong emotion (zežloutla závistí 'she went yellow with jealousy') as Appendix D shows.

These cases are usually spontaneous reactions. They can neither be acted out nor prevented. The most frequent one is blushing, getting red as a symptom of shyness, shame or hesitation, especially amorous. Blushing is a frequent problem of shy girls and boys discussed also by psychologists in the columns of magazines for young people. There are also countless idioms, folk songs, verses etc. with this theme. "Která panna je poctivá, / ta se mládencům vyhýbá. / A když vyhnout nemůže, / začervená se jak růže [The girl that is fair, / avoids boys. / When she cannot avoid them, / she blushes like a rose]", Czech folk song says.

The conceptualisation of shock, horror, fear is connected with getting pale in a similar way: "Když XY uvidél na povolávacím rozkazu své jméno, zbledl [when XY saw his name on the call-up order, he went pale]". It is evident from the context (and also from usual functioning of this model in language) that XY felt strong shock, horror.

When we look up the entry vztekati se 'being furious, in rage' in Haller's dictionary (ČSVS), we find a whole list of synonymous color expressions: být rudý 'to be purple' (červený 'red', bledý 'pale', žlutý 'yellow', zelený 'green', modrý 'blue', fialový 'violet') vztekem 'with anger'. We might be surprised by the fact that practically all colors of the spectrum are involved, even those that we can hardly imagine in connection with the color of the human face. But most frequently red/purple is connected with anger and aggressiveness: we say e.g. zrudnout (vzteky) jako kohout, jako krocan 'get red (with anger) like a cock, a 
turkey' - and we also associate red with phrases like krev se v něm vařila 'his blood was boiling'. An angry person (in both Czech and English) vidí rudě 'sees red'. Again, red is the color of life energy and strength - and also aggressiveness.

If there is an emotion that can deprive us of blood, it is long-lasting, consuming envy, jealousy and hatred, connected in Czech with the adjectives zelený 'green' and žlutý 'yellow', but also with universally usable bledý 'pale' (bledý závistí 'pale with envy', žlutá žárlivost 'yellow jealousy'). But one can get pale also with anger, even when it is less typical in the conceptualisation of anger than getting red.

Bloodlessness (and paleness or whiteness connected with it) is more typical of conceptualization of long lasting trouble, fear, and also sudden shock when a person zbledne jako stěna 'gets white as wall', when he or she is (suddenly) bílý jako plátno 'white as cloth', jako papir 'as paper', jako křída 'as chalk', when he or she is mrtvolně bledý 'deadly pale', sinalý 'pallid', popelavý v obličeji 'ashy in face'.

\section{People, colorless and colorful: conclusion}

Let us return for the last time to more general questions of color and coloring in the Czech conceptualization of world and people. When we talk about coloring in general we have in mind color markedness, deviation from a usual state (i.e. non-color). Let us recall - barevné punčochy 'colored stockings' are stockings other than brown, and barevné sklo 'colored (stained) glass' is glass that is not clear (see above and cf. schema II for more detail).

But when we have in mind the coloring of the human face, the situation is different. Normality is not the same as non-color, bad color in this case. As this coloration is not relevant in communication, it, therefore, has no verbal expression for it in language. Color, color markedness can be of two types here: good and bad. If the state of unmarkedness normality is situated in the middle of the axis dividing good and bad color (for graphical expression see schema III) then color, dobrá barva 'good color' (red or as it may be pink) is on the left side - and negatively perceived non-color (pale, white, green) on the right side.

A person nemá žádnou barvu 'has no color' or má špatnou barvu 'has bad color' when he or she looks unhealthy (is bledy' 'pale' or as it may be zelený 'green', žlutý 'yellow'). After an illness se mu vrací barva do tvář́ 'color returns to his cheeks', už má zase barvu 'he again has color', tváře mu červenají 'his cheeks are getting red', má tváře jako růže 'his cheeks are like roses' or jako slabikáre 'like a spelling book' (spelling book is the first textbook that children learn reading from - and it is usually red and in general very colorful). ${ }^{3}$

In the centre of the semantic field of complexion stands the opposition červený 'red' versus bledý 'pale'. Červený 'red' has (in connection with its prototypes - blood and fire) positive connotations - it is above all life, health, physical beauty and attractiveness, youth, joy, love, but sometimes also too much energy (aggressiveness, anger). On the contrary bledy' 'pale' connotes illness (or possibly death, too), lack of energy, passivity, sadness,

3. In Czech, tváře, cheeks and slabikáře, a rhymed spelling book. 
fear, low physical attractiveness, sometimes a tendency towards spirituality (in opposition to a red that can mean: human, too human). Both redness and paleness take part in the conceptualization of some psycho-physiological states and emotions (e.g. anger, fear).

In this case, other adjectives compete with the semantically neutral adjective bledy 'pale' - bíly 'white', zelený 'green', modrý 'blue', žlutý 'yellow' (and more rarely others) with more specific meanings (e.g. zelený 'green' - short term indisposition; žlutý 'yellow' - more serious long-lasting illness; both zelený 'green' and žlutý 'yellow' - envy; žlutý 'yellow' jealousy, anger).

Paleness often occurs in folk songs as a symptom of unhappy, unrequited or lost love in opposition to it then stand red cheeks, joy and beauty that are connected with the state of being in love, mutual attractiveness and happy love.

P. Eisner mentions the high frequency of the motif of painting and decoration in the Czech and Moravian folk song. She speaks directly about a "painter's polychromy". Motifs of male and female folk painters are frequent, the address můj zlatý obrázku 'my golden picture', and also the related phrasings malovaný zámek 'painted/decorated castle', malovaná brána 'painted/decorated gate', kasárna malovaná 'painted/decorated barracks'. The adjective malovany' 'painted' or 'decorated' has not only the meaning decorated by or with painting (malovaná truhla 'decorated chest', malované vajícko 'decorated egg'), but also nicely red, looking nice, pleasant (malované tváře 'painted', i.e. nice cheeks, huláni-malované děti 'uhlans-painted' - handsome - 'children') as SSJČ says. As we see again, color and coloring plays an important role in conceptualization of a nice appearance, meanwhile that which lacks color is connected with characteristics hinted at by the figurative meanings of the Czech adjective bezbarvý 'colorless': "not having its specific character, without color, grey, inexpressive, inarticulate, indefinite; not interesting, common, everyday" (SSJČ).

\section{References}

Apresjan, J. (1995). Obraz čeloveka po dannym jazyka: popytka sistemnogo opisanija. Voprosy jazykoznanija, 1, 37-67.

Bartmiński, J. (1990). Punkt widzenia, perspektywa, językowy obraz świata. In J. Bartmiński (Ed.), Językowy obraz świata. Lublin.

Berlin, B. \& Kay, P. (1969). Basic color terms: Their universality and evolution. Berkeley: University of California Press.

Blatná, R. (2001). K sémantice slova barva. In I. Vaňková (Ed.), Obraz světa v jazyce (pp. 29-36). Praha.

Eisner, P. (1992). Malované děti. In A. Stich (Ed.), Rady Čechům, jak se hravě přiučiti češtině (pp. 171-180). Praha.

Haller, J. (1969-1986). Český slovník věcný a synonymický (Vols. 1-3). Praha.

Hošek, J. (1994). Cesta $z$ Valdic do Hradce Králové. Předmluva ke knize: V. Renč, Podoben větru. Praha. Johnson, M. (1987). The body in the mind: The bodily basis of meaning, imagination, and reason. Chicago. Jungmann, J. (1835-1839). Slovník česko-německý (J. Petr, Ed.) (Vols. 1-5). Praha.

Karásek ze Lvovic, J. (1995). Venus masculinus. In Básně z konce století (p. 66). Praha.

Lakoff, G. (1987). Women, fire, and dangerous things. Chicago: University of Chicago Press. 
Lakoff, G. \& Johnson, M. (1980). Metaphors we live by. Chicago: University of Chicago Press.

Lotman, Yu. M. (1970). Struktura khudožestvennogo teksta. Moskva.

Machek, V. (1971/1997). Etymologický slovník jazyka českého. Praha.

Němcová, B. (1950). Zlá matka. In Národní báchorky a pověsti II. Praha.

Němcová, B. (1971). Babička.. Praha.

Němec, I., Horálek, J. (1986). Dědictví řeči. Praha.

Neubauer, Z. (2001). O povaze bytí. In Z. Neubauer Smysl a svět (J. Fiala, Ed.) (pp. 15-39). Praha.

Př́ruční slovní jazyka českého. (1935-1957) (Vols 1-9). Praha.

Rakhilina, E. (1995). O cvetnom i bescvetnom. Rusistica Segodnia, 1, 50-69.

Rejzek, J.(2001). Český etymologický slovník. Praha.

Seifert, J. (1981). Píseň o Viktorce. In Zápas s andělem (pp. 191-212). Praha.

Slovník české frazeologie a idiomatiky (SČFI). (1983-1994). (Vols. 1-3). Praha.

Slovník spisovné češtiny pro školu a veřejnost. (1978). Praha 1978 (SSČ I). Vydání druhé, revidované. Praha 1994 (SSČ II)

Slovník spisovného jazyka českého (SSJČ). (1958-1971) (Vols. 1-4). Praha.

Tokarski, R. (1995). Semantyka barw we wspólczesnej polszczyznie. Lublin.

Vaňková, I. (2000). Mienić sie różnymi kolorami. Zabarwienie skóry jako symbolizacja cech $i$ stanów psychofizycznych czlowieka. In R. Grzegorczykowa \& K. Waszakowa (Eds.), Studia z semantyki porównawczej. Nazwy barw. Nazwy wymiarów. Predykaty mentalne (Vol. 1) (pp. 105-124). Warszawa.

Vaňková, I. (2001). Vidět barvy. Souvislosti, 48, 131-143.

Vaňková, I. (2003). Językowe i kulturowe odniesienia czeskiej nazwy barwy zielonej. In R. Grzegorczykowa \& K. Waszakowa (Eds.), Studia z semantyki porównawczej (Vol. 2) (pp. 9-22). Warszawa.

Waszakowa, K. (1999). Jakiego koloru jest człowiek? (O podstawowych nazwach barw używanych na określenie koloru twarzy ludzkiej we współczesnym języku polskim). Prace filologiczne, 44, 545-556.

Wierzbicka, A. (1996). The meaning of color terms and the universals of seeing. In Semantics: Primes and universals (pp. 287-334). New York: Oxford University Press.

\section{Appendix A. What barva 'color' and barevný 'colorful' mean in Czech}

\section{Barva 'color'}

In dictionaries of contemporary Czech, the word barva is listed with several meanings. According to SSJČ, barva is:

1. One of the basic features of matter perceived by sight (barva oblohy 'color of sky', žlutá barva 'yellow color');

2. Dyeing matter (vodová barva 'water color', plechovka s barvou 'tin with color', míchat barvu 'to stir a color');

3. Sum of acoustic characteristics of a tone, sound (hlas př́ijemné barvy 'voice with a nice color');

4. Natural coloring of cheeks, complexion (mit zdravou barvu 'to have healthy color', ztratit barvu 'to lose color', lidé všech barev pleti 'people of all colors of complexion');

5. Symbol, sign of membership (zbrojnoši v královských barvách 'armour-bearers in king's colors', sportovci hájí národní barvy 'sportsmen defend national colors'); 
6. (Bookish) appearance, character, semblance (skvit se barvou lidskosti 'shine with color of humanity', pod barvou prátelství 'under the color of friendship');

7. (Playing cards) all cards with the same signs (ctít barvu 'to honor the color', i.e., to follow suit);

8. Child's game (hrát na barvy/na barvu 'to play on colors/color');

9. (Huntsmen's slang) blood of hunted animals and hunting dogs (pes šel po barvě 'the dog followed the color', i.e. blood).

\section{Barevný 'colorful'}

As far as the adjective barevný is concerned, dictionaries of contemporary Czech list approximately the following:

1. Concerning colors (barevný odstín, shade of color, barevný kontrast, color contrast);

2. Having various colors (SSČ II), having various colors, especially other than white or black (SSČ I), having colors other than white (or black) - SSJČ, colored (PS, SSJČ); for examples see below;

3. Having expressive colors, jolly (barevný kroj, colorful costume, barevná mozaika, colorful mosaic);

4. Poetically jolly, livid, merry, interesting etc. (the opposite of inexpressive, monotonous) - barevná, světlá budoucnost, colorful, bright future, barevný povrch života, colorful surface of life.

\section{Appendix B. Opposition of barevné-nebarevné 'colorful - non-colorful' in Czech}

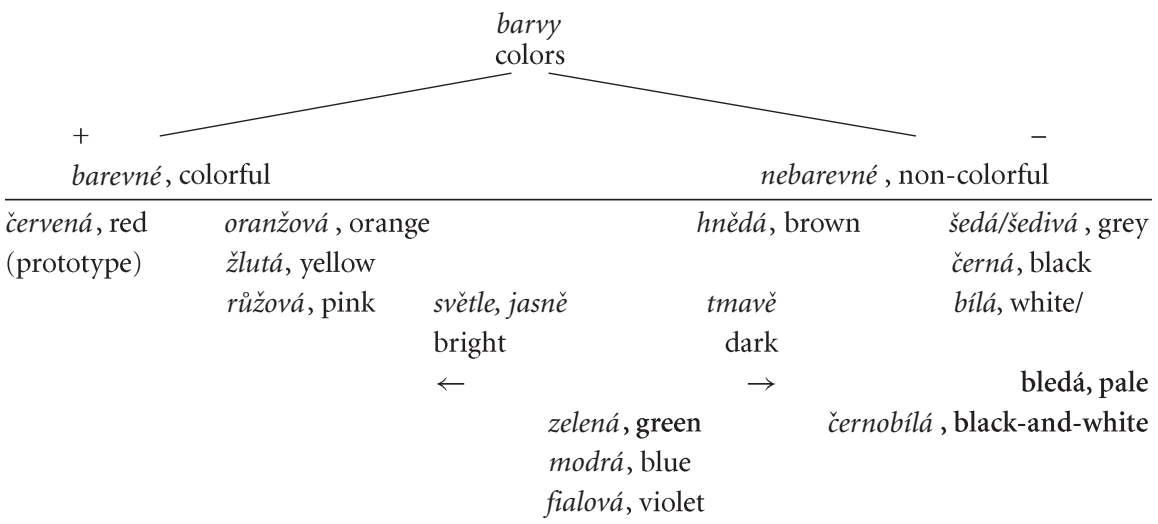

Colourful colors and non-colorful colors: the prototype of colorfulness is červená 'red', indisputable members close to the prototype are oranžová 'orange', žlutá 'yellow', and růžová 'pink'. 
The prototype of non-colorfulness is šedá 'grey', sometimes černá 'black', bílá 'white' in connection with complexion bledá 'pale' and also černobílá 'black and white' sometimes function as the prototype, too. Hnédá 'brown color' (and its shades) is close to the prototype.

Other colors can be placed (according to their shade and brightness) either among colorful or non-colorful colors.

In many cases, the situation is different - but always in agreement with the theory of color markedness: bílá 'white' can behave like a colorful color too (bílá lan̆ 'white hind'), the same may be true of 'black' (černoch 'black man' is often described as barevny 'colorful'), 'brown' (in the sense of a sun-tanned, swarthy complexion - in opposition to bledá 'pale', bílá 'white', which is colorfully non distinct).

\section{Appendix C. Colors of face in Czech}

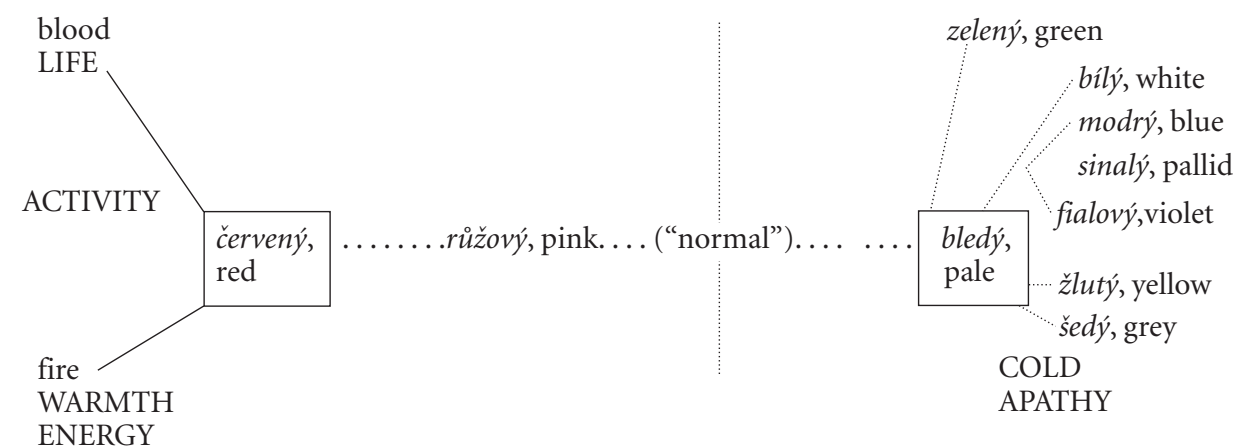




\section{Appendix D. Opposition červený-bledý 'red-pale'}

Basic opposition:

\begin{tabular}{|c|c|c|}
\hline & $\begin{array}{l}\text { červený, red } \\
\text { blood }+ \\
\text { fire }+\end{array}$ & $\begin{array}{l}\text { bledý, pale } \\
\text { blood - } \\
\text { fire - }\end{array}$ \\
\hline \multirow[t]{4}{*}{$\begin{array}{l}\text { life } \\
\text { (animality }\end{array}$} & \multirow[b]{2}{*}{$\begin{array}{l}\text { health, physical } \\
\text { and mental; youth }\end{array}$} & \multirow[b]{2}{*}{$\begin{array}{l}\text { illness, } \\
\text { sickness, old age }\end{array}$} \\
\hline & & \\
\hline & $\begin{array}{l}\text { physical beauty } \\
\text { and attractiveness }\end{array}$ & $\begin{array}{l}\text { inattractiveness or orientation } \\
\text { towards spiritual, surpression of } \\
\text { the bodily (other ideal of } \\
\text { beauty, cf. romanticism) }\end{array}$ \\
\hline & $\begin{array}{l}\text { energy, strength } \\
\text { freshness; activity }\end{array}$ & $\begin{array}{l}\text { torpidity, weakness } \\
\text { passivity, dreaming }\end{array}$ \\
\hline & $\begin{array}{l}\text { orientation towards } \\
\text { the physical and "earthly" }\end{array}$ & $\begin{array}{l}\text { orientation towards } \\
\text { the spiritual }\end{array}$ \\
\hline & optimism, happiness, joy & pessimism, suffering, pain \\
\hline
\end{tabular}

Gradation of the opposition:

červený, red $\mathrm{X}$ zelený, green

červený, red X modrý, blue/ sinalý, pallid 


\title{
Gender, age, and descriptive color terminology in some Caucasus cultures
}

\author{
Liudmila V. Samarina \\ Center of Caucasian Studies, Institute of Ethnology and Anthropology, Russia
}

This paper is based on field data (1989-1993) on color vocabulary from representatives of the ethnic groups of the North Caucasus (Kabardins, Circassians, Adygeis, Karachais, Balkars, Ossetians, Noghais, Abazins, Ingushes), Daghestan (Avars, Dargins), Georgia (Georgians, Azerbaijanis). The main results of the study of the differential impact of gender and age on the use of descriptive color terminology in some Caucasus cultures can be summarized as follows. Both factors turn out to be significant: both the elderly people (irrespective of their gender) and the females (irrespective of their age) tend to use significantly more descriptive rather than abstract color terms. On the other hand, the impact of gender factor is considerably stronger than the one of the age factor.

\section{Introduction}

Following my very first fieldwork in the Caucasus, an impression emerged that color categorization strategies differ considerably between female and male speakers of Caucasus languages. In my publications in Russian (Samarina 1994, 1996), it was hypothesized that the phenomenon has social and cultural origins. In the present study I consider my own field data and their interpretation in relation to conceptions of Western colleagues who study social and cultural influences in the color domain of other ethnicities.

In a number of studies color vocabulary of English-speakers of different gender, age, and occupation was examined (Nowaczyk 1982; Rich 1977; Simpson \& Tarrant 1991). These authors provide evidence that, on average, women elaborate more extensive color vocabularies than men, in particular, with regards to descriptive color terms, designating the entity of all possible means in defining color with the exclusion of abstract color terms (e.g. 'raspberry,' 'yellow as yolk', 'color of pigeon's neck').

In fact, this phenomenon was already observed by Luria during his fieldwork in Uzbekistan in the early 1930s (Luria 1976:22-30). Also, results on gender differences in color description obtained for Gansu (Northwestern China) by Yang (cited in Zollinger 1999: 150) are similar to those for English speakers.

Gender differences in color discrimination were recently studied psychophysically by Bimler, Kirkland, and Jameson (2004), who also consider consequences for between- 
gender differences in color naming. The authors discuss further possible underlying physiological determinants of the phenomenon; these, though, are beyond the scope of my paper.

With respect to the age differences in color vocabulary, there is evidence that older subjects, regardless of gender, use more elaborate names than the younger (Simpson \& Tarrant 1991:57-62). In particular, young teenagers of both genders were found to have a subset of basic color term s that were available for adults (e.g., Davies et al. 1999:179207). For the elementary-school age, a small difference between male and female children in usage of color terms was revealed; however, "older girls used significantly more fancy color terms than did younger girls" (Sleight \& Prinz 1982:78).

In the present context, the conclusion of Bimler et al. $(2004: 128,133)$ is of especial value, who state that "explanations for differences in color experience could be sought at a number of levels, from retinal performance ... to patterns of socialization." The differences could be accounted for by cognitive and social phenomena, whereby "the divergent patterns of socialization for males and females instill a greater awareness of color among women."

\section{Color in some Caucasus cultures: General remarks}

The Caucasian ethnic cultures of the former Soviet Union have been an object of color studies both within the Russian and the Western anthropology only sporadically. For example, Davies, Sosenskaja, and Corbett (1999) investigated color terminology in Tsakhur, a language from Nakh-Daghestanian group.

The considered field data on color vocabulary were collected in 1989-1993 from respondents residing in the North Caucasus, Daghestan, and Georgia; specifically from representatives of the following ethnic groups: Kabardins, Circassians, Adygeis, Karachais, Balkars, Ossetians, Noghais, Abazins, Ingushes (Northern Caucasus); Avars and Dargins (Daghestan); Georgians and Azerbaijanis (Georgia).

Before proceeding to the essence of the present study, I would like to shortly introduce particularities of the Caucasus culture, which are related to the social pattern of using natural colored objects and producing colored artifacts.

In the past, the Caucasus region represented an agrarian society with a strongly pronounced hierarchy and division of social roles, whereby the social roles were separating the two genders. Typical male occupations, especially starting from the beginning of the twentieth century, were and are related to the world of urban trade and business, the world with color constants, so that male representatives became slowly, but steadily estranged from indigenous way of life and, among others, from natural color elements inherent in economy and culture. In spite of the urbanization and modernization processes, the cultures of the region preserved many features of traditional economy and way of life till the end of the twentieth century.

It should be emphasized that a crucial part of the reported data was collected from respondents of a fairly old age, who witnessed pre-industrial period in the history of their 
cultures, the fact that bestows an utmost value to the data. In particular, respondents from highland regions of the Northern Caucasus and Daghestan maintained remembrance of essential information on raw materials for obtaining certain natural (particularly vegetable) dyes and on technology of applying them to dye threads, clothes, and leather. Some of the respondents were monolinguals, mainly women aged 70 or older; their testimony in native languages experienced, though, a minimal influence from other languages, especially from Russian (the then obligatory state language).

\section{A study hypothesis}

While inspecting the field data, it became striking that in many cases respondents' answers correlated more markedly with gender across the investigated ethnicities rather than within the same ethnicity. Specifically, women of different Caucasian cultures interpret the meaning of certain non-abstract color terms in a more similar way than men and women of the same cultural unit.

As noted above, most of respondents in the study were monolingual females of older age. They resided in relatively isolated mountain settlements with preserved traditional lifestyle that makes the information on descriptive color designations most valuable. In contrast to plain-dwellers, who actively interact with various neighboring ethnicities and languages, the old highland women hardly used color names borrowed from other languages, including Russian.

Usually, female respondents not only named in their mother tongue a shown color pattern (in one of the tasks) but also frequently accompanied the description of the color sample with an emotional or esthetic assessment of that color, like 'beautiful-ugly', 'pleasant-unpleasant,' or 'merry-gloomy,' for example. In answering questions on color preferences, women were more active than men, whereby the latter often sought for women's advice or answered that "women know this better."

In general, the data indicate that the "female world" in the Caucasus cultures is richer in color than the "male world": females are more sensitive to fine color nuances; their color vocabulary is more extensive than the male one; and they involve color association more frequently than men. In addition, according to my own observations, female ritual and song folklore is noticeably more satiated with color images.

Supposedly, rich mental representation of color in the Caucasus cultures is related to polychromy in traditional female clothes. The polychrome clothes serve as a gender and social marker, but also is a kind of declaration that color first and foremost is a sphere of women's interest and competence. An expressive illustration is the coloristic richness of female traditional clothes in comparison with less colored and dull male ones (Studenetskaja 1989:214-216). This feature is especially salient in dresses, head-dresses, knitted footwear etc. in the highland population of Daghestan (Bulatova et al. 2001:13129; Sergejeva 1978, 1984). Interestingly, the Daghestan data are in accord with findings for the tribe of Akhas (Northern Thailand). The two populations are very different with respect to both culture and habitat climate zone, but, like Daghestanis, Akhas live in isolated 
Table 1. Sample from ethnic groups of the Northern Caucasus and Georgia (numbers and gender distribution).

\begin{tabular}{lrcc}
\hline People (culture) & N & Females & Males \\
\hline 1. Georgians (Georgia) & 18 & 15 & 3 \\
2. Ossetians (Northern Ossetia, Georgia) & 13 & 9 & 4 \\
3. Ingushes (Northern Ossetia) & 3 & 3 & 0 \\
4. Azerbaijanis (Georgia) & 5 & 3 & 2 \\
5. Adygeis (Adygeja) & 8 & 3 & 5 \\
6. Kabardins (Kabardino-Balkaria) & 3 & 3 & 0 \\
7. Circassians (Karachay-Cherkess) & 7 & 4 & 3 \\
8. Balkars (Kabardino-Balkaria) & 7 & 4 & 5 \\
9. Karachais (Karachay-Cherkess) & 12 & 3 & 2 \\
10. Noghais (Karachay-Cherkess) & 5 & 3 & 0 \\
11. Abazins (Karachay-Cherkess) & 3 & 13 & 7 \\
12. Avars (Daghestan) & 20 & 10 & 2 \\
13. Dargins (Daghestan) & 12 & & 3 \\
\hline
\end{tabular}

highlands; and female handicrafts and clothes indicate, too, women's priority in formation and reproduction of culturally significant color patterns and stereotypes (Goodman 1996: 71-83).

The named above provides grounds for considering social factors as first and foremost affecting female strategy in description of color. In line with this hypothesis, the question posed in present study is of whether there is any relationship between the gender and age in the Caucasus cultures and the usage of descriptive color terms.

\section{Results and Discussion}

The study is based on data collected in the Northern Caucasus and Georgia in 1989-1993. The sample comprised 116 respondents, 85 females and 31 males of different age groups. As shown in Table 1, the respondents represented 13 ethnic groups that belong to four linguistic families, namely Ossetians (the Indo-European family); Georgians (the Kartvelic family); Kabardins, Circassians, Adygeis, Abasins of the Abkhaz-Adygean group and Ingushes, Avars, Dargins of the Nakh-Daghestanian group (the North-Caucasian family); Azerbaijanis, Noghais, Karachais, and Balkars (the Turkic family).

The respondents were interviewed individually in order to elicit the color term list, whereby they were requested to most exhaustively name colors, both abstract and descriptive. For each respondent, her/his ethnicity, gender, and age were noted.

To examine a putative contingency of color vocabulary on the gender, simple regression was carried out with Gender as the independent variable and Percent of descriptive color terms as the dependent variable. The result shows high and significant correlation $(R$ $=0.74 ; p<.001)$; see Table 2 and Figure 1 . 
Table 2. Simple regression (1) with Gender as the independent and Percent of descriptive color terms as dependent variable.

\begin{tabular}{lllccccc}
\hline & \multicolumn{2}{c}{ Non-standardized coefficients } & Standardized coefficients & & \\
& Model & B & SE & Beta & $t$ & $P$ \\
\hline 1 & (Constant) & 25.821 & 1.858 & & & \\
& GENDER & 24.974 & 2.198 & .735 & 11.363 & $<.001$ \\
\hline
\end{tabular}

Dependent variable: Percent of descriptive color terms $\mathrm{R}=0.735 ; \mathrm{R}^{2}=0.540 ;$ Adjusted $\mathrm{R}^{2}=0.536$.

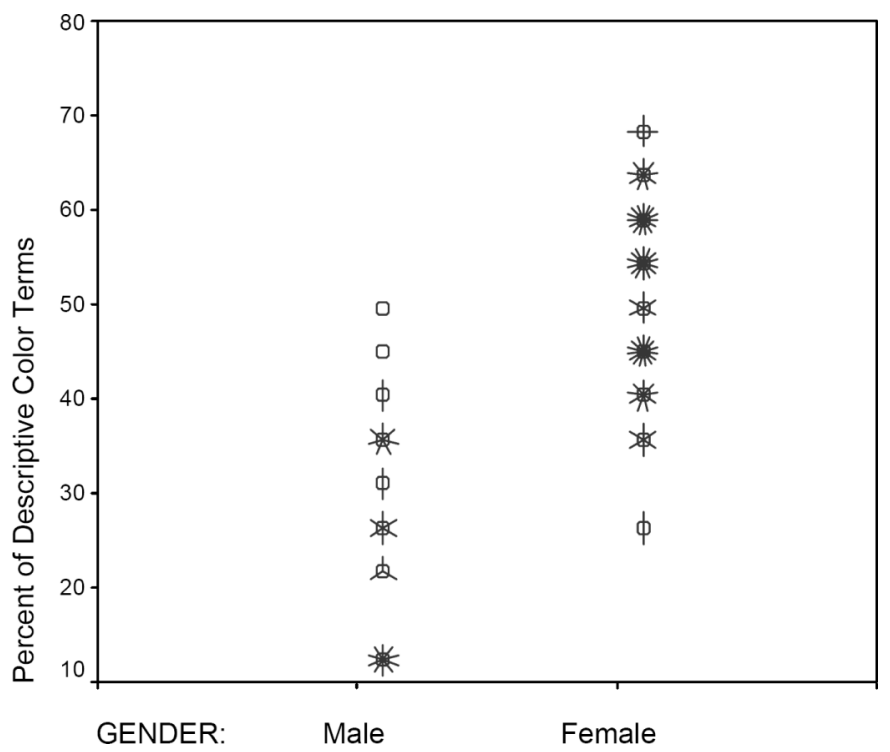

Figure 1. Distribution of percent usage of descriptive color terms between the two genders. The diagram applies the so-called "sunflower" method of data presentation; thus, by using a circle without strokes we mean a single case of using "descriptive color terms"; a circle with two strokes denotes two cases; a circle with three stokes denotes three cases and so on.

Further, simple linear regression with Age as the independent and Percent of descriptive color terms as dependent variable was conducted; its result shows that the correlation also was high $(R=0.50)$ and significant $(p<.001)$, but lower than that with the gender (Table 3 and Figure 2).

Lastly, multiple regression analysis was carried out, with Gender and Age as the independent variables and Percent of descriptive color terms as the dependent variable. Table 4 shows that, as a result of entering both variables into the common regression model, for both variables $b$, the regression coefficients, that indicate the degree of correlation, increased, whereby $R$ reaches 0.922 . This result implies that both age, but especially gender has significant impact on elaboration of the color vocabulary. 
Table 3. Simple regression (2) with Age as the independent and Percent of descriptive color terms as dependent variable.

\begin{tabular}{ccccccc}
\hline & \multicolumn{2}{c}{ Non-standardized coefficients } & Standardized coefficients & & \\
& Model & B & SE & Beta & $t$ & $P$ \\
\hline 1 & (Constant) & 12.548 & 5.344 & & 2.348 & $<.05$ \\
& AGE & .498 & .083 & .496 & 5.994 & $<.001$ \\
\hline
\end{tabular}

Dependent variable: Percent of descriptive color terms $\mathrm{R}=0.496 ; \mathrm{R}^{2}=0.246 ;$ Adjusted $\mathrm{R}^{2}=0.239$.

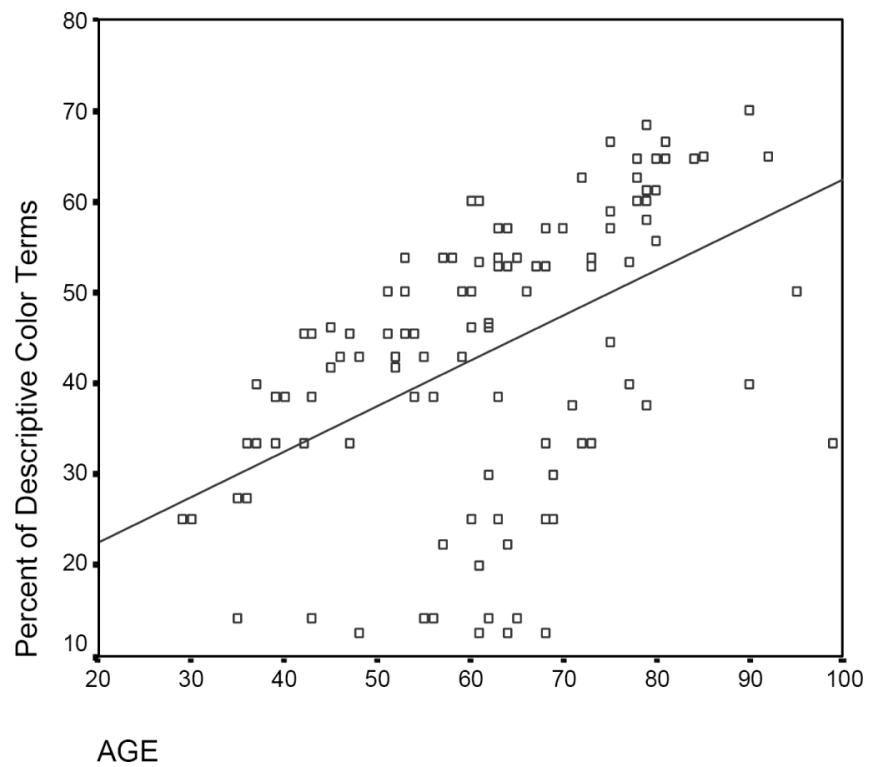

Figure 2. The scattergram of usage percent of descriptive color terms contingent on the age.

Table 4.

\begin{tabular}{ccccccc}
\hline \multicolumn{2}{c}{ Model } & Non-standardized coefficients & Standardized coefficients & & \\
& B & SE & Beta & $t$ & $P$ \\
\hline 1 & (Constant) & -10.271 & 2.633 & & & \\
& AGE & .561 & .037 & .558 & 14.991 & $>.001$ \\
& GENDER & 26.488 & 1.266 & .779 & 20.925 & $>.001$ \\
\hline
\end{tabular}

Dependent variable: Percent of descriptive color terms $\mathrm{R}=0.922 ; \mathrm{R}^{2}=0.850 ;$ Adjusted $\mathrm{R}^{2}=0.847$.

As the statistical analysis indicates, the hypothesis of correlation between usage of descriptive color terminology with gender and age is confirmed by the data elicited from the Caucasus peoples. 
With regards to the gender, the scale of difference is insufficient to prove superior color discrimination in females based on physiological grounds. We are more inclined to suppose the influence of social factors resulting in richer color cognition in Caucasus women. Conceivably this is due to a long practice of labor division, whence the ability to differentiate colors is important within culturally symbolic domain, as in fancy home-crafts and handicrafts. However, in a closed ethnic community it is also vital for the community's survival - suffice to mention distinctions between edible and poisonous plants or ripe and unripe fruits. The role of the adaptive function of color indication in the natural domain is emphasized by Shepard, who maintains that "communication between individuals about the colors of objects may be of biological significance" (1992:523).

Even in the modern industrial society women are more aware than men about the color that indicates ripeness, quality, the degree of readiness while cooking, and even taste of food products. Indeed, when testing the role of various sensory systems in food evaluation, it turned out that color-based assessment provided more veridical results than indicators of test and form (Zollinger 1988:149-164). Discriminative significance of color indicators for food, but also for a larger set of natural objects (e.g. plants) and domestic animals was demonstrated by Kul'pina (2001). Further, the significance of color vision among basic senses is confirmed by the "linguistic scheme of senses" (Williams 1976).

Thus, the sources of formation of special female "color subculture" among the Caucasian peoples can be found in the division of practice domains between men and women. Commonly, the women work out natural dyes from indigenous raw materials, dye threads, textile, and leather intended for clothes, rugs, and such like.

The spectrum of female practices in rural societies and, especially, the demands of these practices with respect to color perception and discrimination make comprehensible why women acquire non-abstract color vocabulary more readily than abstract. Analogues of such a relation can be found in Old-English and Middle-English color vocabulary: "the earliest secondary color term $\mathrm{s}$ were the names of dye-staffs, pigments, and textiles (and earlier entities from which they derived)" (Casson: 1994, 18), that is the Old-English descriptive color terms turns out to be connected just with those spheres of human activities, which in the Caucasus tended to belong to the female domain.

The vocabulary of descriptive colors used by the Caucasian peoples confirms this tendency. Indeed, the most frequent color terms are derived from the names of cultural and wild plants (e.g., 'raspberry,' 'chestnut'), food products ('yellow as yolk, 'liver color'), color terms of domestic animals ('bay,' 'roan'), as well as of animals and birds living next to people ('color of a mouse,' 'color of pigeon's neck'), and natural materials and plants used for obtaining dyes ('color of oak-bark,' 'color of wood resin,' 'madder').

Notably, among the descriptive color terms those designating warm part of the spectrum dominate. This coincides with an observation on color vocabulary in Southern Italy made by Kristol (1980:139), who comments that "primitive rural communities can easily do without 'blue' terms ... this seems to be a color with little practical use outside fashion, art and literature." Worth mentioning is also a witty remark of Woodworth that if cows were blue or green, the history of color lexicon should be quite different (Woodworth 1910:328). 
One more observation speaks in favor of cultural origin of gender differences in colorterm proficiency. Specifically, if one considers separate data from female respondents, it becomes apparent that their informative capacity of color definition correlates not only with the biological age, but also with a division on the time scale, when natural dyes were supplanted by synthetic (aniline) dyes and domestic dying, in its turn, was substituted by consuming industrially dyed goods.

The emergence of a new "chromatic environment" caused by urbanization and invention of synthetic dyes was forcefully demonstrated by Pavlovič (1977). The rigid chromatic permanence of artificial dyes took place of nature's capricious interplays of light and color. Whereas the scope of natural dyes could broadly vary depending on raw materials and processing technology (e.g. Dode 2001; Zollinger 1999), synthetic dyes follow the rule "one shade = one name." As a consequence, the practical need for descriptive color terms diminishes (Pavlovič 1977).

For the Caucasus ethnic groups the beginning of aniline era pertains to the turn and first decades of the twentieth century, the period of rapid industrial development and, hence, destruction of traditional cultures of the Russian empire and, later, the Soviet Union. Our interviews indicate, indeed, that descriptive meanings of color of those respondents, who had been socialized in the 1920s-1930s (i.e., after starting the industrialization), were markedly less exact than those produced by elder women who had been socialized in pre-industrial era. Descriptions of these latter contain notion of colors produced from plants, traditional foods, and wild and domestic animals and birds.

By comparison, the respondents socialized in the 1940s-1950s and later, in general, provided a qualitatively different set of color terms; these terms indicate a weaker relation to the respective indigenous culture and in great degree are borrowings from Russian. For example, when a respondent of middle or youngest age group was asked for the meaning of 'madder,' an archaic color term, very frequently she could not tell which color it designates.

However, in any Caucasus culture where we could compare information from at least three age groups, the content set of color terms in general - with minor exceptions repeated the list provided by the eldest group. (A similar conclusion was arrived at by Alvarado \& Jameson 2005). Probably, this is evidence for the trend, where the language inter-generation transmission still helps to preserve notions, which already do not reflect the reality immediately.

Finally, it should be pointed out that the data were collected in the ethnic groups differing not only in their languages, but also in their geographic habitat - different climatic and landscape zones, or the plain, foot-hill, and highland. It is therefore worth noting that, along with the contingencies on the gender and generation, the results of the study demonstrate a tight correlation of the set of non-abstract color vocabulary with zonal factors: The higher in mountains and, hence, the more isolated is the settlement, the greater is the descriptive color vocabulary and the more it is related to the realities of the indigenous culture, traditional lifestyle, and the degree of their preservation.

Comparing the present results to those obtained from a sample with different ethnocultural origin could provide more insight into the social, cultural, and technological factors that affect elaboration (or, possibly, regression) of an indigenous color inventory. Such 
a study would also gain from a more gender- and age-balanced sample, from taking into account respondents 'level of education and occupation, as well as from keeping a closer eye at color terms and their meaning contingent on variations in climatic-geographical and landscape patterns.

\section{Conclusion}

The main results of the study of the differential impact of gender and age on the use of descriptive color terminology in some Caucasus cultures can be summarized as follows. Both factors turn out to be significant: both the elderly people (irrespective of their gender) and the females (irrespective of their age) tend to use significantly more descriptive rather than abstract color terms. On the other hand, the impact of gender factor is considerably stronger than the one of the age factor.

\section{Acknowledgments}

This research is supported by the Presidium of the Russian Academy of Sciences program "Ethno-cultural interaction in the Eurasia."

This paper to some extent is a continuation of the discussion started in the framework of the workshop "Anthropology of colour: Colour as a phenomenon of culture" at the 7th Biennial Conference of the European Association of Social Anthropologists, Copenhagen, August 15, 2002. The interdisciplinary approach, which was the main idea of the workshop, inspired its participants to publish this volume. As an organizer of the workshop, I express my gratitude to Galina Yavorska, a co-organizer, and all workshop participants, especially to the late Robert MacLaury, to Galina Paramei, and Kimberly Jameson for their friendly support in its realization.

I am deeply grateful to Robert MacLaury, Galina Paramei, and Don Dedrick for valuable suggestions, generous help during my work on the manuscript and for their efforts in the preparation of the present volume.

Thanks go to Andrey Korotayev and Daria Khalturina for statistical advice. Last but not least, enduring support of Sergej Arutiunov, my supervisor, is gratefully appreciated.

\section{References}

Alvarado, Nancy \& Kimberly A. Jameson (2005). Confidence judgments of color category best exemplars. Cross-Cultural Research, 39, 134-158.

Bimler, David L., John Kirkland, \& Kimberly A. Jameson (2004). Quantifying variations in personal color spaces: Are there sex differences in color perception? Color Research \& Application, 29, 128-134. 
Bulatova, Angara G., Sakinat Sh. Gadžyeva, \& Galina A. Sergejeva (2001). Odežda narodov Dagestana. Istoriko-etnografičeskij atlas [Clothes of the folk of Daghestan: Historico-ethnographical atlas]. Puščino: Otdel Nauchnoj Informacii Puščinskogo Nauchnogo Centra Rossijskoj Akademiji Nauk.

Casson, Ronald W. (1994). Russet, rose, and raspberry: The development of English secondary color terms. Journal of Linguistic Anthropology, 4, 5-22.

Davies, Ian R. L., Tat'jana Sosenskaja, \& Greville G. Corbett (1999). Colours in Tsakhur: First account of the basic colour terms of a Nakh-Daghestanian language. Linguistic Typology, 3, 179-207.

Dode, Zvezdana (2001). Srednevekovyj kostjum narodov Severnogo Kavkaza. Ocherki istoriji [Medieval clothes of folks of Northern Caucasus: Historical essays]. Moscow: Vostochnaja literatura.

Goodman, Jim (1996). Meet the Akhas. Bangkok: Cheney.

Kristol, A. M. (1980). Colour systems in Southern Italy: A case for regression. Language, 56, 137-145.

Kul'pina, Valentina G. (2001). Lingvistika cveta: terminy cveta $v$ pol'skom i russkom jasykakh [Linguistics of color: Color terms in Polish and Russian]. Moscow: Moskovskij litsej.

Luria, Aleksandr R. (1976). Cognitive development: Its cultural and social foundations. Cambridge, MA: Harvard University Press.

Nowaczyk, Ronald H. (1982). Sex-related differences in the color lexicon. Language and Speech, 25, 257265.

Pavlović, Zoran (1977). Svet Boje [World of color]. Beograd: Turistička štampa.

Rich, Elaine (1997). Sex-related differences in colour vocabulary. Language and Speech, 20, 404-409.

Samarina, Liudmila V. (1994). Osobennosti ženskogo cvetovosprijatija v tradicionnoj kul'ture [Particularities of female color perception in a traditional culture]. In V.A. Tishkov (Ed.), Ženščina i svoboda: Puti vybora v mire tradicij i peremen [A Woman and freedom. Ways of choice in the world of the tradition and changes] (pp. 384-390). Moscow: Nauka.

Samarina Liudmila V. (1996). Cvet v ženskoj subkul'ture (Severnyj Kavkaz, Dagestan) [Color in a female subculture (Northern Caucasus and Daghestan)]. In Gumanitarnaja nauka v Rossii [Human sciences in Russia] (pp. 378-384). Moscow: Meždunarodnyj nauchnyj fond.

Sergejeva, Galina A. (1978). Osnovnye komplexy tradicionnoj odeždy avarcev i ix transformacija v sovetskoe vremja [Main complexes of Avars traditional clothes and their change in the Soviet period]. In V. K. Gardanov (Ed.), Etničeskie i kul'turno-bytovye processy na Kavkaze [Ethnic and culturaldomestic processes in Caucasus] (pp. 204-254). Moscow: Nauka.

Sergejeva, Galina A. (1984). Vjazanaja obuv' narodov Gornogo Dagestana [Knitted footwear of the peoples of Highland Daghestan]. KES (The Caucasian Ethnographic Collection), 8, 106-140.

Shepard, Roger N. (1992). The perceptual organization of colors: An adaptation to regularities of the terrestrial world? In J. Barkow, L. Cosmides, \& J. Tooby (Eds.), The adapted mind. Evolutionary psychology and the generation of culture (pp. 495-532). New York \& Oxford: Oxford University Press.

Simpson, Jean \& Arthur W. S. Tarrant (1991). Sex- and age-related differences in colour vocabulary. Language and Speech, 34, 57-62.

Sleight, Christine C. \& Philip M. Prinz (1982). Children's color vocabulary. Language and Speech, 25, 75-79.

Studenetskaja, Evgenija N. (1989). Odežda narodov Severnogo Kavkaza. XVIII-XX v. [Clothes of the folks of Northern Caucasus. 18th-20th centuries]. Moscow: Nauka.

Williams, Joseph M. (1976). Synaesthetic adjectives: A possible law of semantic change. Language, 52, 461-478.

Woodworth, Robert S. (1910). The puzzle of color vocabularies. Psychological Bulletin, 7, 325-328.

Zollinger, Heinrich (1988). Biological aspects of color naming. In I. Rentschler, B. Herzberger, \& D. Epstein (Eds.), Beauty and the brain. Biological aspects of aesthetics (pp. 149-164). Basel and Boston: Birkhäuser.

Zollinger, Heinrich (1999). Color: A multidisciplinary approach. Zürich: Verlag Helvetica Chemica Acta. 


\title{
Towards a new topology of color
}

\author{
Barbara Saunders \\ Department of Social Cultural Anthropology, Katholicke Universiteit Leuven, \\ Belgium
}

The mainstream concept of 'color' implicitly contains a theory of unification (through percepts and sensations), about the physical universe and mind. 'Color' is the primal 'glue', the medium of 'in-between-ness' that holds world and mind together. I argue that a re-characterization of this theory and its buried preconceptions could generate, within a discipline like Anthropology, a new topology. In contrast to the theory of Basic Color Terms $(1969,1991)$, my suggestion is to draw on historiography and philosophy, rather than on unilineal, evolutionary science. What is of importance, as Robert MacLaury knew so well, is to question the way mainstream color theory remains disguised by functional coherence and formal systematizations of 'data'. Were that knowledge, hitherto masked by physiological theory, to implode, then it would reveal the structures of history and power congealed within it. That would raise significant questions, especially about the nature of 'cross-cultural' experimentation on 'color.'

\section{Color science, color categories, color semantics}

Following what R. E. MacLaury aimed to do for so much of his life, in this chapter I shall point to alternative perspectives on, and explanations of, the assertions and praxes of Berlin and Kay on color, color naming, and color science. Like MacLaury, I too ventured to the North-west Coast of Canada to do my color-naming fieldwork. MacLaury's (1987) publication on Shuswap was a major resource for my work with the Kwakwaka'wakw of Vancouver Island; and our fieldwork arrives at similar conclusions. His findings, where he argues:

Since the yellow-green-blue category represents the extreme, dropping it into the universal sequence concedes the debate to the relativists.

(MacLaury 1992:2)

are similar to mine (Saunders 1992). Our differences, however, lie in his concession to the relativist explanation. In contrast I believe that the so-called 'universalism/relativism' debate is the same phenomenon where relativism is universalism at a distance, as Latour (1993:105) has shown. This means that I wish to find new paths, beyond the universalism/relativism debate, to characterise the nature of my arguments about color and color naming. 
As befits an anthropologist, I am therefore agnostic about the theoretical entitities of mainstream color science (cf. van Fraassen 2001) exemplified by Berlin and Kay as 'universalistic' (and 'relativistic' by MacLaury), which rest on a framework borrowed from physics, postulating psychophysical-neurophysiological parallelisms. But further I am skeptical about its basic schema of explanation, which requires an enriching 'leap' into cognition, language, and culture. My route, as an anthropologist to a better understanding, is to try to recover the human subject in the constitution of its knowledge, and its knowledge of that knowledge, especially where that is acutely relevant 'in the field.' Both the one who conducts the experiments and the one who is 'experimented upon' become the concern of my anthropological inquiry. MacLaury has tended to remain concerned with serious re-interpretations of Berlin and Kay's theories. It is there that our differences lie.

To state my long-term aims in somewhat different terms, I wish to contribute: first, to histories and philosophies of science which do not sanctify the past or ratify contemporary scientific epistemology: the Boyle-Locke-Quine injunction that philosophy and history act as 'underlaborers' to science (Saunders 2003); second, to the vital and growing area of 'science studies' in the human- and life-sciences; third, to the re-evaluation of 'vernacular' color praxes and 'subjugated knowledges' as distinct from scientific ones.

\section{Consensus}

According to a consensus of psycho-physiological and philosophical theories, color sensations (or qualia) are generated in a cerebral 'space' fed from photon-photoreceptor interaction in the retina of the eye. The resulting 'space' has three dimensions: hue (or chroma), saturation (or 'purity'), and brightness (lightness, value, or intensity) and (in some versions) is further structured by primitive or landmark 'colors' - usually four, or six (when white and black are added to red, yellow, green, and blue). It has also been proposed that there are eleven semantic universals - labeling the previous six plus the intermediaries' of orange, pink, brown, purple, and grey. Versions of this consensus provide ontological, epistemological and semantic blueprints for the brute fact of the reality of color ordained by Nature (evolution). In contrast to this consensus, I shall argue that 'seeing color' is not a matter of light waves impacting on our eyes, which produce sensations to be categorised and labeled in the 'color space' in the brain. While electrochemical events may unproblematically be regarded as the causal precondition for seeing color, the reception of sensations in 'the color space' as semantically labeled natural categories, kinds, or information, is a 'just so' story.

\section{Authority of consensus}

I consider that the authority of this consensus is better regarded not as the result of truth-tracking of 'nature', but as the socio-historical outcome of various philosophical presuppositions, scientific theories, experimental practices, technological apparatus, and their recursive feedback into the lifeworld. The anthropological thesis that I explore is whether, and to what extent, as color science and technology change, we ourselves are 
changed to accord with it. Thus unlike the other authors in this book, I am doing anthropology at two levels: primarily an anthropology of color science itself (and its effect on our own lifeworld), but one which has strong implications for our understanding of those studied by the 'anthropology of color', usually in fact of color language or categories, exemplified by other authors.

\section{Beyond universalism and relativism}

\section{Useful analytic categories}

Being agnostic about the theoretical entities of color science, I can avoid taking them as universals from the start. Hence the existing 'domain of color' and its representations, metrical and mental, that pervade discussions of color in psychology, philosophy, and the cognitive sciences can be critiqued. Such cherished nostrums as 'domain specificity' and 'innateness' can also be unsettled and reconfigured. I propose, in contrast to the Platonic-Cartesian-Lockean universals which science reveals, the notion of 'exosomatic organ' based on Hegelian universals. Hegel's universals are the product of human action in its settings, in which both action and settings evolve in mutuality. This is a dynamic relation as organisms act upon and change their environments by which they are in turn changed through the processes of history.

Thus I suggest that the phenomenal color space and the appeal to unique hues and basic color terms might better be understood as the development of a self-certifying exosomatic organ. It originates in Hegel's insistence on objectification and expression as conditions of humanisation (Innis 1984; Saunders 1998). It was later developed into the view that exosomatic organs - including institutional structures - have their own historical trajectories that anchor the fluidity of perception and apprehension, as tools, which extend or substitute for the inchoate powers of the human body.

The notion of 'exosomatic organ' has been used by Kapp (1877/1978), Lotka (1956), and Gehlen (1980). Its locus classicus is Marx (Capital):

Darwin has interested us in the history of Nature's technology, that is, in the formation of the organs of plants and animals, which organs serve as instruments of production for sustaining life. Does not the history of the productive organs of man, of organs that are the material basis of all social organization, deserve equal attention? (quoted in Innis 1984)

Exosomatic organs are implicit in the works of Nietzsche, Heidegger, and Benjamin as transformations of perceptual structures attendant upon technology in general. In contrast to the Greek separation of the senses, and their divorce from the world, exosomatic organs exist as both an objective and subjective 'fact' in the world. Heelan's 'readable technologies' are relevant here (Heelan 1992, 1983; Heelan \& Schulkin 1998). These are institutionalised structures described under the threefold rubric of substitution, compensation (or extension) and distortion of the potential capacities of the human brain and 
body. Readable technologies, for example alpha-numerical systems, extend the capacities and recursively alter the powers of the body.

Provisionally I explain the creation of the exosomatic organ in terms both of the material and inscriptional culture of science (Carroll-Burke 2001) and of the 'nomological machine' of Cartwright (1999). A nomological machine employs models of phenomena already severely reduced and regimented. Using color appearance laws (Wittgenstein's 'color grammar') as the observational base, more models at the theoretical level are added. The confirming experimental measurements are carried out in increasingly machine-like environments, and their applications tailored to patches of order in a messy world (cf. Danziger 1990, who argues this for the whole of psychology). Equipment and processes are then streamlined to become the right environment for ever-more powerful nomological machines which are governed by laws only applicable to such machines, which are shielded so as to exhibit law-like behavior. Consequently stable classification spaces, such as 'the' color space, become entrenched, while standardised experiments carried out in laboratories or 'the field' become small-scale machines, made to accord with the ground rules. Color science then produces ceteris paribus laws, which are more or less empirically adequate in controlled and limited situations.

\section{What can these analytic categories do?}

These analytic categories are tools that can open up the notion of 'enclosure' which is the major principle that guarantees the domain of color. The task for color scientists is to define that domain, make sense of its diversity, integrate its elements, and thereby 'enclose' it. Closely allied is reliance on the central aim of the standard empiricist interpretation of how scientific theories relate to the world, namely that they 'fit' observable phenomena within the enclosure. But the only way that observation or 'being directly perceived by the senses' can have scientific clout is if the senses themselves and the objects of their sensings are metricized in the first place. In order to judge whether this metricized colorpatch or beam of light is the same or different from that, first my capacity to 'judge color itself' must be metricized. Only data achieved through this praxis will count as scientific. In order for measurements to be confirming instances of metrical correlations predicted by color science, there must be an antecedent acceptance that systematic metricization of 'color judgements', makes sense.

The notion of nomological machine allows me to undertake a critical history and ethnography of these amalgamations, systematicizations and praxes. And they allow the reintroduction of material, political and social concerns, so that what was once understood as a priori may be regarded as constituted historically by the lifeworld of science itself.

\section{A new topology of color science}

Here I state my ideal (although the fulfillment of it is something else...). Just as MacLaury intended with his Vantage Theory, my new topology of color and color science tries to recognise the embeddedness of the observer both in the object of study and in the goals 
of inquiry. This applies to the color scientist's 'standard observer', the color scientist as observer, and the anthropologist 'observing' color science. This stance may be characterised as an ethnography - as grappling with the lifeworld of color science in so far as it studies material culture, as well as attempting to disentangle conceptual confusions. In addition it attempts the stance of the historiographer who describes and interprets the traces, fragments, and coherences of the color archive, as well as drawing insights from James, Husserl, Heidegger, and Wittgenstein on the productive, emergentist 'pull' of science, and its inevitable involvement in its own lifeworld. I use the concept of 'lifeworld' to refer to the 'pregiven' world(s) both of practicing scientists and of people(s) in general; thus what is 'pregiven' in one place and/or time need not be so at another. Both specialist and general lifeworlds constitute the preconditions or subsoil of color science.

\section{Reintegration}

Since Galileo, 'color' and color names have been the core example in epistemology and ontology of 'secondary qualities, 'sensations', and 'ideas'. Locke, in his Essay on Human Understanding (1689) entrenched it to justify positions on the intersecting axes of empiricism and innatism, and culture and nature. Color is held to unify (through percepts and sensations), physical universe and mind. It is the primal 'glue', the medium of 'inbetweenness' or 'tissue' that holds world and mind together: the world of objects being presented to the mind as an array of colored patches. It is by recharacterising this picture and its buried preconceptions that a new topology of color and color science within an integrative discipline such as anthropology, drawing on historiography and philosophy, could be of fundamental importance.

\section{Exemplary case study}

\section{Color categories: Universal or global?}

The theory of basic color semantics or categories developed initially by Berlin and Kay provides an exemplary case study in color science. I have extensively critiqued Berlin and Kay's (1969) programme and its reliance on color science as well as the related work of Rosch on the Dani, arguing that she confused Wittgenstein's notion of 'family resemblence' with a theory of universals (Saunders 1992, 2000a; Saunders \& van Brakel 2001). Although the stated goal was to refute relativism the deeper, unarticulated outcome of this programme, has been to re-entrench the realist and developmentalist agenda set by Locke's notion of secondary qualities with its developmentalist implications (Arneil 1996; Saunders 2003). This has consequences for the evaluation of the programme.

Berlin and Kay originally proposed that in all natural languages, there are words referring to the same group of color foci ('best examples' of red, green, and so on). This sequence of Basic Color Terms expanding from two to eleven in different languages, in a process of evolution, was gratefully received by sociobiologists as 'a primary epigenetic rule serving color category development' (Lumsden 1985). It has since become a favorite 
example in natural kinds, induction, modularity and meme debates and is reproduced in innumerable publications across many disciplines (for references, see Saunders \& van Brakel 1997).

The emerging basic color categories are the development of units of 'information'. The purpose of cross-cultural data is to demonstrate the development of these units as expressed in different languages/cultures. Yet even in so-called 'reformed versions of this programme when the foci are no longer held to be those modeled by the Munsell chart or are claimed to be culture or language 'relative', the same presuppositions are upheld albeit with 'looser' causal connections (Dedrick 2002; Roberson et al. 2000). Thus the universalism-relativism dichotomy is never transcended.

\section{One stadial set of color categories}

Doubts about this programme have arisen (for references see Saunders 1992; Saunders \& van Brakel 1997; van Brakel 2002). One reason is its lack of clarity about what is meant by semantics, categories and concepts (see Brandom 2000 for an attempt at clarification). Another is that it can be seen as a further example of the way colonial linguistics has, for nearly two centuries, regimented the language(s) of the world (Errington 2001). A third is that communities have adjusted to global inscriptional praxes and technology (producing, for example, a global 'grammar of color'). What is needed to counterbalance the onesided color naming and categorization programme is a study of globalisation processes and technology transfer as much as of indigenous practices.

The 'data' produced within the color categorization/semantics paradigm contain countless examples of the influence of prior expectations, colonialism and global standards, as also of 'outliers' discarded because they did not fit the preconceptions of the investigators. The latter 'adjustments' however are invariably attributed to the evolution of natural language. The presumption that the human brain is hardwired to one stadial set of sensations and ideas, is all-pervasive - even when claims to 'relativity' are made - for non-scientists tend to follow mainstream scientific ideas about what determines color perception. This must raise questions both about the ethical norms and the cultural agenda of the programme.

\section{Subjugated knowledges}

I suggest that the discarded data or data forced into a priori pigeon holes by the theory of color categorization/semantics should no longer remain buried and disguised by the functionalist coherence and formal systemization of theory. These data are the immediate emergence of historical contents and of the social relations that produced them; they say something important about the conditions of the production of such 'knowledge'. For were that knowledge, hitherto masked by the body of theory, to irrupt, it would reveal the structures of power concealed within that theory, opening up difficult questions about the nature of 'cross-cultural' experimentation as well as about the purpose and use of the 'data' collected. 
Such conclusions are also something else. They constitute responses in situations that the informant does not understand, or fears, or sees ironically, or just wants to be done with. Yet these responses have been disqualified, as inadequate to their task, or as insufficiently elaborated to be thought of as 'information'. They are treated as naïve responses low down on the hierarchy unable to reach the normative level of cognitive science. The selowranking, inadequate, disqualified, 'subjugated' knowledges, which are particular, local or regional, could however, through their reappearance, act as a critique of the unintended tyranny of the globalising programme of color categories/semantics.

\section{Translation and noise}

'Translation' in the color categorizing programme works as follows: in the prestigious World Color Survey, or WCS (Berlin, Kay, \& Merrifield 1991), although all 110 sets of data contain a column 'field gloss' (for the so-called indigenous 'color' words produced in response to the Munsell system), this column is mostly empty. When empty it means there are no color terms or categories that meet the criteria of the linguist. On the rare occasions when field glosses are given, some seem straightforward 'color' words, but a variety of other glosses occur, which at a later stage of processing are often upgraded as 'basic' or downgraded as 'non-basic' color words. For example, in Kemtuik (Indonesia) 'dark weather' becomes BLACK and basic, 'kind of tree' becomes PURPLE and non-basic; in Kuku-Yalinji (Australia), the field gloss 'unripe' becomes GREEN although it is labelled GRUE in the stage assignments; and in Mantjiltjara (Australia) the field gloss 'bloodblood' becomes the BCT RED, while 'earth' becomes BROWN as a WCS gloss, but is also referred to as PINK (in the stage assignment of speaker \#7). The ensuing set of 'world color names' is then processed further in a variety of ways to become the coded inscriptions of computational data.

Here too are some of the anomalies that have been discarded as 'noise' by the WCS. In 20 of the 110 languages, individual speakers are reported to use the same word in response to white and black chips. That is, where for example a speaker of Waorani (Ecuador) uses cognates of a term waimo for white, speaker \#11 uses a cognate for both black and white. Similarly in Jicaque (Honduras) eight of ten speakers use pje to name white, whereas two speakers use it for both black and white. For Berik (Indonesia), sinsini WHITE is used by three speakers 'as the name for dark or even black chips', while another Berik speaker focused the BLACK term in both black and white. The WCS procedure suggests that such speakers take black and white to be either both white, or both black. It isn't really possible to say that the term used 'anomalously' doesn't mean 'white' (or 'black' as the case may be), because that term (presumably) has passed all the 'operational criteria' for a Basic Color Term. There are similar problems in more than 20 other languages where for example speakers use the same utterance for 'white' and 'red', or for 'pink' and 'black'. In Siriono (Bolivia) for example one speaker (\#16) uses two 'yellow' terms, one for black.

The significance of such anomalies or illogicalities is to open up a rift between the epistemological desiderata (the claim that 'red' is a color) and the ontological presuppositions (a world ontologically independent of the form of all experience - the God's eye 
view), the conflation of which has hitherto permitted special status to be attributed to color categories. To discard the 'anomalies' then is to presume that the sense of color used by the experimenter is 'special', that it constitutes a preordained, primordial anchor to the world, which some languages (namely the experimenter's) have evolved to pick up.

In contrast, we can turn to Benjamin in the 'Task of the translator' (1955/1970) who says:

Our translators have a far greater reverence for the usage of their own language than for the spirit of the foreign works... The basic error of the translator is that he preserves the state in which his own language happens to be instead of allowing his language to be powerfully affected by the foreign tongue. Particularly when translating from a language very remote from his own he must go back to the primal elements of language itself and penetrate to the point where work, image and tone converge. He must expand and deepen his language by means of the foreign language.

As I find the story of evolutionarily emerging inductive generalisations or units of information unconvincing, I suggest that Benjamin's ethical principle might be applied to color nomenclature and categorization as much as to any other domain. Lucy (1997) made a related point, in the more restricted context of linguistics, when he argued that the sine qua non for good comparative linguistics was to start by understanding and respecting the nature of the language you are studying, which may be unimaginably different from your own, and never to assume beforehand that your own semantic domains exist in the other language. This of course would make projects like the WCS impossible. The relation of such a project to the lifeworlds of the peoples it is directed at, to their actual 'practices is like that of the questionnaires that come through our letterboxes, to our personal judgments.

\section{Suggestions for a new approach: Color in the lifeworld}

\section{A skill in a changing world}

I suggest that 'to see color' is not a biological 'given', but a many-stranded socio-historical 'institution'. The complex praxes and generative rules of this institution feed back into, and modify the ontology of the world. This is a process of both selection and transformation. Thus in the global, scientific world, once the closed system of basic color categories is in place, metaphysical precept, optical habit and daily practice combine to incorporate back into the visual universe the scientific field that these categories had been developed to organise and describe. The story of metamers, sensations, and semantic labels then provides the indisputable 'information base' for 'seeing color' (Saunders 2000a, b).

To see color might better be regarded as a tacit skill that has been learned through training in the appropriate judgements (Saunders 1998a, b; Saunders \& van Brakel 2002a). The basic idea was clearly stated by Merleau-Ponty sixty years ago: 
The gaze gets more or less from things according to the way in which it questions them, ranges over or dwells on them. To learn to see colors is to acquire a certain style of seeing, a new use of one's own body; it is to enrich and recast the body image (schema). Whether a system of motor or perceptual powers, our body is not an object for an 'I think', it is a grouping of lived-through meanings which move towards its equilibrium. (Merleau-Ponty 1945: 153 of Eng. Transl.)

Following too the approaches of the late Husserl and Bourdieu, I take the world in which humans live not as a mathematical model stored in the brain, but as constituted by purposes emerging in relational processes, embedded in social and moral orders (a lifeworld). In perception, as Wittgenstein said, the crucial factor is the flow of praxiological orientation arising through corporeally realised intentionality (Coulter \& Parsons 1991). This cannot be reduced to photon-photoreceptor interactions (plus eye and head movements), a color space, opponent neurons, and the encapsulated and mandatory evolutionary level of color semantics. If one adopts the stance that finds the flow of praxiological orientations a more compelling explanation, then the 'sensory core' (Costall 2002) of information on which the Berlin and Kay programme relies, can be left behind.

In the globalized world such technological color-units as paints and dye stuffs, artificial light sources, TV and computer screens, and their underlying standardisations, are built into the scientifically constructed organ-space, and picked up by purposive exposure and training. In this way a stable network has come into being for color perception so that its skills, techniques and grammar have become so naturalised that experimental research automatically confirms its 'truth'. Where such skills have not been stably naturalised, the phenomenon that Berlin and Kay call 'evolutionary stages' appears. 'Seeing color' is analogous to 'speaking correctly' in a literate, schooled population rather than, as color science supposes, to the response of the circulatory system to changes in temperature. The analogy can be worked out in detail: skills, habits, social institutions, history, theory, academic literature, and technological support.

\section{Intentional color}

An alternative is to reintegrate 'vernacular' color praxes and 'subjugated knowledges' in order to recover the human subject in the constitution of that knowledge. Similarly evolutionarily emerging neuro-based informational primitives - the encapsulated modules of color categories - can be displaced, and a quality like Kwakw'ala lhenxa (Saunders 1992; lhenxa 'green', 'yellow'), or Arunta tierga (Spencer \& Gillen 1927; tierga 'yellow', 'green', 'blue') can be relocated into 'vernacular intentional space', characterising it in purposive, indexical, and interactional terms. Then lhenxa and tierga are fragments of meaning and sense only in the normative setting of the customs, beliefs and desires, and appropriate behavior of Kwakwaka'wakw or Arunta speakers, as components of vistas only discernible from their intentional points of view. Concepts of 'color' or whatever, would then be inextricably bound up with engagements which cannot be understood except by those who enjoy the appropriate kind of intentional liaison with the world, that is, by people who have the skills to use terms like lhenxa and tierga in the appropriate contexts - an intentional liaison only secured when one is part of (or becomes part of) a community for 
which it is utterly natural to identify features of the world with these notions. To put it differently, to translate Anbarra:

... gun-gungundja gun-gungaltja; gun-gungundja gun-gungaltja ...

we need to know the context - a setting and a mood shared by the translators which allows them to render the utterance of their Anbarra companion by a line from Dylan Thomas:

... the sloe black, slow black, crow black, fishing boat bobbing sea. (Jones \& Meehan (1978)

The Berlin and Kay programme in contrast seems to believe that Munsell and similar systems provide precisely the unquestionably objective conditions under which the experimenter is empowered to define black' as this, and declare certain statements true ('this is black'), or less than true ('this is gun-gungundja'). John Gage in his pioneering Color and Culture put it simply and clearly: 'From the point of view of the historian of culture, what is most unsatisfying about Berlin and Kay's approach to color-language is the assumption that subjects tested will respond in a 'natural' way to the presentation of small chips of colored plastic from the Munsell System used by the researchers, a system which itself grew out of 19th century assumptions about 'primary' colors.' (Gage 1993:79).

\section{Consequences}

In the Berlin and Kay programme, the color space is foundational. Furthermore through postulating that each species has its own color space, and that where the animal kingdom leaves off, different cultures take over (with one species-specific color space evolutionarily ordered), continuity of the species-arguments are preserved. Kay, Berlin, and Merrifield (1991) for example say that conclusions drawn from the World Color Survey go:

... a substantial way in predicting the composite color categories of the world's languages from properties of color vision that are independent of culture and of language, biological properties which are in fact independent of human experience per se being widespread in genera other than Homo.

Differences in the human organization of color are therefore also biological. The 'composite categories' for example, mentioned above, are every bit as much biological as the different color spaces of different species. They are evidence for hardwired, biological difference. The arguments of the Berlin and Kay programme are therefore that peoples who speak Dani or Aguaruna or Kwakw'ala are perhaps as close to pigeons or goldfish which respond to distal wavelengths, as they are to American English speakers with eleven basic color categories. Or rather these peoples are somewhat less than the avian and aquatic beings because they have not yet reached the full realisation of the potential for their species, i.e. the full lexicalisation/categorization of the human 'color space.'

This full-blooded 'developmentalism' is crucial to the color semantic/categories programme, without which it would be like driving a car without wheels. So unless questions are asked about just how 'color' is defined, what the status of the Munsell and other systems are, how the nomologically necessary, emergent color categories tie up with the idea 
of 'induction' and 'information,' and just where evolution fits in, we too will be held captive by the Berlin and Kay picture.

\section{Conclusion}

Perhaps the first thing that the color naming and categorization programme might do is to treat the people it has hitherto 'experimented on' as Thou, in Buber's sense, not as It, in order to restore an ethical perspective to its inquiries. That this is necessary is shown by the continued use of such phyla-orthogenetic terms as 'Stone Age' to characterise certain peoples (Rosch 1972; Davidoff et al. 1999; Roberson et al. 2000). It remains to be seen whether the programme could survive such a radical move. If it did, perhaps it might learn from Wittgenstein (1956:420) the simple dictum:

... [color names] are finer spun than crude hands have any inkling of.

\section{References}

Arneil, B. (1996). John Locke and America. The Defence of English Colonialism, Oxford: Clarendon.

Benjamin, W. (1955/1970). The task of the translator. In Illuminations, Arendt, H. (ed), pp. 69-82. London: Collins/Fontana.

Berlin, B., P. Kay, and W. Merrifield (1991). The World Color Survey. Dallas: Academic Publications of the Summer Institute of Linguistics.

Berlin, B. \& P. Kay (1969). Basic Color Terms. Their Universality and Evolution, Berkeley: UCP.

Brandom, R.B. (2000). Articulating Reasons. An Introduction to Inferentialism, Cambridge: Harvard UP

Carroll-Burke, P. (2001). Tools, instruments and engines: Getting a handle on the specificity of engine science, Social Studies of Science 31 (4): 593-625.

Cartwright, N. (1999). The Dappled World. A Study of the Boundaries of Science, Cambridge: CUP.

Coulter, J. and E. D. Parsons (1991). The Praxiology of Perception, Inquiry 33: 251-272.

Costall, A. (2002). Why don't children draw what they see? In Saunders and van Brakel, pp. 69-76.

Danziger, K. (1990). Constructing the Subject, Cambridge: CUP.

Davidoff, J., Davies, I. and Roberson, D. (1999). Color categories in a stone-age tribe, Nature, 398: 203204.

Dedrick, D. (2002). The routes/roots of color term reference. In Saunders and van Brakel, pp. 53-68.

Errington, J. (2001). Colonial Linguistics, Annual Review of Anthropology, pp. 19-39.

Fodor, J. (1981). The present status of the Innateness Controversy. In Representations, Cambridge: MIT Press.

Gage, John (1993). Color and Culture. Practice and Meaning from Abstraction to Antiquity, London: Thames and Hudson.

Gehlen, A. (1980). Man in the Age of Technology, intro P. Berger, trans. P. Lipscomb, New York: Columbia UP.

Hardin, C. L. and L. Maffi (eds.) (1997). Color Categories in Thought and Language, New York: CUP.

Heelan, P. (1992). The new relevance of experiment. In Hardy and Lambree (eds.), Phenomenology of Natural Science, Dordrecht: Kluwer. 
Heelan, P. (1983). Space-Perception and the Philosophy of Science, Berkeley: UCP.

Heelan, P. and J. Schulkin (1998). Hermeneutical philosophy and pragmatism: A philosophy of science, Synthese, 115(3): 269-302.

Ingold, T. (1993). Technology, language, intelligence: A reconsideration of basic concepts. In Gibson K. R. and T. Ingold (eds), Tools, Language and Cognition in Human Evolution, pp. 449-472. Cambridge: CUP.

Innis, R. E. (1984). Technics and the bias of perception, Philosophy \& Social Criticism, 10: 67-91.

Jones, R. and B. Meehan (1978). Anbarra concept of color. In Australian Aboriginal Concepts, L. R. Hiatt (ed.), Canberra: Australian Institute of Aboriginal Studies.

Kapp, E. (1877). Grundlinien einer Philosphie der Technik, Dusseldorf: Stern-Verlag Janssen and Co. (1978).

Kay, P., B. Berlin, and W.R. Merrifield (1991). Biocultural implications of color naming, Linguistic Anthropology, 1: 12-25.

Latour, B. (1993). We Have Never Been Modern, transl. by C. Porter, New York and London: Harvester Wheatsheaf.

Lotka, F. (1956). Elements of Mathematical Biology, New York: Dover.

Lucy, J. A. (1997). The linguistics of 'color'. In C. L. Hardin and L. Maffi (eds.), Color Categories in Thought and Language, pp. 320-346. Cambridge: CUP.

Lumsden, C. J. (1985). Color categorization: A possible concordance between genes and culture, Proceedings of the National Academy of Science of the U.S.A., 82: 5805-5808.

MacLaury, R. E. (1987). Color-category evolution and Shuswap yellow-with-green, American Anthropologist, 89: 107-124.

MacLaury, R. E. (1991). Exotic color categories: Linguistic relativity to what extent? Journal of Linguistic Anthropology 1: 26-51.

Roberson, D., Davies, I., and Davidoff, J. (2000). Color categories are not universal: Replications and new evidence from a stone-age culture, Journal of Experimental Psychology, 129: 369-398.

Rosch Heider, E. (1972). Probabilities, sampling, and ethnographic method: The case of Dani color names, Man 7: 448-466.

Saunders, B. A. C. (1992). The Invention of Basic Color Terms, Utrecht: ISOR.

Saunders, B. A. C. (1995). Disinterring Basic Color Terms: A study in the mystique of cognitivism, History of the Human Sciences 8: 19-38.

Saunders, B. A. C. (1998). What is color?, British Journal of Psychology, 89: 697-704.

Saunders, B. A. C. (1999). The spectre of color, Science as Culture, 8: 473-496.

Saunders, B. A. C. (2000a). Revisiting Basic Color Terms, Journal of the Royal Anthropological Institute, formerly Man, N.S., 6: 81-99.

Saunders, B. A. C. (2000b). One machine among many, (commentary on Palmer's 'Color, Consciousness and the Isomorphism Constraint'), Behavioural and Brain Sciences 22: 969-70.

Saunders, B. A. C. (2007). The empire of empiricism, to be published in A New History of Anthropology, H. Kuklick (ed.), Oxford: Blackwell.

Saunders, B. A. C. (2007). Paul Virilio and the technology of 'color', forthcoming.

Saunders, B. A. C. and J. van Brakel (1988). Re-evaluating Basic Color Terms, Cultural Dynamics, 1: 359378.

Saunders, B. A. C. and J. van Brakel (1995). Translating the World Color Survey, Post-Modernism and Anthropology, Geuijen et al. (eds.), pp. 161-78. Assen: van Gorcum.

Saunders, B. A. C. and J. van Brakel (1997). Are there non-trivial constraints on color categorization?, Behavioral and Brain Sciences, 20: 167-232.

Saunders, B. A. C. and J. van Brakel (1999). Color word trouble, Behavioral and Brain Sciences, 22: 725729.

Saunders, B. A. C. and J. van Brakel (2001). Rewriting color, Philosophy of the Social Sciences, 31: 538-556. 
Saunders, B. A. C. and J. van Brakel (2002a). Kleur: een exosomatisch orgaan?, Tijdschrift voor Filosofie, 64: 299-324.

Saunders, B. A. C. and J. van Brakel (2002b). Theories, Technologies, Instrumentalities of Color: Anthropological and Historiographic Perspectives, Maryland: UP of America.

Spencer, B. and F. J. Gillen (1927). The Arunta, vol. 2, London: Macmillan.

Thompson, E. (1995). Color Vision. A Study in Cognitive Science and the Philosophy of Perception, New York: Routledge.

van Brakel, J. (1993). The plasticity of categories: The case of color, British Journal of Philosophy Studies 44: 103-135.

van Brakel, J. (2002a). Chromatic language games and their congeners. In Saunders and van Brakel (2002b), pp. 147-168.

van Brakel, J. (2002b). The coming-to-be of color spaces. In Saunders and van Brakel, pp. 307-326.

van Brakel, J. and B. A. C. Saunders (1997). On the existence of a fixed number of unique opponent hues. In C. M. Dickinson et al. (eds.), John Dalton's Color Vision Legacy (pp. 393-402), London: Taylor \& Francis.

van Brakel, J. and B. A. C. Saunders (2002). Color: An exosomatic organ? In R. Eschbach and G. G. Marcu (eds.), SPIE Proceedings, 4663: 162-176.

Van Fraassen, B. C. (2001). Constructive empiricism now, Philosophical Studies 106: 151-170.

Wittgenstein, L. (1956). Remarks on the Foundations of Mathematics, Von Wright, G. E. H., R. Rhees and G. E. M. Anscombe (eds.), Oxford: Basil Blackwell. 



\section{Index}

\section{A}

Abazins 457, 458, 460

Abkhaz-Adygean 460

Adygeis 457, 458, 460

Afghan 158

African Americans 16

Afroasiatic 213, 225, 226

Aguaruna 476

Akhas 459

Akkadian 229-235, 239-244, 254-256, 258, 263, 271, 282, 287

Alemannisch 320-324

Aleppo 263

alloseme 397, 398, 402, 403

American English 18, 61, 129, $310,379,476$

Amharic 247, 248, 250, 252, 254-256

amplifying function 343

Anbarra 413, 476

Ancient Egyptian 61, 211-214, 216, 225, 226, 231, 232, 236, 237, 239, 242, 282, 285

Ancient Greek 5, 107, 111, 234, 244, 286, 414, 416

Ancient Indian 415, 416

Ancient Slavic 411

Anglo-Normans 185

Anglo-Saxon 171, 174, 176, 177, 180

anomalous trichromats 35

anthropological $6,8,13,20,21$, $56,109,166,175,230,319,379$, 421, 468

Apache 128-130, 134

Arabic 222, 223, 225, 240-243, 250, 254-257, 263-288, 327

Aramaic 253, 255, 256, 263 , 269-272, 282, 286, 287

Argobba 254, 256

Arunta 475

Assyrian (see Akkadian) 263 attributive meanings 101,371 augmentative $395,397,398$ Avars 457, 458, 460

Azerbaijanis 457, 458, 460

B

Babylonian (see Akkadian) 255,276

Badaga 116

Bahrein 263

Balkars 457, 458, 460

Baltic 158, 159

basic color category 20, 136, 332,364

basic color term $8,12,30,57$, $79,126,136,156,167,168,229$, 236, 242, 247, 300, 325, 328-332, 338, 339, 341-343, $358,369,373,458,473$

Batta 116

BCT (see basic color term) 79, $81,98,211,214,227,247-258$, $426,427,429,431,433,473$

Bedouin 263, 264, 266, 267, 269, 272-274, 276, 279, 280, $283,284,287,288$

Belarusian 10

Berber 225, 256, 282, 284

Berik 473

Berlin and Kay hypothesis (see also B\&K hypothesis) 226, 230-232, 236, 240, 242-244, 264, 268, 271-273, 275, 279, 286

best example $30,31,39,43,132$, $248,249,280$

Brazilian 253

bright 59, 67-69, 100, 108-111, $114,117,154,158,160,161$, $171-175,178,179,181,217,223$, $233,235,238,249,255,256$, 263, 268-271, 280, 299, 313, $327,329,331,332,342,349$,
353, 357, 408, 409, 411, 412, $414,415,422,446,447,449$, 454

brightness $13,76,77,83,109$, $110,125,126,129,137,138,154$, $155,157,159-161,171-179$, $181-183,185,186,230,247$, 249, 253, 256-258, 263, 267, 269, 271, 272, 276, 279, 280, 284, 285, 287, 300, 301, 308-310, 327, 347, 352, 360, 372, 380, 395, 398, 399, 402, $415,434,454,468$

British 5, 13, 16, 17, 61, 63, 155, $156,181,348$

British English $13,61,63,155$

C

Cairene 263, 266, 275, 281

Castilian 285

Caucasian 457-459, 463

Celtic 155

Central African 16

Central Salish 395

Chaha 256

Challam 116

Chehalis 396, 401

Chichewa 17

Chilliwack 396

Chinese $61,95,244,277,278$, 296-298, 300, 301, 309, 310, 401, 446

Chroma (see also Munsell Chroma) 30, 59, 126, 133, $285,322,323,428,429,431$, 468

chromatic dimension 37 chromatic sensation 34 chromatic sensitivity 34,36 chromaticity $16,87-89,91,93$ chromaticness $57-60,62,71$, 76,160

Chukchi 118 
Circassians 457, 458, 460

Classical Arabic 265, 282, 283

Classical Greek 5, 234, 235

Classical Latin 180, 234, 264

codability $98,101,135,136$

coextension 131, 135, 144, 310, 311

cognitive model 133, 359, 395,

399

coextensive range 142

cognitive viewpoint (see also

vantage) 56

color association 459

color category $20,49,56,118$,

$125,129,134,136,137,177,182$,

248, 250, 283, 297, 321-323,

328-330, 332, 350-352, 357,

$359,364,371,434,471$

color concept $300,380,386$, $389,390,405,406$

color foci $42,45-49,277,471$

color markedness 442, 451, 454

color naming $3-9,11-15,18-21$,

$29,30,32,34-40,49,50,55$,

57, 60, 79, 99, 116, 137, 145,

$151,157,158,233,240,243$,

256, 295, 337, 421, 432, 434,

$438,458,467,472,477$

color recognition 56

color science $112,467-471,475$

color semantics $166,171-173$,

$186,252,268,363,379,380$,

$413,467,471,475$

color universals 30, 406

color vocabulary $5,6,12,18$,

$174,176,177,182,230-234$,

239-242, 244, 295, 296, 304,

308, 330, 421, 441, 457-461,

463,464

Color-Aid Corporation 76,87 ,

90, 94

color-category system 56

color-normal observer 33

color-opponent axes 33

color-term basicness 56

color-term evolution 110, 156, 230

colored artifacts 458

colorful 241, 279, 344, 441-444, 451-454

colorfulness 441-444, 453

colorless $173,313,329,368$,

441-445, 451, 452 combinability $68,101,363-365$, $369,370,374,375,380,383$

communication accuracy 84 , 98

compound color term (see also compound chromatic term; compound term) 57, 59,

$66-68,70-71,430$

conceptual 21, 37, 99-101, 212, 232, 240, 373, 379, 386, 471

cone-opponent space 37 consensus $40,49,55,57,60,61$, 75, 87-90, 94, 101, 236, 468 construal $13,363,379,382,383$, 386

Coptic 211, 214, 216-220, 223, 224, 226, 227, 242

Cowichan 396

criteria of color term basicness 126

cross-cultural 12, 13, 19-21, 75, $304,355,360,467,472$ cross-linguistic universal 314 cultural environment 34 culturally symbolic 463 culturally-specific concept 374 Cypriot 263, 281, 282

Czech 14, 441-454

D

Daghestanis 459

Damara 116

Damascene 263

Dani 110, 471, 476

Dargin 457, 458, 460

darkness 6, 99, 109, 110, 154, $155,159,161,173,175,176,180$, 181, 237, 254, 257, 270, 300, $301,354,356,407$

denotative consistency 60,63 , 69,71

denotative meaning 55,57

denotative precision $55,57,60$, 70,71

derived color term 426

derivational productivity 153 , $156,163,164,248,267,373$

desaturation category 127,129 , 147

descriptive color terminology $457,462,465$

descriptive function 381

deuteranomalous 35 developmental $3,8-12,20,75$, 79, 80, 83, 101, 266, 269, 274, 286

diminutive $266,395,398-402$ discourse structures 380 distributional potential 153, $373,380,382$

Dutch 6

East Slavic 76

East Slavonic 10

Eblaite 256

Egyptian 16, 61, 211-214, 216-221, 223, 225-227, 230-233, 236-244, 256, 263, $265,279,282,285$

elementary colors 59,68 emergent color categories 476 English 6, 7, 9, 12-14, 18, 19, 29-33, 38, 40, 41, 43-48, 59, $61,63,65,75,77-80,82,85$, 87-91, 93-97, 110, 129, 133, $134,138,145,151-160,163-165$, $167,168,171-186,227$, 242-244, 266, 267, 278, 281, 285, 296-298, 300, 304, 307, $310,311,320,322,323,341,343$, $347,348,350,352,355$, 359-361, 364, 365, 367, 370, $373,379,382,395,397,415$, $425,429,435,445,447,449$, 451, 457, 476, 477

Ennemor 254

Epigraphic South Arabian 254, 256

Erzya 158

Eskimo 321-323

Estonian 158

Ethiosemitic 247, 249, 251-253, 256

ethno-linguistic 55,56

European 7, 11, 12, 16, 109, 211, $242,265,278,281,284,355$. 357, 411

evolutionary sequence (see also color-term evolution) 36 , 229, 230, 232, 243, 275, 295, 306, 307, 309, 330

evolutionary stage $\quad 253,272,274$ Ezha 256 


\section{F}

Fingu 116

Finnic 158, 159

Finnish 153-155, 158-168

Finno-Ugric 158, 167

fixed coordinate $141,144,145$

focal choices 29, 31, 32, 40, 41, 50

focal color $38,40,41,43,45,46$, $59,60,429,436$

folklore $14,367,405,407-412$, 414-418, 459

fovea 15,34

French 6, 111, 145, 155, 158, 159, 240, 241, 243, 266, 281, 285, 370,372

Fulah 116

Futunese 125-127, 134

\section{G}

Gafat 254

Gaika 116

Gansu 457

Geez 247-258

gene dimorphism 35

Georgians 457, 458, 460

German 5, 6, 13, 14, 107, 112, 113, $127,160,212,221,243,285,319$, $320,322,323,340,341,355$, $356,370,414,421-423,425$, $427,429-432,435,445,449$

Germanic 155, 159, 179, 180, 370

Gogot 254

Greek 5, 17, 100, 107, 108, 110, $111,155,223,229,230,234,235$, 239-244, 247-252, 255, 263, 282, 286, 288, 408, 410, 414, 416, 449, 469

grue $7,11-19,31,38,44,84,111$, $183,213,231,232,237,268$, $282,328,371,473$

Guajibo 137

Guarajío 129, 130

Gurage 254-256

Gyeto 256

$\mathrm{H}$

Halkomelem 14, 395-399, 402, 403

Hanunóo 5, 6, 283

Harari 254-256

Harsusi 254
Hebrew 225, 253-256, 263, 268, $271,272,282,285,287$

Hering's opponent-color theory 57

Himba 19

Hindi 12

Hittite 230, 240

Hmong 330, 332

Homeric Greek 234

Huastec 137

Hungarian 61, 159

I

inceptive $395-397,399,401,402$ Indian 16, 38-41, 49, 415, 416

Indo-European 12, 230, 242-244, 370, 371, 411, 413-415, 449, 460

information value $337,341,343$

Ingrian 158

Ingushes $457,458,460$

interdisciplinary semantics 179, 184

Interpoint-Distance Model 98

Irish 17,414

J

Japanese 10, 13, 269, 285, 295-302, 304, 307-314

Jibbali 254

Jicaque 473

K

Kabardins 457, 458, 460

Kanakas 118

Karachais 457, 458, 460

Karelian 158

Kemtuik 473

Koda 116

Krobo 116

Kuku-Yalinji 473

Kurdish 263

Kwakw'ala 475,476

$\mathrm{L}$

Latin $108,155,158,180,230,234$, $244,264,341$

Latvian 158

Lebanese $263,269,275,285$

lens $14,15,18,34-36$

lexical accessibility 101 lexical category boundary (see also Whorfian effect) 87, 101

lightness $6,10,30,33,38$, $41-44,56,58,59,76-78,80$, $83,84,89,91-93,95-97,99$, 109, 110, 133, 135, 137, 146, 154, $160,173,176,256,257,360$, $380,431,468$

lightness dimension $76,91,97$ linguistic picture of the world $441,445,446$

linguistic relativity $21,30,55$, 56,268

Lithuanian 158, 414

Livonian 158

Low German 341

Ludian 158

luminance $33,87,138,154,161$

M

macular density 34

macular pigment 34,35

Madingo 116

Makah 116

Maltese 263, 267, 281, 284, 286

Mandaic 253-257, 263, 271, 272

Mandarin Chinese 61, 244

Mantjiltjara 473

Marathi 38

Mari 159

Masai 128, 131

Masqan 254

Matsqui 396, 401

Mazatec 137

Mehri 254, 271

Mesoamerican Color Survey 129, 147, 308, 312

metaphor $8,13,166,224,283$, $352-354,356,358,360,398$

Mexican Spanish 129

Middle Arabic 277

Middle Eastern 277

Middle English $145,157,185$, 186

Middle Low German 191

Mien (see also Yao) 325-332, 334

Mixtec 127, 130, 134, 135, 144

mobile coordinate 141 
Modern English (ModE) 8, $172,174,175,181,182,186,244$, 266, 381, 391

Modern Standard German 319, 320,322

Moravian 452

Mordvin 158

Moroccan 263

morpho-syntactic marker 383 , 387,389

morphological structure 337

Muher 254, 256

Mulgi 197

Munsell array 41, 99, 131, 139, 146, 396

Munsell Chroma 30, 126

Munsell color chart (see also Munsell chart) 175, 177, 327, 400-402, 472

Munsell color chips (see also Munsell chips) 30, 91, 125, $126,128,129,132,137,139,146$, 256, 322, 323, 395, 396

Munsell Color Solid 98, 125, 380

Munsell Hue 39

Munsell pallette 38

Munsell Value 30, 297

Musqueam 396

Mycenean (see also Mycenean Greek) 229, 230, 234, 235, 239-244

$\mathrm{N}$

Nakh-Daghestanian 458, 460 Nahuatl 134

naming centroid 31

Natural Color System (see also NCS) $57-71,76$

natural colored objects 458

Navaho 128, 129

Nicomen 396

Noghais 457, 458, 460 nominal density 55,57 nominalization of a color term 378

non-abstract color term 459 non-basic color term (see also nonBCT) 57, 65, 71, 266, $379,383,438$

North-Caucasian 460

Numic 127, 128
O

ocular media 14, 34

Old Arabic 263, 265-269, 271, $274,275,279,280,282-288$

Old English (OE) 145, 154, 155, $157,159,171-182,185,186,242$, 243

Old Ethiopic (see also Geez) 247-258

Old Russian 76, 369

Olon 116

Olonetsian 158

opponent-color theory (see also Hering's opponent-color theory) 57

Ossetians 158, 458, 460

Ostwald Color Cone 221

Ovaherero 116

P

Palestinian 255, 256, 263, 264, 269, 271, 273, 279

partition principle 62

peak sensitivity 35

perceptual color $20,55,57,71$, 95

perceptual color space 20,55 , 57,71

perceptual dimension 93, 99

perceptual task 81

Persian 158, 240-242, 263, 288

Phoenician 253, 254, 282

photopigment 35

photoreceptor 14

pigment density 35

Pilaltxw 396

Polish 373, 445-447

Pondo 116

Portuguese 17

post-receptoral stage 33

Pre-Islamic Arabic 268, 271

primary color term 414

Proto-Berber 282

Proto-Finnic 158

Proto-Indo-European 158

Proto-Numic 127

Proto-Semitic 247, 248, 251, 253-258

Proto-Slavic 76

prototype 138, 369-371, 374, 405-408, 411, 412, 414, 416, $418,443,444,448,454,455$ psycholinguistic $21,56,57,59$, $71,75,76,87,363,372,375$, 379, 405, 417

psychophysical 4, 20, 21, 32, 34, $49,98,133,324,430,450$

Punic 254, 255

R

radiance $411-417$

relative basicness $56,151-154$, $157,158,161,165-168$

relativism $5,6,21,467,469,471$ retina $14-16,34,109,110,468$

Rumanian 285

Russian 10, 13, 55-57, 59-71, 75-91, 93-102, 159, 160, 240, 244, 258, 278, 285, 363, 364, 366-375, 381, 405, 408-410, 412, 414, 415, 417, 418, 442, $444,445,457-459,464$

$S$

Sabaic 254-256

Sami 158

Sanskrit 263

Sapir-Whorf hypothesis 95 saturation 5, 13, 30, 59, 76-78, $80,83,84,95,99,126,129,137$, $146,154,155,157,160,161,172$, $175,178,182,183,230,327$, $347,360,372,380,395,398$, 399, 402, 431, 434, 468 saturation dimension 76 Scandinavian 155

Scowlitz 396 secondary color term 312,463 Selti 254

semantic color space $79,83,84$, 101

semantic field $184,212,221$, 226, 348, 411, 446, 451

semiotic $8,100,147,337,338$, 412, 421, 430, 435, 437

Semitic 225, 233, 238, 242, 247, $248,252,257,258,263-265$, $268,271,282,286,287$

Serbian 405, 408-412, 414-417

Seri 137

short-wavelength sensitivity 35

Sioux 116

Siriono 473

Slavic 14, 76, 371, 405, 409, 411, 413-415, 449 
Snake 116

social marker 459

Soddo 254

Soqotri 254

South African 18, 116

Spanish 6, 17, 79, 126, 129, 138, $285,319,320,322,323$

spectral sensitivity $16,34,35$

stative 395-397, 399-402

stereotype 405-408, 412, 416-418

Sumas 396, 401

Sumerian 282

Swedish 61, 76, 159, 160, 288, $337-340,342$

Syrian $225,263,269$

\section{$\mathrm{T}$}

Tait 396

Tambukki 116

Tamil 38, 39

Tangiers 263

Telugu 116

tetrachromacy 35

Tigre 247, 249, 250, 254, 255

Tigrinya 247-249, 254-256

Tlapanec 135, 137

Toda 116

Tovarese (see also Tovarense) $127,319-323$

trichromacy $5,19,37$

Tsakhur 458

Turkish 263, 288

Tzeltal 32, 134

Tzotzil 129
U

Ugaritic 253-256, 263

Ukrainian 10, 370, 371, 373, 405, 408-411, 414, 417, 418

unique hue 38

universal color categories 30

universalism (see also Berlin and Kay hypothesis) 6, 151, 226, 467, 469

Uralic 158

US English (see also American English) 18, 61, 84, 90, 95, 129, 310, 379, 476

UV exposure 35

V

Value (see Munsell Value) 18, $30,31,37,49,59,60,128,133$, $152,153,156,157,162-164,167$, 222, 232, 238, 278-280, 284, 297, 298, 311, 320, 322, 323, $337,340,341,343,380,423$, $428,429,431,436,445,458$, 459, 468

vantage $8,9,13,56,131,136$, 138-144, 147, 309, 375, 399, 403, 470

vantage theory $8,9,13,56,131$, 136, 138-142, 147, 309, 375, 399,470

Venezuelan Spanish 322

Vepsian 158

Vietnamese $83-85,446$

visible spectrum $4,13,14,33$

visual environment 34,98 visual sensitivity 13,35

Volgan 158, 159

Võru 193, 197

Votic 189, 191-193, 198, 197-199, 207

W

Walbiri 127

Waorani 473

weak linguistic relativity 56

West African 17

Whorf-Sapir (see also

Sapir-Whorf hypothesis) 5, 55, 95, 211

Whorfian effect $79,87,93,101$

Whorfian hypothesis 5

Wolane 254

World Color Survey (see also WCS) 7, 29-32, 40, 41, 43-48, 50, 91, 99, 145-147, 305, $308,312,319,473,474,476$

Y

Yao (see Mien) 325-332, 334

Yemeni 263, 269, 282, 285

Z

Zapotec 125-127, 137

Zinacantecan 17

zooming 141, 142

Zway 254 
The field of color categorization has always been intrinsically multi- and inter-disciplinary, since its beginnings in the nineteenth century. The main contribution of this book is to foster a new level of integration among different approaches to the anthropological study of color. The editors have put great effort into bringing together research from anthropology, linguistics, psychology, semiotics, and a variety of other fields, by promoting the exploration of the different but interacting and complementary ways in which these various perspectives model the domain of color experience. By so doing, they significantly promote the emergence of a coherent field of the anthropology of color.

"Nobody can tell where Rob MacLaury's inquisitive mind would have led him in years to come in his passionate quest for an anthropology of color. But this collection is certainly a testament to what he had set out to accomplish. Galina Paramei and Don Dedrick are to be commended for seeing it through to publication and making it available to an interdisciplinary and international public that will benefit from it and further advance this complex and fascinating field."

Luisa Maffi, in the Foreword to the volume

"This is an important volume for the breadth of collected papers it presents which share the focus of culture and perceptual color experience. Some of its articles present analyses of phenomena from languages and dialects that are quickly disappearing from the world; whereas others catalog more esoteric or ancient features of studied languages - quite possibly presenting the first thorough accounting of such features in an English language volume. The collected information contained in this volume is essential for understanding universal features in color perception and cognition as distinct from the culturally relative factors inherent in the phenomena."

Kimberly A. Jameson, University of California, Irvine

"This collection is remarkable for its breadth: from individual perception and cognition to social construction, from the evolution of color terms in Greek, Coptic, and Japanese to the uses of color terms in fashion. Here there is much to learn, to argue, and to ponder."

Larry Hardin, Syracuse University

"Discussions of colour cognition must be grounded in empirical evidence about how people actually use colour words. This volume contains such evidence, drawn from a range of language families and aspects of culture, and a variety of contemporary and historical sources."

David Bimler, Massey University, New Zealand

"An essential addition to the personal libraries of workers in this very broad area and a mandatory acquisition for academic libraries. It will function as a handbook, selected chapters will be used to guide new researchers, and even the curious general reader will find it tantalising."

Ian Davies, University of Surrey, UK

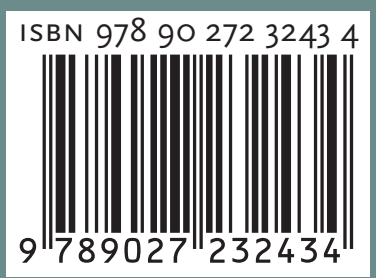

\section{JOHN BENJAMINS PUBLISHING COMPANY}

